TOWARDS A CHARACTERISTIC EQUATION FOR PERMEABILITY

\author{
A Thesis \\ by \\ ADIL AHMED SIDDIQUI \\ Submitted to the Office of Graduate Studies of \\ Texas A\&M University \\ in partial fulfillment of the requirements for the degree of \\ MASTER OF SCIENCE
}

August 2008

Major Subject: Petroleum Engineering 


\title{
TOWARDS A CHARACTERISTIC EQUATION FOR PERMEABILITY
}

\author{
A Thesis \\ by \\ ADIL AHMED SIDDIQUI \\ Submitted to the Office of Graduate Studies of \\ Texas A\&M University \\ in partial fulfillment of the requirements for the degree of \\ MASTER OF SCIENCE
}

\begin{abstract}
Approved by:
Chair of Committee, Committee Member, Thomas A. Blasingame Walter B. Ayers Richard Gibson

Head of Department, Stephen A. Holditch
\end{abstract}

August 2008

Major Subject: Petroleum Engineering 


\author{
ABSTRACT \\ Towards a Characteristic Equation for Permeability. (August 2008) \\ Adil Ahmed Siddiqui, B.E., National University of Sciences \& Technology, Pakistan \\ Chair of Advisory Committee: Dr. Thomas A. Blasingame
}

The characterization of reservoir permeability $(k)$ remains the elusive challenge in reservoir engineering. This work considers prior developments in an evolutionary sense, and, as with prior work, our goal is the development of a "characteristic permeability relation" $(C P R)$. To this end, we have developed $5 C P R$ formulations - 3 of which could be considered modifications of "historical" models and 2 of which are "weighted" power law-exponential models.

In this work, we consider permeability to be only a function of two variables; $k=f(\phi, z)$ - porosity $(\phi)$ and $z$, where $z$ is either the water saturation $\left(S_{w}\right)$ or the Archie Formation Factor $(F)$. Our rationale in considering $k=f(\phi, z)$ is two-fold — first, such a formulation is a fundamental extension of the $k=f(\phi)$ correlation work by Archie (and countless others); and second, our validation datasets are limited to literature cases and cases obtained from industry sources - none of which would be considered suitable for extension beyond porosity and another variable.

We demonstrate and validate our concept of a characteristic permeability relation using various datasets obtained from the literature and from industry sources. In this work we show that each proposed relation has a unique character and performance - depending on primarily on the data, rather than the functional form of the permeability relation. Using the characteristic permeability relations developed in this work - the proposed permeability relations can be extended to other and other data types. It may also be possible to develop so-called "hydraulic flow unit" methods which segregate petrophysical data into depositional flow sequences. 


\section{DEDICATION}

This thesis is dedicated to my family members and friends for their help and support.

There is no man living that can not do more than he thinks he can.

- Henry Ford

Patience and perseverance have a magical effect before which difficulties disappear and obstacles vanish.

— John Quincy Adams 


\section{ACKNOWLEDGEMENTS}

I would like to express my gratitude to Dr. Tom Blasingame for his patience, support and guidance throughout my research and graduate studies at Texas A\&M University. I would also like to thank Drs. Walter Ayers and Richard Gibson for serving as members of my advisory committee. 


\section{TABLE OF CONTENTS}

ABSTRACT

\section{CHAPTER}

I INTRODUCTION

Statement of the Problem ......................................................................

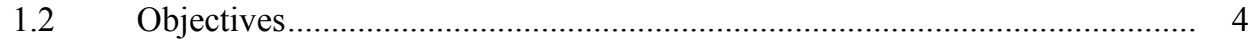

$1.3 \quad$ Validation ....................................................................................... 5

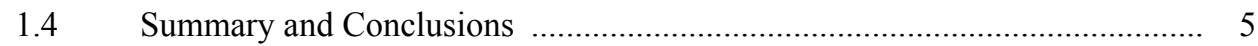

Organization of the Thesis ..................................................................... 6

II CHARACTERISTIC PERMEABILITY RELATIONS …....................................... 7

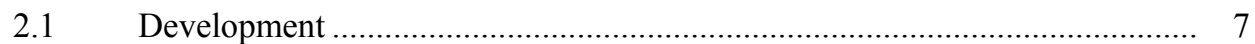

2.2 Characteristic Behavior ........................................................................ 7

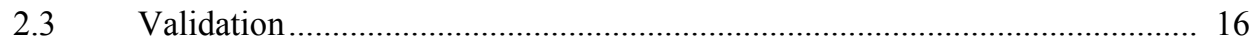

$2.4 \quad$ Results and Discussion ..................................................................... 24

III SUMMARY, CONCLUSIONS, AND RECOMMENDATIONS FOR FUTURE

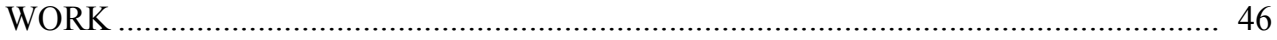

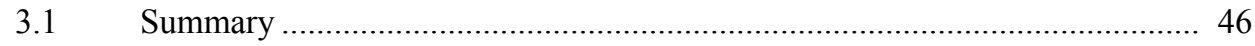

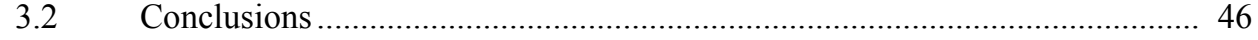

Recommendations for Future Work ............................................... 48

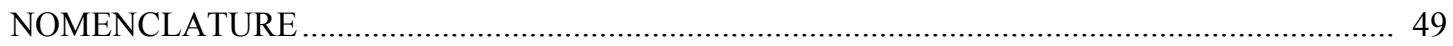

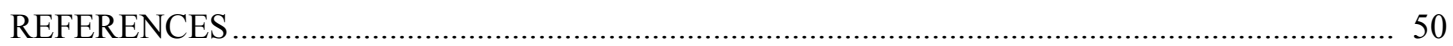


APPENDIX A - DERIVATION OF THE "PAPE" MULTI-POWER LAW

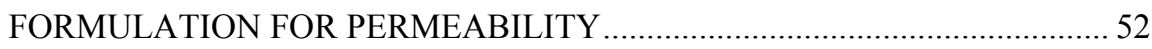

APPENDIX B - RESULTS FOR HOWELL WELL (E. TEXAS — USA) …………………….... 59

APPENDIX C - RESULTS FOR MIOCENE SAND (S. LOUISIANA — USA) ….................... 173

APPENDIX D - RESULTS FOR VICKSBURG SAND (S. TEXAS — USA) ……………....... 213

APPENDIX E - RESULTS FOR WILCOX SAND (S. TEXAS — USA) ………....................... 253

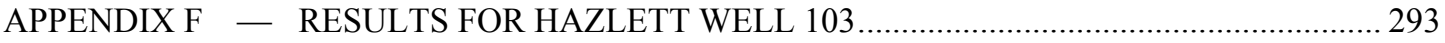

APPENDIX G — RESULTS FOR DEVONIAN LIMESTONE (TEXAS — USA)....................... 324

APPENDIX H — FOR FRIO SHALY SANDSTONE (TEXAS — USA) ……………………..... 355

APPENDIX I - RESULTS FOR LOWER WILCOX SHALY SANDSTONE (TEXAS -

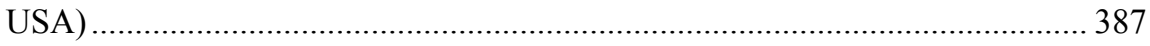

APPENDIX J - RESULTS FOR LOWER PALUXY CLEAN SANDSTONE (TEXAS -

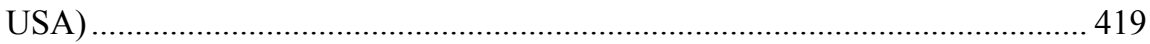

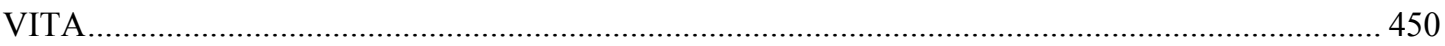




\section{LIST OF FIGURES}

2.1 Model CPR-A: $k=a(\phi-c)^{b} \quad\left(c=c_{\max } \exp \left[-c_{1} \phi^{c_{2}} z^{c_{3}}\right]\right)$ - Log-log correlation of permeability $(k)$ and porosity $(\phi)$

2.2 Model CPR-B: $k=a(\phi+c)^{b} \quad\left(c=c_{\max } \exp \left[-c_{1} \phi^{c_{2}} z^{c_{3}}\right]\right)$ - Log-log correlation of permeability $(k)$ and porosity $(\phi)$

2.3 Model $C P R-C: \quad k=c \exp [\beta \phi] \quad c=c_{\max } \exp \left[-c_{1} \phi^{c_{2}} z^{c_{3}}\right]-$ Semilog correlation plot of permeability $(k)$ and porosity $(\phi)$.

2.4a "Clean Sand" Plot — Archie "Clean Sand" trend is given by the straight-line trend at the far left of the data (power law model).

2.4b "Dirty Sand" Plot - Archie "Dirty Sand" trend is given by the straight-line trend at the far right of the data (exponential model).

2.5a Model CPR-A: $k=a(\phi-c)^{b}\left(c=c_{\max } \exp \left[-c_{1} \phi^{c_{2}} F^{c_{3}}\right]\right)$ - "Clean Sand" Plot (log-log format) - Archie "Clean Sand" trend is given by the straight-line trend at the far left of the data (power law model).....

2.5b Model CPR-A: $k=a(\phi-c)^{b}\left(c=c_{\max } \exp \left[-c_{1} \phi^{c_{2}} F^{c_{3}}\right]\right)$ - "Dirty Sand" Plot (semilog format). Archie "Clean Sand" trend is given by the curved trend at the far left of the data (power law model)

2.6a Model CPR-A: calculated versus measured permeability

2.6b Model CPR-A: Permeability calculated using Model CPR-A versus permeability calculated using the GRACE Algorithm ....

2.6c Model $C P R$-A: Calculated $c$-function values versus measured $c$-function values

2.7a Model CPR-B: $\quad k=a(\phi+c)^{b} \quad c=c_{\max } \exp \left[-c_{1} \phi^{c_{2}} F^{c_{3}}\right]$ - "Clean Sand" Plot (log-log format) - Archie "Dirty Sand" trend is given by the straight-line trend at the far right of the data (power law model)

2.7b Model CPR-B: $k=a(\phi+c)^{b} \quad c=c_{\max } \exp \left[-c_{1} \phi^{c_{2}} F^{c 3}\right]$ - "Dirty Sand" Plot (semilog format) - Archie "Dirty Sand" trend is given by the curved trend at the far right of the data (power law model)

2.8a Model CPR-B: calculated versus measured permeability.

2.8b Model $C P R-B$ : Permeability calculated using Model $C P R-B$ versus permeability calculated using the GRACE Algorithm ...... 
2.9a Model CPR-C: $k=c \exp [\beta \phi] \quad c=c_{\max } \exp \left[-c_{1} \phi^{c 2} F^{c 3}\right]$ - "Clean Sand" Plot (log-log format) — Archie "Dirty Sand" trend is given by the curved trend at the far right of the data (exponential model).

2.9b Model $C P R-C: \quad k=c \exp [\beta \phi] \quad c=c_{\max } \exp \left[-c_{1} \phi^{c 2} F^{c_{3}}\right]$ - "Dirty Sand" Plot (semilog format) — Archie "Dirty Sand" trend is given by the straight-line trend at the far right of the data (exponential model)

2.10a Model CPR-C: calculated versus measured permeability

2.10b Model CPR-C: Permeability calculated using Model $C P R-C$ versus permeability calculated using the GRACE Algorithm .....

2.10c Model CPR-C: Calculated $c$-function values versus measured $c$-function values

2.11a Model CPR-D: $k=x a \phi^{b}+(1-x) \alpha \exp [\beta \phi] \quad[0 \leq x \leq 1] \quad$ - Weighted Power LawExponential Model used to correlate permeability $(k)$ and porosity $(\phi)$

2.11b Model CPR-D: $k=x a \phi^{b}+(1-x) \alpha \exp [\beta \phi] \quad[0 \leq x \leq 1] \quad$ - Weighted Power LawExponential Model used to correlate permeability $(k)$ and porosity $(\phi)$................................. 35

2.12a Model CPR-DxL: Calculated versus measured permeability ............................................... 36

2.12b Model CPR-DxL: Permeability calculated using Model CPR-C versus permeability calculated using the GRACE Algorithm

2.12c Model CPR-DxL: Calculated $x$-function weight values versus measured $x$-function weight values 38

2.13a Model CPR-DxQ: Calculated versus measured permeability

2.13b Model CPR-DxQ: Permeability calculated using Model $C P R-C$ versus permeability calculated using the GRACE Algorithm

2.13c Model CPR-DxQ: Calculated $x$-function weight values versus measured $x$-function weight values

2.14a "Modified Timur Model:" Calculated versus measured permeability.

2.14b "Modified Timur Model:" Permeability calculated using the "Modified Timur Model" versus permeability calculated using the GRACE Algorithm 43

2.15 GRACE Model: Calculated versus measured permeability. 
2.16 All Models: Calculated versus measured permeability ................................................ 45 


\section{CHAPTER I}

\section{INTRODUCTION}

\subsection{Statement of the Problem}

There have been exhaustive attempts to correlate permeability with porosity and other variables — this is one of the most intractable problems in the petroleum industry. Our goal is not to provide a "universal" relation or concept, but rather, to provide "characteristic relations" that can accurately represent a correlation of permeability with other variables. A typical "characteristic relation" will use either the power law or exponential models proposed by Archie (1950).

$$
\begin{array}{ll}
k_{\text {Archie-PLM }}=a \phi^{b} & (\text { "Power Law Model")............. } \\
k_{\text {Archie-EM }}=\alpha \exp [\beta \phi] & (\text { "Exponential Relation") }
\end{array}
$$

Our motivation for this work is our observation that many correlations of permeability, porosity, and other variables might yield inconsistent and/or inaccurate results, especially for low permeability rock samples. As we are pursuing opportunities in very low permeability $(k<0.01 \mathrm{md})$ and ultra-low permeability $(k<0.001 \mathrm{md})$ reservoirs, the potential for such correlations to establish/verify reservoir quality becomes significant. We believe that for ultra-low permeability samples, it will increasingly be difficult to measure permeability on a routine basis making the correlation of permeability a main concern (using a few key measurements of permeability).

Unfortunately, there is no single relation written primarily in terms of porosity that can be valid as a permeability estimator across different types of rocks and/or different scales. As part of our literature review, we found similar attempts - the most recent of which is a series of articles by Pape et al. (1999, 2000) which focus on a fractal-based derivation of permeability from porosity. The Pape et al. result is presented as an additive power law relation, typically of the form: $k=a \phi+b \phi^{2}+c \phi^{10}$. We do not believe that the Pape et al. model will find significant utility in the petroleum industry, apart from unconsolidated materials and rocks of very uniform grain sizes. We do not wish to diminish the work of Pape et al. rather we just do not see the same behavior in our rock sample data (i.e., extremely well-defined data trends where permeability is essentially only a function of porosity). For reference, we rederived the Pape et al. fractal model for permeability in Appendix A.

This thesis follows the style and format of the SPE Journal. 
Another modified power law model written for permeability as a univariate function of porosity is the "Bryant-Finney" model (Finney (1970), Bryant et al. (1993a, 1993b), and Bryant et al. (1993c)).

The "Bryant-Finney" model is given as.

$$
k=a(\phi-c)^{b}
$$

We labeled models such as Eq. 1.3 to be "modified power law" formulations, since these models employ a "correction" term in the original power law formula. As a side note, we also established the "BryantFinney" model independently when working on our own modified power law relations, but we did not consider the $c$-term to be constant. Regardless of origin, the Bryant-Finney model is one of our characteristic permeability relations. The negative (-) sign in front of the $c$-term is a minor issue (negative numbers must be avoided in the argument of the power law formulation), but we found a few cases where this model performs very well.

In general, applying the Bryant-Finney model with the power law straight line at the leftmost portion of the data, seems to be somewhat limiting as the negative $c$-term has a profound impact when the $(\phi-c)$ quantity approaches zero (and then negative numbers). We find that when Eq. 1.3 is recast using $(\phi+c)$, and then the straight-line power law trend is placed at the rightmost portion of the data, we instead achieve a "J" type of shape in the data function, which seems to mimic the behavior of the Pape et al. fractal model. This formulation (in terms of $(\phi+c)$ ) is given by:

$$
k=a(\phi+c)^{b}
$$

However - historically, engineers and geoscientists have by far preferred an exponential type relationship to correlate permeability as a function of porosity — as given below:

$$
k=\alpha \exp [\beta \phi]
$$

Eq. 1.5 has been used almost exclusively in the correlation of permeability, based primarily on the rationalization that "permeability is a logarithmically-scaled variable." We are concerned that Eq. 1.5 is uniquely an empirical result - as opposed to the generalized power law scaling of permeability and porosity (Eq. 1.1), which has had considerable validation by theory and by experimental study primarily with unconsolidated sediments/materials and very uniform pore distributions.

We cite references by Beard and Weyl (1973), Berg (1970), and Morrow et al. (1969) to support our contention of a power law formulation for the permeability of unconsolidated sediments, and we note (without references) that this characteristic behavior has also been observed in fluidized beds, powders, ceramics, cements, and sintered metals. The power law scaling of permeability is widely accepted (under specific conditions), but the exponential scaling has, to our knowledge, never been proven theoretically. 
There are suggestions that the exponential scaling of permeability has to do with the tendency for permeability to be logarithmically distributed, and perhaps this is sufficient as "validation." We believe that the exponential correlation of permeability and porosity is most likely an empirical representation of a more complex process - one where the power law model is not directly applicable due to "non-ideal" conditions (e.g., shaliness, poor grain sorting, diagenesis, etc.). However; we employ the exponential model in two of our $C P R$ models.

Based on the discussion, we presently know (or accept) the following points:

\section{Power Law Model for Permeability: Unconsolidated Sediments/Materials}

Eq. 1.1 provides the base relation for a power law scaling of permeability using porosity (i.e., $k=a \phi^{b}$ ). This formulation is validated in a form that includes the square of grain size by Beard and Weyl (1973) and Morrow et al. (1969) for unconsolidated sediments and for (theoretical) packings of perfect spheres [Berg (1970)].

$$
k_{\mathrm{Berg}}=a \phi^{b} d_{p}^{2} \quad \text { ("Berg Equation" (general form) }
$$

Further, variations of the simple power law formulation have been given which include saturation as a correlation variable by Timur (1968) (referencing his own relation) and Ahmed et al. (1989) (referencing the Coates-Denoo (1981) permeability relation) — these relations are:

$$
\begin{array}{ll}
k_{\text {Timur }}=a \phi^{b} S_{w}^{c} & (\text { "Timur Model").................. } \\
k_{\text {Coates }}=\left[100 \phi^{2} \frac{\left(1-S_{w}\right)}{S_{w}}\right]^{2} & (\text { "Coates-Denoo Model") }
\end{array}
$$

\section{Modified Power Law Model for Permeability: Proposed for Consolidated Sediments}

Eqs. 1.3 and 1.4 are proposed intuitively as "modifications" of the power law model for permeability (i.e., Eq. 1.1) - where these formulations are intended to compensate for non-ideal behavior due to the influence of variables other than porosity. Eqs. 1.3 and 1.4 are repeated for clarity in this discussion:

$$
\begin{aligned}
& k_{\text {Bryant-1 }}=a(\phi-c)^{b} \quad(\text { Bryant-Finney Model (straight-line on left of data) }) \\
& k_{\text {Bryant-2 }}=a(\phi+c)^{b} \quad \text { (Modified Bryant-Finney Model (straight-line on right of data) }
\end{aligned}
$$

As we do not believe that the Pape et al. model $\left(k=a \phi+b \phi^{2}+c \phi^{10}\right)$ is viable in practice, we mention this model as only as a possible (analytical) solution for relating permeability to porosity in near-ideal situations. 


\section{Modified Exponential Model for Permeability: Proposed for Consolidated Sediments}

Eq. 1.5 is the "basis" formulation for the exponential model, where Eq. 1.5 is repeated for clarity.

$$
k=\alpha \exp [\beta \phi] \quad(\text { "Exponential Model") }
$$

Our contention is that the slope term $(\beta)$ is constant, but the intercept term varies from an original value to a maximum value. For convenience, we write this modified exponential form as.

$$
k=c\left(\phi, F, V_{s h}, \text { etc. }\right) \exp [\beta \phi]
$$

A " $c_{\min }$ to $c_{\max }$ " formulation could be developed using the "error function" (erf[x]), and would be written as:

$$
k=c \exp [\beta \phi] \quad \text { where } c=c_{\min }+\left(c_{\max }-c_{\min }\right) \operatorname{erf}\left[c_{1} \phi^{c 2} z^{c 3}\right]
$$

However, the erf[x] term would be potentially difficult to resolve as the $c$-function may not be particularly similar to erf[x]. We have found that the following "exponential" form for the $c$-function is well-behaved and generally provides accurate estimates:

$$
k=c \exp [\beta \phi] \quad c=c_{\max } \exp \left[-c_{1} \phi^{c_{2}} z^{c_{3}}\right]
$$

In addition to the (somewhat) theoretical relations proposed by Eqs. 1.3-1.11, we also employ a "hybrid" relationship between the Archie power law and exponential models (Eqs. 1.1 and 1.2, respectively). We have labeled this the Weighted Power Law-Exponential Model, which is defined as:

$$
k=x a \phi^{b}+(1-x) \alpha \exp [\beta \phi] \quad[0 \leq x \leq 1] \quad(\text { basic form) }
$$

\subsection{Objectives}

The objectives of this work are:

- To propose and develop a family of "characteristic permeability relations" - specifically, functional forms based on the original observations of Archie (power law and exponential behavior of numerous permeability-porosity data sets).

- To validate these "characteristic permeability relations" using data from literature and industry sources for consolidated rocks, as well as data for other materials.

- To provide a general methodology for applying the "characteristic permeability relations" in practice, including methods to distinguish poor data from correlation efforts. 


\subsection{Validation}

Data obtained from the literature and industry resources were used to validate the models (i.e., "characteristic permeability relations") proposed in this work. The (measured) permeability of the samples (from both carbonate and sandstone reservoirs) ranges from low (tight gas sands) to high permeability reservoir rocks. One example validation case (the "Lower Wilcox" Well, located in South Texas (USA) is presented in the next chapter and the other nine example validation cases are presented in the appendices (see Appendix B - Appendix J). We present the characteristic plots for each validation case where we attempt to validate the "power law" and/or "exponential" nature of the data corresponding to the characteristic permeability relations. In each validation case, the proposed characteristic permeability relations (CPRs) A-D are applied. In addition, we use the "Alternating Conditional Expectations" (ACE) algorithm (see Xue et al. 1997), which is a non-parametric regression method to obtain the optimum correlation, to check the consistency of the proposed models (i.e., CPRs).

\subsection{Summary and Conclusions}

In this work we attempt to develop, demonstrate and validate our concept of a characteristic permeability relation $(C P R)$ for various different models where we allow the reservoir permeability to be a function of only two variables — namely, porosity and water saturation or Archie Formation Factor.

We evaluate each characteristic permeability relation systematically to identify the strengths of each proposed relation. Specifically, our objective is to demonstrate the characteristic (or dominant) behavior of each characteristic permeability relation.

In conclusion, we recognize that each characteristic permeability relation has a distinctive "signature," and we note that some CPRs perform better than others depending on the nature of the problem. It is also worth to note that the systematic application of the CPRs as proposed and demonstrated in this work should be functional in the correlation of a given set of permeability data with other variables. 


\subsection{Organization of the Thesis}

The outline of the proposed research thesis is as follows:

- Chapter I - Introduction

- Statement of the Problem

- Objectives

- Validation

- Summary and Conclusions

- Organization of the Thesis

- Chapter II - Characteristic Permeability Relations

- Development

- Characteristic Behavior

- Validation

- Results and Discussion

- Chapter III — Summary, Conclusions, and Recommendations for Future Work

- Summary

- Conclusions

- Recommendation for Future Work

- Nomenclature

- References

- Appendices

- Appendix A - Derivation of the "Pape" Multi Power Law Formulation for Permeability

- Appendix B - Results for Howell Well (E. Texas - USA)

- Appendix C - Results Miocene Sand (S. Louisiana - USA)

- Appendix D - Results for Vicksburg Sand (S. Texas - USA)

- Appendix E - Results for Wilcox Sand (S. Texas - USA)

- Appendix F - Results for Hazlett Well 103

- Appendix G - Results for Devonian Limestone (Texas - USA)

- Appendix H - Results for Frio Shaly Sandstone (Texas - USA)

- Appendix I - Results for Lower Wilcox Shaly Sandstone (Texas - USA)

- Appendix J - Results for Paluxy Clean Sandstone (Texas - USA)

- Vita 


\section{CHAPTER II}

\section{CHARACTERISTIC PERMEABILITY RELATIONS}

\subsection{Development}

In this section we present the five characteristic permeability relations that we have developed in this work. As stated before, three of the proposed characteristic permeability relations could be considered as modifications of "historical" models and two of the characteristic permeability relations are hybrid formulations which use both exponential and power law relations. The characteristic permeability relations are given by:

CPR-A — Modified Archie "Clean Sand" Model: (Bryant-Finney Model)

$$
k=a(\phi-c)^{b} \quad c=c_{\max } \exp \left[-c_{1} \phi^{c 2} z^{c 3}\right]
$$

CPR-B — Modified Archie "Dirty Sand" Model: (Power-Law Basis)

$$
k=a(\phi+c)^{b} \quad c=c_{\max } \exp \left[-c_{1} \phi^{c_{2}} z^{c_{3}}\right]
$$

CPR-C — Modified Archie "Dirty Sand" Model: (Exponential Basis)

$$
k=c \exp [\beta \phi] \quad c=c_{\max } \exp \left[-c_{1} \phi^{c 2} z^{c_{3}}\right]
$$

CPR-DxL - Weighted Power Law-Exponential Model: (Linear logarithmic weights model)

$$
k=x a \phi^{b}+(1-x) \alpha \exp [\beta \phi] \quad[0 \leq x \leq 1] \quad x=\exp \left[-\ln \left(x_{0}\right)+x_{1} \ln (\phi)+x_{2} \ln (z)+x_{3} \ln (\phi) \ln (z)\right]
$$

CPR-DxQ — Weighted Power Law-Exponential Model: (Quadratic logarithmic weights model)

$$
\begin{aligned}
& k=x a \phi^{b}+(1-x) \alpha \exp [\beta \phi] \quad[0 \leq x \leq 1] \\
& x=\exp \left[\begin{array}{l}
-\ln \left(x_{0}\right)+x_{1} \ln (\phi)+x_{2} \ln (z)+x_{3} \ln (\phi) \ln (z)+x_{4} \ln (\phi)^{2} \\
+x_{5} \ln (z)^{2}+x_{6} \ln (\phi)^{2} \ln (z)+x_{7} \ln (\phi) \ln (z)^{2}+x_{8} \ln (\phi)^{2} \ln (z)^{2}
\end{array}\right]
\end{aligned}
$$

\subsection{Characteristic Behavior}

In our next effort we consider the "characteristic behavior" of Eqs. 2.1-2.5 which is the so-called "characteristic permeability relations" (CPRs). Our goal is to establish the characteristic or dominant behavior of each relation - but not the behavior of the individual " $c$ or $x$-functions," these function will be illustrated in the "Validation" section. 
We utilize field examples to illustrate the behavior of the characteristic permeability relations (CPRs) (i.e., Eqs. 2.1-2.5) - and we attempt to "connect" each relation to an aspect of theory or accepted empirical behavior. Our "historical" characteristic permeability relations (i.e., CPR-A, CPR-B, and $C P R-C$ ) were formulated using the work by others as follows:

- Archie (1950): $\quad$ Logarithm of permeability as a function of porosity (exponential model) and permeability as a function of the Archie Formation Factor, which leads directly to a power law relationship of permeability with porosity (i.e., Eq. 1.1). According to the data presented by Archie, the power law relationship of permeability with porosity is primarily valid for unconsolidated/weakly consolidated rocks.

- Morrow et al. (1969): Correlation of permeability with porosity and grain size for unconsolidated sediments using statistical representations of the grain size distribution.

- Berg (1970): $\quad$ Correlation of permeability with porosity and grain size using theoretical (systematic) packings of perfect spheres.

- Beard and Weyl (1973): Correlation of permeability with porosity and grain size for unconsolidated sediments using sieved sand samples to represent the influence of both grain size and sorting.

- Pape et al. (1999): Correlation of permeability with porosity using a "multiple power law" model based on fractal theory.

These contributions lead us to the definition of characteristic power law permeability-porosity relations based on modifications of the Archie "Clean Sand" model (Eq. 1.1). In particular, relations CPR-A (Eq. 3.1) and $C P R-B$ (Eq. 3.2) are direct modifications of the Archie "Clean Sand" model. As a coincidence, Bryant (1993a, 1993b, 1993c) and Finney (1970) also proposed the CPR-A formulation (Eq. 2.1), so we will also refer to the CPR-A model as the Bryant-Finney model. We note that Eqs. 1.1 and 2.1 are both "clean sand" models, implying that the Archie (clean sand) power law trend will lie to the left of the data body (see Fig. 2.1). Also the " $c_{\max }$ " trend is constructed by substitution of a constant value of " $c_{\max }$ " into Eq. 2.1 until trend lies along the rightmost portion of the data (see Fig. 2.1).

In order to use the $C P R-A$ equation, first we plot $\log (k)$ versus $\log (\phi)$. Then we locate the Archie "Clean Sand" trend - we use the graphical method (i.e., hand analysis) to establish the $a$ and $b$ parameters. We establish the $c_{\max }$ trend by substitution of $c_{\max }$ values, and the $c_{\max }$ trend should be just beyond the rightmost body of data. Next, we use regression methods to estimate the $c_{1}, c_{2}$, and $c_{3}$ parameters. 
It is worth to note that regression methods should not be used to estimate the $a$ and $b$ parameters. As $C_{\max }$ is subtracted in this model, care must be taken to avoid negative values in the solution. For many cases, the $b$-parameter is on the order of 8 [suggested from the work of Morrow et al (1969) and Beard and Weyl (1974)].

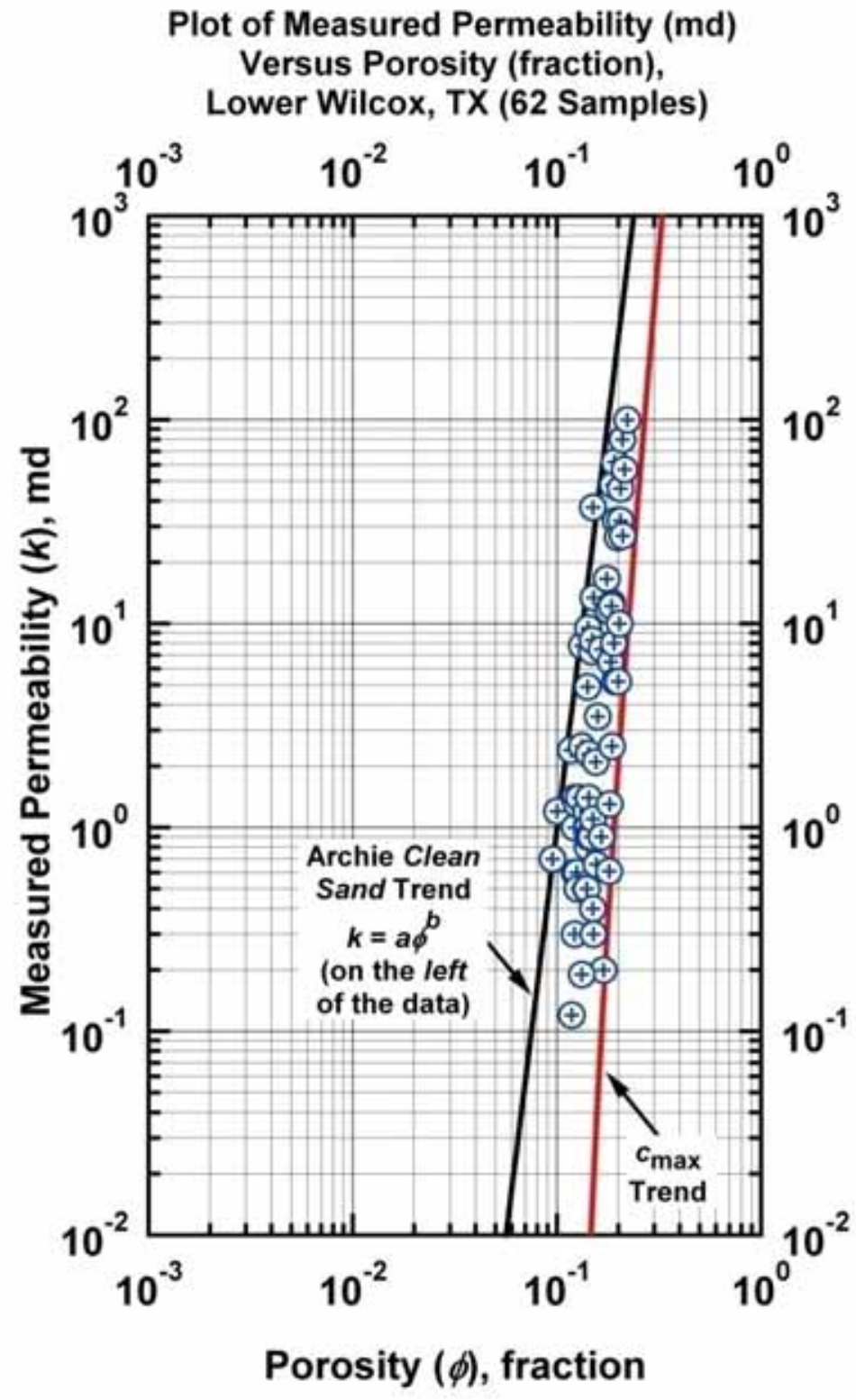

Figure 2.1 - Model CPR-A: $k=a(\phi-c)^{b}\left(c=c_{\max } \exp \left[-c_{1} \phi^{c_{2}} z^{c_{3}}\right]\right)-$ Log-log correlation of permeability $(k)$ and porosity $(\phi)$. Archie "clean sand" trend is constructed to the left of the body of data. 
Generally speaking, we strongly recommend against using statistical methods for locating the base trends for all CPR models - these trends should be constructed (carefully) by the analyst. Regression can and should be used to estimate the model parameters (e.g., the $c_{i}$ and $x_{i}$ coefficients), but never to locate the base trends (i.e., the power law or exponential trends).

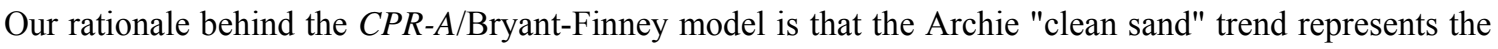
highest permeability value for a given value of porosity, hence the position of this line to the left of the data body. By induction, any point not lying on the "clean sand" trend must be affected by some factor(s) which mitigate permeability — for example: shaliness, poor sorting, diagenesis, etc.

In contrast, we define $C P R-B$ (Eq. 2.2) as the Archie "dirty sand" power law model and $C P R-C$ (Eq. 2.3) as the Archie "dirty sand" exponential model. CPR-B implies that the "dirty sand" trend on a log-log plot of permeability versus porosity will lie along the far rightmost trend of data (see Fig. 2.2).

In order to use the $C P R-B$ equation first we plot $\log (k)$ versus $\log (\phi)$. Then we locate the Archie "Dirty Sand" trend - we use the graphical method (i.e., hand analysis) to establish the $a$ and $b$ parameters. The $c_{\max }$ trend is established by substitution of $c_{\max }$ values, we note that the $c_{\max }$ trend should be just beyond the leftmost body of data. We finally use regression methods to estimate the $c_{1}, c_{2}$, and $c_{3}$ parameters.

We should not use regression methods to estimate the $a$ and $b$ parameters. As $c_{\max }$ is added in this model, you can note the unique "J"-shape of the $c_{\max }$ trend towards the left. A best "hand fit" of the $b$-parameter should always work well.

Similarly, CPR-C implies that the "dirty sand" trend on a plot of logarithm of permeability versus porosity will also lie along the far rightmost trend of data (see Fig. 2.3). Archie (1950) showed some of the first correlations of $\log (k)$ versus $\phi$, and although Archie provided an average trend through the data, we can infer that (perhaps) the exponential trend $(k=\alpha \exp [\beta \phi])$ could represent the lowest (i.e., far rightmost) correlation of permeability and porosity on the semilog plot. The "dirty sand" trend illustrated in Fig. 2.3 is plausible in all of the cases we consider.

In order to use the $C P R-C$ equation first we plot $\log (k)$ versus $\phi$. Then we locate the Archie "Dirty Sand" trend - we use the graphical method (i.e., hand analysis) to establish the $\beta$-parameter (i.e., the slope). We establish the $c_{\max }$ (straight-line) trend by substitution of $c_{\max }$ values, similarly, the $c_{\max }$ trend should be just beyond the leftmost body of data. We finally use regression methods to estimate the $c_{1}, c_{2}$, and $c_{3}$ parameters.

It is very important to note that regression methods to should not be employed to estimate the $\beta$-parameter. $c_{\max }$ simply represents a "shift" of the straight-line (exponential) trend to the left-most portion of the data. An alternate formulation of this problem could be written as: 


$$
\begin{aligned}
& k=c \exp [\beta \phi] \\
& c=c_{\text {min }}+\left(c_{\text {max }}-c_{\text {min }}\right) \operatorname{erf}\left[c_{1} \phi^{c_{2}} z^{c_{3}}\right]
\end{aligned}
$$

Although we do not believe that such a form will be necessary in practice, it does have certain attractive features (e.g., $c_{\min }<c<c_{\max }$ is explicitly stated).

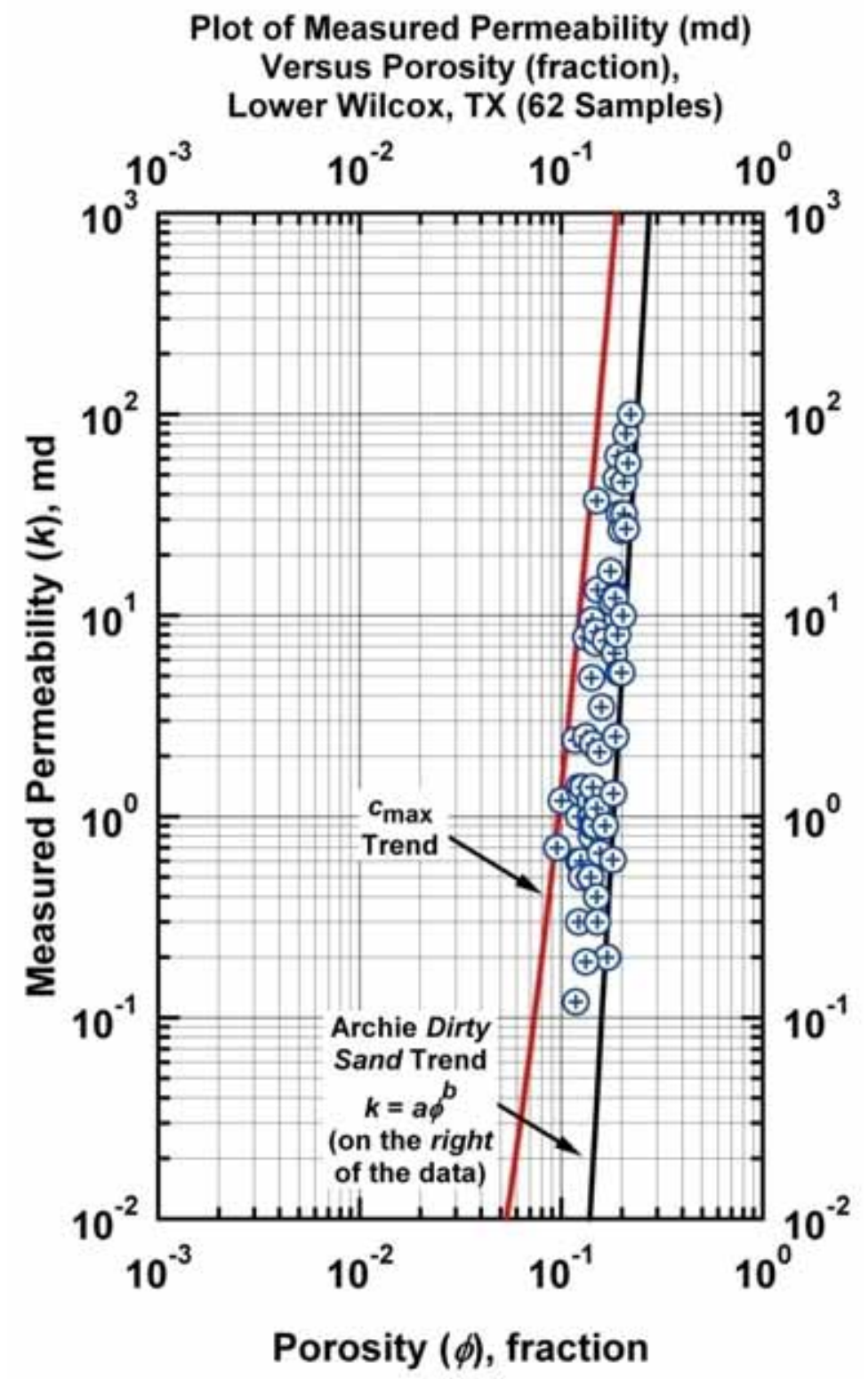

Figure 2.2 - Model CPR-B: $k=a(\phi+c)^{b} \quad\left(c=c_{\max } \exp \left[-c_{1} \phi^{c_{2}} z^{c 3}\right]\right)$ - Log-log correlation of permeability $(k)$ and porosity $(\phi)$. Archie "dirty sand" trend is constructed to the right of the body of data. 
Our final approach, the Weighted Power Law-Exponential Model is a simple weighting of the historical power law and exponential trends, the generic form of this "weighted" model is given as:

$$
k=x a \phi^{b}+(1-x) \alpha \exp [\beta \phi] \quad[0 \leq x \leq 1]
$$

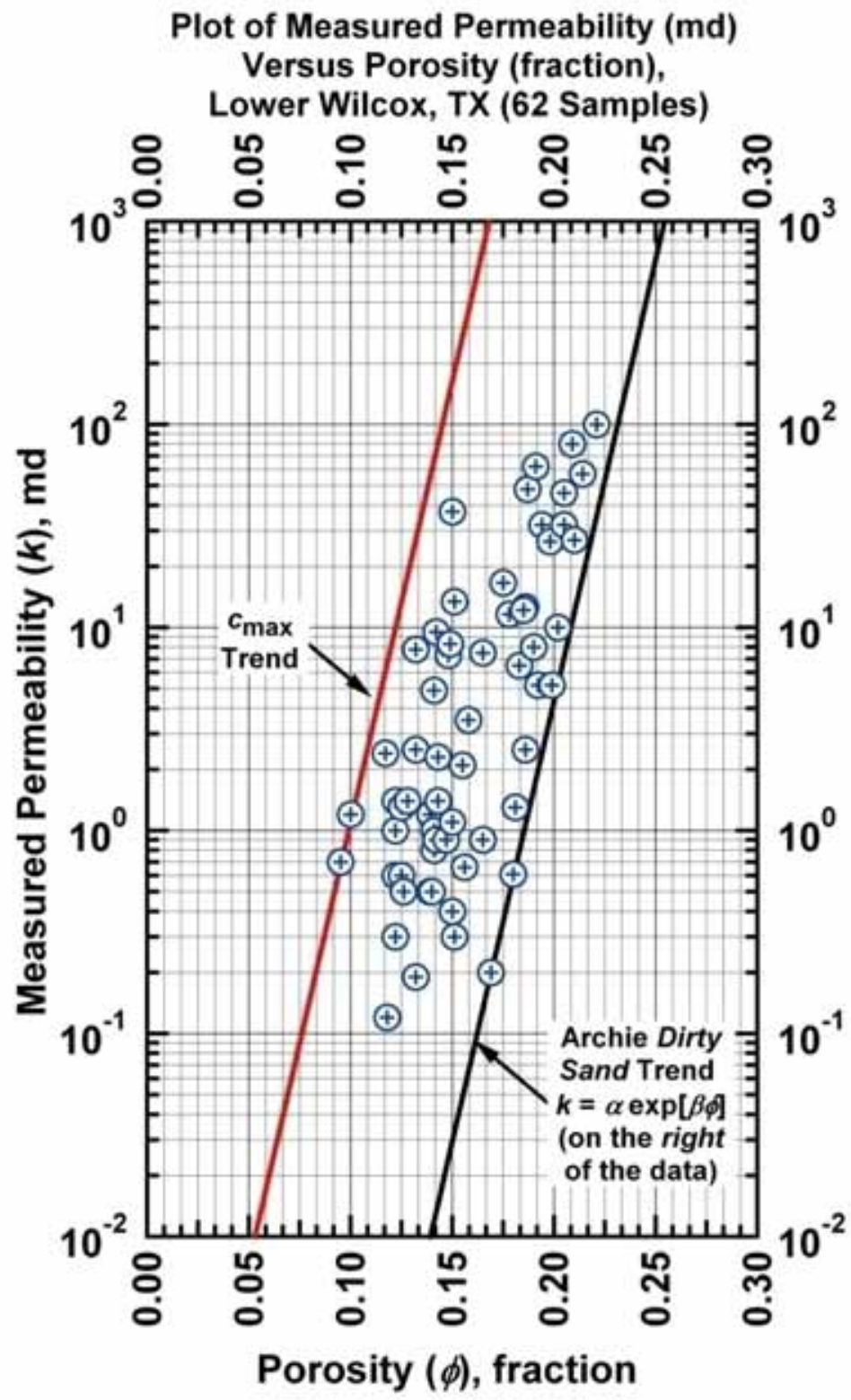

Figure 2.3 - Model $C P R-C: k=c \exp [\beta \phi] \quad c=c_{\max } \exp \left[-c_{1} \phi^{c_{2}} z^{c_{3}}\right]$ - Semilog correlation plot of permeability $(k)$ and porosity $(\phi)$. Archie "dirty sand" trend is constructed to the right of the body of data. 
Our approach has been to define the power law relation $\left(a \phi^{b}\right)$ as the Archie "clean sand" trend (the same as using CPR-A (Eq. 2.1) on the leftmost portion of the data on a $\log (k)$ versus $\log (\phi)$ plot); and to define the exponential relation $(\alpha \exp [\beta \phi])$ as the Archie "dirty sand" trend (the same as in $C P R-C$ (Eq. 2.3) on the rightmost portion of the data on $\log (k)$ versus $\phi$ plot)). Once the trends are defined, and the parameters $(a, b, \alpha, \beta)$ are known, we then solve Eq. 2.7 for the weights ( $x$-values). This process gives us a basis for establishing the weights (somewhat) directly from the data. Obviously, the weights ( $x$-values) then must be correlated with independent variables (e.g., $\phi, S_{w}, F, V_{s h}$, etc.). Solving Eq. 2.7 for the weights (i.e., the $x$-values), we have:

$$
x=\frac{(k-\alpha \exp [\beta \phi])}{\left(a \phi^{b}-\alpha \exp [\beta \phi]\right)}
$$

Our procedure is to estimate $x$ using data, and then to attempt a correlation of $x=f\left(\phi, S_{w}, F, V_{\text {sh }}\right.$, etc.). As such, our best effort to correlate the $x$-values has led to the following logarithmic polynomial forms:

1. $C P R-D x L$ : (Linear logarithmic weights model)

$$
x=\exp \left[-\ln \left(x_{0}\right)+x_{1} \ln (\phi)+x_{2} \ln (z)+x_{3} \ln (\phi) \ln (z)\right]
$$

2. CPR-DxL: (Quadratic logarithmic weights model)

$$
x=\exp \left[\begin{array}{l}
-\ln \left(x_{0}\right)+x_{1} \ln (\phi)+x_{2} \ln (z)+x_{3} \ln (\phi) \ln (z)+x_{4} \ln (\phi)^{2} \\
+x_{5} \ln (z)^{2}+x_{6} \ln (\phi)^{2} \ln (z)+x_{7} \ln (\phi) \ln (z)^{2}+x_{8} \ln (\phi)^{2} \ln (z)^{2}
\end{array}\right]
$$

As noted earlier in Eqs. 2.9 and 2.10, the z-variable represents $S_{w}$ or $F$, as prescribed by available data. The $z$-variable could also be $V_{\text {sh }}$, well log responses, etc. As comment, there are certainly other forms that could be used to represent the $x$-variable, but for the purposes of this work, we are quite satisfied with the performance of the logarithmic polynomial forms (Eqs. 2.9 and 2.10) as correlating functions.

Our rationale in creating the Weighted Power Law-Exponential Model was to "capture" the most significant features of the power law (Archie "clean sand") and the exponential (Archie "dirty sand") models. We recognize that such a "hybrid" model may not have a strong theoretical basis, but our intention is to provide this model as a mechanism to correlate permeability. A graphical presentation of the Weighted Power Law-Exponential Model is illustrated in Fig. 2.4. We note that the power law and exponential relations form an "envelope" around the permeability-porosity data (the log-log format is shown in Fig. 2.4a and the semilog format is shown in Fig. 2.4b). The construction of the envelope is by design as we wish to capture all of the data (or at least, all of the relevant data). The weighting function $(x)$ is then used to correlate the "interior" behavior of the data envelope. 


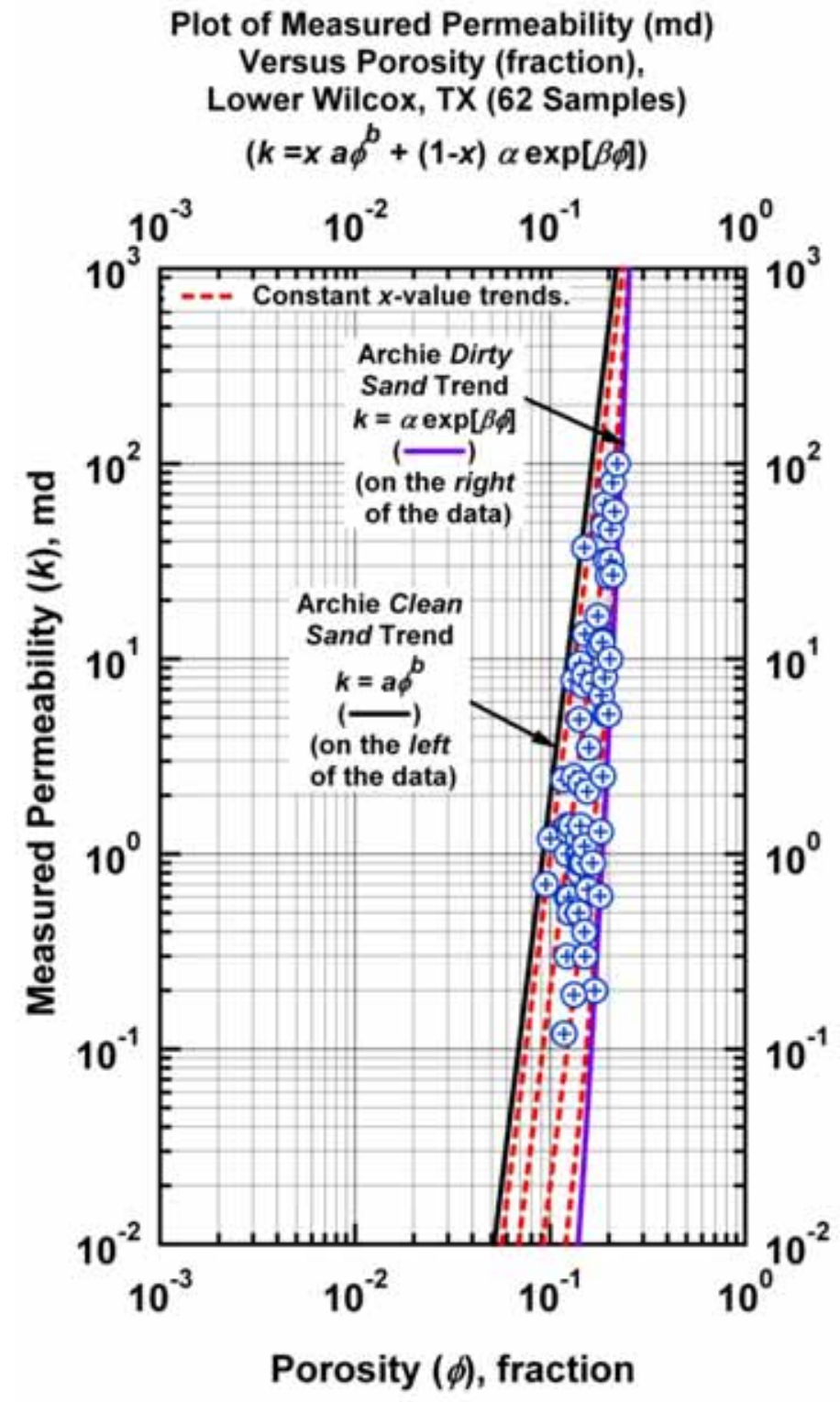

Figure 2.4a - "Clean Sand" Plot — Archie "Clean Sand" trend is given by the straight-line trend at the far left of the data (power law model). 


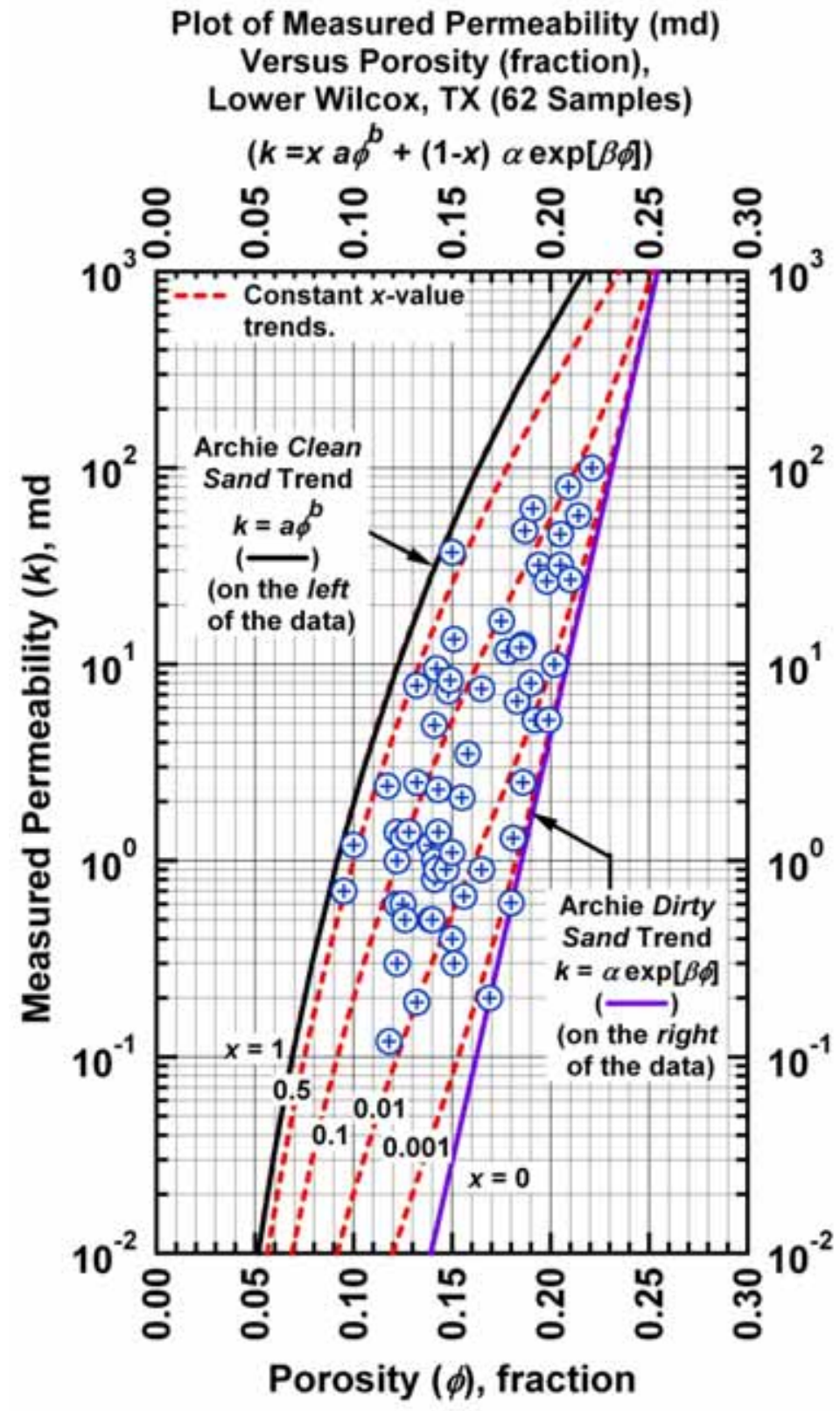

Figure 2.4b _ _ "Dirty Sand" Plot — Archie "Dirty Sand" trend is given by the straight-line trend at the far right of the data (exponential model) 
Because of the Cartesian scaling of the porosity axis in Fig. 2.4b, this plot provides perhaps the best resolution of the power law and exponential "boundaries" of the data envelope, as well as the selected values of the $x$-function, which illustrate the interior behavior.

\subsection{Validation}

In this section we present an example validation - in particular, the "Lower Wilcox" Well, located in South Texas (USA), where this is a tight gas reservoir system comprised of shaly (clastic) sediments (Ellis, 1987). The characteristic plots for this case were shown in Figs. 2.5-2.7, where "characteristic" implies that we attempted to validate the "power law" and/or "exponential" nature of the data relative to the characteristic permeability relations (CPRs) A-D (i.e., Eqs. 2.1-2.5).

As in every validation case, we apply CPRs A-D (and the Archie "Clean Sand" Model) to the field data, in this case the field data were obtained from the "Lower Wilcox" Well:

For completeness, we also use a modification of the "Timur" model (Timur, 1968) - where this model is optimized to a particular dataset using non-linear regression methods. For this case (i.e., and other cases where we have $F$ but not $S_{w}$ ), the "Modified Timur Model" is defined as:

$$
k_{\text {Timur }}=a \phi^{b} F^{c} \quad(\text { "Modified Timur Model"). }
$$

In this work we also utilize the so-called "Alternating Conditional Expectations" (or ACE) algorithm (see Xue et al, 1997) - this is a non-parametric regression method referred to in this work as the "GRACE" solution. 


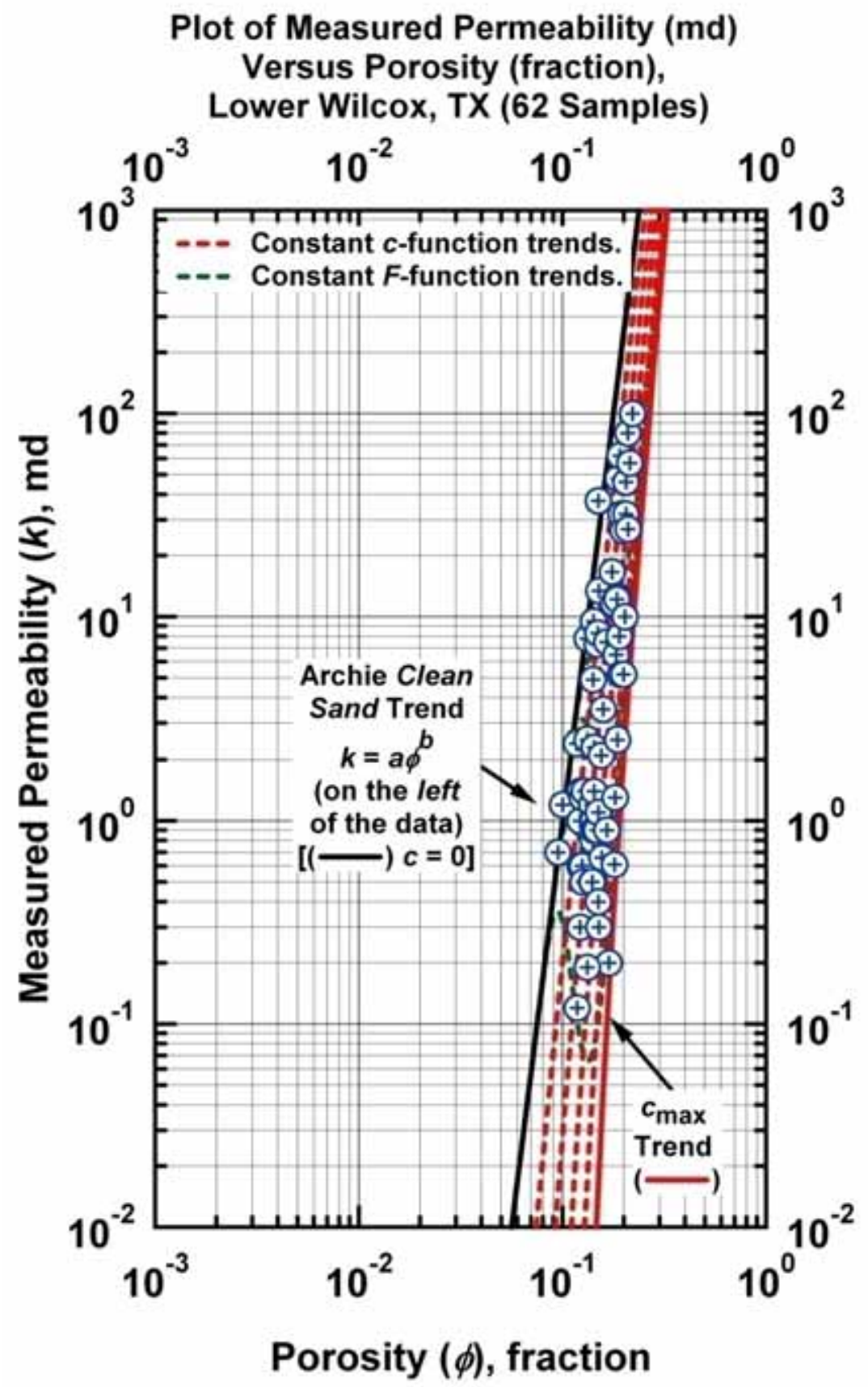

Figure 2.5a - Model CPR-A: $k=a(\phi-c)^{b}\left(c=c_{\max } \exp \left[-c_{1} \phi^{c_{2}} F^{c_{3}}\right]\right)$ - "Clean Sand" Plot (log-log format) - Archie "Clean Sand" trend is given by the straight-line trend at the far left of the data (power law model). [Case: Lower Wilcox S. TX (USA)] 


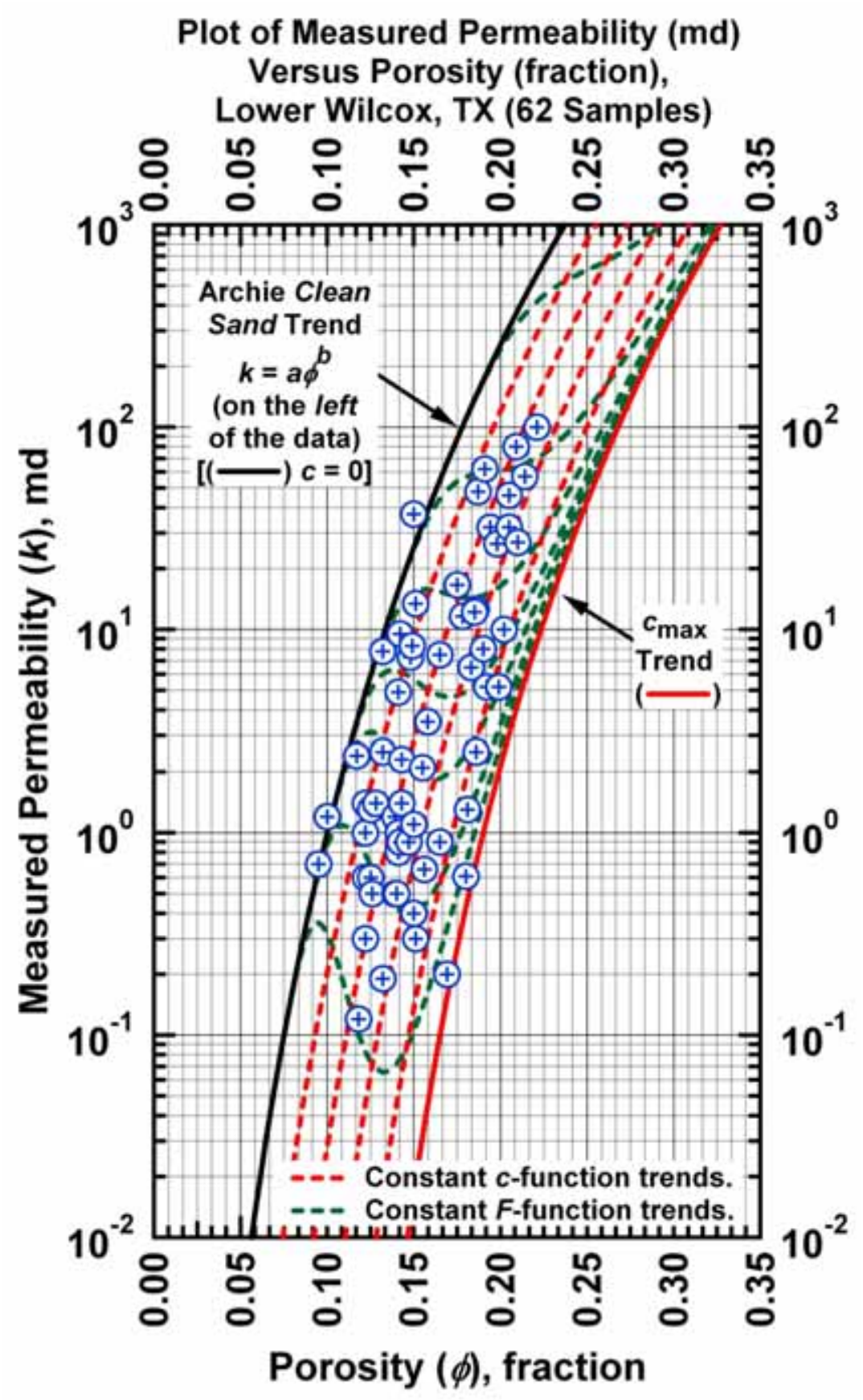

Figure 2.5b - Model $C P R-A: \quad k=a(\phi-c)^{b}\left(c=c_{\max } \exp \left[-c_{1} \phi^{c_{2}} F^{c_{3}}\right]\right)$ — "Dirty Sand" Plot (semilog format). Archie "Clean Sand" trend is given by the curved trend at the far left of the data (power law model). [Case: Lower Wilcox S. TX (USA)] 
Comparison of Permeability (md) Calculated Using the "CPR-A" Model Versus the Measured Permeability (md), Lower Wilcox, TX (62 Samples)

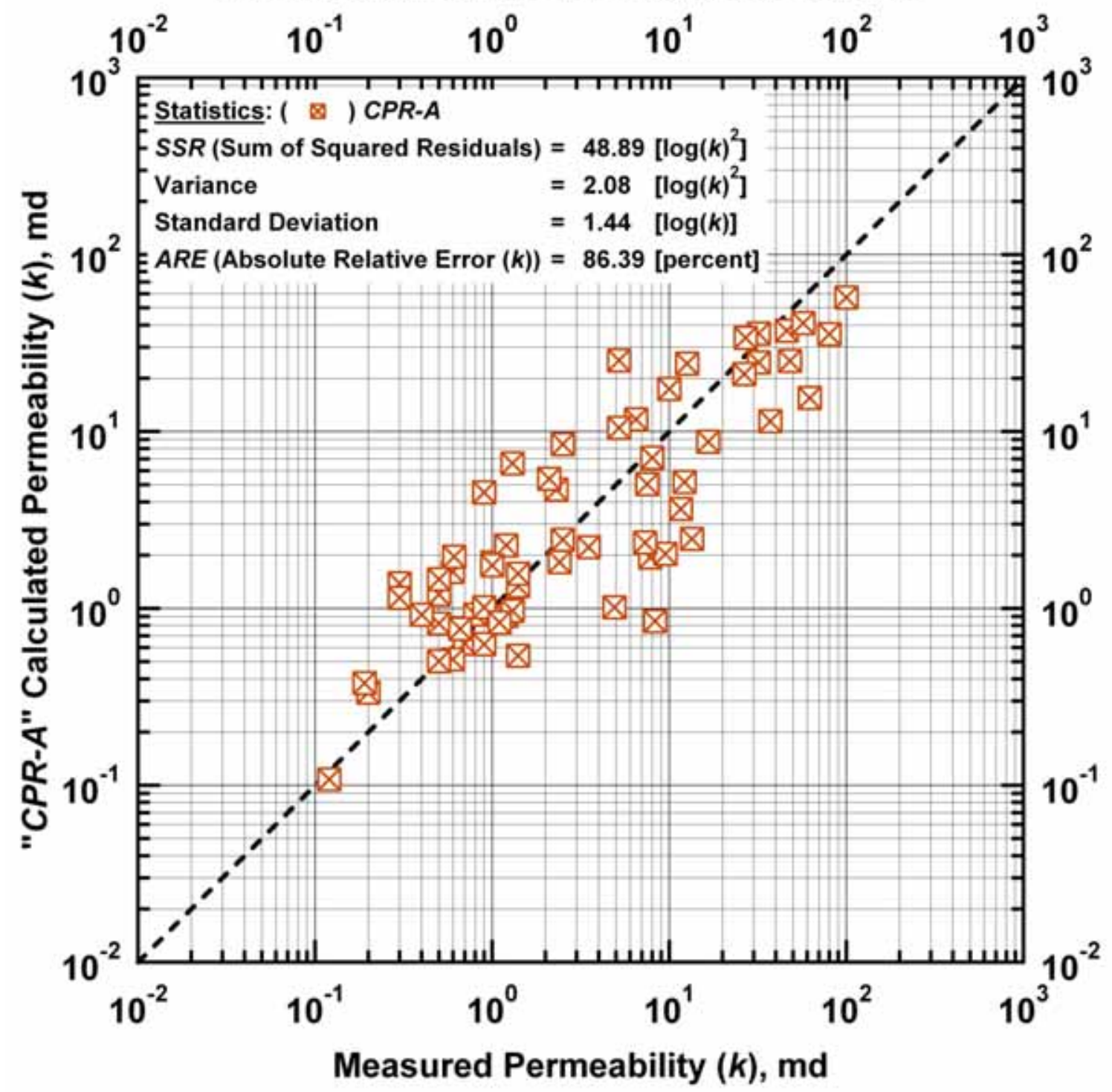

Figure 2.6a - Model CPR-A: calculated versus measured permeability. [Case: Lower Wilcox S. TX (USA)] 


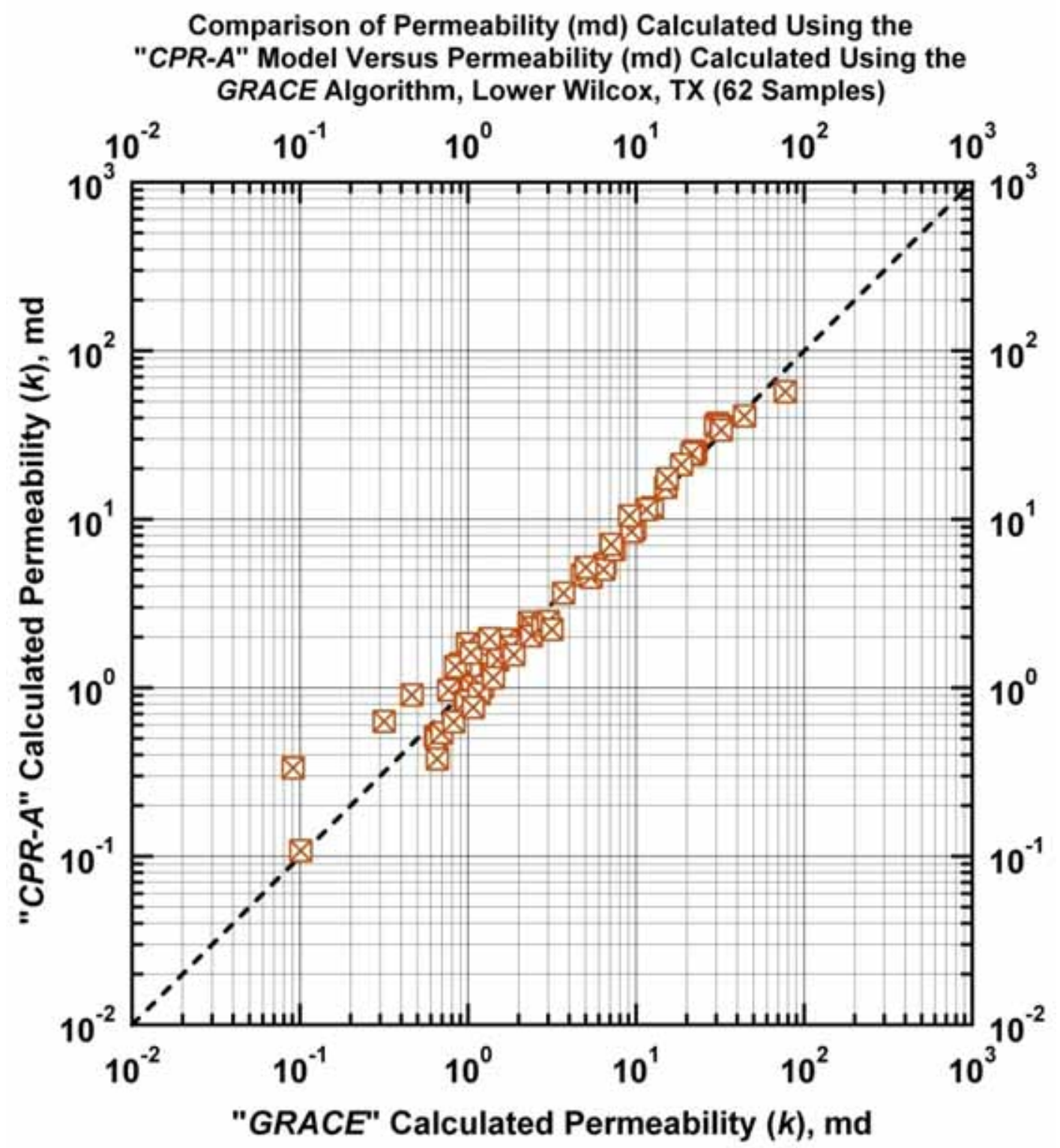

Figure 2.6b - Model CPR-A: Permeability calculated using Model $C P R-A$ versus permeability calculated using the GRACE Algorithm. [Case: Lower Wilcox S. TX (USA)] 


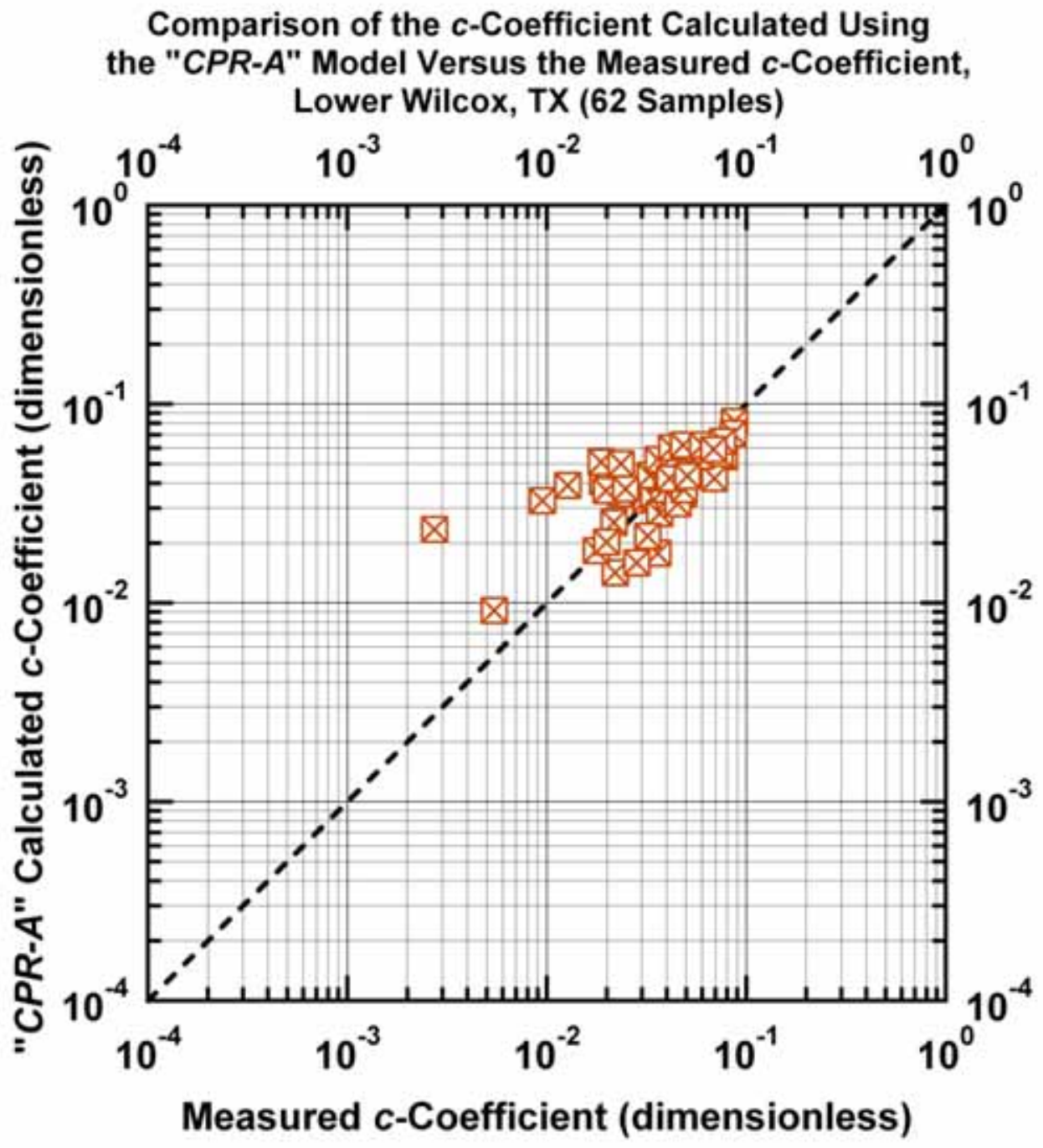

Figure 2.6c - Model $C P R$ - $A$ : Calculated $c$-function values versus measured $c$-function values. [Case: Lower Wilcox S. TX (USA)] 


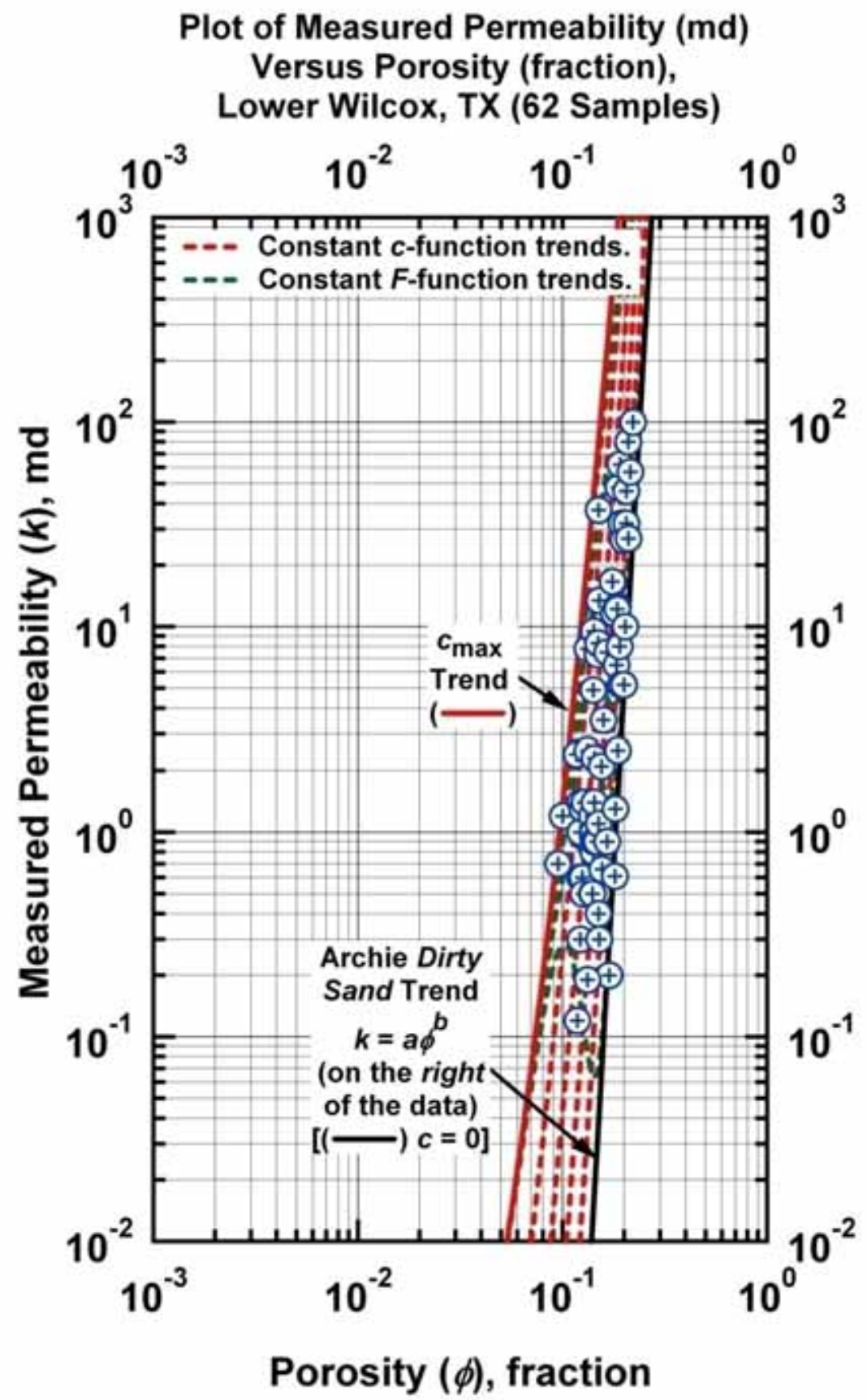

Figure 2.7a - Model CPR-B: $k=a(\phi+c)^{b} \quad c=c_{\max } \exp \left[-c_{1} \phi^{c_{2}} F^{c_{3}}\right]$ - "Clean Sand" Plot (log-log format) - Archie "Dirty Sand" trend is given by the straight-line trend at the far right of the data (power law model). [Case: Lower Wilcox S. TX (USA)] 


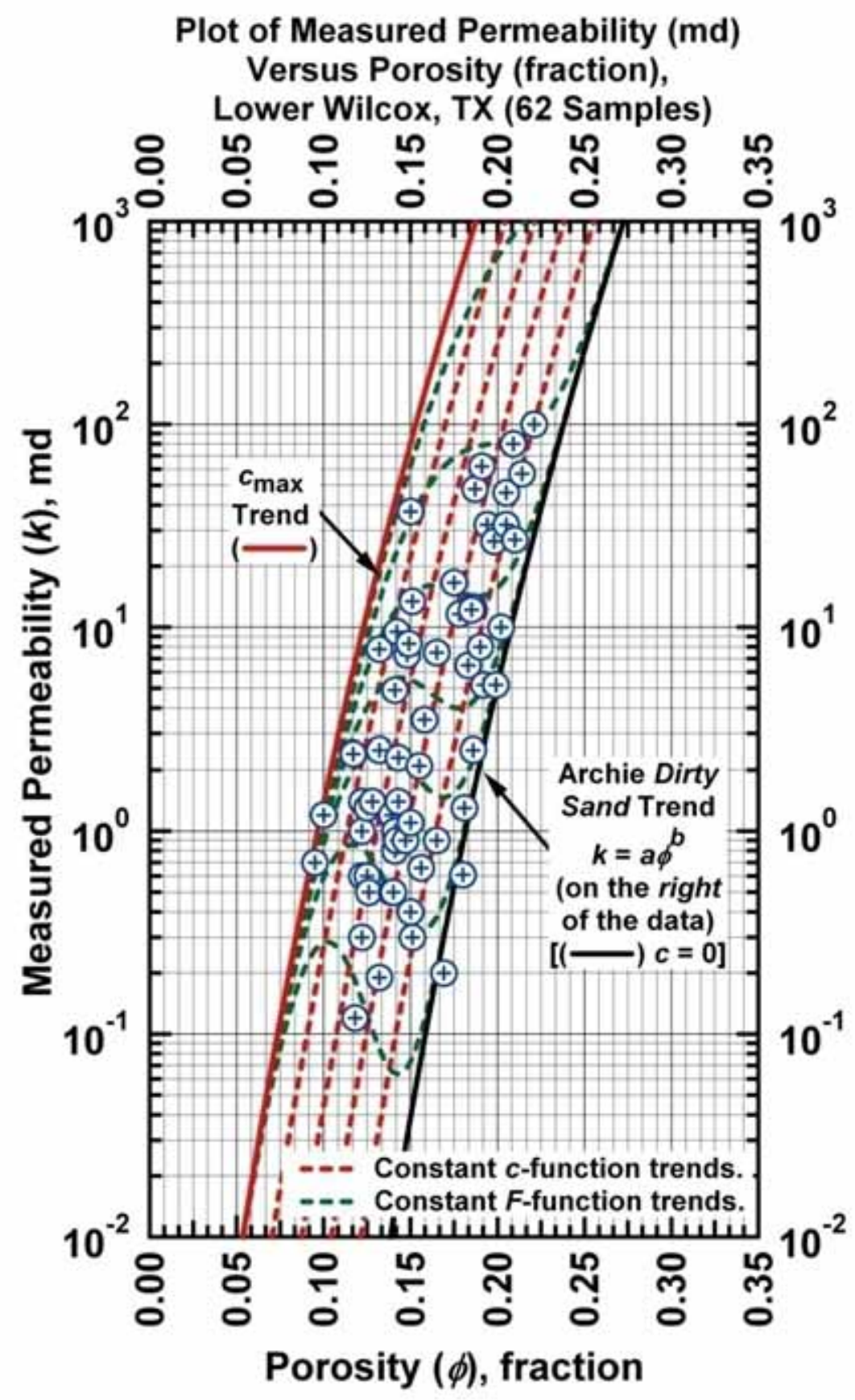

Figure 2.7b - Model CPR-B: $k=a(\phi+c)^{b} \quad c=c_{\max } \exp \left[-c_{1} \phi^{c 2} F^{c 3}\right]$ — "Dirty Sand" Plot (semilog format) - Archie "Dirty Sand" trend is given by the curved trend at the far right of the data (power law model). [Case: Lower Wilcox S. TX (USA)] 


\subsection{Results and Discussion}

In Fig. 2.5 we observe very good behavior of the $C P R$ - $A$ model for this case — we clearly note the power law straight-line trend in Fig. 2.5a (log-log format), as well as the clarity and uniqueness of the fit in Fig. 2.5b (semilog format). In Fig. 2.6 we provide the "error" plots for this case, where measured and computed data are compared systematically. Of particular value is Fig. 2.6b (GRACE comparison), a very good correlation.

We now consider the case of CPR-B - the Modified Archie "Dirty Sand" Model (power law base). In this case the $c$-function is added to the power law relation, and the rightmost data become the basis for the power law straight-line, and the leftmost data are used to define $c_{\max }$. The results for this case are presented in Figs. 2.7 and 2.8. In Fig. 2.7a (log-log format) we note a very good "envelope" is formed by the $C P R-B$ model, essentially all of the data on the right and left flanks are well-matched (i.e., the "dirty sand" power law relation and its correction function, respectively). Similarly, in Fig. 2.7b (semilog format), we observe an excellent distribution of the power law model across the body of the data. We can also note the "constant $c$-function" lines which provide orientation as to the influence of the $c$-function, as well as the constant " $F$-function" lines which indicate the behavior of the Formation Factor relative to this correlation and data set. The error analysis for this case is shown in Fig. 2.8 and all of the component plots suggest that the $C P R-B$ model has given excellent performance with the only significant errors/deviations in the lower permeability values.

The CPR-C model — the Modified Archie "Dirty Sand" Model (exponential base) is shown in Figs. 2.9 and 2.10 and, as with the $C P R-B$ model, we note very good performance of the base function - in this case an exponential relation. In particular, Fig. 2.9b illustrates the excellent conformance of the $C P R-C$ model for this case (again recalling that this model has an exponential relationship with porosity as its basis). In Fig. 2.10 we review the error analyses plots for this case, and we note good performance (visually) in terms of the correlation of the results using model CPR-C. We do note that in this case, the overall (statistical) error is somewhat high, but we have to remember that we are proposing "characteristic models" in this work, and as such, this case appears (again, based on visual inspections) to have good clustering of the correlated results. Also, the $c$-function in this case (see Fig. 2.10c) is not a correction term, but rather the instantaneous intercept for the exponential basis. Again, we are approaching this work from the perspective that the primary value is the characteristic model, not the statistical "best fit" correlation. We believe that the $C P R-C$ model has performed well for this case, and the plots in Fig. 2.10 confirm the value of this model as a characteristic relation.

We next apply the Weighted Power Law-Exponential Models - Model CPR-DxL (linear weights) and Model $C P R-D x Q$ (quadratic weights). The base results using Models $C P R-D x L$ and $C P R-D x Q$ are presented in Fig. 2.11 - where the power law and exponential basis functions are simply fitted to the 
appropriate portion of the data. The power law equation represents the "Archie clean sand" trend and is fitted to the leftmost data as shown in Fig. 2.11a. In contrast, the exponential equation is thought to represent the "Archie dirty sand" trend and the exponential is fitted to the far rightmost portion of the data (see Fig. 2.11b).

In Figs. 2.12 and 2.13 we present the error analysis which includes the weighting functions (Eqs. 2.9 or 2.10) as appropriate. In Fig. 2.12a we note a fairly good correlation of permeability, and in Fig. 2.12b we note that the results obtained using Model CPR-DxL does "drift" slightly from the GRACE solution, indicating some inconsistency. The computed weight function $(x)$ shown for the $C P R-D x L$ model in Fig. 2.12c does have more scatter than expected, but the trend is centered on the perfect correlation line. The results obtained using Model $C P R-D x Q$ are presented in Fig. 2.13, and we note substantially improved behavior over that of Model $C P R-D x L$.

In Fig. 2.14 we present the results of the "Modified Timur Model" which is essentially just a generalized power function relation in terms of $k, \phi$, and $F$. Somewhat surprisingly, as shown in Figs. 2.14a and 2.14b, the "Modified Timur Model" provides the best correlation of the data for this case.

In Fig. 2.15 we present the results predicted by the GRACE algorithm, which is a non-parametric regression approach (see Xue, et al (1997) for details). In theory, the GRACE algorithm should provide the most unbiased correlation of the data - i.e., the GRACE algorithm is designed not to "fit the errors" as other regression approaches may. It is our contention that the GRACE algorithm is the statistical standard — and any algorithm/approach/model which achieves better regression statistics than the GRACE algorithm is actually "fitting the errors" in the data. The only case which has significantly better regression statistics than the GRACE algorithm is the "Modified Timur Model" — hence, we must label this case as "over-fitted" in a statistical sense.

Our final graphic, Fig. 2.16, illustrates all of the models on a single plot of calculated versus measured permeability. We also present a table of all statistical results in Table 2.1 below.

Table 2.1 — Statistical Results for all Models (Lower Wilcox Case, $n=62$ ).

\begin{tabular}{|c|c|c|c|c|}
\hline Model & $\begin{array}{c}\text { Sum of } \\
\text { Squared } \\
\text { Residuals } \\
{\left[\log (k)^{2}\right]}\end{array}$ & $\begin{array}{l}\text { Variance } \\
{\left[\log (k)^{2}\right]}\end{array}$ & $\begin{array}{c}\text { Standard } \\
\text { Deviation } \\
{[\log (k)]}\end{array}$ & $\begin{array}{c}\text { Absolute } \\
\text { Relative } \\
\text { Error } \\
\text { [percent] }\end{array}$ \\
\hline$C P R-A$ & 48.89 & 2.08 & 1.44 & 86.39 \\
\hline$C P R-B$ & 48.78 & 2.11 & 1.45 & 90.10 \\
\hline$C P R-C$ & 57.32 & 2.20 & 1.48 & 99.03 \\
\hline$C P R-D x L$ & 51.74 & 2.36 & 1.54 & 102.77 \\
\hline$C P R-D x Q$ & 49.10 & 2.15 & 1.46 & 93.05 \\
\hline Modified Timur & 47.83 & 2.11 & 1.45 & 84.41 \\
\hline GRACE Algorithm & 50.47 & 2.17 & 1.47 & 88.14 \\
\hline
\end{tabular}




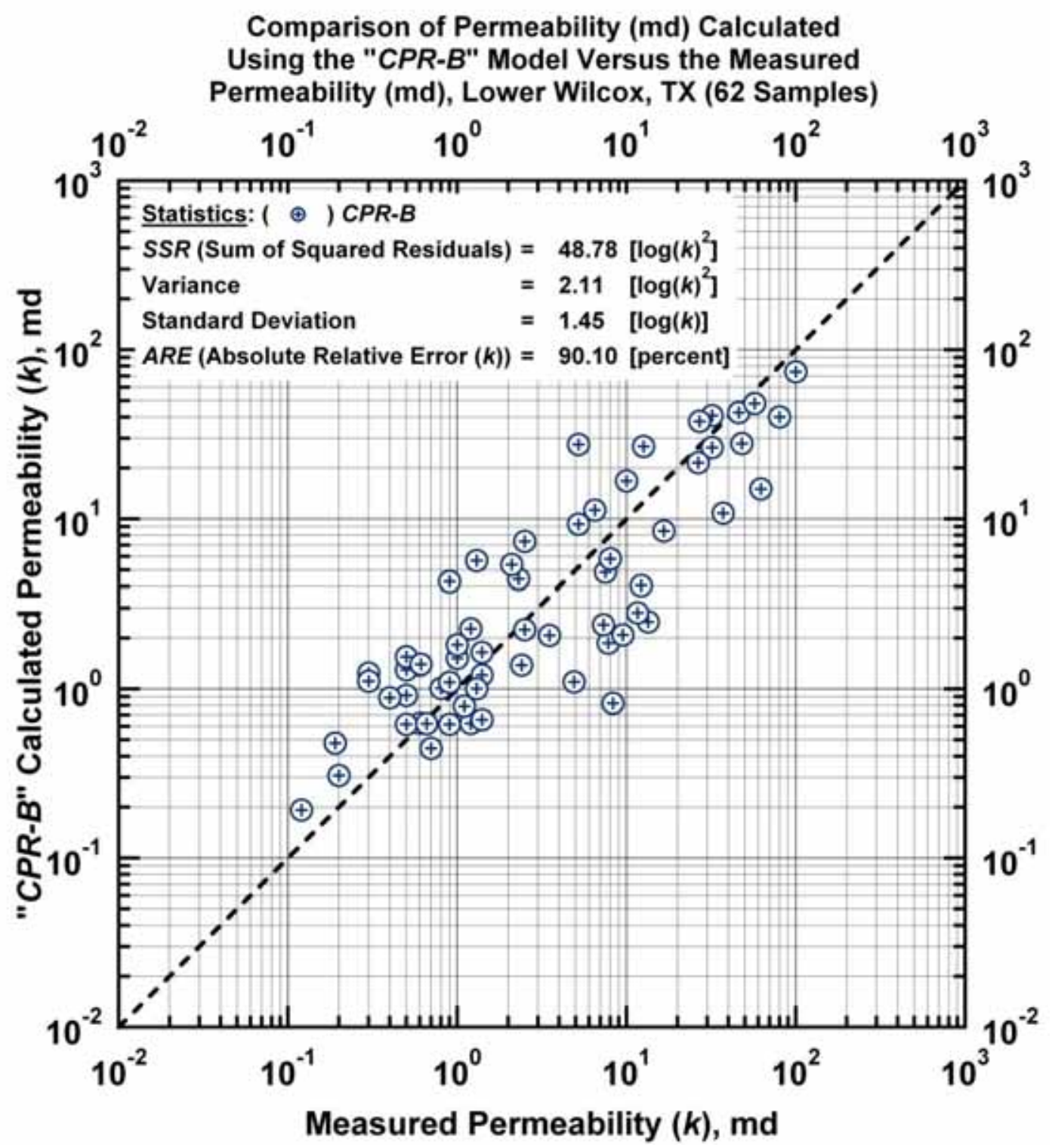

Figure 2.8a - Model $C P R-B$ : calculated versus measured permeability. [Case: Lower Wilcox S. TX (USA)] 


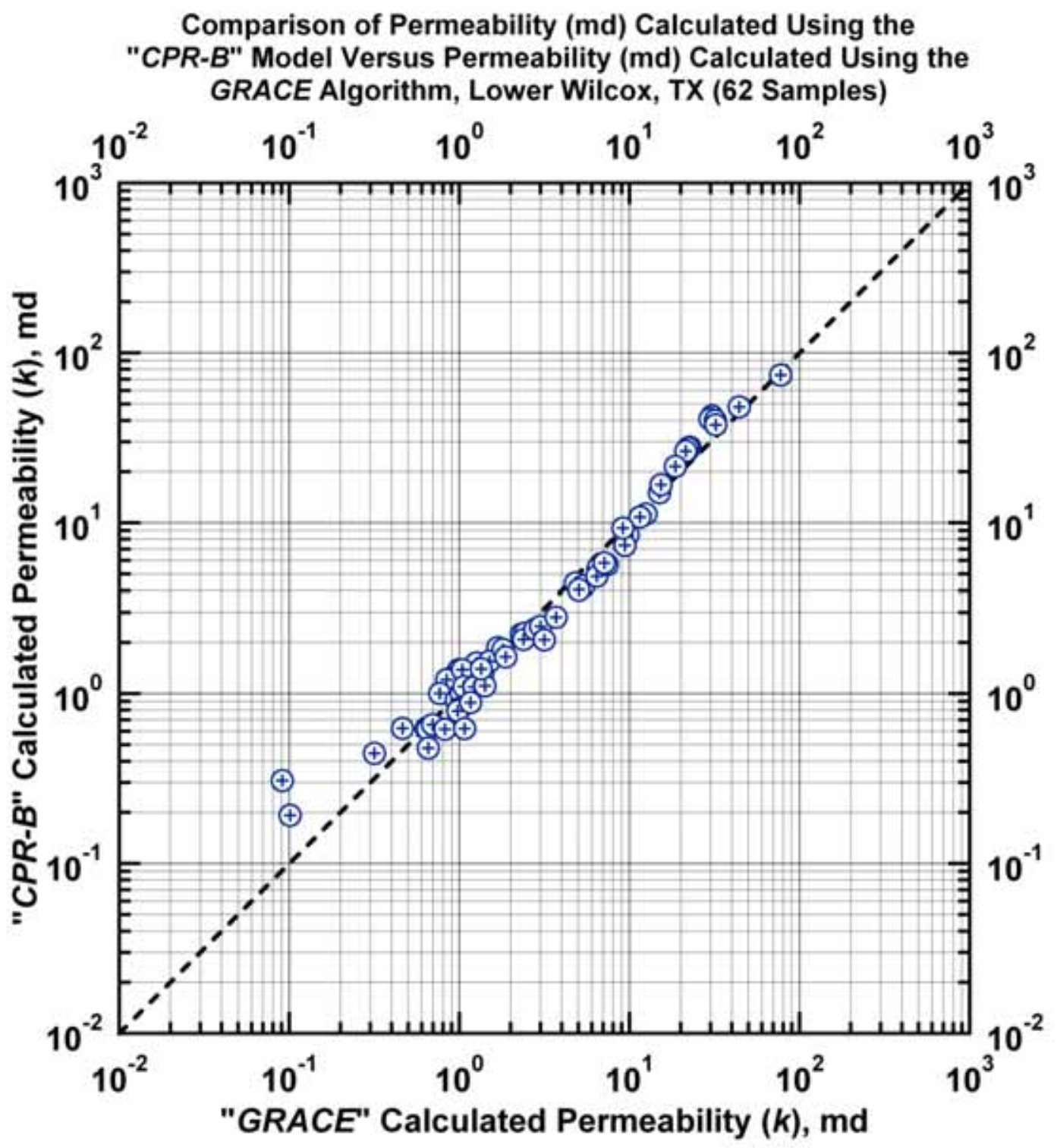

Figure 2.8b - Model $C P R-B$ : Permeability calculated using Model $C P R-B$ versus permeability calculated using the GRACE Algorithm. [Case: Lower Wilcox S. TX (USA)] 


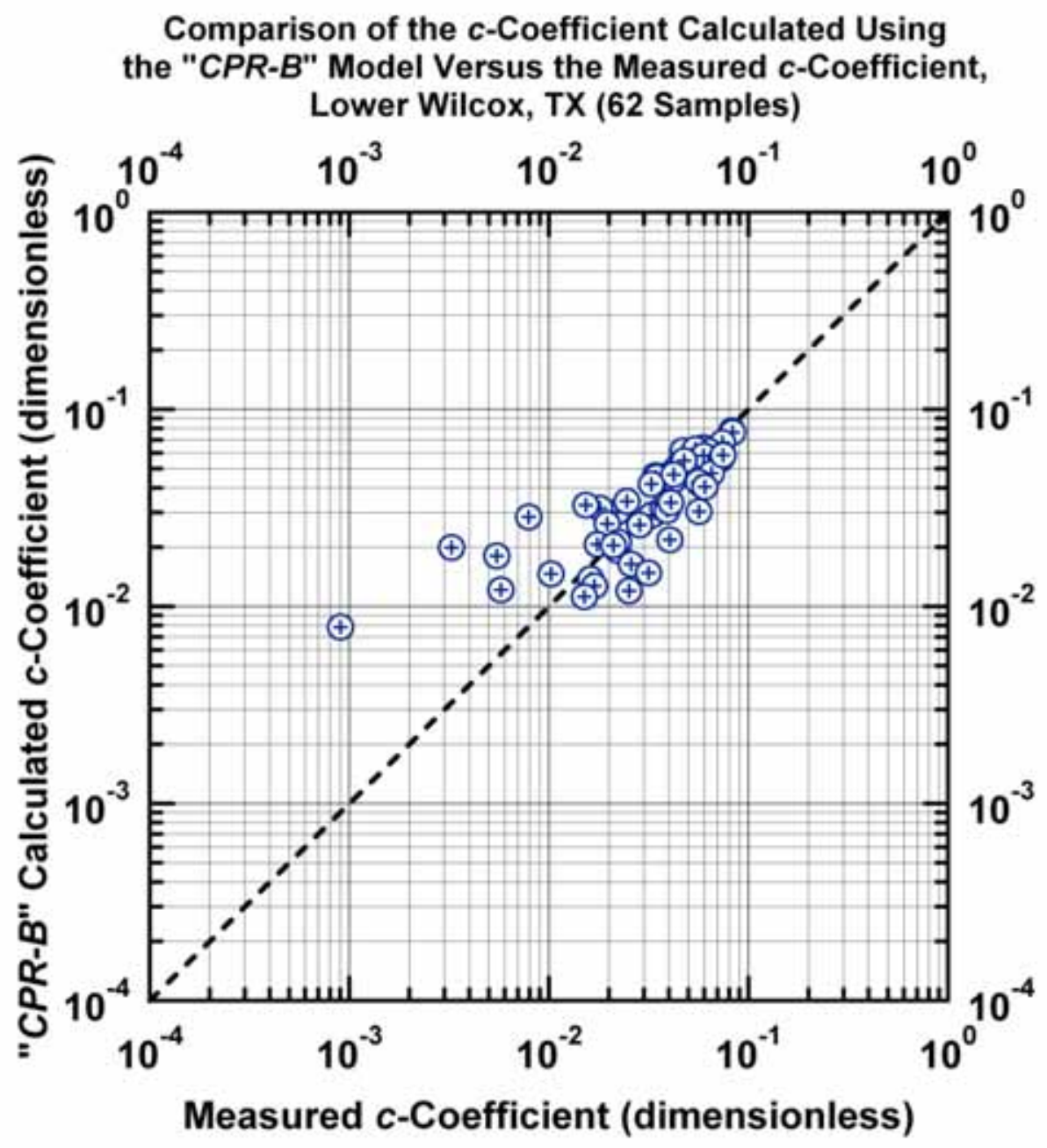

Figure 2.8c - Model $C P R$ - $B$ : Calculated $c$-function values versus measured $c$-function values. [Case: Lower Wilcox S. TX (USA)] 


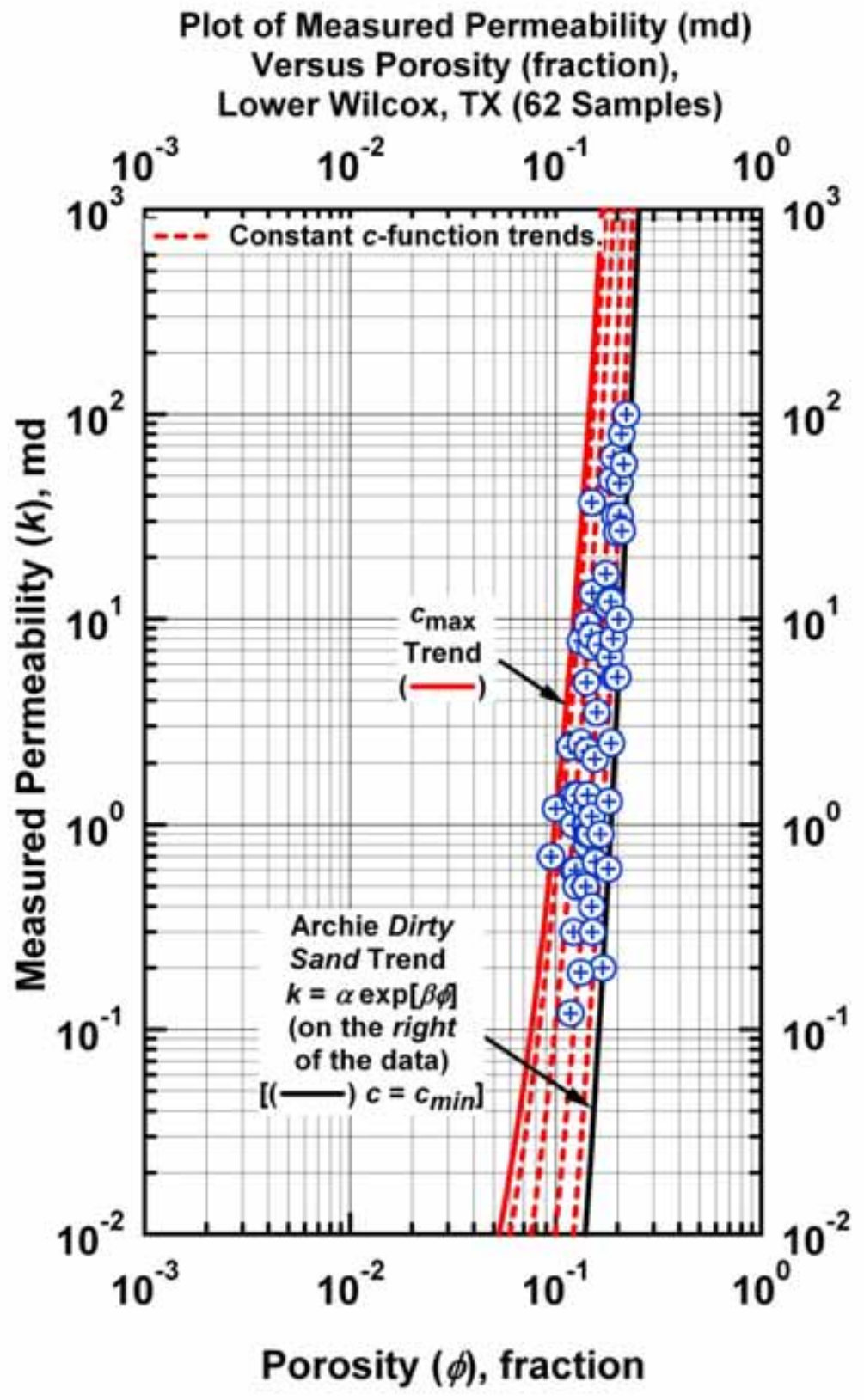

Figure 2.9a - Model $C P R-C: \quad k=c \exp [\beta \phi] \quad c=c_{\max } \exp \left[-c_{1} \phi^{c_{2}} F^{c_{3}}\right]$ - "Clean Sand" Plot (log-log format) - Archie "Dirty Sand" trend is given by the curved trend at the far right of the data (exponential model). [Case: Lower Wilcox S. TX (USA)] 


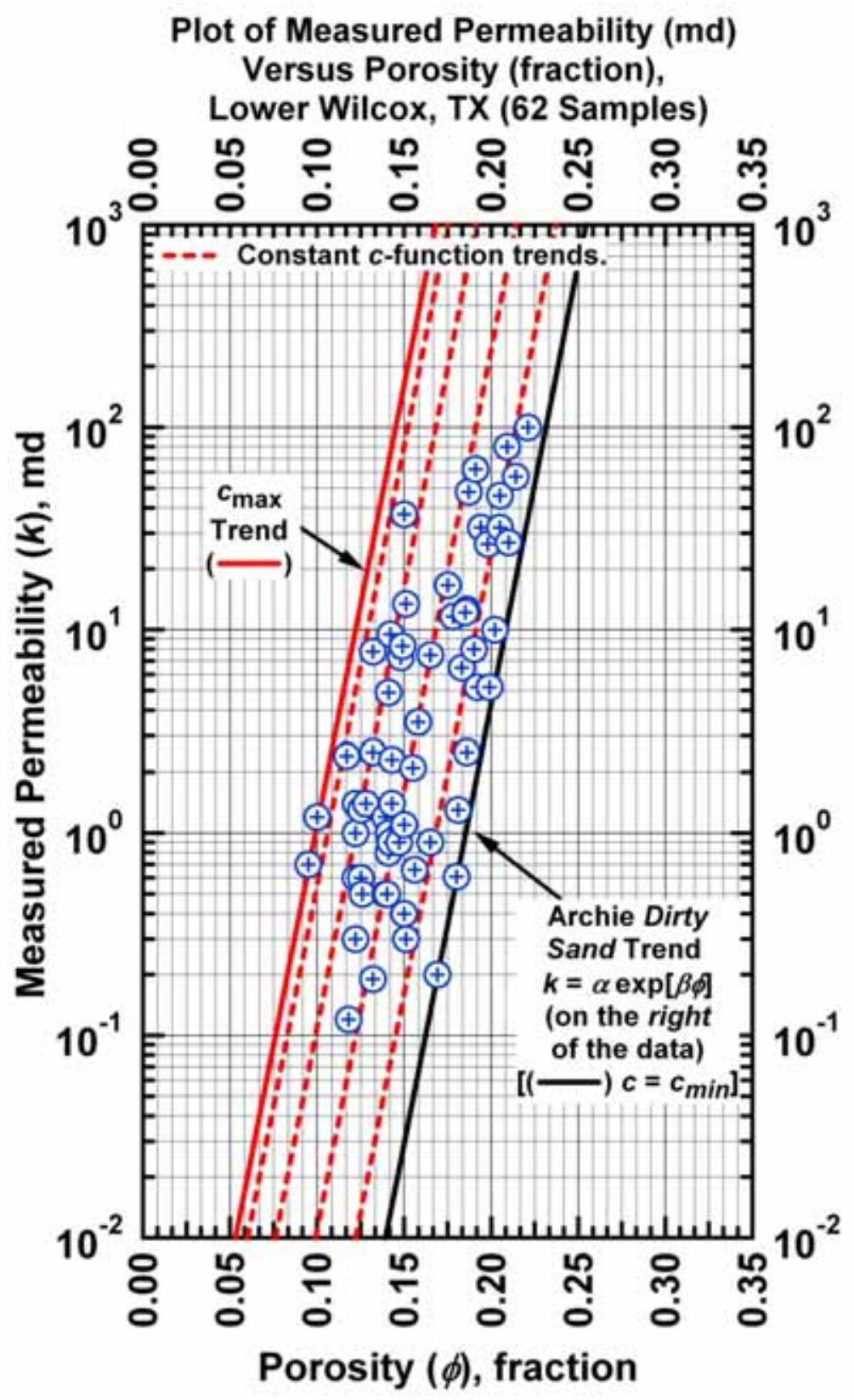

Figure 2.9b - Model $C P R-C: \quad k=c \exp [\beta \phi] \quad c=c_{\max } \exp \left[-c_{1} \phi^{c_{2}} F^{c_{3}}\right]$ - "Dirty Sand" Plot (semilog format) — Archie "Dirty Sand" trend is given by the straight-line trend at the far right of the data (exponential model). [Case: Lower Wilcox S. TX (USA)] 
Comparison of Permeability (md) Calculated Using the "CPR-C" Model Versus the Measured Permeability (md), Lower Wilcox, TX (62 Samples)

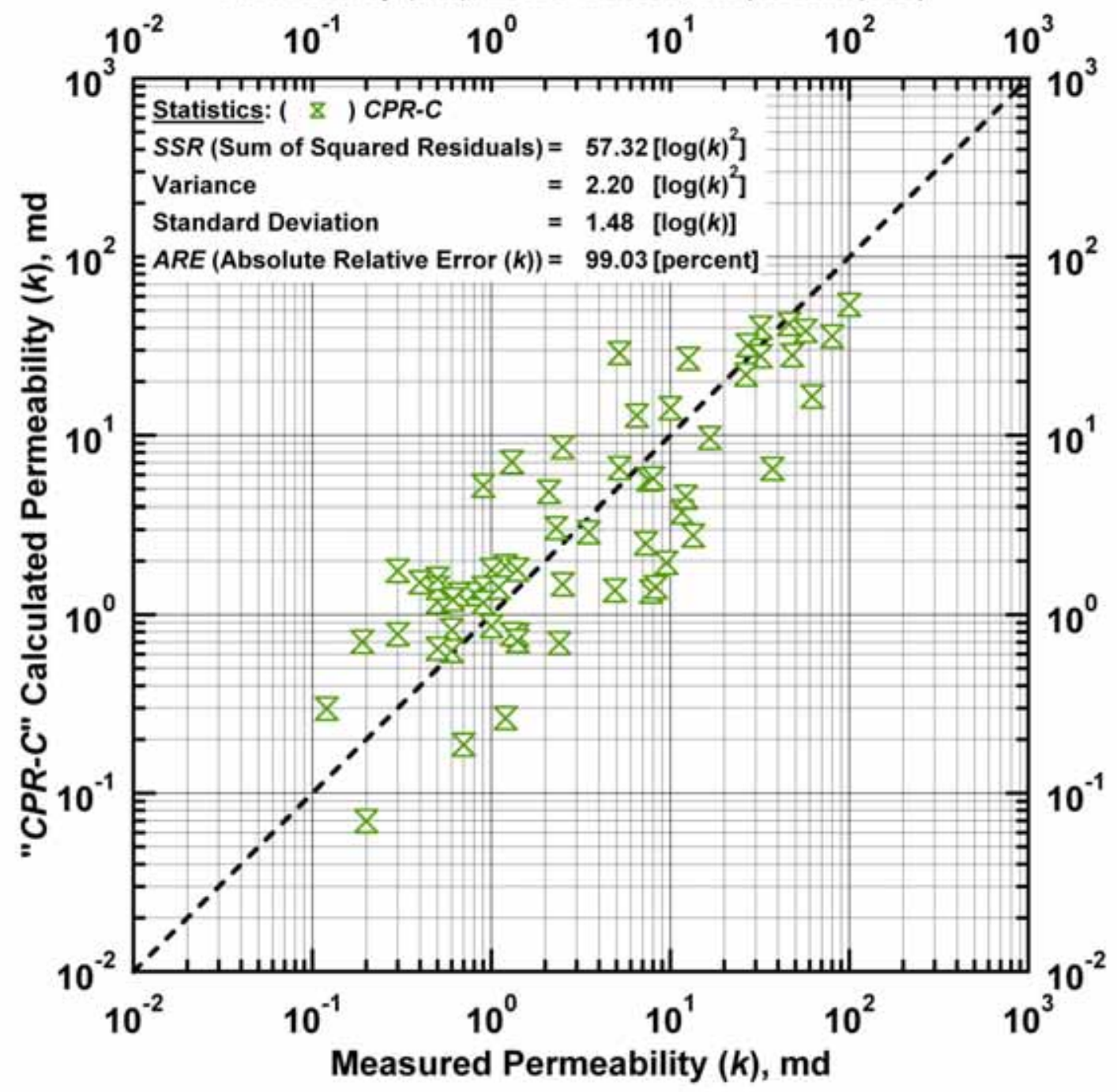

Figure 2.10a - Model CPR-C: calculated versus measured permeability. [Case: Lower Wilcox S. TX (USA)] 


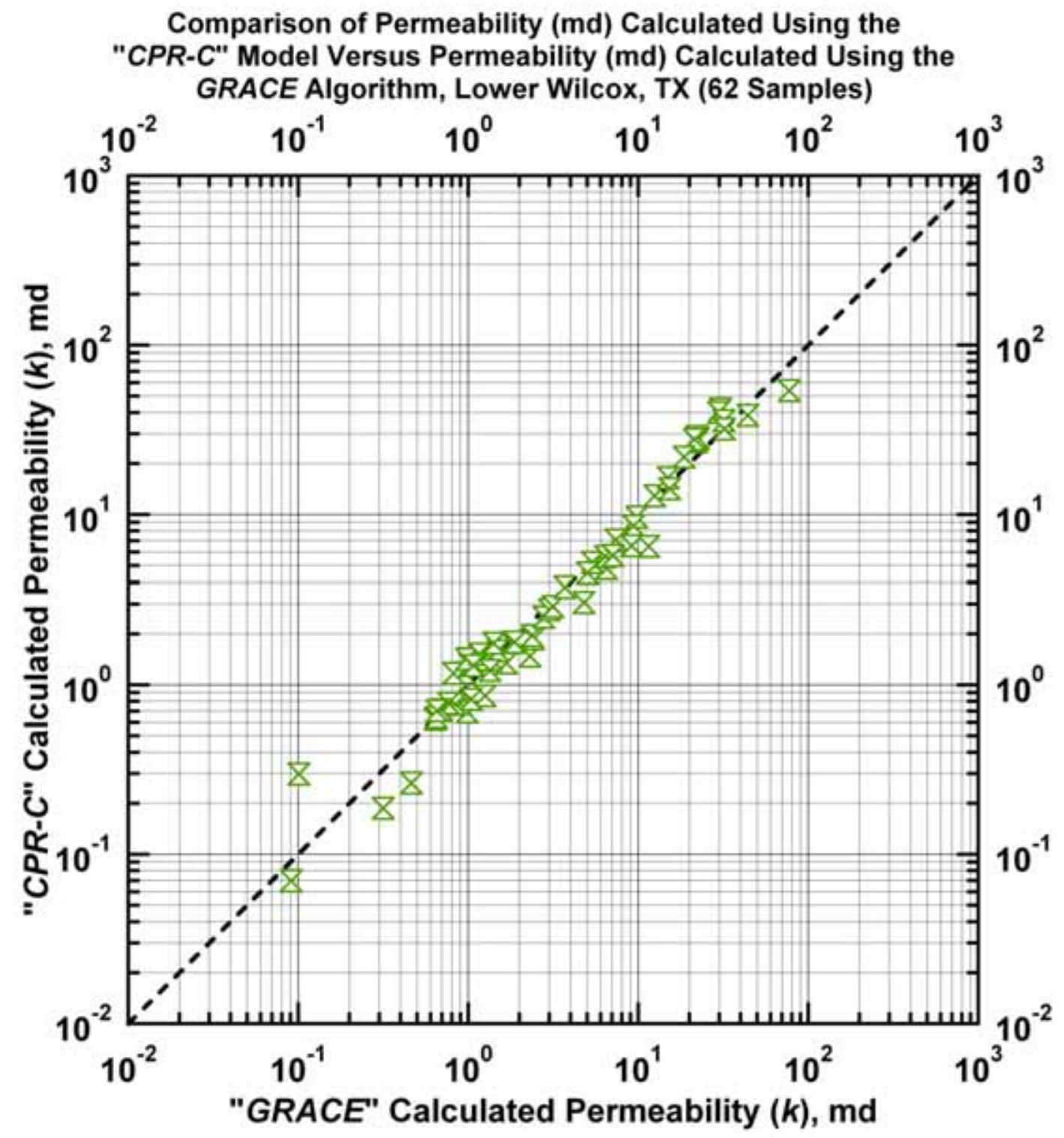

Figure 2.10b - Model $C P R-C$ : Permeability calculated using Model $C P R-C$ versus permeability calculated using the GRACE Algorithm. [Case: Lower Wilcox S. TX (USA)] 


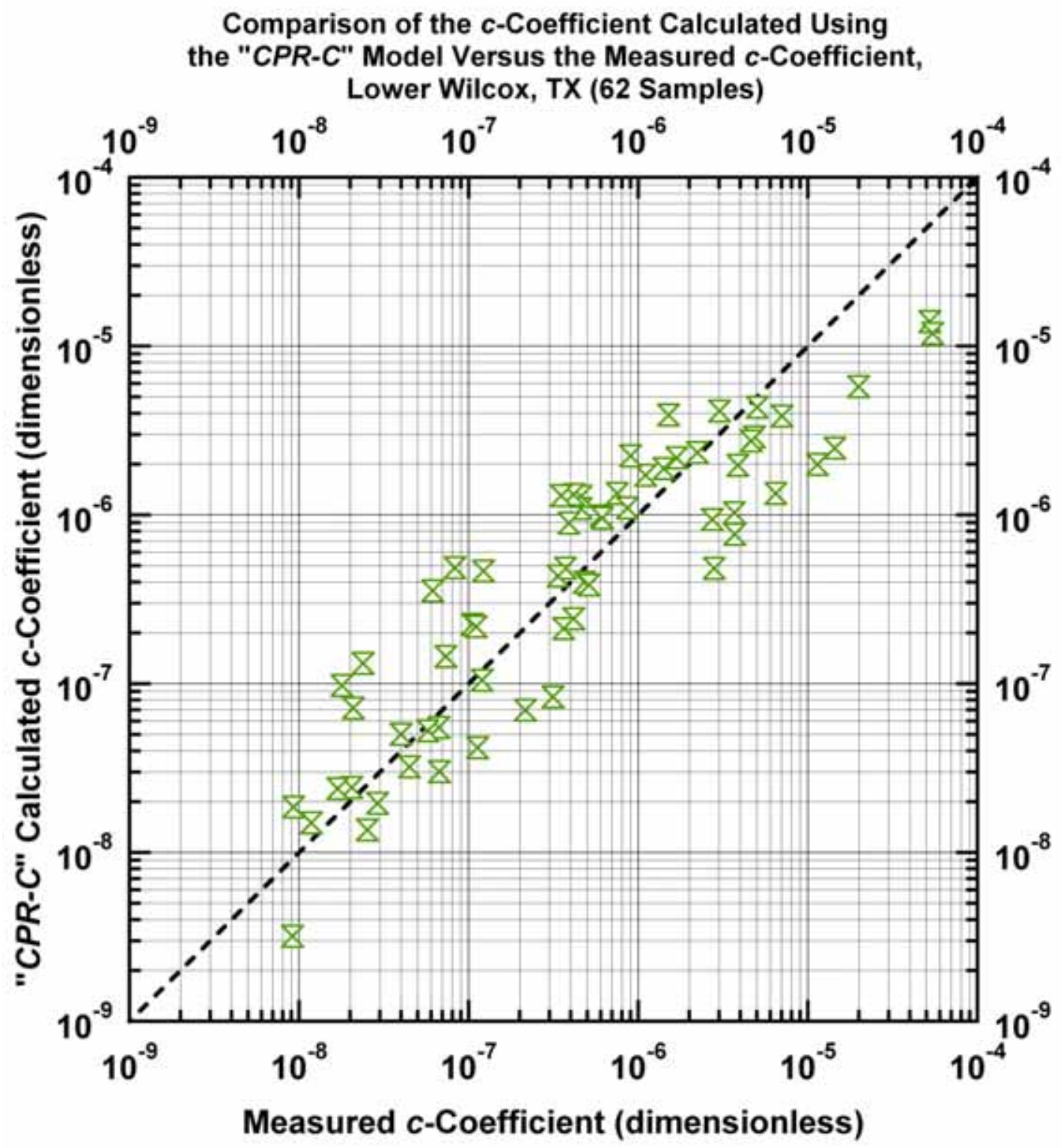

Figure 2.10c - Model $C P R$ - $C$ : Calculated $c$-function values versus measured $c$-function values. [Case: Lower Wilcox S. TX (USA)] 


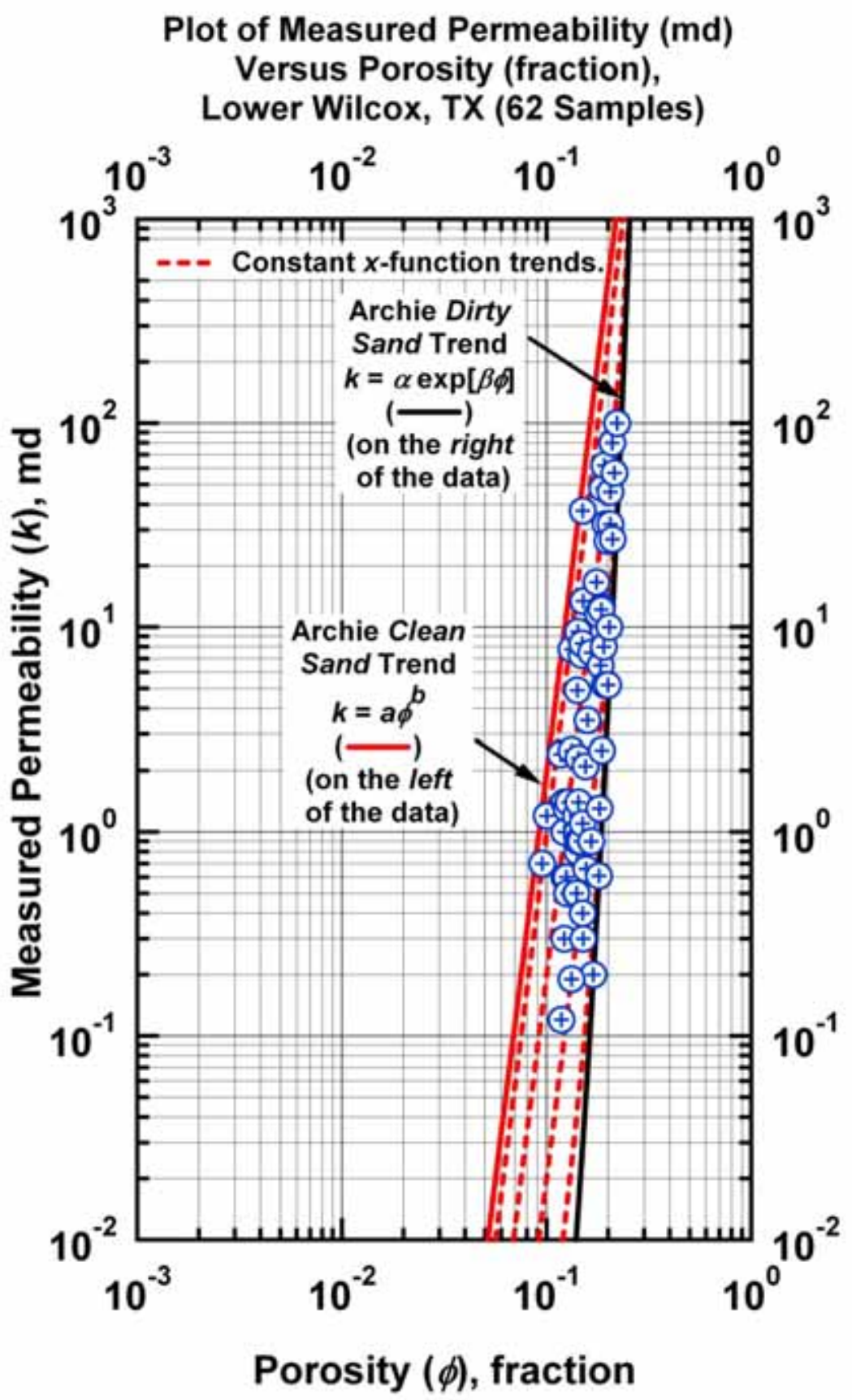

Figure 2.11a - Model CPR-D: $k=x a \phi^{b}+(1-x) \alpha \exp [\beta \phi] \quad[0 \leq x \leq 1]$ - Weighted Power Law-Exponential Model used to correlate permeability $(k)$ and porosity $(\phi)$. "Clean Sand" Plot - Archie "Clean Sand" trend is given by the straight-line trend at the far left of the data (power law model). [Case: Lower Wilcox S. TX (USA)] 


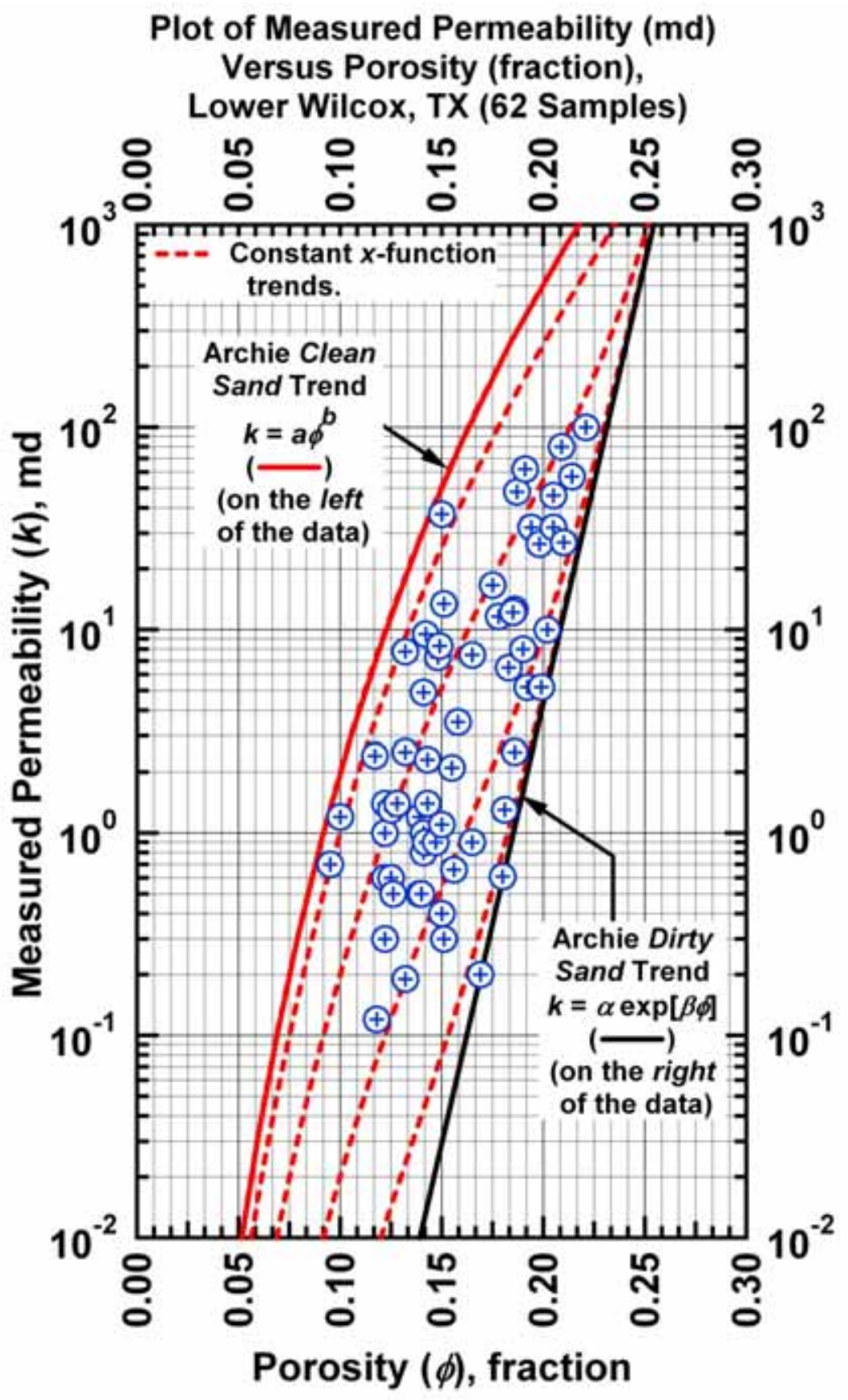

Figure 2.11b - Model CPR-D: $k=x a \phi^{b}+(1-x) \alpha \exp [\beta \phi] \quad[0 \leq x \leq 1]$ - Weighted Power Law-Exponential Model used to correlate permeability $(k)$ and porosity $(\phi)$. "Dirty Sand" Plot - Archie "Dirty Sand" trend is given by the straight-line trend at the far right of the data (exponential model). [Case: Lower Wilcox S. TX (USA)] 


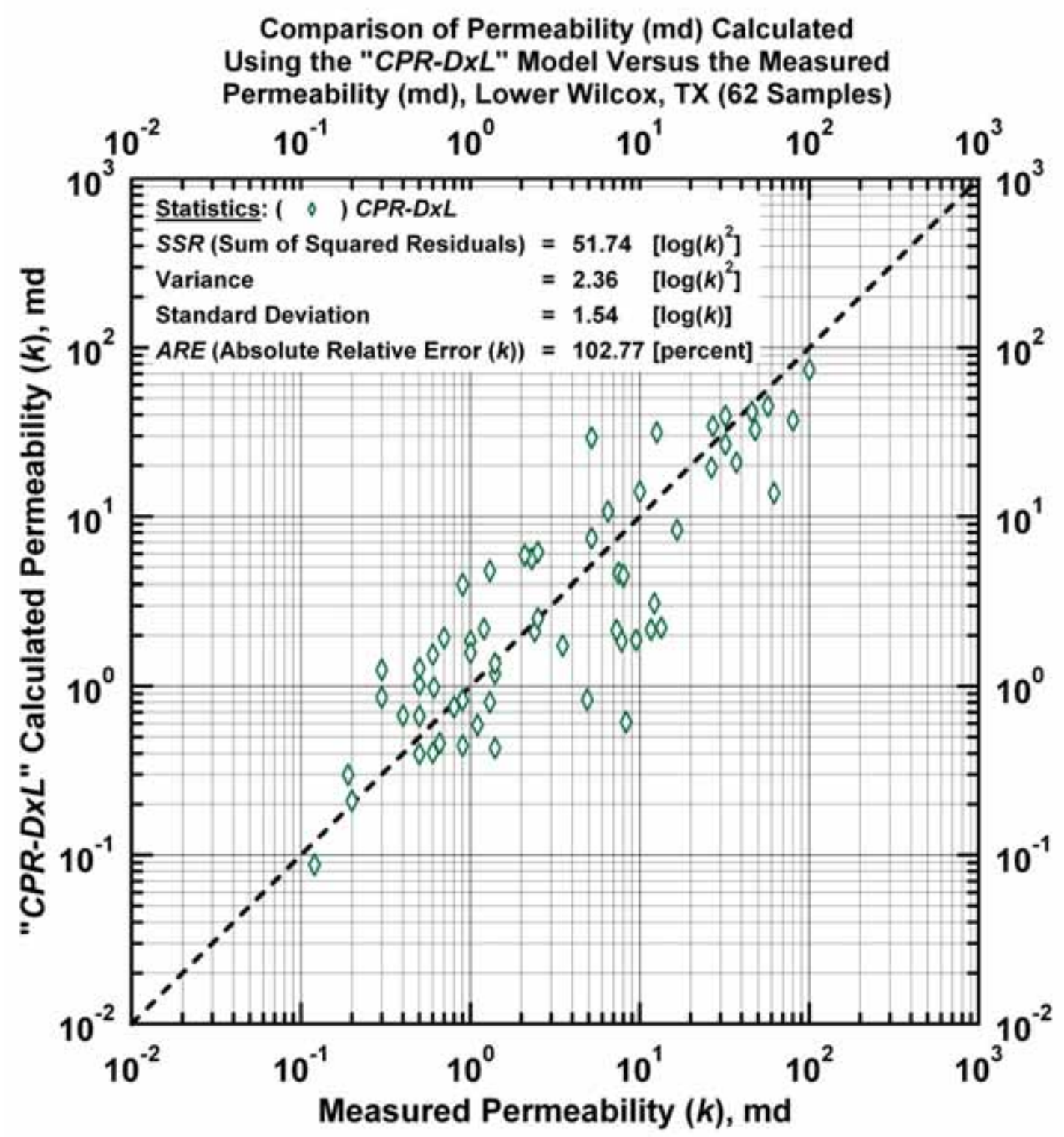

Figure 2.12a - Model CPR-DxL: Calculated versus measured permeability. [Case: Lower Wilcox S. TX (USA)] 


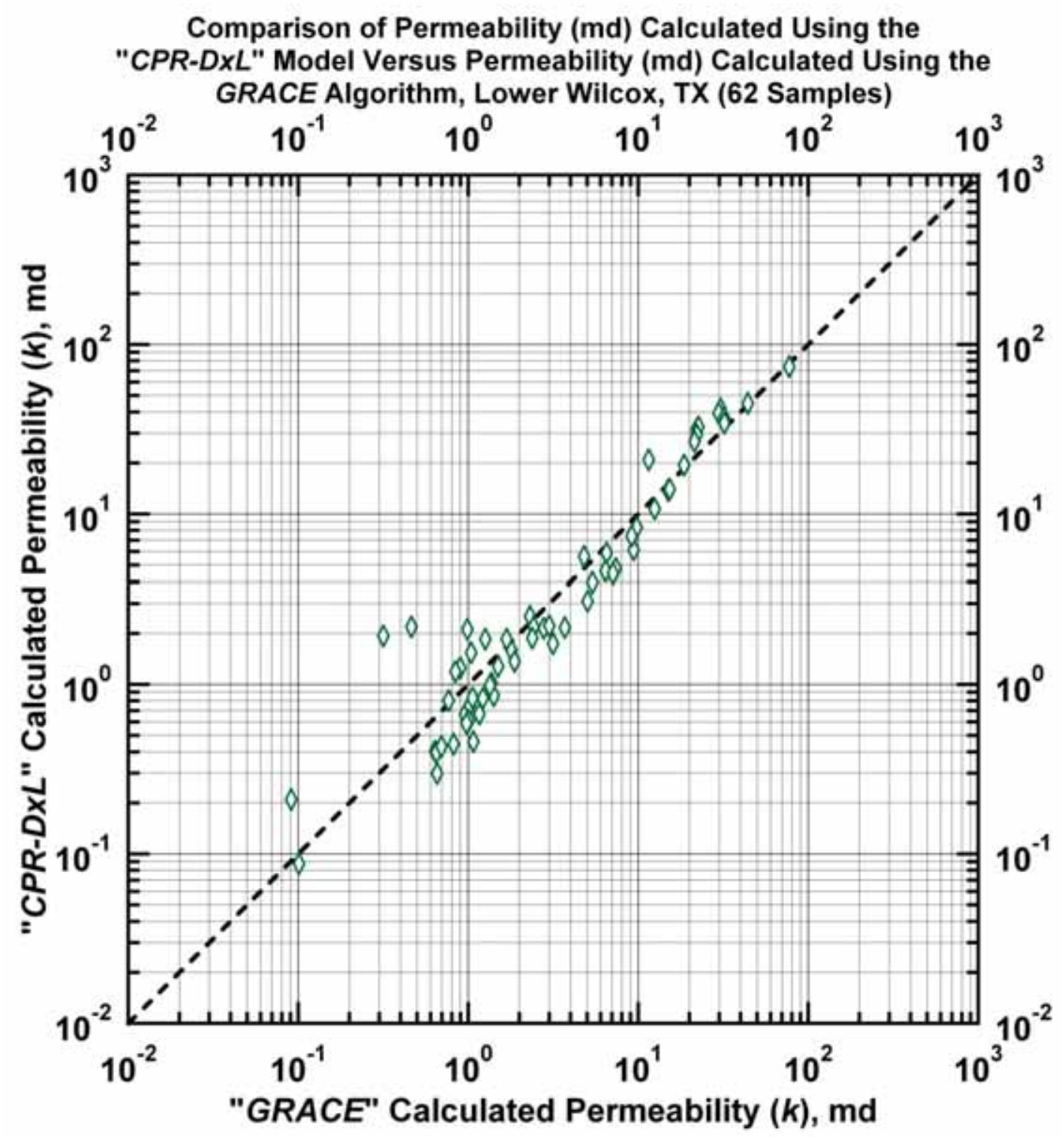

Figure 2.12b - Model $C P R-D x L$ : Permeability calculated using Model $C P R-C$ versus permeability calculated using the GRACE Algorithm. [Case: Lower Wilcox S. TX (USA)] 
Comparison of the $x$-Coefficient Calculated Using the "CPR-DxL" Model Versus the Measured $x$-Coefficient, Lower Wilcox, TX (62 Samples)

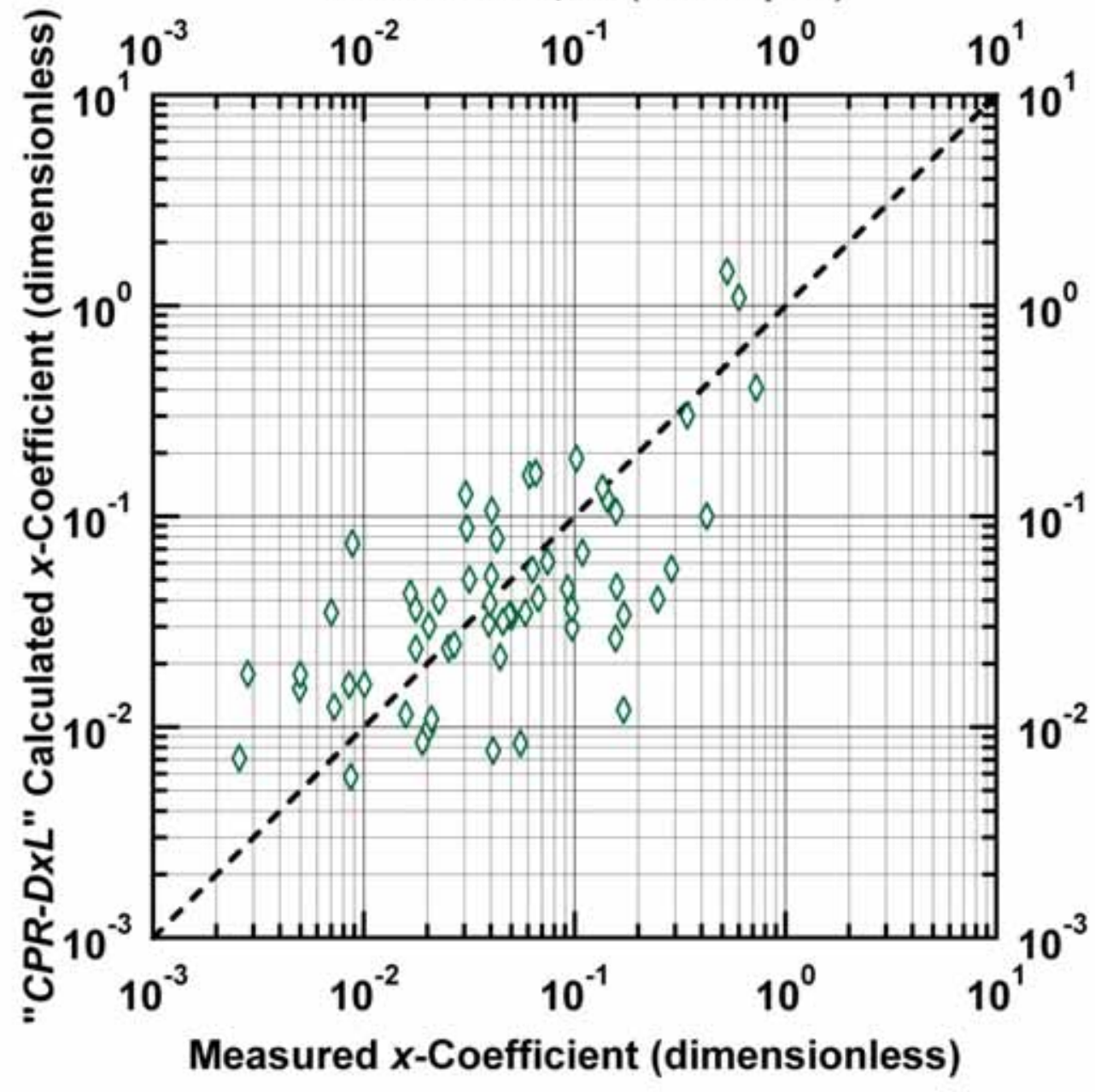

Figure 2.12c - Model CPR-DxL: Calculated $x$-function weight values versus measured $x$ function weight values. [Case: Lower Wilcox S. TX (USA)] 
Comparison of Permeability (md) Calculated Using the "CPR-DxQ" Model Versus the Measured Permeability (md), Lower Wilcox, TX (62 Samples)

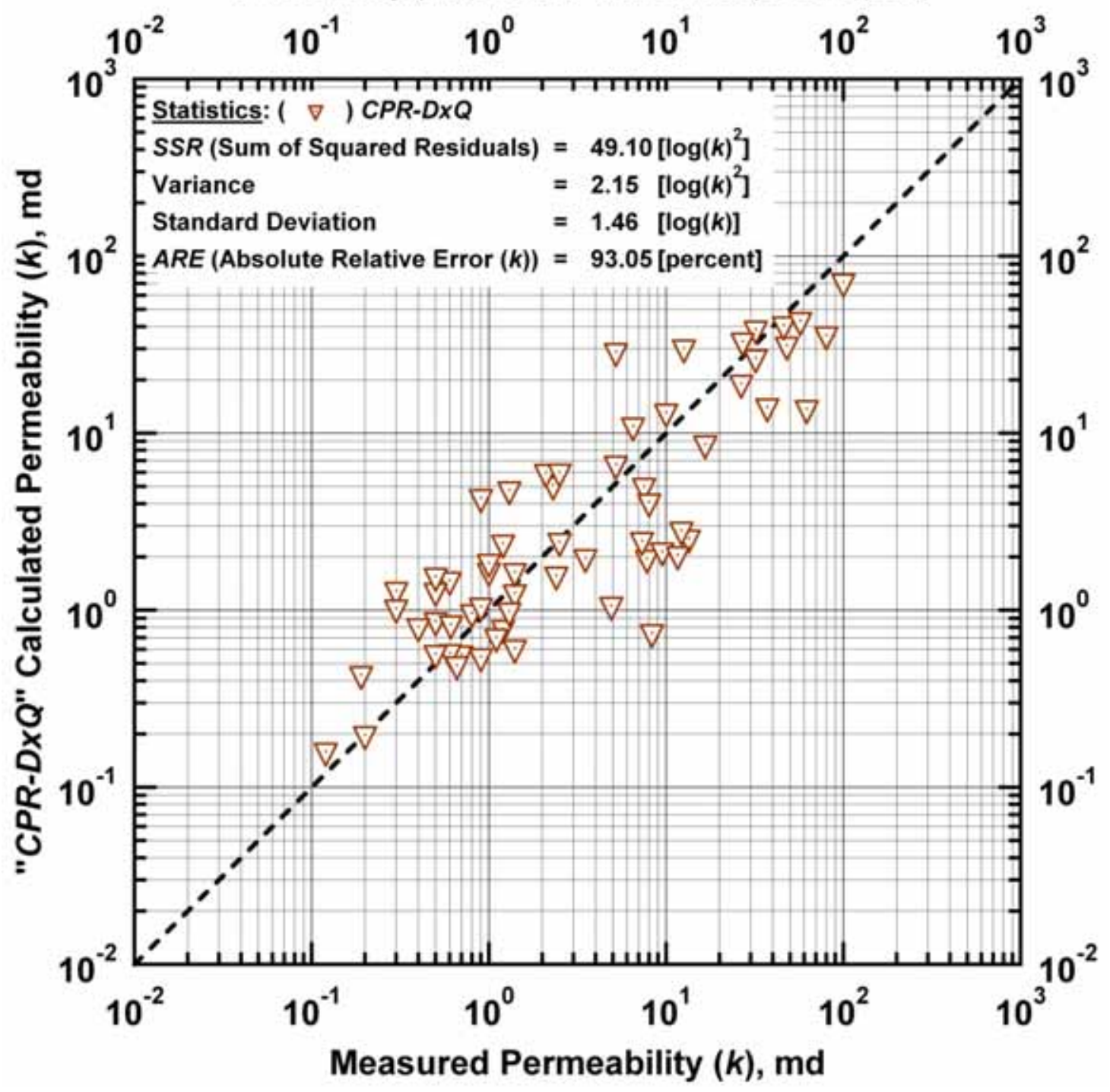

Figure 2.13a - Model CPR-DxQ: Calculated versus measured permeability. [Case: Lower Wilcox S. TX (USA)] 


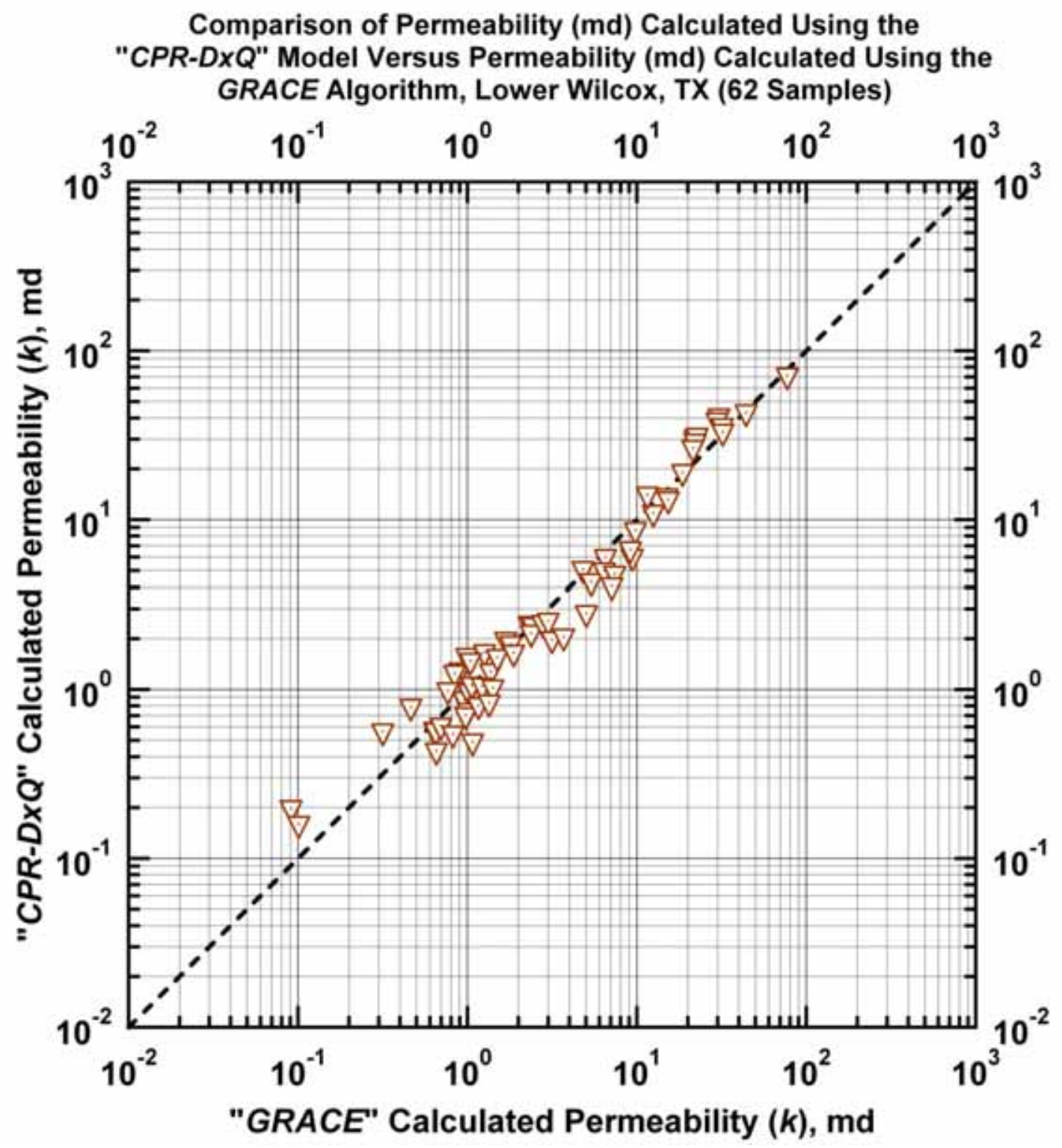

Figure 2.13b - Model $C P R-D x Q$ : Permeability calculated using Model $C P R-C$ versus permeability calculated using the GRACE Algorithm. [Case: Lower Wilcox S. TX (USA)] 


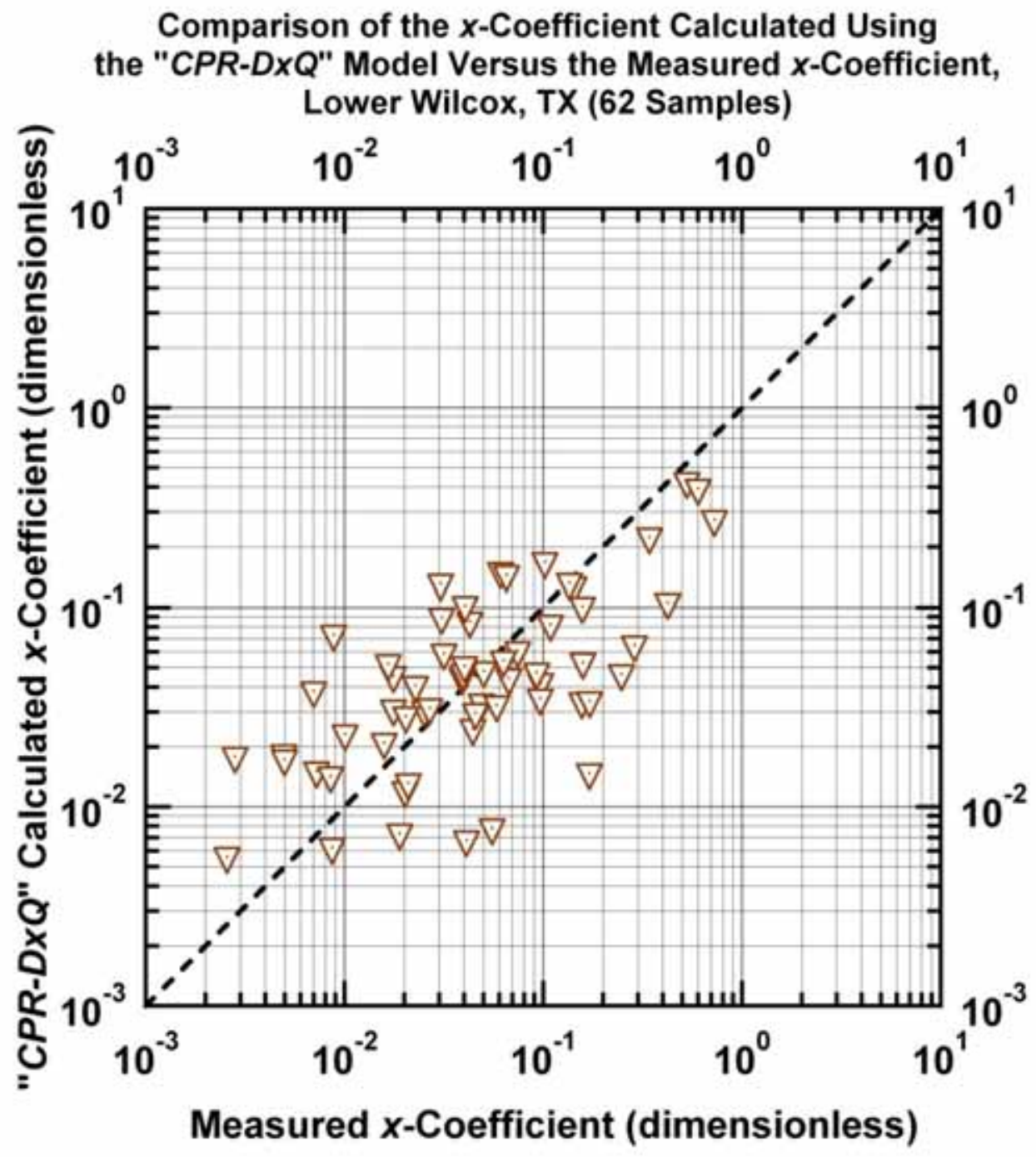

Figure 2.13c - Model $C P R-D x Q$ : Calculated $x$-function weight values versus measured $x$ function weight values. [Case: Lower Wilcox S. TX (USA)] 


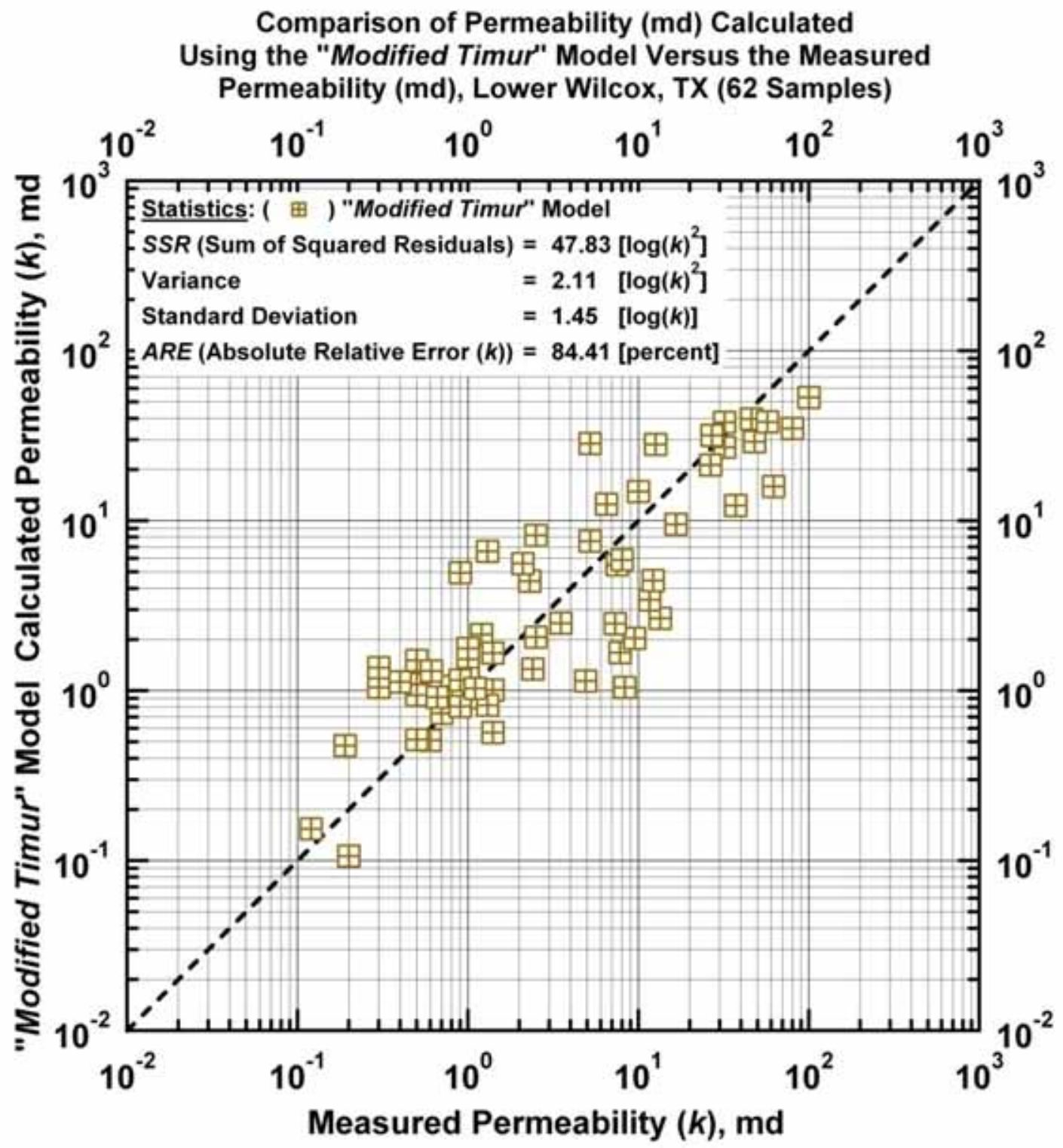

Figure 2.14a - "Modified Timur Model:" Calculated versus measured permeability. [Case: Lower Wilcox S. TX (USA)] 


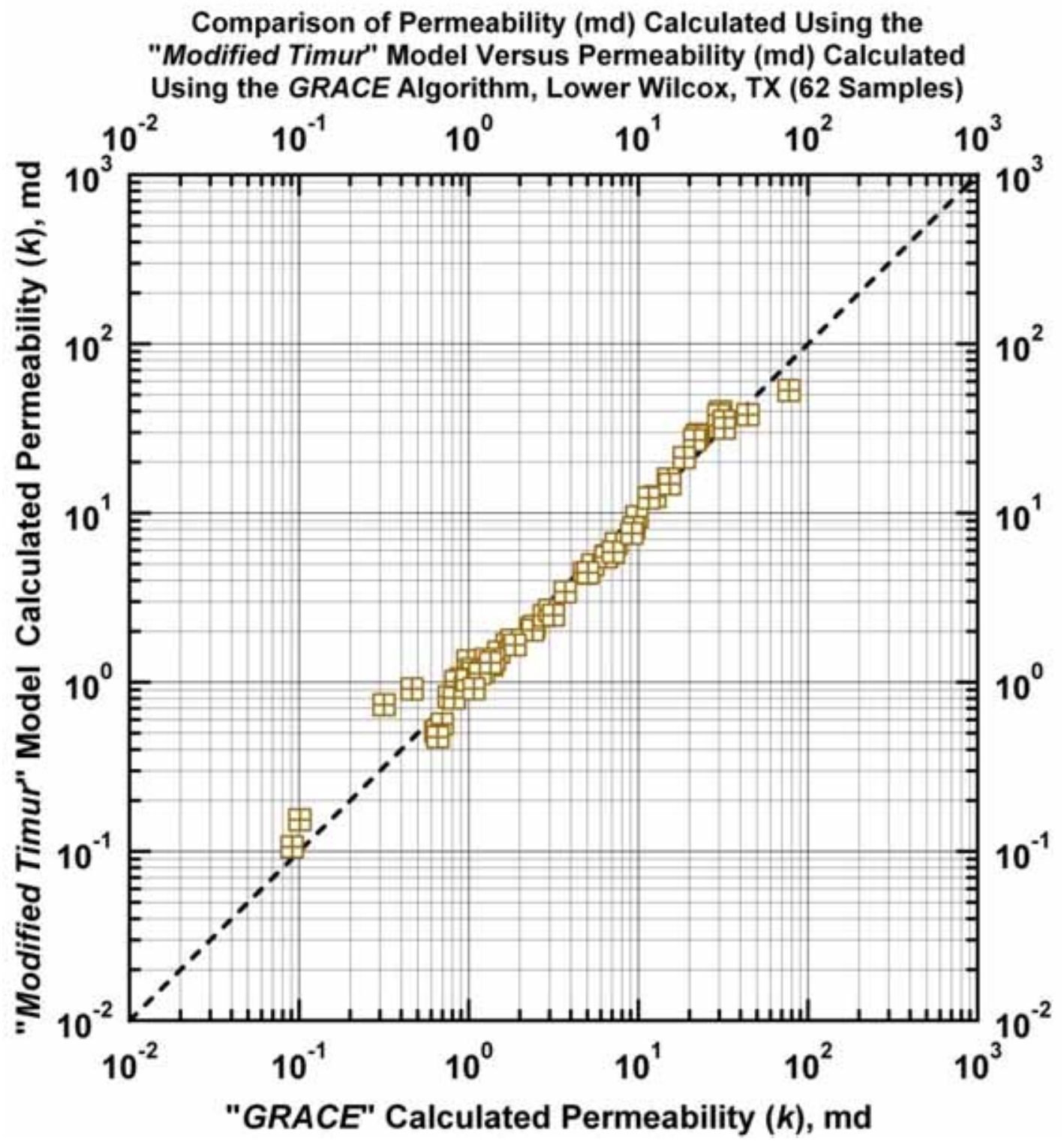

Figure 2.14b — "Modified Timur Model:" Permeability calculated using the "Modified Timur Model" versus permeability calculated using the GRACE Algorithm. [Case: Lower Wilcox S. TX (USA)] 


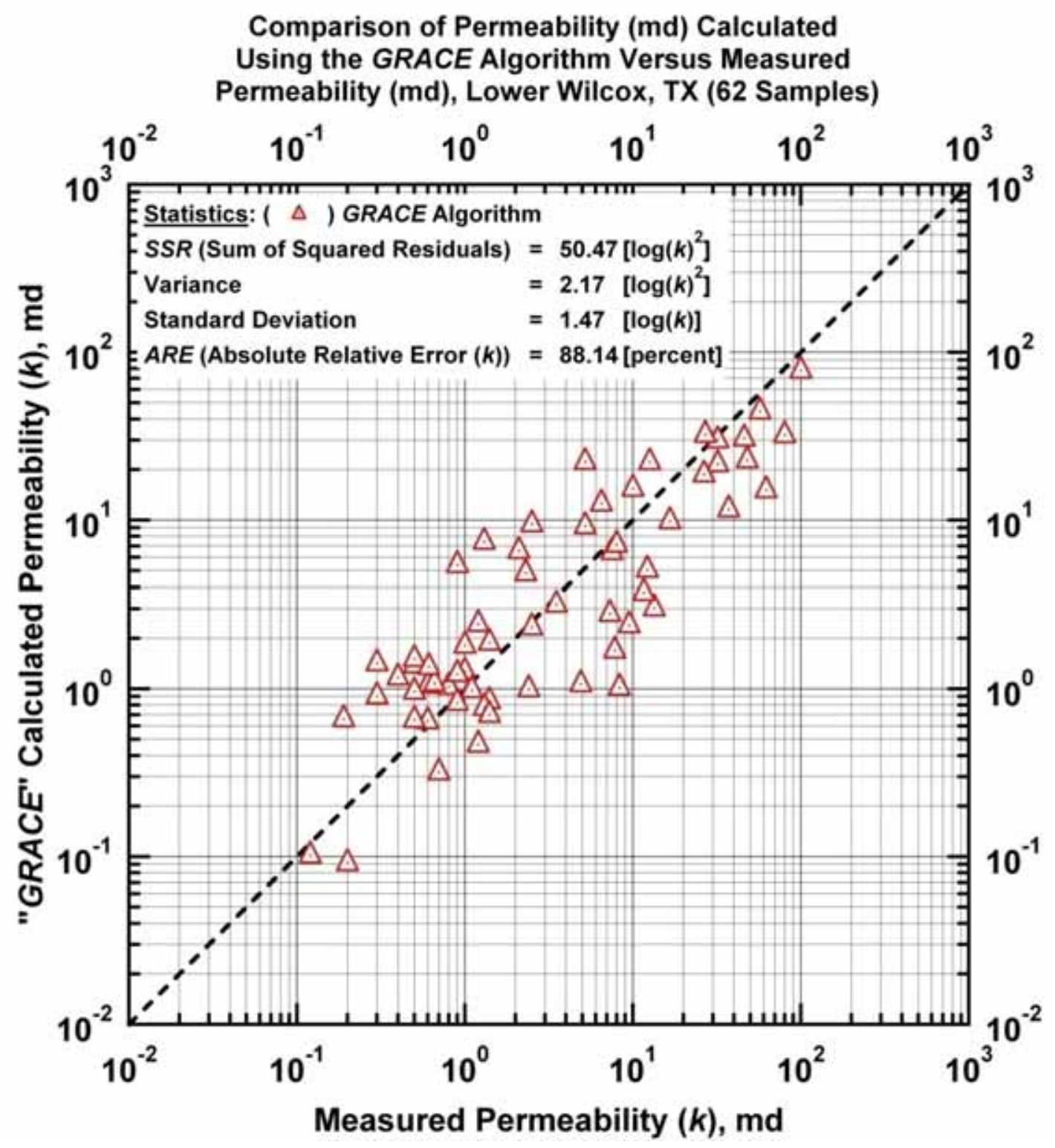

Figure 2.15 - GRACE Model: Calculated versus measured permeability. [Case: Lower Wilcox S. TX (USA)] 


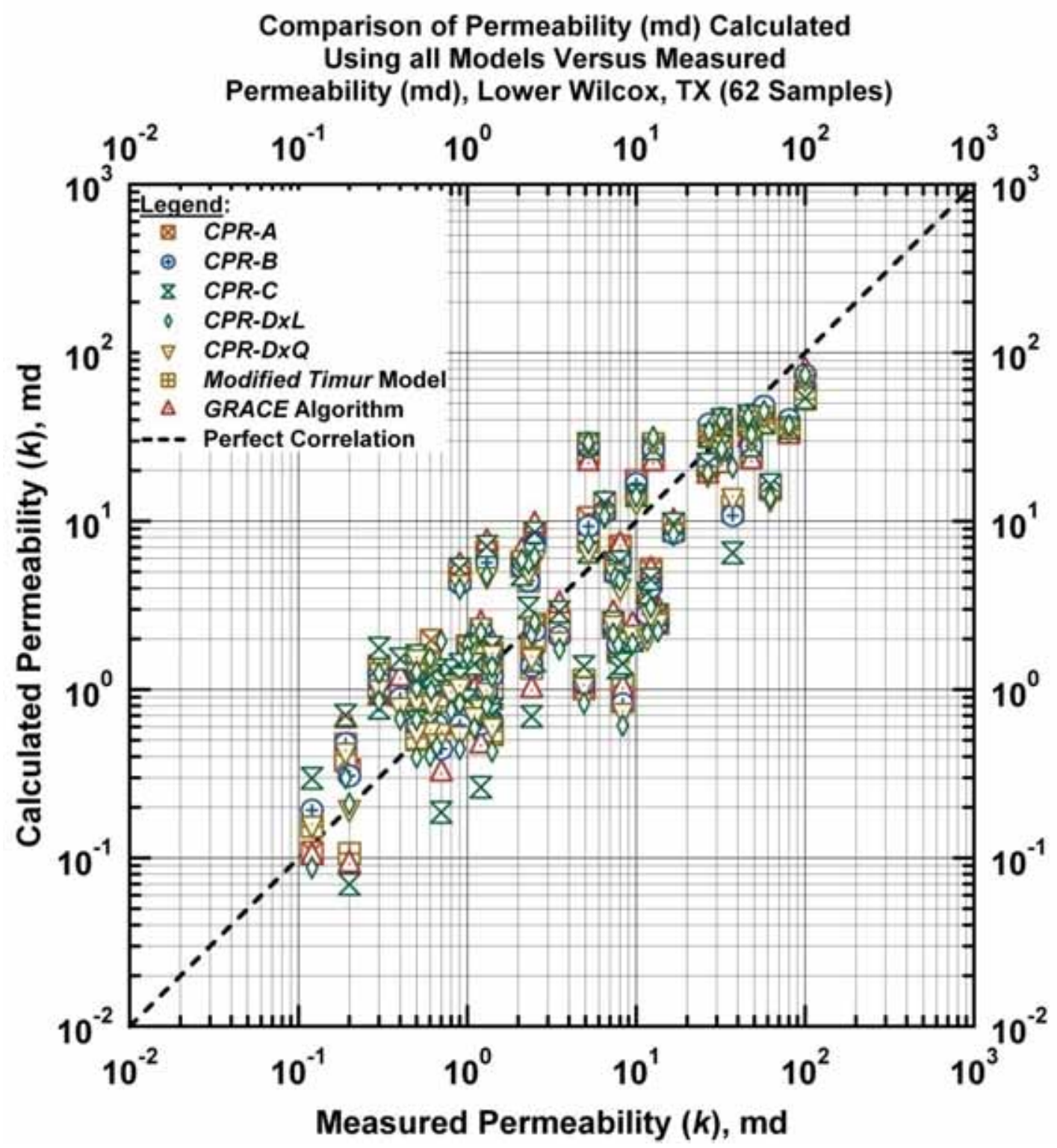

Figure 2.16 - All Models: Calculated versus measured permeability. [Case: Lower Wilcox S. TX (USA)] 


\section{CHAPTER III \\ SUMMARY, CONCLUSIONS, AND RECOMMENDATIONS FOR FUTURE WORK}

\subsection{Summary}

In this work, we have successfully developed, demonstrated, and validated our concept of a characteristic permeability relation $(C P R)$ for several different models where $k=f\left(\phi, S_{w}\right.$ or $\left.F\right)$. Our goal was not to achieve a "universal" $C P R$, but to identify the strengths of each of our proposed relations. Perhaps the most important contribution of this work is the systematic evaluation of each $C P R-$ in particular, the illustration of the dominant (or characteristic) behavior of each CPR.

\subsection{Conclusions}

We present the following conclusions for our characteristic permeability relations (CPRs) (i.e., permeability models):

- Archie "Clean Sand" Model:

$$
k=a \phi^{b}
$$

- Base Plot: $\log (k)$ versus $\log (\phi)$.

- This is the basis relation for all "power law" CPRs.

- This $C P R$ is uniquely suited to highly-sorted unconsolidated sediments.

- CPR-A - Modified Archie "Clean Sand" Model: (Bryant-Finney Model)

$$
k=a(\phi-c)^{b} \quad \text { where } c=c_{\max } \exp \left[-c_{1} \phi^{c_{2}} z^{c 3}\right]
$$

Base Plot: $\log (k)$ versus $\log (\phi)$.

- This $C P R$ represents negative deviation from the Archie "Clean Sand" power law model - i.e., the permeability profile is less than the Archie "Clean Sand" CPR.

- The straight-line power law trend $(c=0)$ must be placed just to the left of the data.

- This $C P R$ has the potential for negative values in the power law argument - therefore, $c_{\max }$ must be chosen carefully. 
- CPR-B - Modified Archie "Dirty Sand" Model: (Power-Law Basis)

$$
k=a(\phi+c)^{b} \text { where } c=c_{\max } \exp \left[-c_{1} \phi^{c_{2}} z^{c_{3}}\right]
$$

Base Plot: $\log (k)$ versus $\log (\phi)$.

- This $C P R$ represents positive deviation from a straight-line power law trend (i.e., $c=0$ ), placed just beyond the far rightmost portion of the data trend.

- The straight-line power law trend $(c=0)$ must be placed just to the right of the data.

- For our data sets, this $C P R$ was the most "universal" in terms of the proposed "simple" power law or exponential relations ( $C P R-D x L$ and $C P R-D x Q$ were often quite comparable to this relation).

- CPR-C - Modified Archie "Dirty Sand" Model: (Exponential Basis)

$$
k=c \exp [\beta \phi] \text { where } c=c_{\max } \exp \left[-c_{1} \phi^{c_{2} z^{c 3}}\right]
$$

Base Plot: $\log (k)$ versus $\phi$.

- This $C P R$ is initiated with a straight-line placed on the rightmost data trend, and only the intercept (c) changes (as prescribed by Eq. 2.3).

- For our data sets, this $C P R$ also performed very well in general.

- CPR-DxL - Weighted Power Law-Exponential Model: (Linear logarithmic weights model)

$$
k=x a \phi^{b}+(1-x) \alpha \exp [\beta \phi] \quad[0 \leq x \leq 1] \quad x=\exp \left[-\ln \left(x_{0}\right)+x_{1} \ln (\phi)+x_{2} \ln (z)+x_{3} \ln (\phi) \ln (z)\right]
$$

- Base Plots: $\log (k)$ versus $\log (\phi)$ and $\log (k)$ versus $\phi$.

- This $C P R$ performed well for most cases, the $x$-function model prescribed in Eq. 2.8 was, in a few cases, insufficient to model the character of the $x$-function defined by data. 
- CPR-DxQ - Weighted Power Law-Exponential Model: (Quadratic logarithmic weights model)

$$
\begin{aligned}
& k=x a \phi^{b}+(1-x) \alpha \exp [\beta \phi] \quad[0 \leq x \leq 1] \\
& x=\exp \left[\begin{array}{l}
-\ln \left(x_{0}\right)+x_{1} \ln (\phi)+x_{2} \ln (z)+x_{3} \ln (\phi) \ln (z)+x_{4} \ln (\phi)^{2} \\
+x_{5} \ln (z)^{2}+x_{6} \ln (\phi)^{2} \ln (z)+x_{7} \ln (\phi) \ln (z)^{2}+x_{8} \ln (\phi)^{2} \ln (z)^{2}
\end{array}\right]
\end{aligned}
$$

- Base Plots: $\log (k)$ versus $\log (\phi)$ and $\log (k)$ versus $\phi$.

- This $C P R$ performed well for all cases, often providing the best correlation. We have some concern that the flexible character of the $x$-function model prescribed in Eq. 2.5 may be "over-fitting" the data (i.e., fitting the errors in the data). This should not be a significant issue, but the analyst should be aware of the possibility of this condition.

In addition to the conclusions given above - we note the following generic conclusions:

- The proposed "characteristic permeability relations" each have a unique "signature," and clearly some $C P R s$ provide better correlation of data than others - this is a problem-dependent issue, there is no single best or worst $C P R$.

- The systematic application of the CPRs as proposed and demonstrated in this work should provide the ability to correlate a given set of permeability data with other variables (e.g., porosity, water saturation, Archie Formation Factor, shale fraction, individual well log responses, etc.).

\subsection{Recommendations for Future Work}

We put forward the following recommendations to extend this work:

- Application/validation of the CPRs to samples from specific carbonate reservoirs.

- Application/validation of the CPRs to samples from low to ultra-low permeability reservoirs.

- Extension of the CPRs to the identification and analysis of "hydraulic flow units."

- Integration of the CPRs with relations for capillary pressure and relative permeability.

- Utilization of the CPRs for reservoir scaling (heterogeneity). 


\section{NOMENCLATURE}

$a=$ Constant coefficient in the characteristic permeability relation

$b=$ Constant coefficient in the characteristic permeability relation

$c=$ Function in the characteristic permeability relation

$d_{p}=$ Average grain diameter, $\mathrm{m}$ (or appropriate consistent units)

$F=$ Archie Formation Factor (dimensionless)

$k=$ Formation Permeability, md (or any consistent units)

$S_{w}=$ Water Saturation (fraction)

$V_{\text {sh }}=$ Shale Volume (fraction)

$x=$ Weighting Function

$z \quad=$ Variable (Water saturation or Archie Formation Factor)

$\alpha=$ Constant coefficient in the characteristic permeability relation $(C P R-C, C P R-D x L, C P R-D x Q)$

$\beta=$ Constant coefficient in the characteristic permeability relation $(C P R-C, C P R-D x L, C P R-D x Q)$

$\phi \quad=$ Porosity, fraction 


\section{REFERENCES}

Ahmed, U., Crary, S.F., and Coates, G.R. 1989. Permeability Estimation: The Various Sources and Their Interrelationship. Paper SPE 19604 presented at the SPE Annual Technical Conference and Exhibition, San Antonio, Texas, 8-11 October.

Archie, G.E. 1942. Electrical Resistivity Log as an Aid in Determining Some Reservoir Characteristics. Trans., AIME 146: 54-62.

Archie, G.E. 1950. Introduction to Petrophysics of Reservoir Rocks. Bull. AAPG 34: 943-961.

Beard, D.C., and Weyl, P.K. 1973. Influence of Texture on Porosity and Permeability of Unconsolidated Sand. Bull., AAPG 57: 349-69.

Berg, R.R. Method for Determining Permeability from Reservoir Rock Properties. 1970. Trans., GCAGS 20: 303-317.

Bryant, S., Cade, C., and Mellor, D. 1993a. Permeability Prediction from Geologic Models. Bull., AAPG 77: 1338-1350.

Bryant, S., Cade, C., and Mellor, D. 1993b. Physically Representative Network Models of Transport in Porous Media. AIChE 39: 387-396.

Bryant, S.C., King, P.R., and Mellor, D.W. 1993c. Network Model Evaluation of Permeability and Spatial Correlation in a Real Random Sphere Packing. Transport in Porous Media 11: 53-70.

Coates, G.R., and Denoo, S. 1981. The Producibility Answer Product. Schlumberger Technical Review 29 (2): 55-63.

Ellis, K.W. 1987. Extended Correlations of Porosity, Permeability, and Formation Resistivity Factor. MS thesis, Texas A\&M U., College Station, Texas.

Finney, J. 1970. Random Packings and the Structure of Simple Liquids: I. The Geometry of Random Close Packing. Proceedings of the Royal Society of London Series A-Mathematical \& Physical Sciences 319: 479-493.

Hazlett, W.G. 1989. Correlation of Reservoir Rock Properties in Porous Media. PhD dissertation, Texas A\&M U., College Station, Texas.

Jennings, J.W., and Lucia, J. 2001. Predicting Permeability from Well Logs in Carbonates with a Link to Geology for Interwell Permeability Mapping. Paper SPE 71336 presented at the SPE Annual Technical Conference and Exhibition, New Orleans, Louisiana, September 30-October 3.

Morrow, N.M, Huppler, J.D., and Simmons III, A.B. 1969. Porosity and Permeability of Unconsolidated, Upper Miocene Sands from Grain-Size Analysis. J. Sed. Pet. 39 (1): 312-321. 
Pape, H., Clauser, C., Iffland, J. 1999. Permeability Prediction Based on Fractal Pore-Space Geometry. Geophysics 64: 1447-1460.

Pape, H., Clauser, C., Iffland, J. 2000. Variation of Permeability with Porosity in Sandstone Diagenesis Interpreted with a Fractal Pore Space Model. Pure Applied Geophysics 157: 603-619.

Timur, A. An Investigation of Permeability, Porosity, and Residual Water Saturation Relationships. 1968. Trans., SPWLA Symposium Paper I.

Travis Peak Formation Core Report — Well Howell No. 5, S.A. Holditch, (1986).

Travis Peak Formation Core Report - Well Cargill No. 15, Mobil Exploration \& Production Company, (1989).

Xue, G., Datta-Gupta, A., Valko, P., and Blasingame, T.A. 1997. Optimal Transformations for Multiple Regression: Application to Permeability Estimation from Well Logs. SPEFE, 85-93. 


\section{APPENDIX A \\ DERIVATION OF THE "PAPE" MULTI POWER LAW FORMULATION FOR PERMEABILITY}

Pape et al. (1999) developed a fractal model to predict the permeability of sedimentary as well as metamorphic rocks. The Pape et al. permeability model consists of a number of power law relations (typically with integer exponents) simply added together (usually 3 ). This approach is theoretically based on a fractal model for the internal structure of a porous medium [Pape et al. (1984, 1987a, 1987b) Pape and Schopper (1988), and Pape et al. 1999)]. The proposed "fractal" permeability relation is a completely general formulation that links porosity to permeability — where permeability can be estimated using either porosity data, or from the pore-radius distribution. Pape et al. (1999) propose that this general relation can be tuned to the entire range of sandstones - from clean to shaly sands.

\section{A.1 Tortuosity}

The expression for the permeability of a porous medium is given by the modified Kozeny-Carman equation [Carman (1956) and Kozeny (1927)]:

$$
k=\frac{r_{\text {eff }}^{2}}{8} \frac{1}{F}
$$

Where $F$ is the formation factor as defined by Archie (1942) — and is defined as the ratio of tortuosity $(T)$ and the porosity $(\phi)$ :

$$
F=\frac{T}{\phi}
$$

The formation (resistivity) factor was defined as the ratio of the electrical resistivity of a porous medium saturated (100 percent) with an electrolyte compared to the resistivity of the electrolyte, and is written as:

$$
F=\frac{R_{O}}{R_{w}}
$$

Considering Eq. A-3, it is obvious that $R_{o}$ will always be greater than $R_{w}$, hence $F$ will always be greater than unity. $F$ can be related to permeability via the Kozeny-Carman relation (i.e., Eq. A-1). An empirical relationship between $F$ and $\phi$ is given by "Archie's first law" — which is given as:

$$
F=\frac{a}{\phi^{m}}
$$

In Eq. A-4, $a$ is a factor depending on lithology and $m$ is the cementation (or tortuosity) factor, which depends on rock structure. From Serra (1984) the parameters $a$ and $m$ typically vary along the ranges: 


$$
\begin{aligned}
& 0.6<a<2 \\
& 1<m<3
\end{aligned}
$$

Pape et al (1987b) showed that the tortuosity behaves as a fractal and depends on the ratio of $r_{\text {eff }}$ and $r_{\text {grain }}$, with an exponent involving the fractal dimension (D). The Pape et al (1987b) formulation is given as:

$$
T=1.34\left[\frac{r_{\text {grain }}}{r_{\text {eff }}}\right]^{0.67(D-2)}
$$

Eq. A-5 implies that the tortuosity increases with increasing fractal dimension. Pape et al (1987b) suggest that Eq. A-5 is only valid for the range: $2<D<2.4$ (common rock samples). Pape and Schopper (1988) suggest that strongly fractured rocks (and/or claystones) demonstrate fractal dimensions in the range of 2.4 $<D<3$ - Pape and Schopper note that these rocks are characterized by a high degree of connectivity within the pore system, which in turn reduces the tortuosity.

At this stage $r_{\text {eff }}$ is unknown, so we replace $r_{\text {eff }}$ in Eq. A-5 by the pore radius at the 50th-percentile $\left(r_{50}\right)$ and the $r_{\text {grain }}$ variable is replaced by the median grain radius $d_{m}$. Also, as noted by Kulenkampff (1994), reservoir sandstones are limited in tortuosity - therefore Eq. A-5 is modified such that for large pore radii ( $r_{\text {eff }}$ or $r_{50}$ ), then we set $T=T_{\max }=10$ as the limit when $r_{\text {eff }}\left(\right.$ or $\left.r_{50}\right)$ approaches zero. This substitution avoids extreme and unrealistic tortuosity values. Making these substitutions into Eq. A-5, and solving for the reciprocal of tortuosity using data taken on Rotliegend sandstones in northwestern Germany, Köhler et al (1993) presented the following result:

$$
\frac{1}{T}=0.1+0.5\left[\frac{r_{50}}{d_{m}}\right]^{0.67(D-2)}
$$

\section{A.2 Permeability-Porosity Relationship}

Consider again the Kozeny-Carman (Eq. A-1) and the expression for the Archie Formation Factor (Eq. A2), if we combine Eqs. A-1 and A-2, then we can express permeability as a function of the effective pore $\operatorname{radius}\left(r_{\text {eff }}\right)$, the tortuosity $(T)$, and the porosity $(\phi)$ as:

$$
k=\frac{r_{\text {eff }}^{2}}{8} \frac{1}{F}=\frac{r_{\text {eff }}^{2}}{8} \frac{1}{(T / \phi)}=\frac{r_{\text {eff }}^{2}}{8} \frac{\phi}{T}
$$

Using Eq. A-5, and assuming that $D=2.36$ (the "average" value for hydrocarbon-bearing formations in northwestern German), Pape et al (1984) present the following relations for $T$ and $\phi$ :

$$
T=1.34\left[\frac{r_{\text {grain }}}{r_{\text {eff }}}\right]^{0.67(D-2)}=1.34\left[\frac{r_{\text {grain }}}{r_{\text {eff }}}\right]^{0.24}
$$

and 


$$
\phi=0.534\left[\frac{r_{\text {grain }}}{r_{\text {eff }}}\right]^{0.39(D-3)}=0.5\left[\frac{r_{\text {grain }}}{r_{\text {eff }}}\right]^{-0.25}
$$

If we neglect the slight differences in the exponents, solving Eqs. A-8 and A-9 for an approximation only in terms of tortuosity and porosity, we have:

$$
T \approx \frac{0.67}{\phi}
$$

and solving A-9 as an identity for $r_{\text {eff }}$ and $r_{\text {grain }}$ in terms of $\phi$, we obtain:

$$
r_{\text {eff }}^{2}=r_{\text {grain }}^{2}(2 \phi)^{8}
$$

Substituting Eqs. A-2, A-10, and A-11 into Eq. A-1 - and using a default value of $r_{\text {grain }}=200,000 \mathrm{~nm}$ (again, an average grain radius in the German sandstone data sets), $k$ can now be written as

$$
\begin{aligned}
k & =\frac{r_{\text {eff }}^{2}}{8} \frac{1}{F} \\
& =\frac{r_{\text {grain }}^{2}}{8} \frac{(2 \phi)^{8}}{(T / \phi)} \\
& =\frac{r_{\text {grain }}^{2}}{8} \frac{(2 \phi)^{8}}{\left[\frac{0.67}{\phi^{2}}\right]} \\
& =\frac{(200,000 \mathrm{~nm})^{2}}{8} \frac{(2)^{8}}{(0.67)} \phi^{10} \\
& =1.91045 \times 10^{12} \phi^{10} \mathrm{~nm}^{2}
\end{aligned}
$$

Eq. A-12 defines a linear trend of slope 10 on a $\log -\log$ plot of $k$ versus $\phi$. Eq. A-12 is presumed to be valid for porosities larger than 0.1 - Pape et al (1987a) and Pape et al (1987b) noted that the effective pore radii of the samples they considered do not decrease as rapidly with decreasing porosity as suggested by Eq. A-11. Pape et al suggest that the permeability estimates can be improved for cases where $0.01<\phi$ $<0.1$ by assigning a fixed value to the effective hydraulic pore radius in Eq. A-1 - i.e., $r_{\text {eff }}=r_{\text {eff.fix }}=200$ $\mathrm{nm}$. This substitution is equal or near to the mean of $r_{\text {eff }}$ value determined from measured permeabilities and porosities using Eqs. A-1, A-2, and A-10 for $0.01<\phi<0.1$. Substituting $r_{\text {eff.fix }}=200 \mathrm{~nm}$ into the combination of Eqs. A-1, A-2, and A-10 yields 


$$
\begin{aligned}
k & =\frac{r_{\text {eff }}^{2}}{8} \frac{1}{F} \\
& =\frac{(200 \mathrm{~nm})^{2}}{8} \frac{1}{\left[\frac{0.67}{\phi^{2}}\right]} \\
& =7462.69 \phi^{2} \mathrm{~nm}^{2}
\end{aligned}
$$

However, for $\phi<0.01$ this expression still yields permeabilities which are much lower than the measured data. A limiting value for $r_{\text {eff }}$ in this range is $r_{\text {eff,min }}=50 \mathrm{~nm}$ - substituting $r_{\text {eff,min }}=50 \mathrm{~nm}$ as $r_{50}$ into Eq. A-6 yields a maximum value for tortuosity, $T_{\max }=10$. Using $r_{\text {eff }}=r_{\text {eff, } \min }=50 \mathrm{~nm}$ and $T=T_{\max }=10$; substituting into Eqs. A-1 and A-2 and solving for permeability yields:

$$
\begin{aligned}
k & =\frac{r_{\text {eff }}^{2}}{8} \frac{1}{F} \\
& =\frac{r_{\text {eff }, \min }^{2}}{8} \frac{1}{\left(T_{\max } / \phi\right)} \\
& =\frac{r_{\text {eff }, \min }^{2}}{8} \frac{1}{T_{\max }} \phi \\
& =\frac{(50 \mathrm{~nm})^{2}}{8} \frac{1}{(10)} \phi \\
& =31.25 \phi \mathrm{nm}^{2}
\end{aligned}
$$

Pape et al (1999) propose a generalized expression for permeability as a summation of Eqs. A-12, A-13, and A-14 — with the rationale that this definition provides an "average permeability-porosity" relationship for the entire porosity range. The generalized Pape et al (1999) result is:

$$
k=31.25 \phi+7462.69 \phi^{2}+1.91045 \times 10^{12} \phi^{10} \mathrm{~nm}^{2} \text { (Pape Analytical) }
$$

Pape et al (1999) state that the addition of the expressions for the low-, medium-, and high-porosity ranges is reasonable because, for a given porosity, the expressions for the other two porosity ranges do not contribute significantly due to the difference in the exponents of porosity used in Eq. A-15.

Obviously, Eq. A-15 is an idealized formulation, and the coefficients of the three power law terms in Eq. A-15 must be calibrated when applied to a specific formation or reservoir. For the case of Rotliegend samples (northeastern Germany), Pape et al (1999) provide the following result, obtained by regression:

$$
k=155 \phi+37315 \phi^{2}+6.30 \times 10^{12} \phi^{10} \mathrm{~nm}^{2} \text { (Rotliegend) }
$$


Recall that the "analytical" relations where derived assuming a fractal dimension of $D=2.36$ - obviously, if the fractal dimension changes, so must the exponents (as well as the multiplicative constants). As such, Pape et al (1987a) propose the following general result:

$$
k=A \phi+B \phi^{m}+C \phi^{\frac{m+2}{c_{1}(3-D)}} \mathrm{nm}^{2} \quad\left(\text { where } 0.39<c_{1}<1\right)
$$

Where $D$ is the fractal dimension, and $m$ is the exponent (cementation factor) in Archie's first law (i.e., Eq. A-4). The parameter $c_{1}$ relates the effective pore radius $r_{\text {eff }}$ to the pore radius $r_{p}$ and the grain radius $r_{\text {grain }}$ as follows:

$$
\frac{r_{\text {grain }}}{r_{p}}=\left[\frac{r_{\text {grain }}}{r_{\text {eff }}}\right]^{c_{1}} \text { with } \mathrm{c}_{1}=0.39 \text {, if } \frac{r_{\text {grain }}}{r_{\text {eff }}}>30
$$

Pape et al (1987a) propose Eq. A-18 as an empirical result, for which Pape et al suggest Eq. A-18 is valid for a wide variety of sandstones.

Another formulation, the "shaly sandstone" relation is developed by Pape et al (1999) to represent shaly sand samples. This result is given as:

$$
k=6.2 \phi+1493 \phi^{2}+5.8 \times 10^{11} \phi^{10} \mathrm{~nm}^{2} \text { (Shaly Sandstone) }
$$

Finally, Pape et al (1999) present a formulation that matches data measured on high-porosity kaolinite samples and on low-porosity shales, this is the so-called "shale" equation, and is given below:

$$
k=0.1 \phi+26 \phi^{2}+1 \times 10^{11} \phi^{10} \mathrm{~nm}^{2} \text { (Shale) }
$$




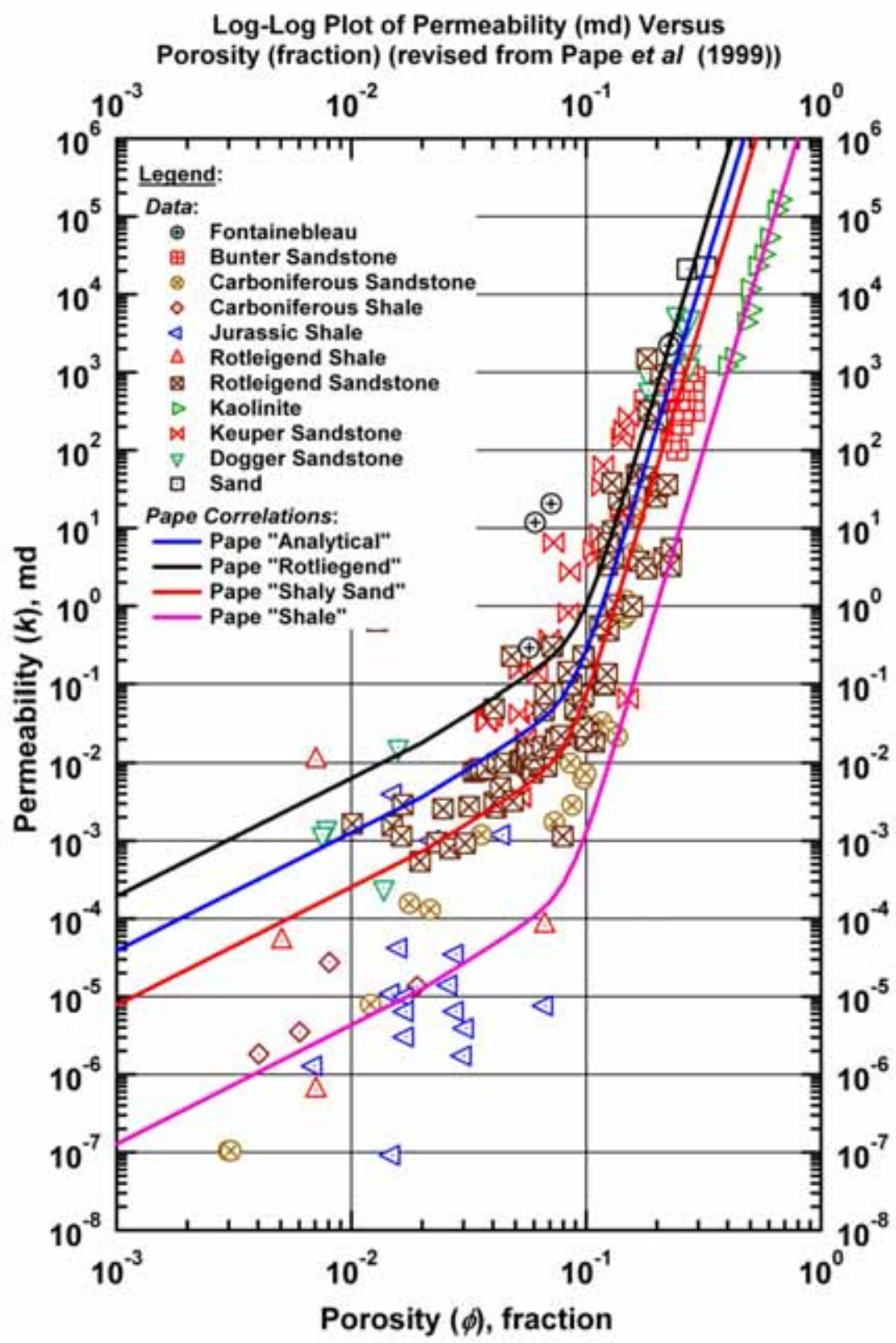

Figure A.1 — Pape et al (1999) fractal correlation of permeability and porosity — various data sources (modified from Pape et al (1999)). 


\section{Nomenclature: Pape Derivation}

$a \quad=$ Intercept term in Archie's First Law (dimensionless)

$D=$ Fractal dimension (dimensionless)

$d_{m}=$ Median grain radius, $\mathrm{nm}$

$F=$ Archie Formation Factor (dimensionless)

$k=$ Formation Permeability, $\mathrm{nm}^{2}$

$m$ = Slope term in Archie's First Law (i.e., the "cementation factor") (dimensionless)

$S_{w}=$ Water Saturation (fraction)

$r_{p}=$ Pore radius, $\mathrm{nm}$

$r_{\text {eff }}=$ Effective pore radius, $\mathrm{nm}$

$r_{\text {grain }}=$ Effective grain radius, $\mathrm{nm}$

$r_{50}=$ Pore radius at the 50th-percentile, $\mathrm{nm}$

$R_{o}=$ Resistivity of the sample at $S_{w}=1$, ohm-m

$R_{w}=$ Resistivity of the electrolyte (usually salt water), ohm-m

$T=$ Tortuosity (dimensionless)

$\phi \quad=$ Porosity, fraction

\section{References: Pape Derivation}

Archie, G. E. 1942. The Electrical Resistivity Log as an Aid in Determining Some Reservoir Characteristics. Trans. AIME. 146: 54-61.

Carman, P. C. 1956. Flow of Gases Through Porous Media. Butterworth Scientific Publication

Köhler, M., Pape, H., Pasternak, M., and Schmidt, R. 1993. Natural Gas Reserves in the Ems Estuary Region, Part I. Reservoir engineering (in German): Geological Survey of Lower Saxony, internal report no. 110986, Hannover, Germany.

Kozeny, J. 1927. Über die kapillare Leitung des Wassers im Boden (Aufstieg Versickerung und Anwendung auf die Bewässerung). Sitz. Ber. Akad. Wiss. Wien, Math. Nat. (Abt. IIa). 136a: 271-306.

Kulenkampff, J. 1994. Die komplexe elektrische Leitfähigkeit poröser Gesteine im Frequenz-bereich von $10 \mathrm{~Hz}$ bis $1 \mathrm{Mhz}$ : Einflüsse von Porenstruktur und Porenfüllung. Ph.D. dissertation, Tech. U. Clausthal.

Pape, H., Riepe, L., and Schopper, J. R. 1984. The Role of Fractal Quantities, as Specific Surface and Tortuosities, for Physical Properties of Porous Media. Particle Character 1: 66-73.

Pape, H., Riepe, L., and Schopper, J. R. 1987a. Interlayer Conductivity of Rocks - a Fractal Model of Interface Irregularities for Calculating Interlayer Conductivity of Natural Porous Mineral Systems. Colloids and Surfaces 27: 97-122.

Pape, H., Riepe, L., and Schopper, J. R. 1987b. Theory of self-similar network structures in sedimentary and igneous rocks and their investigation with microscopical methods. J. Microscopy 148: 121-147 (1987b).

Pape, H., and Schopper, J. R. 1988. Relations Between Physically Relevant Geometrical Properties of a Multifractal Porous System. In Characterization of porous solids, eds. K. K. Unger, J. Rouquerol, K.S.W. Sing, and H. Kral, 473-482, Elsevier Science Publ. Co., Inc.

Pape, H., Clauser, C., And Iffland, J. 1999. Permeability Prediction Based on Fractal Pore Space Geometry. Geophysics 64 (5): 1447-1460.

Serra, O. 1984. Fundamentals of Well-Log Interpretation: 1. The Acquisition of Data. Elsevier Science Publ. Co., Inc. 


\section{APPENDIX B \\ RESULTS FOR HOWELL WELL \\ (E. TEXAS - USA)}

In this Appendix we present the "Howell" Well, located in East Texas (USA), where this is a sandstone reservoir (Holditch (1986)). First we do the correlation taking the entire interval in the correlation then we divide the interval into two zones based on the depositional environment. The correlation works good for all the cases but as expected it performs better when we divide the interval into different zones.

\section{Model CPR-A: (Appendix B)}

The "characteristic permeability relation" (or CPR) Model CPR-A (i.e., the modified Archie "Clean Sand" Model) is given as:

$$
k=a(\phi-c)^{b} \quad c=c_{\max } \exp \left[-c_{1} \phi^{c_{2}} S_{w}^{c_{3}}\right]
$$

Where a plot of $\log (k)$ versus $\log (\phi)$ is used to graphically calibrate Eq. B-1 in terms of the coefficients $a, b$, and $c_{\max }$. Fig. B.1 shows that the $C P R-A$ model performs quite well for this case. Figs. B.1a and $\mathbf{b}$ show the $\log -\log$ and the semilog format of permeability versus porosity, respectively. In Fig. B.2 we provide the "error" plots for this case, where the measured and computed data are compared systematically. In Fig. B.2a we note a good correlation of the results obtained using Model CPR-A versus the measured permeability data - there is good agreement with the perfect correlation trend (i.e., the 45degree line). In Fig. B.2b we provide a comparison with the GRACE algorithm correlation (Xue et al, 1997)), and we note that the CPR-A model results correlate very well with the GRACE algorithm results for this case. In Fig. B.2c we present the calculated and measure $c$-function - and again we note a very good correlation of the data about the perfect correlation trend. Lastly, in Fig. B.2d and e we present the measured and calculated permeability with depth.

\section{Model CPR-B: (Appendix B)}

The "characteristic permeability relation" (or CPR) Model CPR-B (i.e., the modified Archie "Dirty Sand" Model (power-law basis)) is given as:

$$
k=a(\phi+c)^{b} \quad c=c_{\max } \exp \left[-c_{1} \phi^{c_{2}} S_{w}^{c_{3}}\right]
$$

Where a plot of $\log (k)$ versus $\log (\phi)$ is used to graphically calibrate Eq. B-2 in terms of the coefficients $a, b$, and $c_{\max }$. The results for this case are presented in Figs. B.3 and 4. In Fig. B.3a (log-log format) we note that a very distinct "envelope" is formed by the $C P R-B$ model, essentially all of the data on the right and left flanks are well-matched (i.e., the "dirty sand" power law relation (applied on the right portion of the data) and its correction function (applied on the left portion of the data). Similarly, in Fig. B.3b 
(semilog format), we observe an excellent distribution of the power law model across the body of the data. The error analyses for this case are shown in Fig. B.4. In Fig. B.4a we present the results obtained using Model $C P R-B$ versus the measured permeability data and in this case there is good agreement with the perfect correlation trend (i.e., the 45-degree line). We provide a comparison with the GRACE algorithm correlation in Fig. B.4b - the results using Model CPR-B and the GRACE algorithm compare very well for this case. We compare the computed and data-derived values of the $c$-function for this case in Fig. B.4c and we note a reasonably "tight" correlation, except for few outliers of the $c$-function. Lastly, in Fig. B.4d and e we present the measured and calculated permeability with depth. In summary, we believe that Model $C P R-B$ is a very effective correlation model for this particular data case.

\section{Model CPR-C: (Appendix B)}

The "characteristic permeability relation" (or CPR) Model CPR-C (i.e., the modified Archie "Dirty Sand" Model (exponential basis)) is given as:

$$
k=c \exp [\beta \phi] \quad c=c_{\max } \exp \left[-c_{1} \phi^{c_{2}} S_{w}^{c_{3}}\right]
$$

Where a plot of $\log (k)$ versus $\phi$ is used to graphically calibrate Eq. B-3 in terms of the coefficients - $\beta$ and $c_{\max }$. Model $C P R-C$ is fundamentally different than Models $C P R-A$ and $C P R-B$, as the basis for Model $C P R-C$ is an exponential function, not a power law relation. In Figs. B.5 and 6 we present the results of applying Model $C P R-B$ for this case, and in general, we observe a very consistent performance of Model $C P R-B$. Fig. B.5b illustrates the excellent conformance of Model $C P R-C$ for this case - again recalling that this model has an exponential relationship with porosity as its basis.

In Figs. B.6a, B.6b, and B.6c we review the error analyses plots for this case, and we note good performance (visually) in terms of the correlation of the results using Model $C P R-C$. In this case, the $c$ function (in Eq. B-3) is not a correction term, but rather the instantaneous intercept for the exponential basis. The correlation of the $c$-function (see Fig. B.6c) appears to be consistent — but scatter and a slight off-center trend is evident. However, as we are approaching this work from the perspective that the primary value of the work is the characteristic model, not the statistical "best fit" correlation, we believe that the $C P R-C$ model has performed well for this case, and the plots in Fig. B.6 confirm the value of this model as a "characteristic relation" for permeability.

\section{Model CPR-D: (Appendix B)}

The "characteristic permeability relation" (or $C P R$ ) Model CPR-D (i.e., the weighted power law-exponential model) is given in its fundamental form as:

$$
\left.k=x a \phi^{b}+(1-x) \alpha \exp [\beta \phi] \quad[0 \leq x \leq 1]\right] .
$$

For Eq. B-4 there are two proposed models for the $x$-function (i.e., the weighting function) - these are:

$$
x=\exp \left[-\ln \left(x_{0}\right)+x_{1} \ln (\phi)+x_{2} \ln \left(S_{w}\right)+x_{3} \ln (\phi) \ln \left(S_{w}\right)\right](\text { Model CPR-DxL)........ }
$$




$$
x=\exp \left[\begin{array}{l}
-\ln \left(x_{0}\right)+x_{1} \ln (\phi)+x_{2} \ln \left(S_{w}\right)+x_{3} \ln (\phi) \ln \left(S_{w}\right) \\
+x_{4} \ln (\phi)^{2}+x_{5} \ln \left(S_{w}\right)^{2}+x_{6} \ln (\phi)^{2} \ln \left(S_{w}\right) \\
+x_{7} \ln (\phi) \ln \left(S_{w}\right)^{2}+x_{8} \ln (\phi)^{2} \ln \left(S_{w}\right)^{2}
\end{array}\right] \text { (Model CPR-DxL) }
$$

As noted in Eq. B-4b, Model CPR-DxL utilizes a logarithmic linear model for the weights ( $x$-values) and as seen in Eq. B-4c, Model CPR-DxQ utilizes a logarithmic quadratic model for the weights ( $x$-values). While these models (Eqs. B-4b and B-4c) are empirical, we have found generally good application of these relations for all cases. In this case, the base results using Models $C P R-D x L$ and $C P R-D x Q$ are presented in Fig. B.7 - where the power law and exponential basis functions are fitted to the appropriate portion of the data. The power law equation represents the "Archie clean sand" trend and is fitted to the leftmost data as shown in Fig. B.7a and in contrast, the exponential equation is thought to represent the "Archie dirty sand" trend and the exponential is fitted to the far rightmost portion of the data (see Fig. B.7b).

In Figs. B.8 and 9 we present the error analyses for this case, where we have employed the weighting functions (Eqs. B-4b and B-4c) as appropriate. In Fig. B.8a we find a good correlation of permeability (with the out-lying points noted in the middle of the trend). The comparison of the Model CPR-DxL results are compared to the results obtained from the GRACE algorithm in Fig. B.8b, we note that the results obtained using Model $C P R-D x L$ does "drift" slightly from the GRACE solution at middle values of permeability, indicating some inconsistency. The computed weight function ( $x$-values) shown for the $C P R$-DxL model in Fig. B.8c does exhibit more scatter than expected, but the trend is (relatively) centered about the perfect correlation line. Fig. B.8d and e provide the measured and calculated permeability versus depth.

The results obtained using Model $C P R-D x Q$ are presented in Fig. B.9, and we note substantially improved behavior over that of Model CPR-DxL. In particular, the base correlation of computed and measured permeabilities (Fig. B.9a) for Model $C P R-D x Q$ does indicate a slightly better correlation than that of Model $C P R-D x L$. Also, the comparison of Model CPR-DxQ with the results from the GRACE algorithm (Fig. B.9b) exhibits better agreement than the results of Model CPR-DxL. Finally, the correlation of the $x$ function (Fig. B.9c) for Model CPR-DxQ is better than the correlation for Model CPR-DxL. These comparisons suggest that Model CPR-DxQ (i.e., the combination of Eqs. B-4a and B-4c) has provided a better correlation of data, for this case, than Model CPR-DxL. Fig. B.9d and e provide the measured and calculated permeability versus depth. 
"Timur Model": (Appendix B)

The "Timur Model" [Timur (1968)] is given as:

$$
k_{\text {Timur }}=a \phi^{b} S_{w}^{c}
$$

In Fig. B.10 we present the results of the "Timur Model" (Eq. B-5) which is essentially just a generalized power function relation in terms of $k, \phi$, and $S_{w}$. As shown in Figs. B.10a and b, the "Timur Model" also provides a good correlation of the data for this case. The comparison of results using the "Timur Model" with the results of the GRACE algorithm (Fig. B.10b).

\section{"Coates Model": (Appendix B)}

The "Coates Model" [referenced by Ahmed et al (1989)] is given as:

$$
k_{\text {Coates }}=\left[100 \phi^{2} \frac{\left(1-S_{w}\right)}{S_{w}}\right]^{2} .
$$

In Fig. B.11 we present the results of the "Coates Model" (Eq. B-6) which is essentially just a modified power function relation in terms of $k, \phi$, and $S_{w}$. The results are shown in Figs. B.11a and $\mathbf{b}$ of the data for this case. The comparison of results using the "Coates Model " with the results of the GRACE algorithm (Fig. B.11b) is also shown.

\section{GRACE Algorithm: (Appendix B)}

In Fig. B.12 we present the results predicted by the GRACE algorithm, which is a non-parametric regression approach (see Xue, et al (1997) for details). In theory, the GRACE algorithm should provide the most unbiased correlation of the data - i.e., the GRACE algorithm is designed not "fit the errors" as other regression approaches may. It is our contention that the GRACE algorithm is the statistical standard - and any algorithm/approach/model which achieves better regression statistics than the GRACE algorithm is actually "fitting the errors" in the data. Since none of the cases are better statistically than the GRACE algorithm so none of the cases are "over-fitted" in a statistical sense.

\section{Comparison of All Models: (Appendix B)}

Fig. B.13, illustrates all of the models on a single plot of calculated versus measured permeability. We also present a table of all statistical results in Table B.1.

As mentioned before we perform the same procedure but this time we make two zones of the entire interval and the results are presented from Figs. B.14 to B.39, and we note substantially improvement in the correlation. 
We present a table of all statistical results taking into account all of the interval in Tables B.1.

Table B.1 - Statistical Results for all Models (Howell Well, E. TX (USA), $n=352)$.

\begin{tabular}{|c|c|c|c|c|}
\hline Model & $\begin{array}{c}\text { Sum of } \\
\text { Squared } \\
\text { Residuals } \\
{\left[\log (k)^{2}\right]}\end{array}$ & $\begin{array}{l}\text { Variance } \\
{\left[\log (k)^{2}\right]}\end{array}$ & $\begin{array}{c}\text { Standard } \\
\text { Deviation } \\
{[\log (k)]}\end{array}$ & $\begin{array}{c}\text { Absolute } \\
\text { Relative } \\
\text { Error } \\
\text { [percent] }\end{array}$ \\
\hline$C P R-A$ & 813.37 & 5.46 & 2.34 & 291.48 \\
\hline$C P R-B$ & 806.50 & 4.95 & 2.23 & 278.37 \\
\hline$C P R-C$ & 795.47 & 5.45 & 2.33 & 288.73 \\
\hline$C P R-D x L$ & 781.67 & 5.46 & 2.34 & 274.74 \\
\hline$C P R-D x Q$ & 696.09 & 5.63 & 2.37 & 288.62 \\
\hline Timur & 878.58 & 5.24 & 2.29 & 310.91 \\
\hline Coates & 1085.36 & 4.69 & 2.16 & 352.45 \\
\hline GRACE Algorithm & 779.84 & 5.31 & 2.30 & 267.90 \\
\hline
\end{tabular}

The coefficients for the models used in this case are provided in Table B.2.

Table B.2a - Model Coefficients (Howell Well, E. TX (USA), $n=352$ ).

\begin{tabular}{|c|c|c|c|c|c|c|}
\hline Model & $a$ or $\alpha$ & $b$ or $\beta$ & $c$ or $c_{\max }$ & $c_{1}$ & $c_{2}$ & $c_{3}$ \\
\hline$C P R-A$ & $7.00 \times 10^{9}$ & 8.00 & 0.100 & $7.55 \times 10^{-3}$ & -1.79 & -0.42 \\
\hline$C P R-B$ & $4.00 \times 10^{9}$ & 12.00 & 0.090 & 77.17 & 1.61 & 1.02 \\
\hline$C P R-C$ & $5.00 \times 10^{-8}$ & 100.00 & 0.006 & 48.74 & 0.73 & 0.42 \\
\hline Timur & $1.41 \times 10^{2}$ & 3.71 & 2.574 & - & - & - \\
\hline Coates & $6.27 \times 10^{4}$ & 5.54 & 0.781 & - & _- & - \\
\hline
\end{tabular}

Table B.2b - Model Coefficients (Howell Well, E. TX (USA), $n=352$ ).

\begin{tabular}{|c|c|c|c|c|c|c|c|c|c|}
\hline & \multicolumn{2}{|c|}{ Model } & $a$ & $b$ & $\alpha$ & \multicolumn{2}{|l|}{$\beta$} & & \\
\hline & & & $7.00 \times 10$ & 8.00 & $5.00 \times 10^{-}$ & 100.00 & & & \\
\hline & & $D x Q$ & $7.00 \times 10^{5}$ & 8.00 & $5.00 \times 10^{-}$ & 100.00 & & & \\
\hline Model & $c_{0}$ & $c_{1}$ & $c_{2}$ & $c_{3}$ & $C_{4}$ & $c_{5}$ & $c_{6}$ & $c_{7}$ & $C_{8}$ \\
\hline$C P R-D x L$ & $2.85 \times 10^{9}$ & -5.88 & -9.00 & -2.68 & - & - & - & - & - \\
\hline$C P R-D x Q$ & $1.75 \times 10^{7}$ & -0.08 & -12.22 & 2.14 & 1.47 & -5.05 & 2.51 & 0.06 & 0.92 \\
\hline
\end{tabular}

These are the results for Zone 1.

Table B.3 - Statistical Results for all Models (Howell Well, E. TX (USA), $n=228)$.

\begin{tabular}{|c|c|c|c|c|}
\hline Model & $\begin{array}{c}\text { Sum of } \\
\text { Squared } \\
\text { Residuals } \\
{\left[\log (k)^{2}\right]}\end{array}$ & $\begin{array}{l}\text { Variance } \\
{\left[\log (k)^{2}\right]}\end{array}$ & $\begin{array}{c}\text { Standard } \\
\text { Deviation } \\
{[\log (k)]}\end{array}$ & $\begin{array}{c}\text { Absolute } \\
\text { Relative } \\
\text { Error } \\
\text { [percent] }\end{array}$ \\
\hline$C P R-A$ & 408.58 & 5.56 & 2.36 & 133.32 \\
\hline$C P R-B$ & 438.75 & 5.40 & 2.32 & 138.72 \\
\hline$C P R-C$ & 423.02 & 5.50 & 2.34 & 132.08 \\
\hline$C P R-D x L$ & 431.05 & 5.48 & 2.34 & 131.80 \\
\hline$C P R-D x Q$ & 368.09 & 5.61 & 2.37 & 119.80 \\
\hline Timur & 535.73 & 5.22 & 2.28 & 167.91 \\
\hline Coates & 622.28 & 4.83 & 2.20 & 180.68 \\
\hline GRACE Algorithm & 431.32 & 5.69 & 2.39 & 115.13 \\
\hline
\end{tabular}


The coefficients for the models used in this case are provided in Table B.4.

Table B.4a - Model Coefficients (Howell Well, E. TX (USA), $n=228$ ).

\begin{tabular}{|c|c|c|c|c|c|c|}
\hline Model & $a$ or $\alpha$ & $b$ or $\beta$ & $C$ or $C_{\max }$ & $c_{1}$ & $c_{2}$ & $c_{3}$ \\
\hline$C P R-A$ & $1.00 \times 10^{11}$ & 9.00 & 0.100 & 0.010 & -1.83 & -0.22 \\
\hline$C P R-B$ & $4.80 \times 10^{10}$ & 12.50 & 0.050 & 5970.45 & 3.36 & 1.25 \\
\hline$C P R-C$ & $5.00 \times 10^{-8}$ & 100.00 & 0.001 & 40.00 & 0.76 & 0.31 \\
\hline Timur & $7.38 \times 10^{2}$ & 4.27 & 1.908 & - & - & - \\
\hline Coates & $4.05 \times 10^{9}$ & 11.34 & 0.426 & - & - & - \\
\hline
\end{tabular}

Table B.4b - Model Coefficients (Howell Well, E. TX (USA), $n=228$ ).

\begin{tabular}{|c|c|c|c|c|}
\hline Model & $a$ & $b$ & $\alpha$ & $\beta$ \\
\hline$C P R-D x L$ & $1.00 \times 10^{\Pi 1}$ & 9.00 & $5.00 \times 10^{-8}$ & 100.00 \\
\hline$C P R-D x Q$ & $1.00 \times 10^{11}$ & 9.00 & $5.00 \times 10^{-8}$ & 100.00 \\
\hline
\end{tabular}

\begin{tabular}{|c|c|c|c|c|c|c|c|c|c|}
\hline Model & $c_{0}$ & $c_{1}$ & $c_{2}$ & $c_{3}$ & $c_{4}$ & $c_{5}$ & $c_{6}$ & $c_{7}$ & $C_{8}$ \\
\hline$C P R-D x L$ & $9.71 \times 10^{9}$ & -6.45 & -10.71 & -3.88 & - & - & - & - & - \\
\hline$C P R-D x Q$ & $3.00 \times 10^{6}$ & 1.17 & -31.63 & -14.51 & 1.75 & -6.22 & -0.63 & 0.03 & 1.27 \\
\hline
\end{tabular}

These are the results for Zone 2.

Table B.5 - $\quad$ Statistical Results for all Models (Howell Well, E. TX (USA), $n=124)$.

\begin{tabular}{|c|c|c|c|c|}
\hline Model & $\begin{array}{c}\text { Sum of } \\
\text { Squared } \\
\text { Residuals } \\
{\left[\log (k)^{2}\right]}\end{array}$ & $\begin{array}{l}\text { Variance } \\
{\left[\log (k)^{2}\right]}\end{array}$ & $\begin{array}{c}\text { Standard } \\
\text { Deviation } \\
{[\log (k)]}\end{array}$ & $\begin{array}{c}\text { Absolute } \\
\text { Relative } \\
\text { Error } \\
\text { [percent] }\end{array}$ \\
\hline$C P R-A$ & 302.72 & 2.30 & 1.52 & 133.30 \\
\hline$C P R-B$ & 281.99 & 2.52 & 1.59 & 129.49 \\
\hline$C P R-C$ & 275.54 & 2.53 & 1.59 & 121.95 \\
\hline$C P R-D x L$ & 278.43 & 2.60 & 1.61 & 126.13 \\
\hline$C P R-D x Q$ & 251.05 & 2.67 & 1.63 & 137.16 \\
\hline Timur & 330.38 & 2.13 & 1.46 & 120.01 \\
\hline Coates & 363.65 & 2.30 & 1.52 & 144.04 \\
\hline GRACE Algorithm & 277.97 & 2.29 & 1.51 & 114.20 \\
\hline
\end{tabular}

The coefficients for the models used in this case are provided in Table B.6.

Table B.6a - Model Coefficients (Howell Well, E. TX (USA), $n=124$ ).

\begin{tabular}{|c|c|c|c|c|c|c|}
\hline Model & $a$ or $\alpha$ & $b$ or $\beta$ & $C$ or $C_{\max }$ & $c_{1}$ & $C_{2}$ & $c_{3}$ \\
\hline CPR-A & $7.00 \times 10^{9}$ & 8.00 & 0.060 & $5.26 \times 10^{-4}$ & -2.47 & -0.82 \\
\hline$C P R-B$ & $1.00 \times 10^{20}$ & 21.00 & 0.060 & 1193.25 & 2.55 & 0.65 \\
\hline$C P R-C$ & $5.00 \times 10^{-8}$ & 130.00 & 0.001 & 112.62 & 1.01 & 0.53 \\
\hline Timur & $8.33 \times 10^{-1}$ & 1.87 & 3.336 & - & - & - \\
\hline Coates & $1.68 \times 10^{2}$ & 2.62 & 1.441 & - & - & - \\
\hline
\end{tabular}


Table B.6b - Model Coefficients (Howell Well, E. TX (USA), $n=124)$.

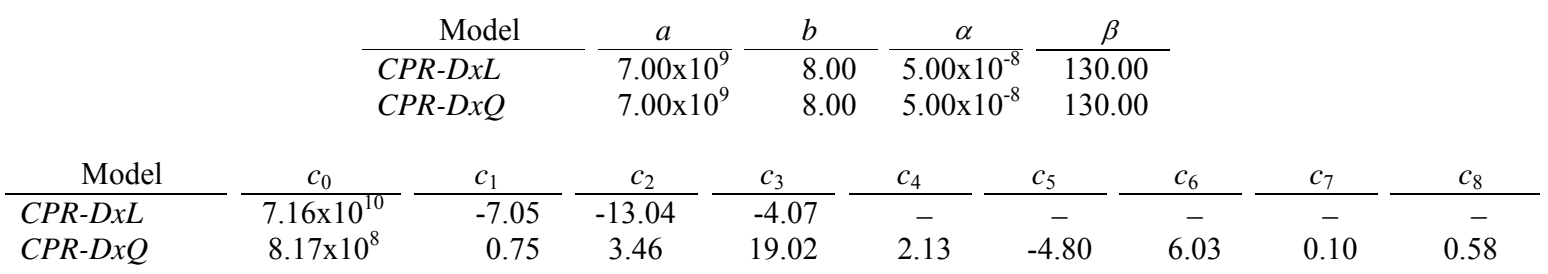

\section{Nomenclature: (Appendix B)}

$$
\begin{aligned}
S_{w} & =\text { Water Saturation (fraction) } \\
k & =\text { Formation Permeability, md (or any consistent units) } \\
\phi & =\text { Porosity, fraction }
\end{aligned}
$$

\section{References: (Appendix B)}

Ahmed, U., Crary, S.F., and Coates, G.R: "Permeability Estimation: The Various Sources and Their Interrelationship," paper SPE 19604 presented at the 1989 SPE Annual Technical Conference and Exhibition, San Antonio, TX, 8-11 Oct., 649-662.

Timur, A.: "An Investigation of Permeability, Porosity, and Residual Water Saturation Relation-ships," Trans., SPWLA Symposium (1968) paper I.

Travis Peak Formation Core Report - Well Howell No. 5, S.A. Holditch, (1986).

Xue, G., Datta-Gupta, A., Valko, P., and Blasingame, T.A.: "Optimal Transformations for Multiple Regression: Application to Permeability Estimation from Well Logs," SPEFE (June 1997), 85-93. 


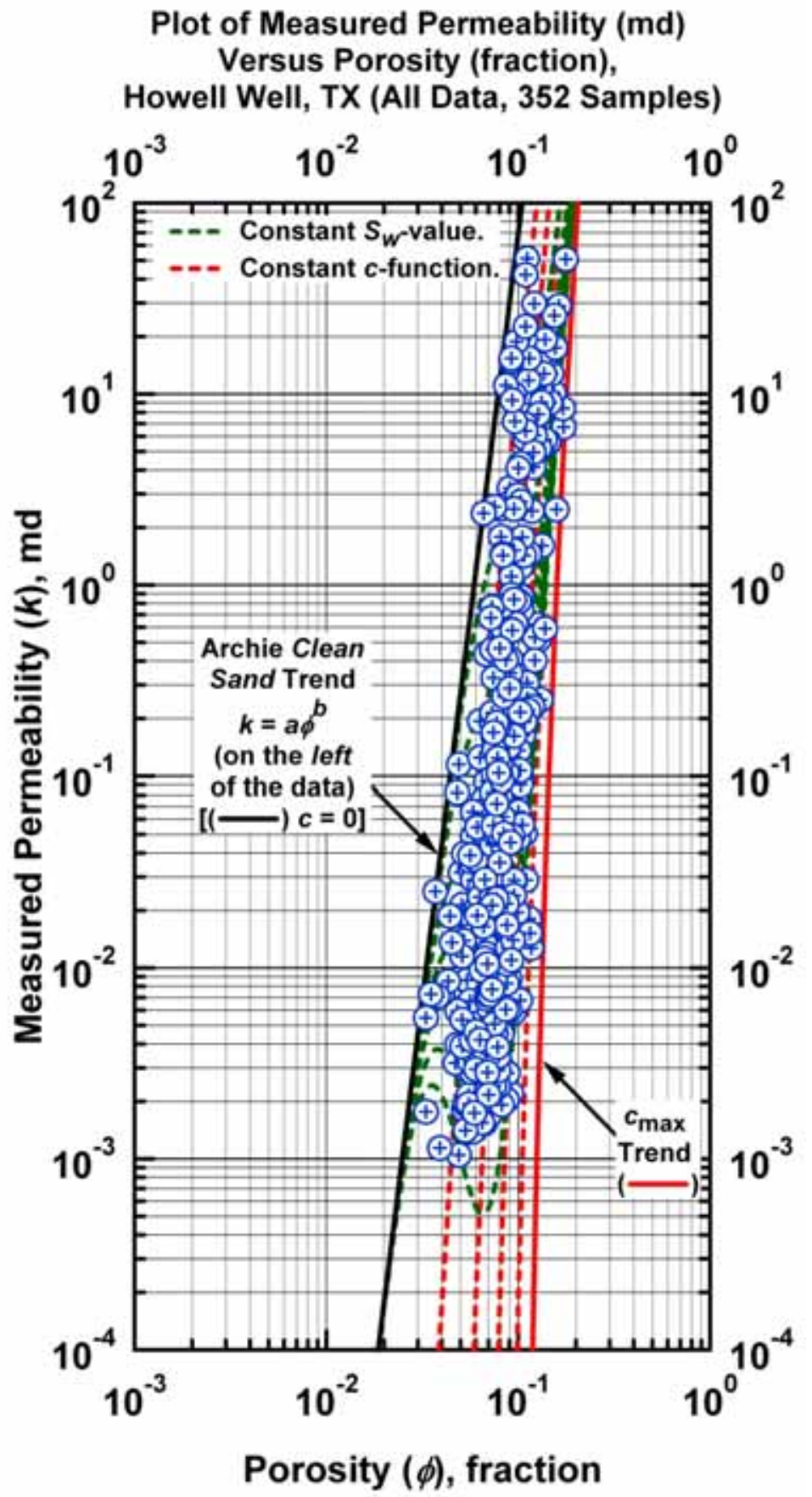

Figure B.1a - Model CPR-A: $\quad k=a(\phi-c)^{b} \quad c=c_{\max } \exp \left[-c_{1} \phi^{c_{2}} S_{w}^{c_{3}}\right]$ - "Clean Sand" Plot (log-log format) - Archie "Clean Sand" trend is given by the straight-line trend at the far left of the data (power law model). [Case: Howell Well, E. TX (USA)] 


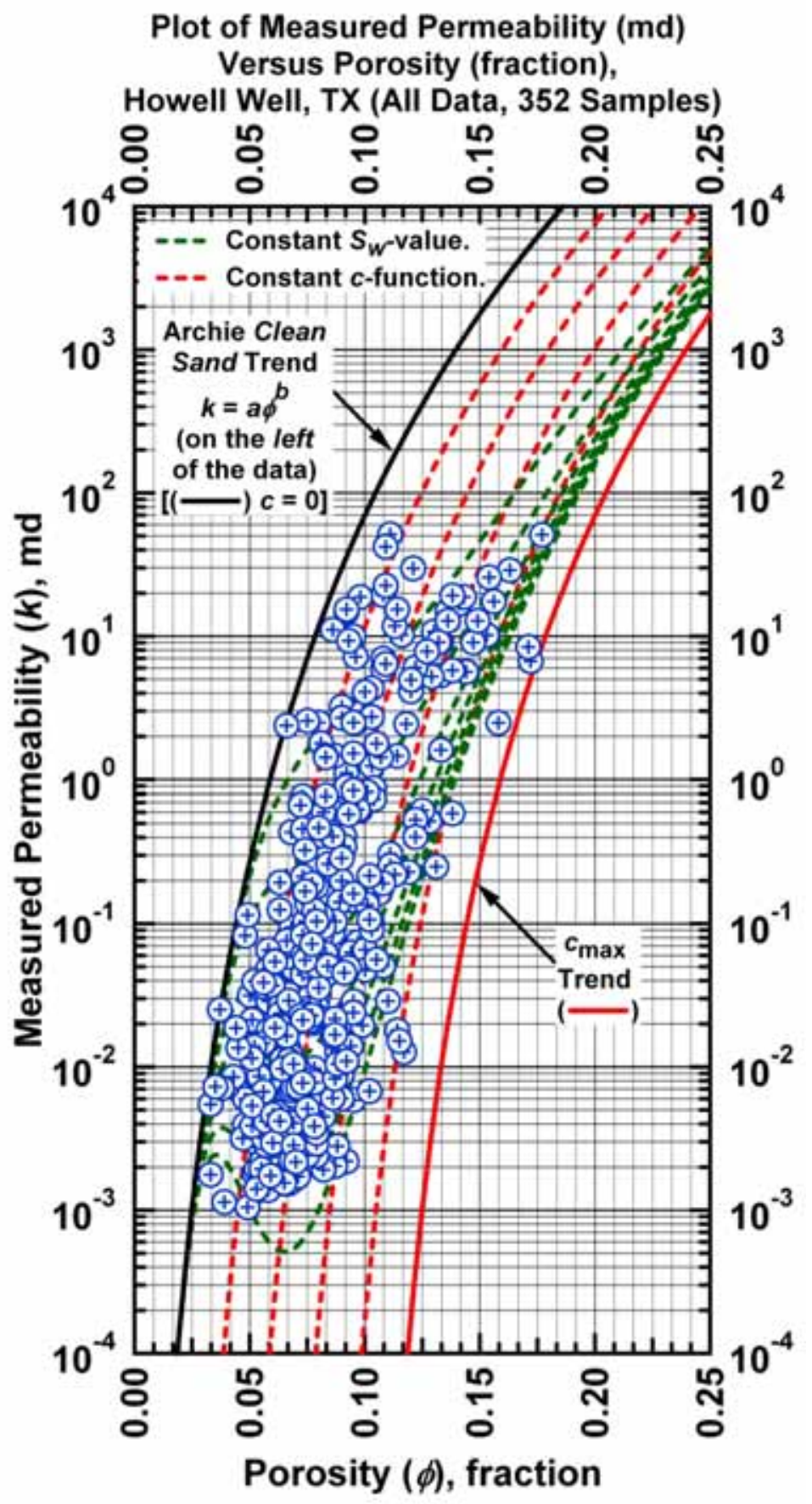

Figure B.1b - Model CPR-A: $k=a(\phi-c)^{b} \quad c=c_{\max } \exp \left[-c_{1} \phi^{C_{2}} S_{w}^{C_{3}}\right]$ - "Dirty Sand" Plot (semilog format). Archie "Clean Sand" trend is given by the curved trend at the far left of the data (power law model). [Case: Howell Well, E. TX (USA)] 
Comparison of Permeability (md) Calculated

Using the "CPR-A" Model Versus the Measured

Permeability (md), Howell Well, TX (All Data, 352 Samples)

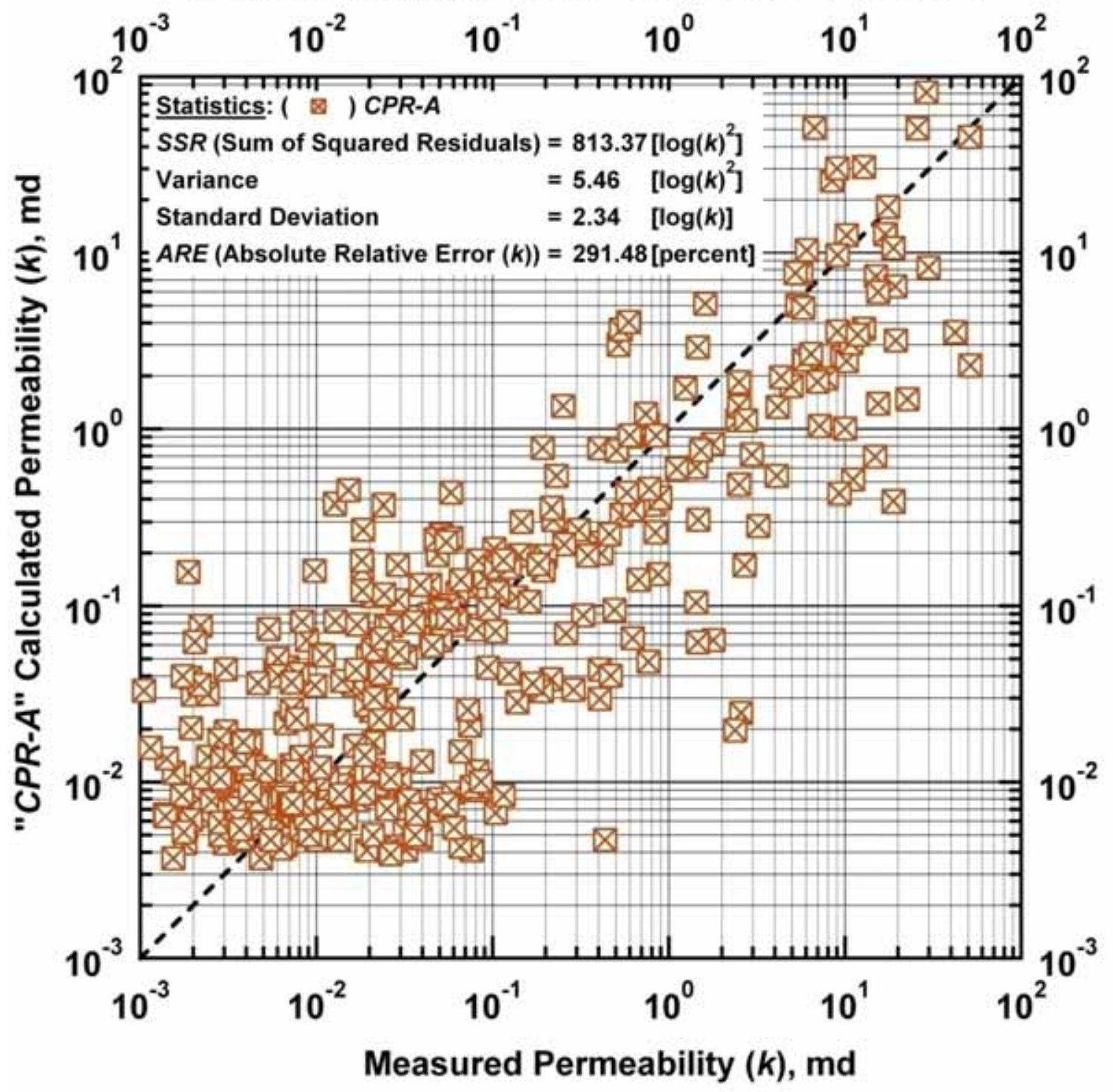

Figure B.2a - Model CPR-A: calculated versus measured permeability. [Case: Howell Well, E. TX (USA)] 


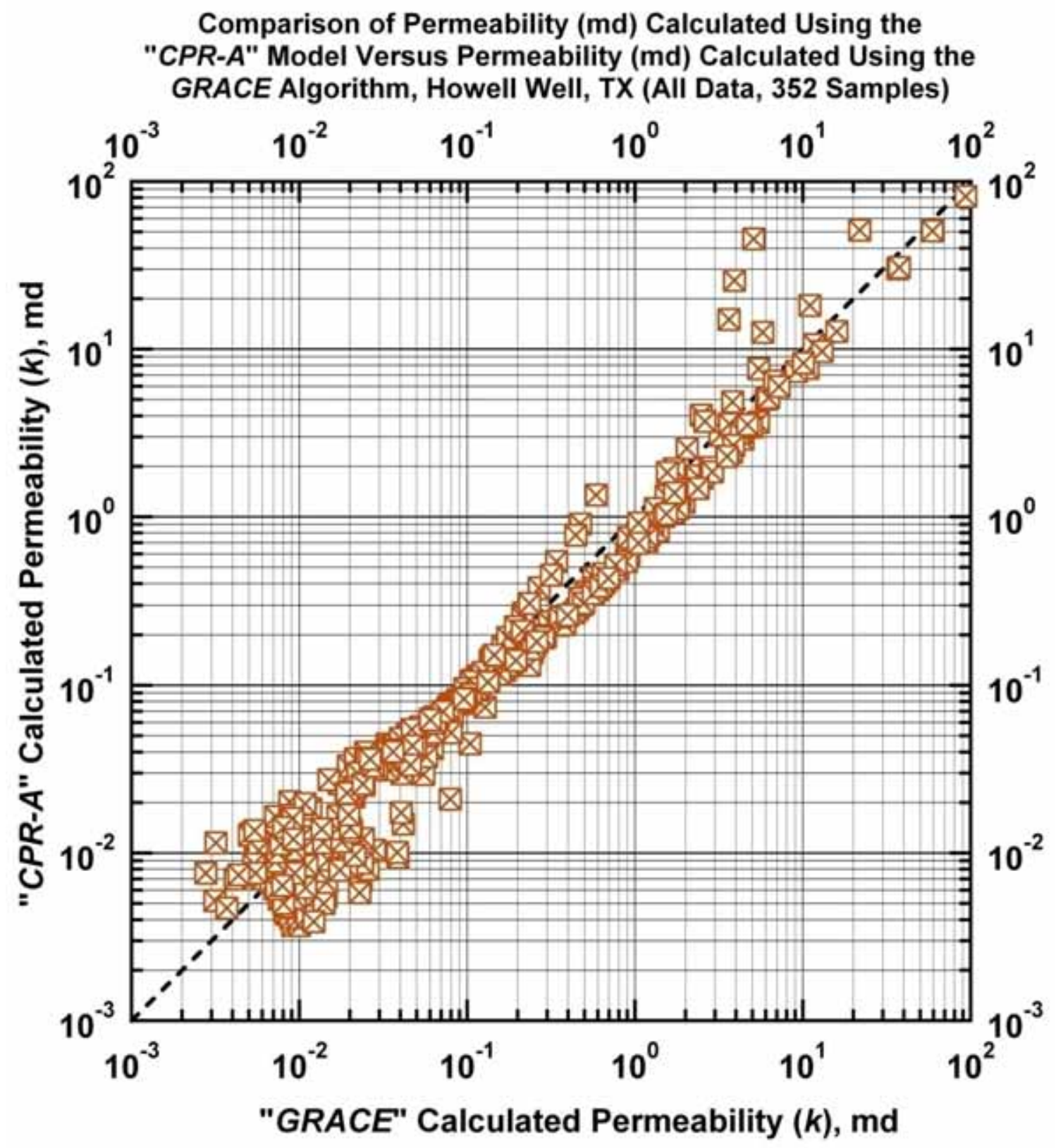

Figure B.2b - Model CPR-A: Permeability calculated using Model CPR-A versus permeability calculated using the GRACE Algorithm. [Case: Howell Well, E. TX (USA)] 
Comparison of the $c$-Coefficient Calculated Using the "CPR-A" Model Versus the Measured $c$-Coefficient, Howell Well, TX (All Data, 352 Samples)

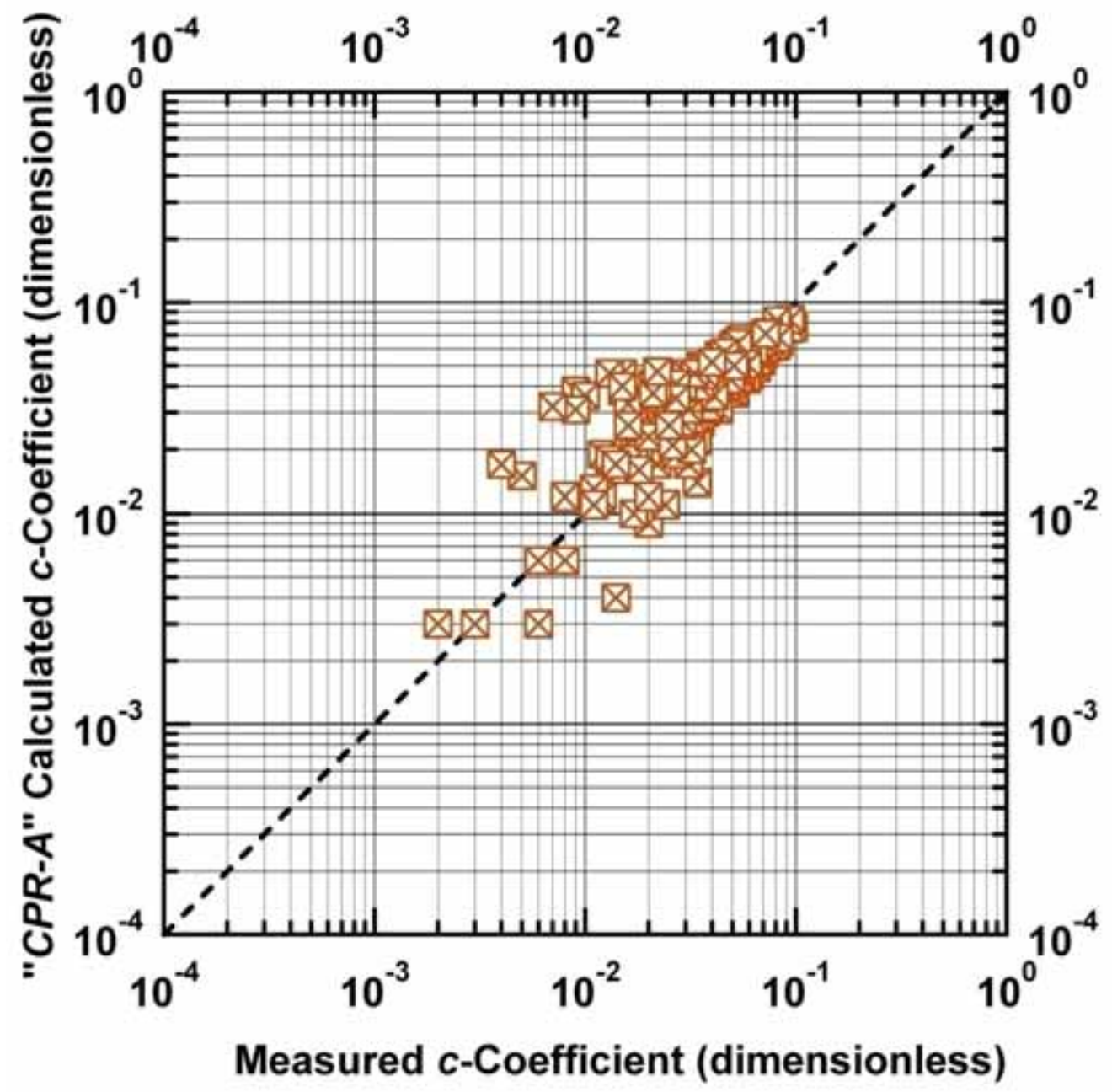

Figure B.2c - Model CPR-A: Calculated $c$-function values versus measured $c$-function values. [Case: Howell Well, E. TX (USA)] 
Plot of Depth (ft) Versus Measured and Calculated Permeability (md), Howell Well, TX

(All data used in correlation, Zone 1)

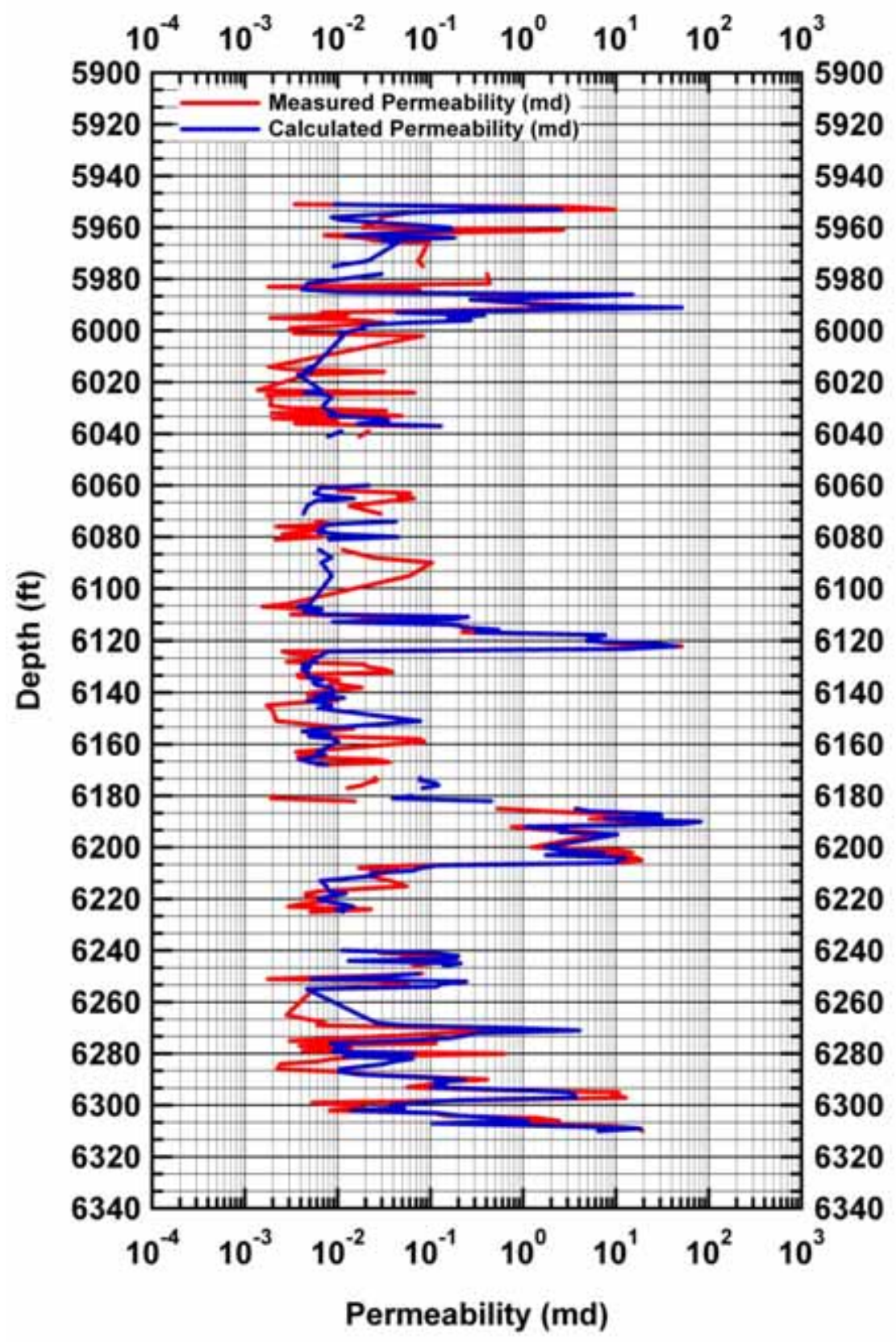

Figure B.2d - Model CPR-A: Calculated and measured permeability values versus depth. [Case: Howell Well, E. TX (USA)] 


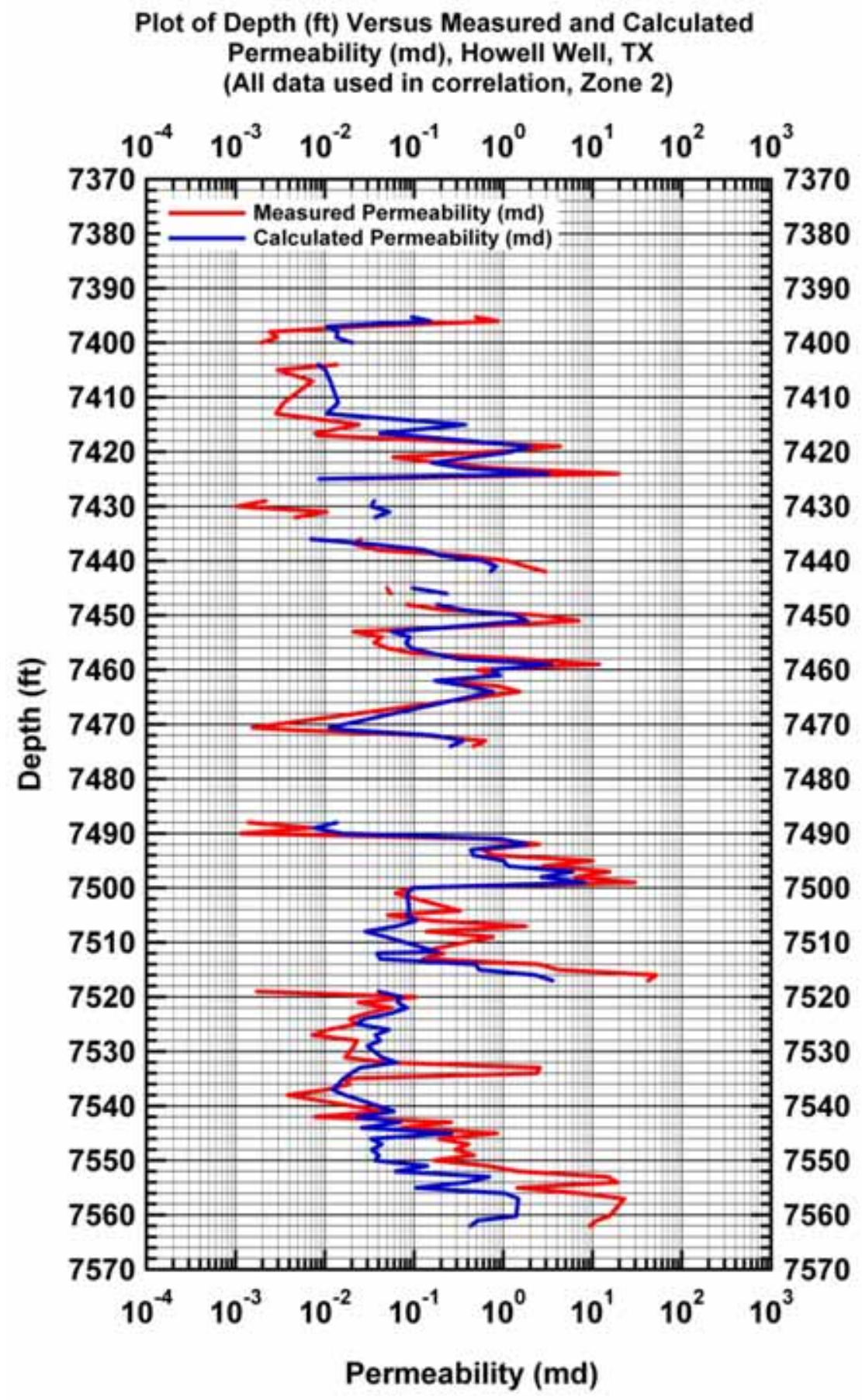

Figure B.2e - Model CPR-A: Calculated and measured permeability values versus depth. [Case: Howell Well, E. TX (USA)] 


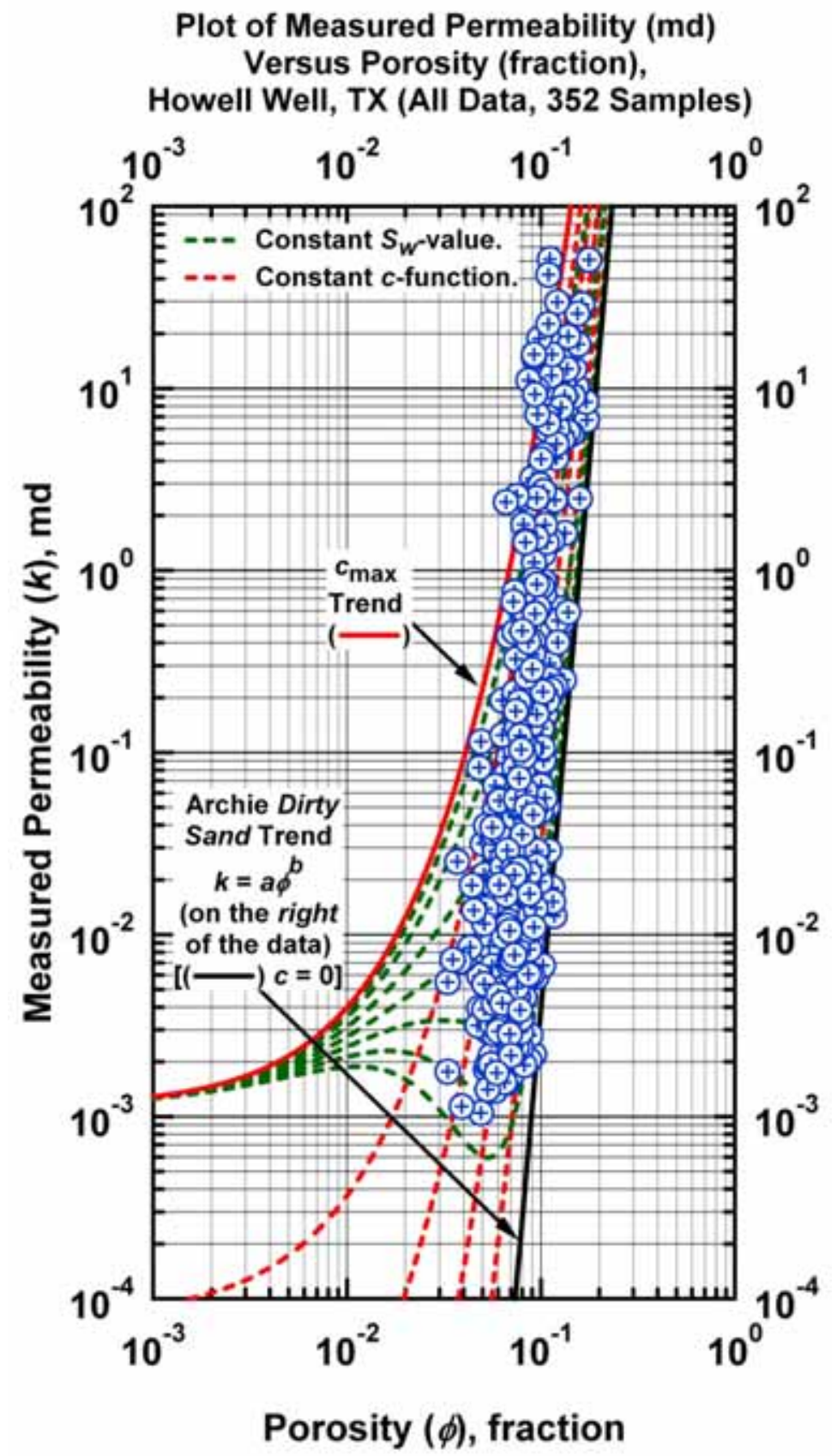

Figure B.3a - Model $C P R-B: \quad k=a(\phi+c)^{b} \quad c=c_{\max } \exp \left[-c_{1} \phi^{c_{2}} S_{W}^{c_{3}}\right]$ — "Clean Sand" Plot (log-log format) — Archie "Dirty Sand" trend is given by the straight-line trend at the far right of the data (power law model). [Case: Howell Well, E. TX (USA)] 


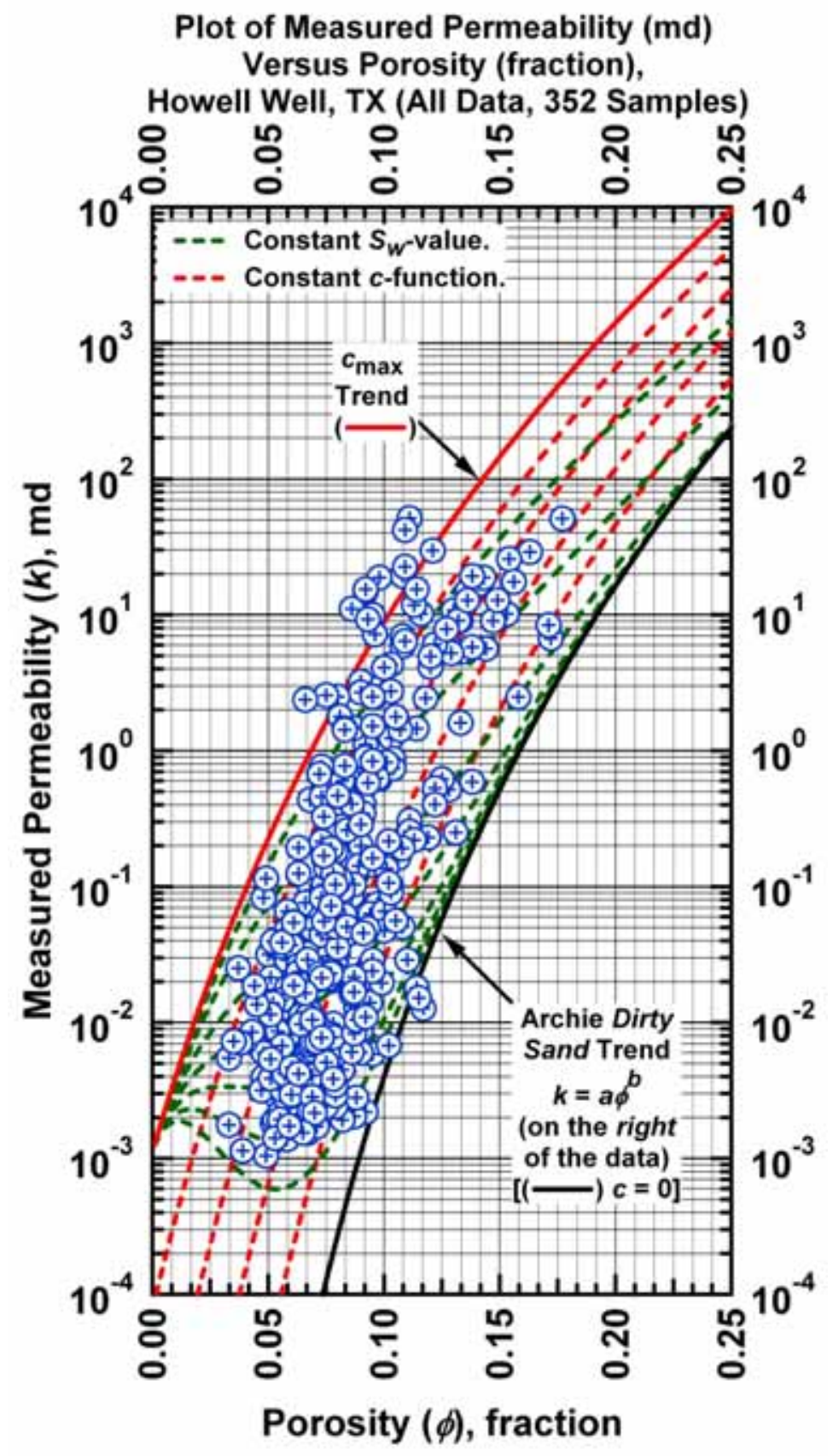

Figure B.3b - Model CPR-B: $k=a(\phi+c)^{b} \quad c=c_{\max } \exp \left[-c_{1} \phi^{c_{2}} S_{w}^{c_{3}}\right]$ — "Dirty Sand" Plot (semilog format) - Archie "Dirty Sand" trend is given by the curved trend at the far right of the data (power law model). [Case: Howell Well, E. TX (USA)] 


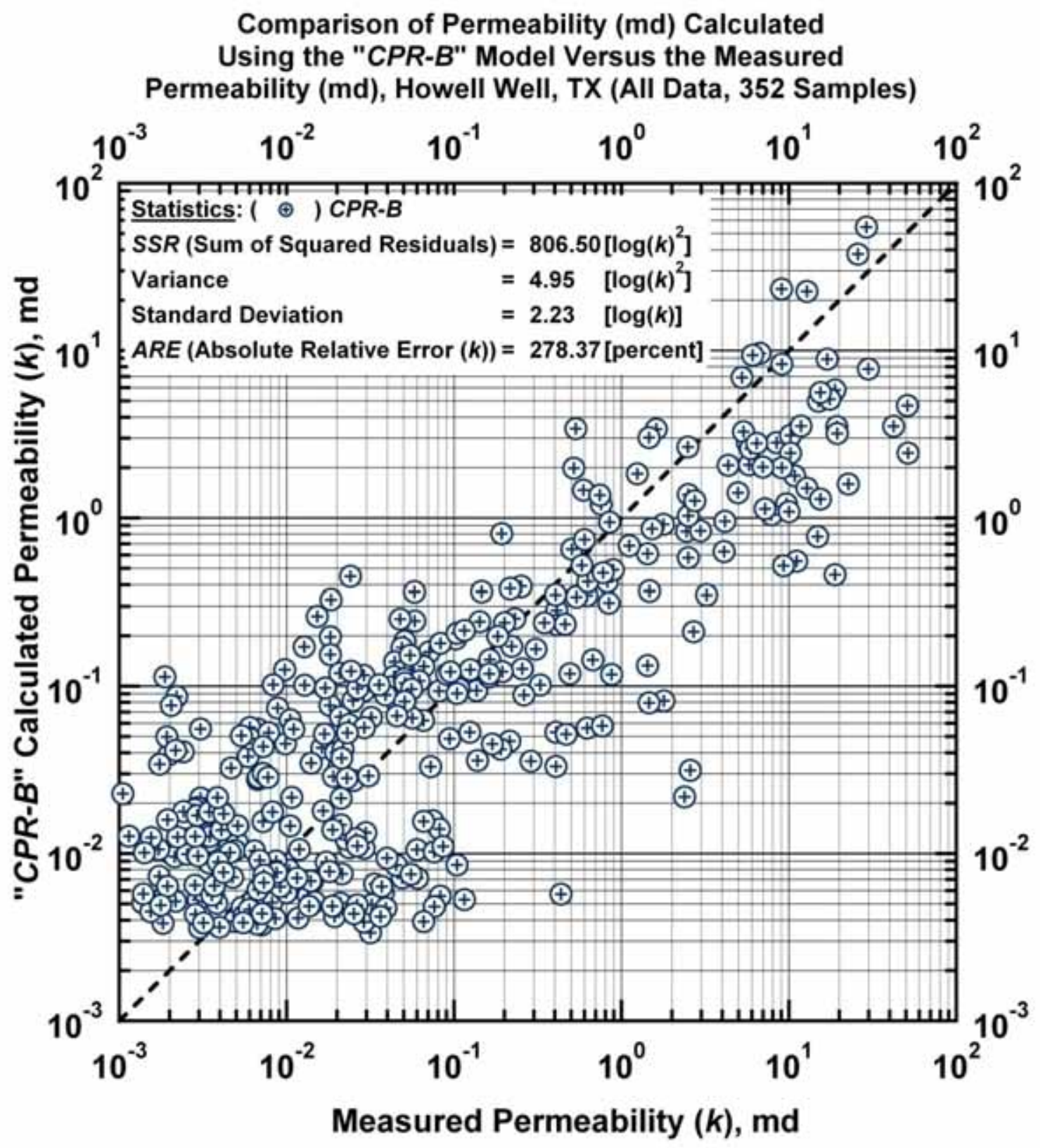

Figure B.4a - Model CPR-B: calculated versus measured permeability. [Case: Howell Well, E. TX (USA)] 


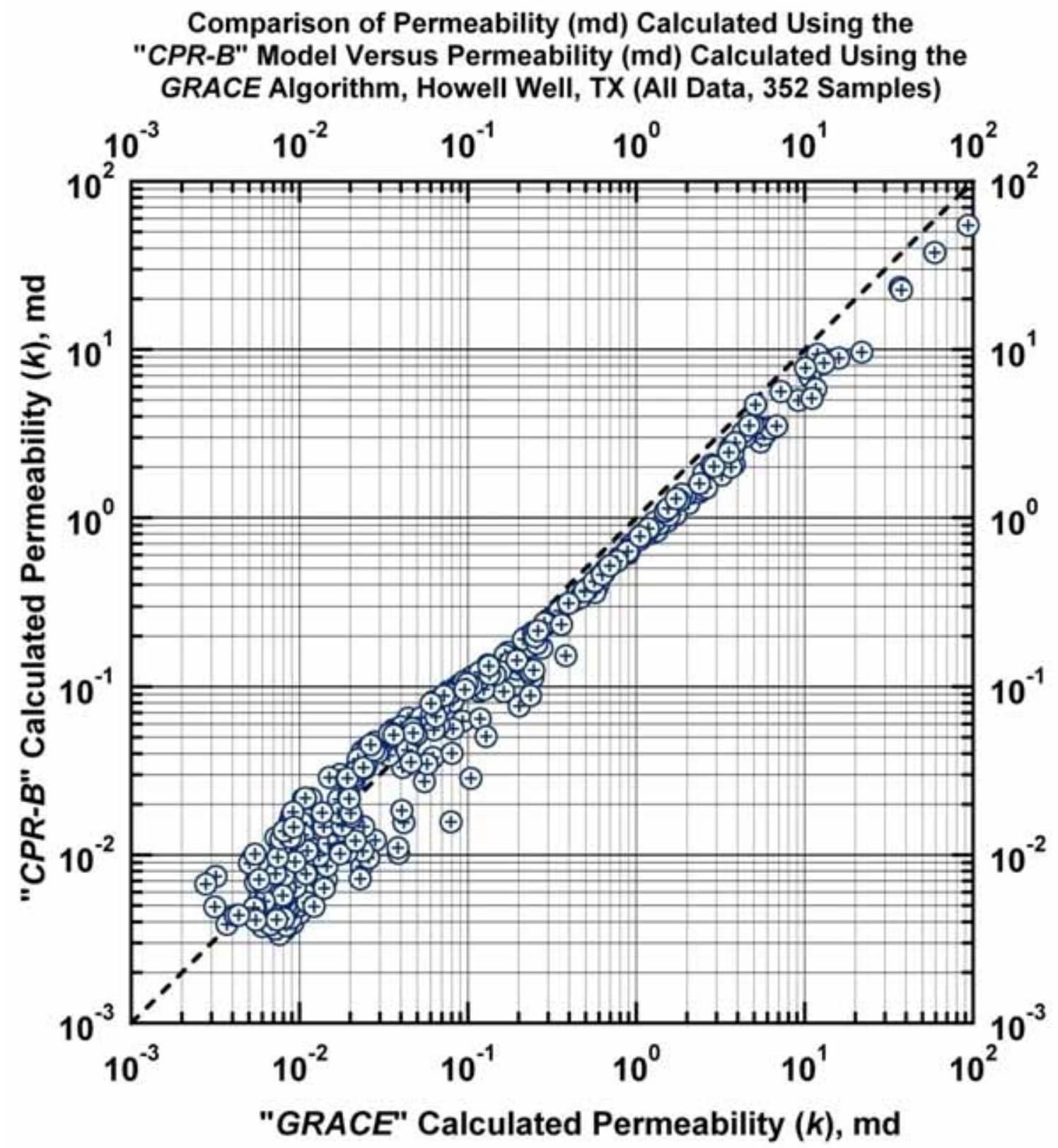

Figure B.4b - Model CPR-B: Permeability calculated using Model $C P R-B$ versus permeability calculated using the GRACE Algorithm. [Case: Howell Well, E. TX (USA)] 


\section{Comparison of the $c$-Coefficient Calculated Using} the "CPR-B" Model Versus the Measured $c$-Coefficient, Howell Well, TX (All Data, 352 Samples)

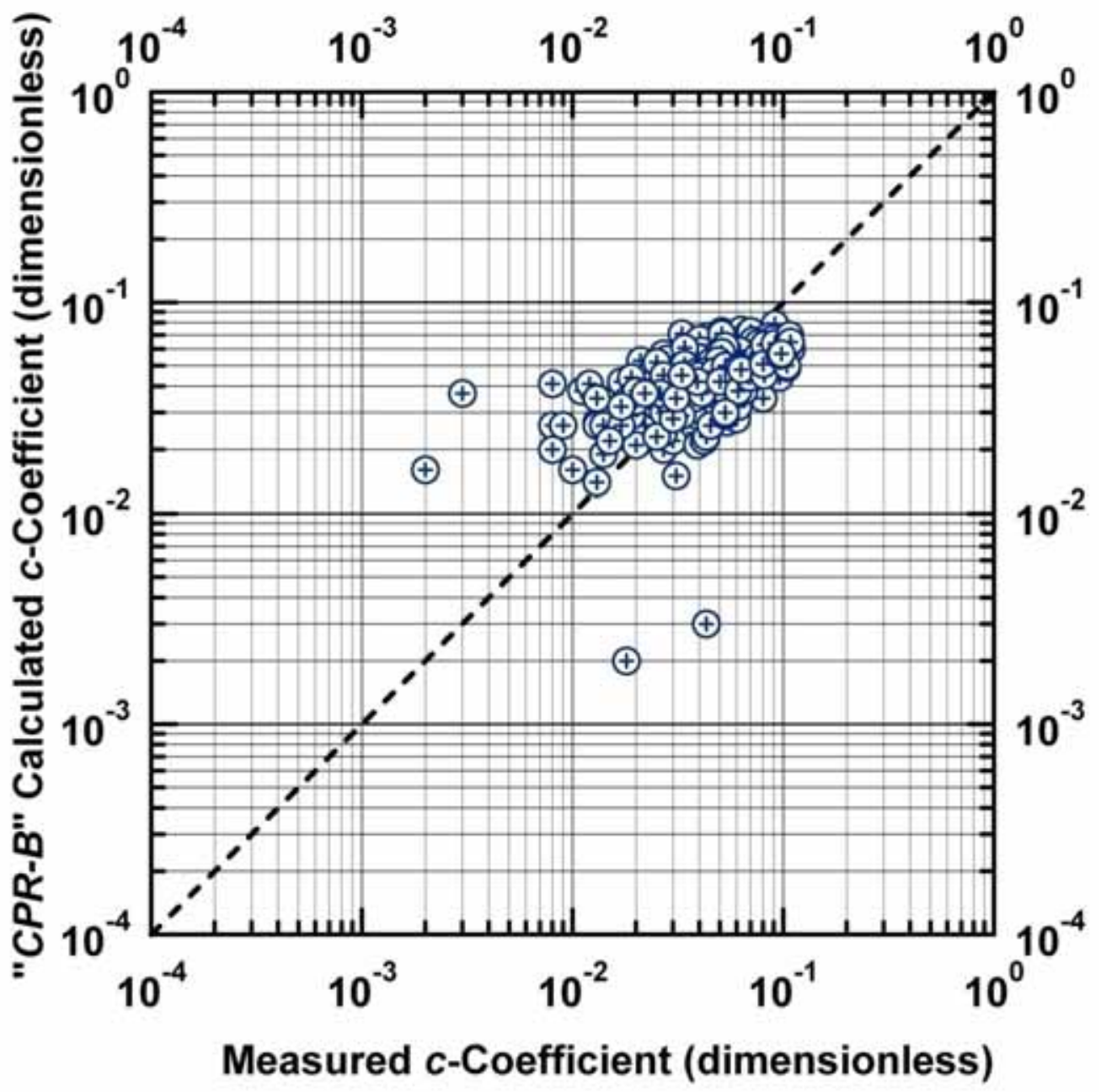

Figure B.4c - Model $C P R$ - $B$ : Calculated $c$-function values versus measured $c$-function values. [Case: Howell Well, E. TX (USA)] 


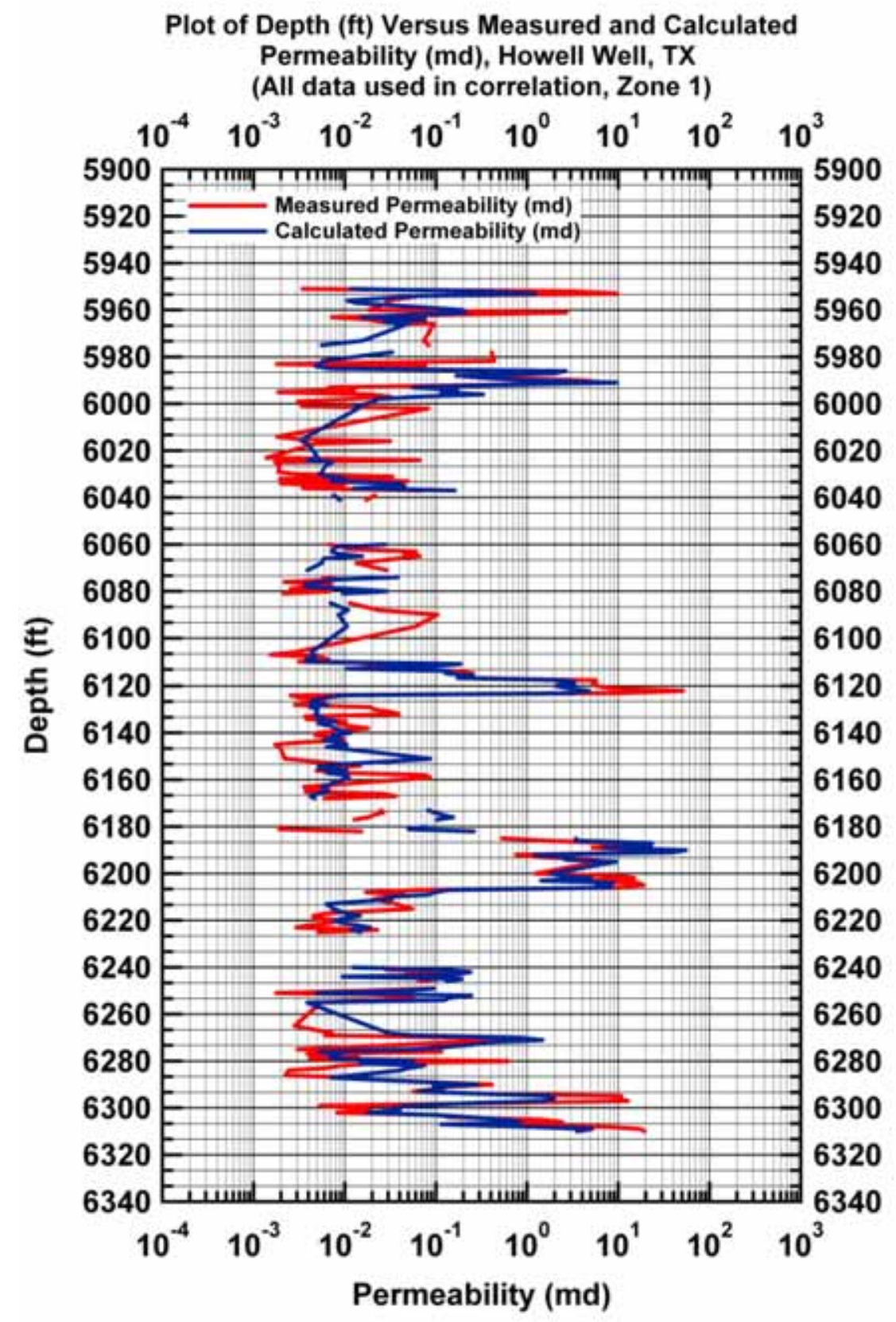

Figure B.4d - Model CPR-B: Calculated and measured permeability values versus depth. [Case: Howell Well, E. TX (USA)] 


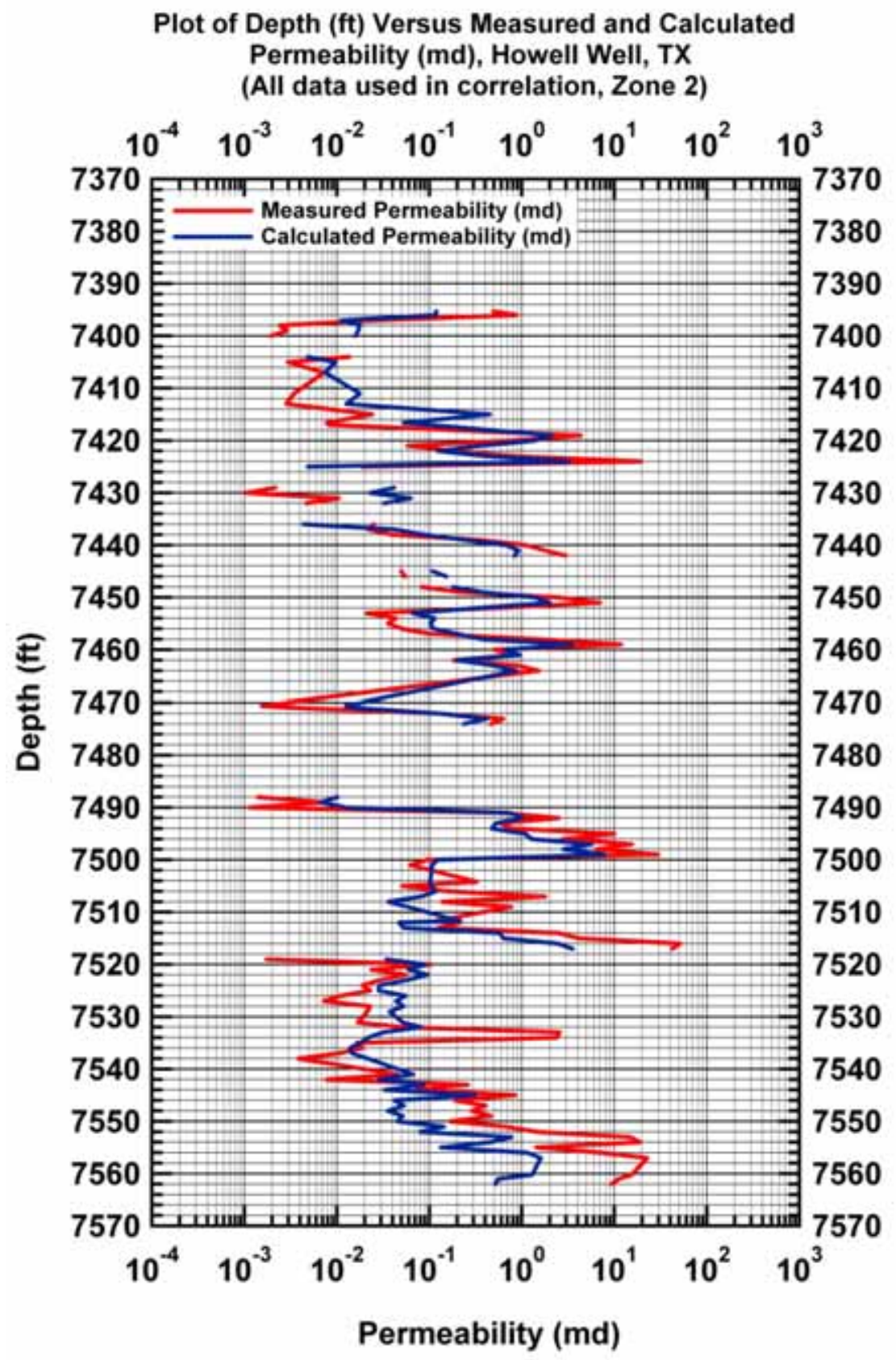

Figure B.4e - Model CPR-B: Calculated and measured permeability values versus depth. [Case: Howell Well, E. TX (USA)] 


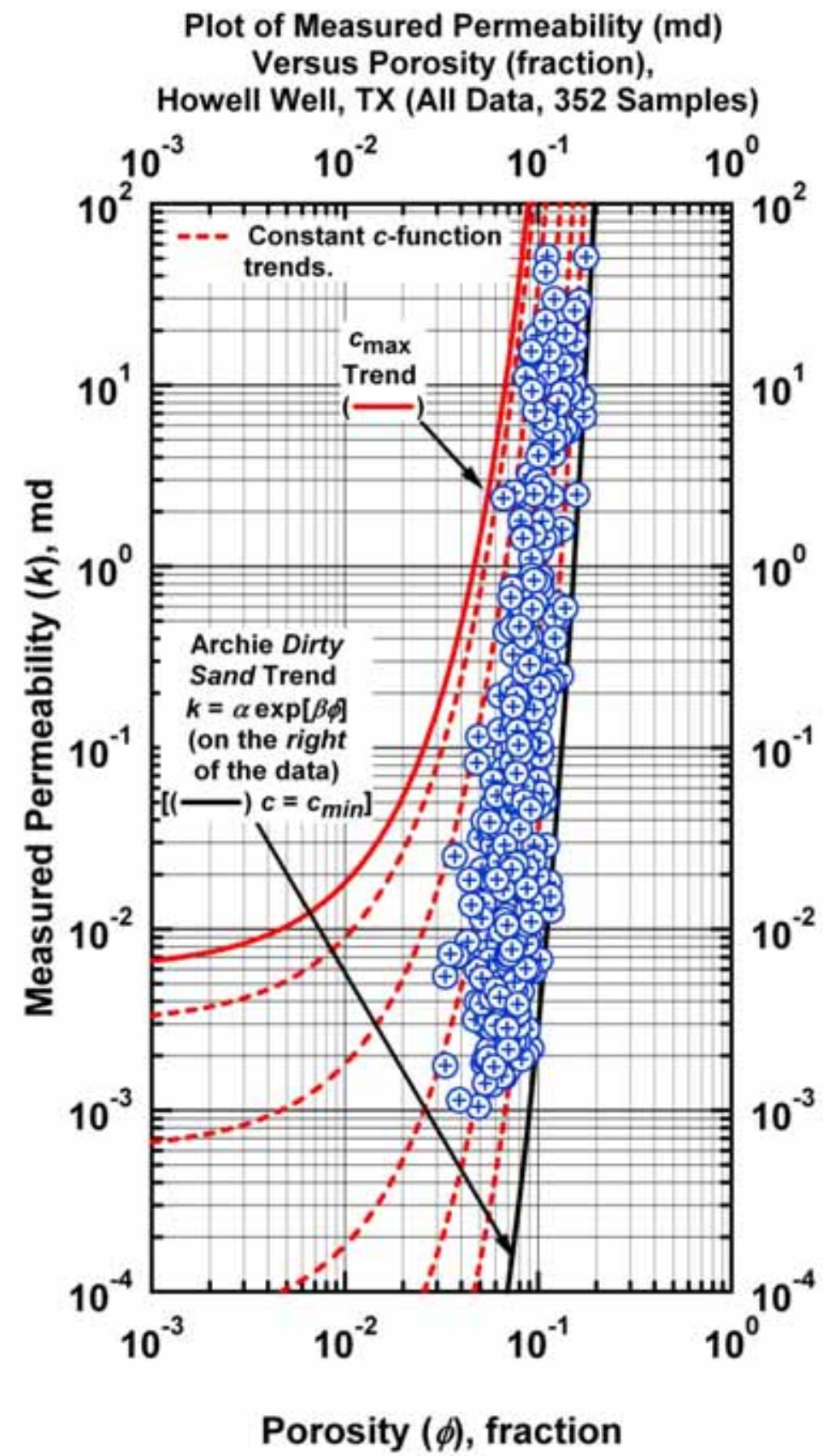

Figure B.5a - Model $C P R-C: \quad k=c \exp [\beta \phi] \quad c=c_{\max } \exp \left[-c_{1} \phi^{c_{2}} S_{w}^{c_{3}}\right]-$ "Clean Sand" Plot (log-log format) - Archie "Dirty Sand" trend is given by the curved trend at the far right of the data (exponential model). [Case: Howell Well, E. TX (USA)] 


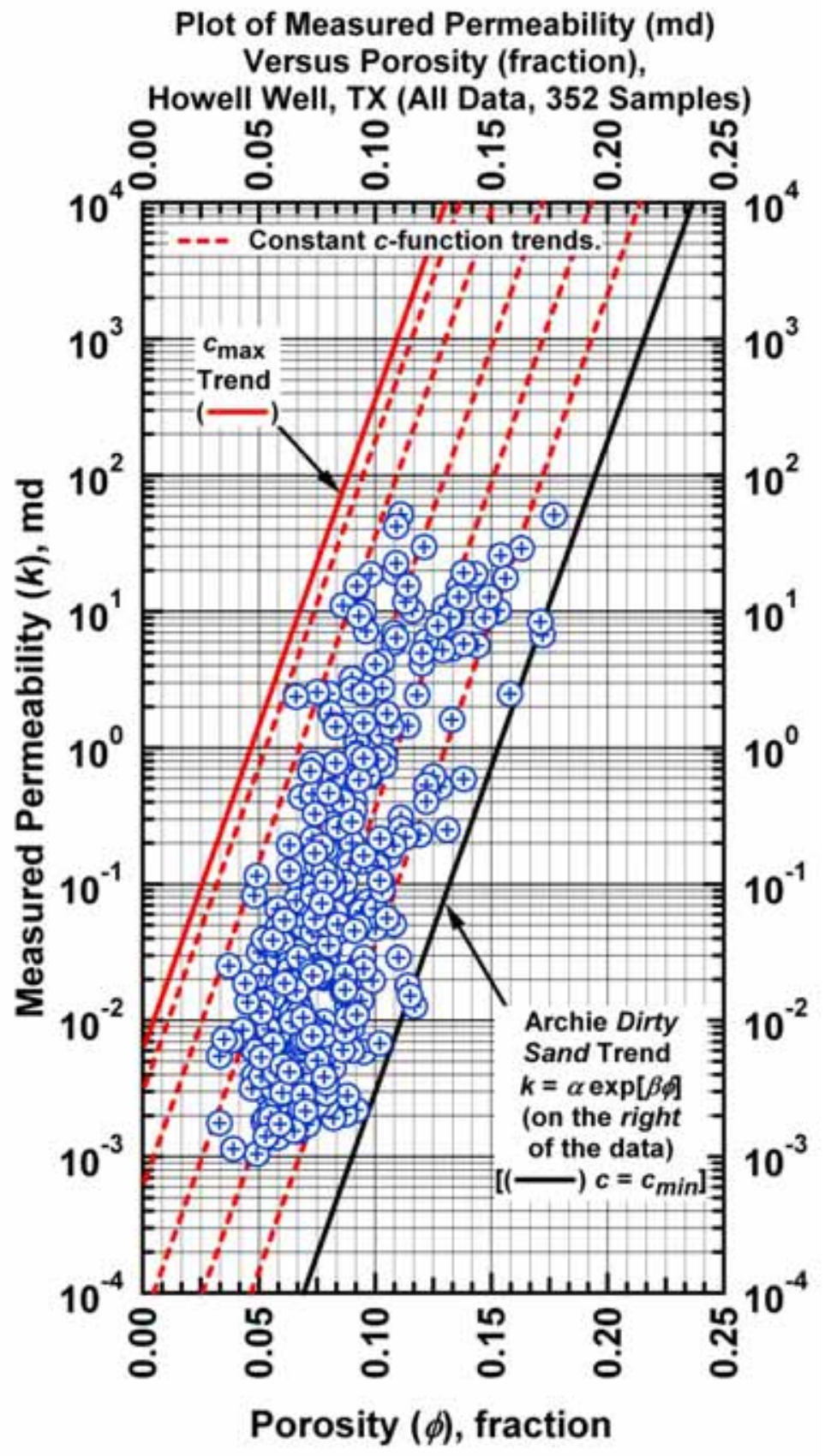

Figure B.5b - Model $C P R-C: k=c \exp [\beta \phi] \quad c=c_{\max } \exp \left[-c_{1} \phi^{c_{2}} S_{w}^{C_{3}}\right]$ - "Dirty Sand" Plot (semilog format) - Archie "Dirty Sand" trend is given by the straight-line trend at the far right of the data (exponential model). [Case: Howell Well, E. TX (USA)] 


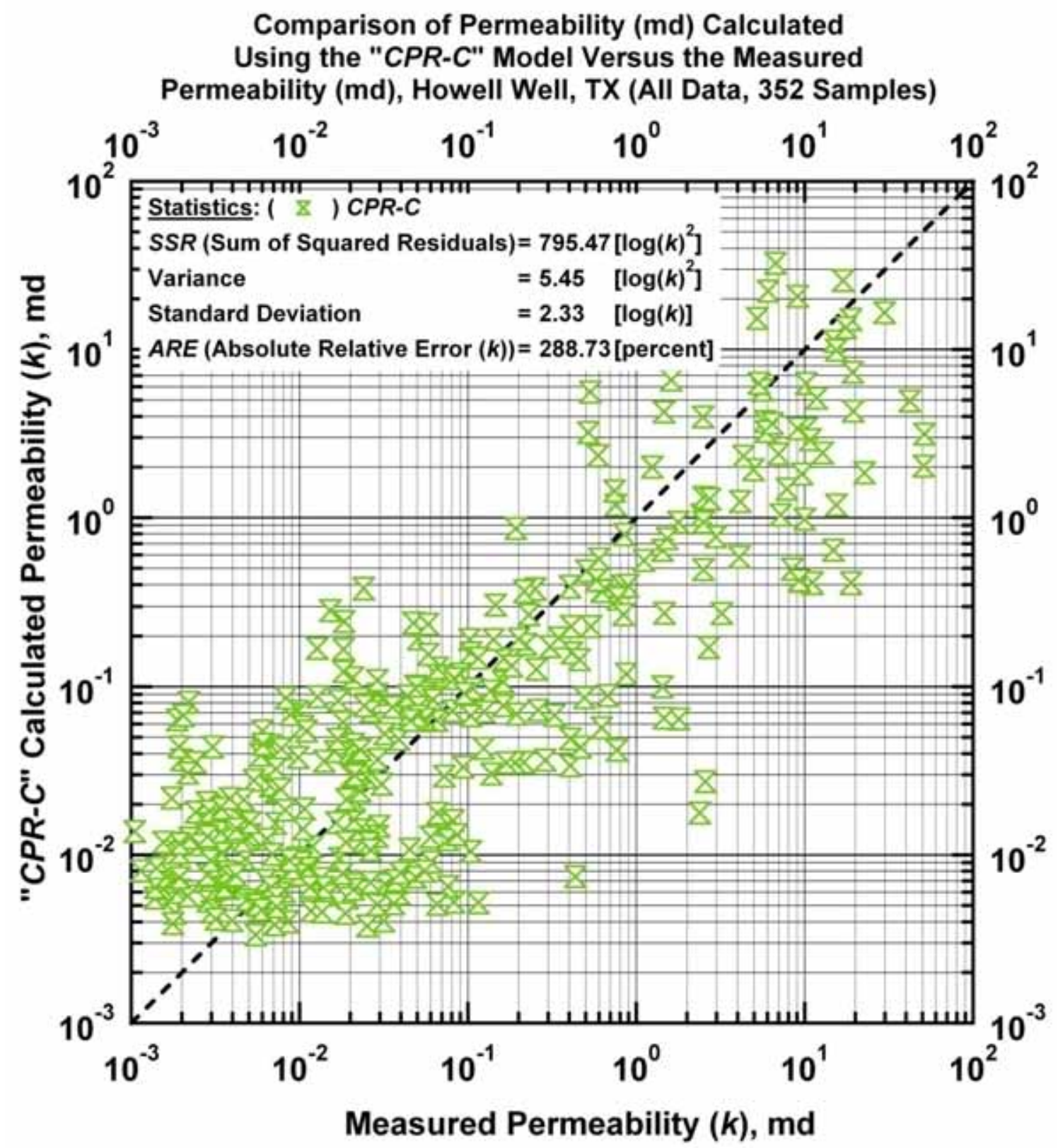

Figure B.6a - Model CPR-C: calculated versus measured permeability. [Case: Howell Well, E. TX (USA)] 


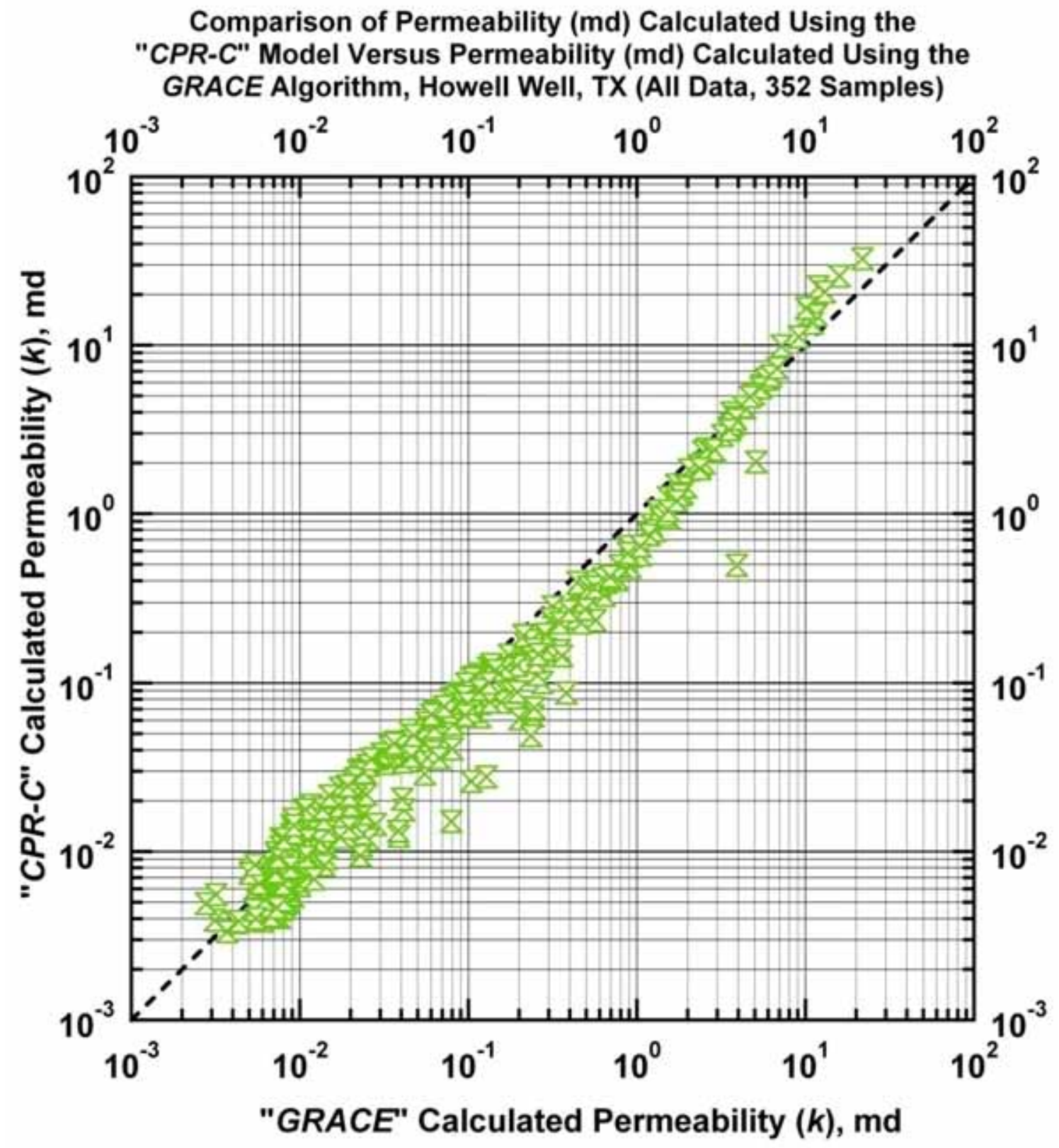

Figure B.6b - Model $C P R-C$ : Permeability calculated using Model $C P R-C$ versus permeability calculated using the GRACE Algorithm. [Case: Howell Well, E. TX (USA)] 


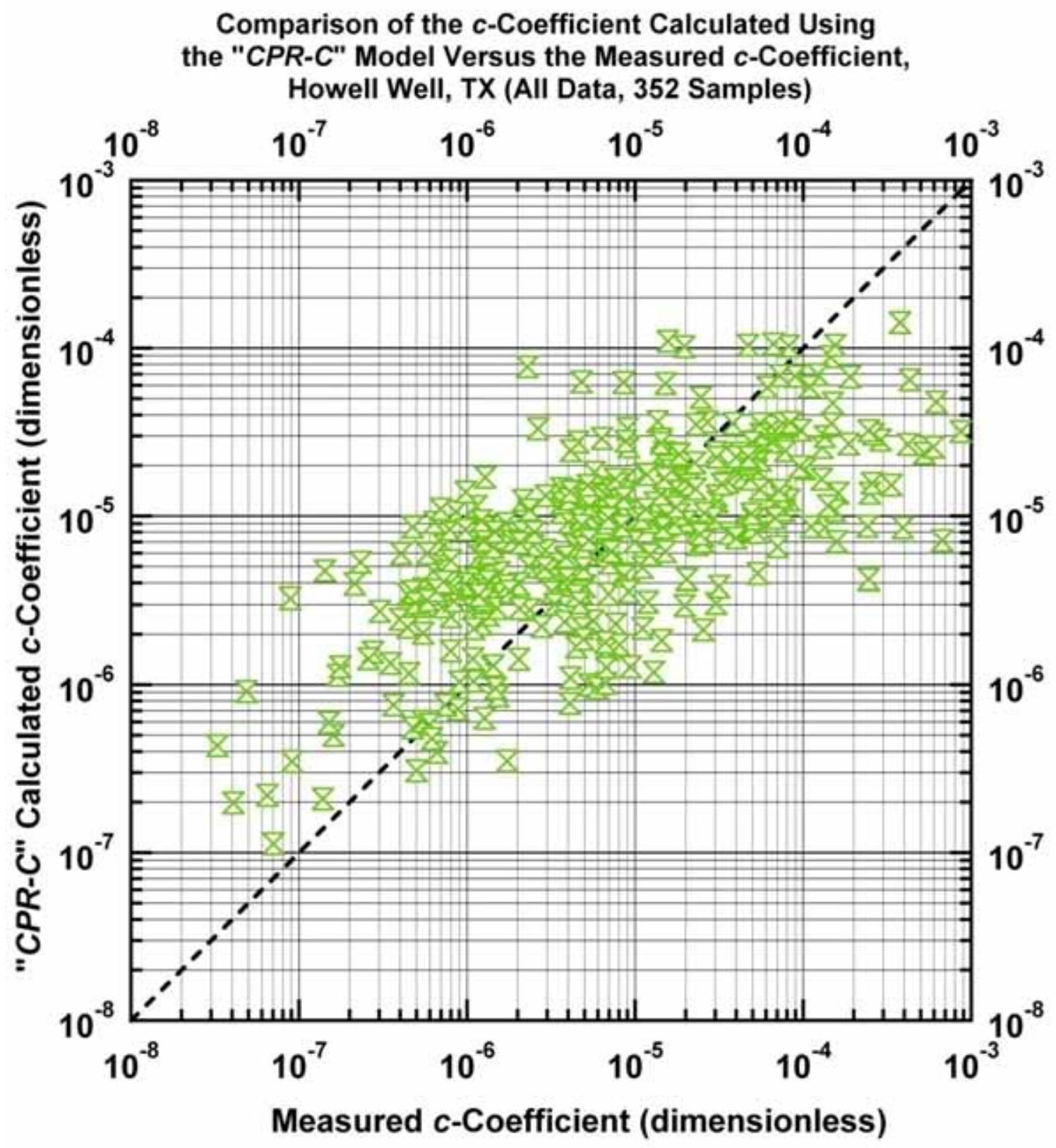

Figure B.6c - Model $C P R$-C: Calculated $c$-function values versus measured $c$-function values. [Case: Howell Well, E. TX (USA)] 


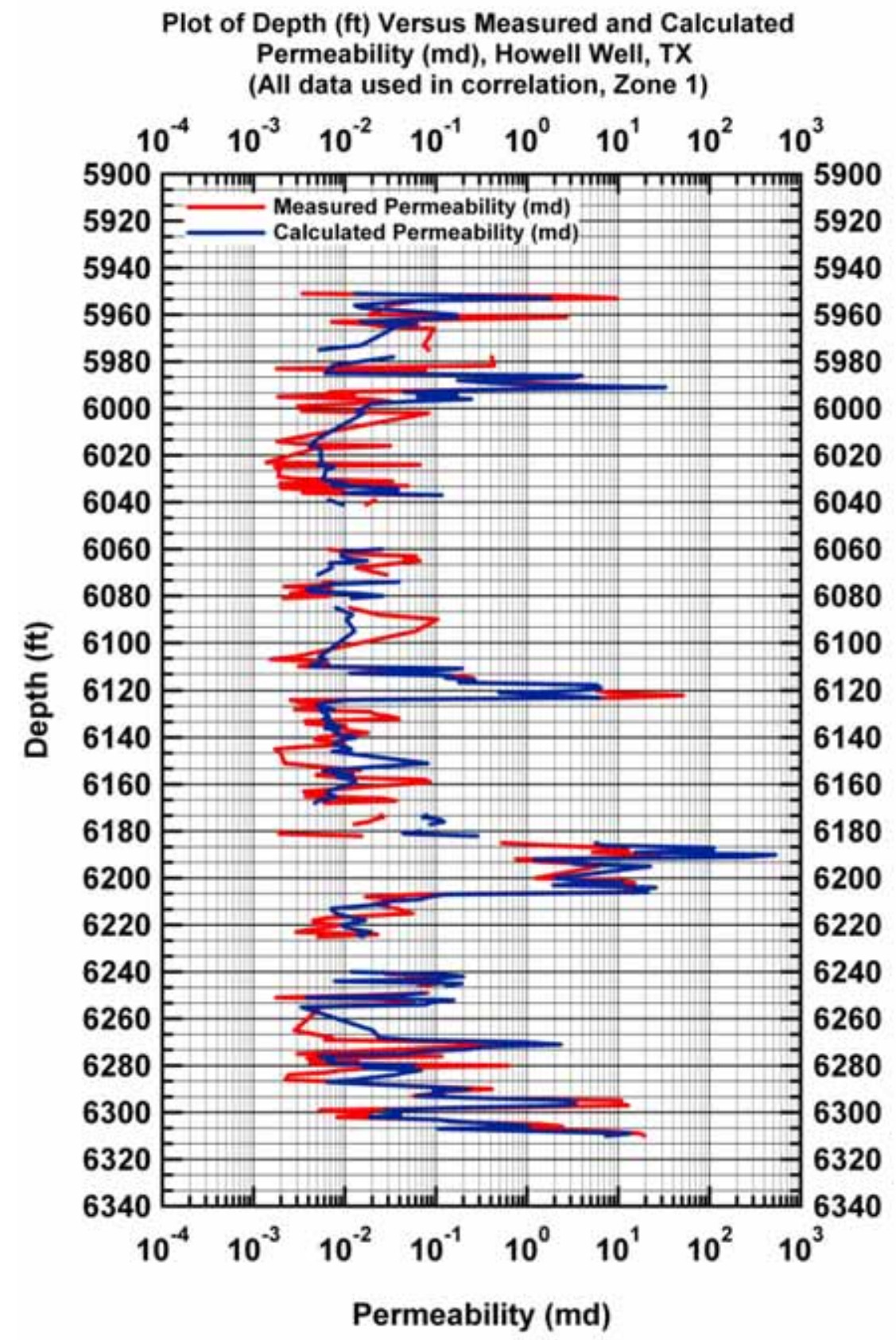

Figure B.6d - Model $C P R-C$ : Calculated and measured permeability values versus depth. [Case: Howell Well, E. TX (USA)] 


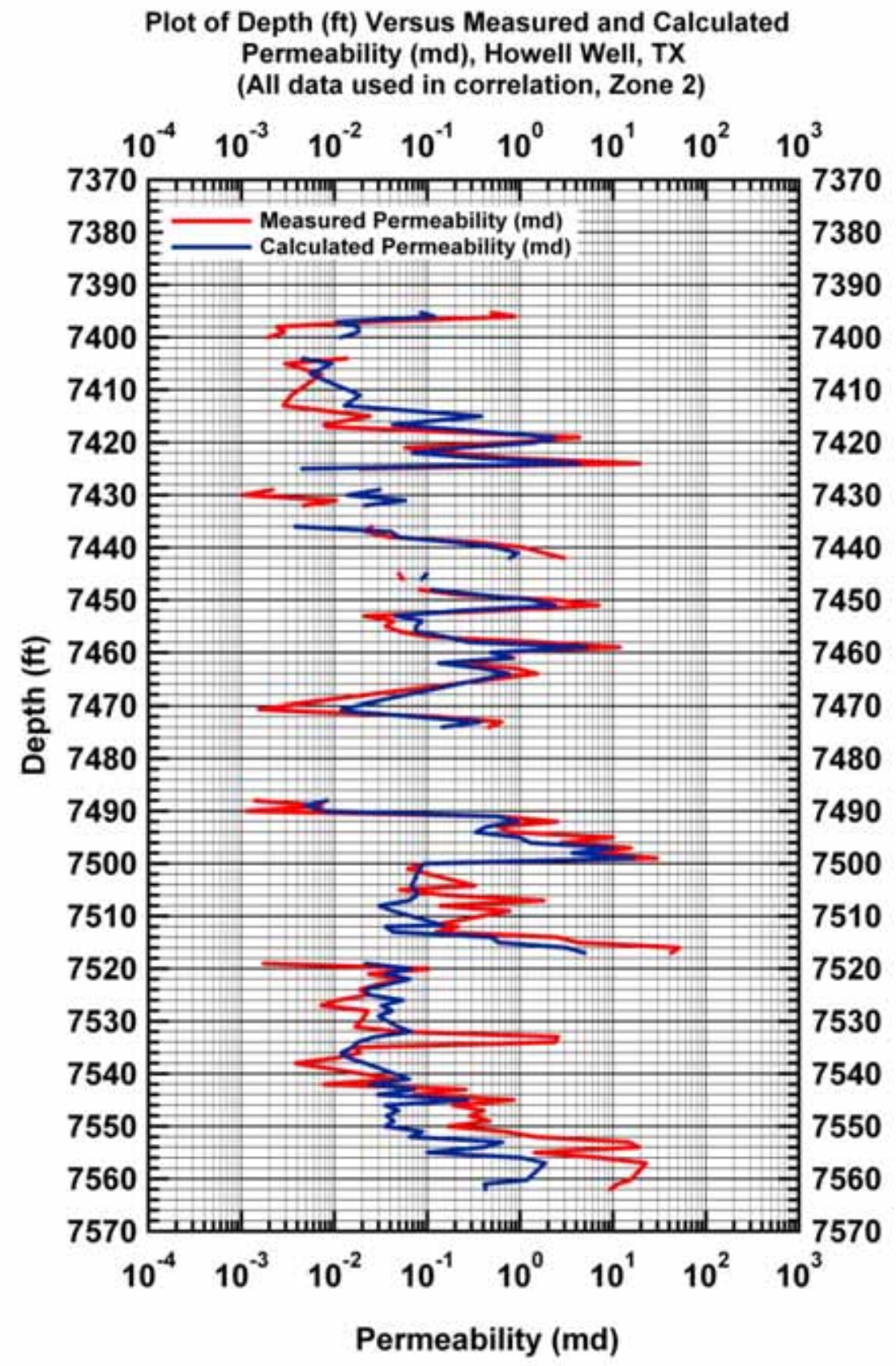

Figure B.6e - Model CPR-C: Calculated and measured permeability values versus depth. [Case: Howell Well, E. TX (USA)] 


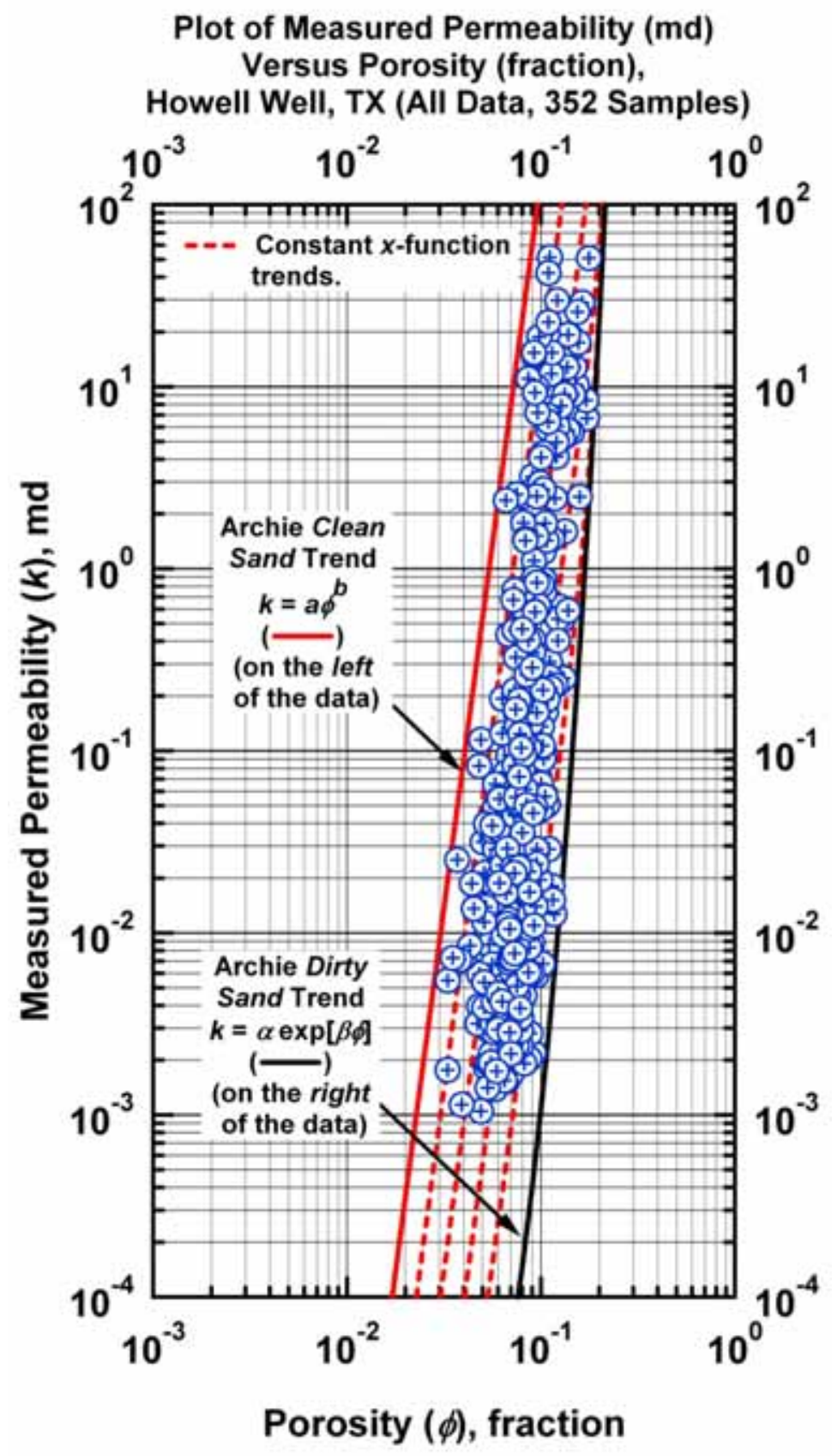

Figure B.7a - Model CPR-D: $k=x a \phi^{b}+(1-x) \alpha \exp [\beta \phi] \quad[0 \leq x \leq 1] \quad$ - Weighted Power Law-Exponential Model used to correlate permeability $(k)$ and porosity $(\phi)$. "Clean Sand" Plot - Archie "Clean Sand" trend is given by the straight-line trend at the far left of the data (power law model). [Case: Howell Well, E. TX (USA)] 


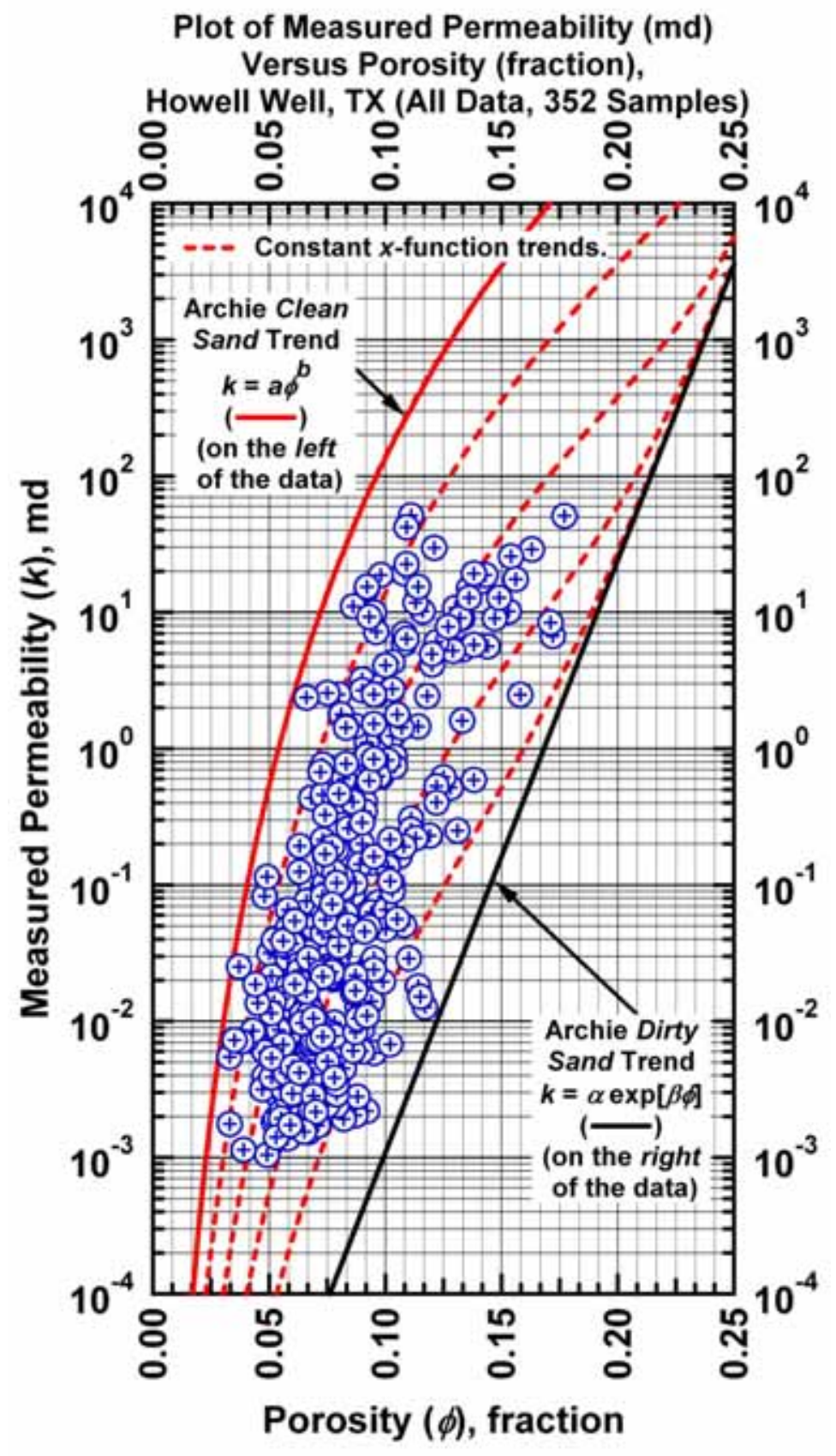

Figure B.7b - Model CPR-D: $k=x a \phi^{b}+(1-x) \alpha \exp [\beta \phi][0 \leq x \leq 1]$ - Weighted Power Law-Exponential Model used to correlate permeability $(k)$ and porosity $(\phi)$. "Dirty Sand" Plot - Archie "Dirty Sand" trend is given by the straight-line trend at the far right of the data (exponential model). [Case: Howell Well, E. TX (USA)] 


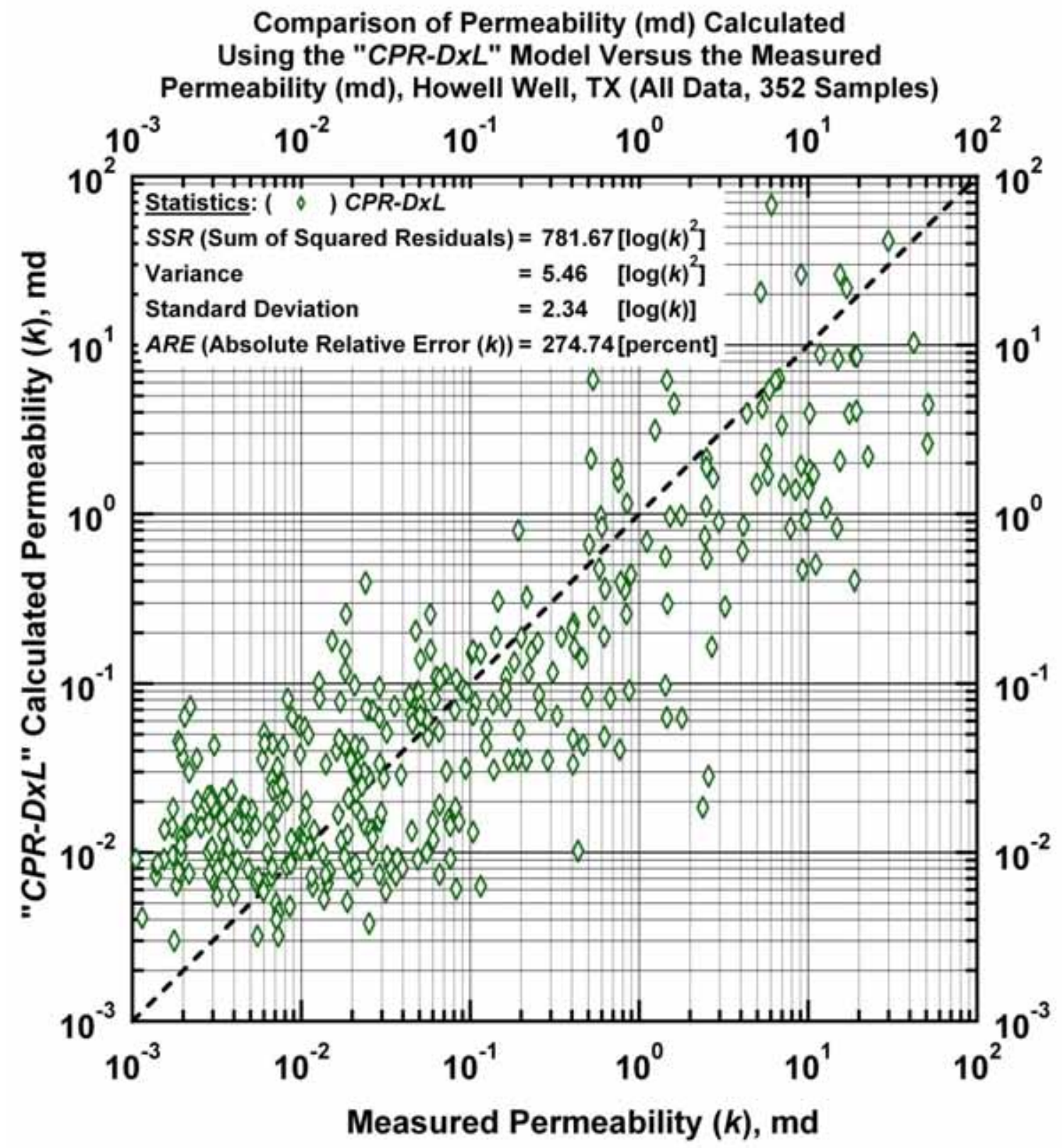

Figure B.8a - Model CPR-DxL: Calculated versus measured permeability. [Case: Howell Well, E. TX (USA)] 


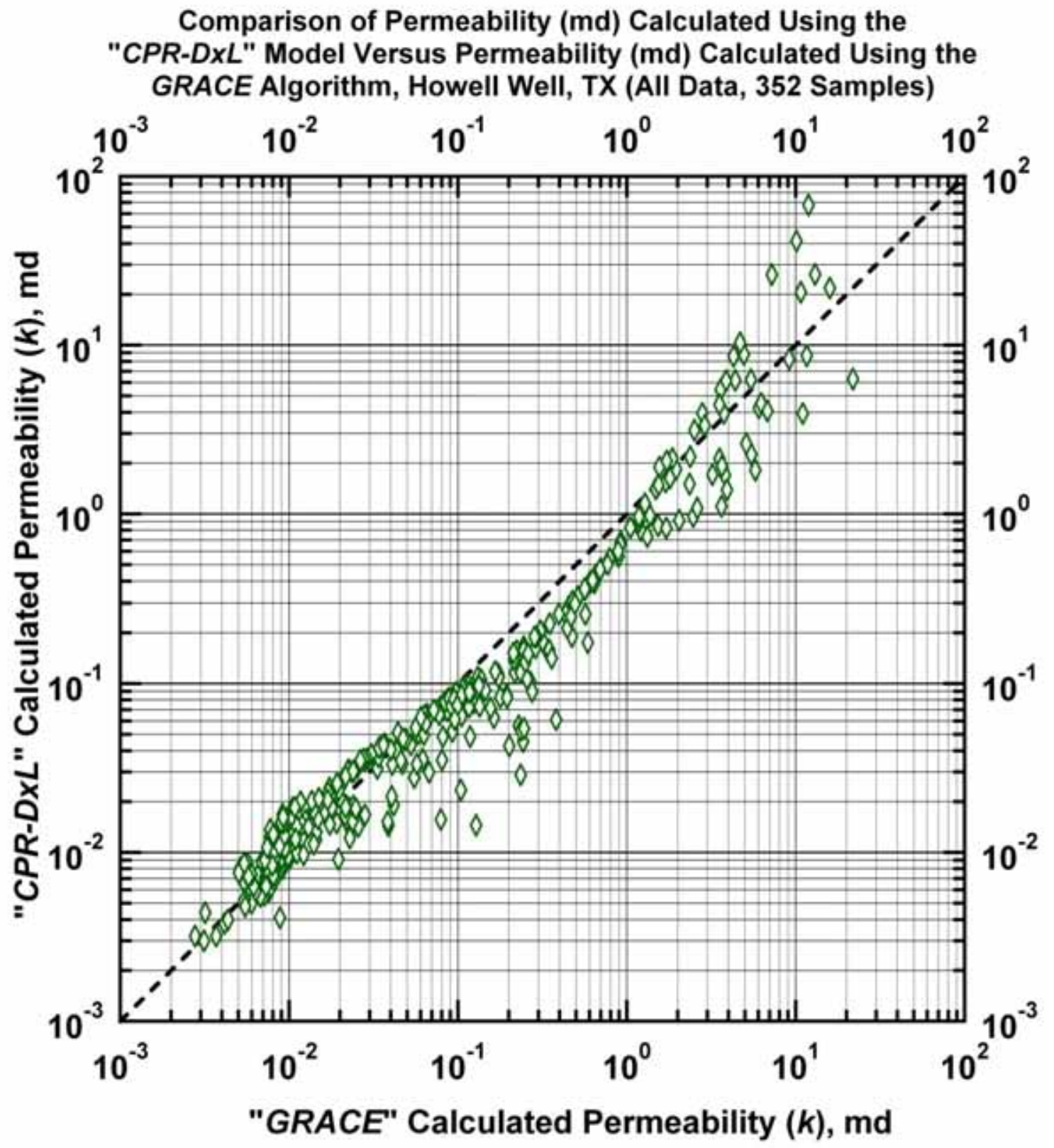

Figure B.8b - Model $C P R-D x L$ : Permeability calculated using Model $C P R-C$ versus permeability calculated using the GRACE Algorithm. [Case: Howell Well, E. TX (USA)] 


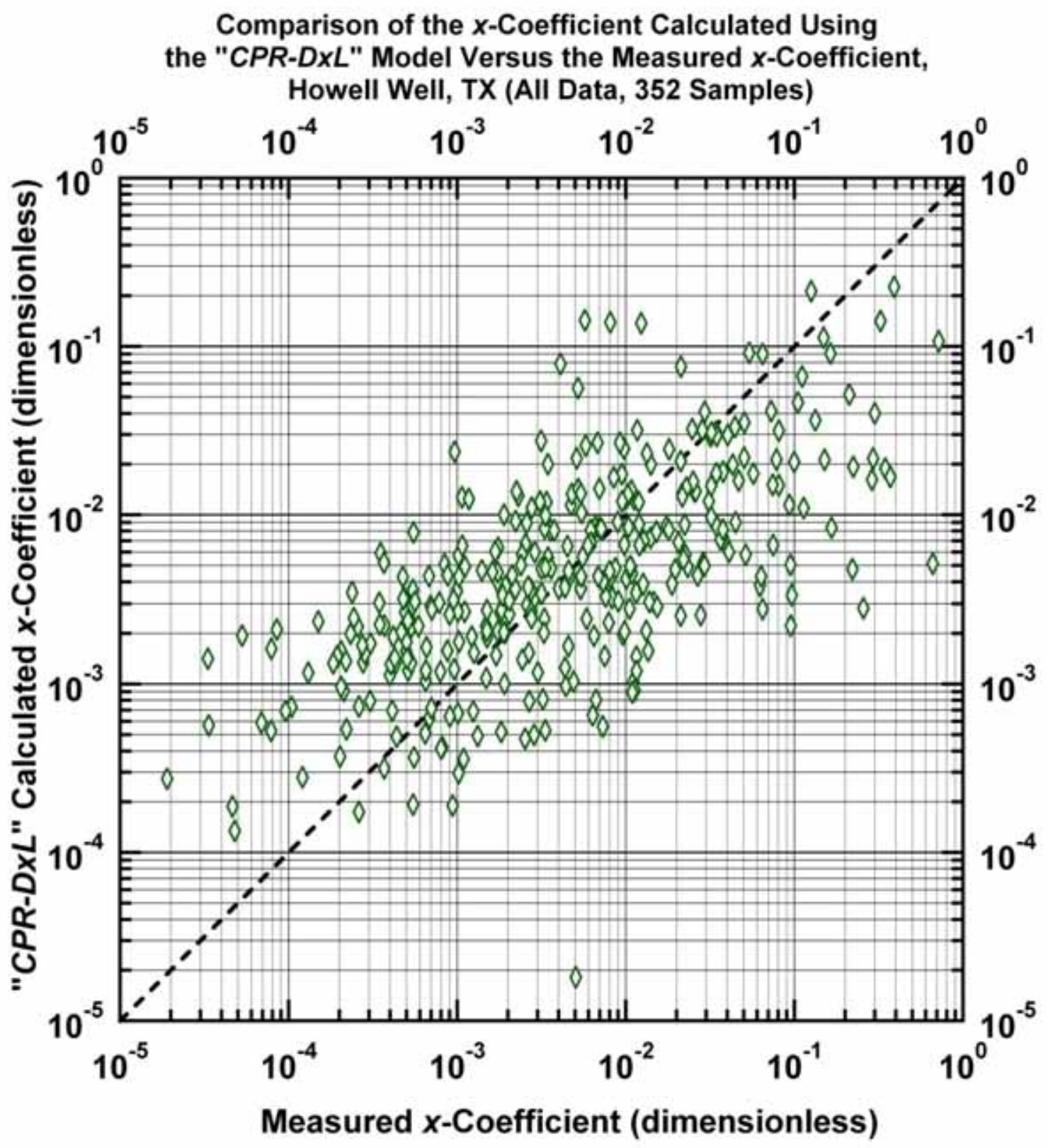

Figure B.8c - Model $C P R-D x L$ : Calculated $x$-function weight values versus measured $x$ function weight values. [Case: Howell Well, E. TX (USA)] 


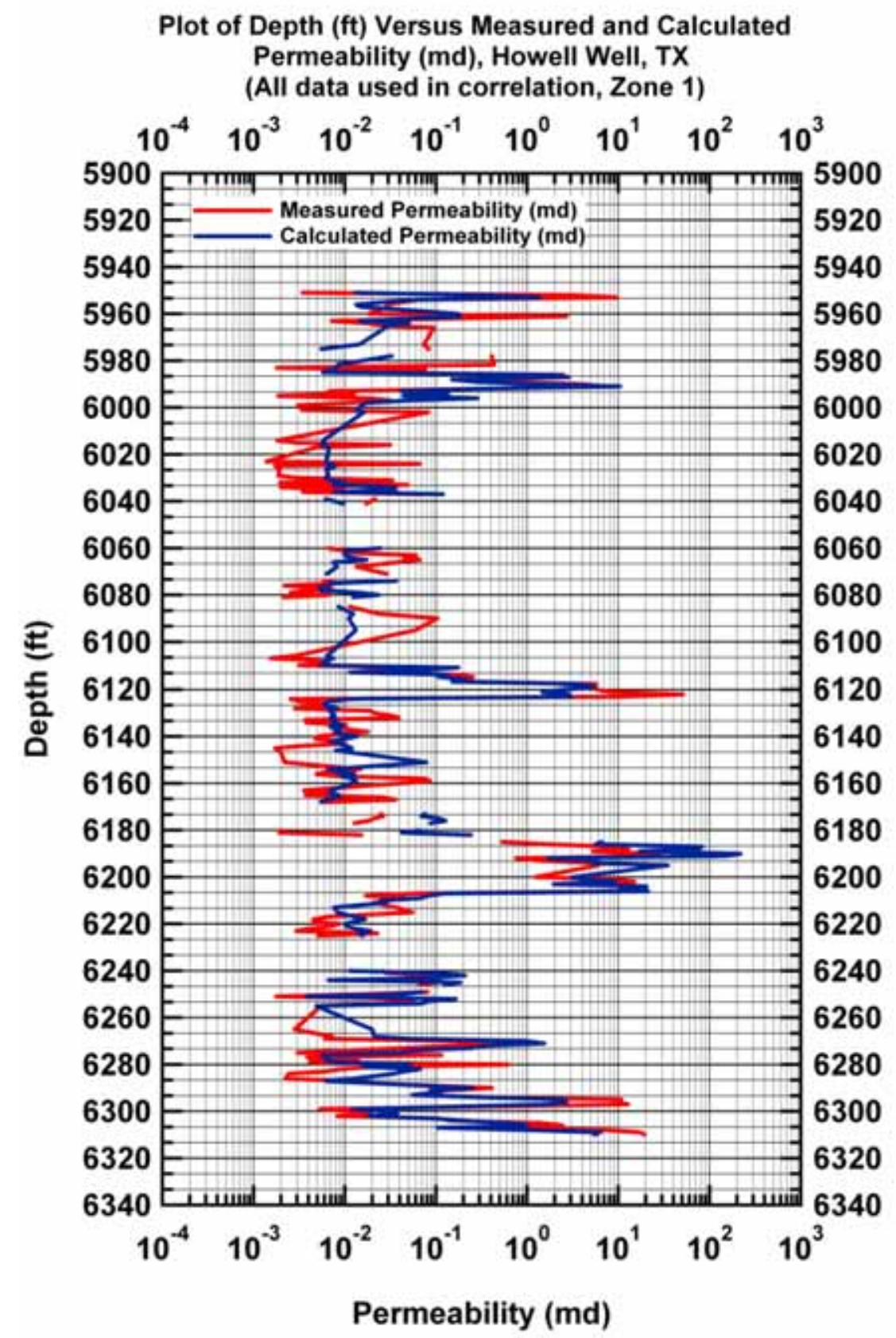

Figure B.8d - Model $C P R-D x L$ : Calculated and measured permeability values versus depth. [Case: Howell Well, E. TX (USA)] 


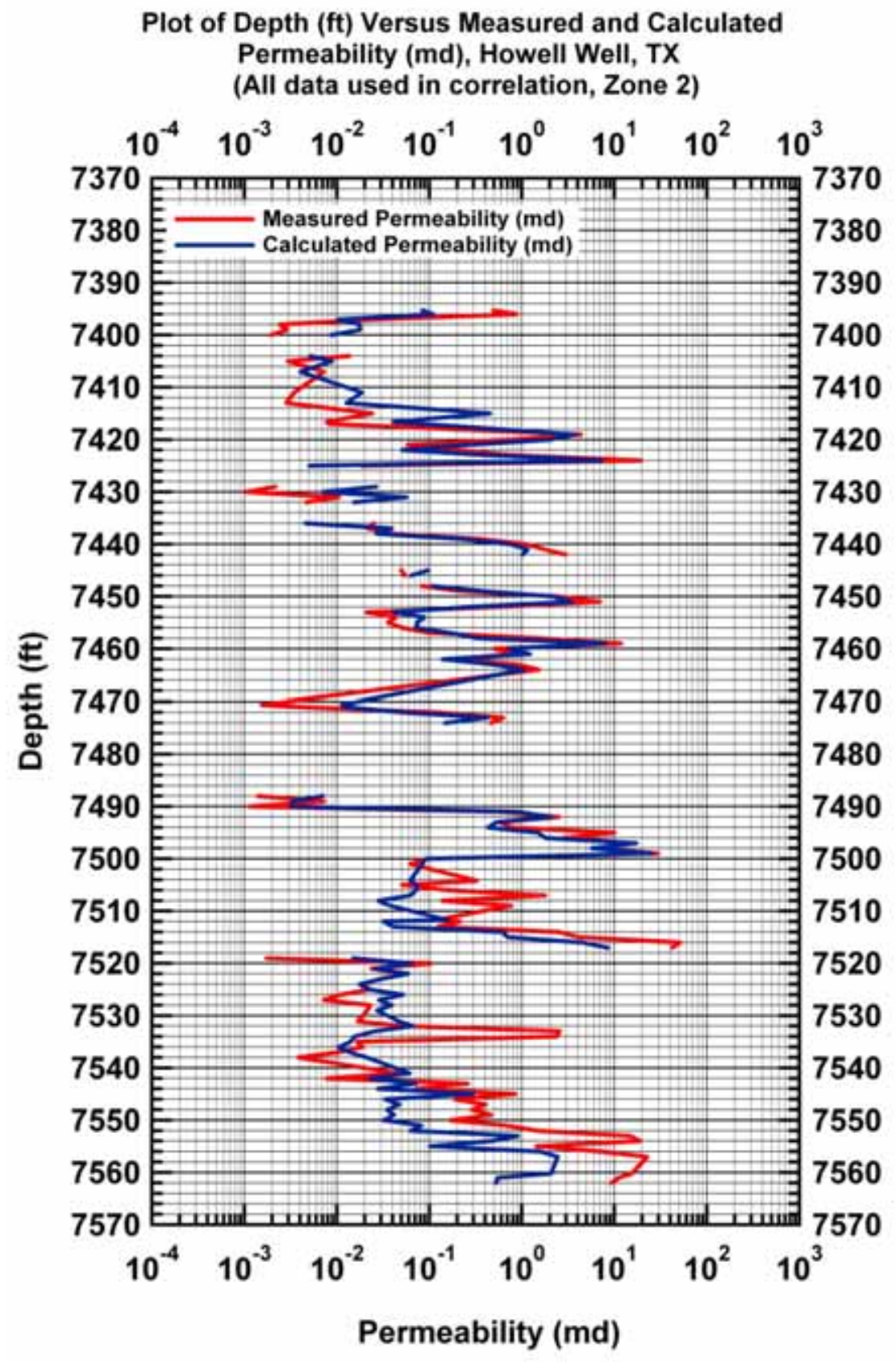

Figure B.8e - Model CPR-DxL: Calculated and measured permeability values versus depth. [Case: Howell Well, E. TX (USA)] 


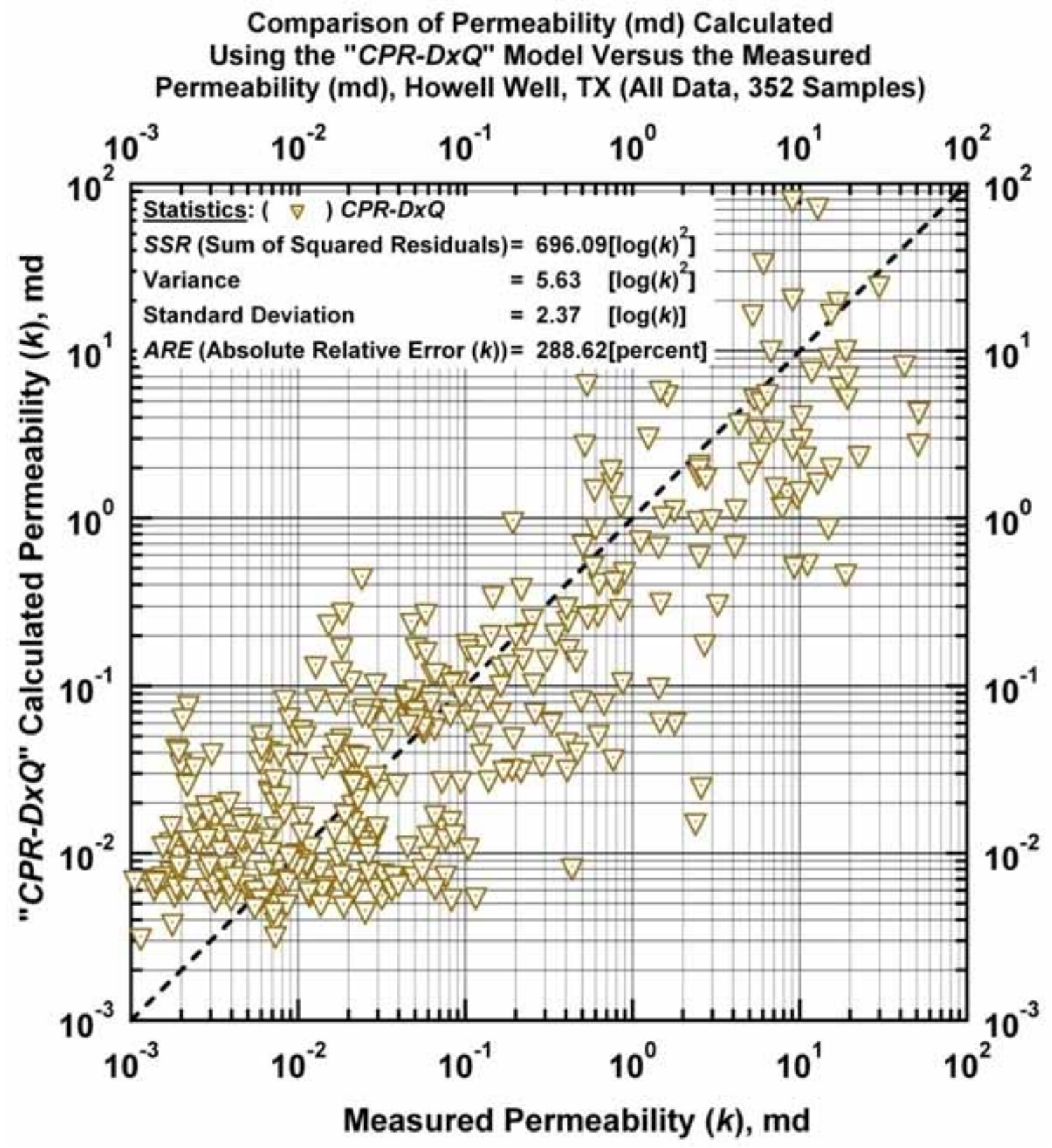

Figure B.9a - Model CPR-DxQ: Calculated versus measured permeability. [Case: Howell Well, E. TX (USA)] 


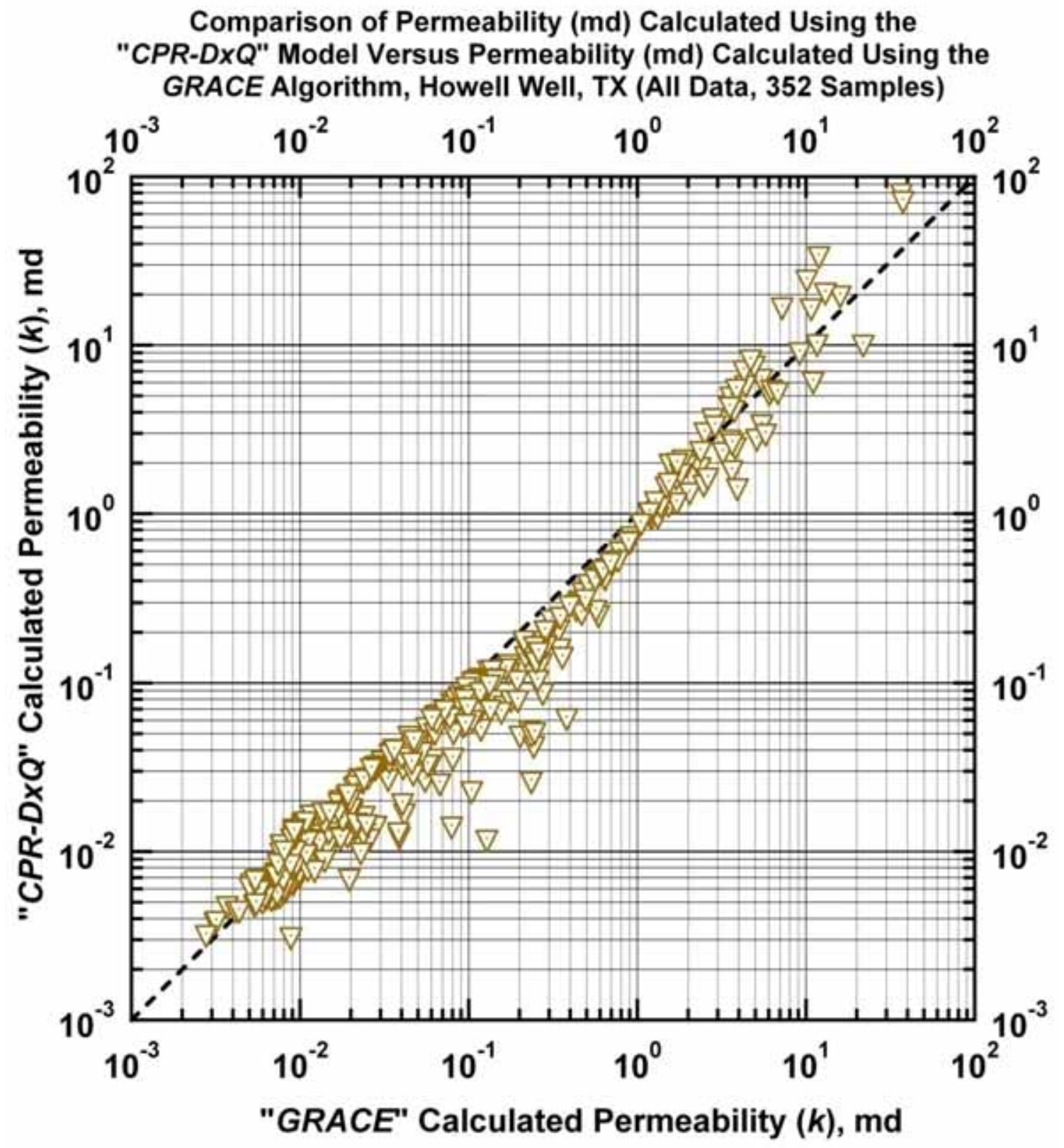

Figure B.9b - Model $C P R-D x Q$ : Permeability calculated using Model $C P R-C$ versus permeability calculated using the GRACE Algorithm. [Case: Howell Well, E. TX (USA)] 
Comparison of the $x$-Coefficient Calculated Using the "CPR-DxQ" Model Versus the Measured $x$-Coefficient, Howell Well, TX (All Data, 352 Samples)

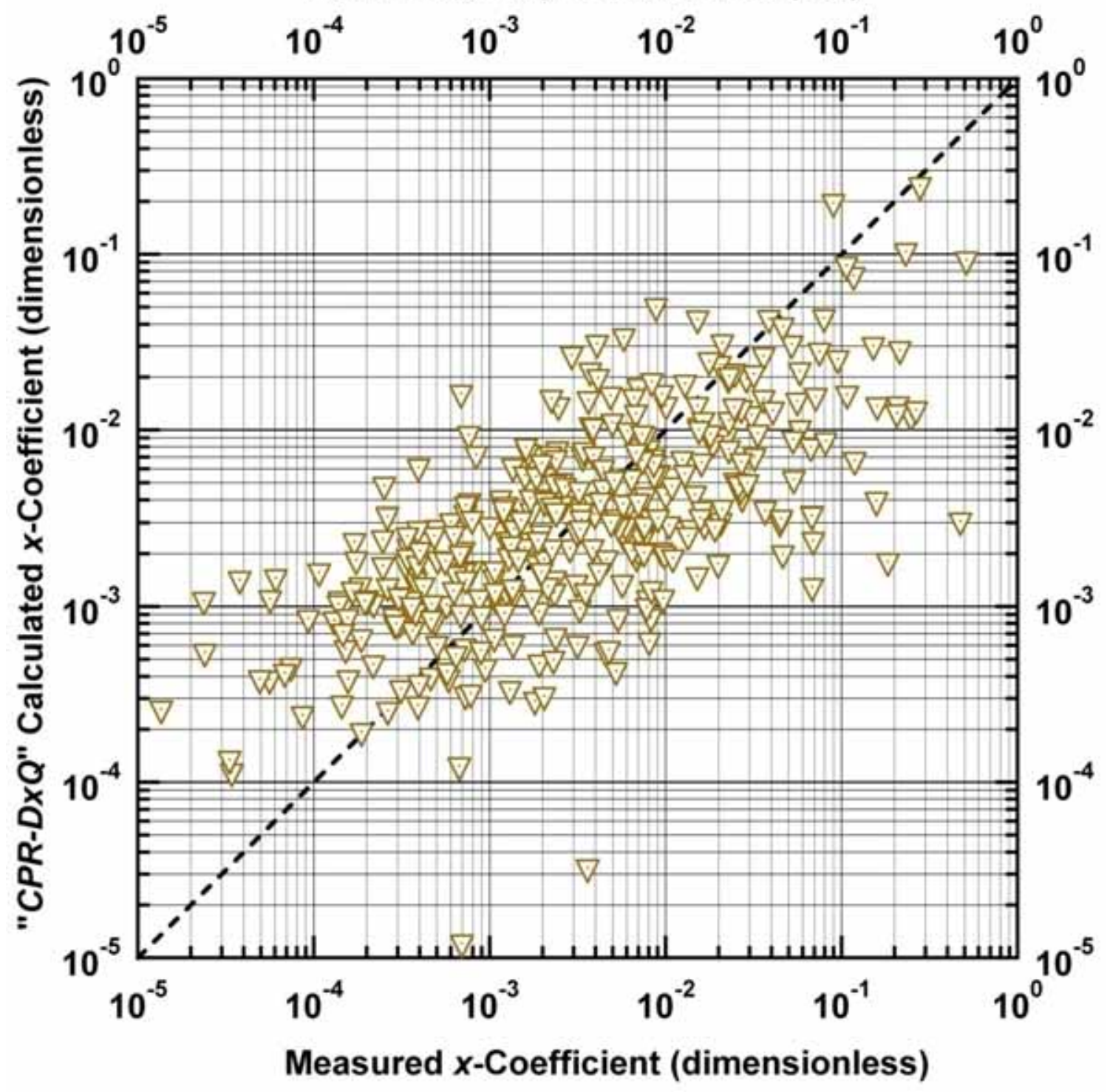

Figure B.9c - Model CPR-DxQ: Calculated $x$-function weight values versus measured $x$ function weight values. [Case: Howell Well, E. TX (USA)] 


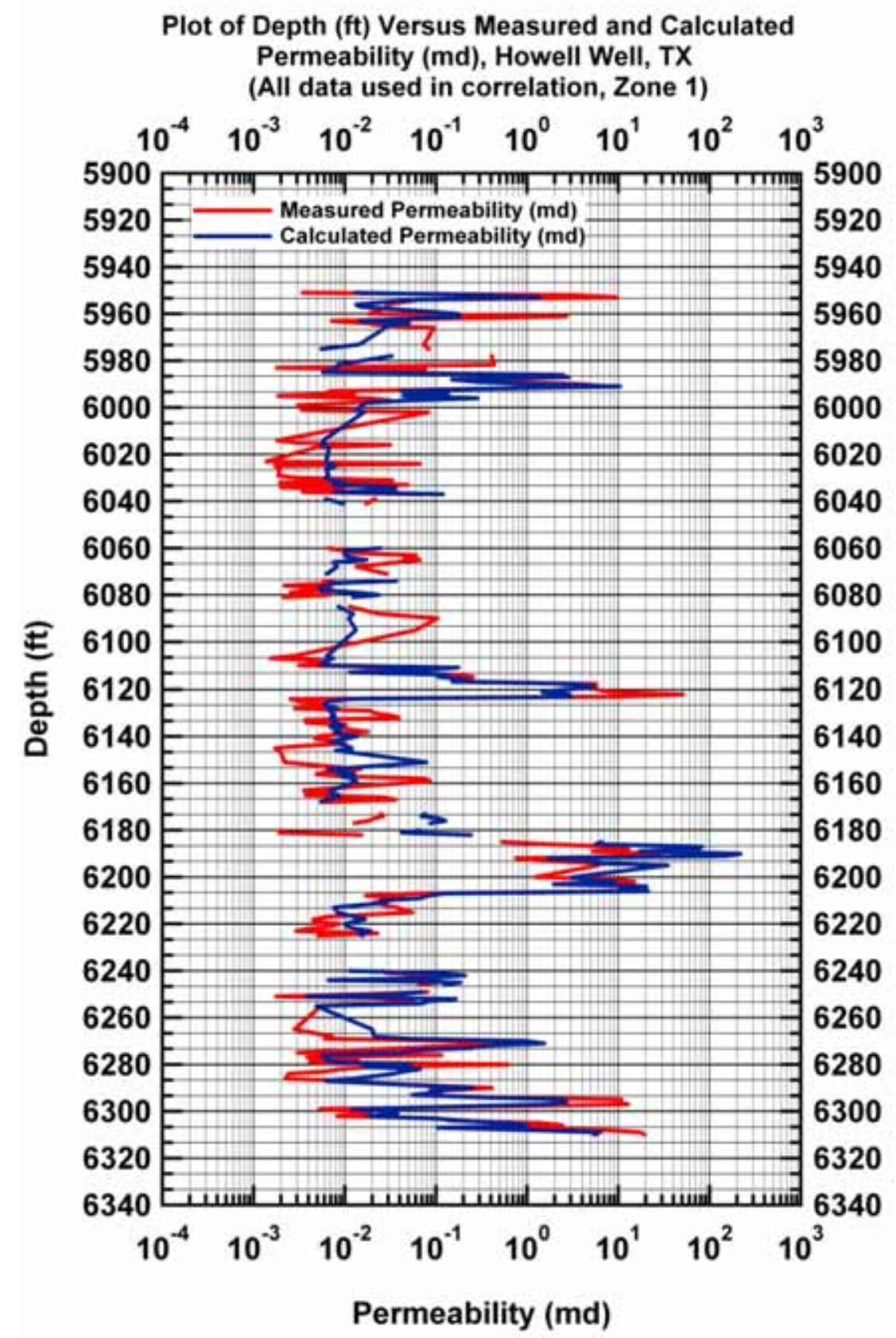

Figure B.9d - Model CPR-DxQ: Calculated and measured permeability values versus depth. [Case: Howell Well, E. TX (USA)] 


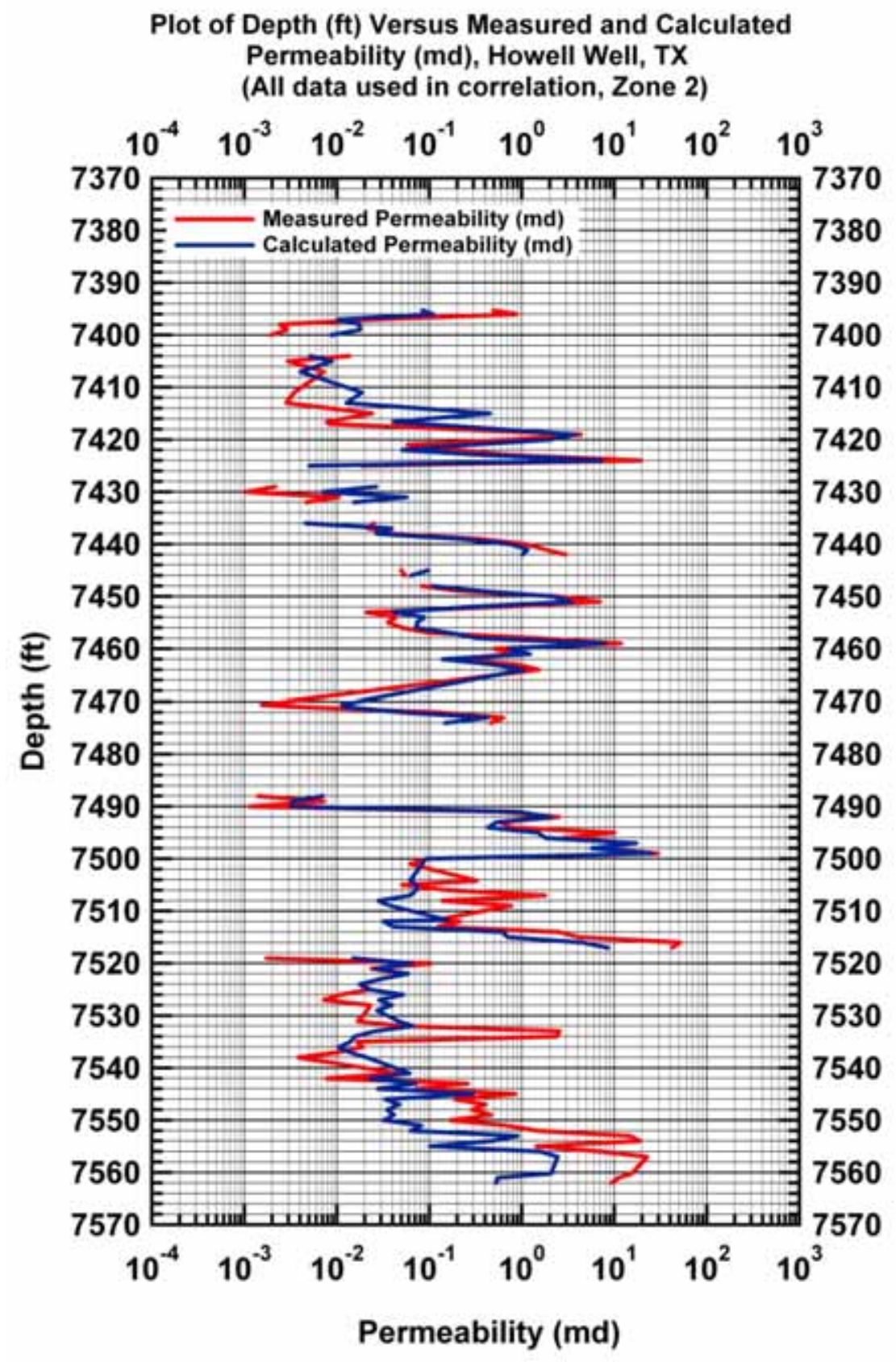

Figure B.9e - Model $C P R-D x Q$ : Calculated and measured permeability values versus depth. [Case: Howell Well, E. TX (USA)] 


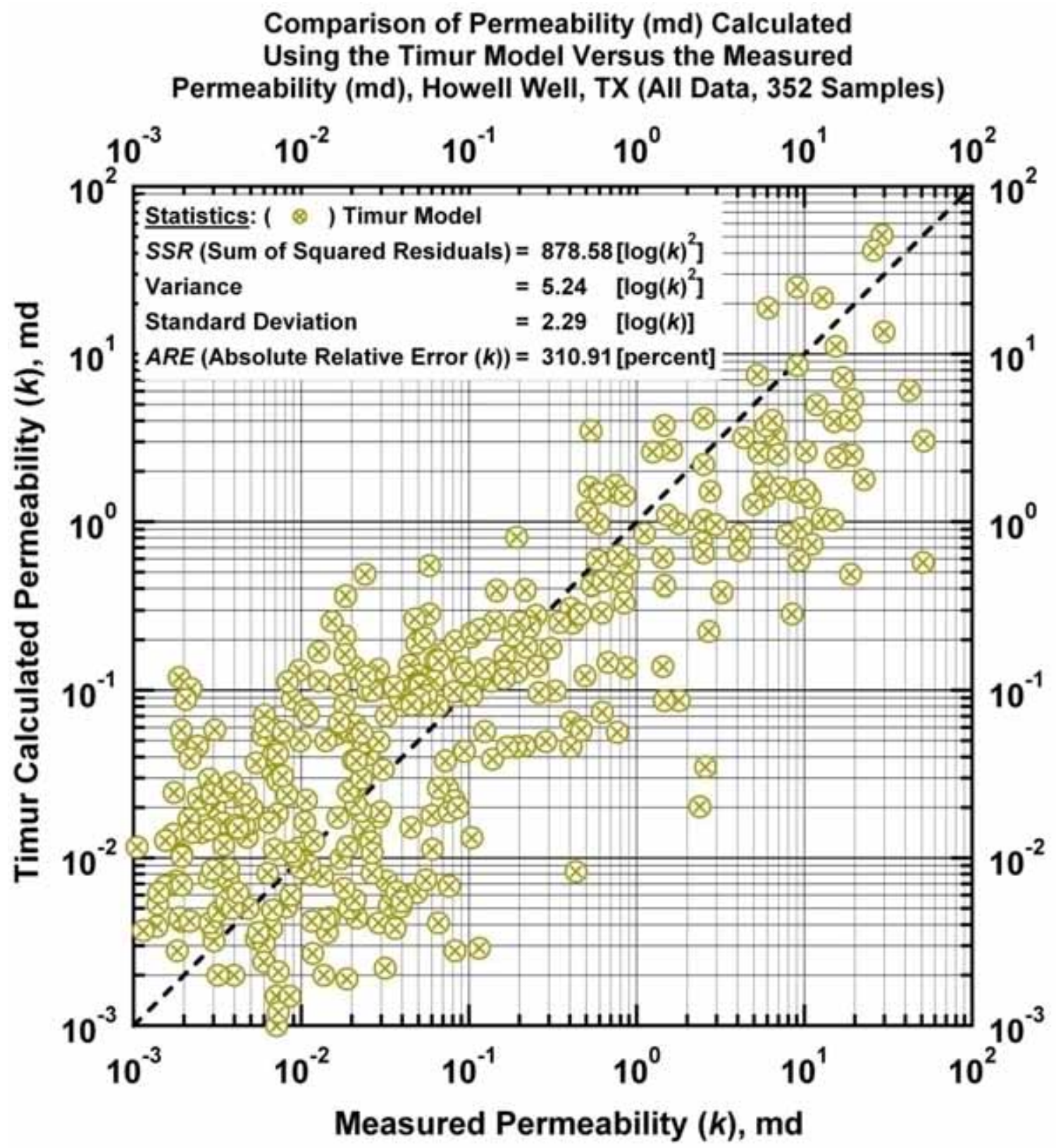

Figure B.10a - "Timur Model:" Calculated versus measured permeability. [Case: Howell Well, E. TX (USA)] 


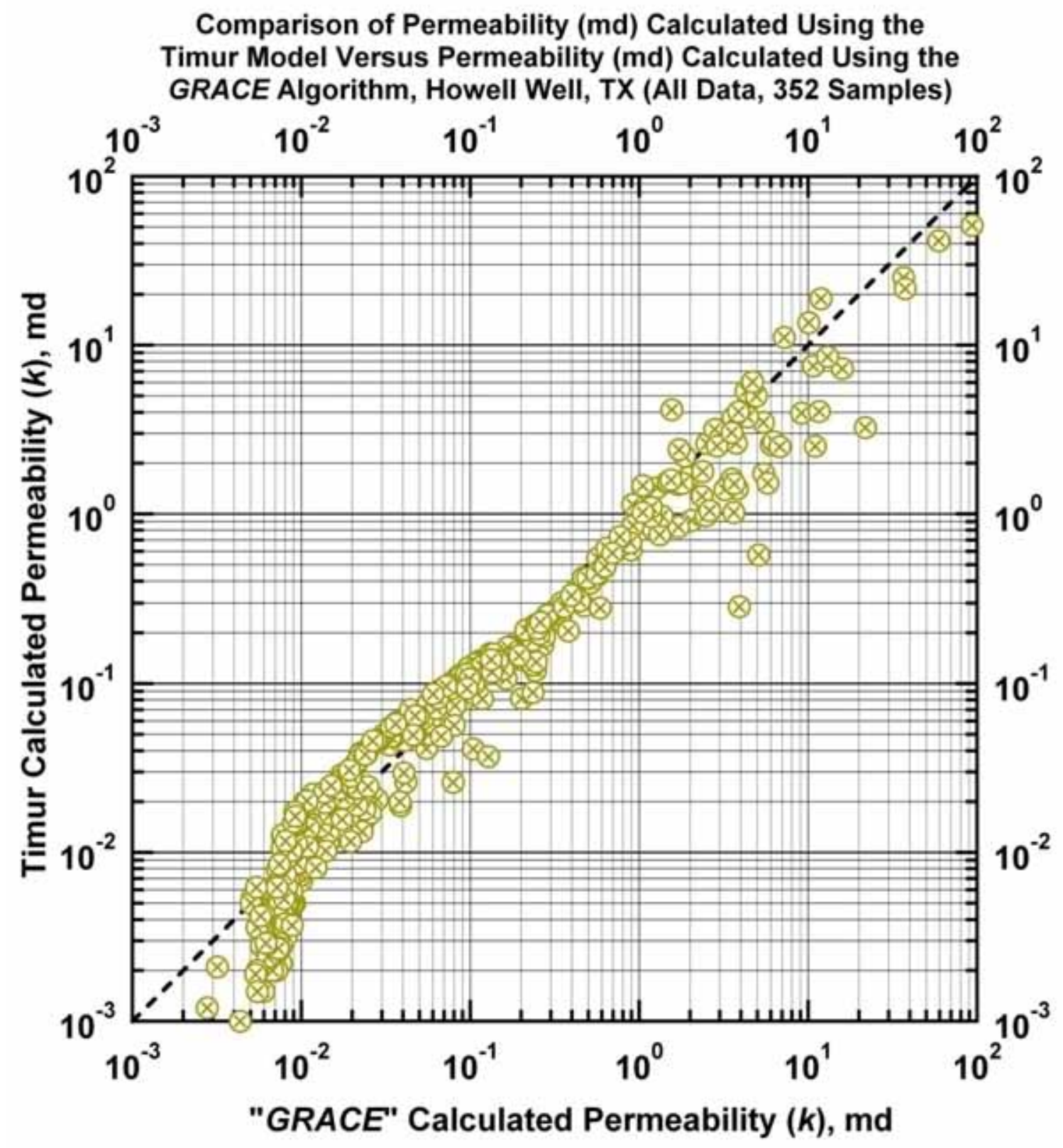

Figure B.10b — "Timur Model:" Permeability calculated using the "Timur Model" versus permeability calculated using the GRACE Algorithm. [Case: Howell Well, E. TX (USA)] 


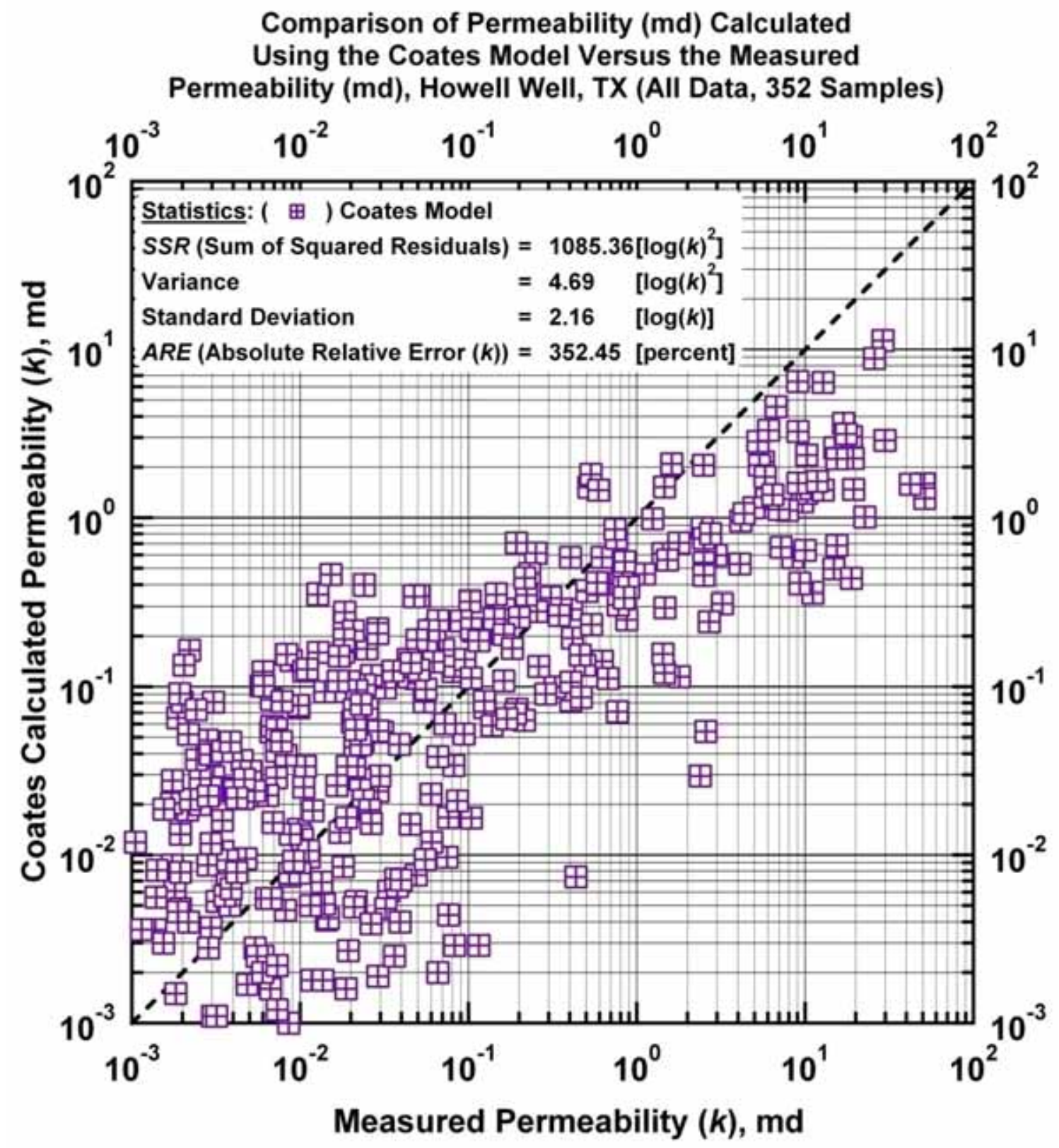

Figure B.11a - "Coates Model:" Calculated versus measured permeability. [Case: Howell Well, E. TX (USA)] 


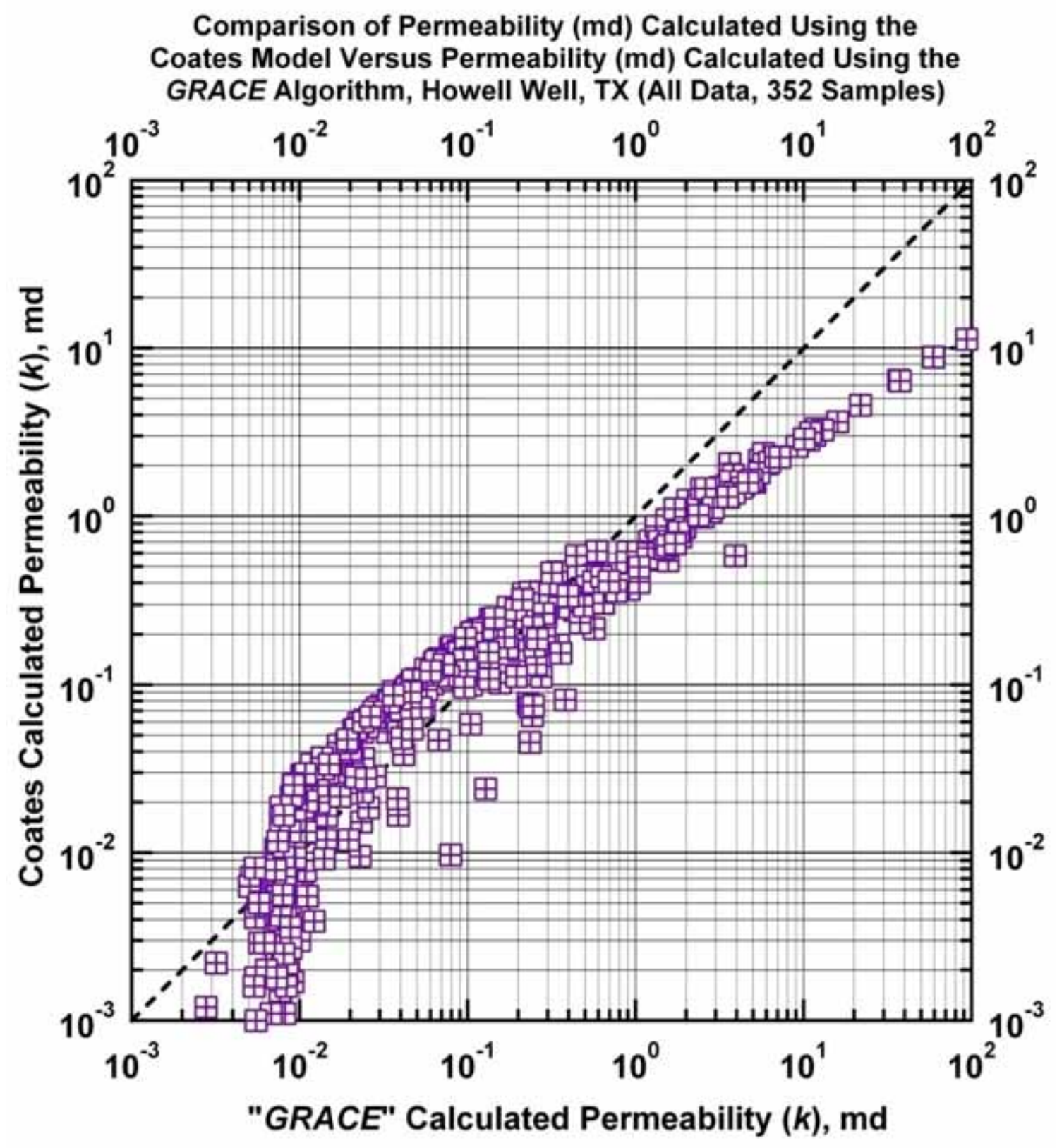

Figure B.11b — "Coates Model:" Permeability calculated using the "Coates Model " versus permeability calculated using the GRACE Algorithm. [Case: Howell Well, E. TX (USA)] 


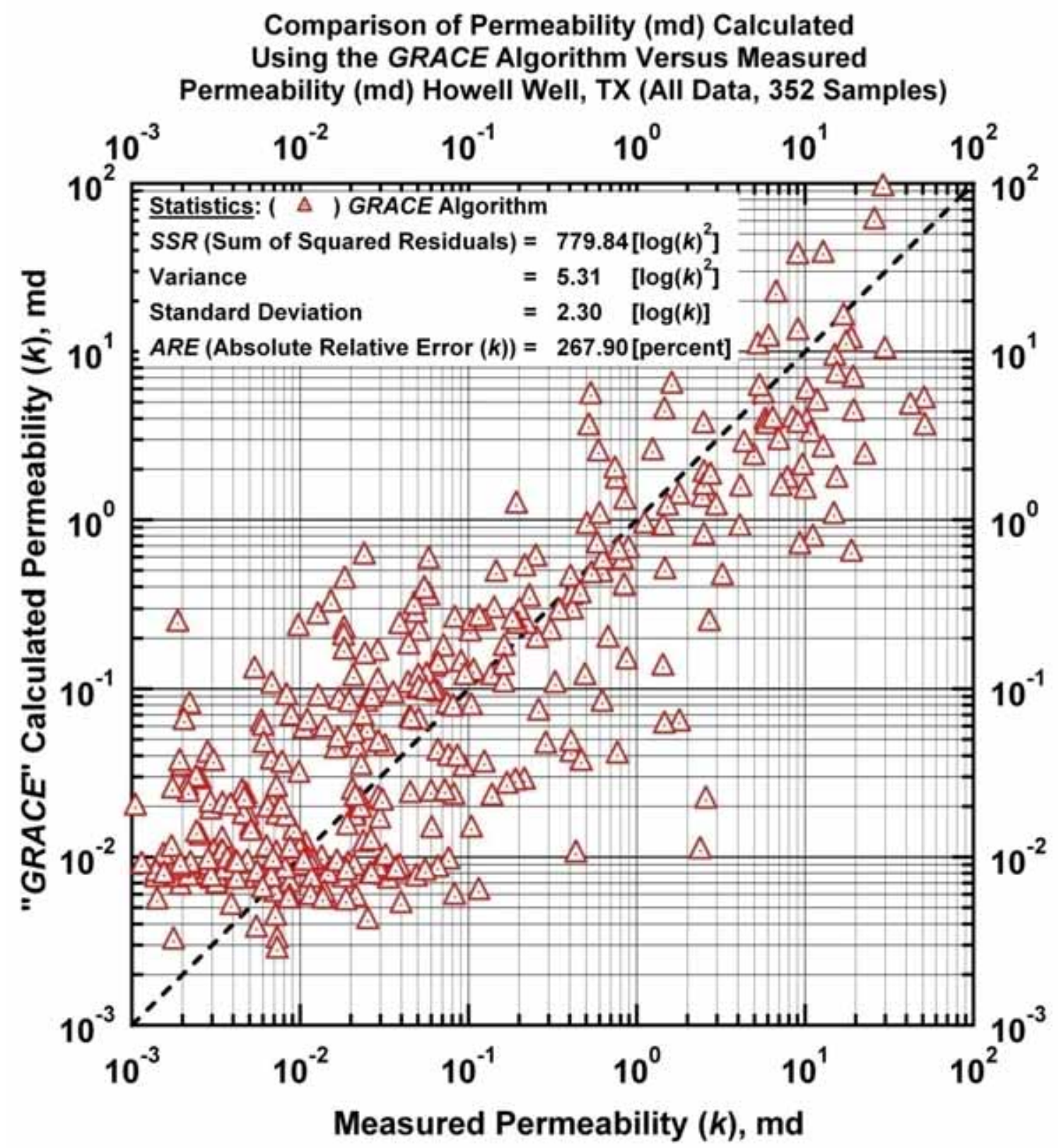

Figure B.12 - GRACE Model: Calculated versus measured permeability. [Case: Howell Well, E. TX (USA)] 


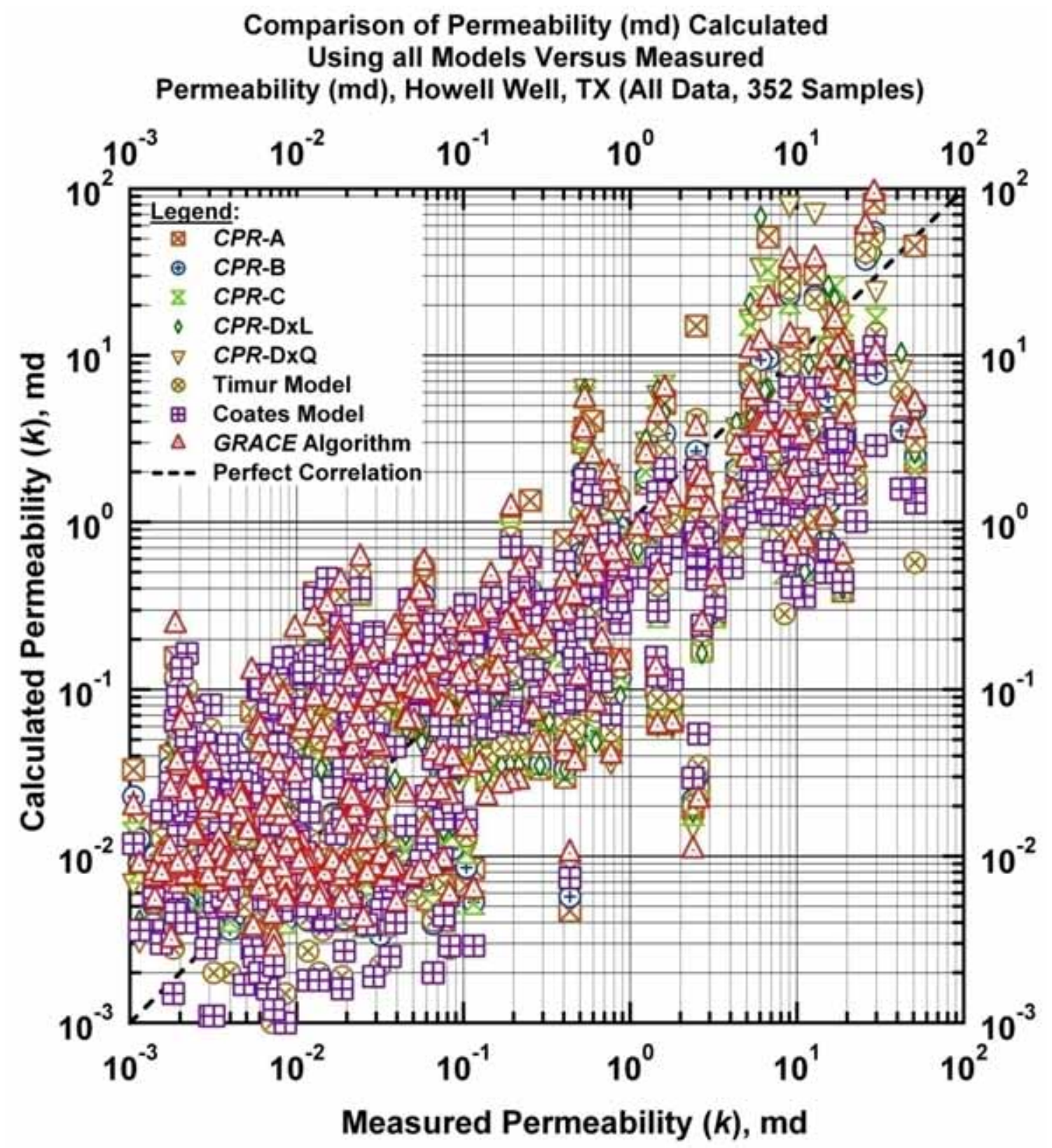

Figure B.13 - All Models: Calculated versus measured permeability. [Case: Howell Well, E. TX (USA)] 


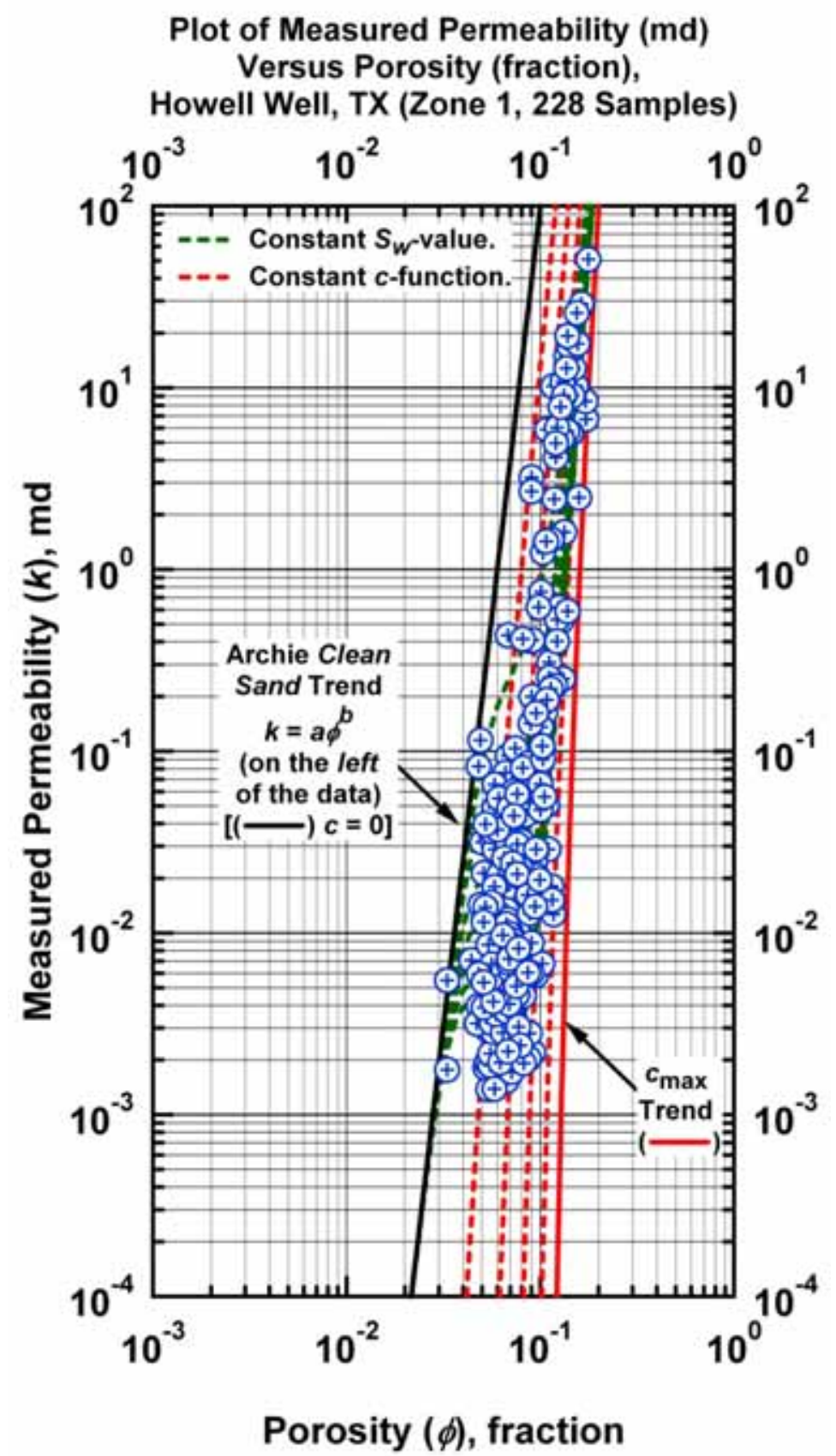

Figure B.14a - Model $C P R-A: \quad k=a(\phi-c)^{b} \quad c=c_{\max } \exp \left[-c_{1} \phi^{C_{2}} S_{w}^{C_{3}}\right]$ - "Clean Sand" Plot (log-log format) - Archie "Clean Sand" trend is given by the straight-line trend at the far left of the data (power law model). [Case: Howell Well, E. TX (USA)] 


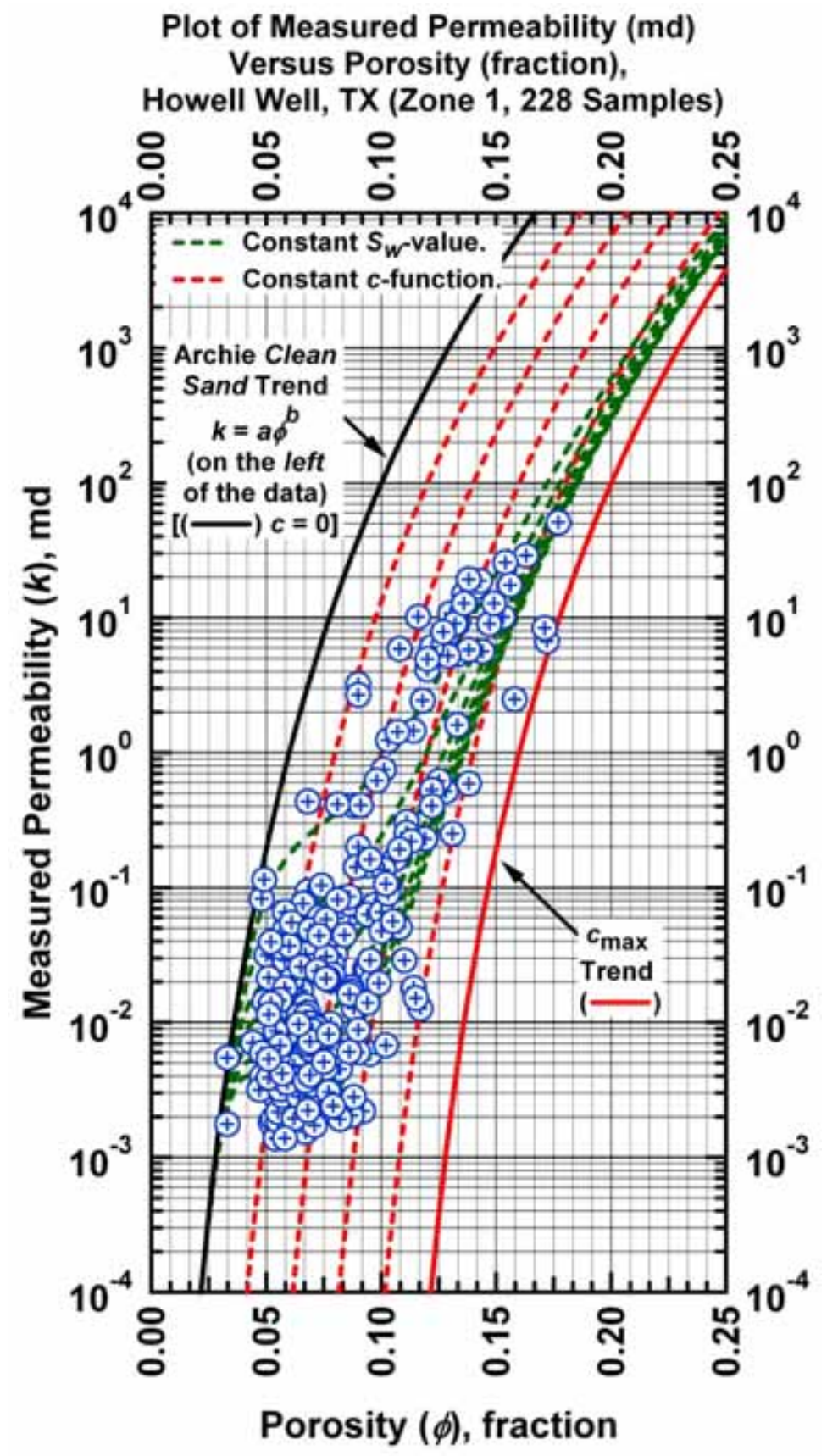

Figure B.14b - Model CPR-A: $k=a(\phi-c)^{b} \quad c=c_{\max } \exp \left[-c_{1} \phi^{c_{2}} S_{W}^{c_{3}}\right]$ - "Dirty Sand" Plot (semilog format). Archie "Clean Sand" trend is given by the curved trend at the far left of the data (power law model). [Case: Howell Well, E. TX (USA)] 


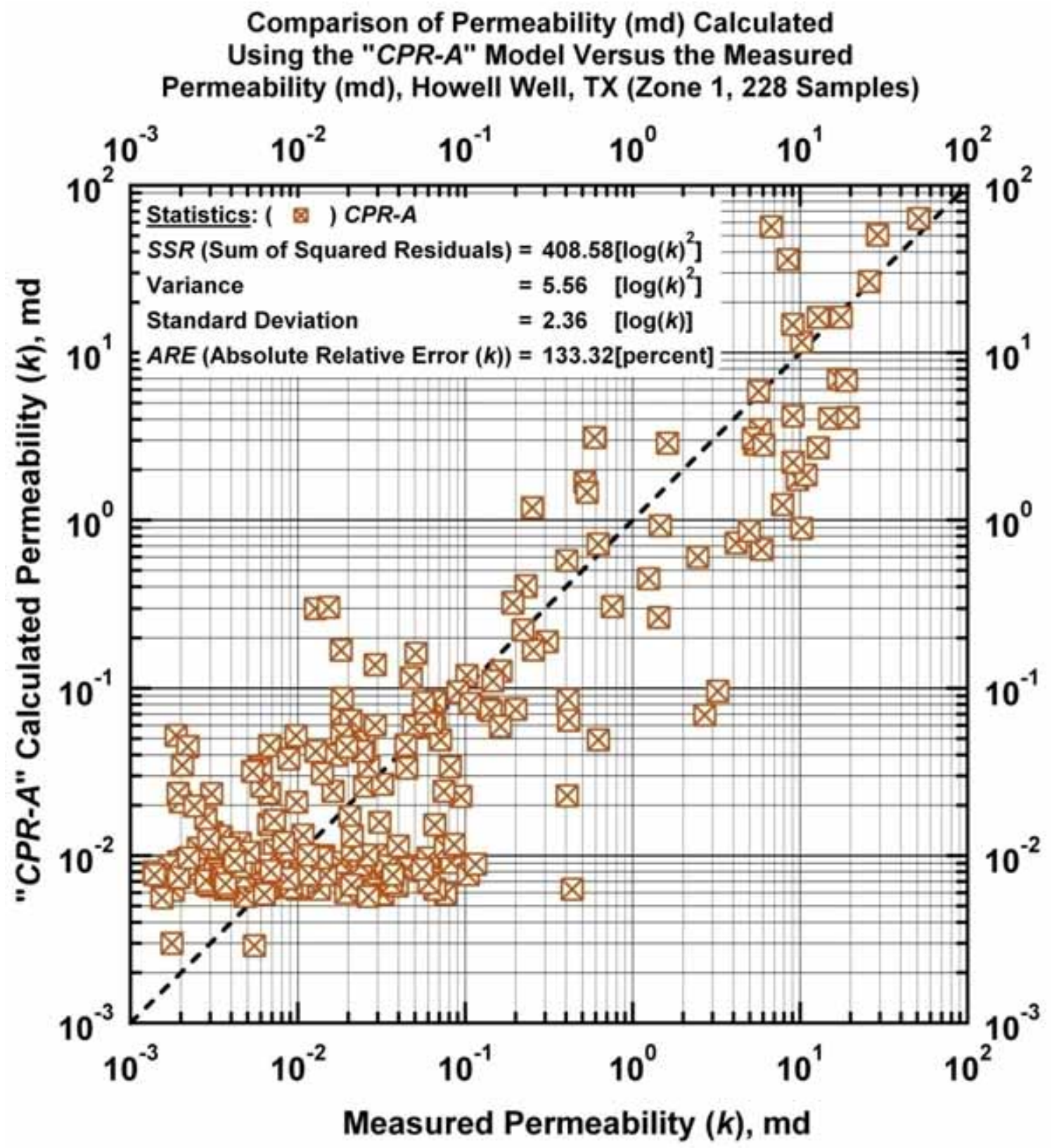

Figure B.15a - Model CPR-A: calculated versus measured permeability. [Case: Howell Well, E. TX (USA)] 


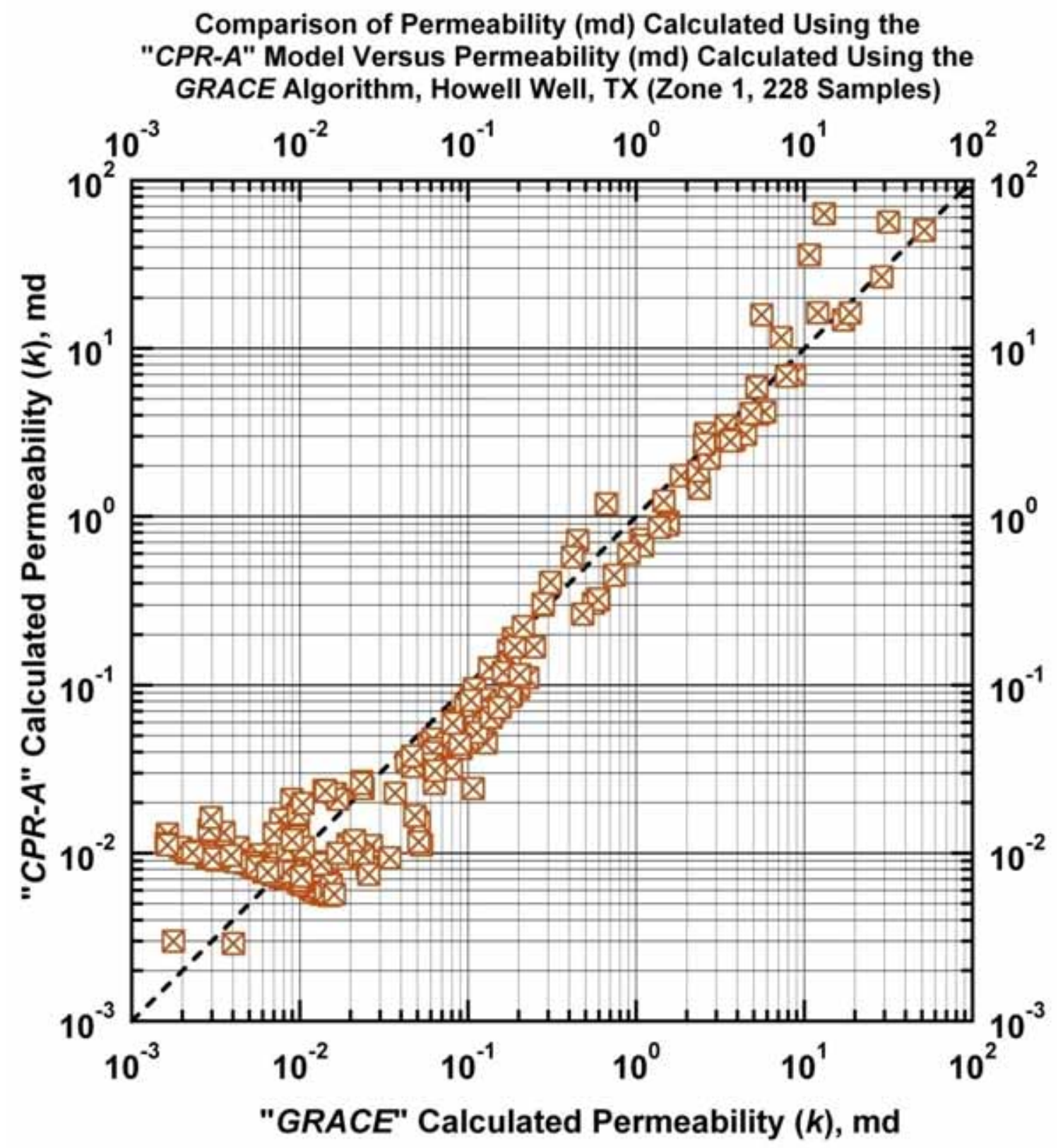

Figure B. 15b - Model CPR-A: Permeability calculated using Model CPR-A versus permeability calculated using the GRACE Algorithm. [Case: Howell Well, E. TX (USA)] 


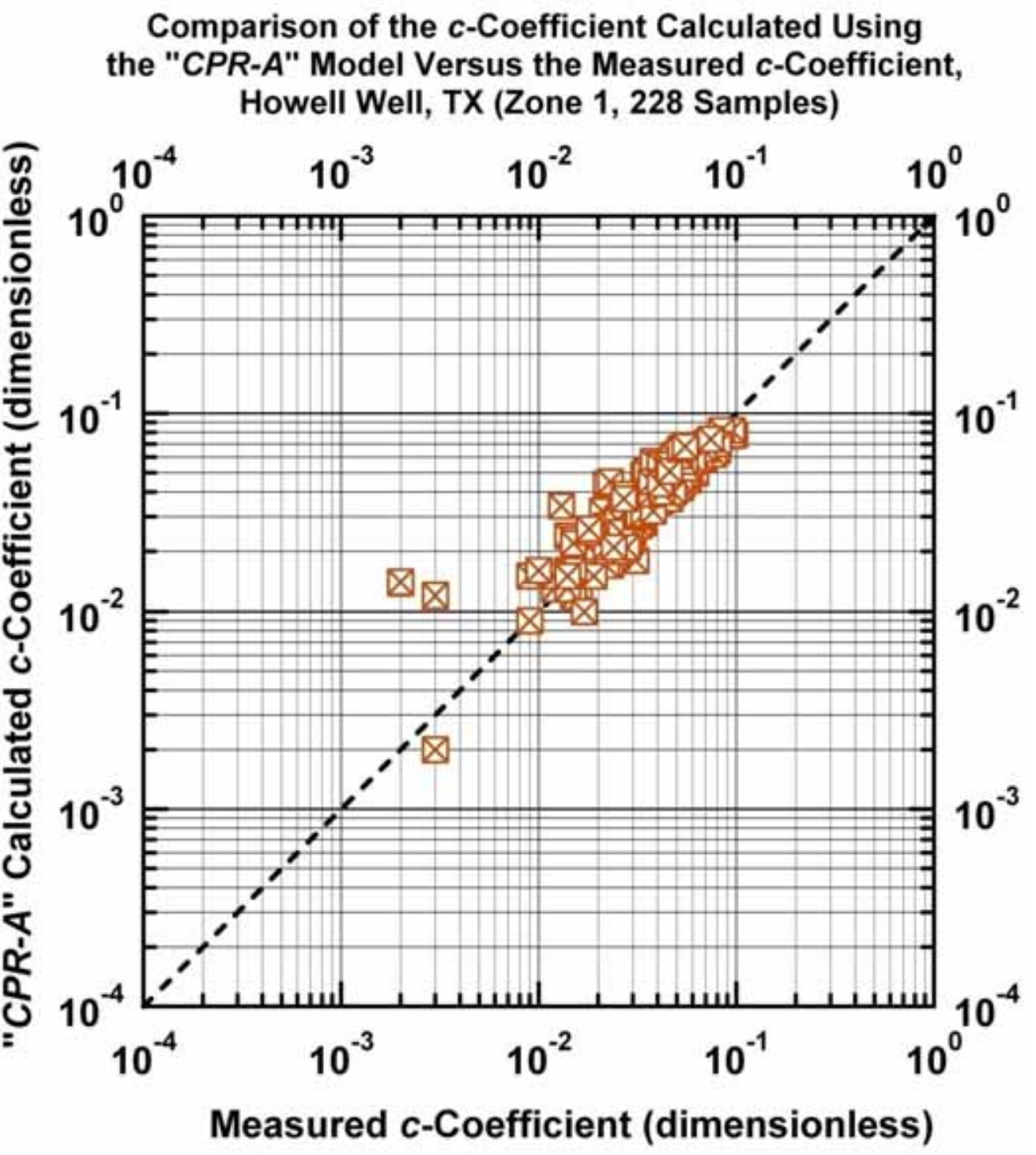

Figure B. 15c - Model $C P R$ - $A$ : Calculated $c$-function values versus measured $c$-function values. [Case: Howell Well, E. TX (USA)] 


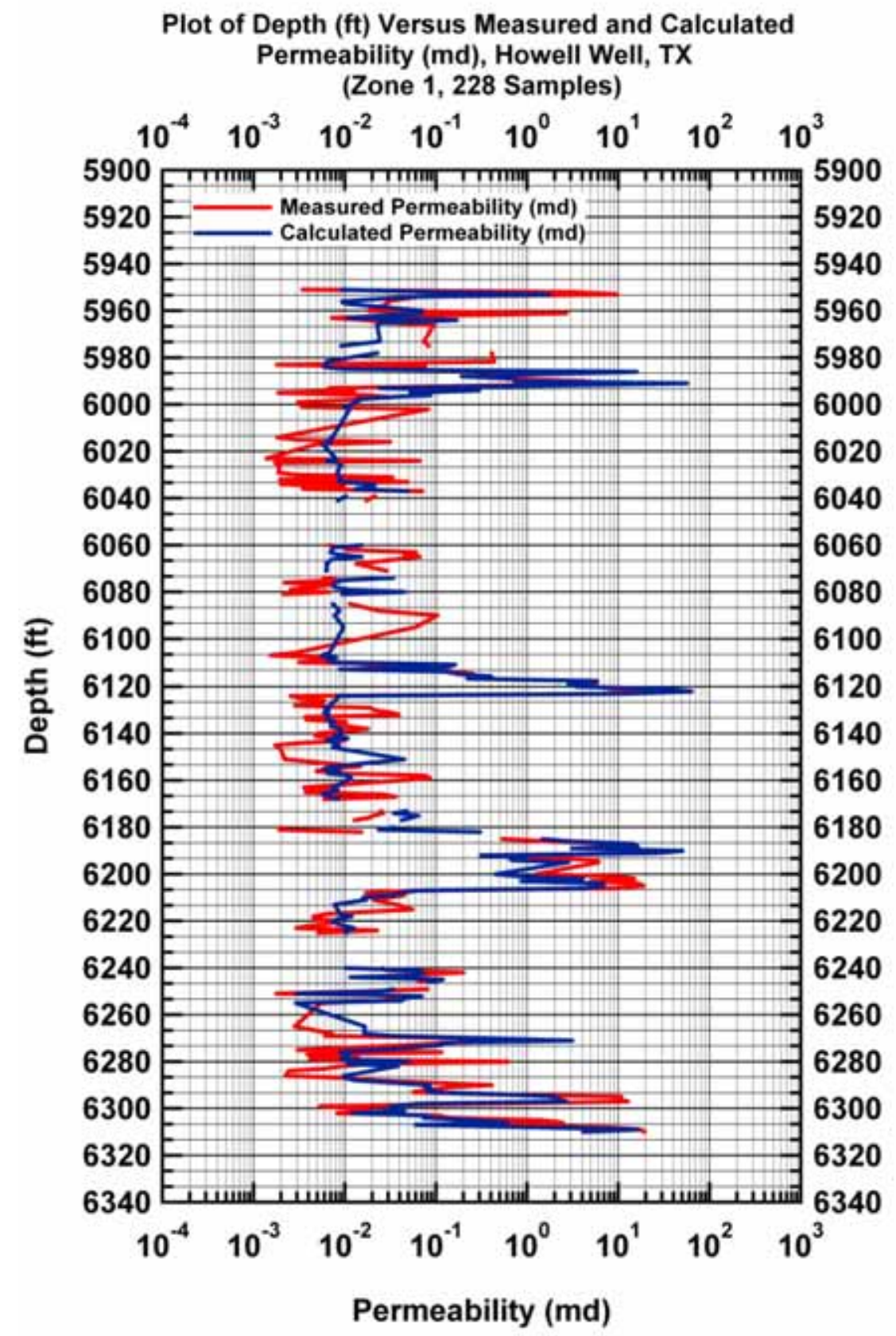

Figure B. 15d - Model CPR-A: Calculated and measured permeability values versus depth. [Case: Howell Well, E. TX (USA)] 


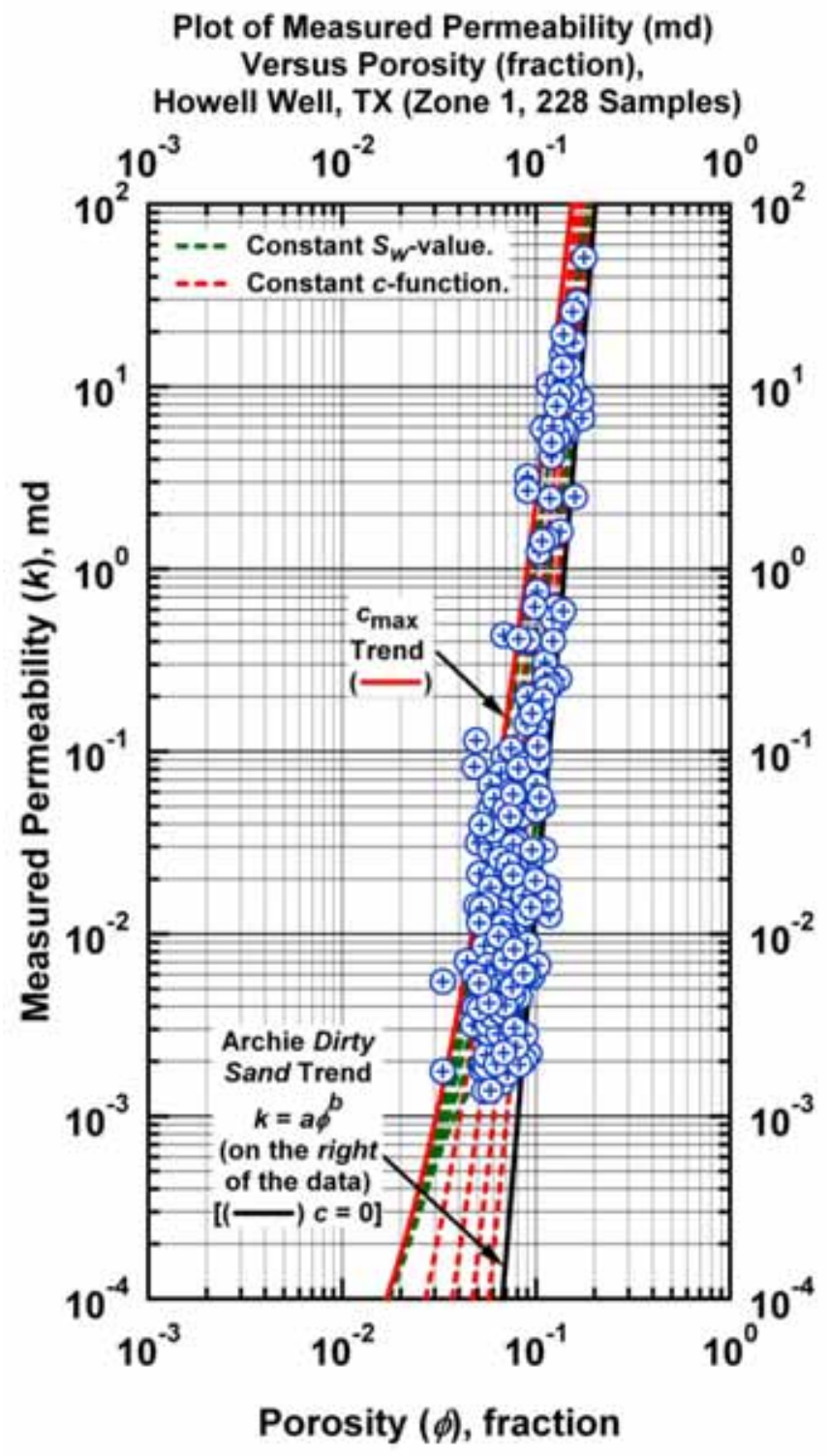

Figure B.16a - Model CPR-B: $\quad k=a(\phi+c)^{b} \quad c=c_{\max } \exp \left[-c_{1} \phi^{c_{2}} S_{w}^{c_{3}}\right]$ - "Clean Sand" Plot (log-log format) - Archie "Dirty Sand" trend is given by the straight-line trend at the far right of the data (power law model). [Case: Howell Well, E. TX (USA)] 


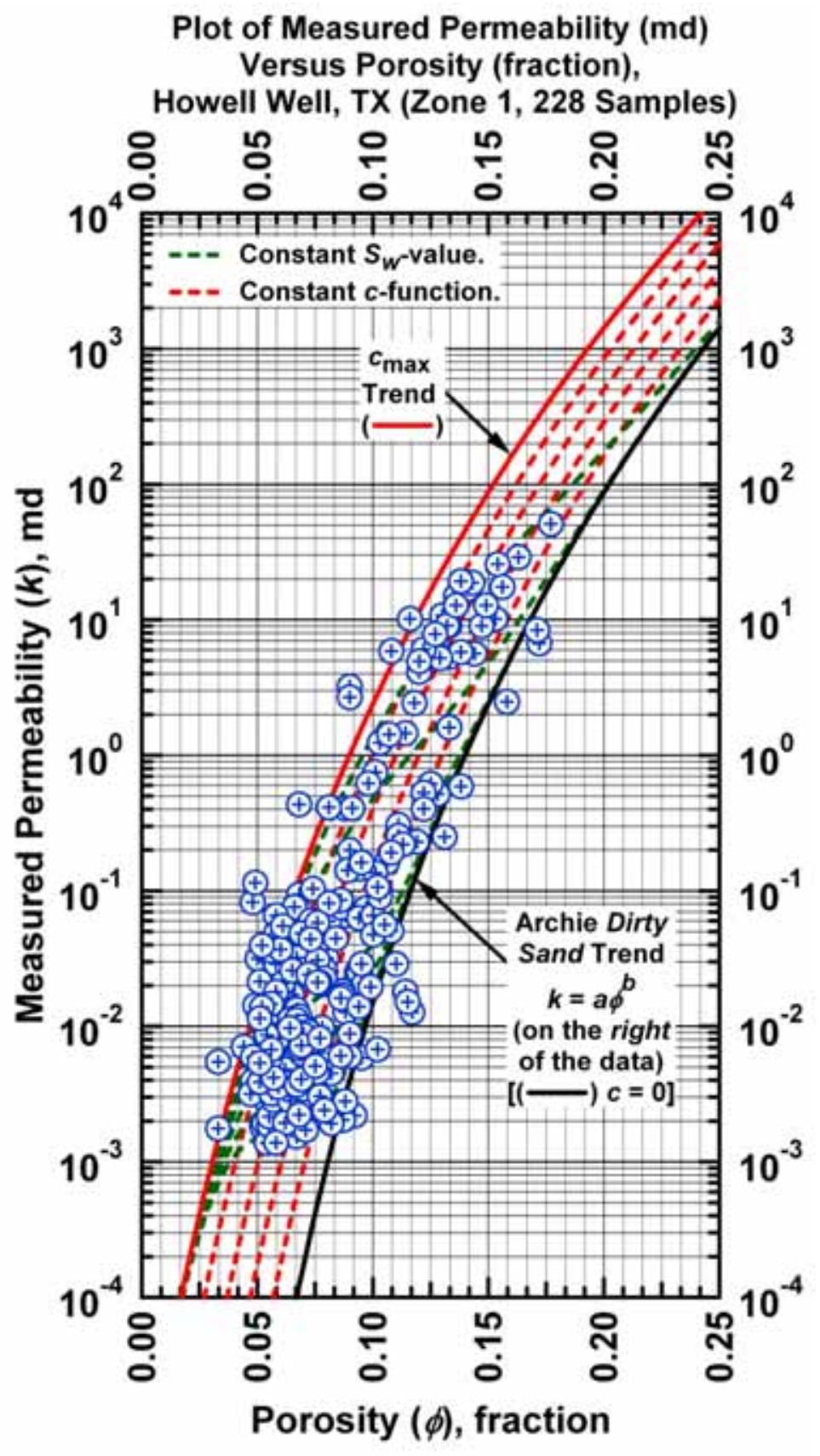

Figure B.16b - Model $C P R-B: k=a(\phi+c)^{b} \quad c=c_{\max } \exp \left[-c_{1} \phi^{c_{2}} S_{W}^{c_{3}}\right]$ - "Dirty Sand" Plot (semilog format) - Archie "Dirty Sand" trend is given by the curved trend at the far right of the data (power law model). [Case: Howell Well, E. TX (USA)] 


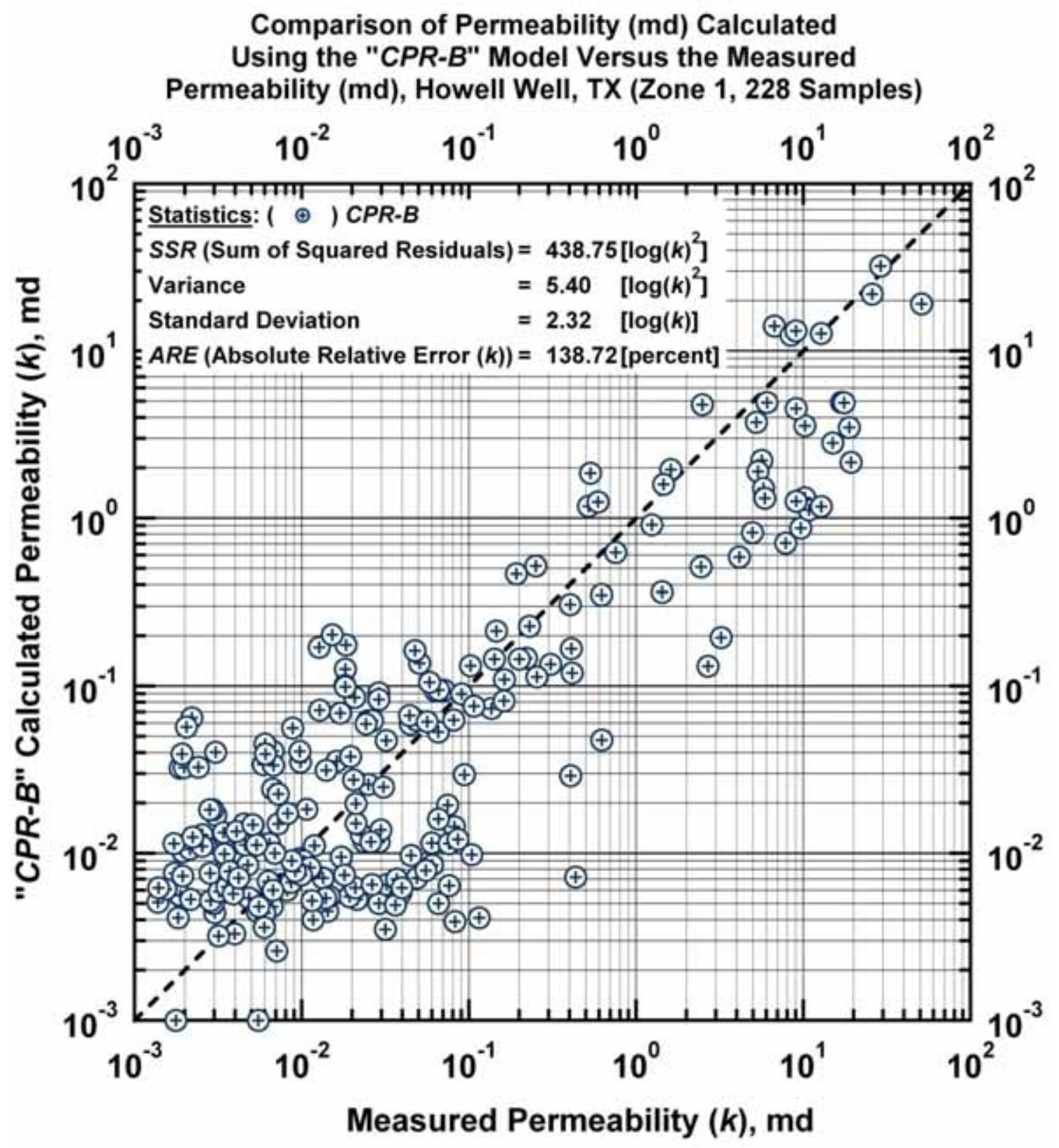

Figure B.17a - Model CPR-B: calculated versus measured permeability. [Case: Howell Well, E. TX (USA)] 


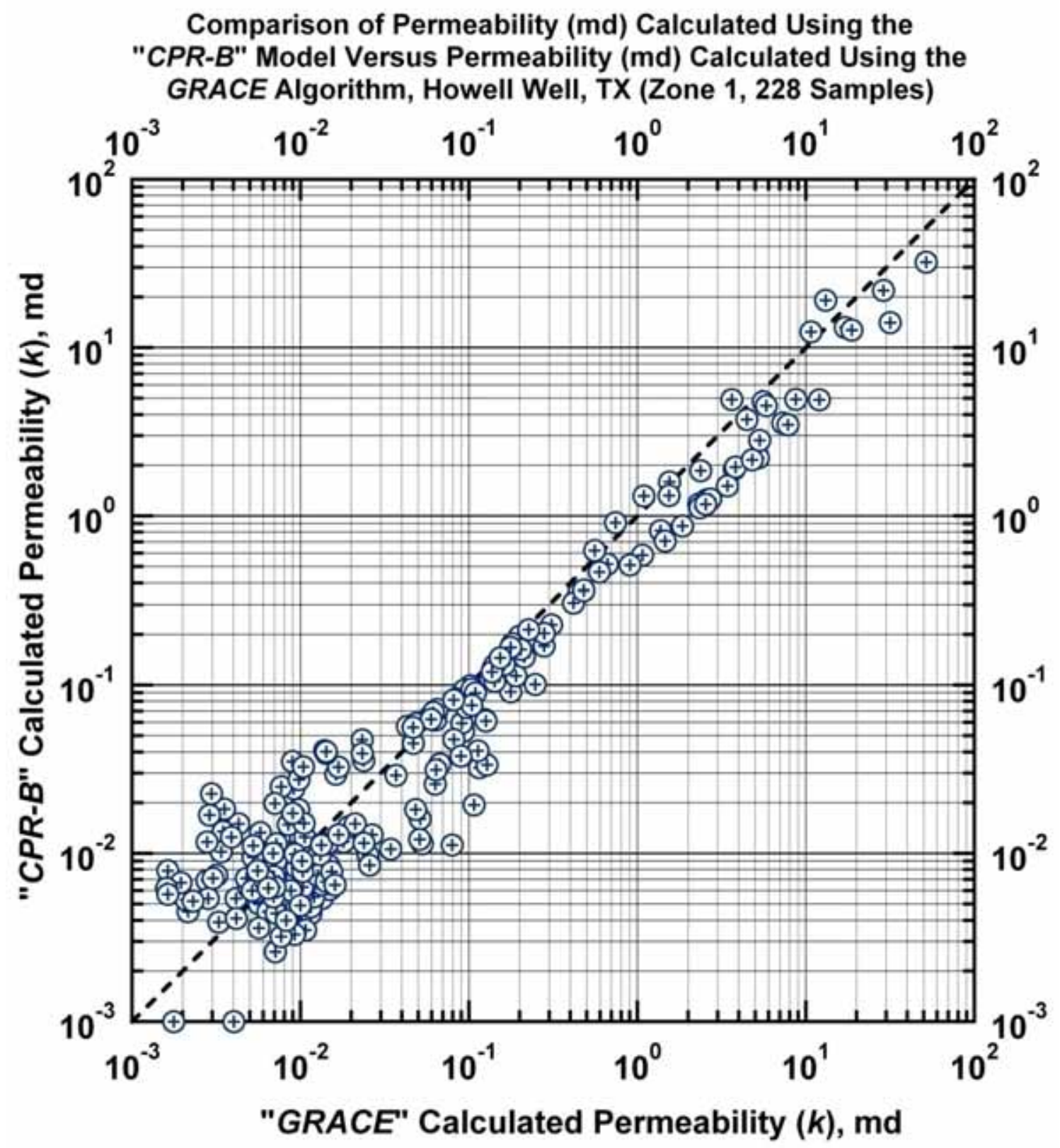

Figure B. 17b - Model CPR-B: Permeability calculated using Model $C P R-B$ versus permeability calculated using the GRACE Algorithm. [Case: Howell Well, E. TX (USA)] 


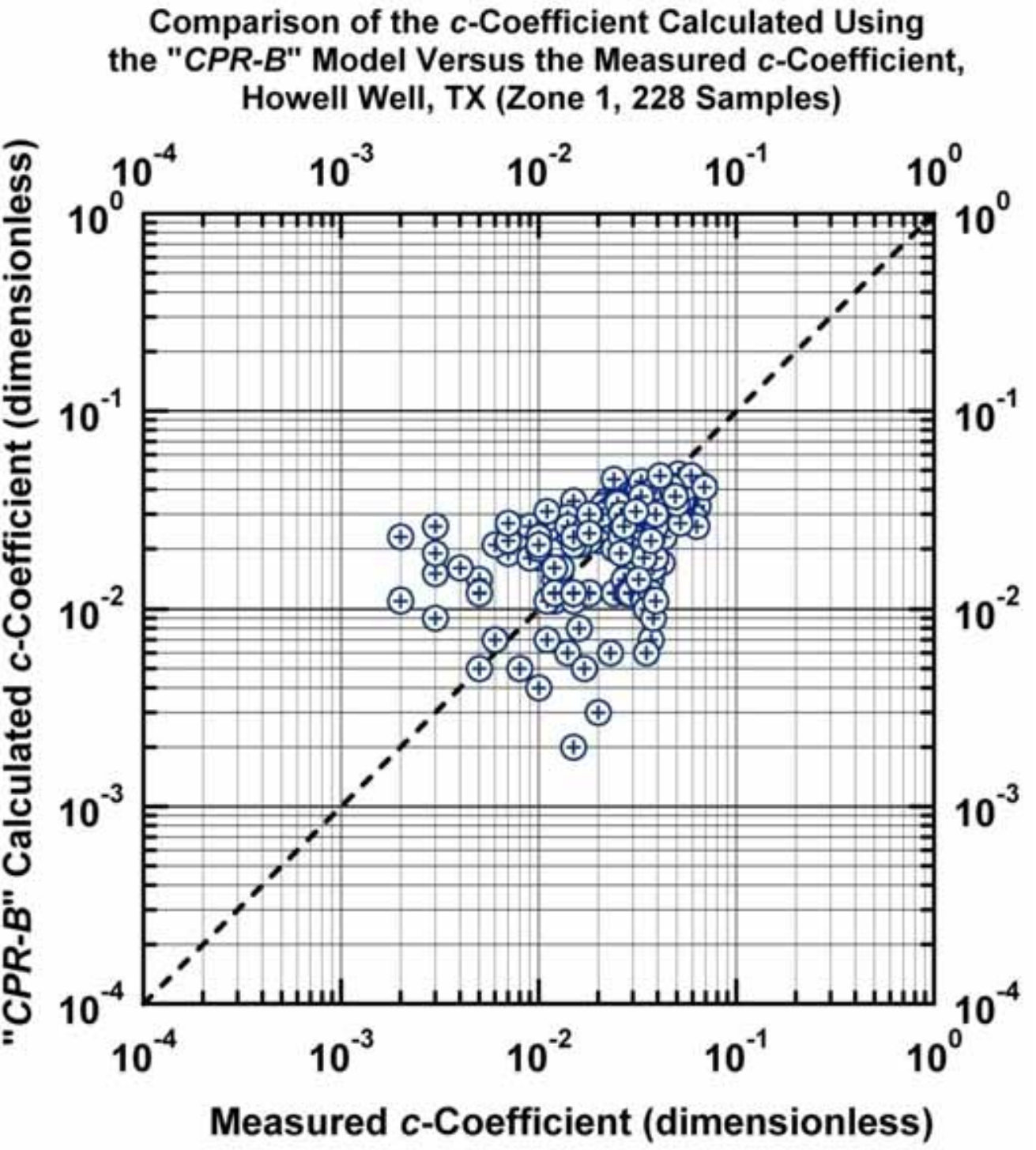

Figure B. 17c - Model CPR-B: Calculated $c$-function values versus measured $c$-function values. [Case: Howell Well, E. TX (USA)] 


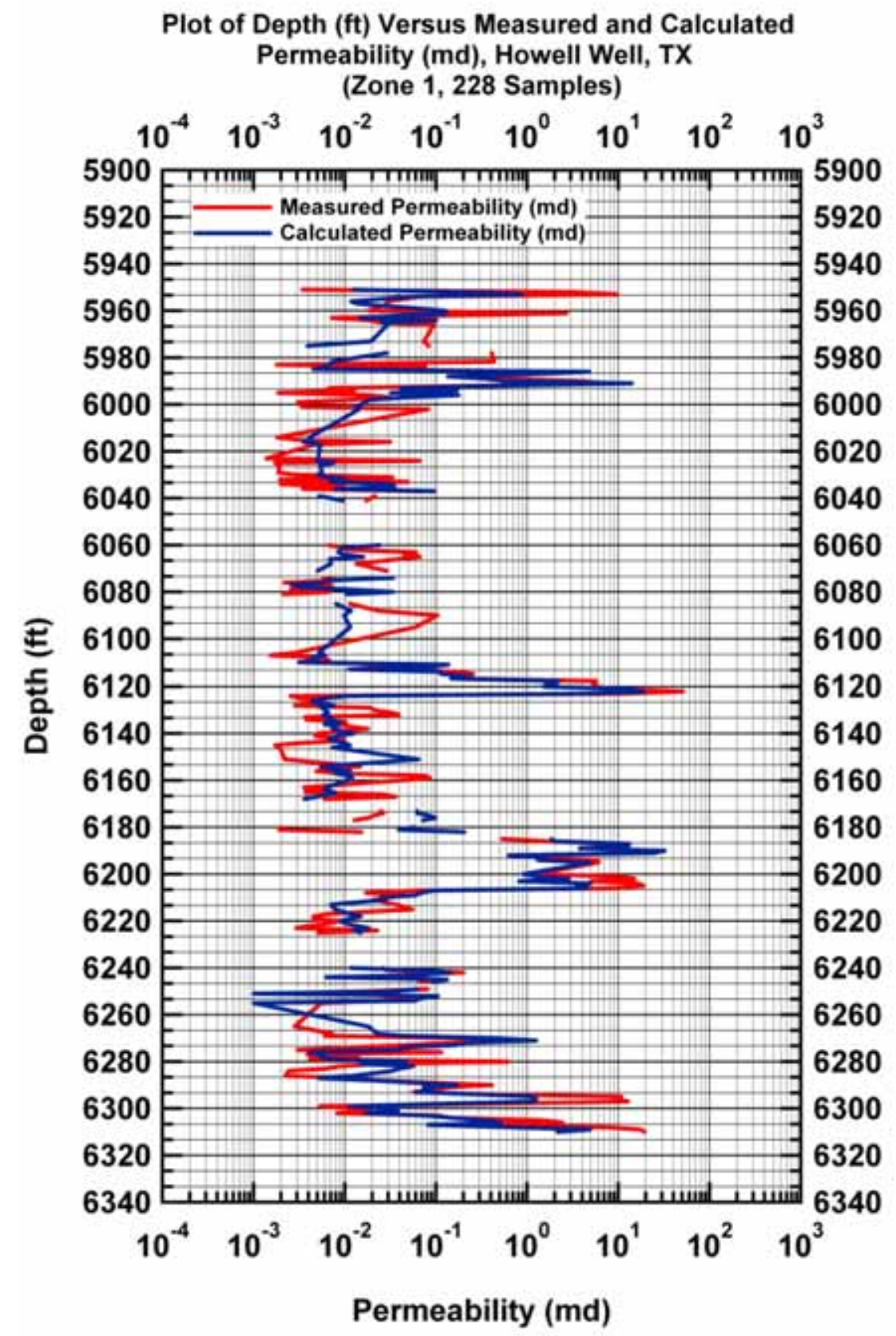

Figure B. 17d - Model CPR-B: Calculated and measured permeability values versus depth. [Case: Howell Well, E. TX (USA)] 


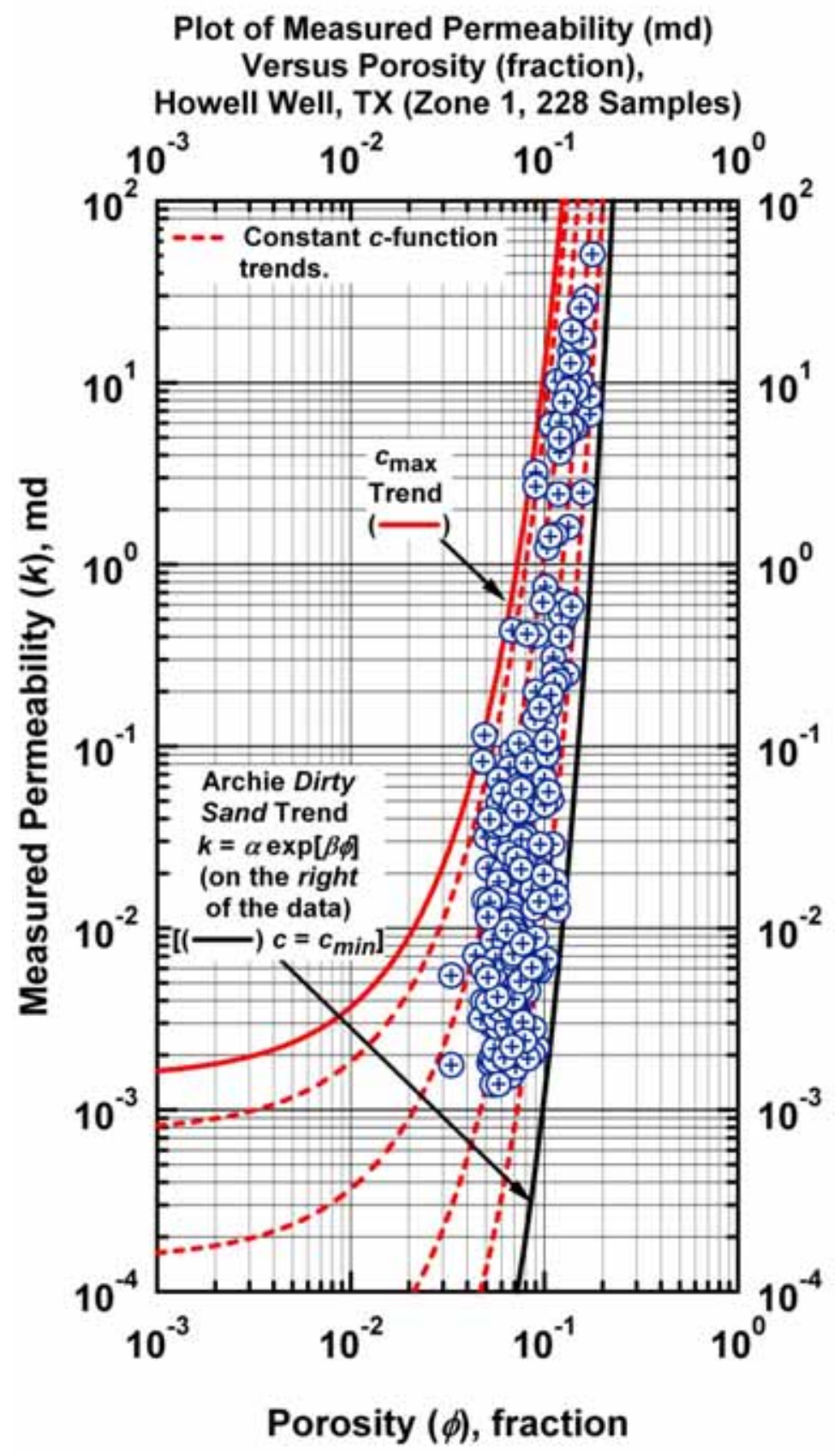

Figure B.18a - Model $C P R-C: \quad k=c \exp [\beta \phi] \quad c=c_{\max } \exp \left[-c_{1} \phi^{c_{2}} S_{w}^{c_{3}}\right]$ - "Clean Sand" Plot (log-log format) - Archie "Dirty Sand" trend is given by the curved trend at the far right of the data (exponential model). [Case: Howell Well, E. TX (USA)] 


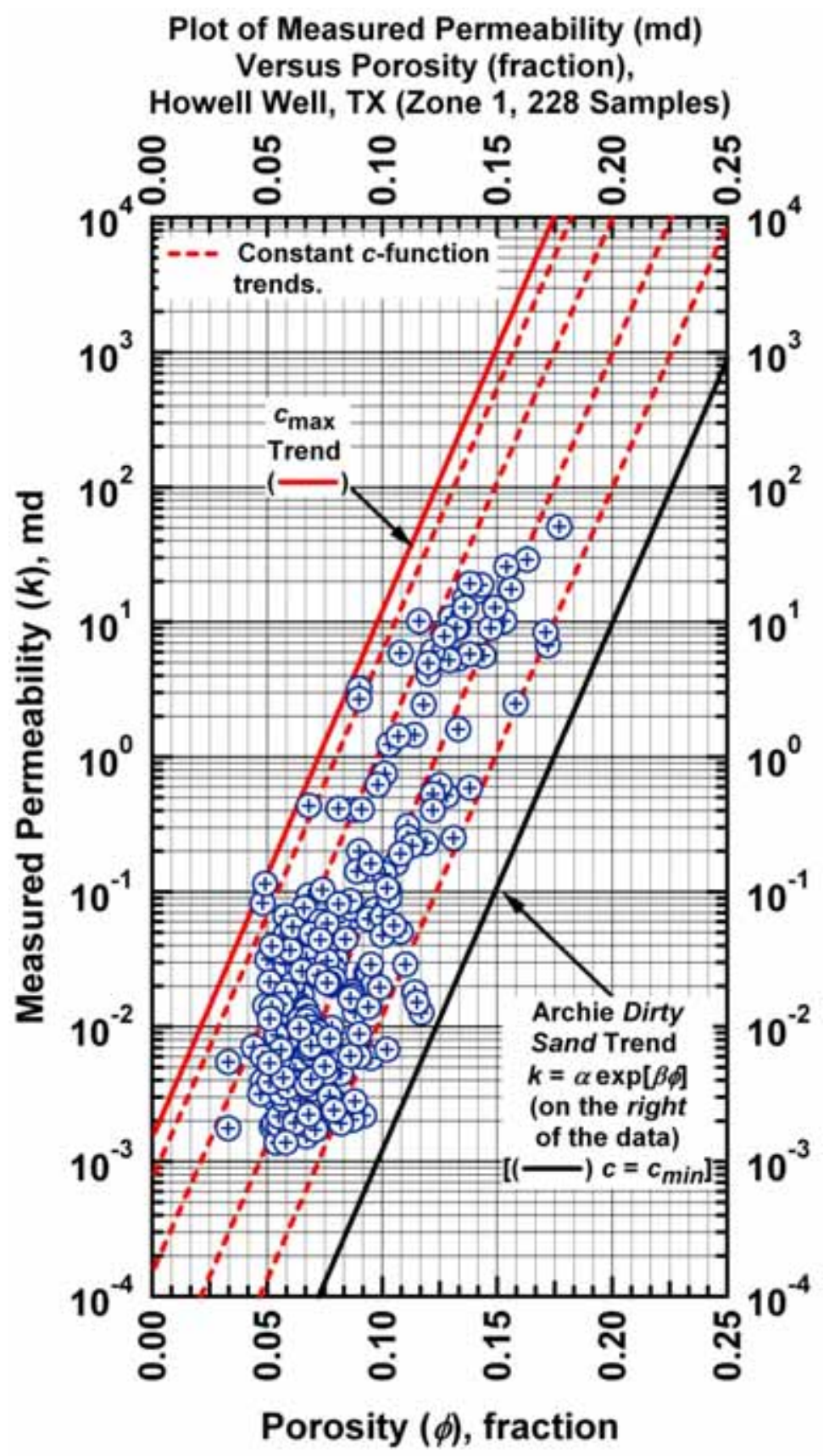

Figure B.18b - Model $C P R-C: k=c \exp [\beta \phi] \quad c=c_{\max } \exp \left[-c_{1} \phi^{c_{2}} S_{w}^{C_{3}}\right]$ - "Dirty Sand" Plot (semilog format) - Archie "Dirty Sand" trend is given by the straight-line trend at the far right of the data (exponential model). [Case: Howell Well, E. TX (USA)] 


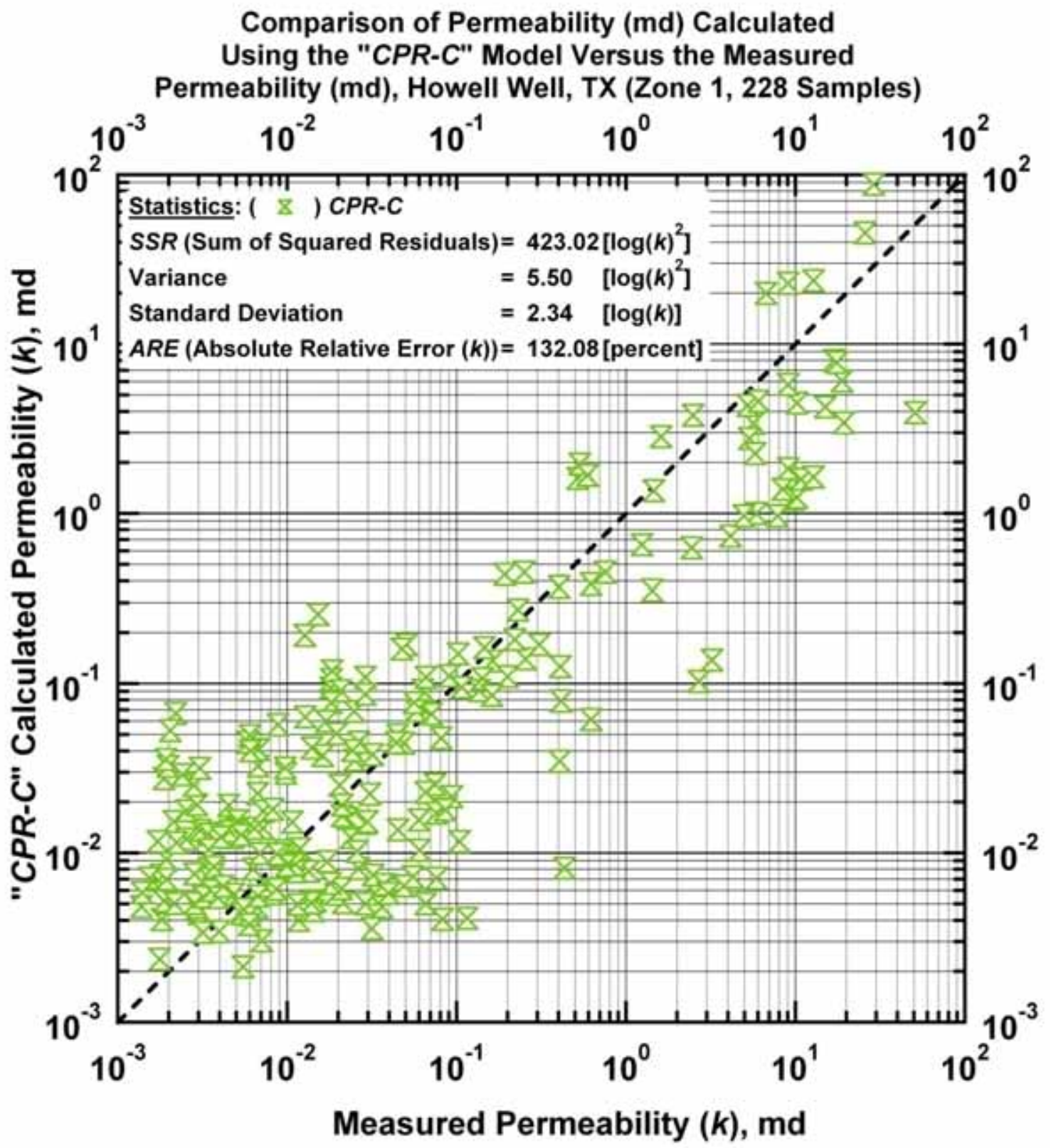

Figure B.19a - Model CPR-C: calculated versus measured permeability. [Case: Howell Well, E. TX (USA)] 


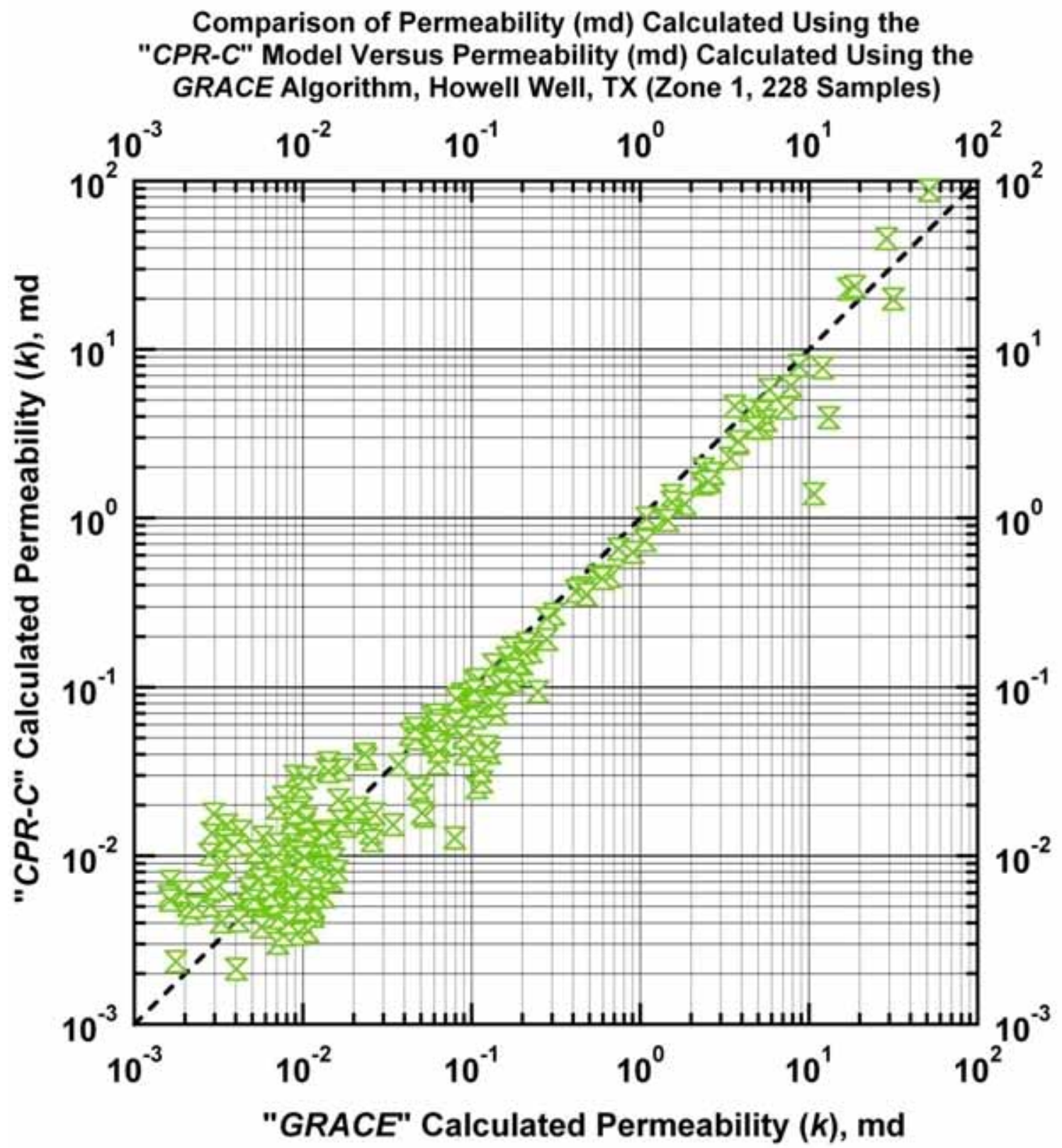

Figure B. 19b - Model $C P R-C$ : Permeability calculated using Model $C P R-C$ versus permeability calculated using the GRACE Algorithm. [Case: Howell Well, E. TX (USA)] 


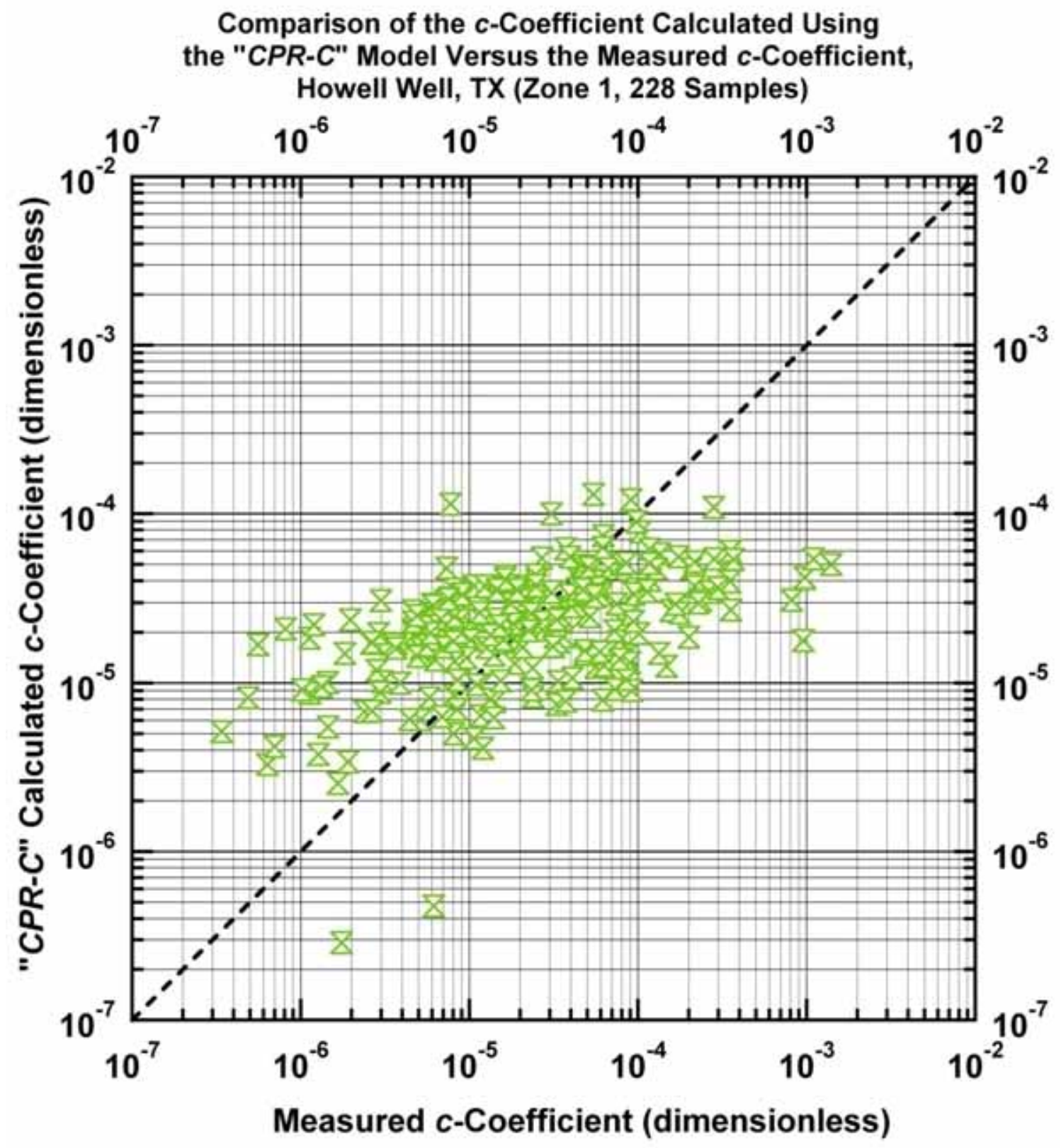

Figure B. 19c - Model $C P R$-C: Calculated $c$-function values versus measured $c$-function values. [Case: Howell Well, E. TX (USA)] 


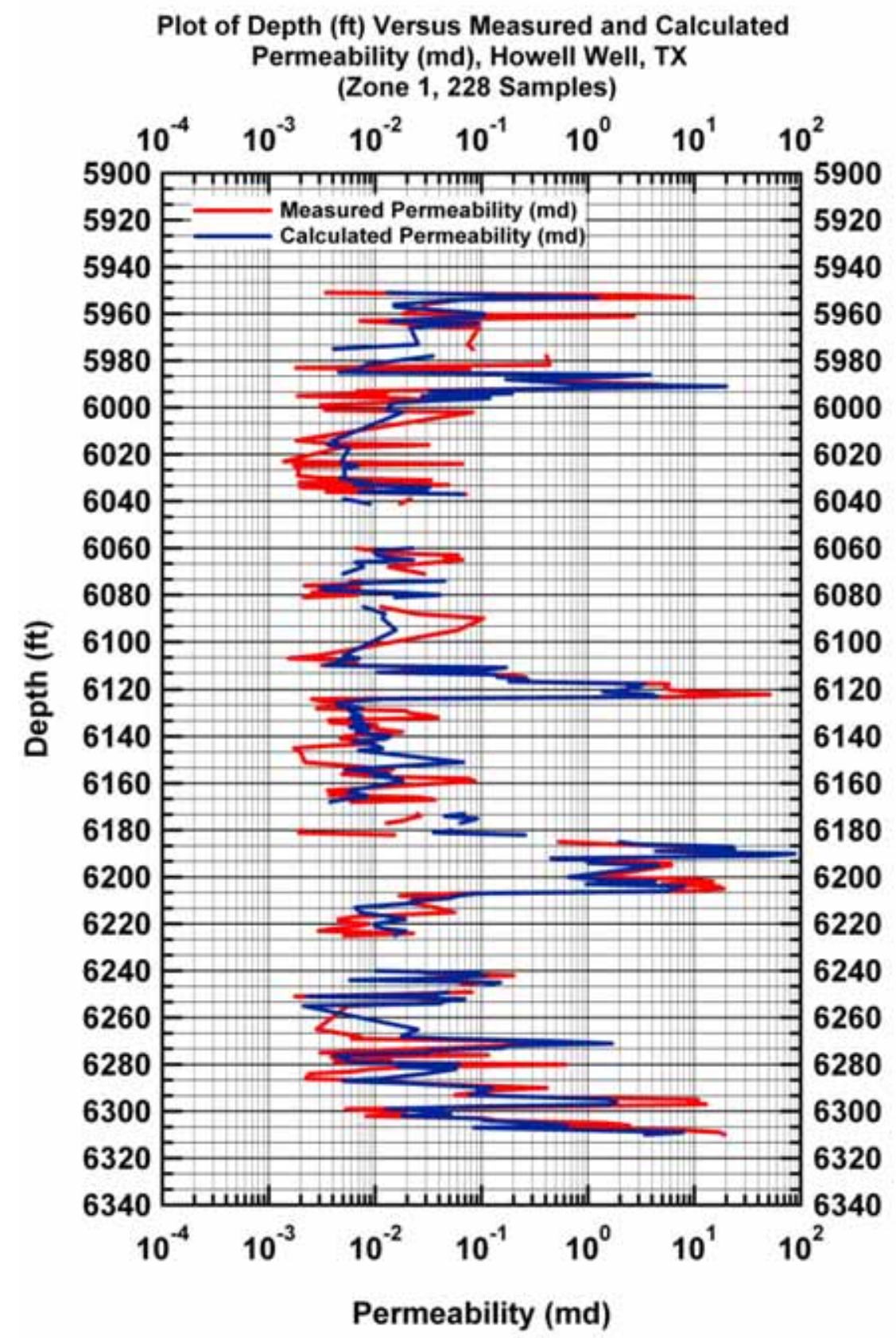

Figure B. 19d - Model CPR-C: Calculated and measured permeability values versus depth. [Case: Howell Well, E. TX (USA)] 


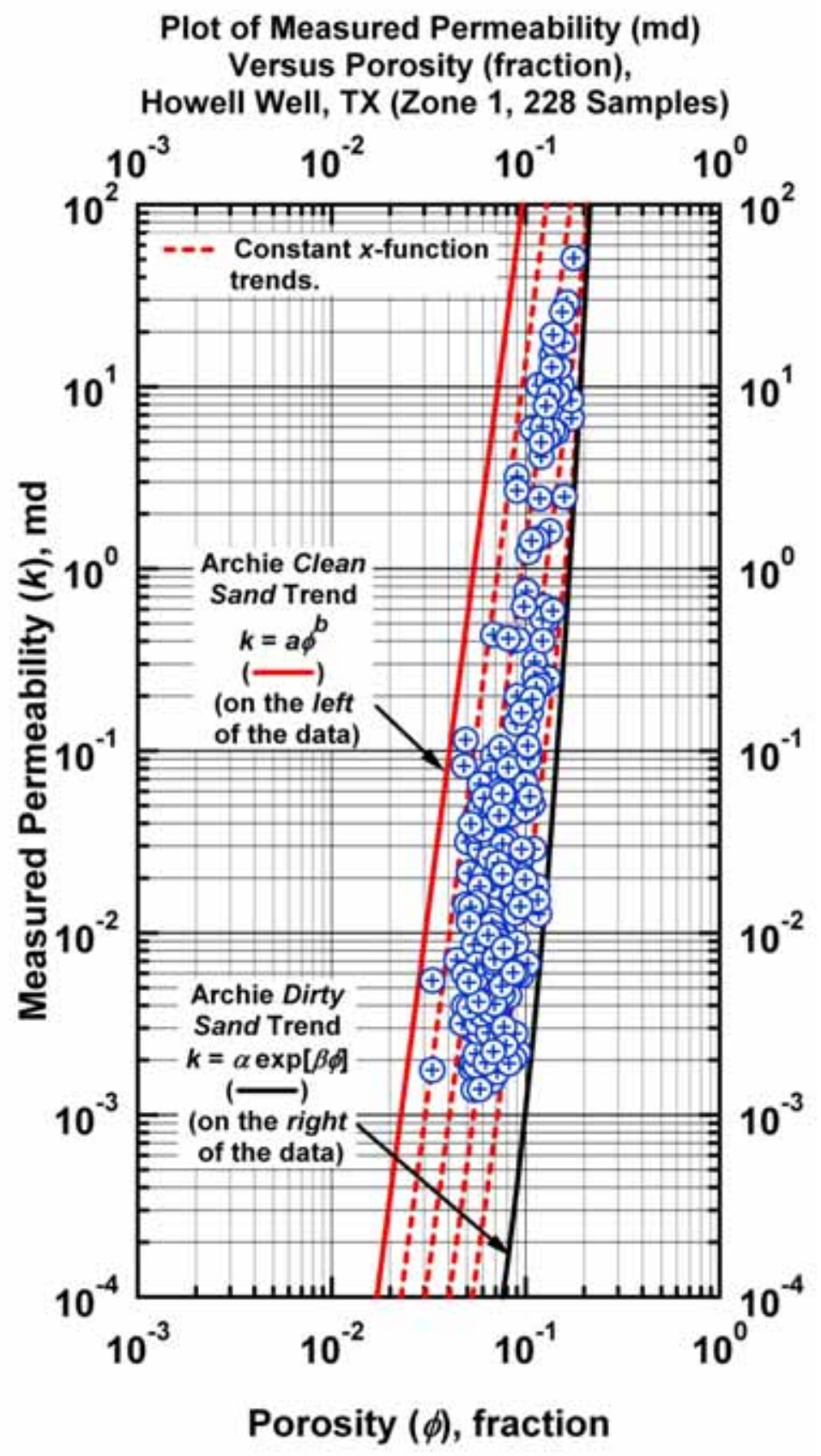

Figure B.20a - Model CPR-D: $k=x a \phi^{b}+(1-x) \alpha \exp [\beta \phi] \quad[0 \leq x \leq 1]$ - Weighted Power Law-Exponential Model used to correlate permeability $(k)$ and porosity $(\phi)$. "Clean Sand" Plot - Archie "Clean Sand" trend is given by the straight-line trend at the far left of the data (power law model). [Case: Howell Well, E. TX (USA)] 


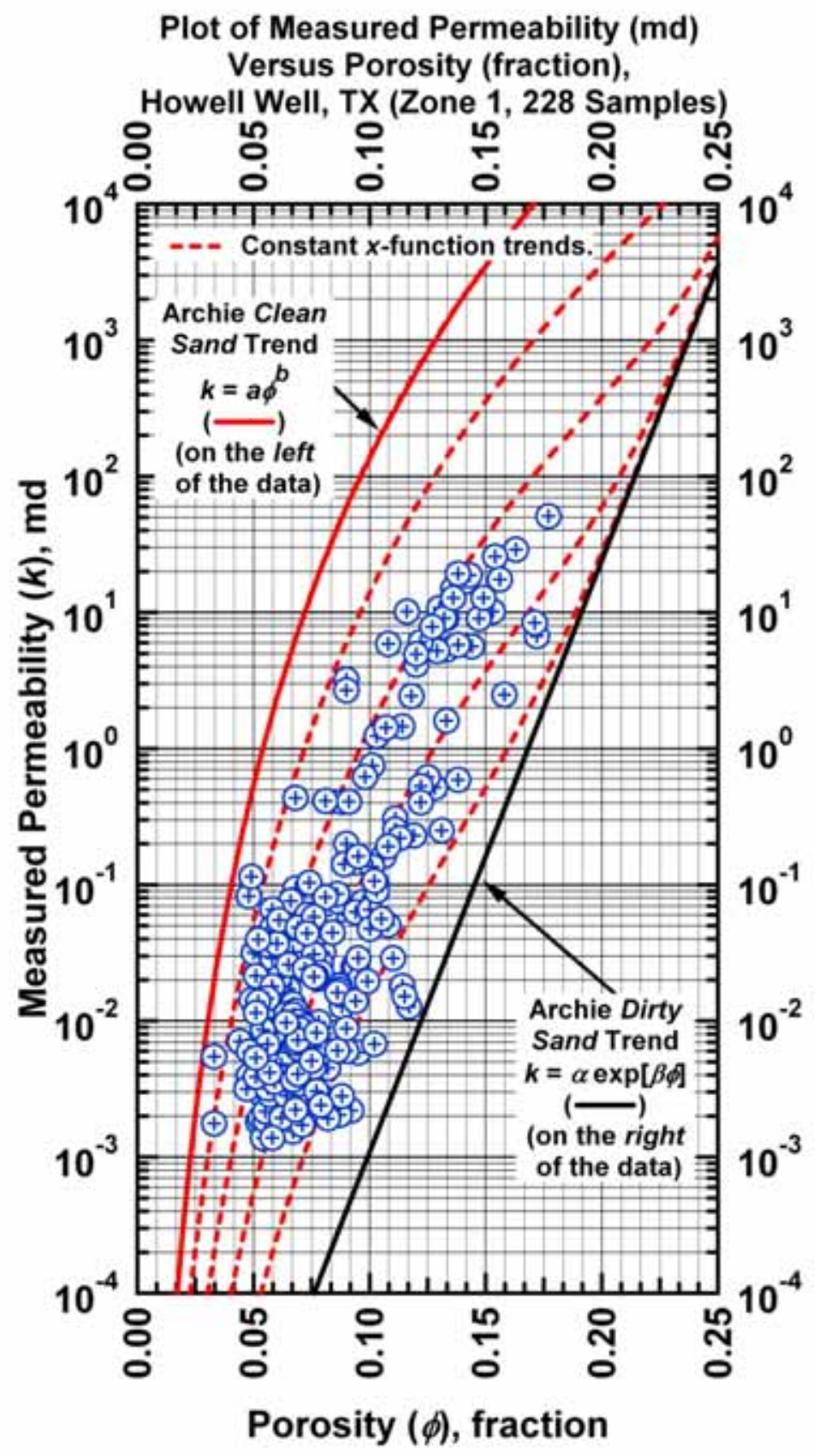

Figure B.20b - Model CPR-D: $k=x a \phi^{b}+(1-x) \alpha \exp [\beta \phi] \quad[0 \leq x \leq 1]$ - Weighted Power Law-Exponential Model used to correlate permeability $(k)$ and porosity $(\phi)$. "Dirty Sand" Plot - Archie "Dirty Sand" trend is given by the straight-line trend at the far right of the data (exponential model). [Case: Howell Well, E. TX (USA)] 


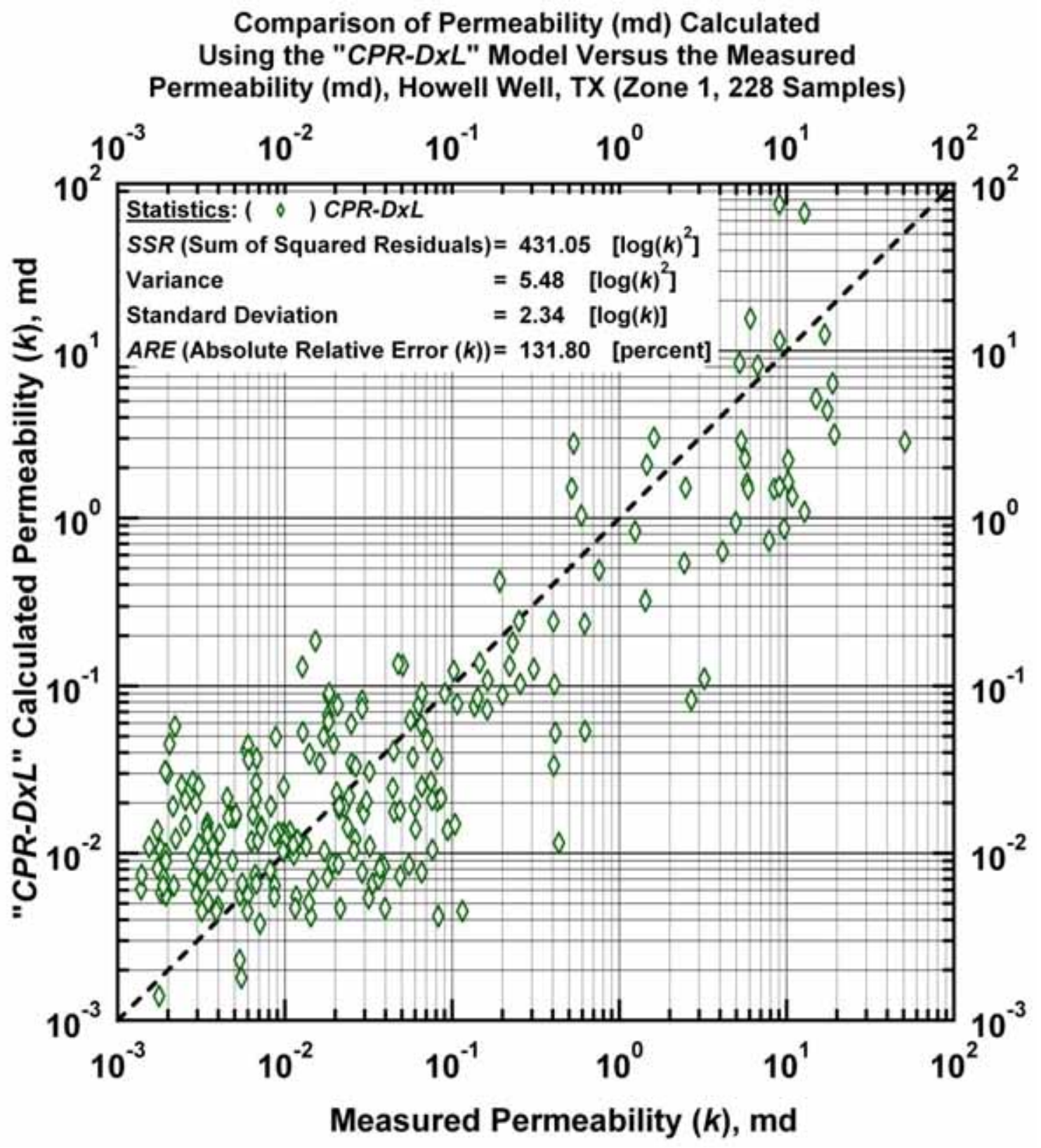

Figure B.21a - Model CPR-DxL: Calculated versus measured permeability. [Case: Howell Well, E. TX (USA)] 


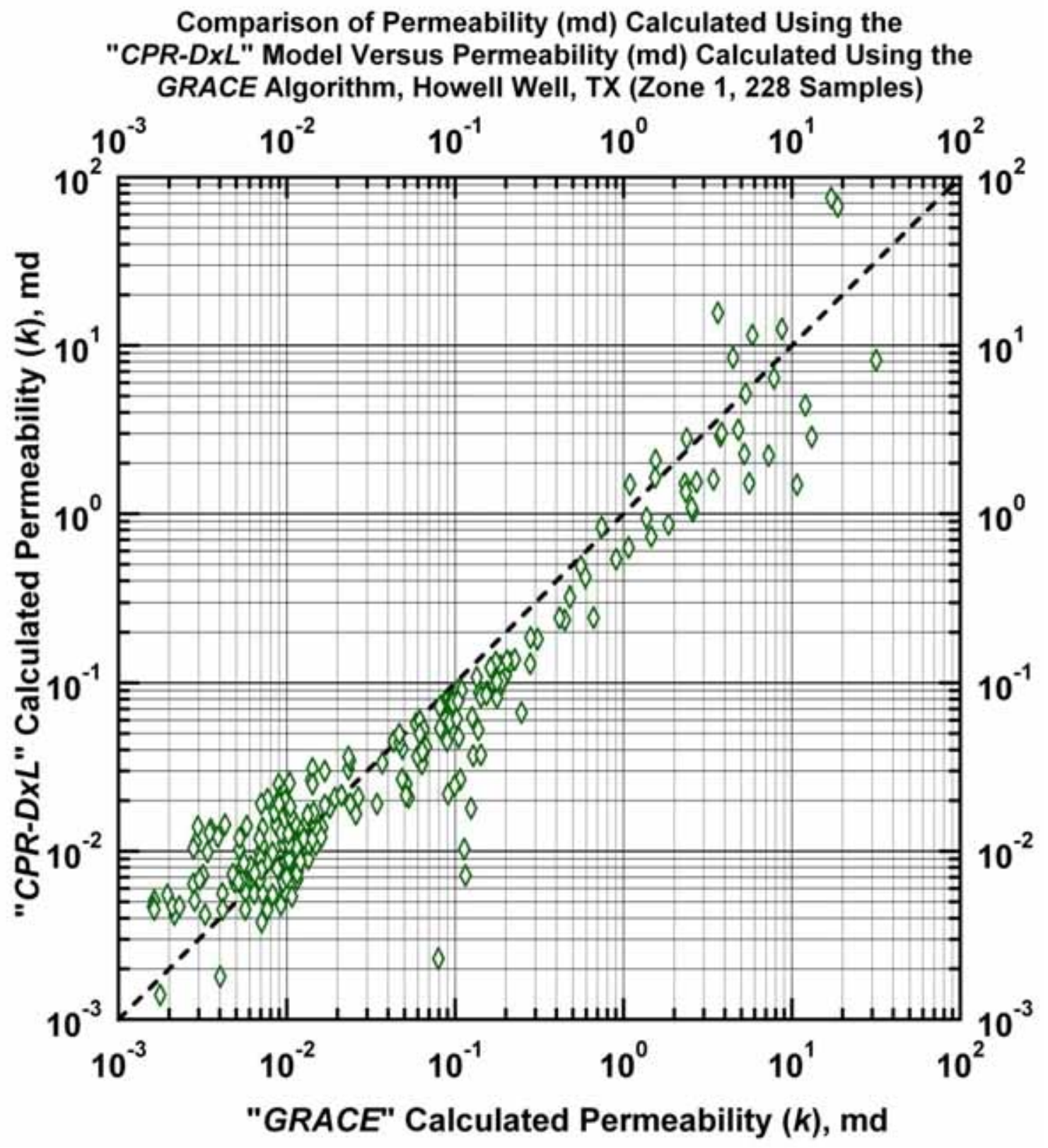

Figure B. 21b - Model $C P R-D x L$ : Permeability calculated using Model $C P R-C$ versus permeability calculated using the GRACE Algorithm. [Case: Howell Well, E. TX (USA)] 


\section{Comparison of the $x$-Coefficient Calculated Using} the "CPR-DxL" Model Versus the Measured $x$-Coefficient, Howell Well, TX (Zone 1, 228 Samples)

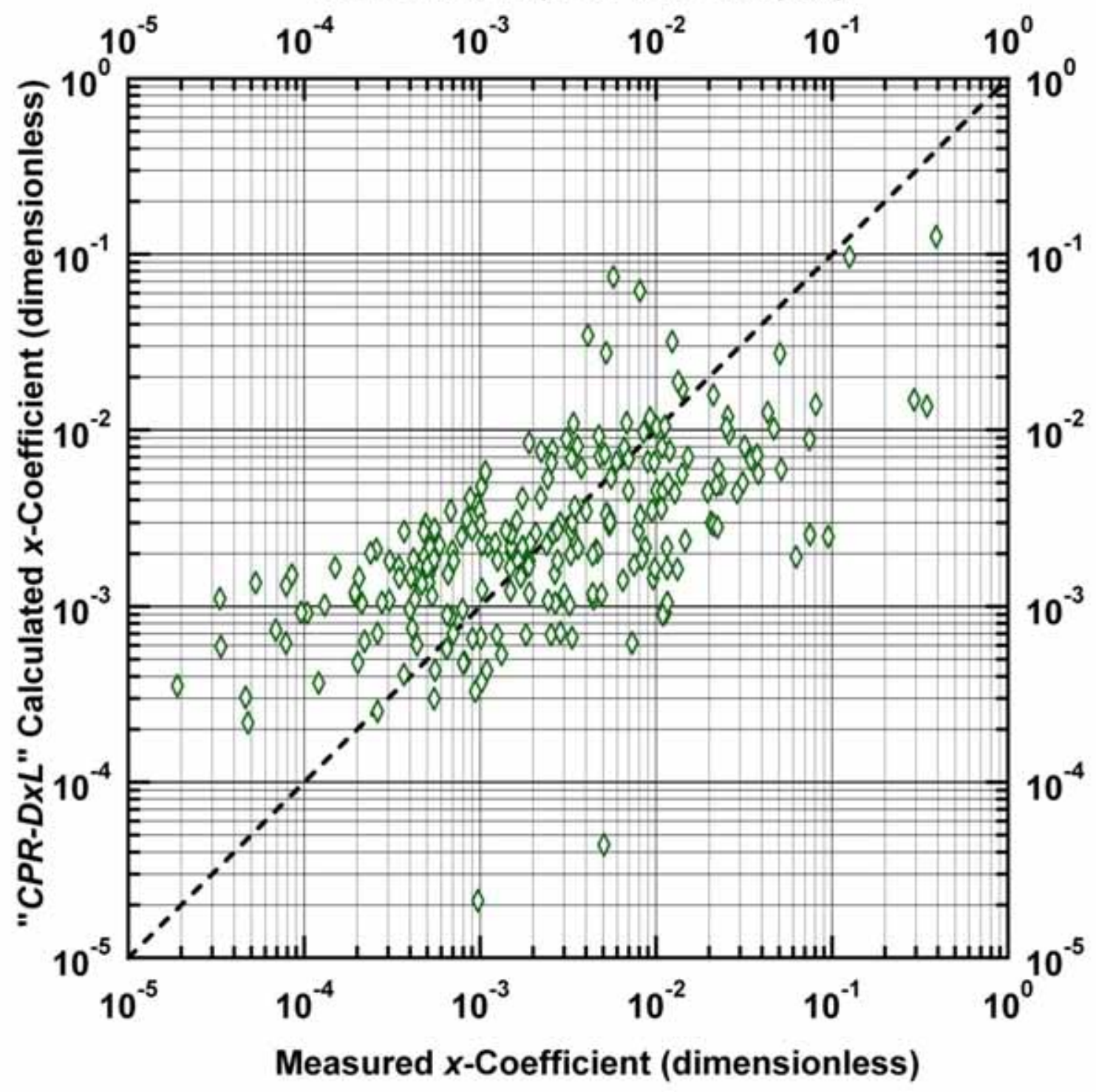

Figure B. 21c - Model $C P R$ - DxL: Calculated $x$-function weight values versus measured $x$ function weight values. [Case: Howell Well, E. TX (USA)] 


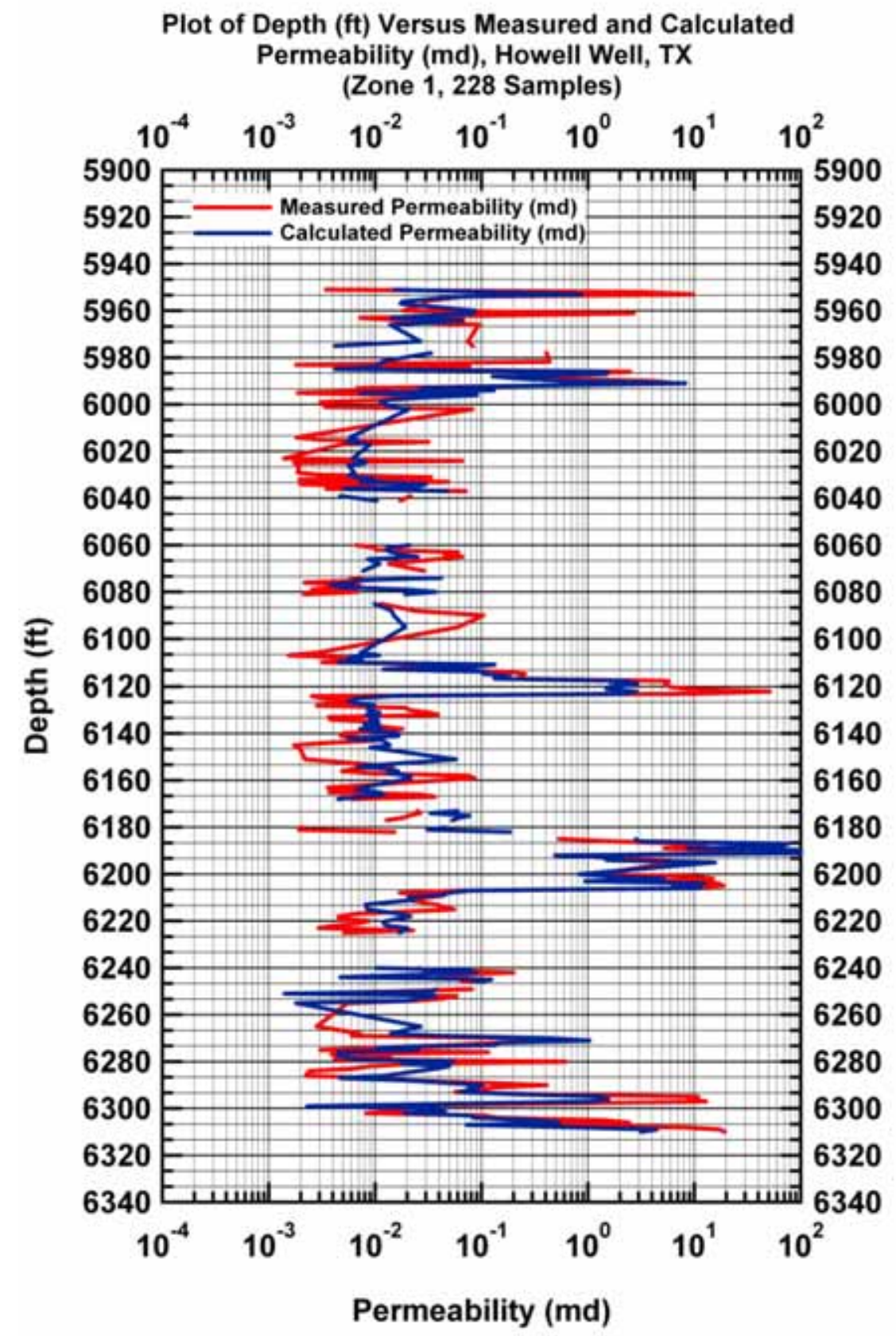

Figure B. 21d - Model $C P R-D x L$ : Calculated and measured permeability values versus depth. [Case: Howell Well, E. TX (USA)] 


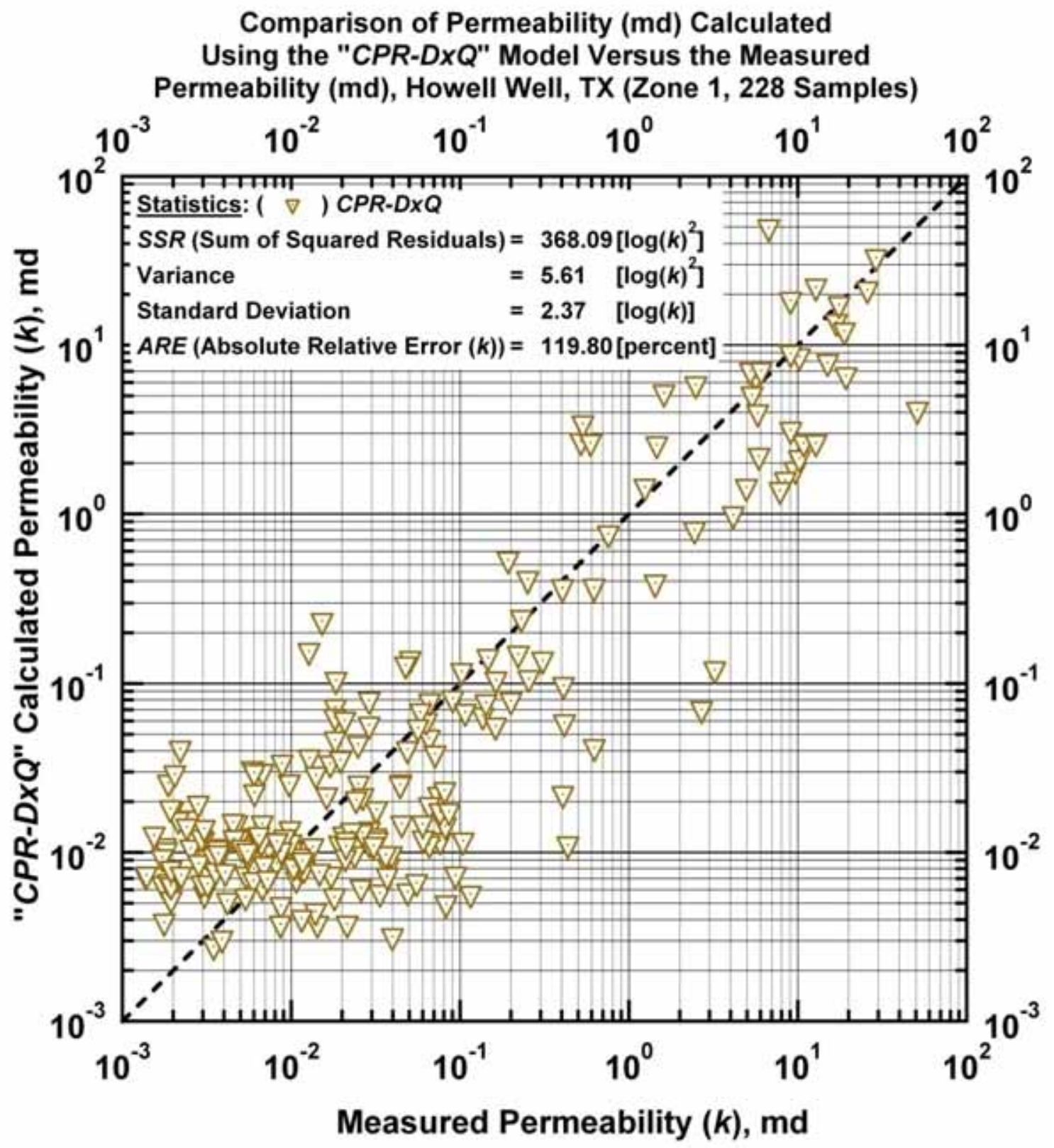

Figure B.22a - Model CPR-DxQ: Calculated versus measured permeability. [Case: Howell Well, E. TX (USA)] 


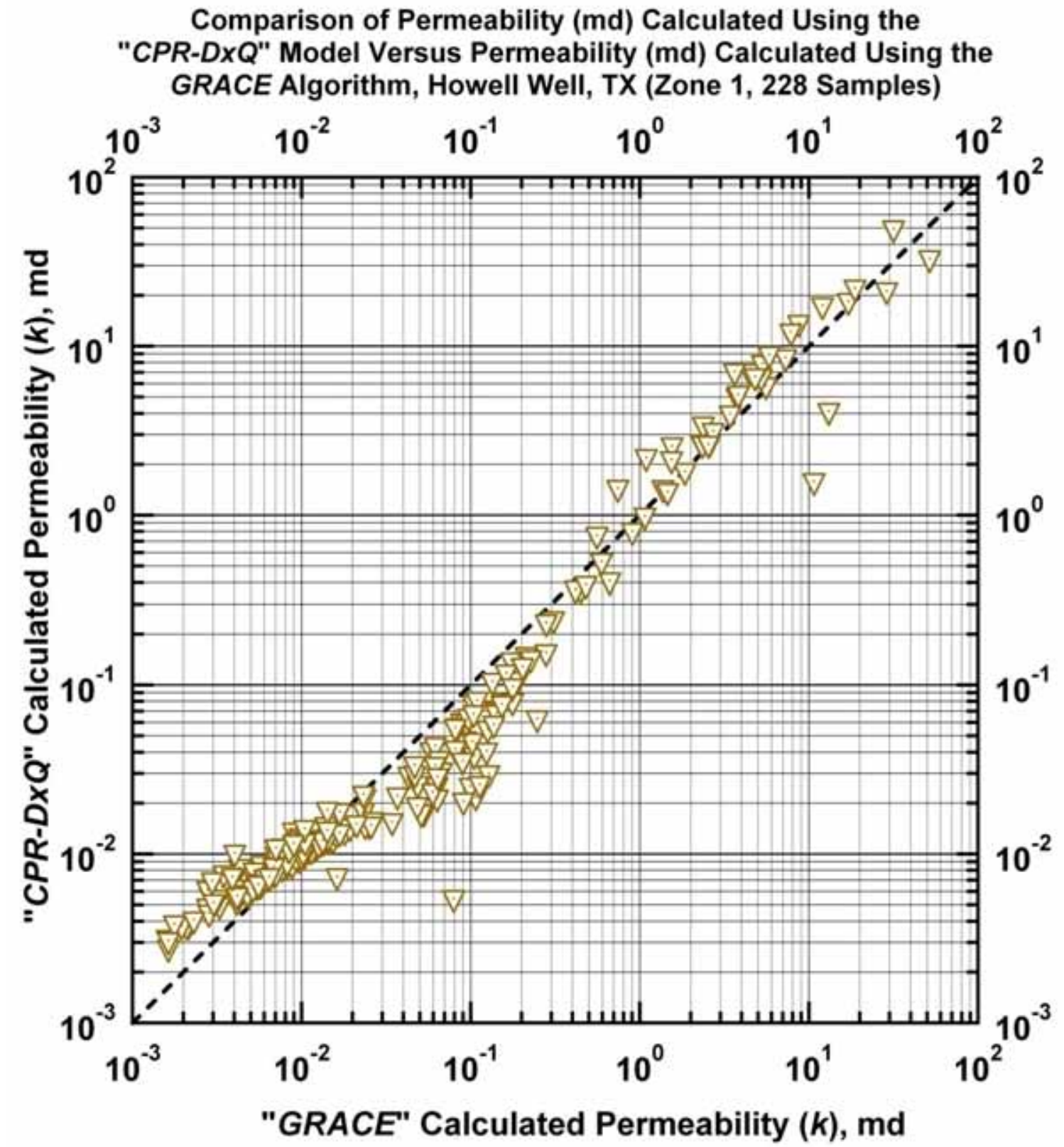

Figure B.22b - Model $C P R-D x Q$ : Permeability calculated using Model $C P R-C$ versus permeability calculated using the GRACE Algorithm. [Case: Howell Well, E. TX (USA)] 


\section{Comparison of the $x$-Coefficient Calculated Using} the "CPR-DxQ" Model Versus the Measured $x$-Coefficient, Howell Well, TX (Zone 1, 228 Samples)

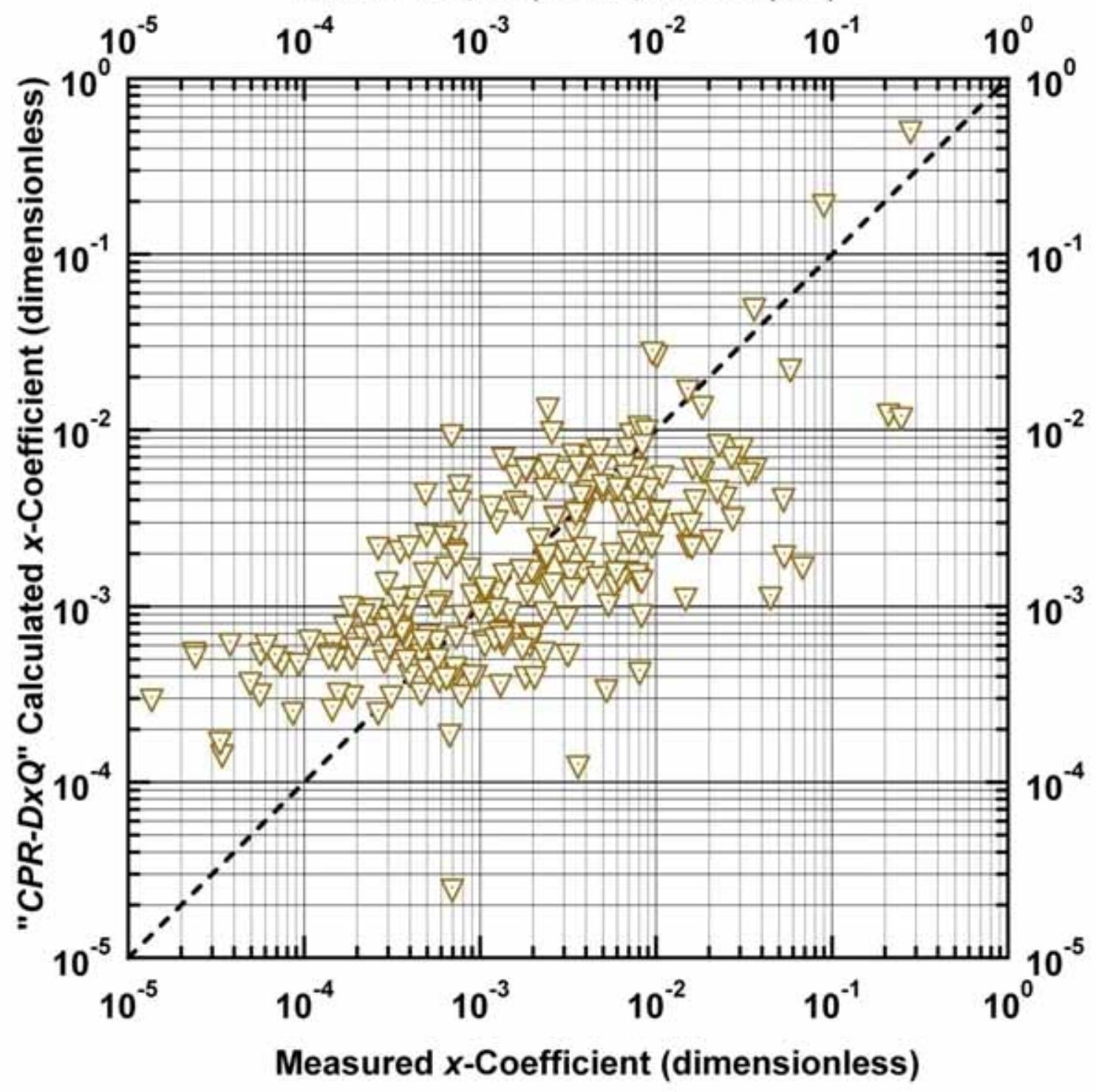

Figure B.22c - Model CPR-DxQ: Calculated $x$-function weight values versus measured $x$ function weight values. [Case: Howell Well, E. TX (USA)] 


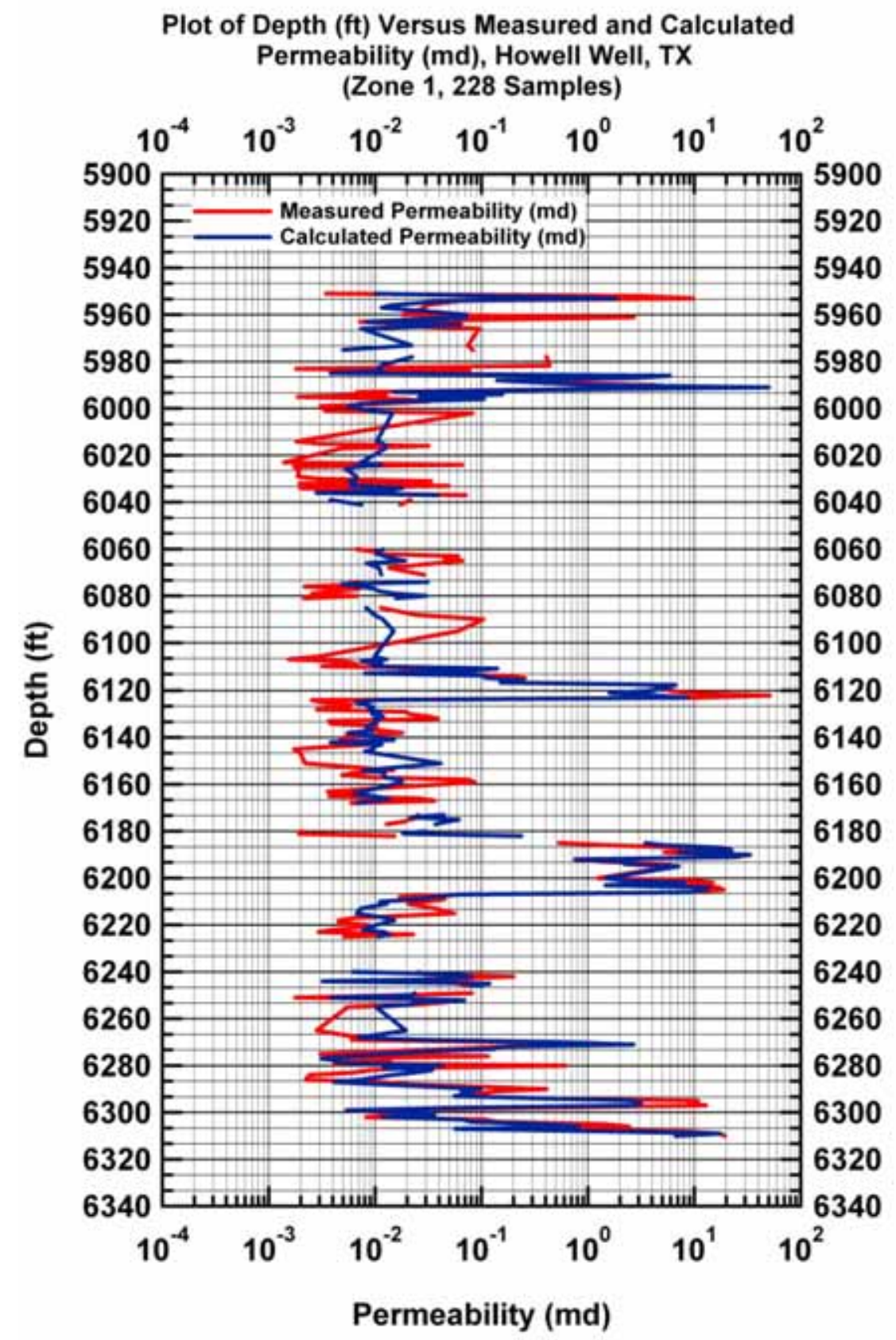

Figure B.22d - Model $C P R-D x Q$ : Calculated and measured permeability values versus depth. [Case: Howell Well, E. TX (USA)] 


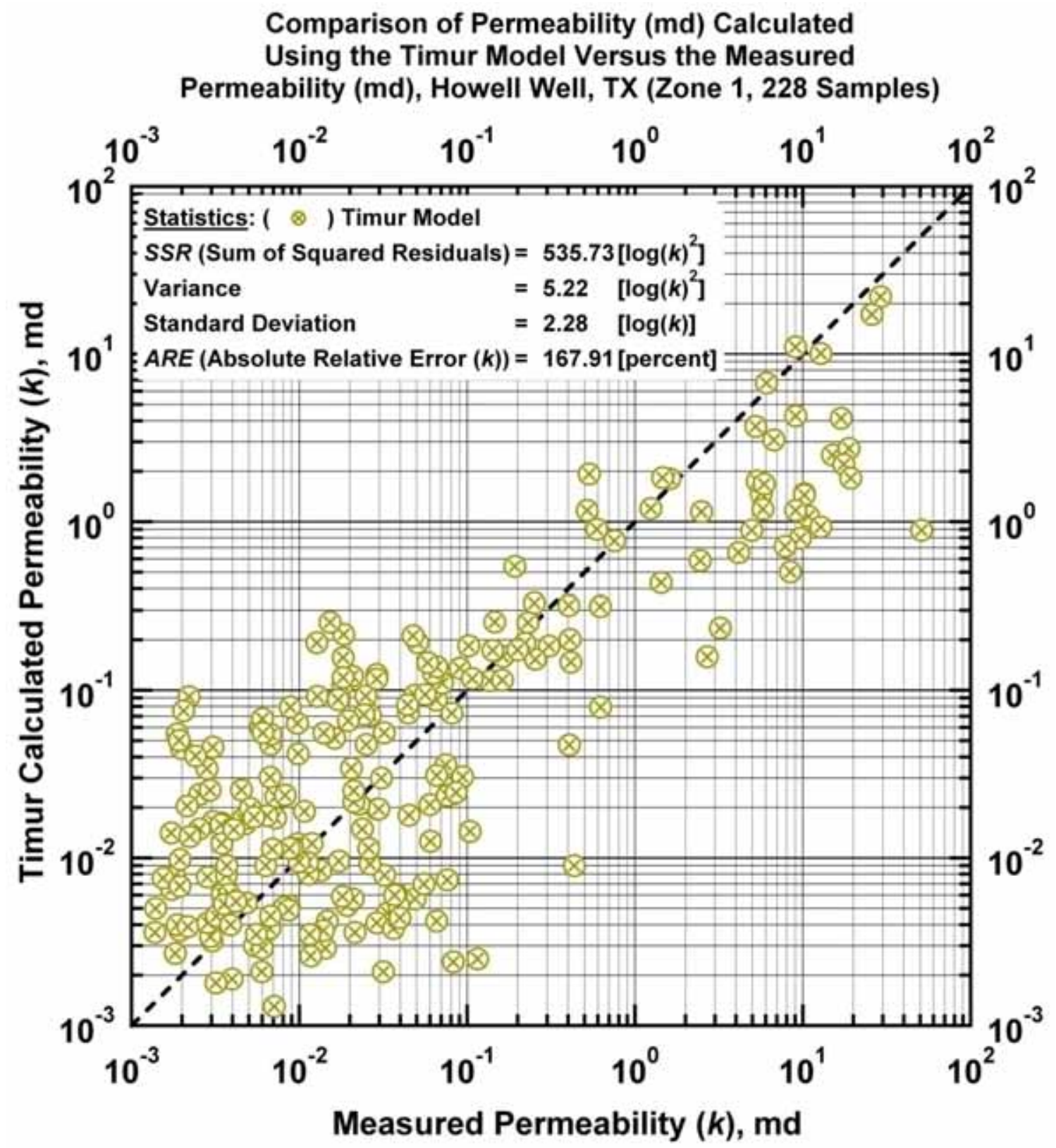

Figure B.23a - "Timur Model:" Calculated versus measured permeability. [Case: Howell Well, E. TX (USA)] 


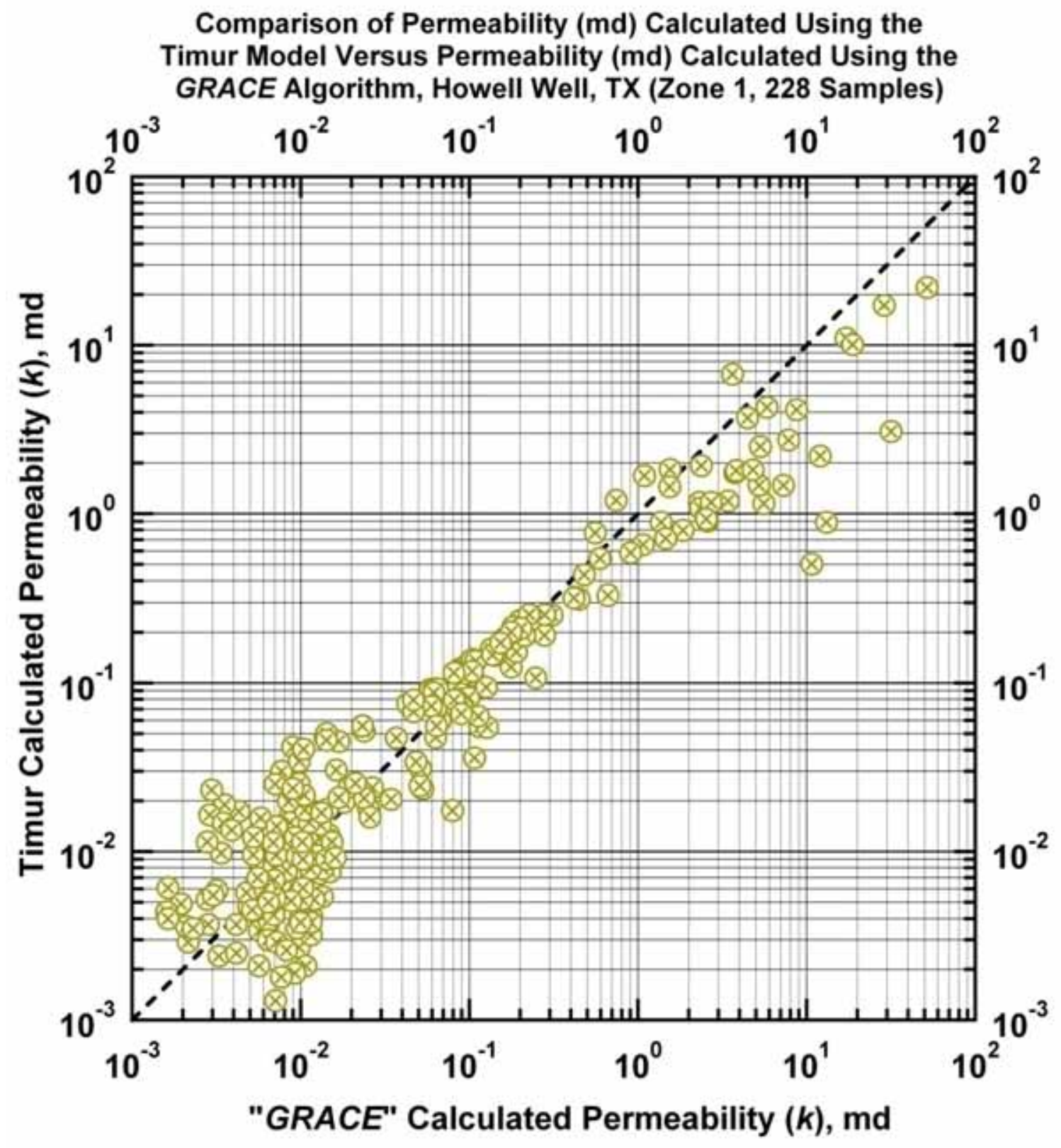

Figure B.23b — "Timur Model:" Permeability calculated using the "Timur Model" versus permeability calculated using the GRACE Algorithm. [Case: Howell Well, E. TX (USA)] 


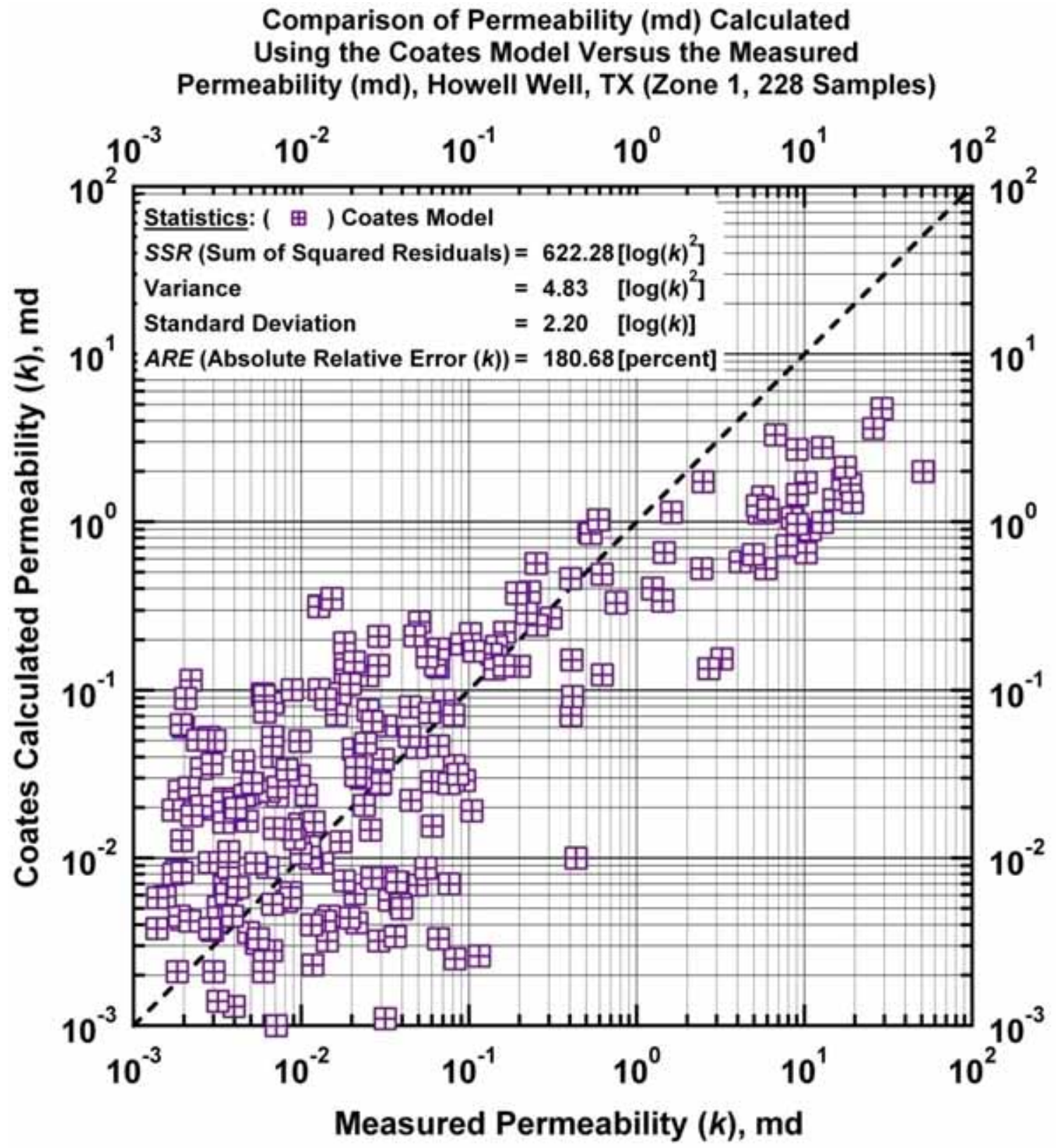

Figure B.24a - "Coates Model:" Calculated versus measured permeability. [Case: Howell Well, E. TX (USA)] 


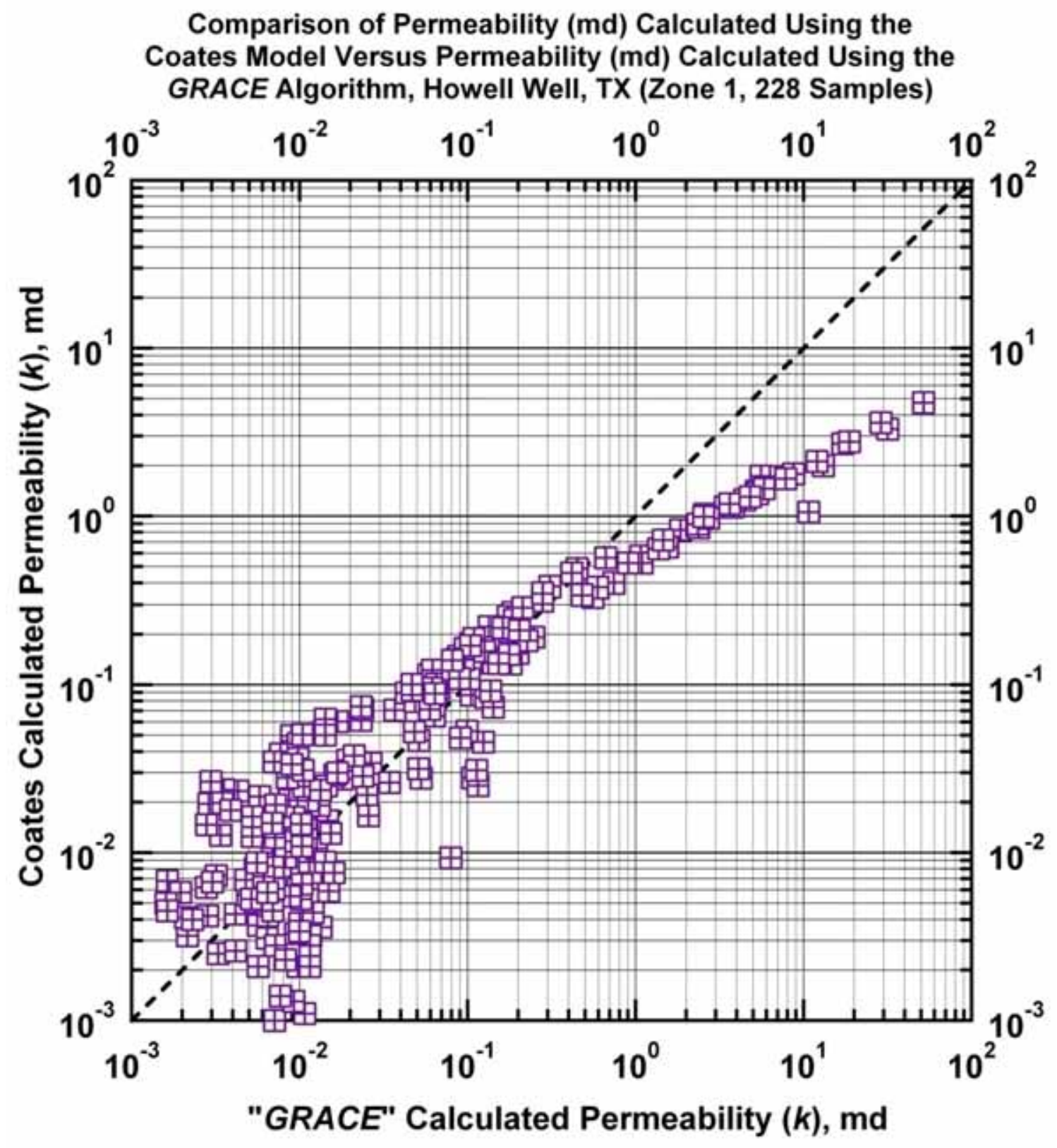

Figure B.24b — "Coates Model:" Permeability calculated using the "Coates Model" versus permeability calculated using the GRACE Algorithm. [Case: Howell Well, E. TX (USA)] 


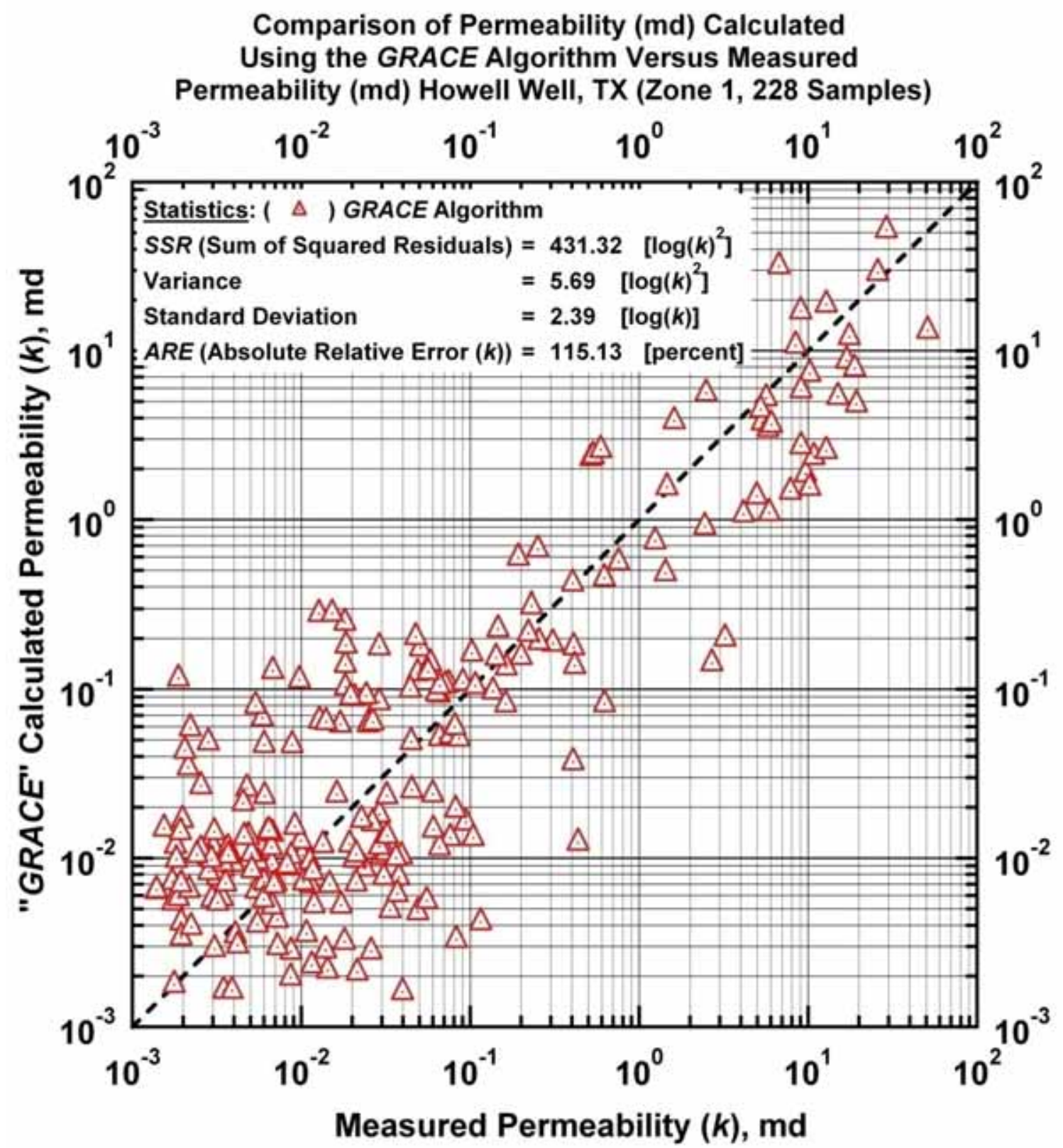

Figure B.25 - GRACE Model: Calculated versus measured permeability. [Case: Howell Well, E. TX (USA)] 


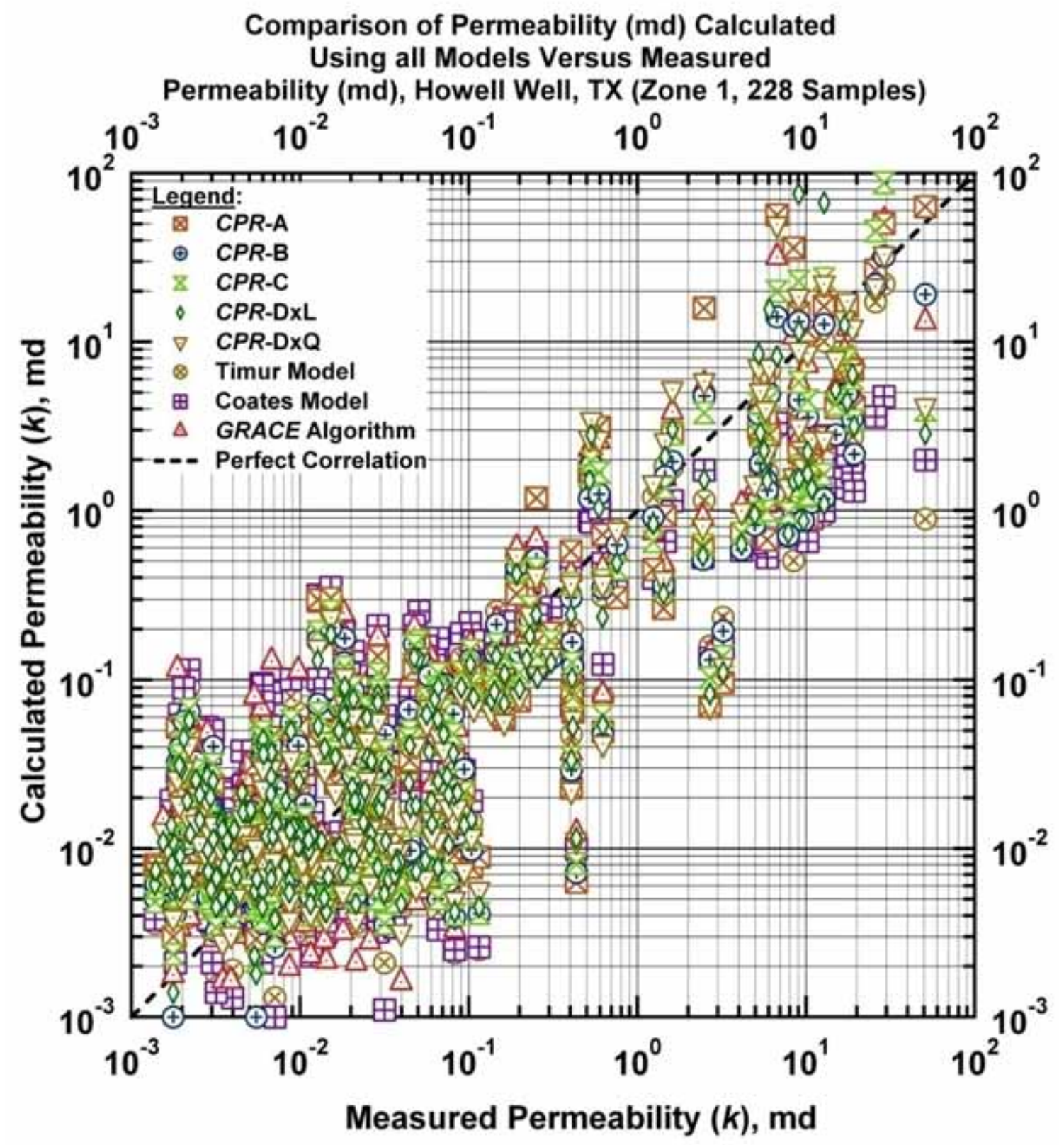

Figure B.26 - All Models: Calculated versus measured permeability. [Case: Howell Well, E. TX (USA)] 


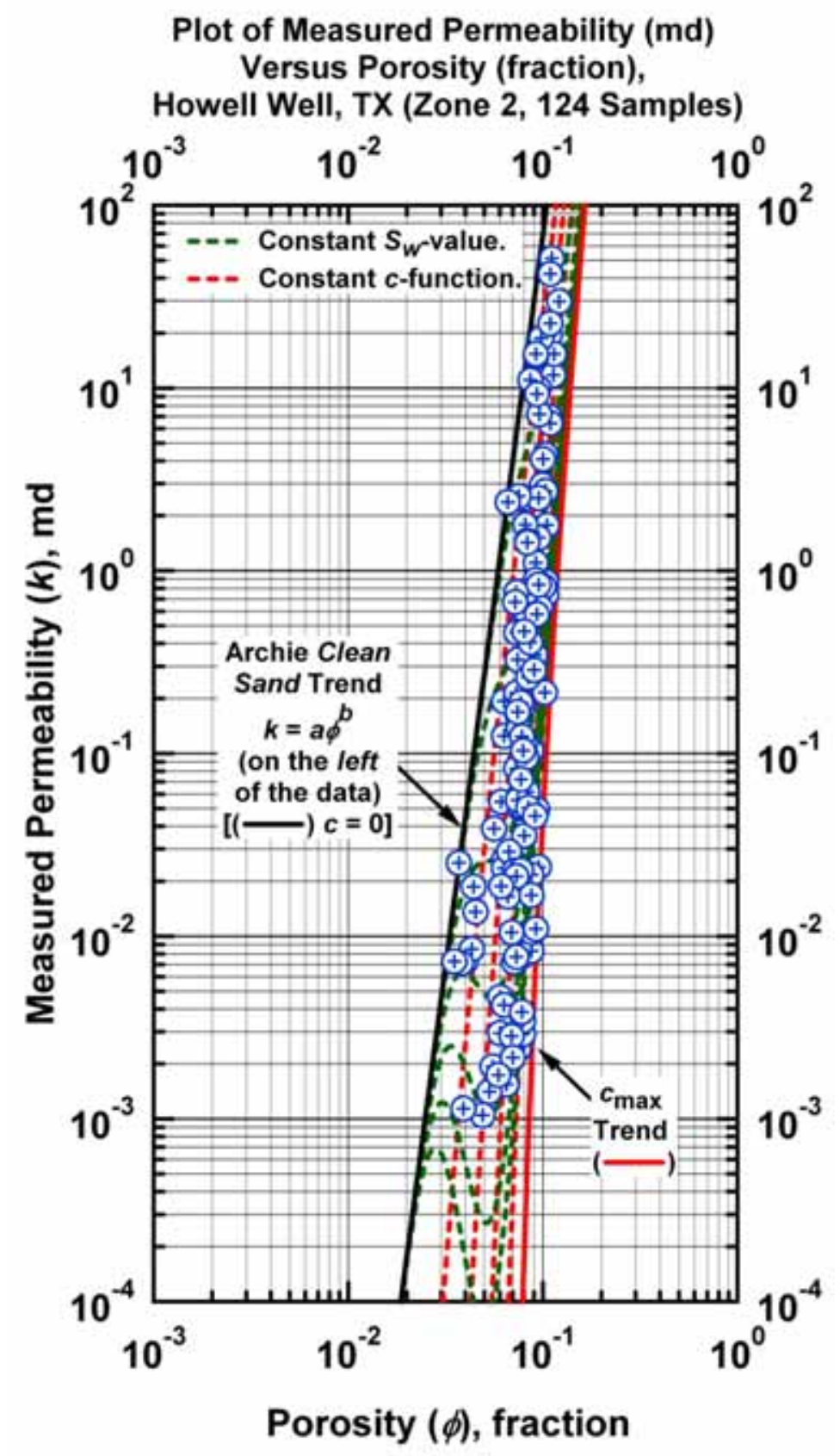

Figure B.27a - Model $C P R-A: \quad k=a(\phi-c)^{b} \quad c=c_{\max } \exp \left[-c_{1} \phi^{c_{2}} S_{w}^{c_{3}}\right]$ - "Clean Sand" Plot (log-log format) - Archie "Clean Sand" trend is given by the straight-line trend at the far left of the data (power law model). [Case: Howell Well, E. TX (USA)] 


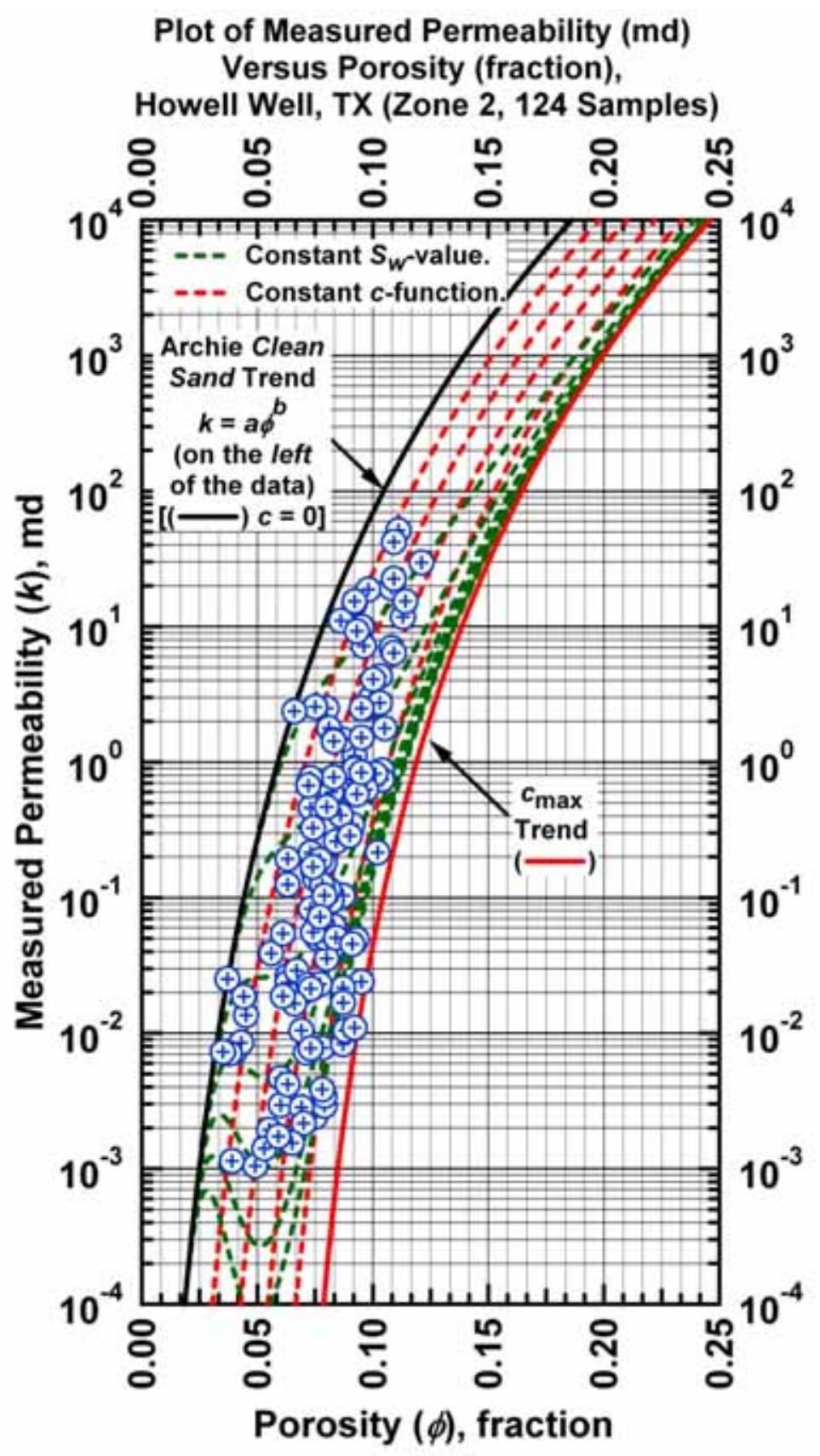

Figure B.27b - Model CPR-A: $k=a(\phi-c)^{b} \quad c=c_{\max } \exp \left[-c_{1} \phi^{c_{2}} S_{W}^{c_{3}}\right]$ - "Dirty Sand" Plot (semilog format). Archie "Clean Sand" trend is given by the curved trend at the far left of the data (power law model). [Case: Howell Well, E. TX (USA)] 


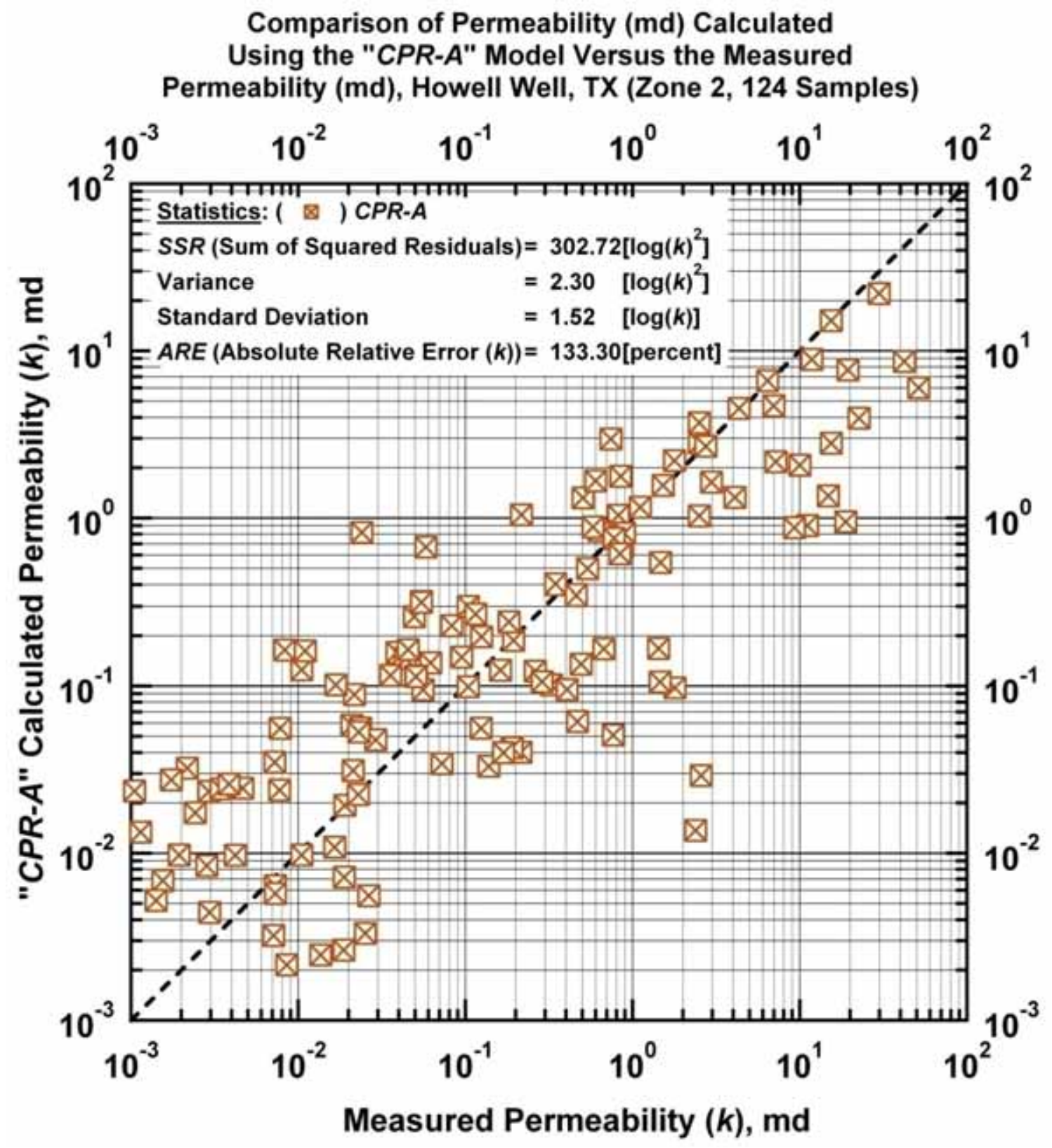

Figure B.28a - Model CPR-A: calculated versus measured permeability. [Case: Howell Well, E. TX (USA)] 


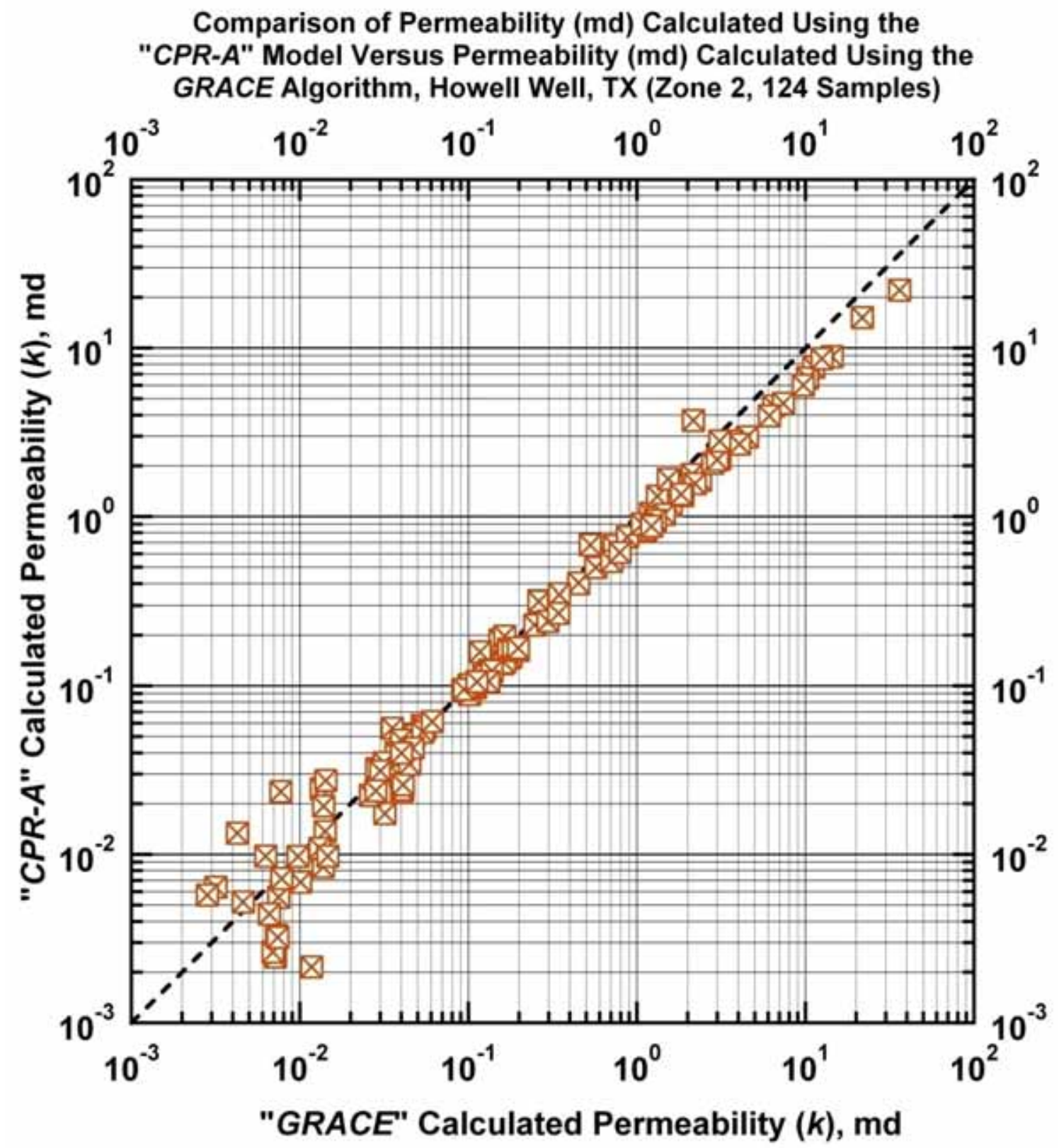

Figure B. 28b - Model CPR-A: Permeability calculated using Model CPR-A versus permeability calculated using the GRACE Algorithm. [Case: Howell Well, E. TX (USA)] 
Comparison of the $c$-Coefficient Calculated Using the "CPR-A" Model Versus the Measured $c$-Coefficient, Howell Well, TX (Zone 2, 124 Samples)

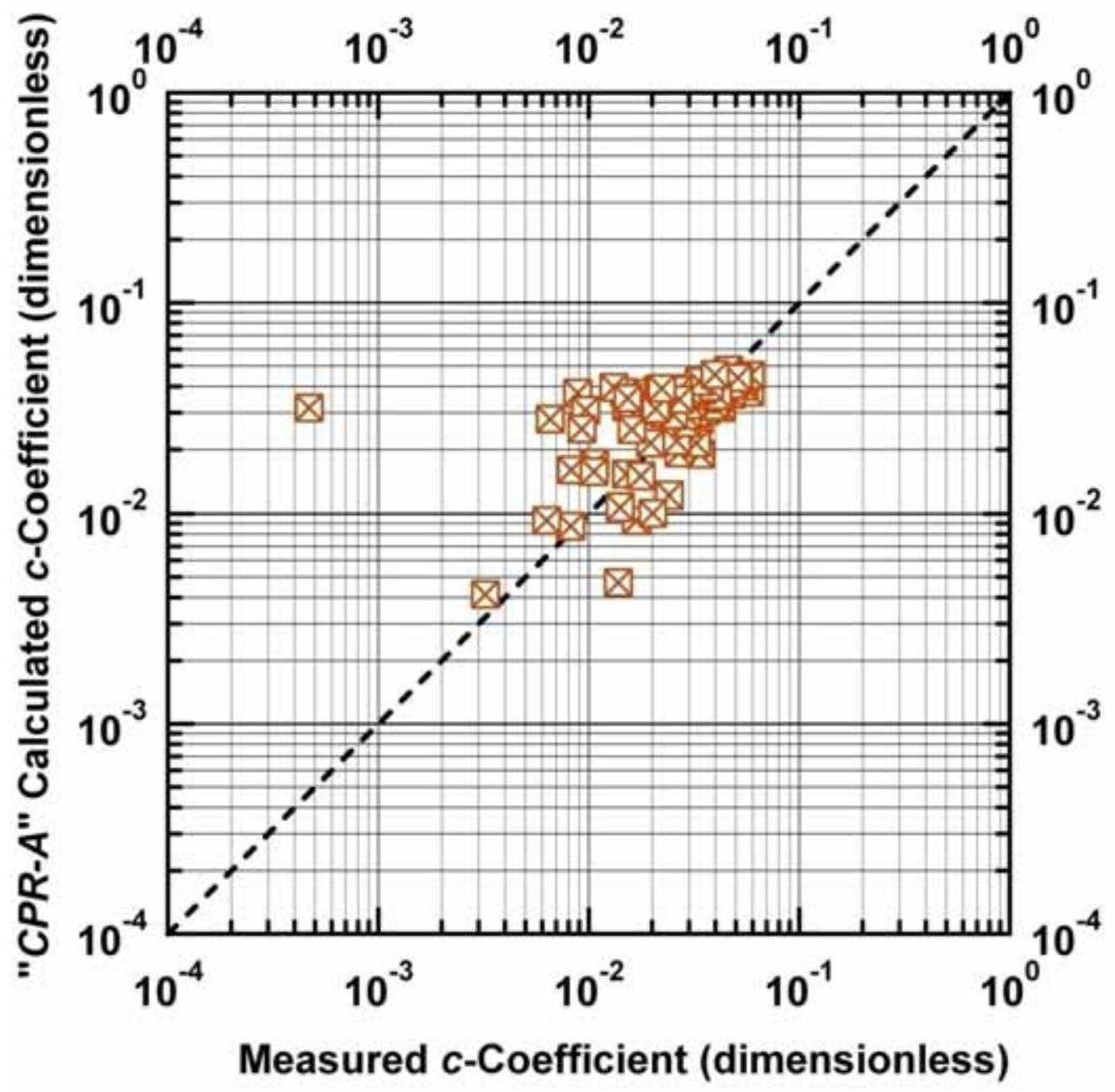

Figure B. 28c - Model $C P R$ - $A$ : Calculated $c$-function values versus measured $c$-function values. [Case: Howell Well, E. TX (USA)] 


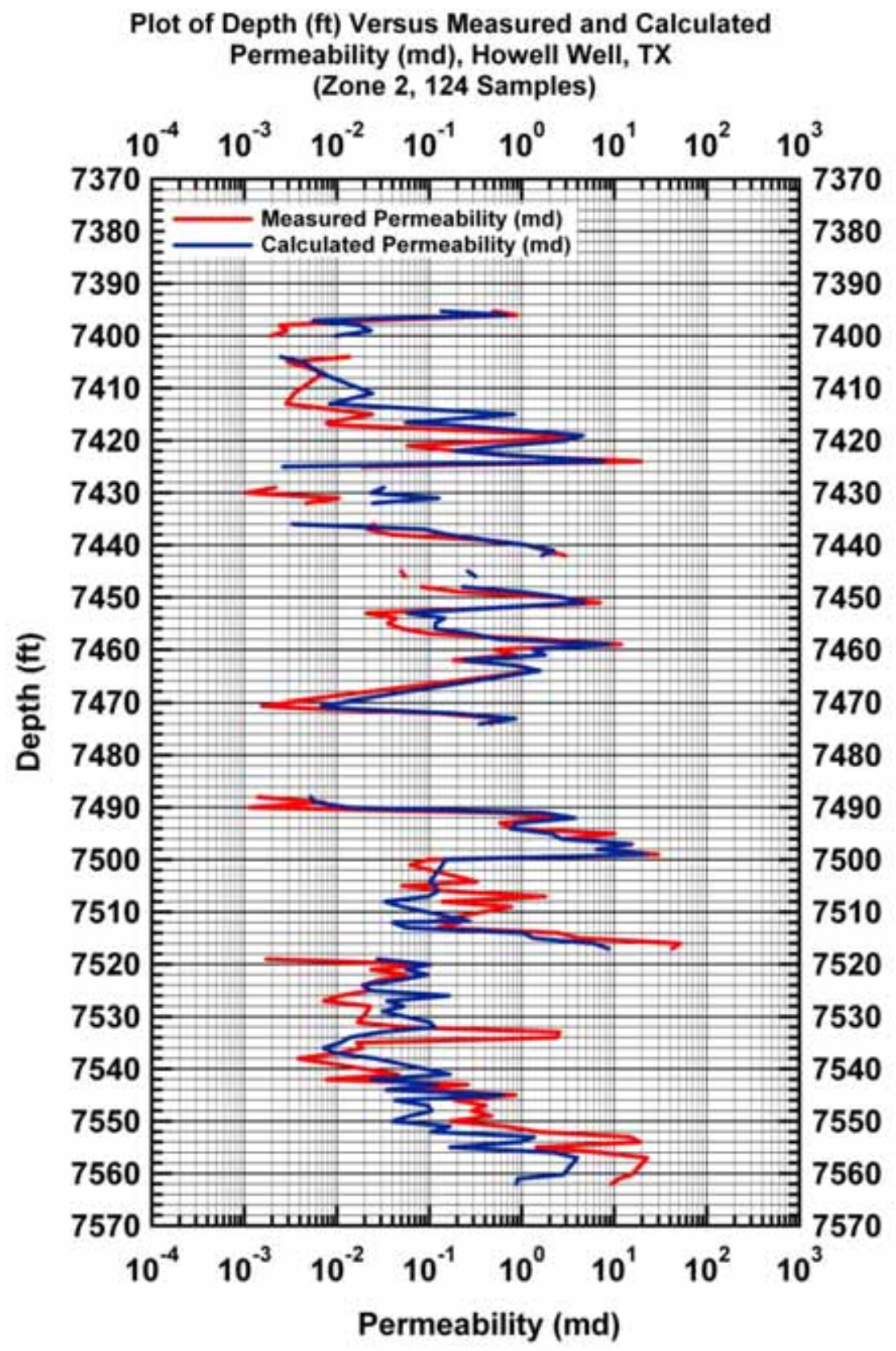

Figure B. 28d - Model CPR-A: Calculated and measured permeability values versus depth. [Case: Howell Well, E. TX (USA)] 


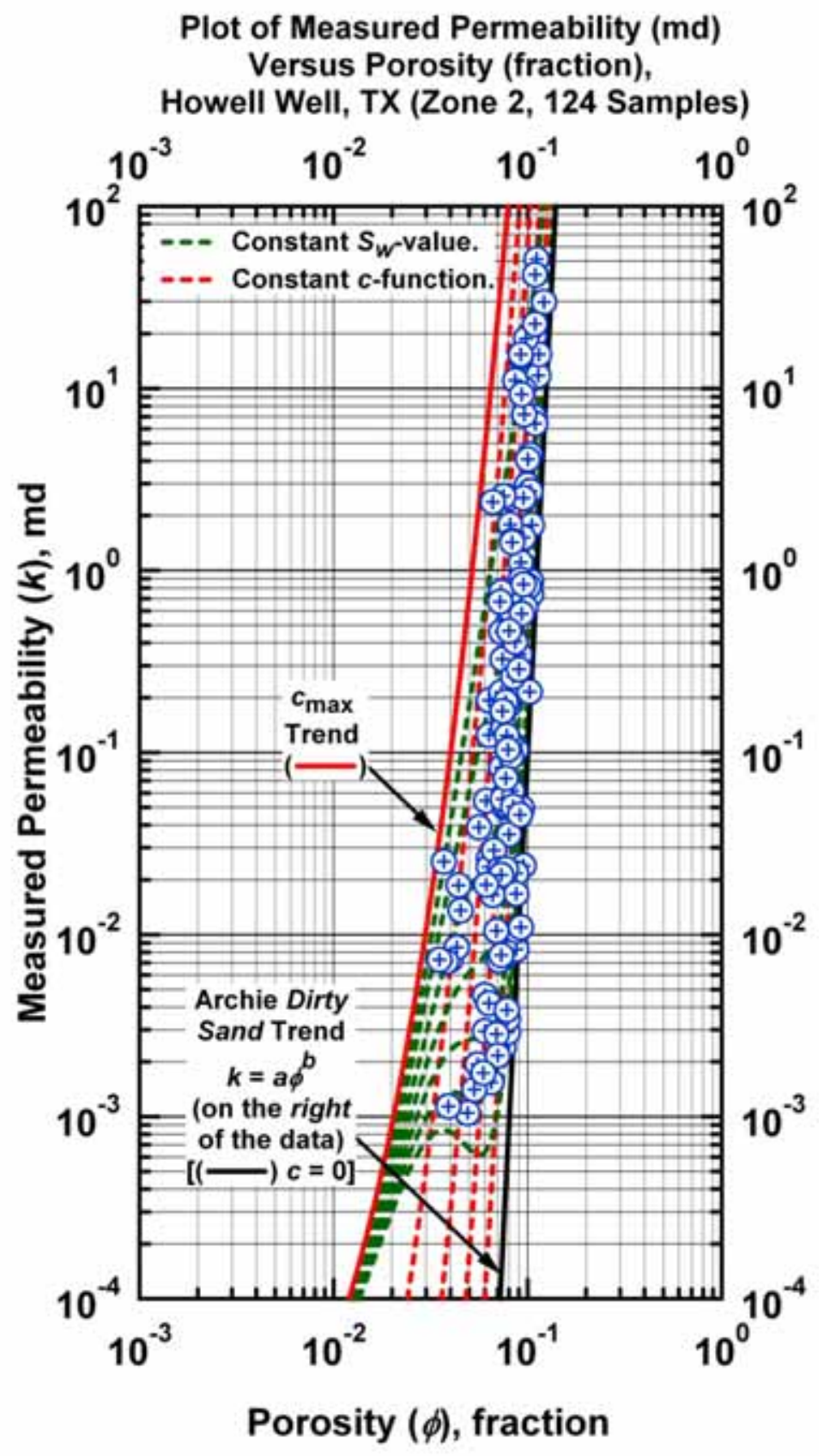

Figure B.29a - Model CPR-B: $\quad k=a(\phi+c)^{b} \quad c=c_{\max } \exp \left[-c_{1} \phi^{c_{2}} S_{w}^{c_{3}}\right]$ - "Clean Sand" Plot (log-log format) - Archie "Dirty Sand" trend is given by the straight-line trend at the far right of the data (power law model). [Case: Howell Well, E. TX (USA)] 


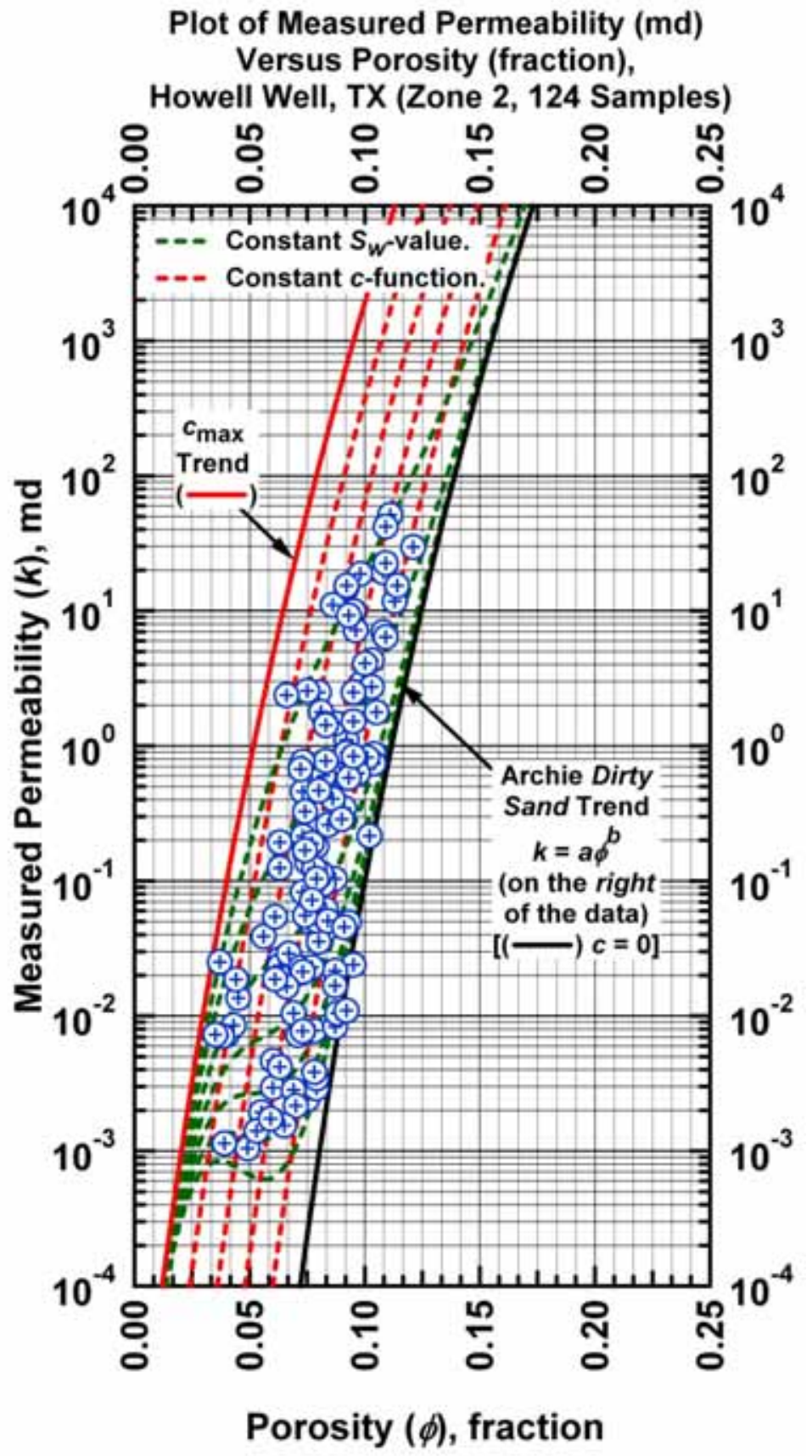

Figure B.29b - Model CPR-B: $k=a(\phi+c)^{b} \quad c=c_{\max } \exp \left[-c_{1} \phi^{c_{2}} S_{W}^{C_{3}}\right]-$ "Dirty Sand" Plot (semilog format) - Archie "Dirty Sand" trend is given by the curved trend at the far right of the data (power law model). [Case: Howell Well, E. TX (USA)] 


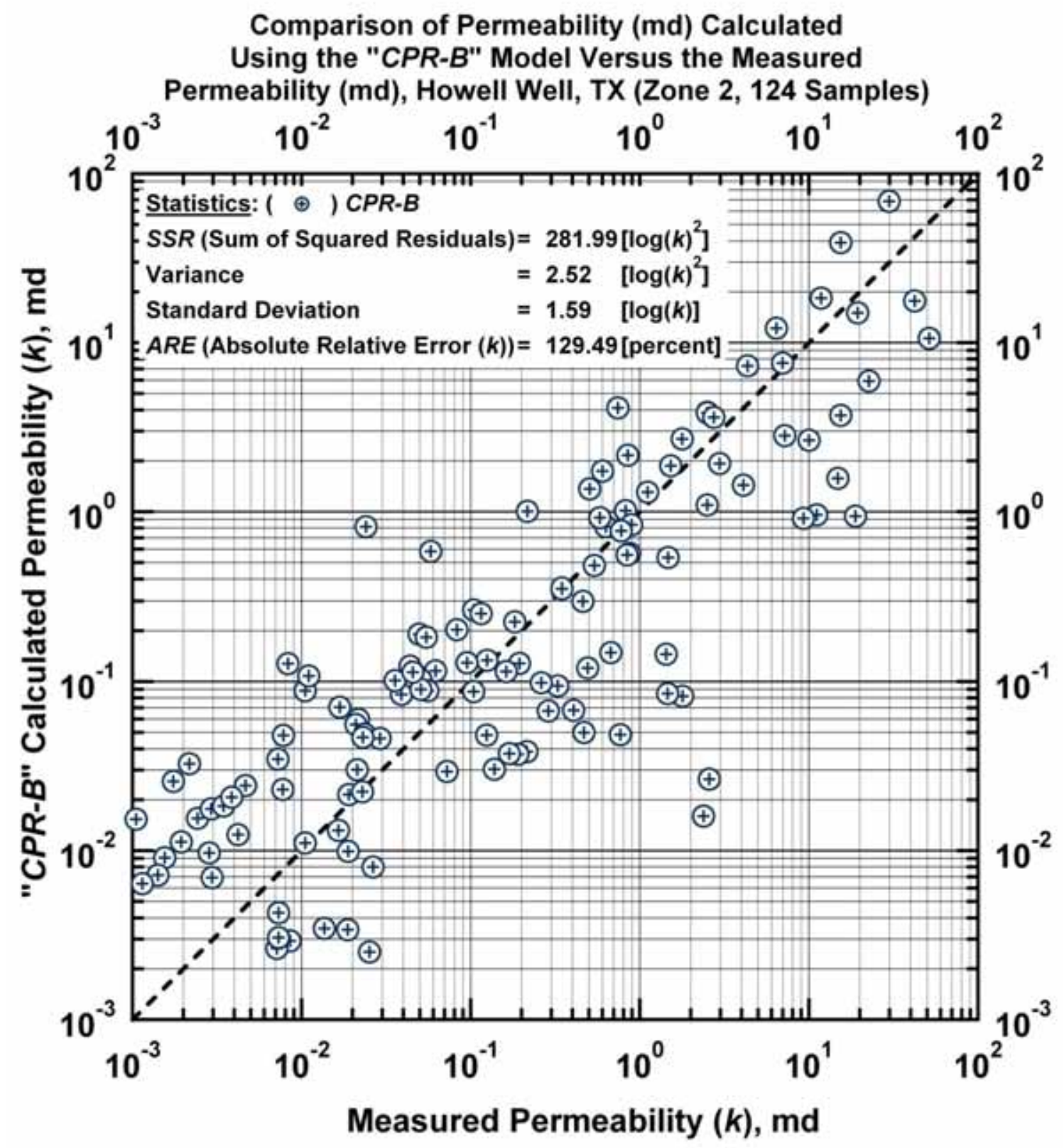

Figure B.30a - Model CPR-B: calculated versus measured permeability. [Case: Howell Well, E. TX (USA)] 


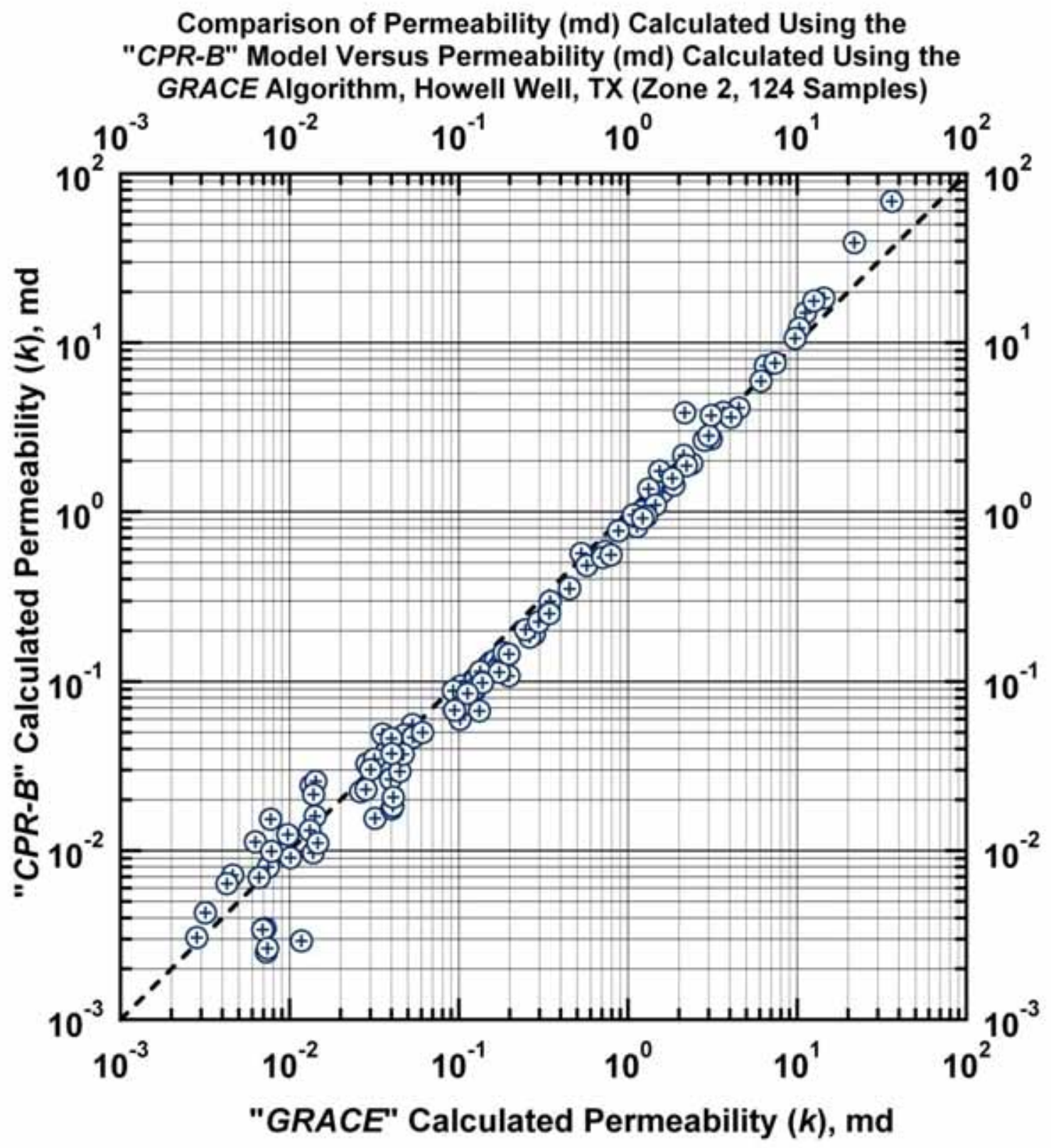

Figure B. 30b - Model CPR-B: Permeability calculated using Model $C P R-B$ versus permeability calculated using the GRACE Algorithm. [Case: Howell Well, E. TX (USA)] 
Comparison of the $c$-Coefficient Calculated Using the "CPR-B" Model Versus the Measured $c$-Coefficient, Howell Well, TX (Zone 2, 124 Samples)

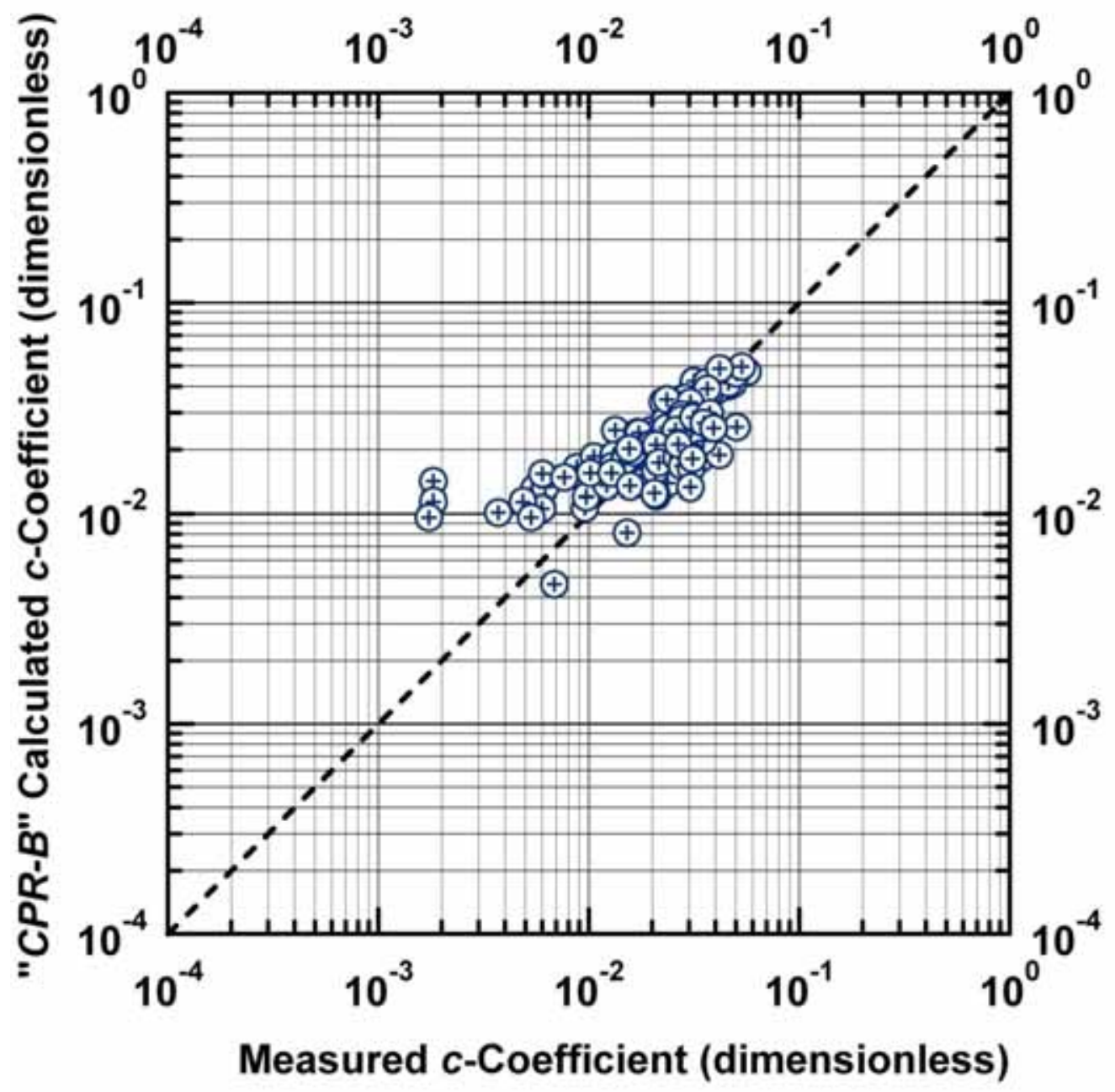

Figure B. 30c - Model $C P R$ - $B$ : Calculated $c$-function values versus measured $c$-function values. [Case: Howell Well, E. TX (USA)] 


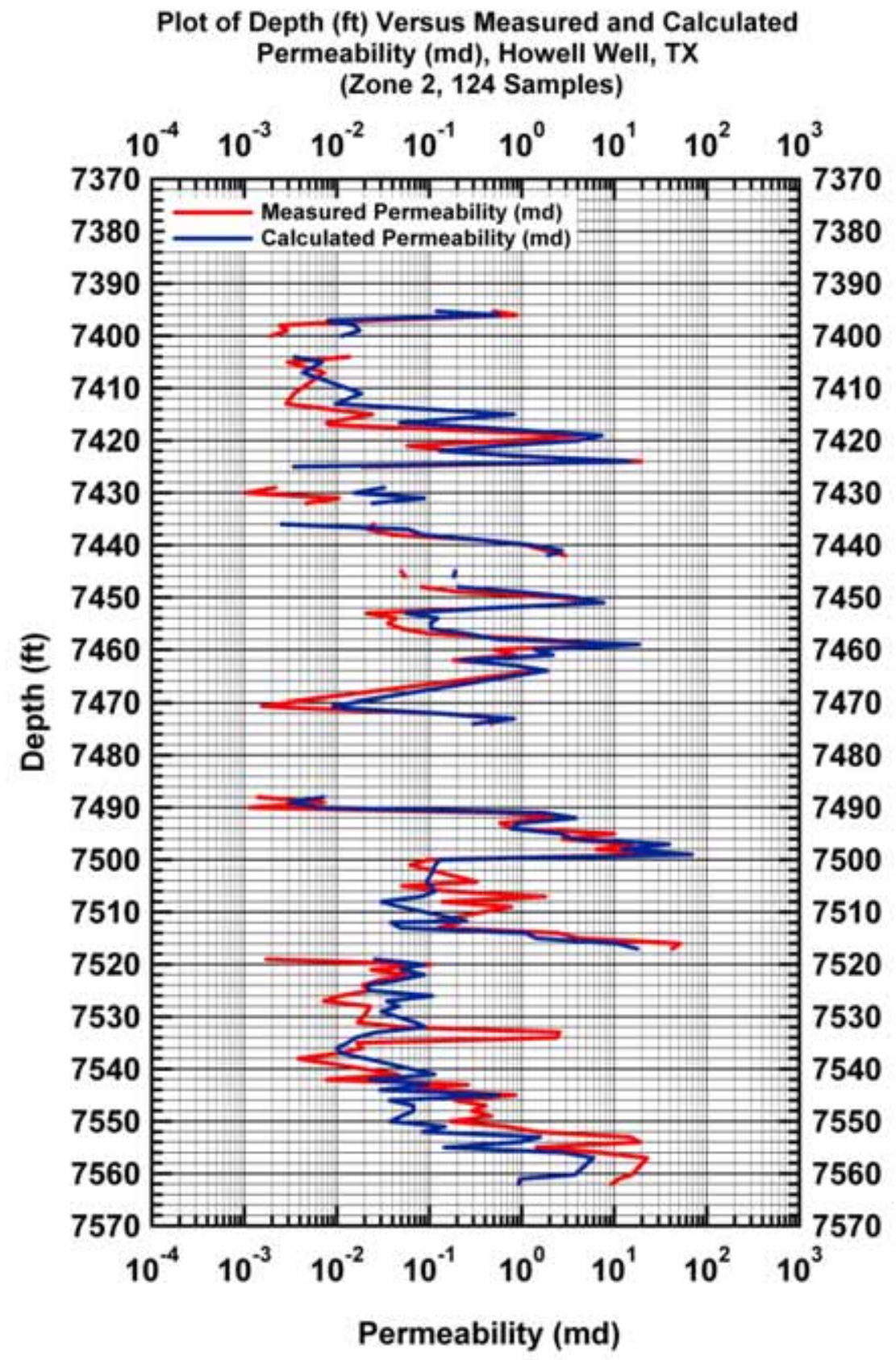

Figure B. 30d - Model CPR-B: Calculated and measured permeability values versus depth. [Case: Howell Well, E. TX (USA)] 


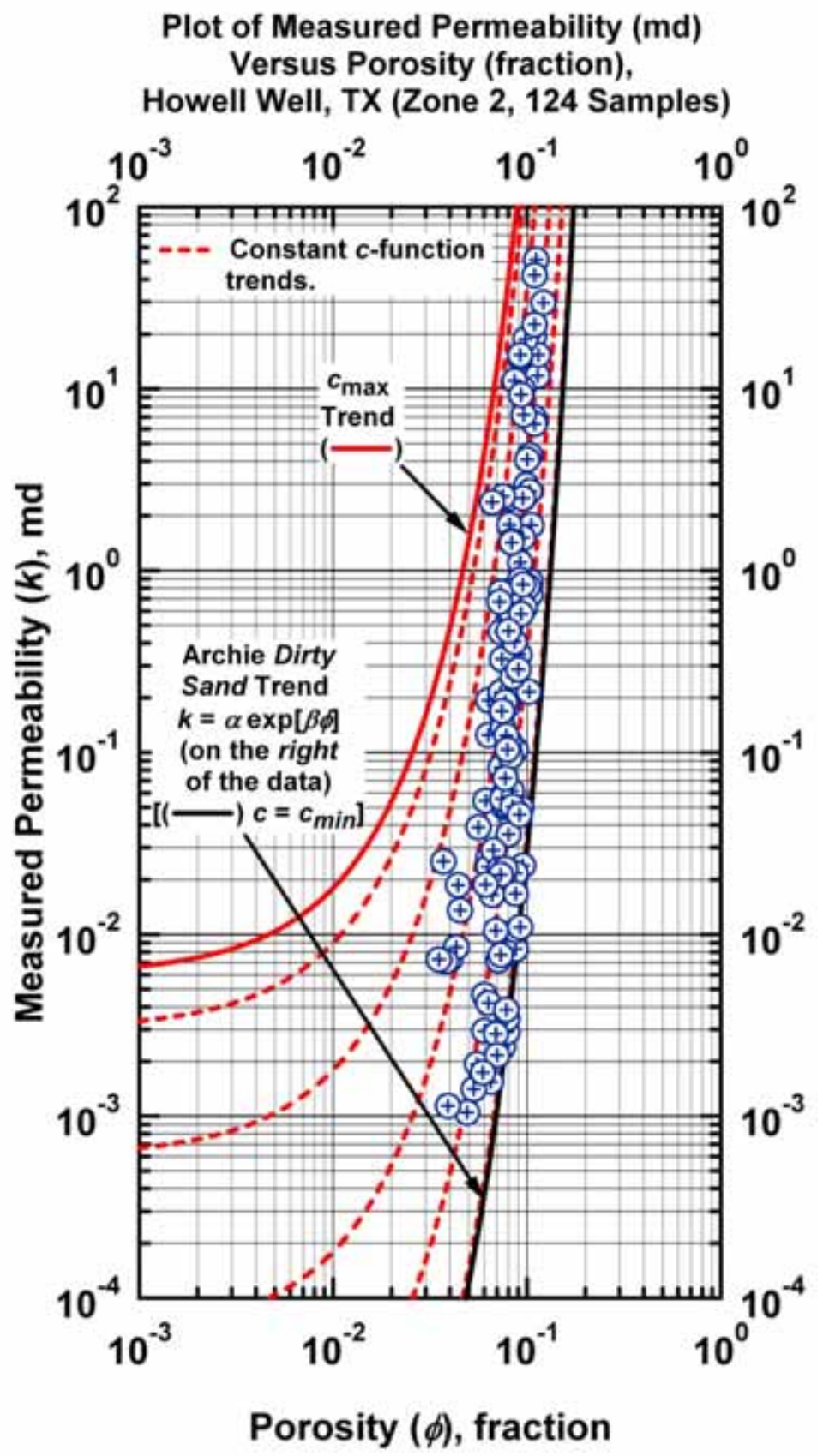

Figure B.31a - Model $C P R-C: \quad k=c \exp [\beta \phi] \quad c=c_{\max } \exp \left[-c_{1} \phi^{c_{2}} S_{w}^{c_{3}}\right]$ - "Clean Sand" Plot (log-log format) - Archie "Dirty Sand" trend is given by the curved trend at the far right of the data (exponential model). [Case: Howell Well, E. TX (USA)] 


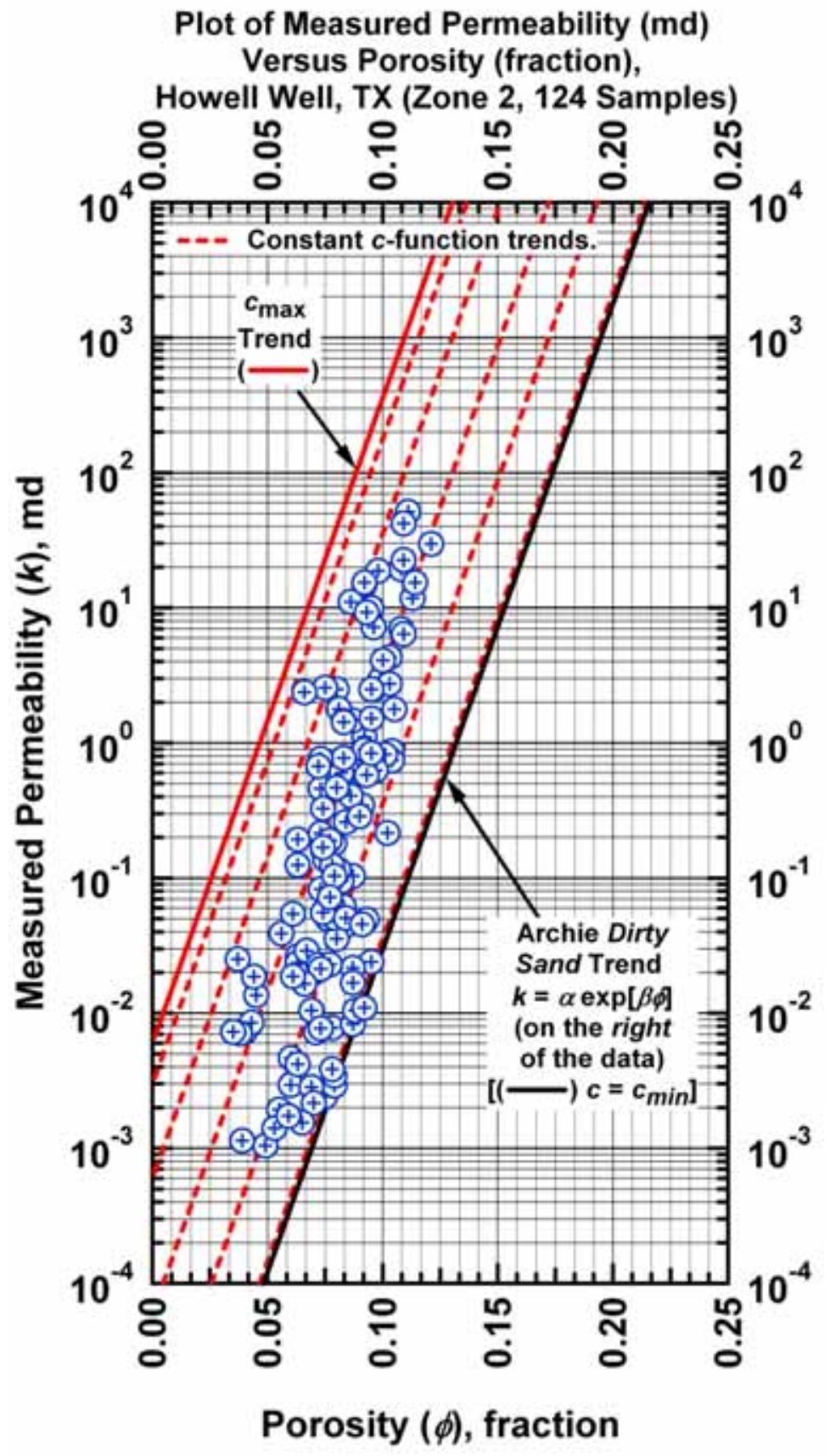

Figure B.31b - Model $C P R-C: k=c \exp [\beta \phi] \quad c=c_{\max } \exp \left[-c_{1} \phi^{c_{2}} S_{w}^{C_{3}}\right]$ - "Dirty Sand" Plot (semilog format) - Archie "Dirty Sand" trend is given by the straight-line trend at the far right of the data (exponential model). [Case: Howell Well, E. TX (USA)] 


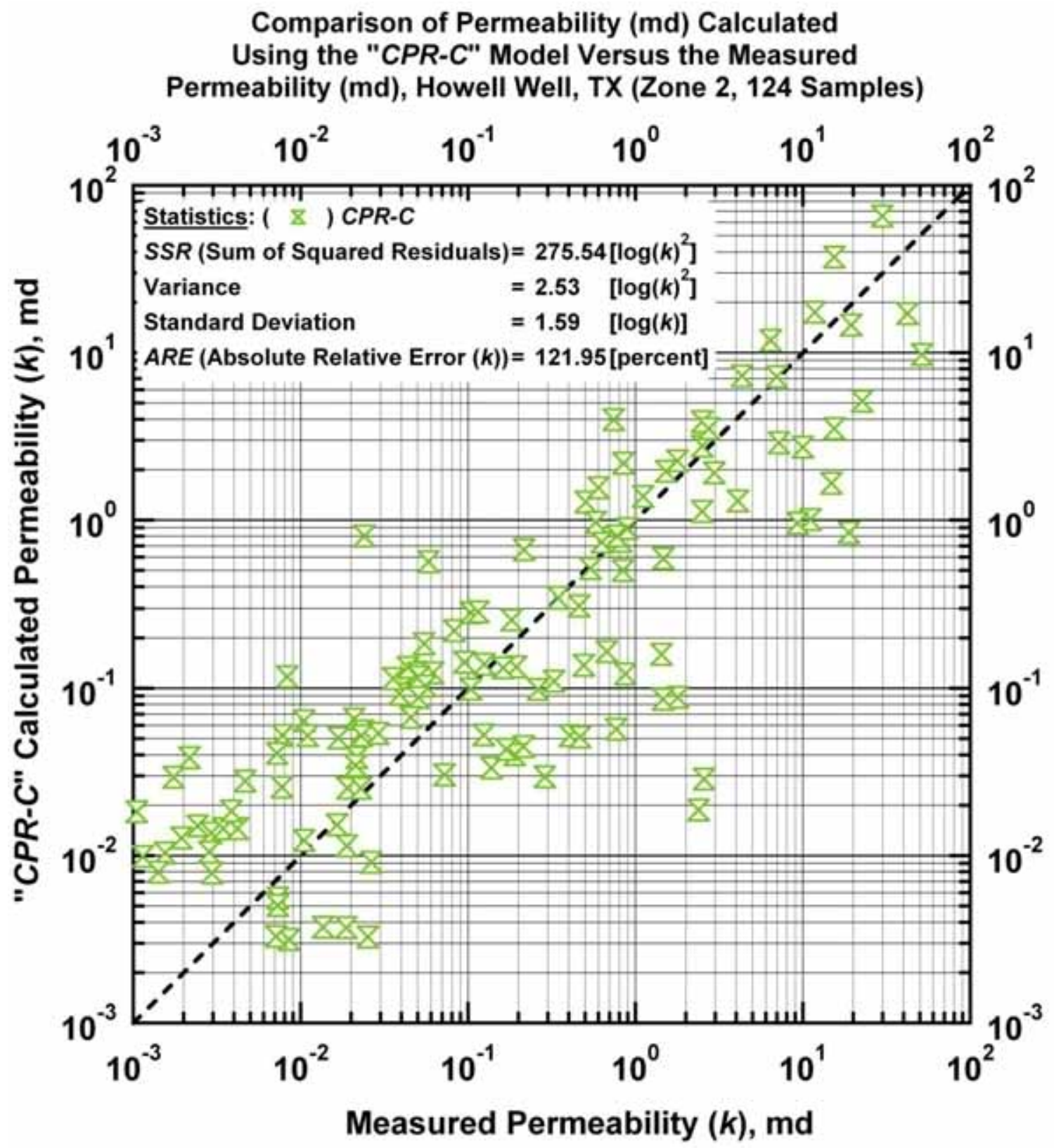

Figure B.32a - Model CPR-C: calculated versus measured permeability. [Case: Howell Well, E. TX (USA)] 


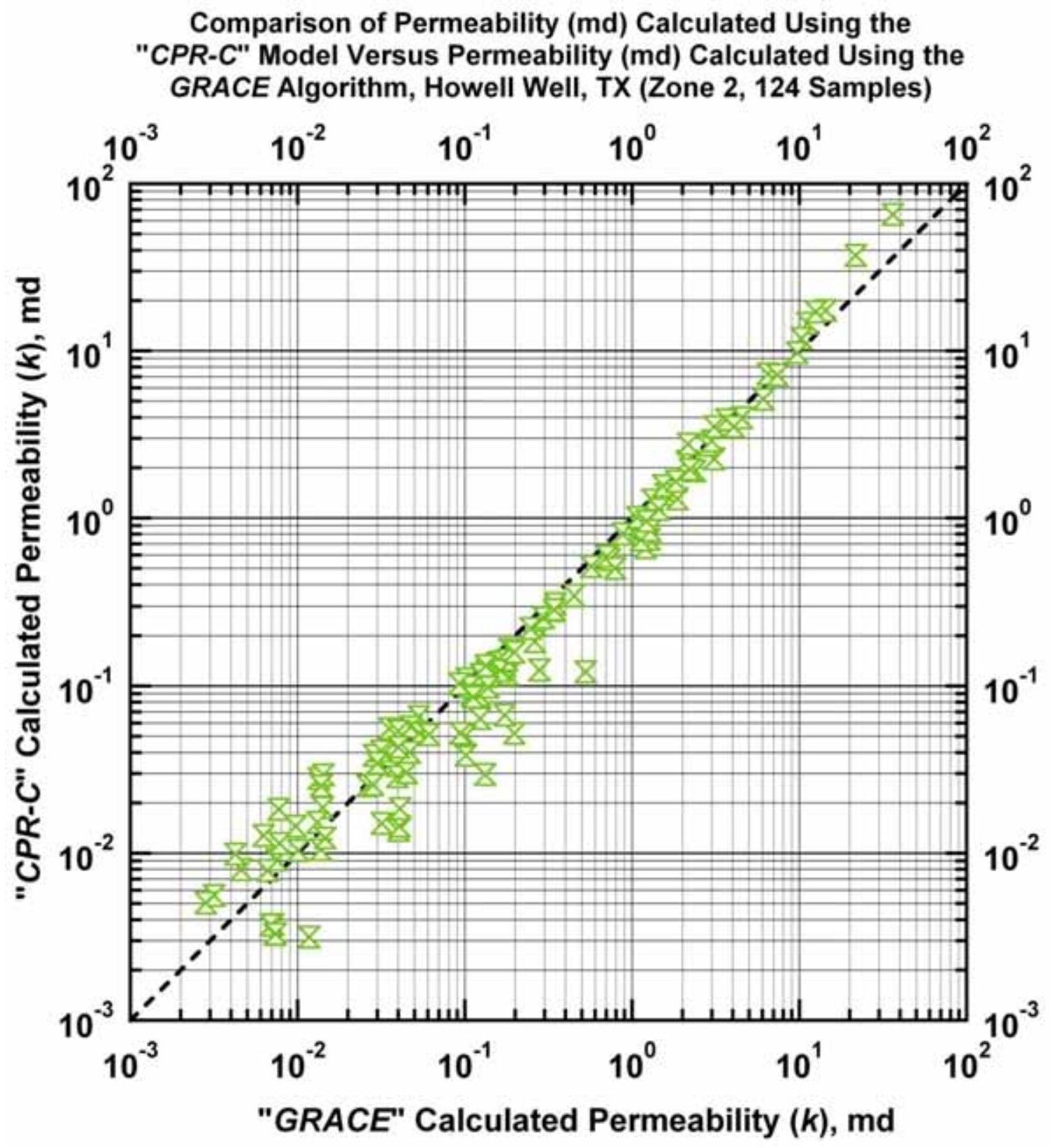

Figure B. 32b - Model $C P R-C$ : Permeability calculated using Model $C P R-C$ versus permeability calculated using the GRACE Algorithm. [Case: Howell Well, E. TX (USA)] 


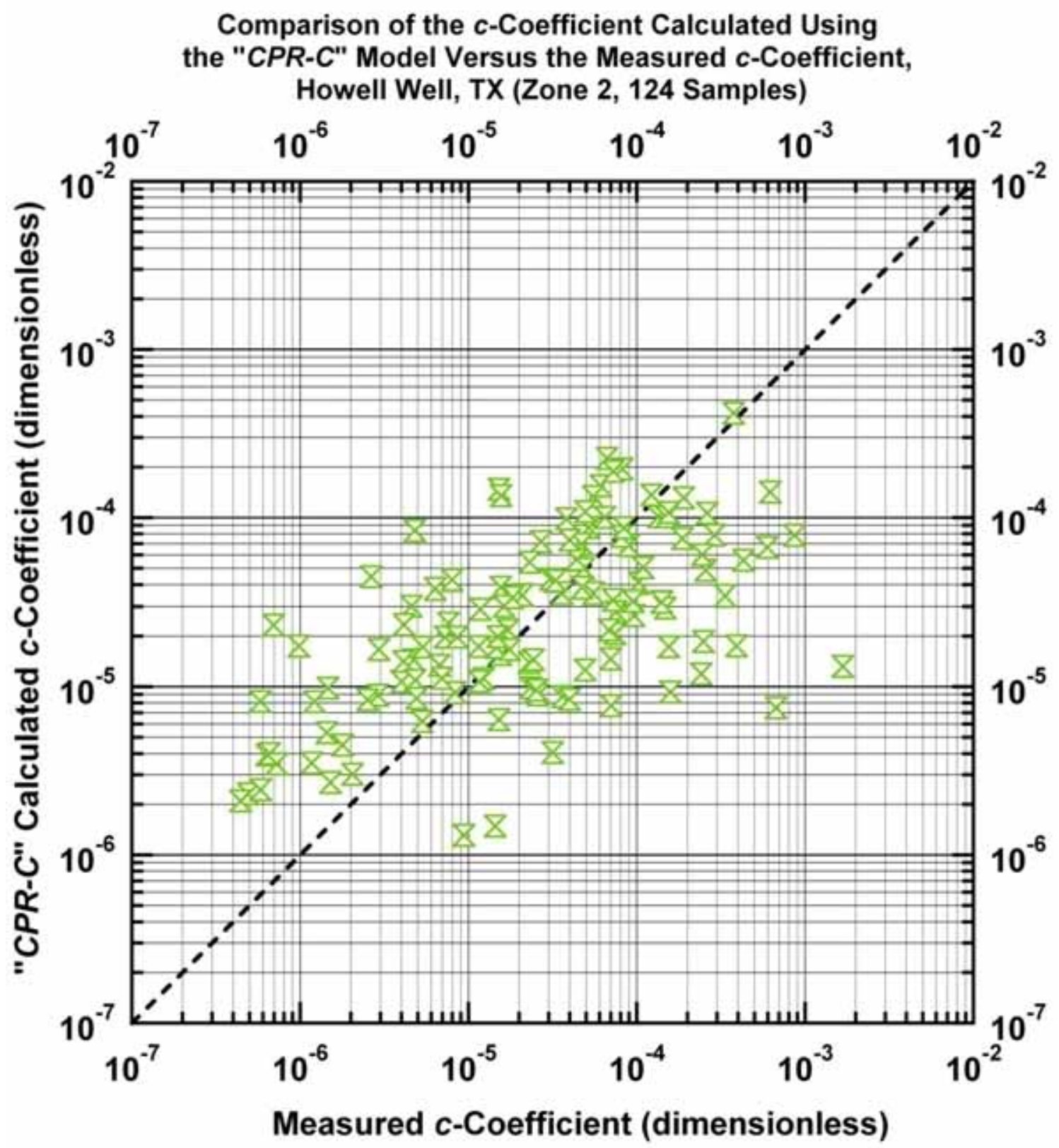

Figure B. 32c - Model $C P R$-C: Calculated $c$-function values versus measured $c$-function values. [Case: Howell Well, E. TX (USA)] 
Plot of Depth (ft) Versus Measured and Calculated Permeability (md), Howell Well, TX

(Zone 2, 124 Samples)

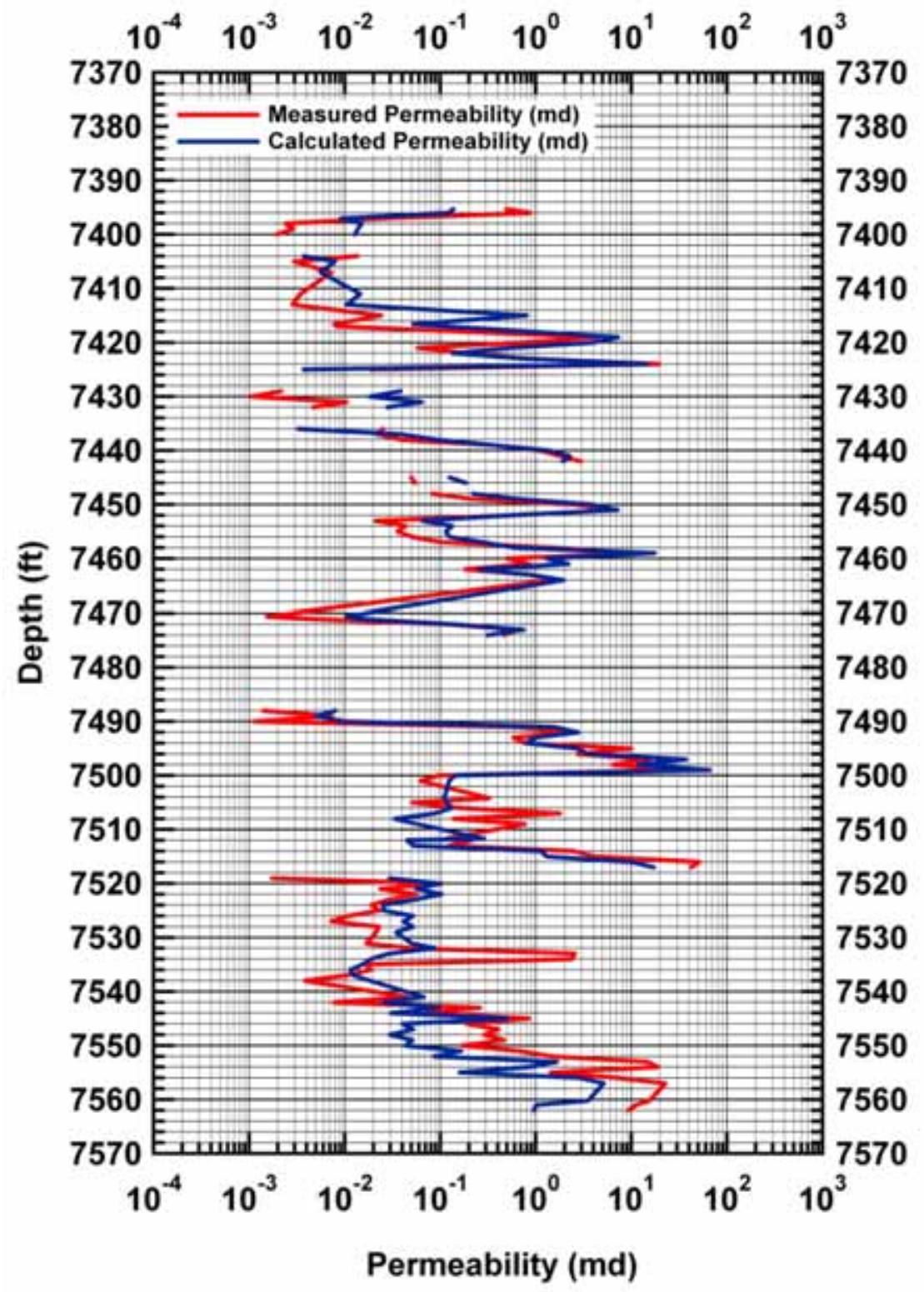

Figure B. 32d - Model CPR-C: Calculated and measured permeability values versus depth. [Case: Howell Well, E. TX (USA)] 


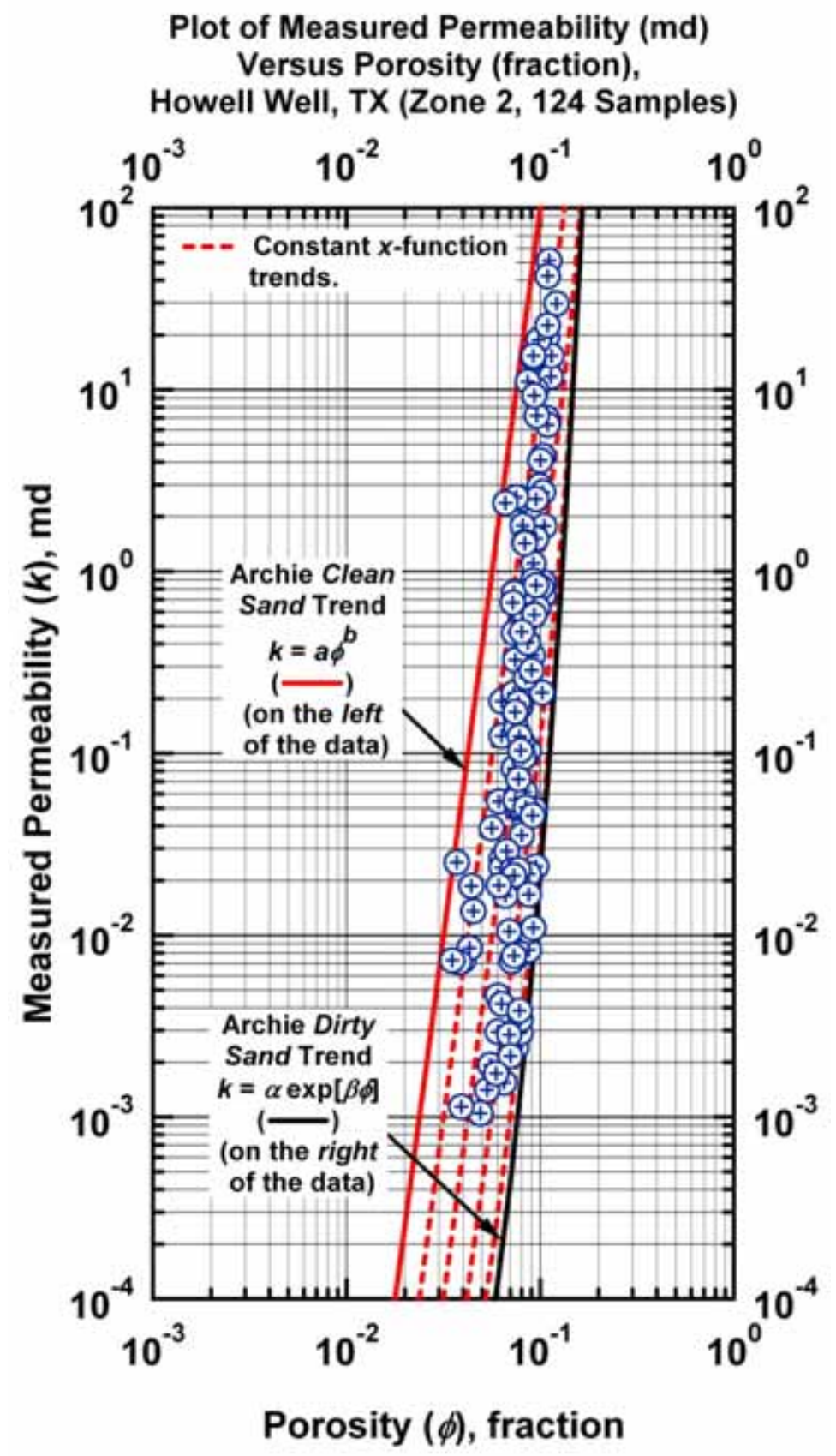

Figure B.33a - Model $C P R-D: k=x a \phi^{b}+(1-x) \alpha \exp [\beta \phi] \quad[0 \leq x \leq 1]$ - Weighted Power Law-Exponential Model used to correlate permeability $(k)$ and porosity $(\phi)$. "Clean Sand" Plot - Archie "Clean Sand" trend is given by the straight-line trend at the far left of the data (power law model). [Case: Howell Well, E. TX (USA)] 


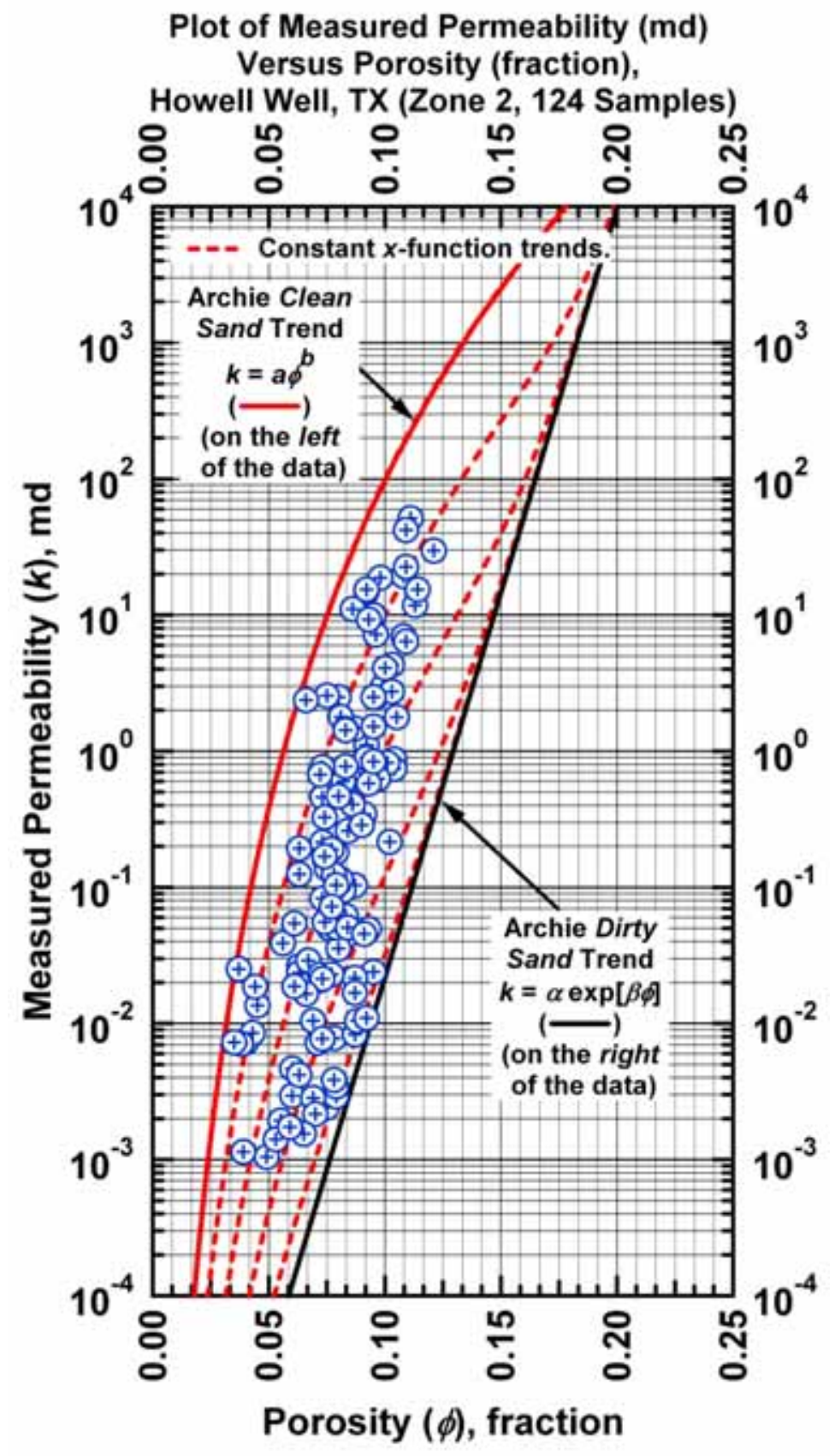

Figure B.33b - Model CPR-D: $k=x a \phi^{b}+(1-x) \alpha \exp [\beta \phi] \quad[0 \leq x \leq 1]$ - Weighted Power Law-Exponential Model used to correlate permeability $(k)$ and porosity $(\phi)$. "Dirty Sand" Plot - Archie "Dirty Sand" trend is given by the straight-line trend at the far right of the data (exponential model). [Case: Howell Well, E. TX (USA)] 


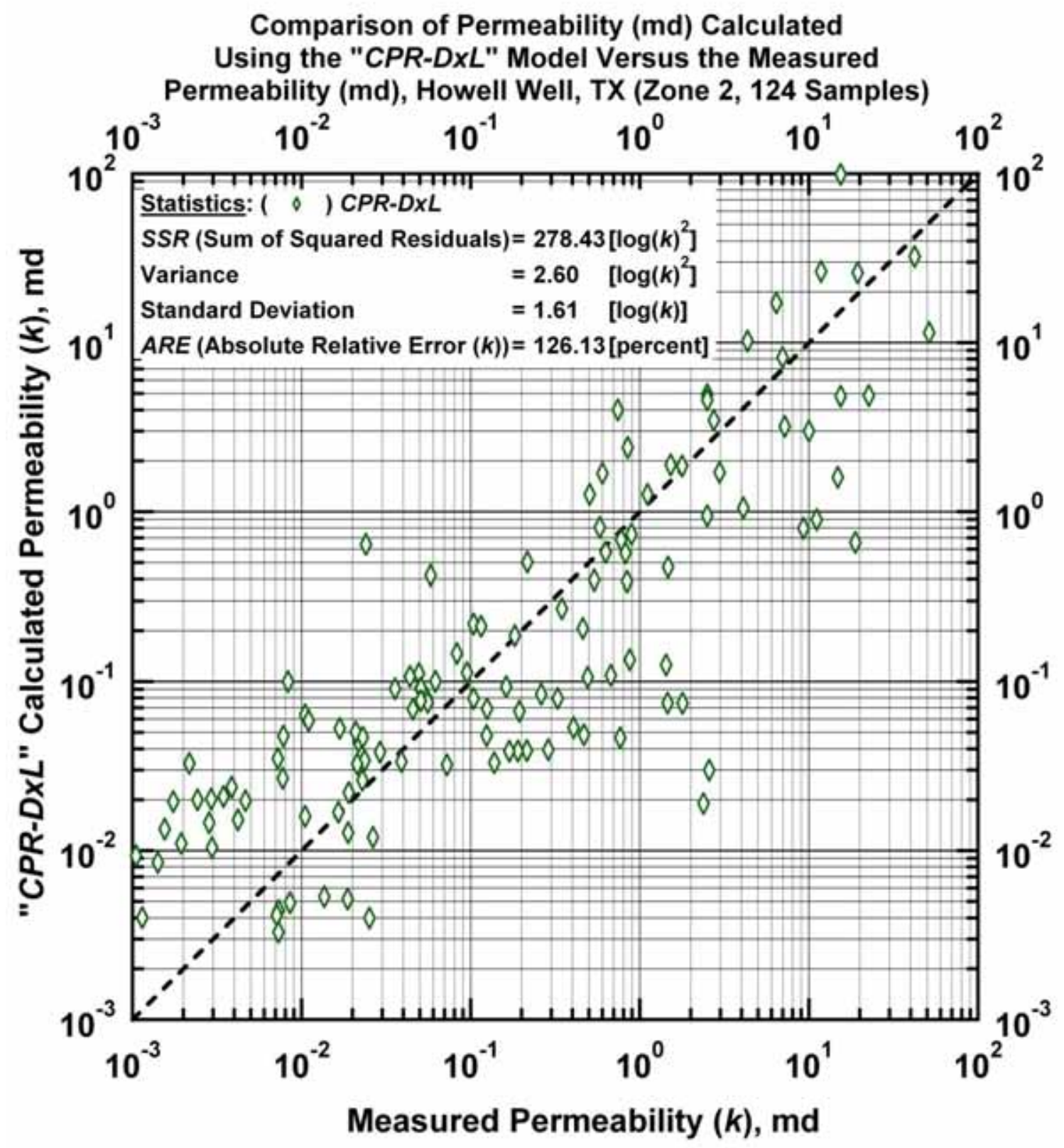

Figure B.34a - Model CPR-DxL: Calculated versus measured permeability. [Case: Howell Well, E. TX (USA)] 


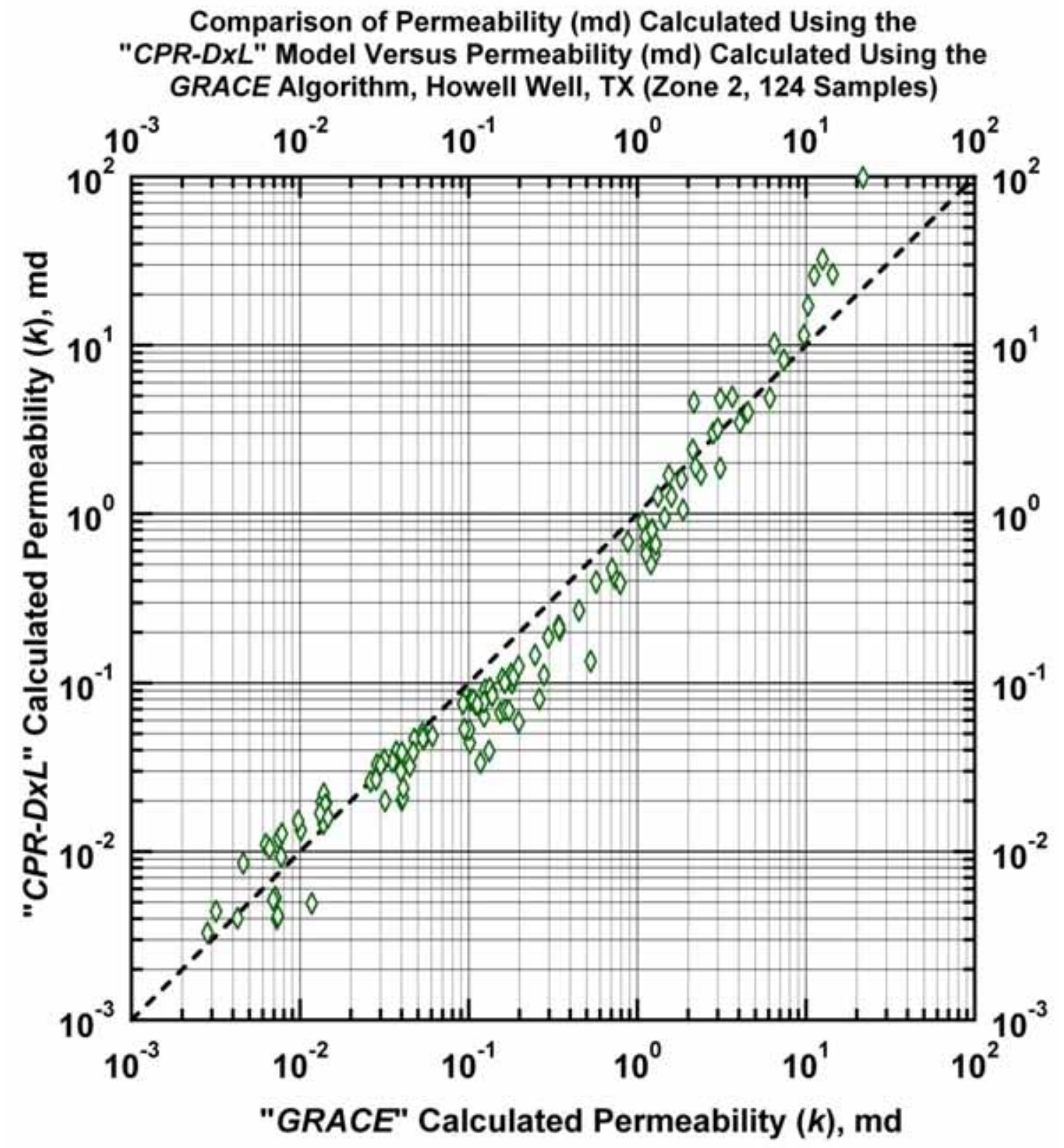

Figure B. 34b - Model $C P R-D x L$ : Permeability calculated using Model $C P R-C$ versus permeability calculated using the GRACE Algorithm. [Case: Howell Well, E. TX (USA)] 
Comparison of the $x$-Coefficient Calculated Using the "CPR-DXL" Model Versus the Measured $x$-Coefficient, Howell Well, TX (Zone 1, 228 Samples)

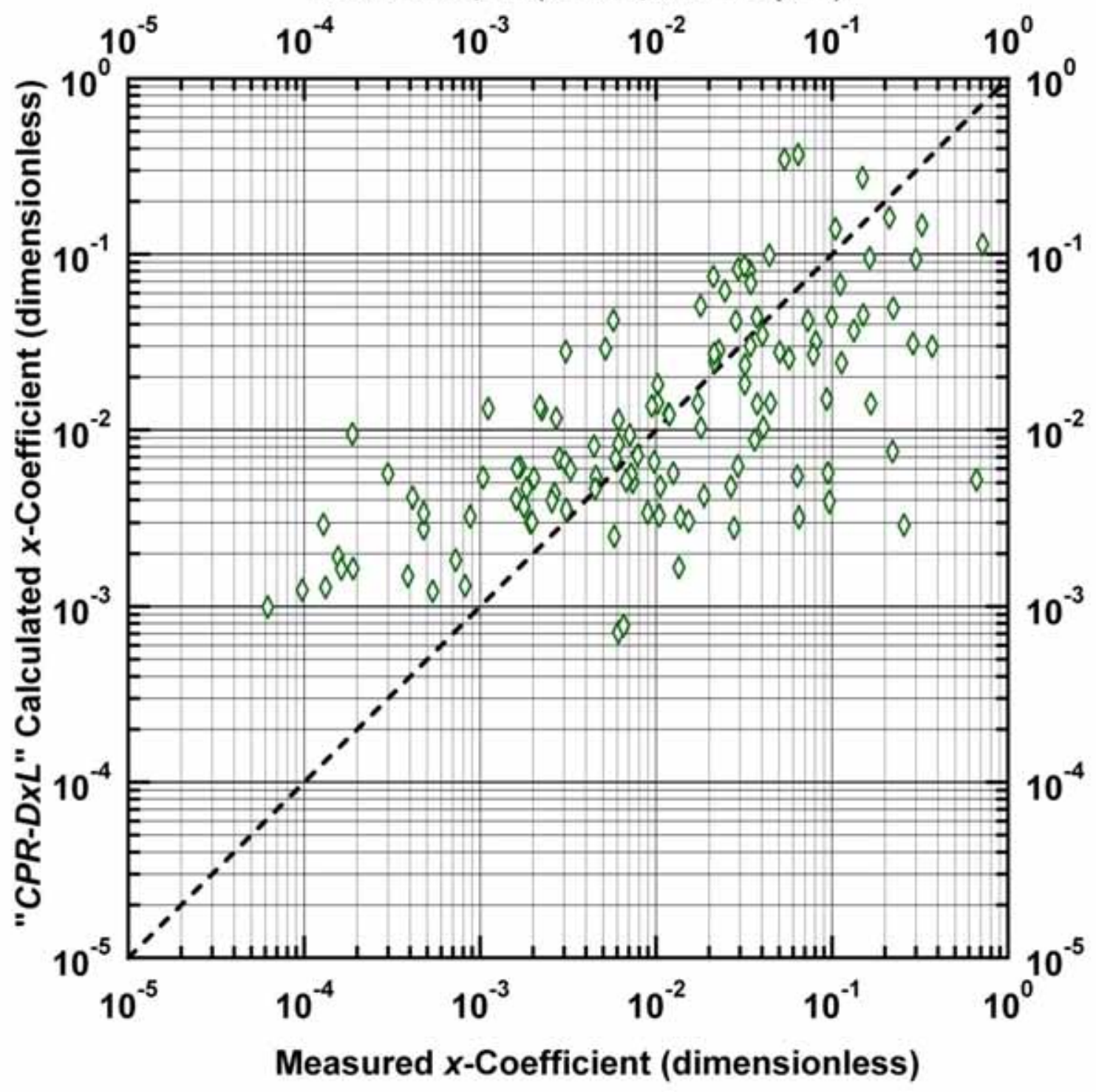

Figure B. 34c - Model CPR-DxL: Calculated $x$-function weight values versus measured $x$ function weight values. [Case: Howell Well, E. TX (USA)] 
Plot of Depth ( $\mathrm{ft}$ ) Versus Measured and Calculated Permeability (md), Howell Well, TX

(Zone 2, 124 Samples)

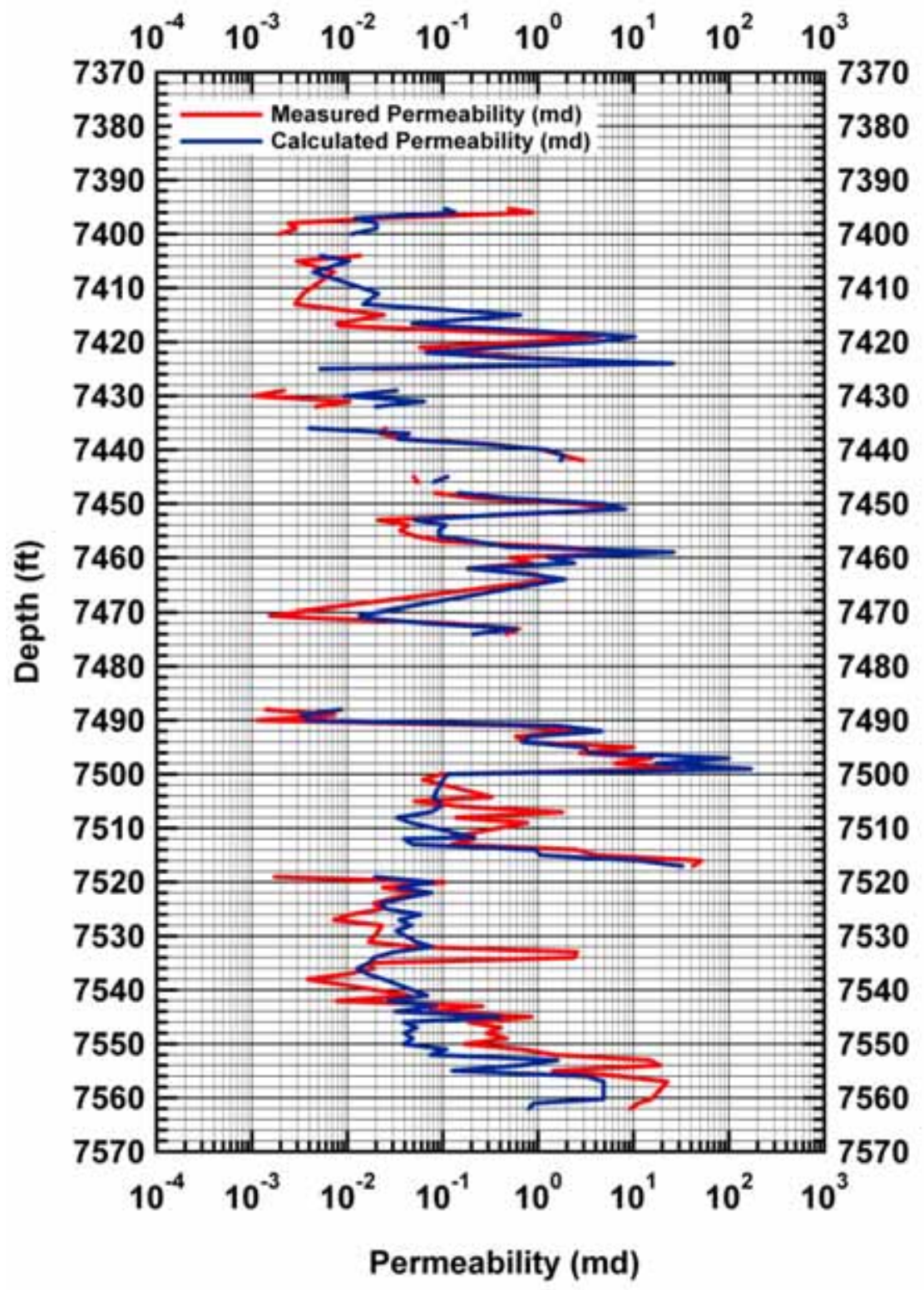

Figure B. 34d - Model CPR-DxL: Calculated and measured permeability values versus depth. [Case: Howell Well, E. TX (USA)] 


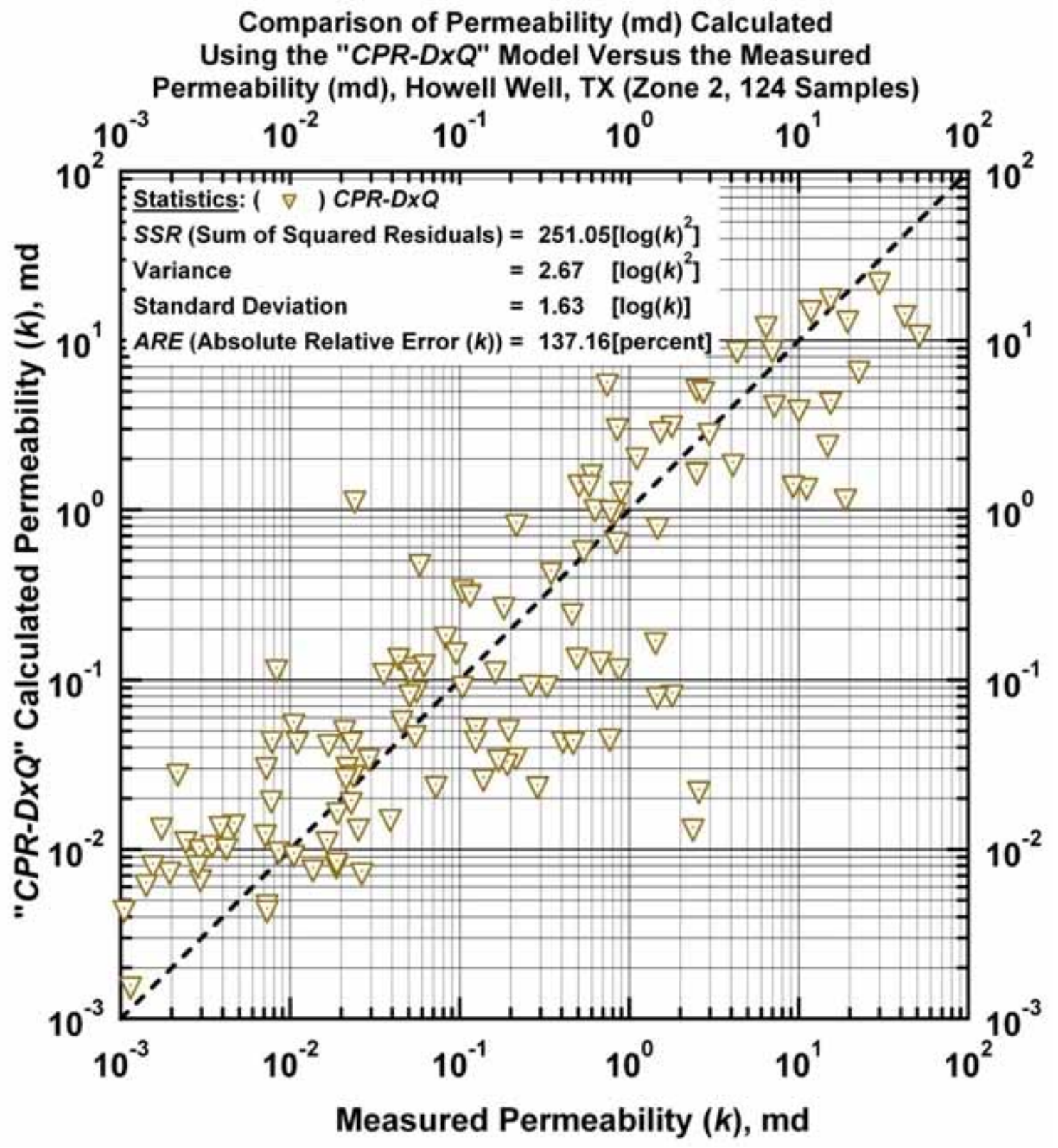

Figure B.35a - Model CPR-DxQ: Calculated versus measured permeability. [Case: Howell Well, E. TX (USA)] 


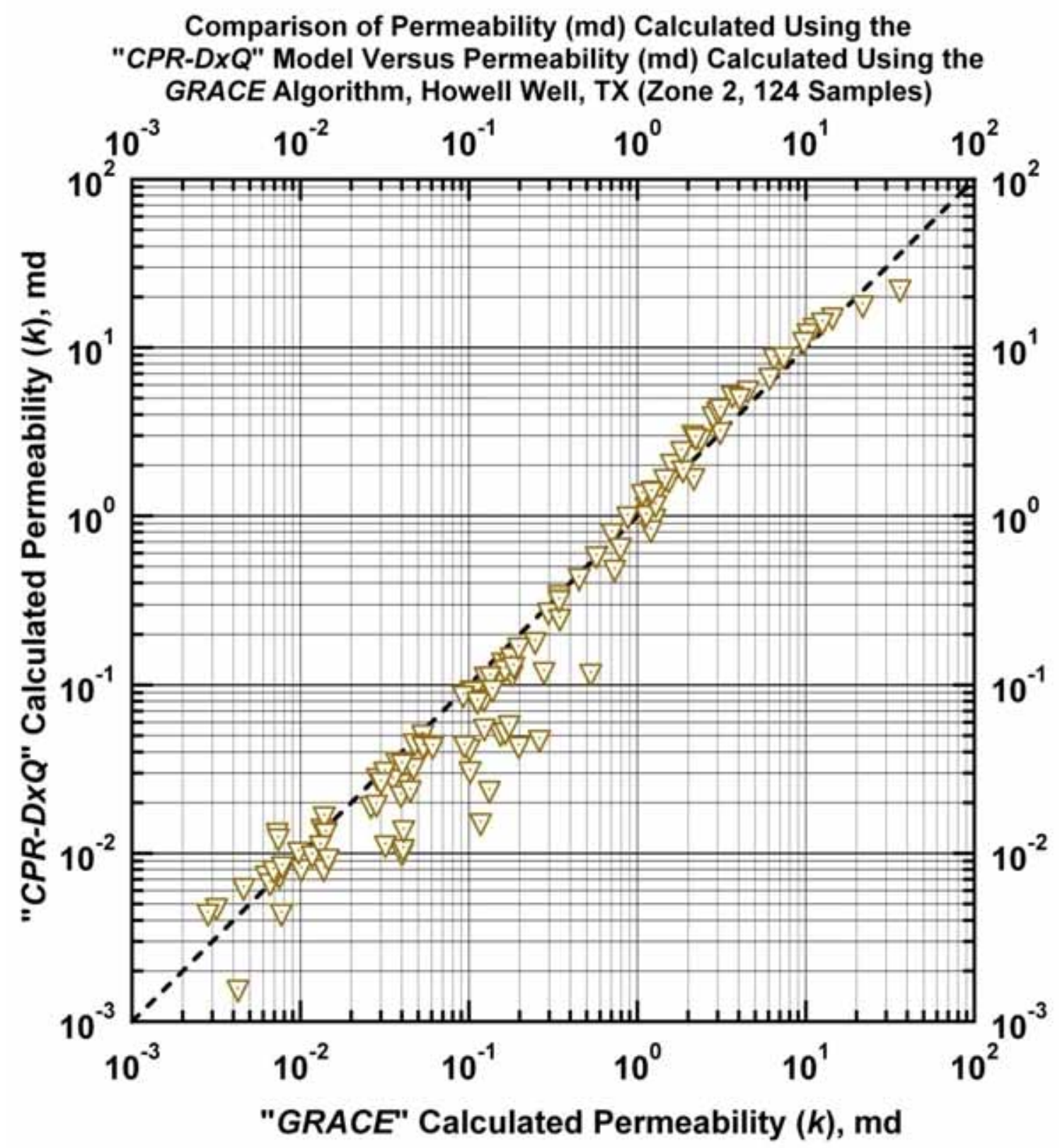

Figure B.35b - Model CPR-DxQ: Permeability calculated using Model $C P R-C$ versus permeability calculated using the GRACE Algorithm. [Case: Howell Well, E. TX (USA)] 


\section{Comparison of the $x$-Coefficient Calculated Using} the "CPR-DxQ" Model Versus the Measured $x$-Coefficient, Howell Well, TX (Zone 2, 124 Samples)

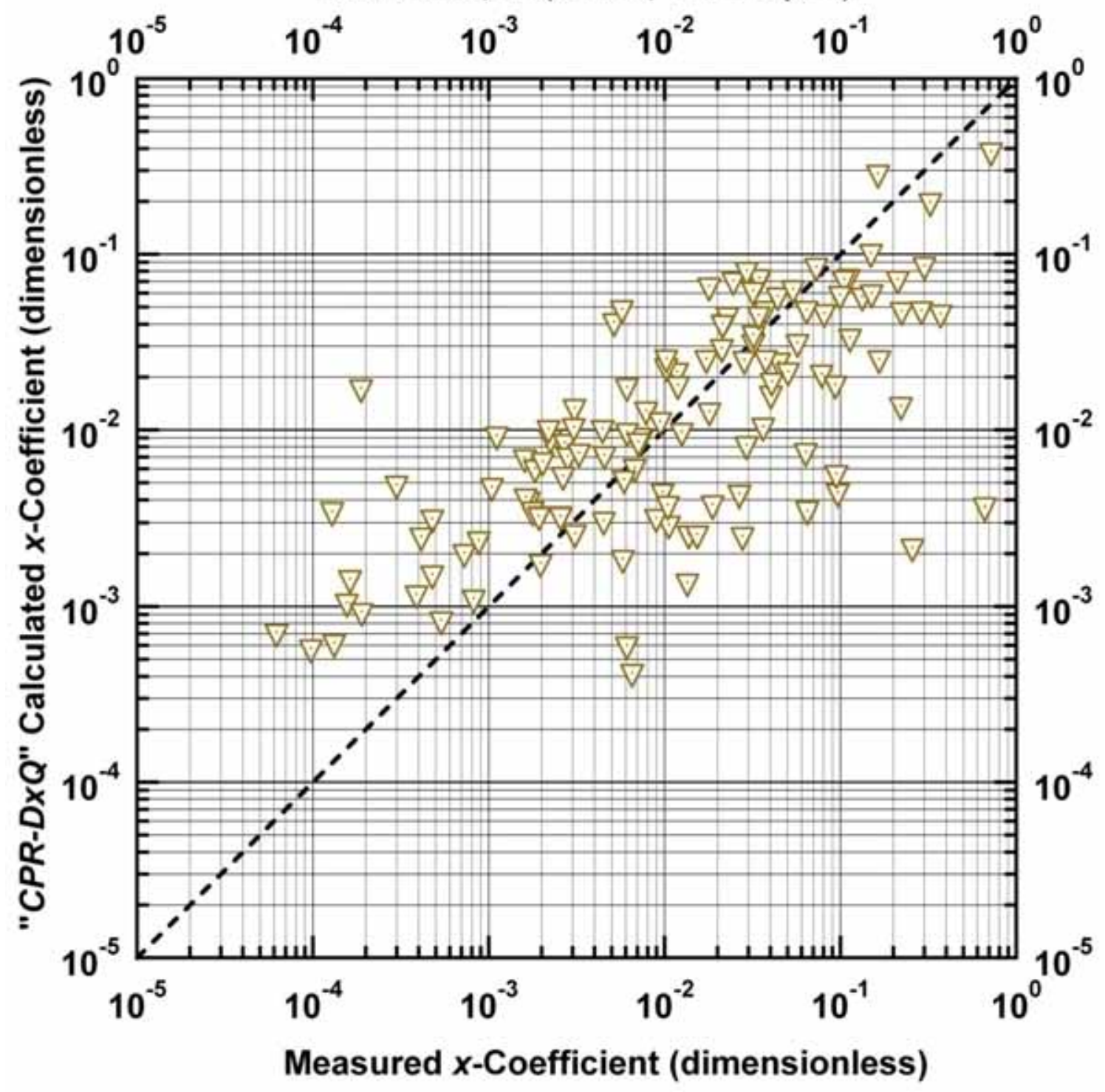

Figure B.35c - Model CPR-DxQ: Calculated $x$-function weight values versus measured $x$ function weight values. [Case: Howell Well, E. TX (USA)] 


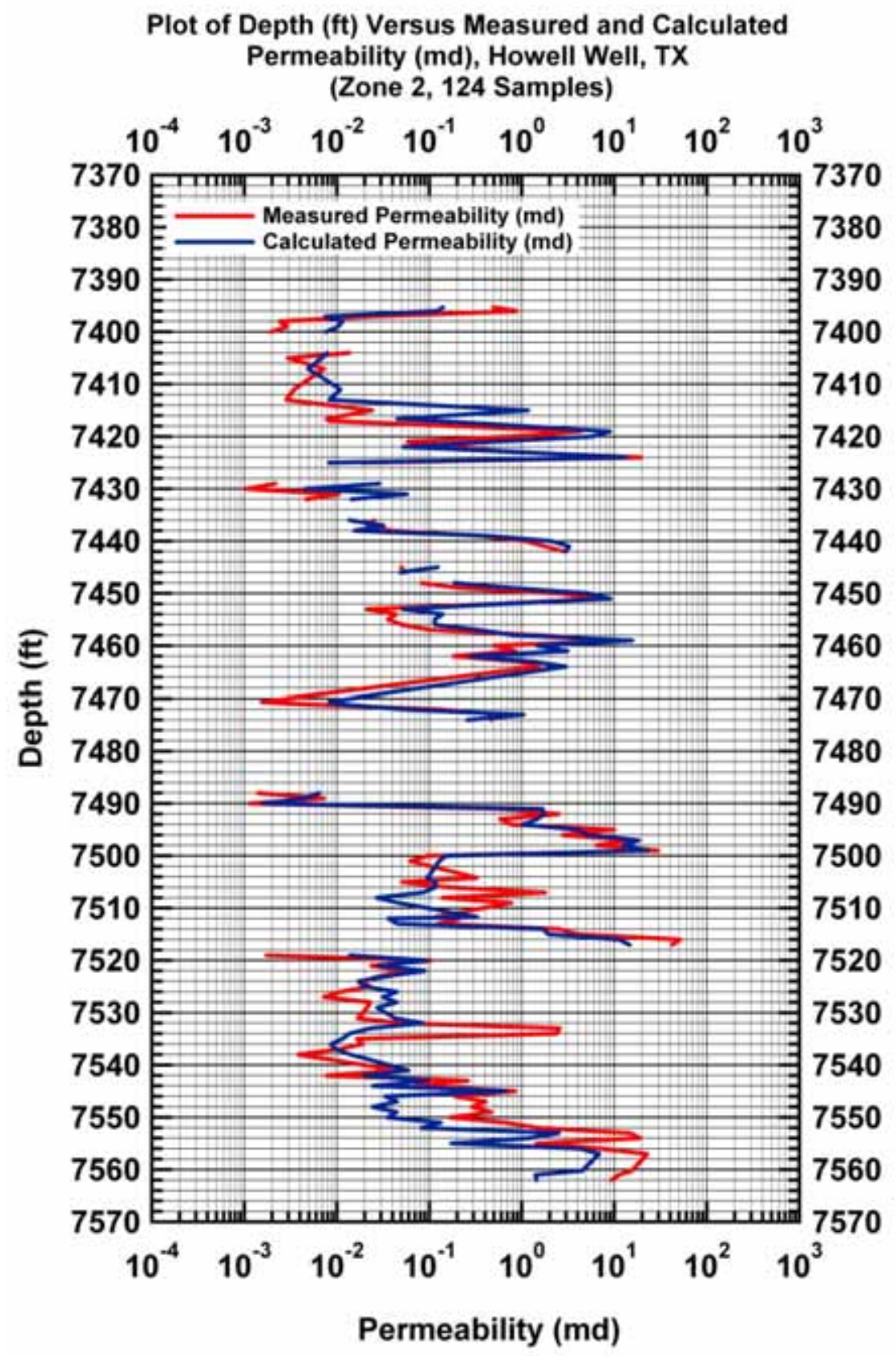

Figure B.35d - Model CPR-DxQ: Calculated and measured permeability values versus depth. [Case: Howell Well, E. TX (USA)] 


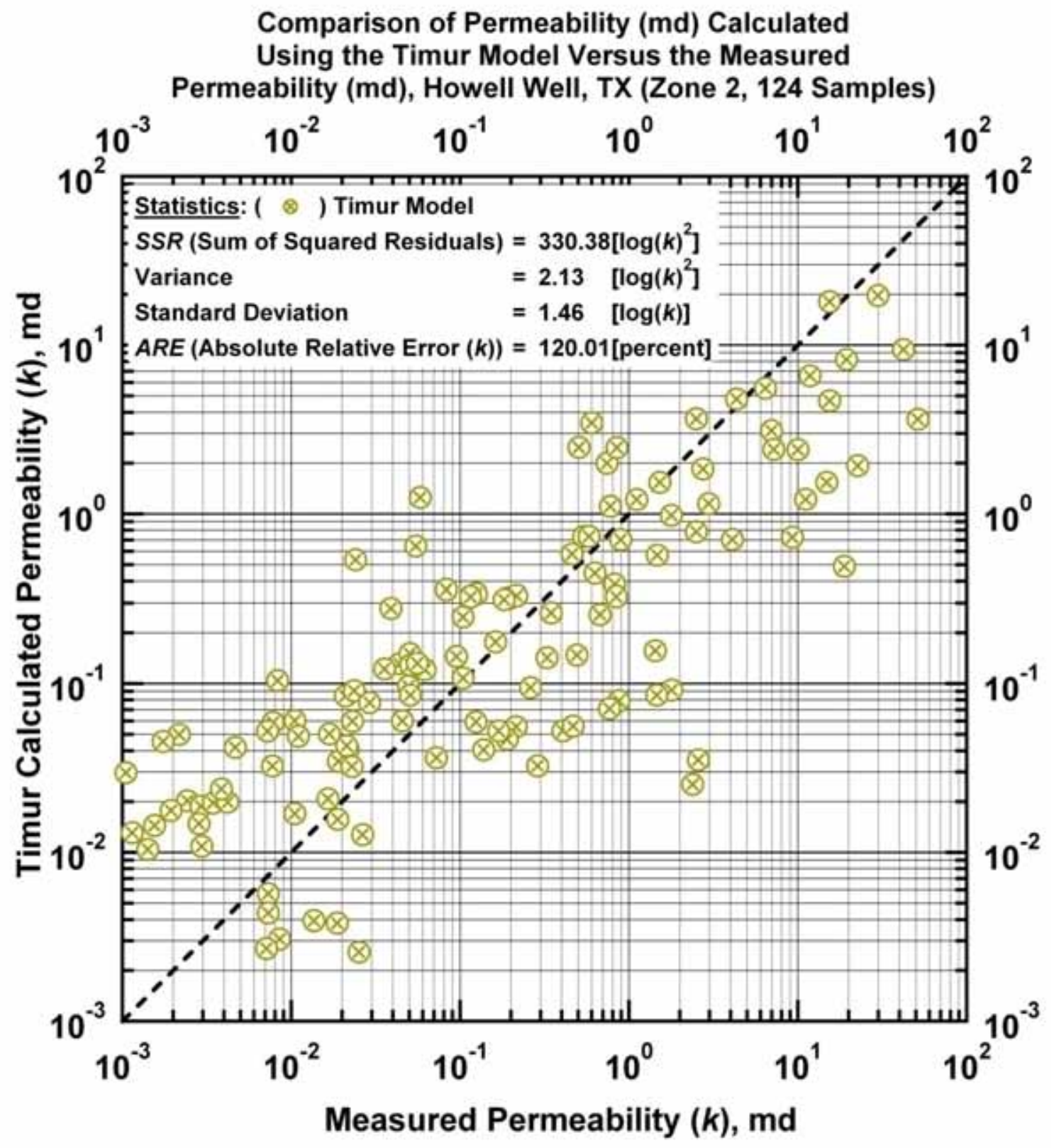

Figure B.36a - "Timur Model:" Calculated versus measured permeability. [Case: Howell Well, E. TX (USA)] 


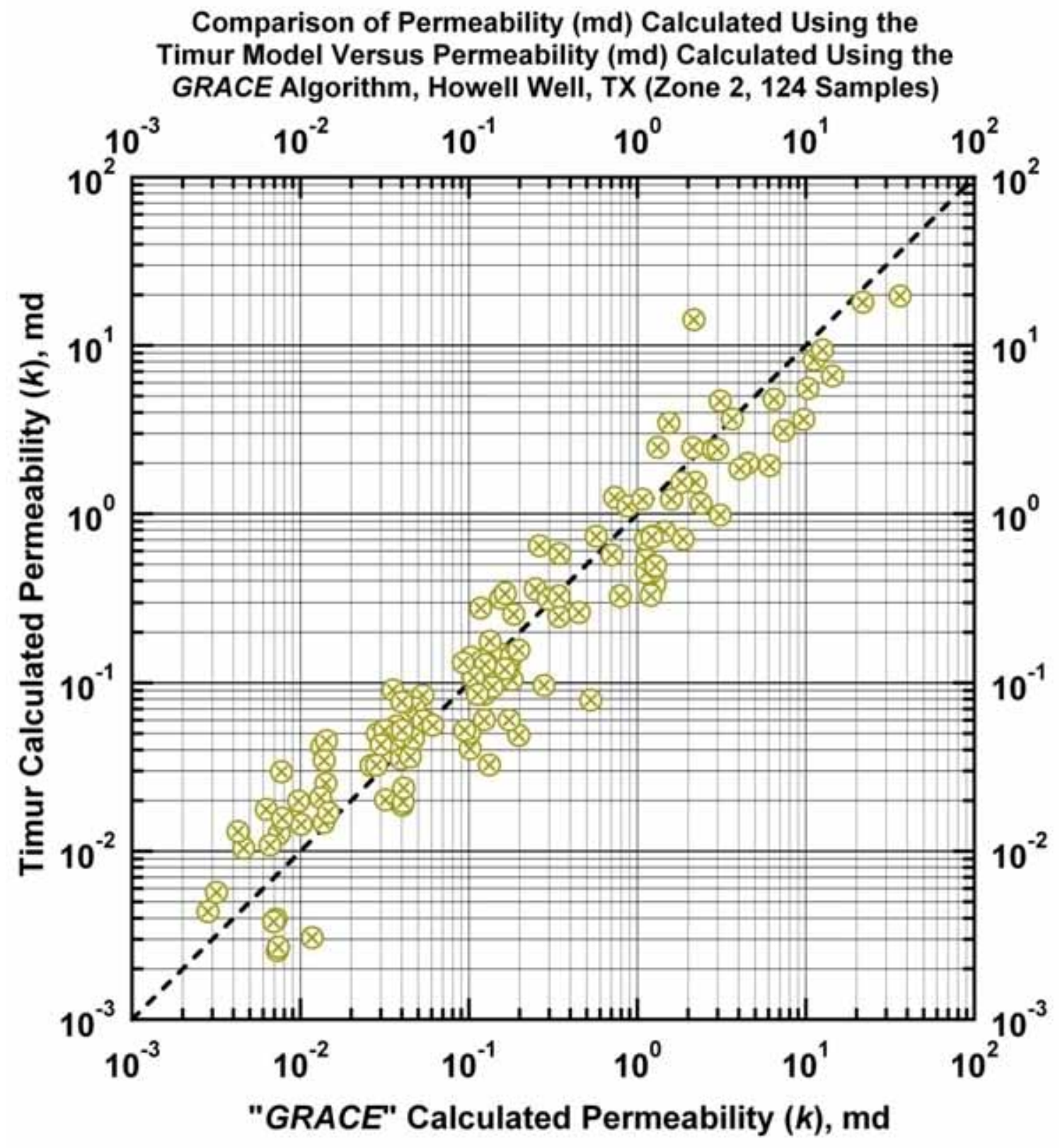

Figure B.36b — "Timur Model:" Permeability calculated using the "Timur Model" versus permeability calculated using the GRACE Algorithm. [Case: Howell Well, E. TX (USA)] 


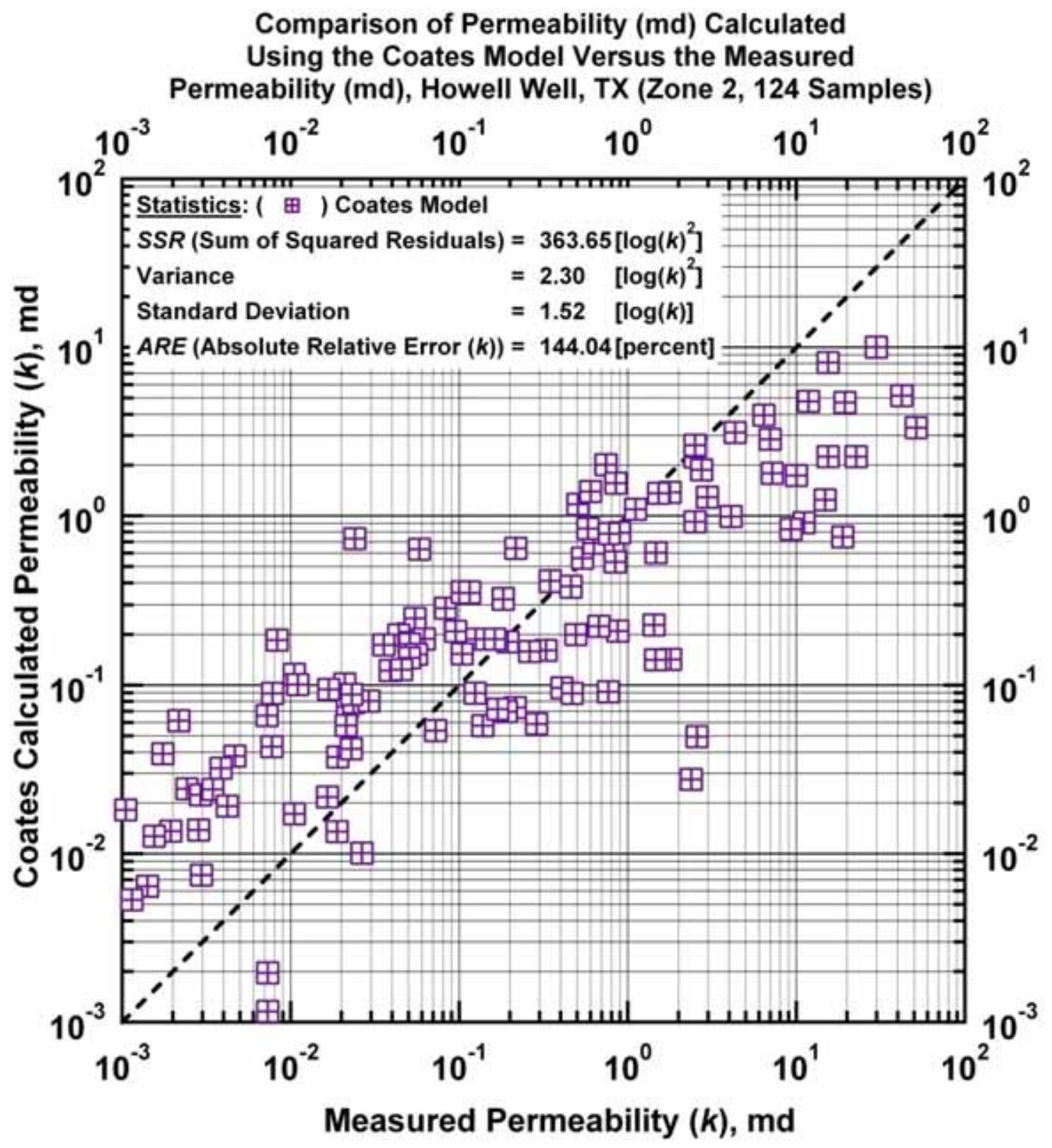

Figure B.37a - "Coates Model:" Calculated versus measured permeability. [Case: Howell Well, E. TX (USA)] 


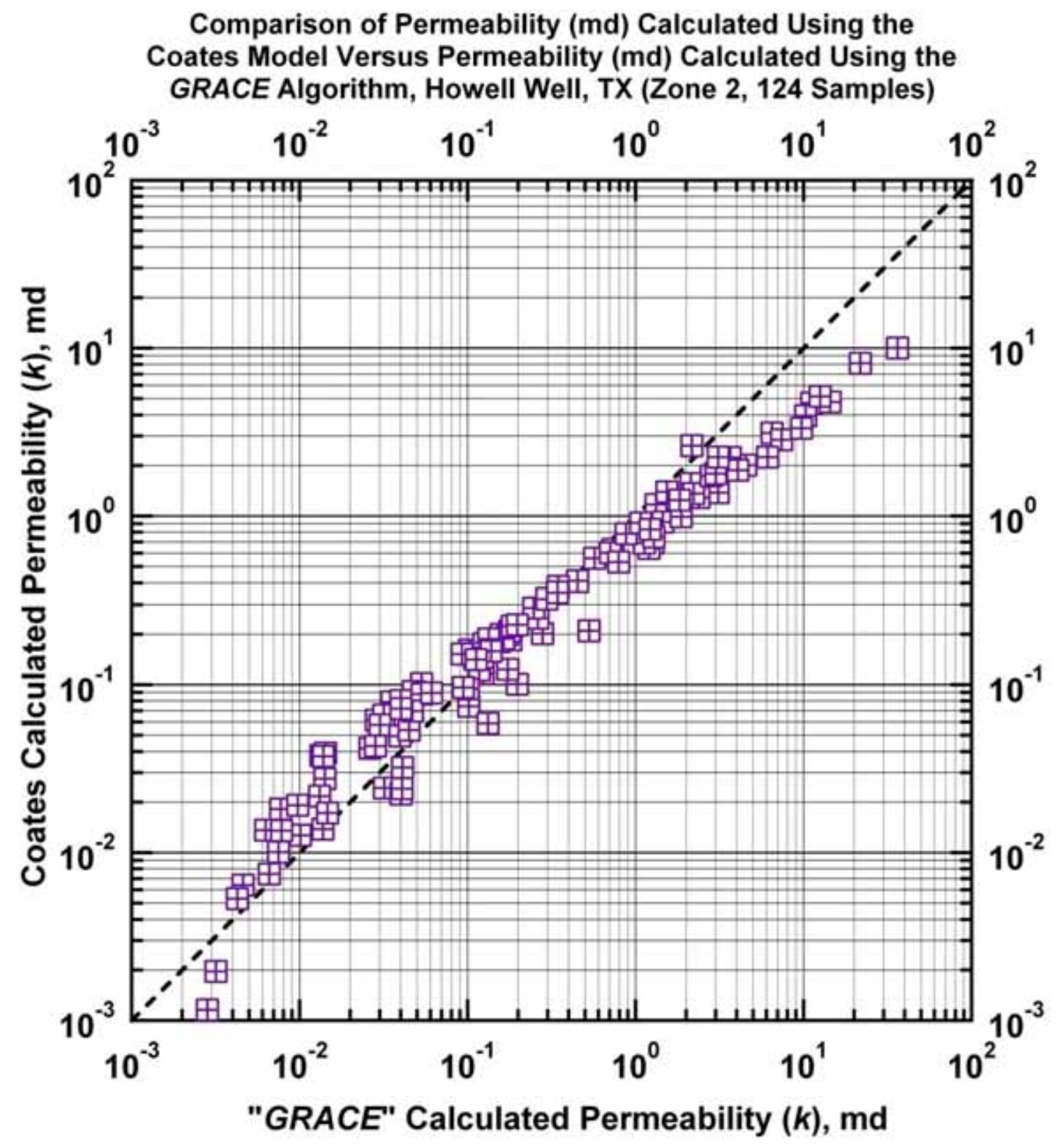

Figure B.37b — "Coates Model:" Permeability calculated using the "Coates Model" versus permeability calculated using the GRACE Algorithm. [Case: Howell Well, E. TX (USA)] 


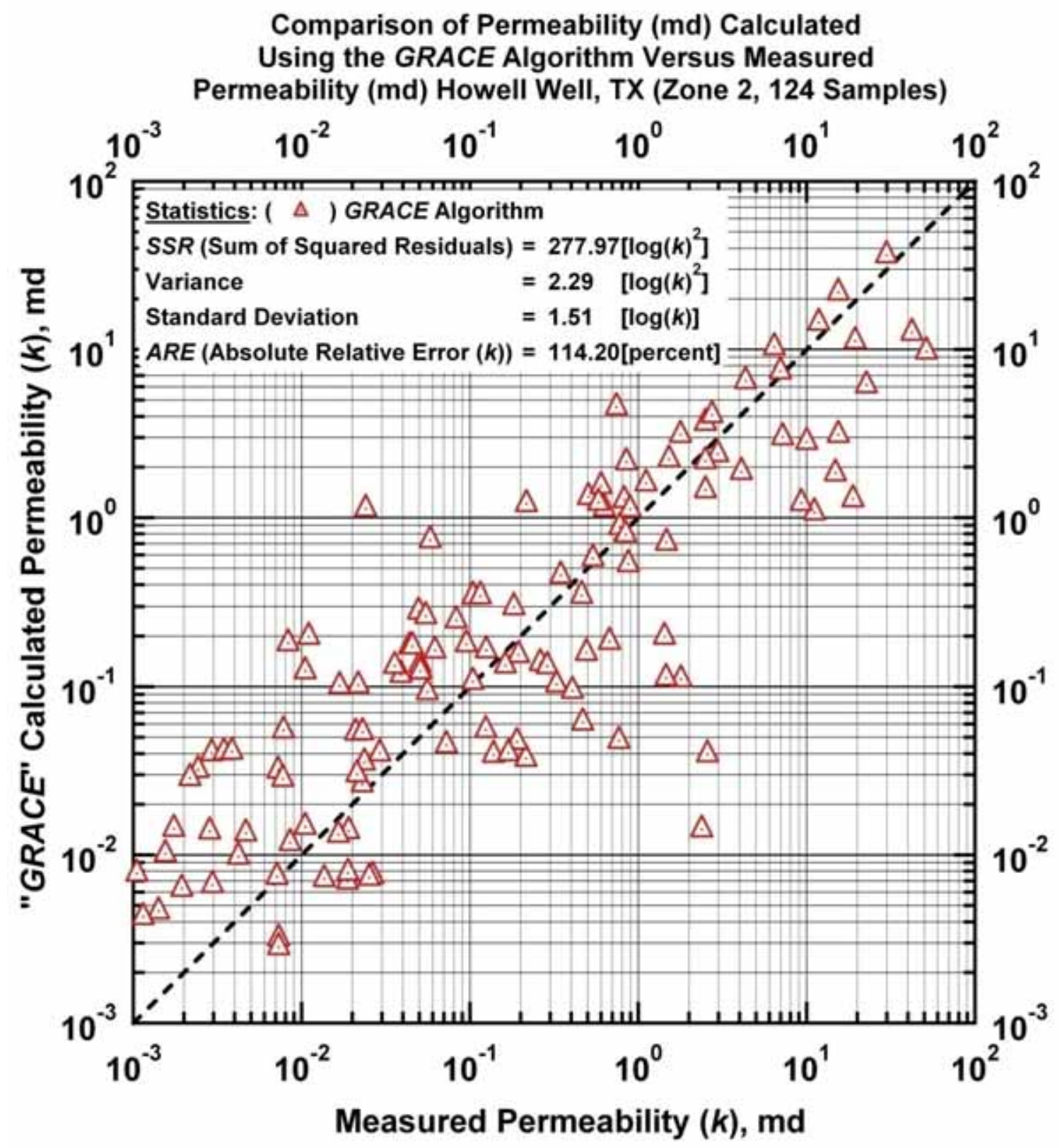

Figure B.38 - GRACE Model: Calculated versus measured permeability. [Case: Howell Well, E. TX (USA)] 


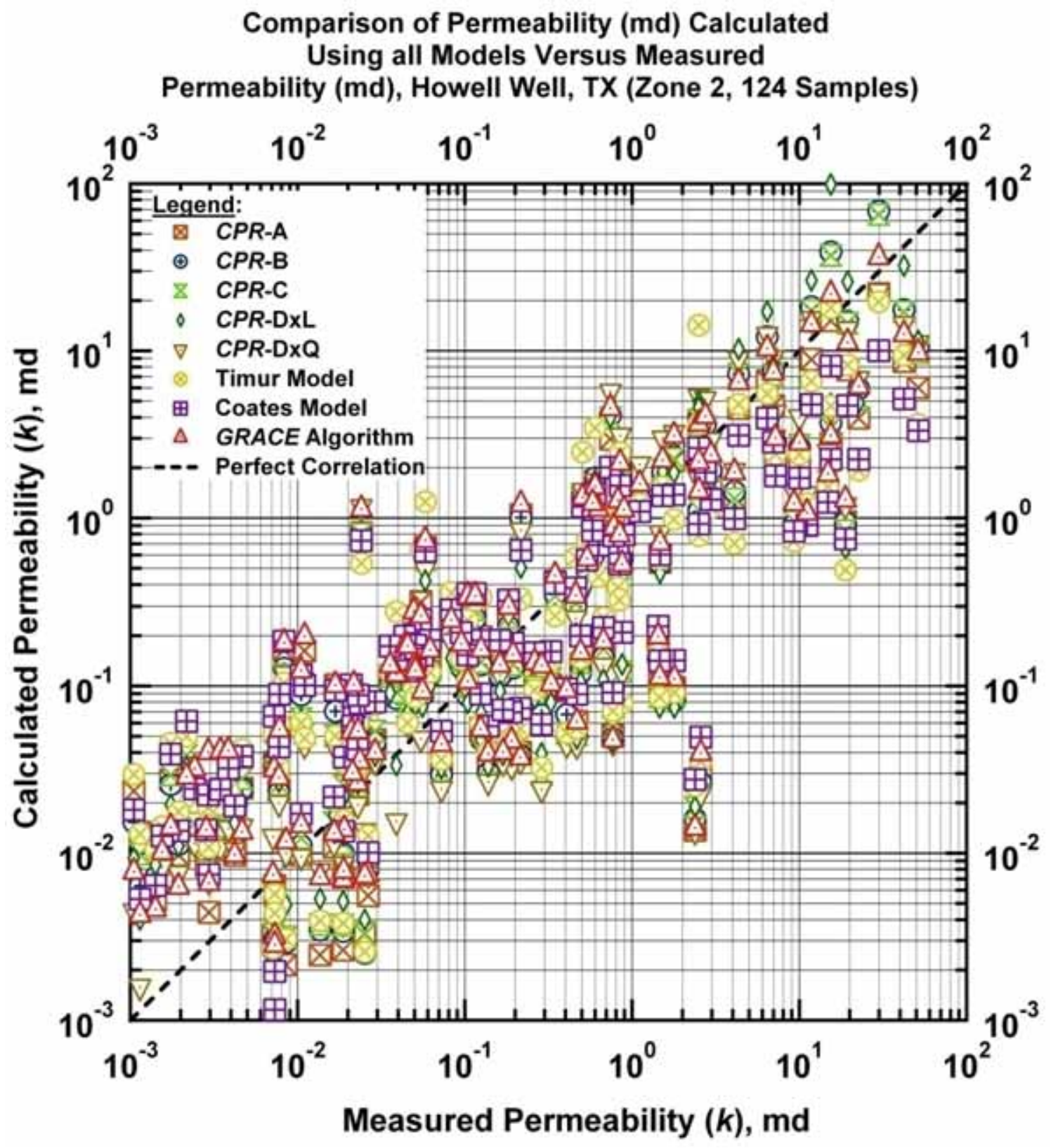

Figure B.39 - All Models: Calculated versus measured permeability. [Case: Howell Well, E. TX (USA)] 


\section{APPENDIX C \\ RESULTS FOR MIOCENE SAND \\ (S. LOUISIANA - USA)}

In this Appendix we present the Miocene Sand, located in S. Louisiana (USA - this is a sandstone reservoir (Granberry and Wilshusen (1962)).

\section{Model CPR-A: (Appendix C)}

The "characteristic permeability relation" (or $C P R$ ) Model CPR-A (i.e., the modified Archie "Clean Sand" Model) is given as:

$$
k=a(\phi-c)^{b} \quad c=c_{\max } \exp \left[-c_{1} \phi^{c_{2}} S_{W}^{c_{3}}\right]
$$

Where a plot of $\log (k)$ versus $\log (\phi)$ is used to graphically calibrate Eq. C-1 in terms of the coefficients $a, b$, and $c_{\max }$. Fig. C.1 shows that the CPR-A model performs quite well for this case. Figs. C.1a and $\mathbf{b}$ show the log-log and the semilog format of permeability versus porosity, respectively. In Fig. C.2 we provide the "error" plots for this case, where the measured and computed data are compared systematically. In Fig. C.2a we note a good correlation of the results obtained using Model CPR-A versus the measured permeability data - there is good agreement with the perfect correlation trend (i.e., the 45degree line). In Fig. C.2b we provide a comparison with the GRACE algorithm correlation (Xue et al. 1997)), and we note that the CPR-A model results correlate very well with the GRACE algorithm results for this case. In Fig. C.2c we present the calculated and measured $c$-function - and we note a deviation of the correlation of the data about the perfect correlation trend. Lastly, in Fig. C.2d we present the measured and calculated permeability with depth.

\section{Model CPR-B: (Appendix C)}

The "characteristic permeability relation" (or CPR) Model CPR-B (i.e., the modified Archie "Dirty Sand" Model (power-law basis)) is given as:

$$
k=a(\phi+c)^{b} \quad c=c_{\max } \exp \left[-c_{1} \phi^{c_{2}} S_{w}^{c_{3}}\right] .
$$

Where a plot of $\log (k)$ versus $\log (\phi)$ is used to graphically calibrate Eq. C-2 in terms of the coefficients $a, b$, and $c_{\max }$. The results for this case are presented in Figs. C.3 and 4. In Fig. C.3a (log-log format) we note that a very distinct "envelope" is formed by the CPR-B model, essentially all of the data on the right and left flanks are well-matched (i.e., the "dirty sand" power law relation (applied on the right portion of the data) and its correction function (applied on the left portion of the data). Similarly, in Fig. C.3b (semilog format), we observe an excellent distribution of the power law model across the body of the data. The error analyses for this case are shown in Fig. C.4. In Fig. C.4a we present the results obtained using 
Model $C P R-B$ versus the measured permeability data and in this case there is good agreement with the perfect correlation trend (i.e., the 45-degree line). We provide a comparison with the GRACE algorithm correlation in Fig. C.4b - the results using Model CPR-B and the GRACE algorithm compare very well for this case. We compare the computed and data-derived values of the $c$-function for this case in Fig. C.4c and we note a reasonably "tight" correlation. Lastly, in Fig. C.4d we present the measured and calculated permeability with depth. In summary, we believe that Model CPR-B is a very effective correlation model for this particular data case.

\section{Model CPR-C: (Appendix C)}

The "characteristic permeability relation" (or CPR) Model CPR-C (i.e., the modified Archie "Dirty Sand" Model (exponential basis)) is given as:

$$
k=c \exp [\beta \phi] \quad c=c_{\max } \exp \left[-c_{1} \phi^{c_{2}} S_{w}^{c_{3}}\right] .
$$

Where a plot of $\log (k)$ versus $\phi$ is used to graphically calibrate Eq. C-3 in terms of the coefficients - $\beta$ and $c_{\max }$. Model $C P R-C$ is fundamentally different than Models $C P R-A$ and $C P R-B$, as the basis for Model $C P R-C$ is an exponential function, not a power law relation. In Figs. C.5 and 6 we present the results of applying Model $C P R-C$ for this case, and in general, we observe a very consistent performance of Model $C P R-C$. Fig. C.5b illustrates the excellent conformance of Model $C P R-C$ for this case - again recalling that this model has an exponential relationship with porosity as its basis.

In Figs. C.6a, C.6b, and C.6c we review the error analyses plots for this case, and we note good performance (visually) in terms of the correlation of the results using Model CPR-C. We believe that the $C P R-C$ model has performed well for this case, and Fig. C.6 confirms the value of this model as a "characteristic relation" for permeability.

\section{Model CPR-D: (Appendix C)}

The "characteristic permeability relation" (or $C P R$ ) Model CPR-D (i.e., the weighted power law-exponential model) is given in its fundamental form as:

$$
\left.k=x a \phi^{b}+(1-x) \alpha \exp [\beta \phi] \quad[0 \leq x \leq 1]\right]
$$

For Eq. C-4 there are two proposed models for the $x$-function (i.e., the weighting function) - these are:

$$
\begin{aligned}
& x=\exp \left[-\ln \left(x_{0}\right)+x_{1} \ln (\phi)+x_{2} \ln \left(S_{w}\right)+x_{3} \ln (\phi) \ln \left(S_{w}\right)\right](\text { Model CPR-DxL) } \\
& x=\exp \left[\begin{array}{l}
-\ln \left(x_{0}\right)+x_{1} \ln (\phi)+x_{2} \ln \left(S_{w}\right)+x_{3} \ln (\phi) \ln \left(S_{w}\right) \\
+x_{4} \ln (\phi)^{2}+x_{5} \ln \left(S_{w}\right)^{2}+x_{6} \ln (\phi)^{2} \ln \left(S_{w}\right) \\
+x_{7} \ln (\phi) \ln \left(S_{w}\right)^{2}+x_{8} \ln (\phi)^{2} \ln \left(S_{w}\right)^{2}
\end{array}\right] \text { (Model CPR-DxL) }
\end{aligned}
$$

As noted in Eq. C-4b, Model CPR-DxL utilizes a logarithmic linear model for the weights ( $x$-values) and as seen in Eq. C-4c, Model CPR-DxQ utilizes a logarithmic quadratic model for the weights ( $x$-values). While these models (Eqs. C-4b and C-4c) are empirical, we have found generally good application of 
these relations for all cases. In this case, the base results using Models CPR-DxL and CPR-DxQ are presented in Fig. C.7 - where the power law and exponential basis functions are fitted to the appropriate portion of the data. The power law equation represents the "Archie clean sand" trend and is fitted to the leftmost data as shown in Fig. C.7a and in contrast, the exponential equation is thought to represent the "Archie dirty sand" trend and the exponential is fitted to the far rightmost portion of the data (see Fig. C.7b).

In Figs. C.8 and 9 we present the error analyses for this case, where we have employed the weighting functions (Eqs. C-4b and C-4c) as appropriate. In Fig. C.8a we find a good correlation of permeability (with the out-lying points noted in the middle of the trend). The comparison of the Model CPR-DxL results are compared to the results obtained from the GRACE algorithm in Fig. C.8b, we note the very good the results obtained using Model CPR-DxL. The computed weight function ( $x$-values) shown for the $C P R-D x L$ model in Fig. C.8c does exhibit deviation from the perfect correlation line. Fig. C.8d provides the measured and calculated permeability versus depth.

The results obtained using Model $C P R-D x Q$ are presented in Fig. C.9, and we note substantially improved behavior over that of Model $C P R-D x L$. In particular, the base correlation of computed and measured permeabilities (Fig. C.9a) for Model CPR-DxQ does indicate a slightly better correlation than that of Model $C P R-D x L$. Also, the comparison of Model CPR-DxQ with the results from the GRACE algorithm (Fig. C.9b) exhibits better agreement than the results of Model CPR-DxL. Finally, the correlation of the $x$-function (Fig. C.9c) for Model CPR-DxQ is better than the correlation for Model CPR-DxL. These comparisons suggest that Model CPR-DxQ (i.C., the combination of Eqs. C-4a and C-4c) has provided a better correlation of data, for this case, than Model CPR-DxL. Fig. C.9d provides the measured and calculated permeability versus depth.

\section{"Timur Model": (Appendix C)}

The "Timur Model" [Timur (1968)] is given as:

$$
k_{\text {Timur }}=a \phi^{b} S_{w}^{c}
$$

In Fig. C.10 we present the results of the "Timur Model" (Eq. C-5) which is essentially just a generalized power-law function relation in terms of $k, \phi$, and $S_{w}$. As shown in Figs. C.10a and b, the "Timur Model" also provides a good correlation of the data for this case. The comparison of results using the "Timur Model" with the results of the GRACE algorithm is presented in Fig. C.10b.

\section{"Coates Model": (Appendix C)}

The "Coates Model" [referenced by Ahmed et al. (1989)] is given as:

$$
k_{\text {Coates }}=\left[100 \phi^{2} \frac{\left(1-S_{w}\right)}{S_{w}}\right]^{2}
$$


In Fig. C.11 we present the results of the "Coates Model" (Eq. C-6) which is essentially just a modified power-law function relation in terms of $k, \phi$, and $S_{w}$. The results are shown in Figs. C.11a and $\mathbf{b}$ of the data for this case. The comparison of results using the "Coates Model" with the results of the GRACE algorithm (Fig. C.11b) is also shown in the same plot.

\section{GRACE Algorithm: (Appendix C)}

In Fig. C.12 we present the results predicted by the GRACE algorithm, which is a non-parametric regression approach (see Xue et al. (1997) for details). In theory, the GRACE algorithm should provide the most unbiased correlation of the data - i.e., the GRACE algorithm is designed not "fit the errors" as other regression approaches may. It is our contention that the GRACE algorithm is the statistical standard - and any algorithm/approach/model which achieves better regression statistics than the GRACE algorithm is actually "fitting the errors" in the data. The only cases which have significantly better regression statistics than the GRACE algorithm are the CPR-A, CPR-DxQ, and "Timur Model" - hence, we must label these cases as "over-fitted" in a statistical sense.

\section{Comparison of All Models: (Appendix C)}

Fig. C.13, illustrates all of the models on a single plot of calculated versus measured permeability. We also present a table of all statistical results in Table C.1. Our final plot, Fig. C.14 shows the source of the original data set.

Table C.1 - Statistical Results for all Models (Miocene Sand, S. LA (USA), $n=24)$.

\begin{tabular}{|c|c|c|c|c|}
\hline Model & $\begin{array}{c}\text { Sum of } \\
\text { Squared } \\
\text { Residuals } \\
{\left[\log (k)^{2}\right]}\end{array}$ & $\begin{array}{l}\text { Variance } \\
{\left[\log (k)^{2}\right]}\end{array}$ & $\begin{array}{c}\text { Standard } \\
\text { Deviation } \\
{[\log (k)]}\end{array}$ & $\begin{array}{c}\text { Absolute } \\
\text { Relative } \\
\text { Error } \\
\text { [percent] }\end{array}$ \\
\hline CPR-A & 7.18 & 3.50 & 1.87 & 48.68 \\
\hline$C P R-B$ & 7.37 & 3.43 & 1.85 & 50.71 \\
\hline$C P R-C$ & 7.54 & 3.74 & 1.93 & 50.03 \\
\hline$C P R-D x L$ & 7.56 & 3.35 & 1.83 & 55.51 \\
\hline$C P R-D x Q$ & 6.30 & 3.42 & 1.85 & 48.03 \\
\hline Timur & 7.16 & 3.72 & 1.93 & 45.13 \\
\hline Coates & 8.05 & 3.56 & 1.89 & 54.08 \\
\hline GRACE Algorithm & 11.02 & 2.02 & 1.42 & 49.81 \\
\hline
\end{tabular}

The coefficients for the models used in this case are provided in Table C.2.

Table C.2a - Model Coefficients (Miocene Sand, S. LA (USA), $n=24)$.

\begin{tabular}{|c|c|c|c|c|c|c|}
\hline Model & $a$ or $\alpha$ & $b$ or $\beta$ & $c$ or $c_{\max }$ & $c_{1}$ & $c_{2}$ & $c_{3}$ \\
\hline$C P R-A$ & $3.00 \times 10^{8}$ & 9.00 & 0.060 & 1.38 & 1.44 & -0.97 \\
\hline$C P R-B$ & $5.00 \times 10^{6}$ & 8.00 & 0.100 & $3.71 \times 10^{-4}$ & -6.60 & 1.38 \\
\hline$C P R-C$ & $5.00 \times 10^{-8}$ & 83.00 & $2.00 \times 10^{-6}$ & 576.54 & 3.77 & 0.44 \\
\hline Timur & $8.49 \times 10^{9}$ & 12.93 & 0.738 & - & - & - \\
\hline Coates & $5.67 \times 10^{6}$ & 8.74 & 1.383 & - & - & - \\
\hline
\end{tabular}


Table C.2b - Model Coefficients (Miocene Sand, S. LA (USA), $n=24)$.

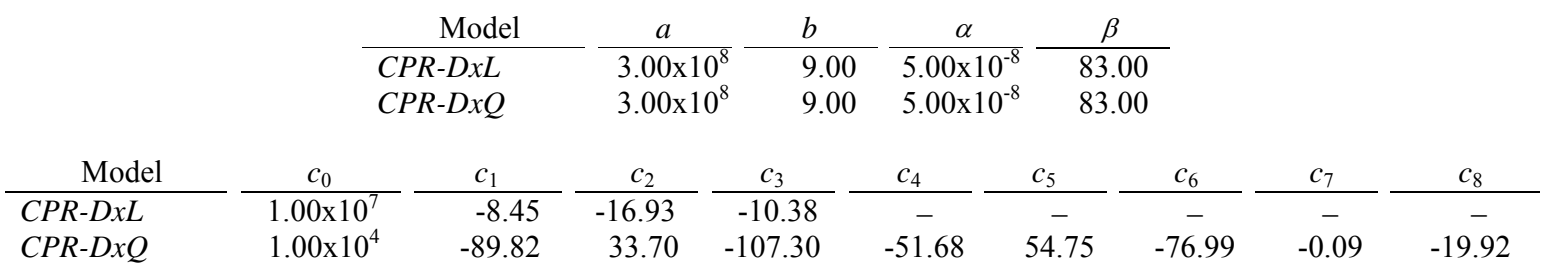

\section{Nomenclature: (Appendix C)}

$$
\begin{array}{ll}
S_{w} & =\text { Water Saturation (fraction) } \\
k & =\text { Formation Permeability, md (or any consistent units) } \\
\phi & =\text { Porosity, fraction }
\end{array}
$$

\section{References: (Appendix C)}

Ahmed, U., Crary, S.F., and Coates, G.R: "Permeability Estimation: The Various Sources and Their Interrelationship," paper SPE 19604 presented at the 1989 SPE Annual Technical Conference and Exhibition, San Antonio, TX, 8-11 Oct., 649-662.

Granberry, R.J., and Wilshusen, R.C.: "Improved Interpretation of Formation Productivity by Combined Use of Core Analysis and Electronic Log Data," Trans., Gulf Coast Association of Geological Societies (1962) 13, 213-221.

Timur, A.: "An Investigation of Permeability, Porosity, and Residual Water Saturation Relation-ships," Trans., SPWLA Symposium (1968) paper I.

Xue, G., Datta-Gupta, A., Valko, P., and Blasingame, T.A.: "Optimal Transformations for Multiple Regression: Application to Permeability Estimation from Well Logs," SPEFE (June 1997), 85-93. 


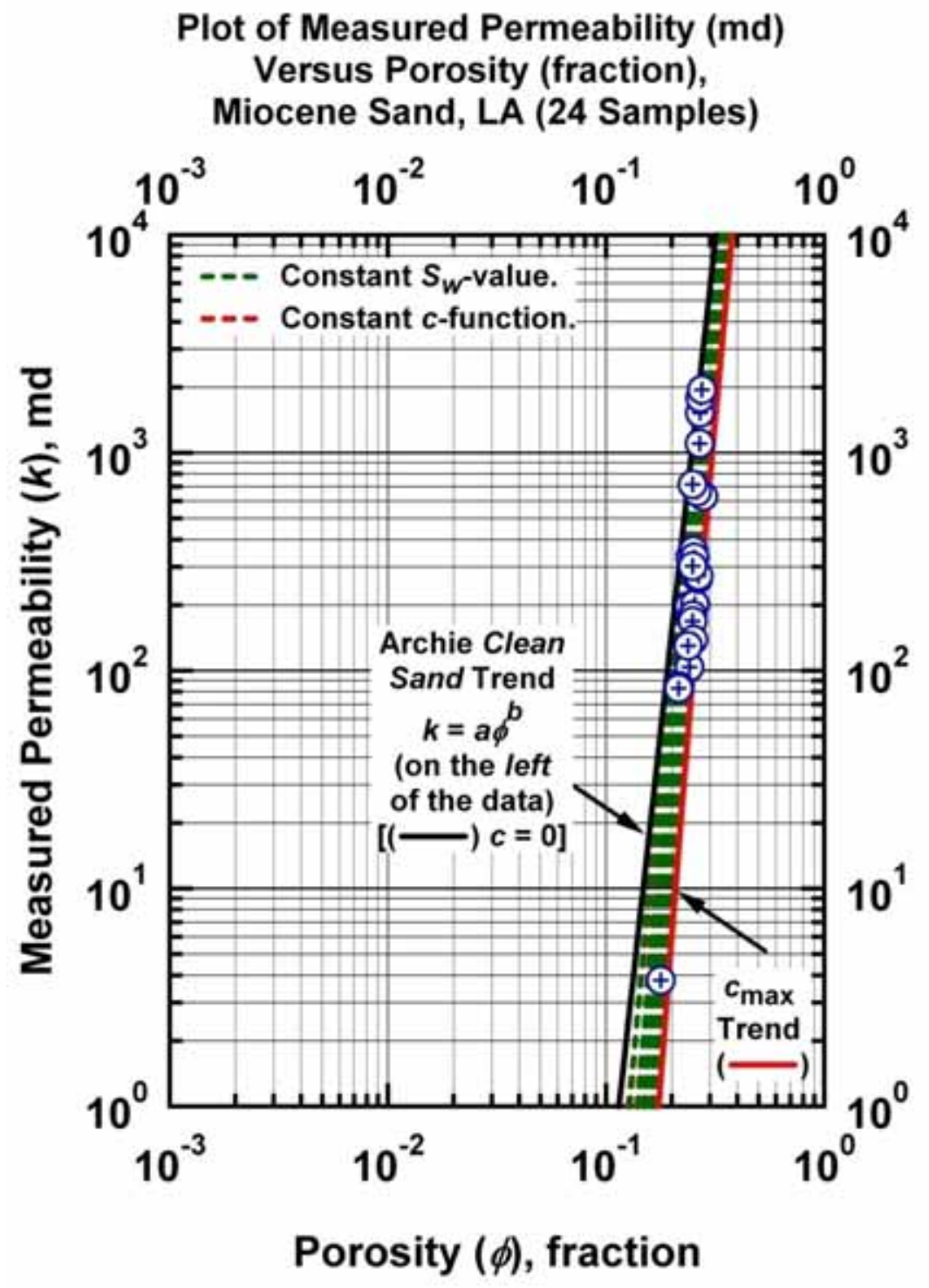

Figure C.1a - Model CPR-A: $\quad k=a(\phi-c)^{b} \quad c=c_{\max } \exp \left[-c_{1} \phi^{c_{2}} S_{w}^{c_{3}}\right] \quad$ - "Clean Sand" Plot (log-log format) - Archie "Clean Sand" trend is given by the straight-line trend at the far left of the data (power law model). [Case: Miocene Sand, S. LA (USA)] 


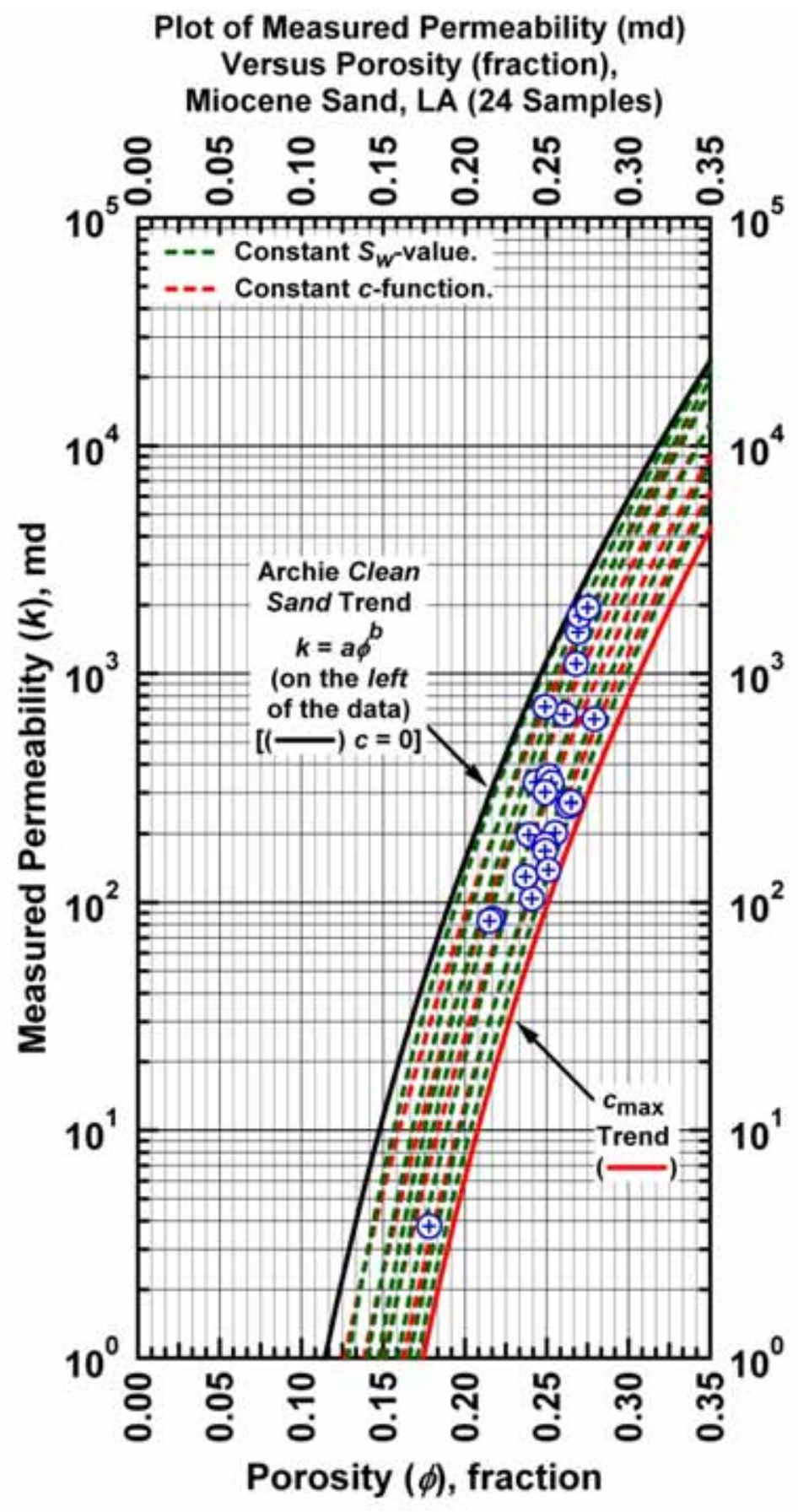

Figure C.1b - Model CPR-A: $k=a(\phi-c)^{b} \quad c=c_{\max } \exp \left[-c_{1} \phi^{c_{2}} S_{w}^{c_{3}}\right]-$ "Dirty Sand" Plot (semilog format). Archie "Clean Sand" trend is given by the curved trend at the far left of the data (power law model). [Case: Miocene Sand, S. LA (USA)] 


\section{Comparison of Permeability (md) Calculated} Using the "CPR-A" Model Versus the Measured Permeability (md), Miocene Sand, LA (24 Samples)

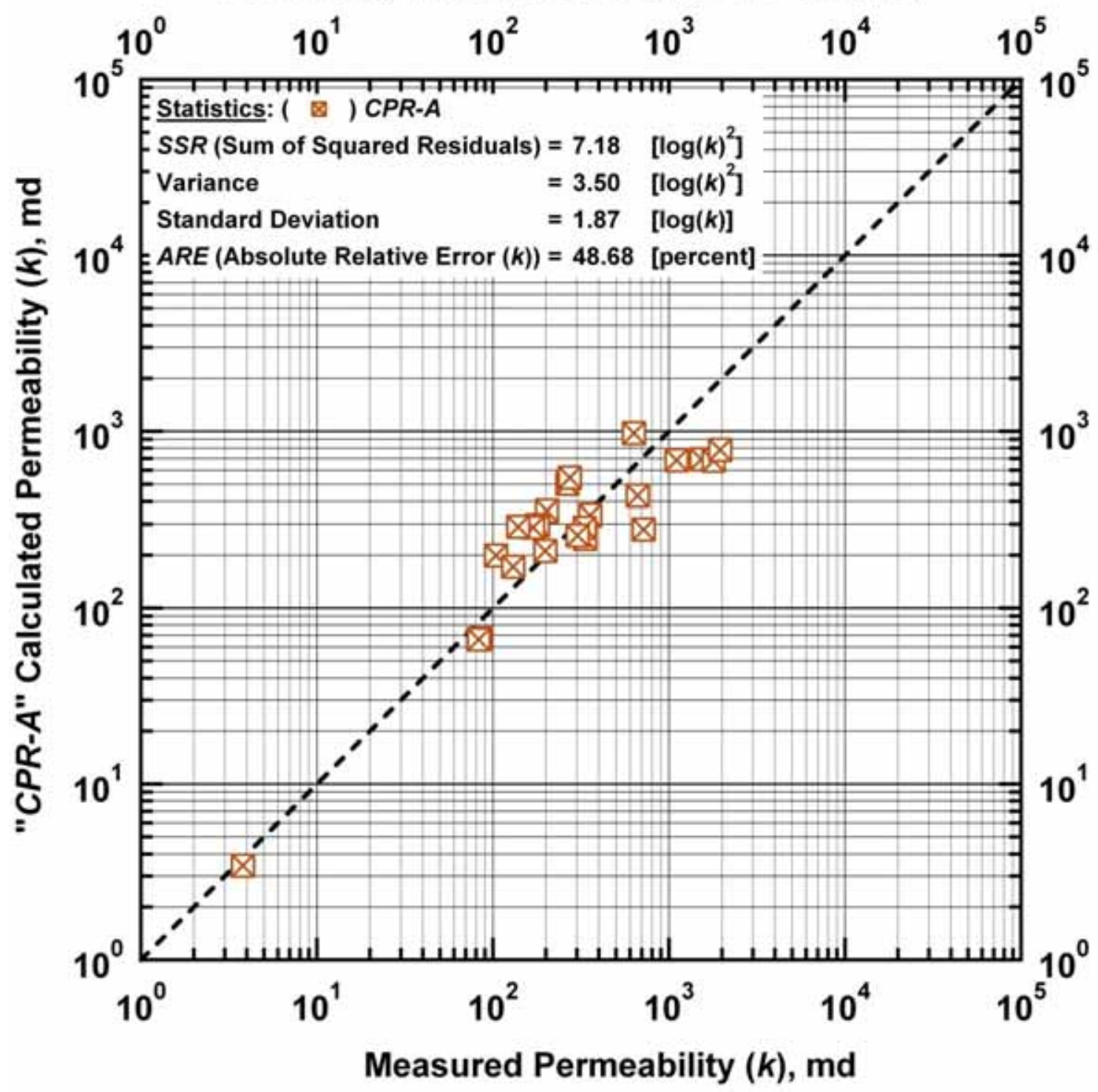

Figure C.2a - Model CPR-A: calculated versus measured permeability. [Case: Miocene Sand, S. LA (USA)] 


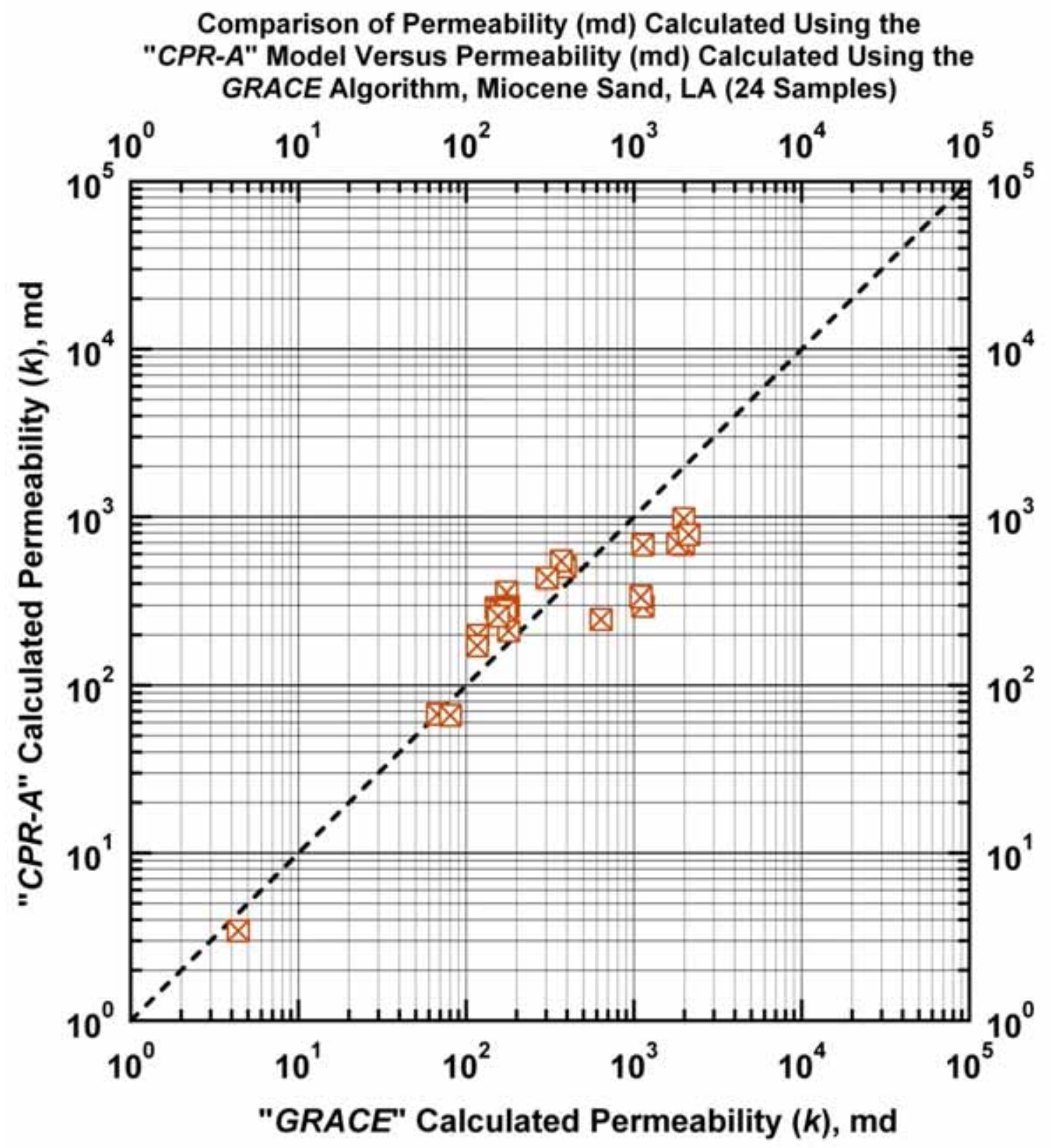

Figure C.2b - Model CPR-A: Permeability calculated using Model CPR-A versus permeability calculated using the GRACE Algorithm. [Case: Miocene Sand, S. LA (USA)] 
Comparison of the $c$-Coefficient Calculated Using the "CPR-A" Model Versus the Measured $c$-Coefficient, Miocene Sand, LA (24 Samples)

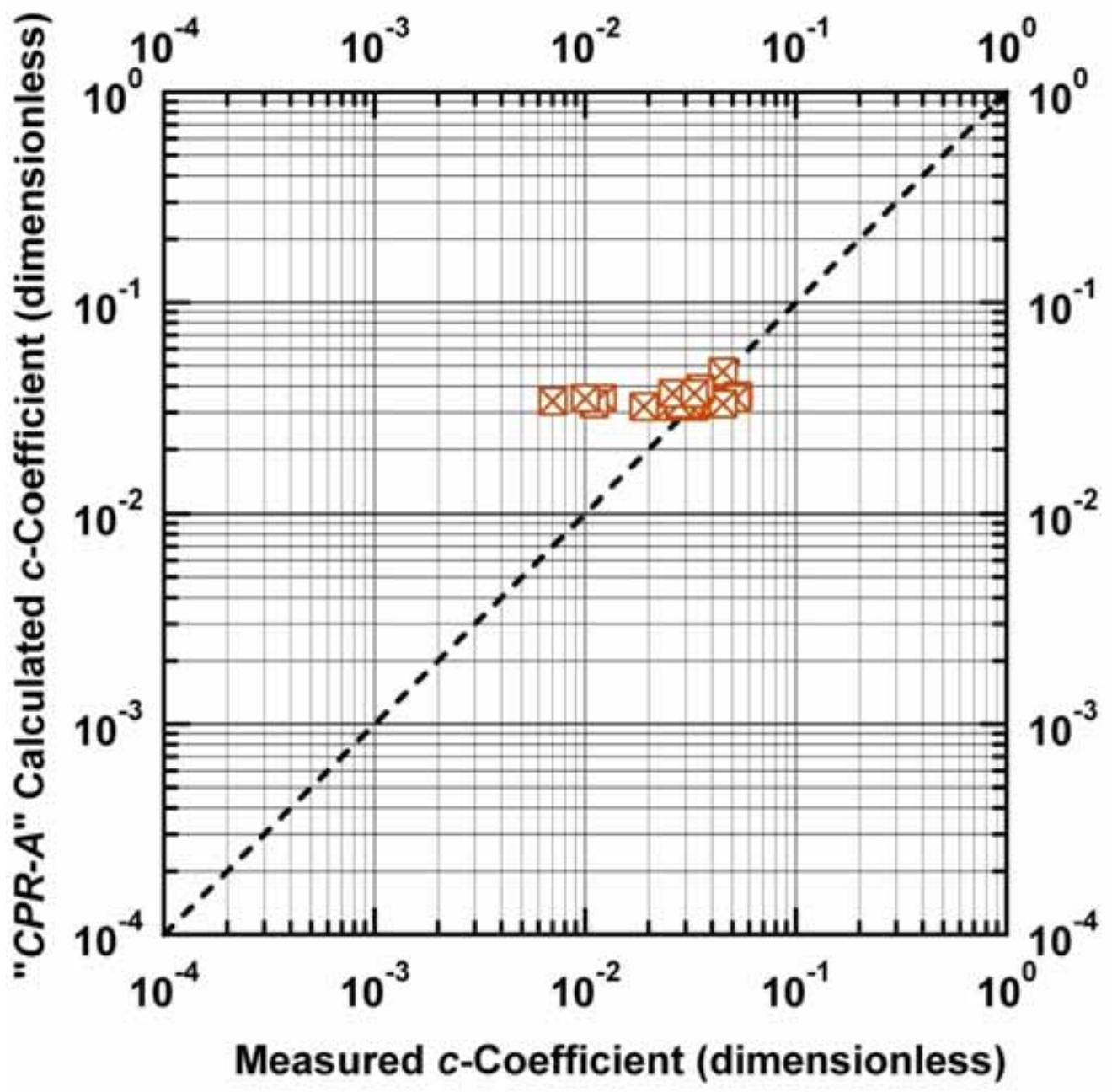

Figure C.2c - Model CPR-A: Calculated $c$-function values versus measured $c$-function values. [Case: Miocene Sand, S. LA (USA)] 
Plot of Depth ( $\mathrm{ft}$ ) Versus Measured and Calculated Permeability (md), Miocene Sand, LA (24 Samples)

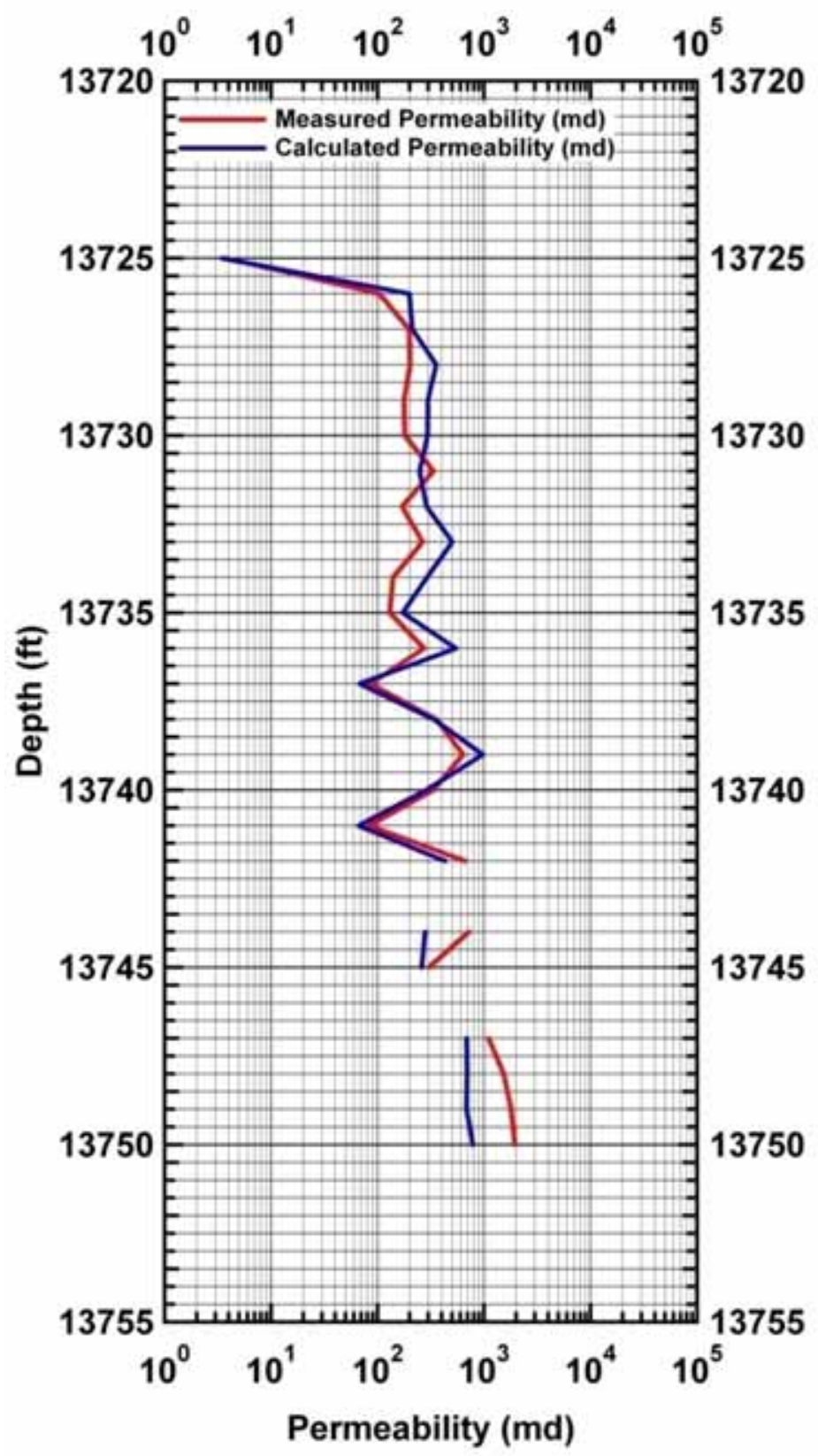

Figure C.2d - Model CPR-A: Calculated and measured permeability values versus depth. [Case: Miocene Sand, S. LA (USA)] 


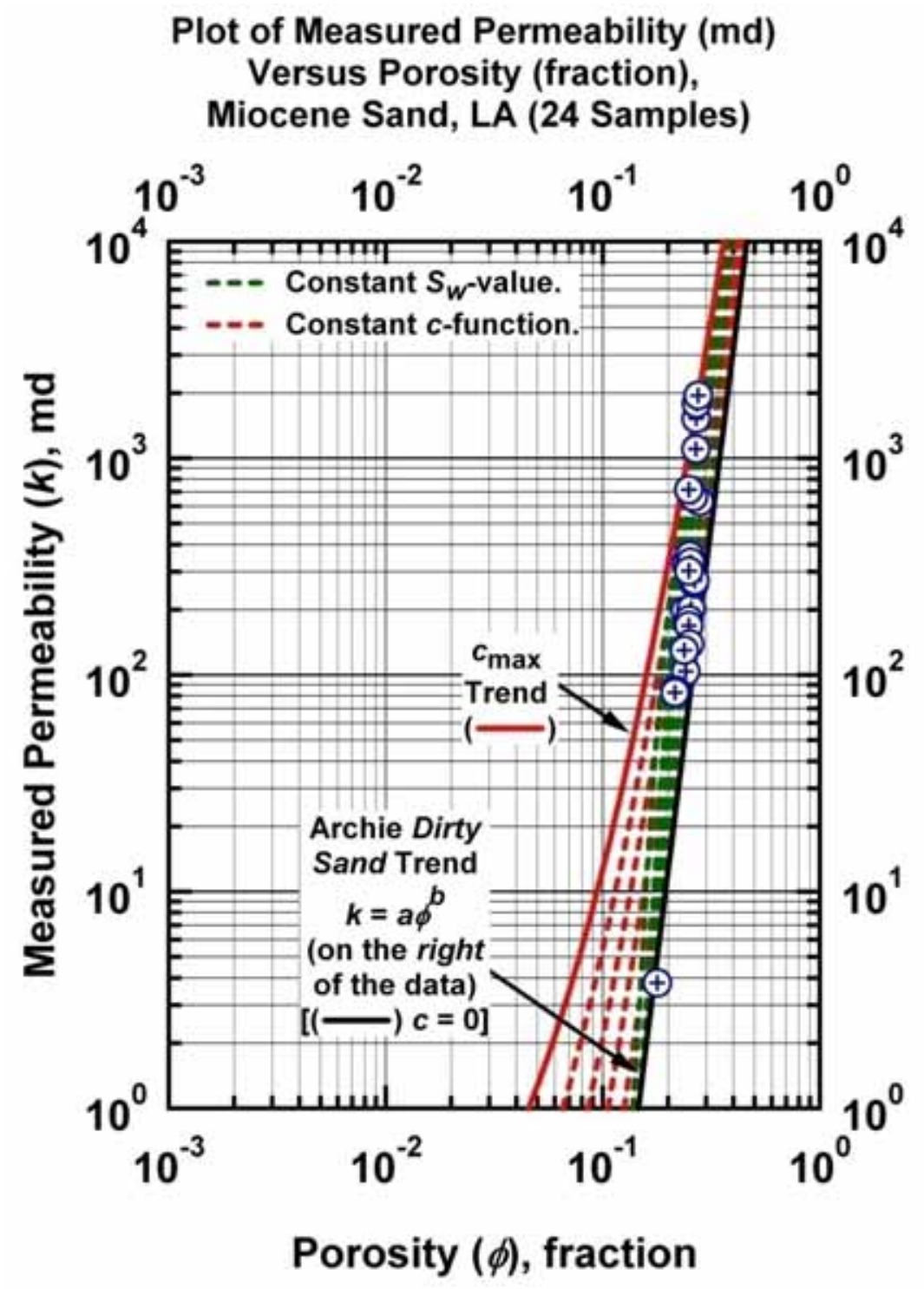

Figure C.3a - Model $C P R-B: \quad k=a(\phi+c)^{b} \quad c=c_{\max } \exp \left[-c_{1} \phi^{c_{2}} S_{w}^{c_{3}}\right]$ - "Clean Sand" Plot (log-log format) - Archie "Dirty Sand" trend is given by the straight-line trend at the far right of the data (power law model). [Case: Miocene Sand, S. LA (USA)] 


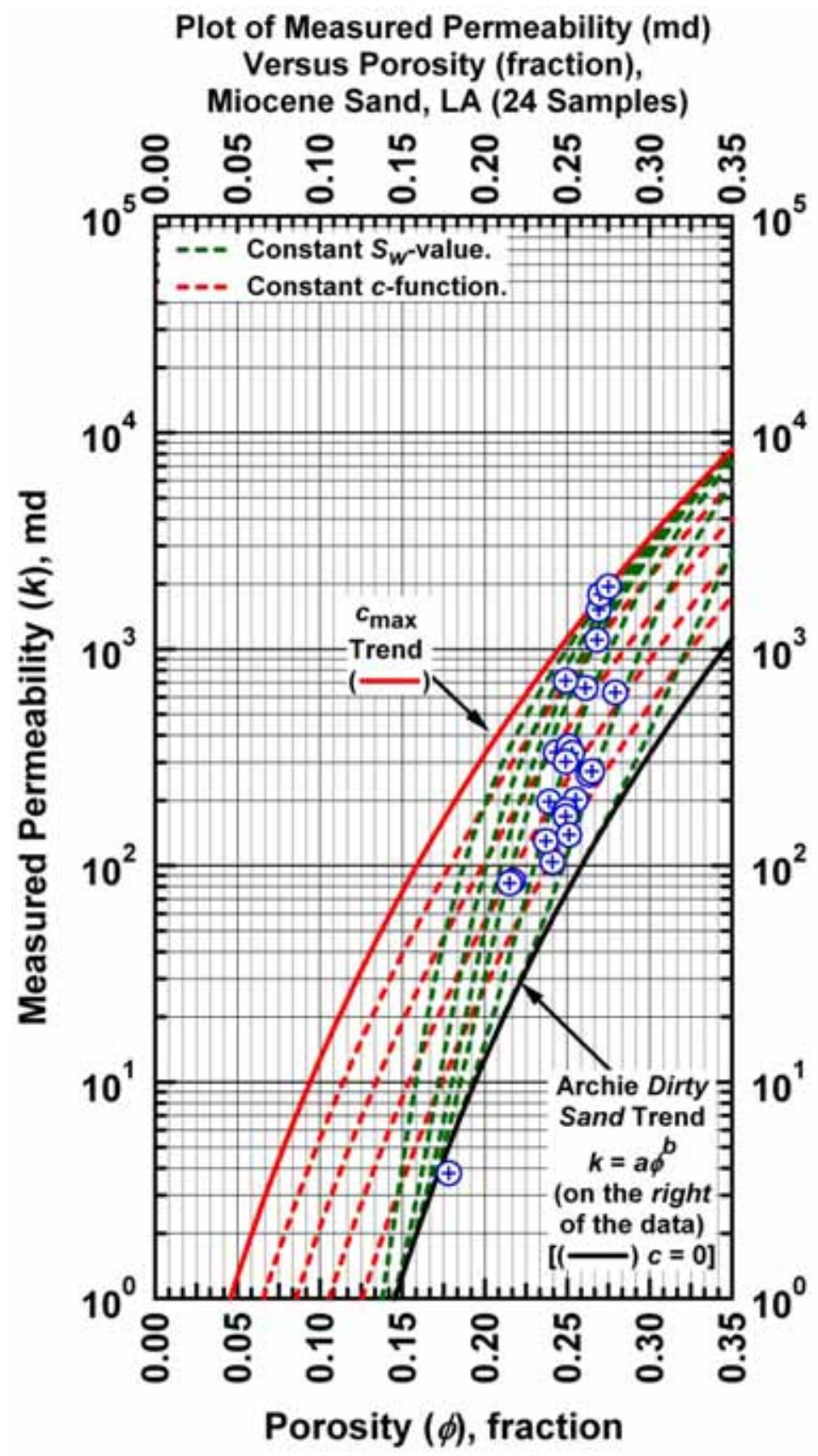

Figure C.3b - Model CPR-B: $k=a(\phi+c)^{b} \quad c=c_{\max } \exp \left[-c_{1} \phi^{c_{2}} S_{w}^{c_{3}}\right]$ - "Dirty Sand" Plot (semilog format) - Archie "Dirty Sand" trend is given by the curved trend at the far right of the data (power law model). [Case: Miocene Sand, S. LA (USA)] 


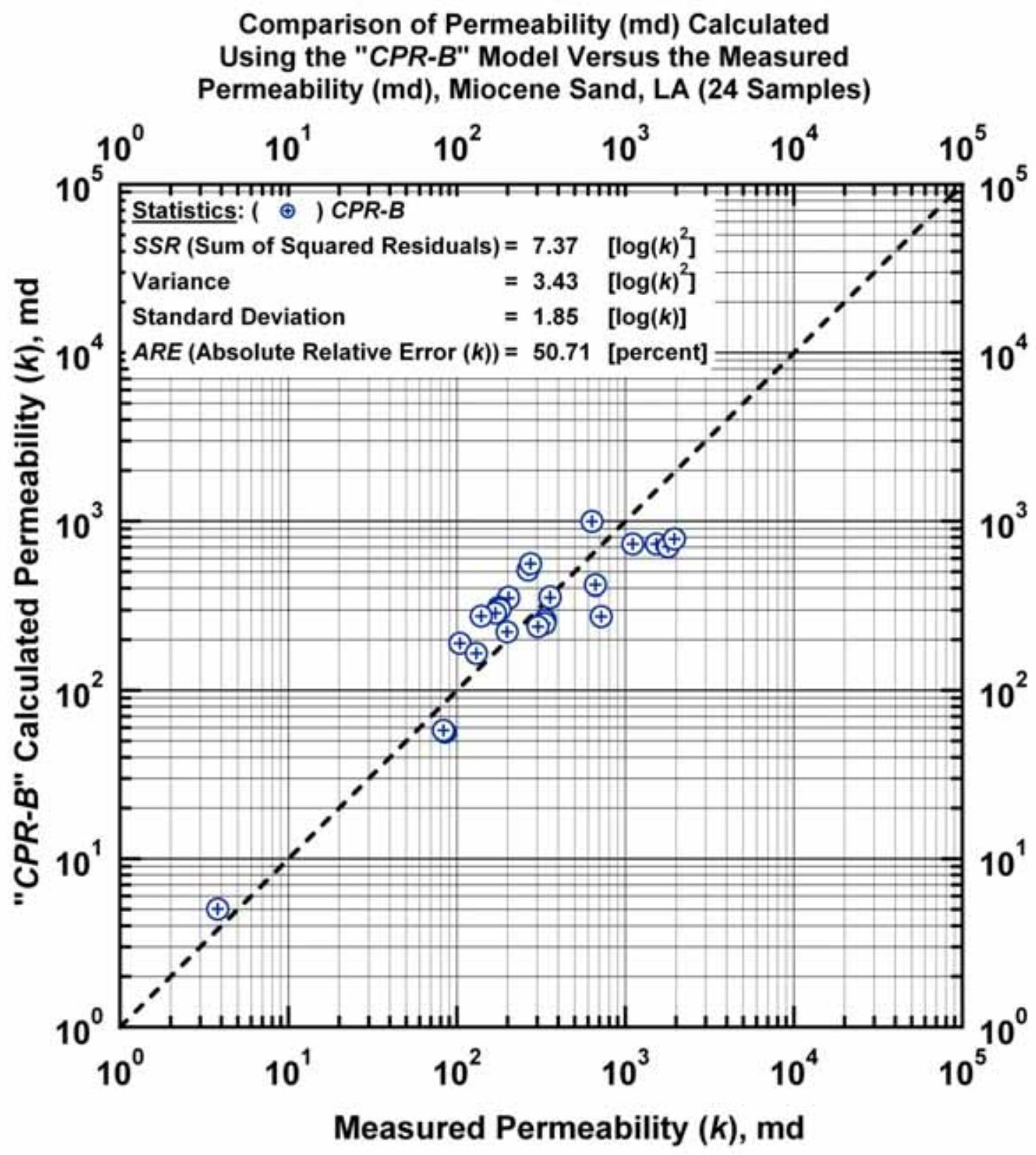

Figure C.4a - Model CPR-B: calculated versus measured permeability. [Case: Miocene Sand, S. LA (USA)] 


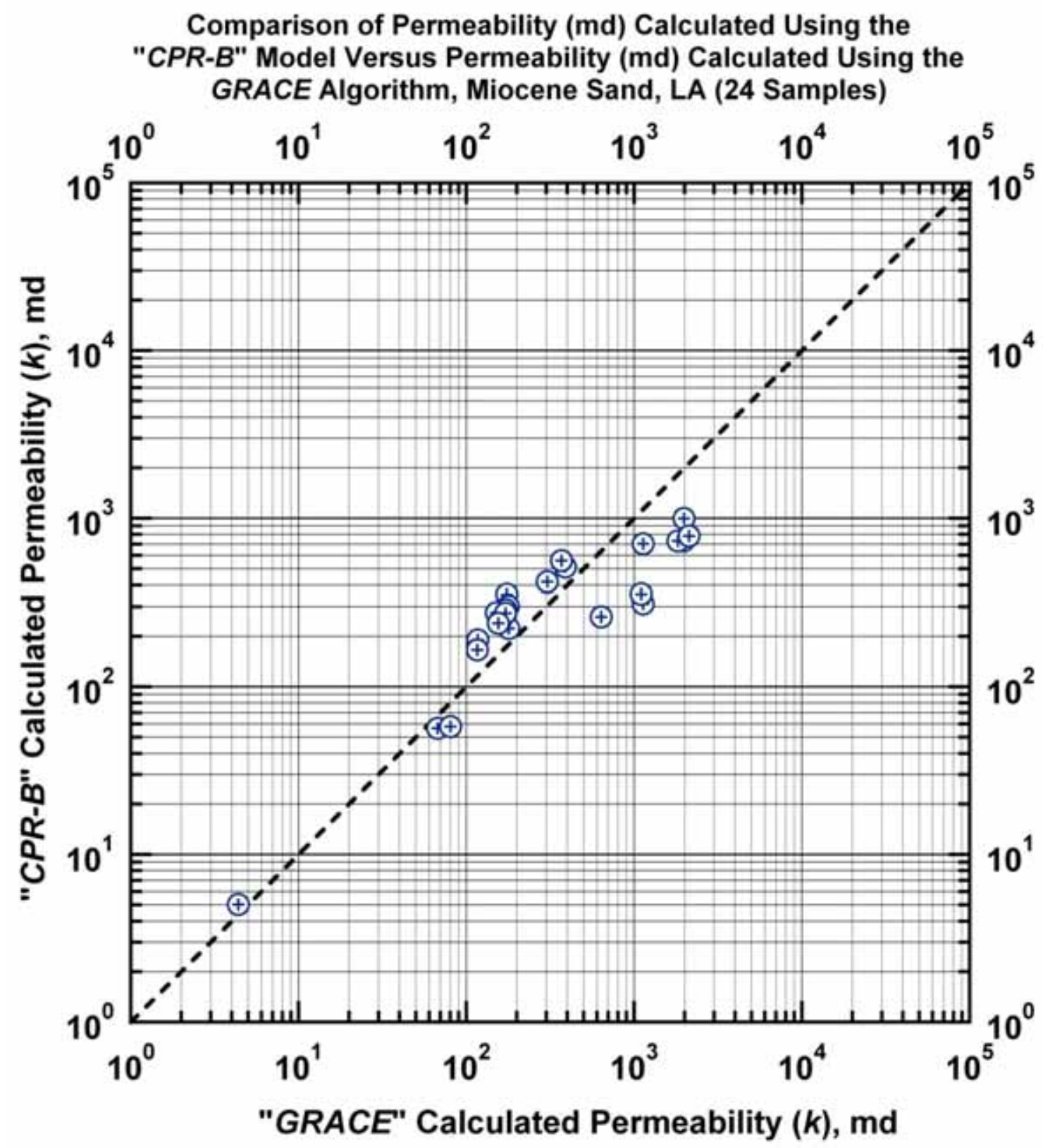

Figure C.4b - Model CPR-B: Permeability calculated using Model $C P R-B$ versus permeability calculated using the GRACE Algorithm. [Case: Miocene Sand, S. LA (USA)] 


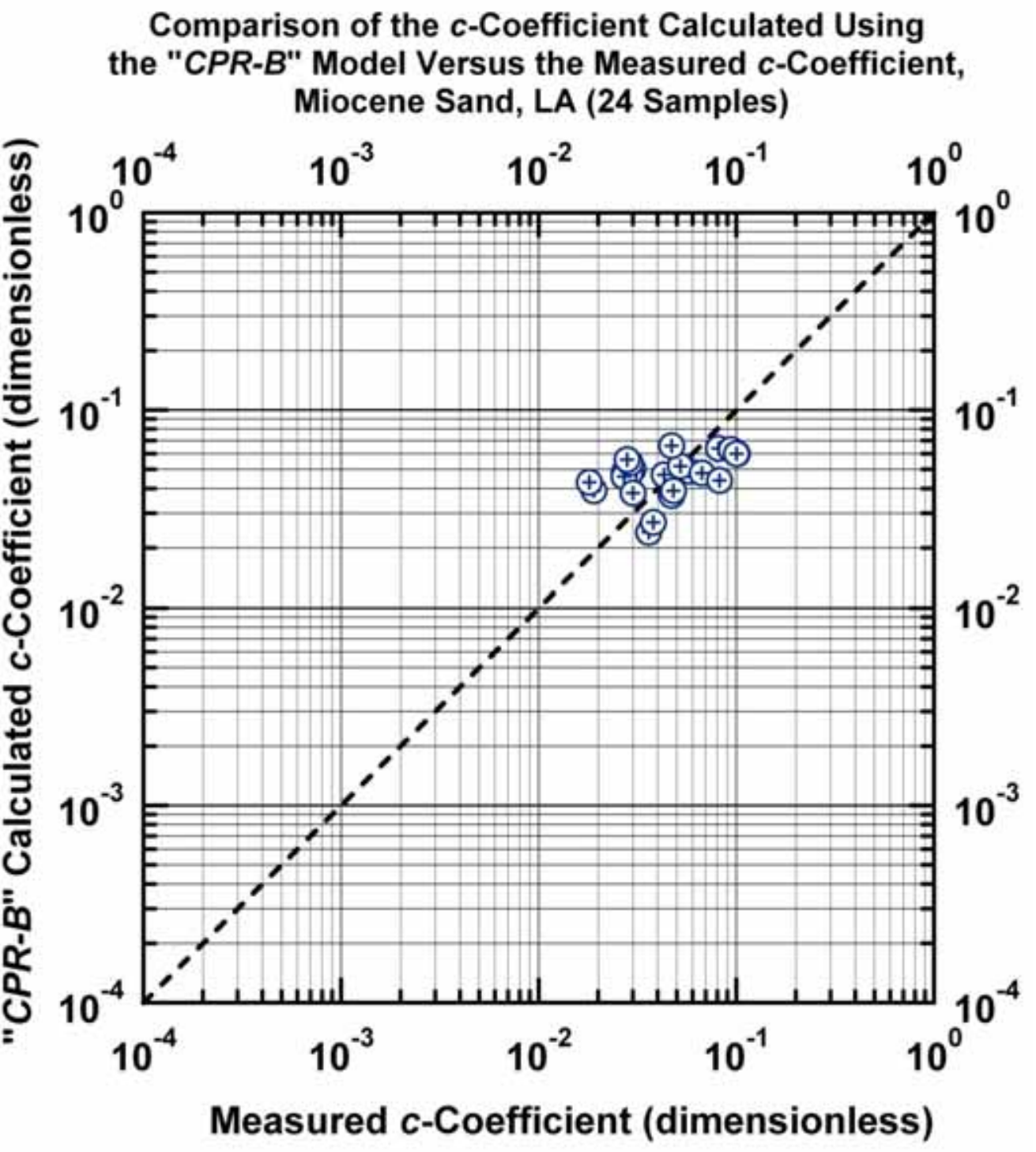

Figure C.4c - Model CPR-B: Calculated $c$-function values versus measured $c$-function values. [Case: Miocene Sand, S. LA (USA)] 
Plot of Depth ( $\mathrm{ft}$ ) Versus Measured and Calculated Permeability (md), Miocene Sand, LA (24 Samples)

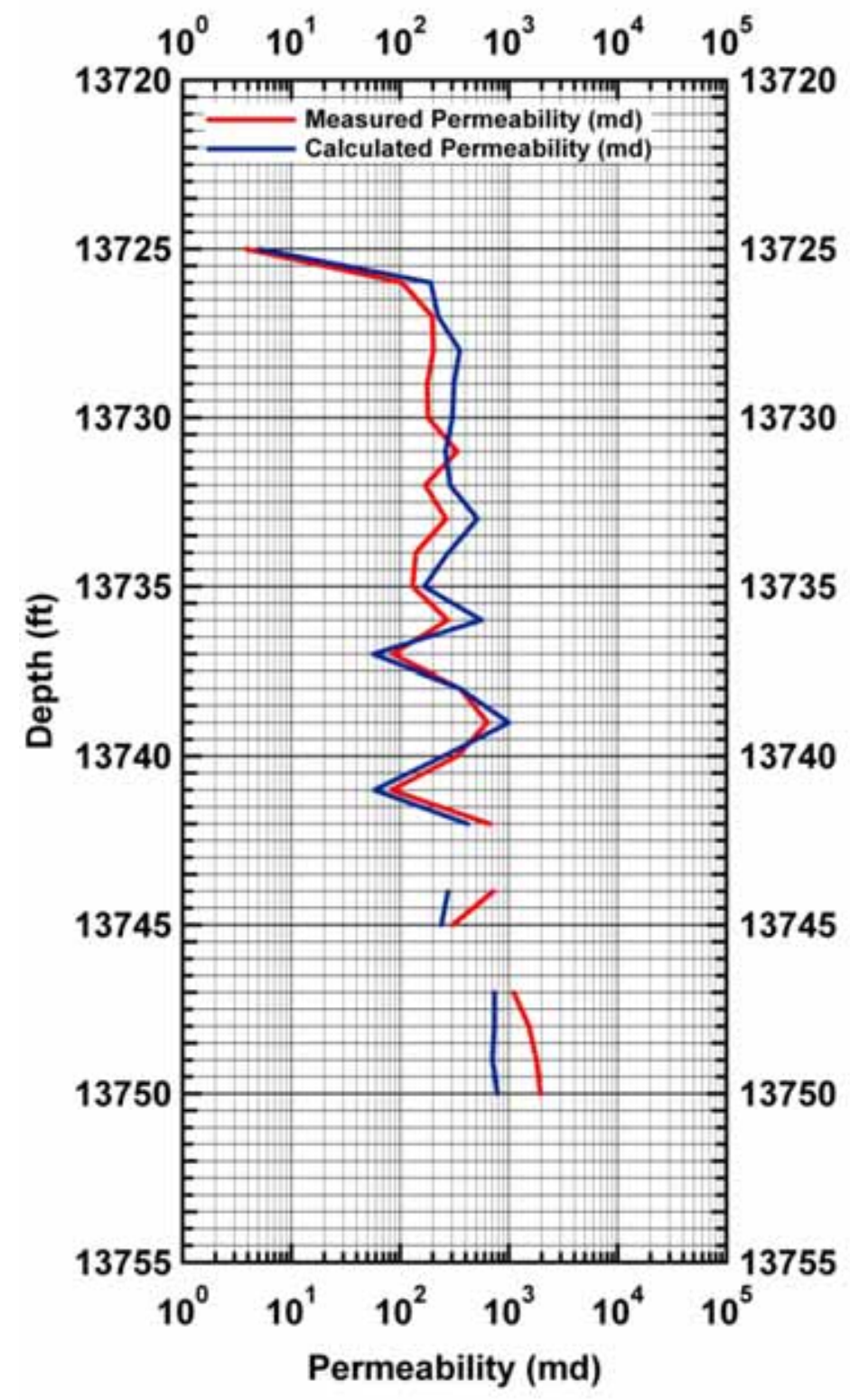

Figure C.4d - Model CPR-A: Calculated and measured permeability values versus depth. [Case: Miocene Sand, S. LA (USA)] 


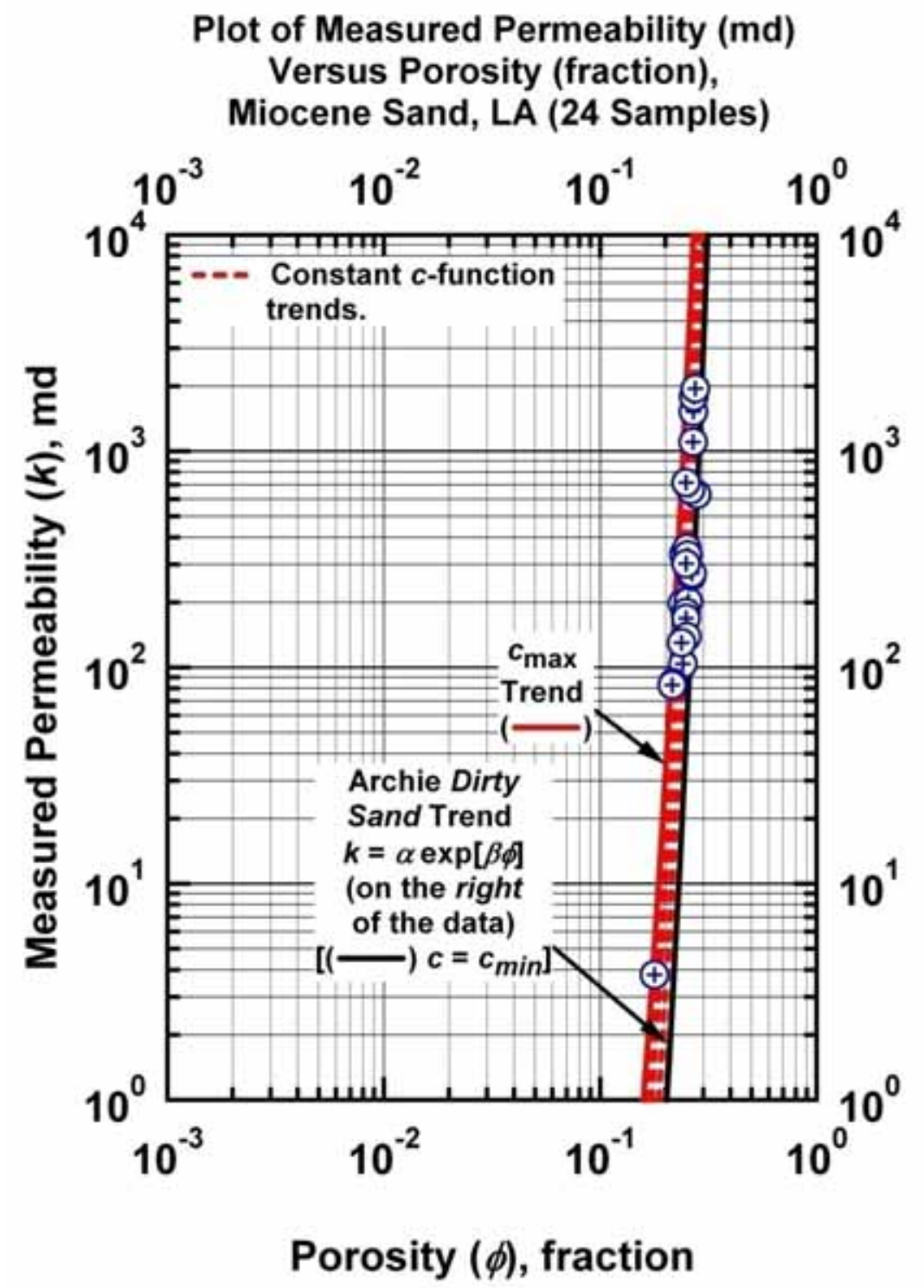

Figure C.5a - Model $C P R-C: \quad k=c \exp [\beta \phi] \quad c=c_{\max } \exp \left[-c_{1} \phi^{c_{2}} S_{w}^{c_{3}}\right]-$ "Clean Sand" Plot (log-log format) - Archie "Dirty Sand" trend is given by the curved trend at the far right of the data (exponential model). [Case: Miocene Sand, S. LA (USA)] 


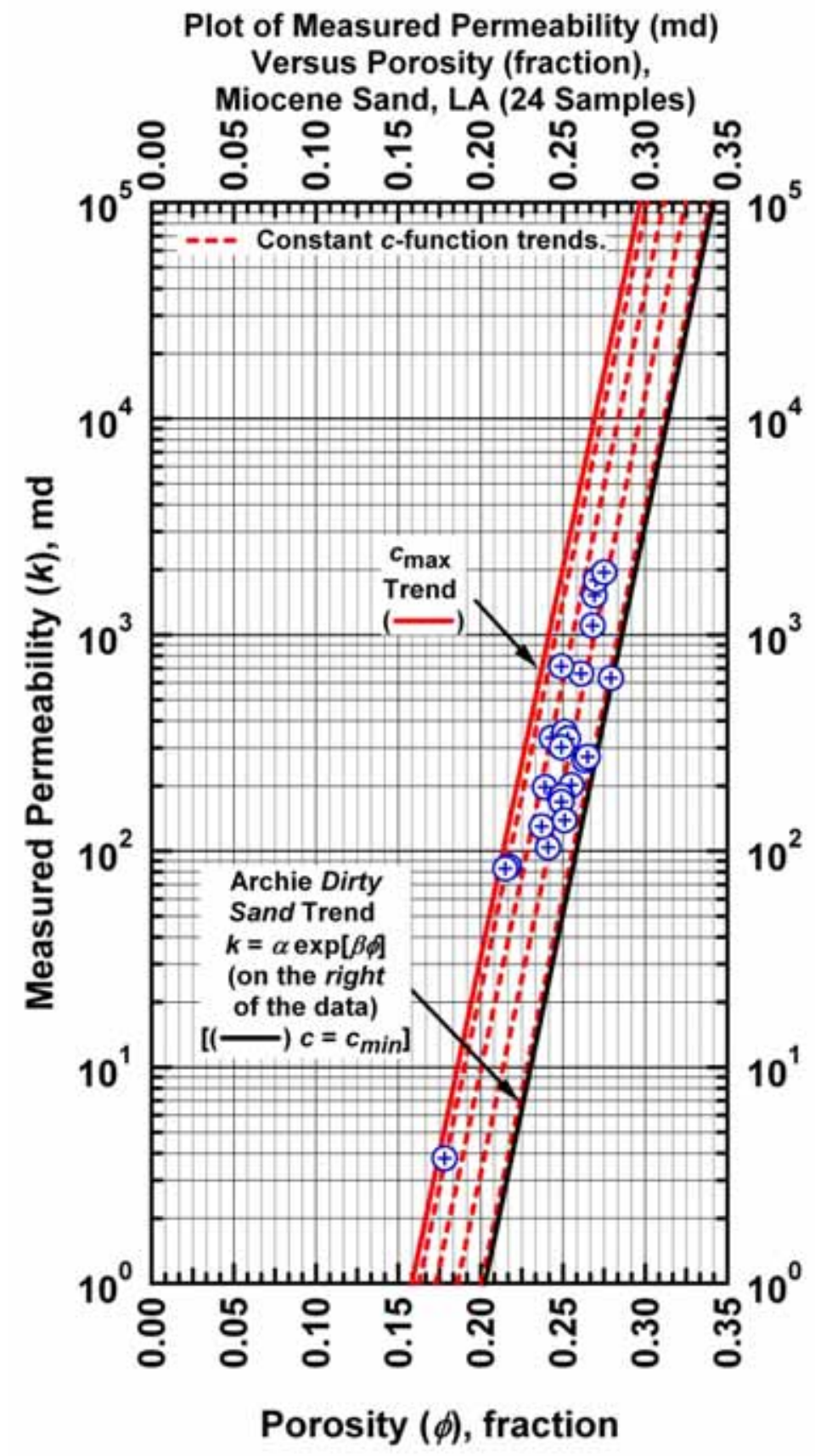

Figure C.5b - Model CPR-C: $k=c \exp [\beta \phi] \quad c=c_{\max } \exp \left[-c_{1} \phi^{c_{2}} S_{w}^{C_{3}}\right]-$ "Dirty Sand" Plot (semilog format) - Archie "Dirty Sand" trend is given by the straight-line trend at the far right of the data (exponential model). [Case: Miocene Sand, S. LA (USA)] 


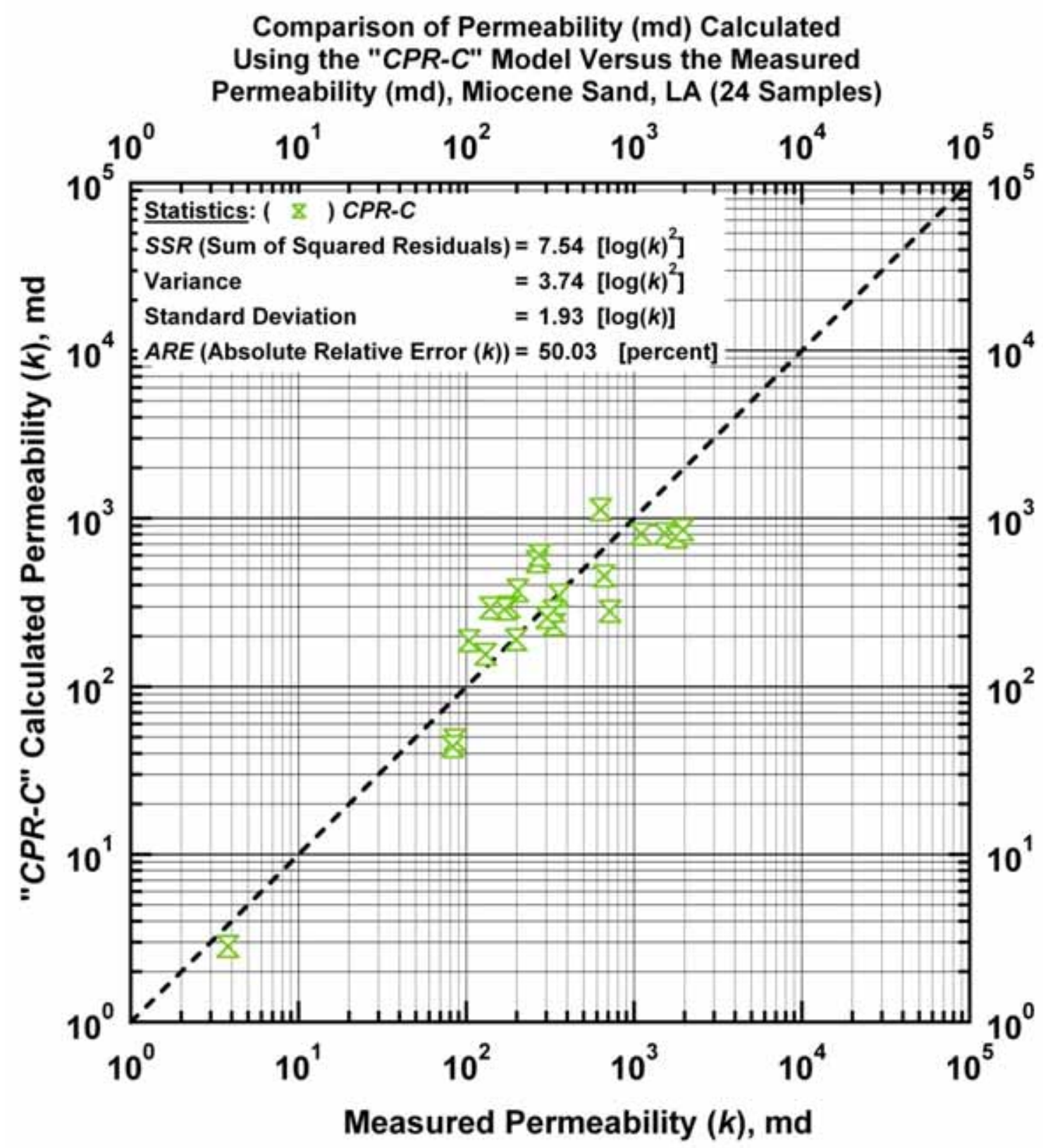

Figure C.6a - Model CPR-C: calculated versus measured permeability. [Case: Miocene Sand, S. LA (USA)] 


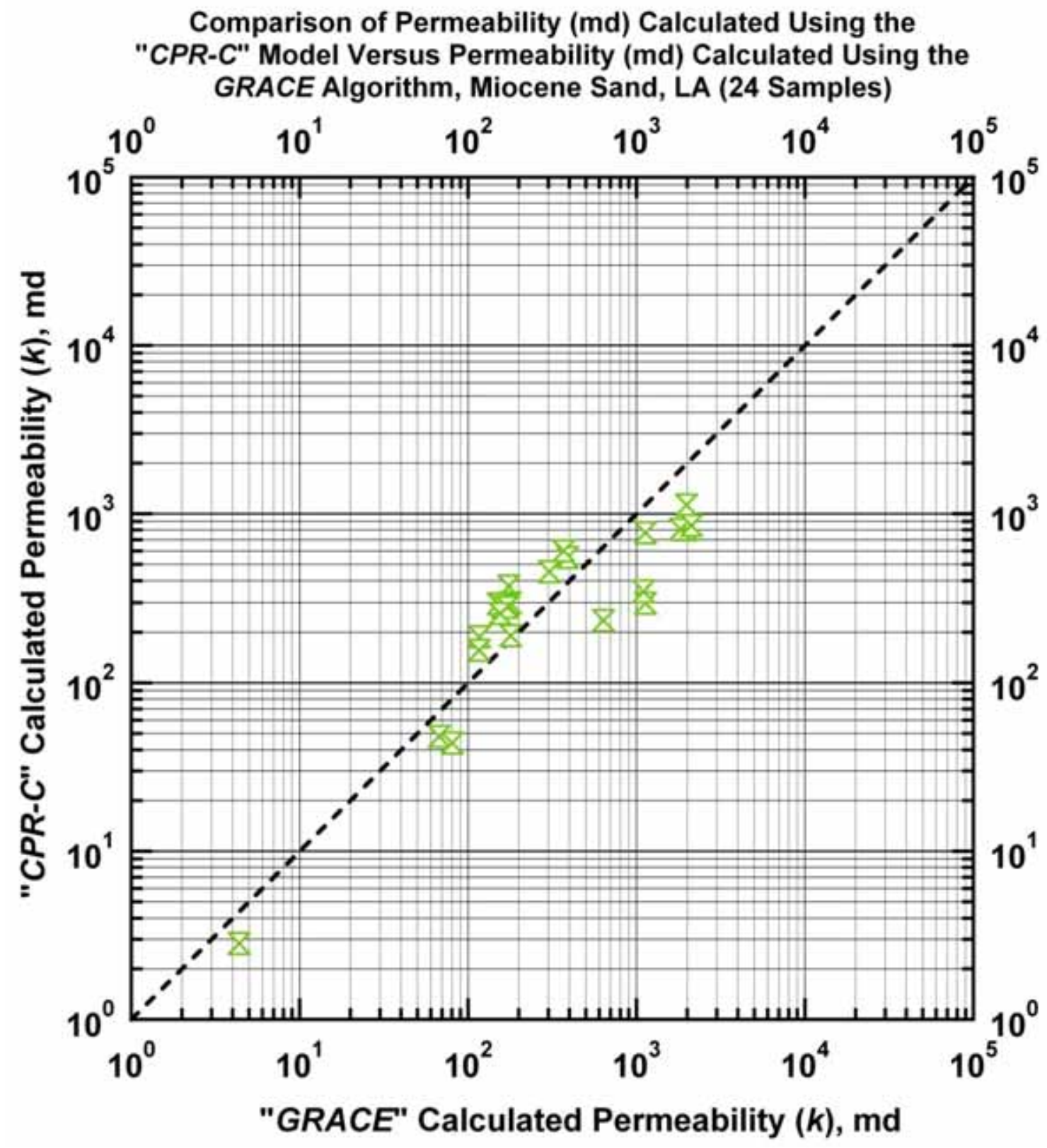

Figure C.6b - Model $C P R-C$ : Permeability calculated using Model $C P R-C$ versus permeability calculated using the GRACE Algorithm. [Case: Miocene Sand, S. LA (USA)] 


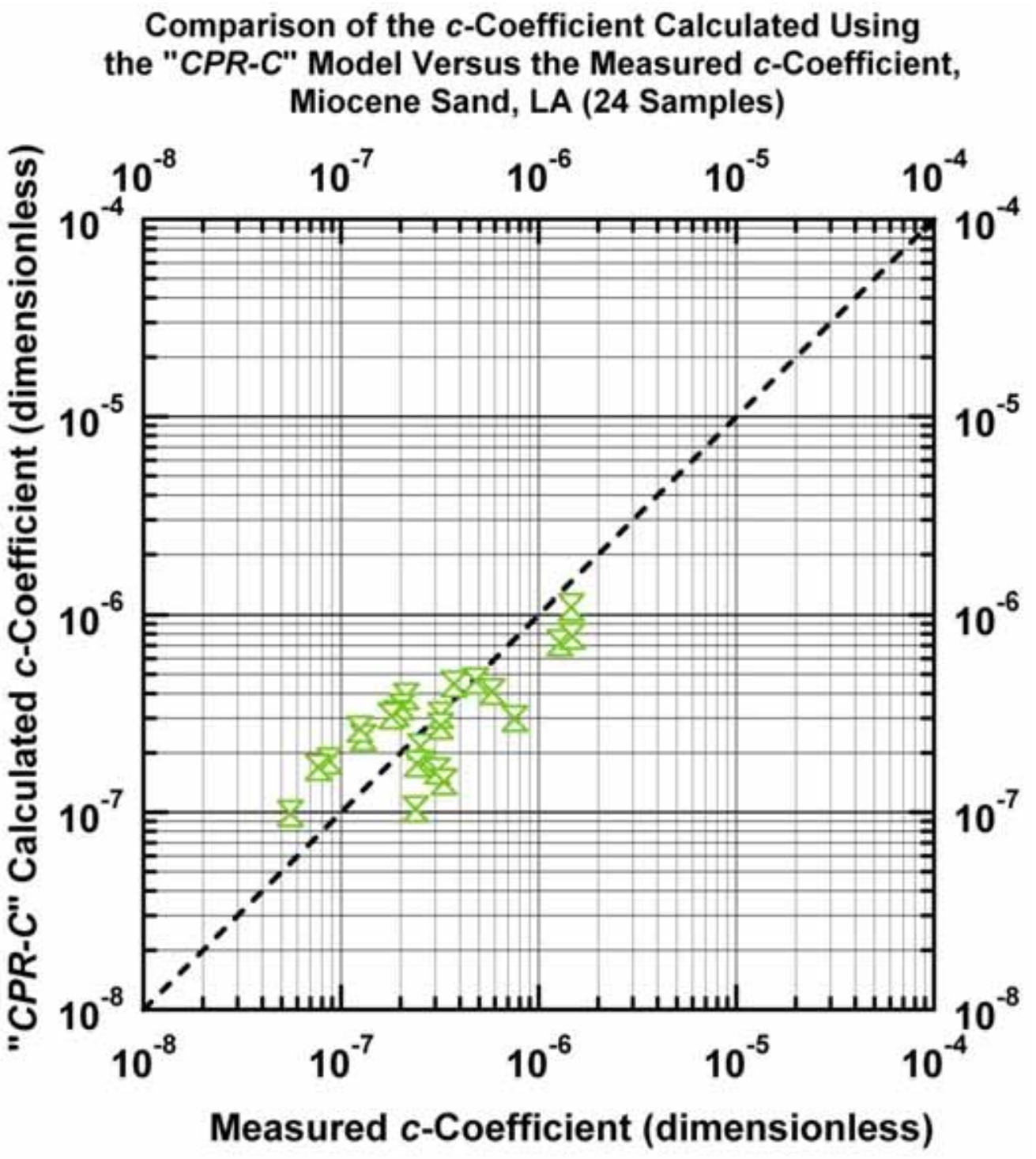

Figure C.6c - Model $C P R-C$ : Calculated $c$-function values versus measured $c$-function values. [Case: Miocene Sand, S. LA (USA)] 


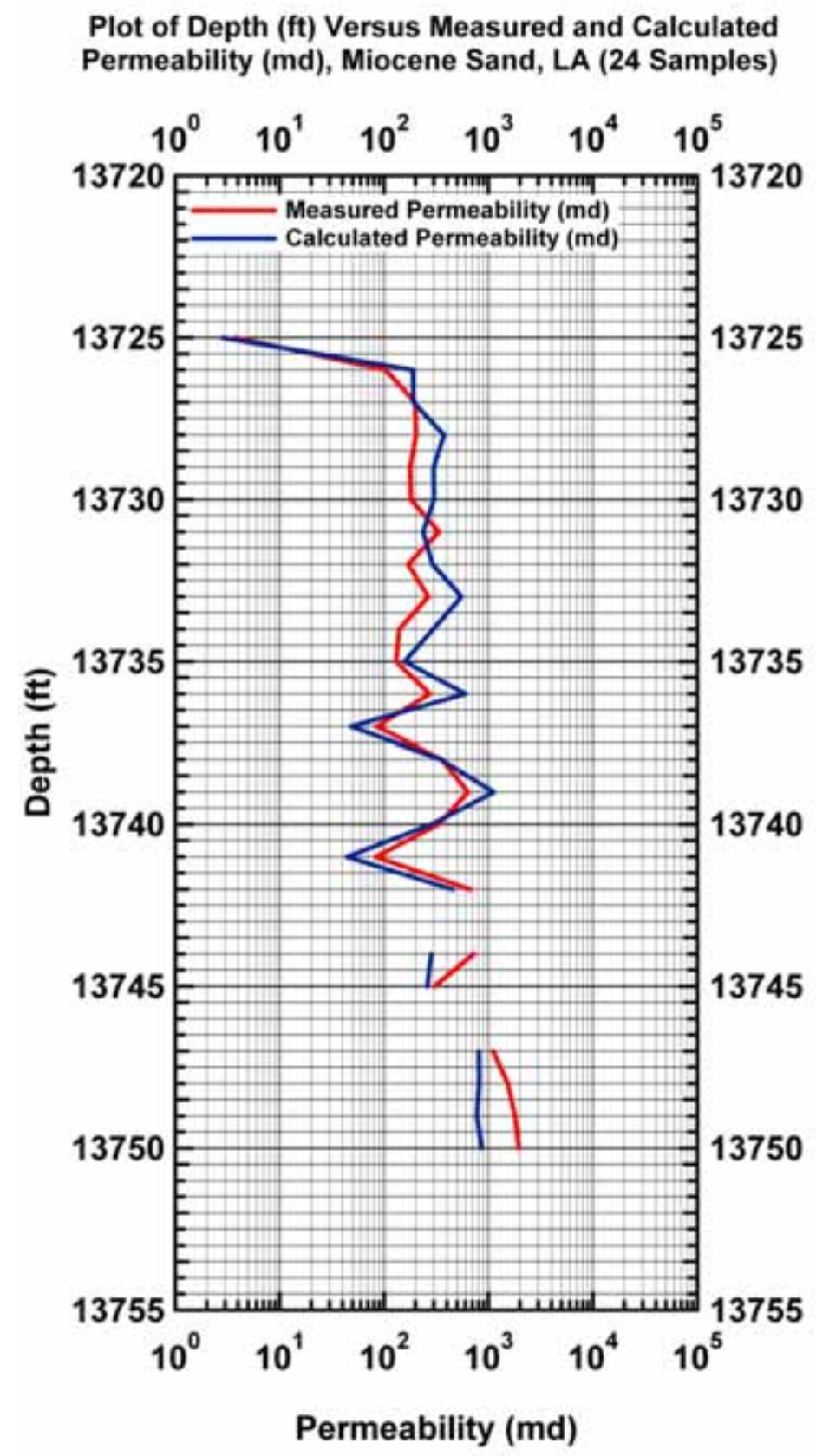

Figure C.6d - Model CPR-A: Calculated and measured permeability values versus depth. [Case: Miocene Sand, S. LA (USA)] 


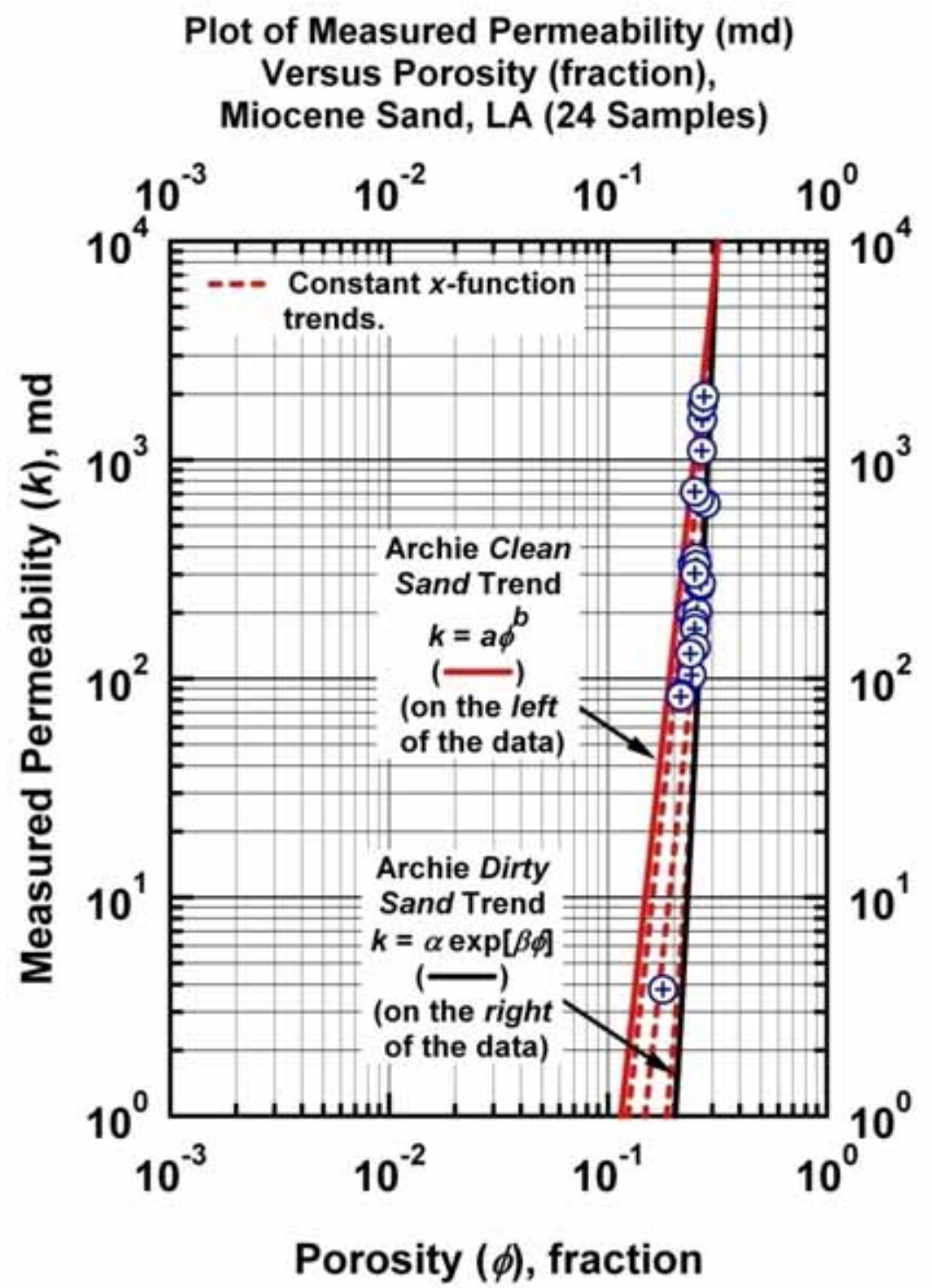

Figure C.7a - Model CPR-D: $k=x a \phi^{b}+(1-x) \alpha \exp [\beta \phi] \quad[0 \leq x \leq 1]$ - Weighted Power Law-Exponential Model used to correlate permeability $(k)$ and porosity $(\phi)$. "Clean Sand" Plot - Archie "Clean Sand" trend is given by the straight-line trend at the far left of the data (power law model). [Case: Miocene Sand, S. LA (USA)] 


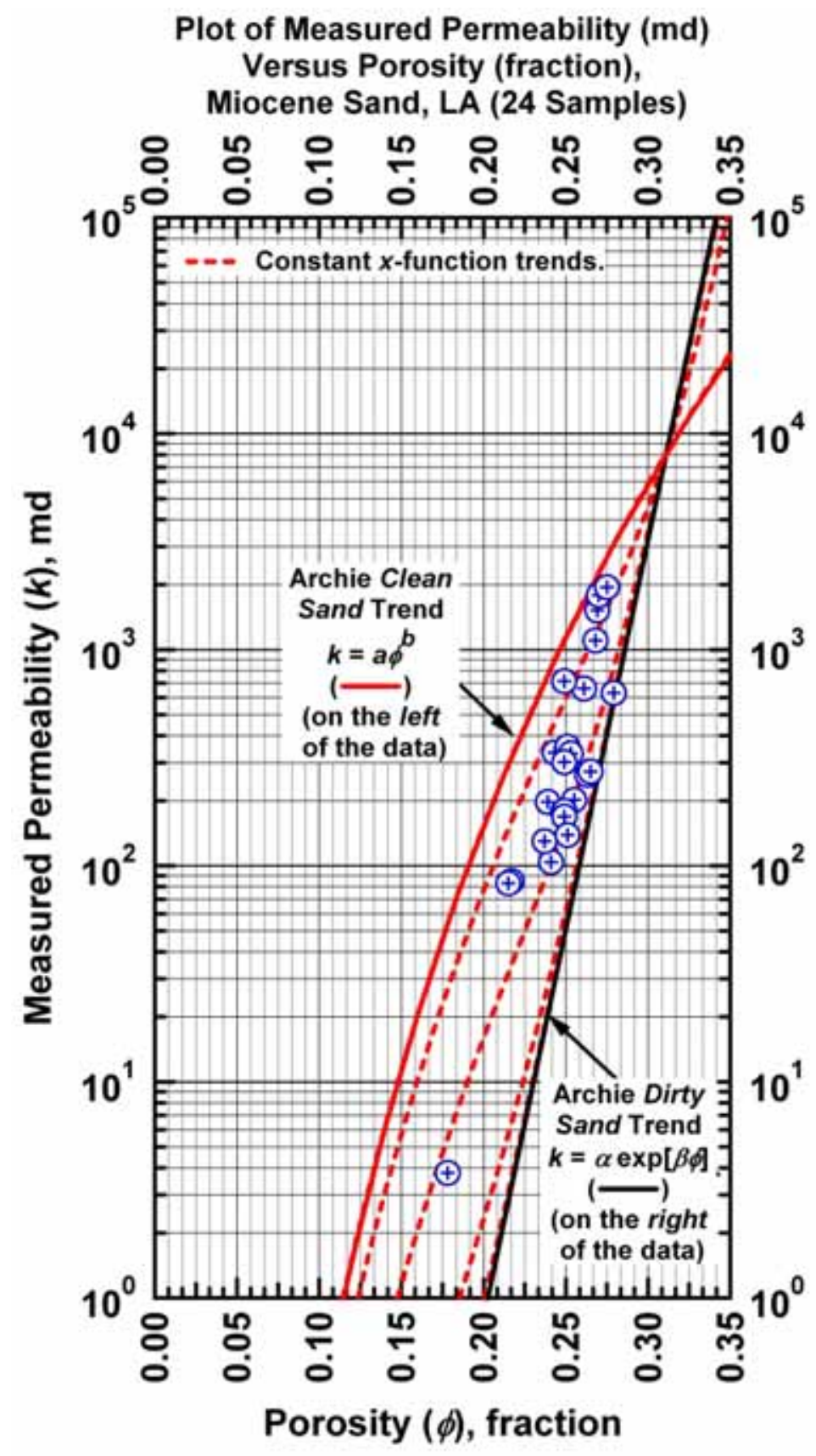

Figure C.7b - Model CPR-D: $k=x a \phi^{b}+(1-x) \alpha \exp [\beta \phi] \quad[0 \leq x \leq 1]$ - Weighted Power Law-Exponential Model used to correlate permeability $(k)$ and porosity $(\phi)$. "Dirty Sand" Plot - Archie "Dirty Sand" trend is given by the straight-line trend at the far right of the data (exponential model). [Case: Miocene Sand, S. LA (USA)] 


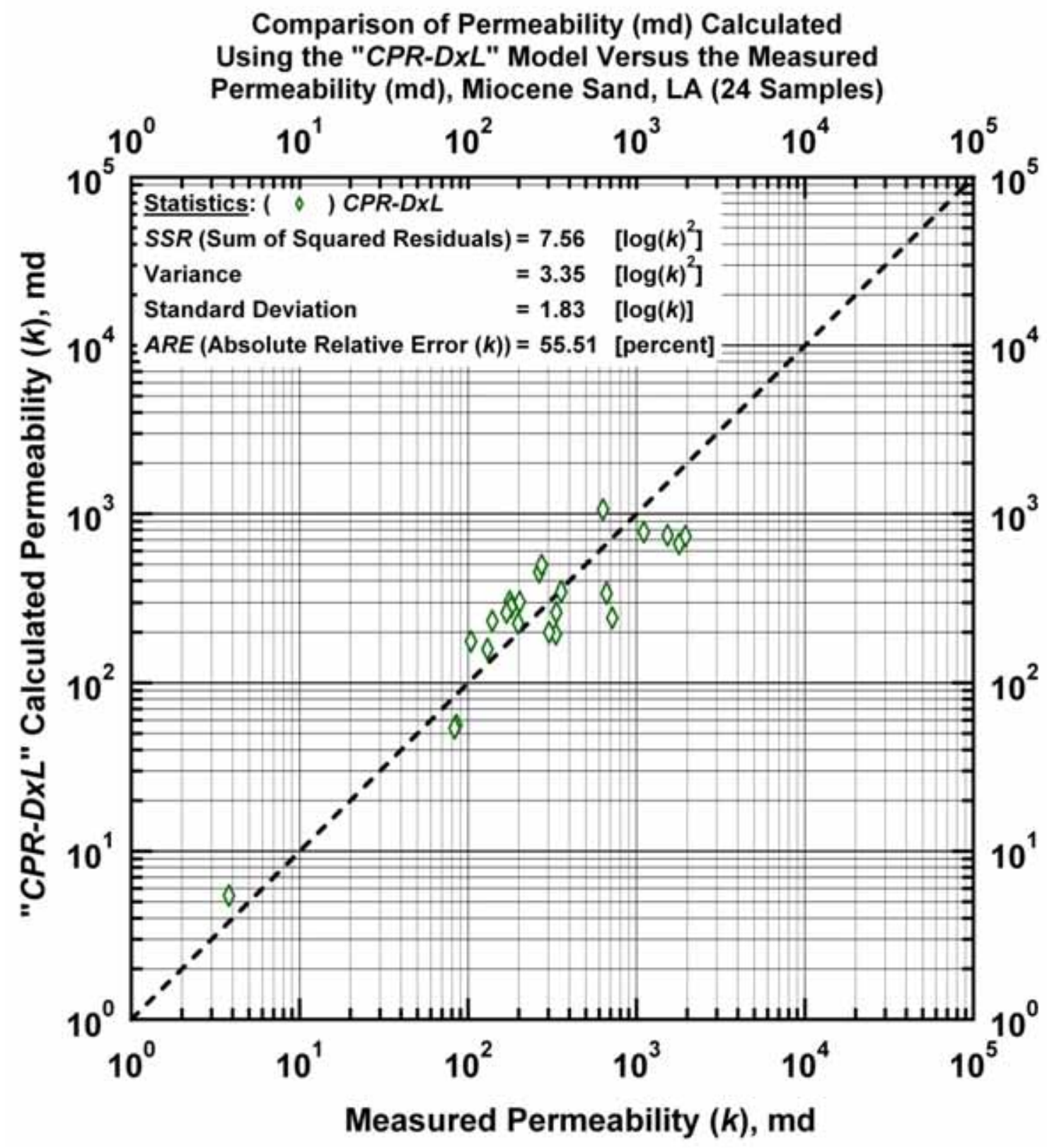

Figure C.8a - Model CPR-DxL: Calculated versus measured permeability. [Case: Miocene Sand, S. LA (USA)] 


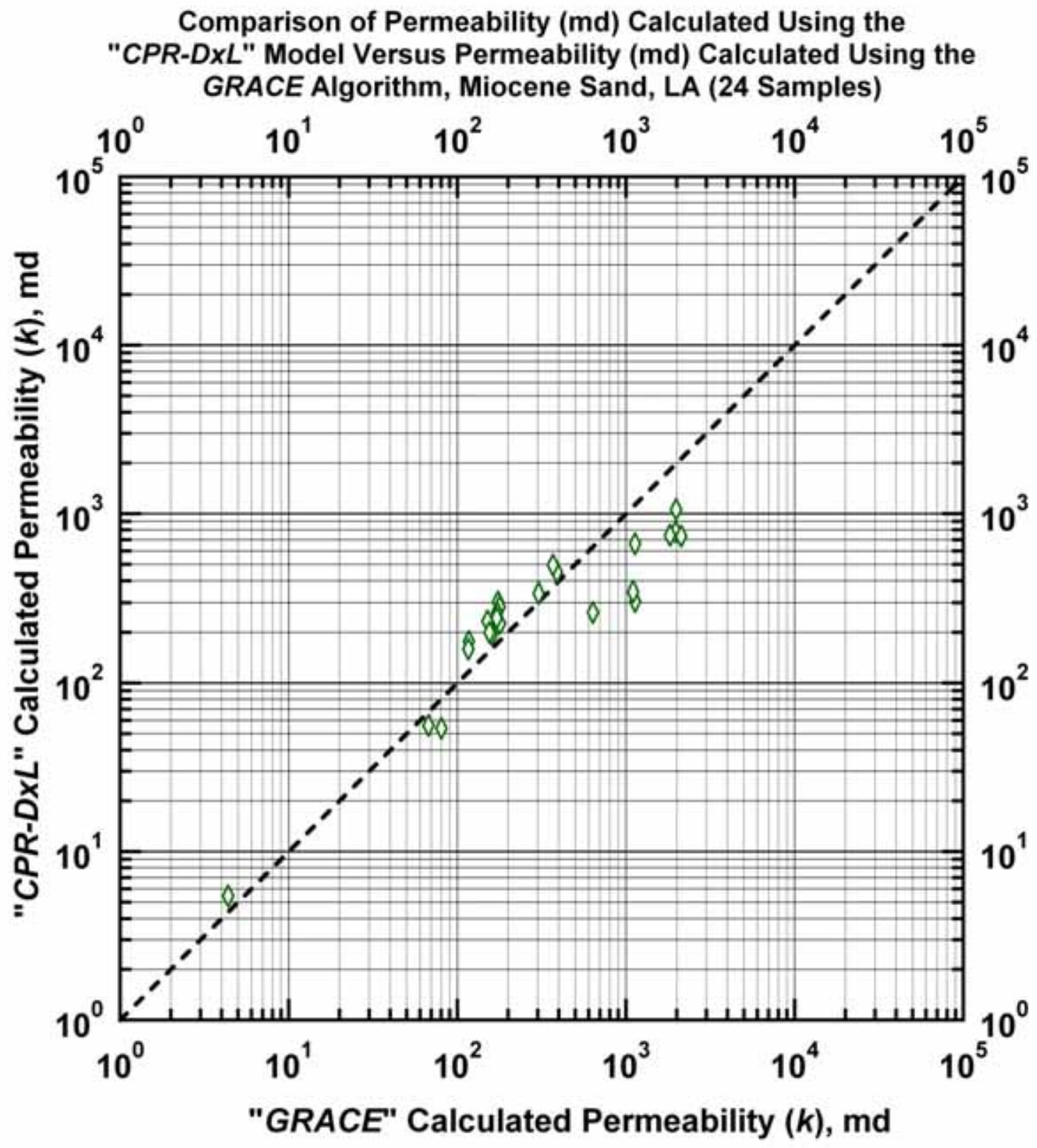

Figure C.8b - Model $C P R-D x L$ : Permeability calculated using Model $C P R-C$ versus permeability calculated using the GRACE Algorithm. [Case: Miocene Sand, S. LA (USA)] 


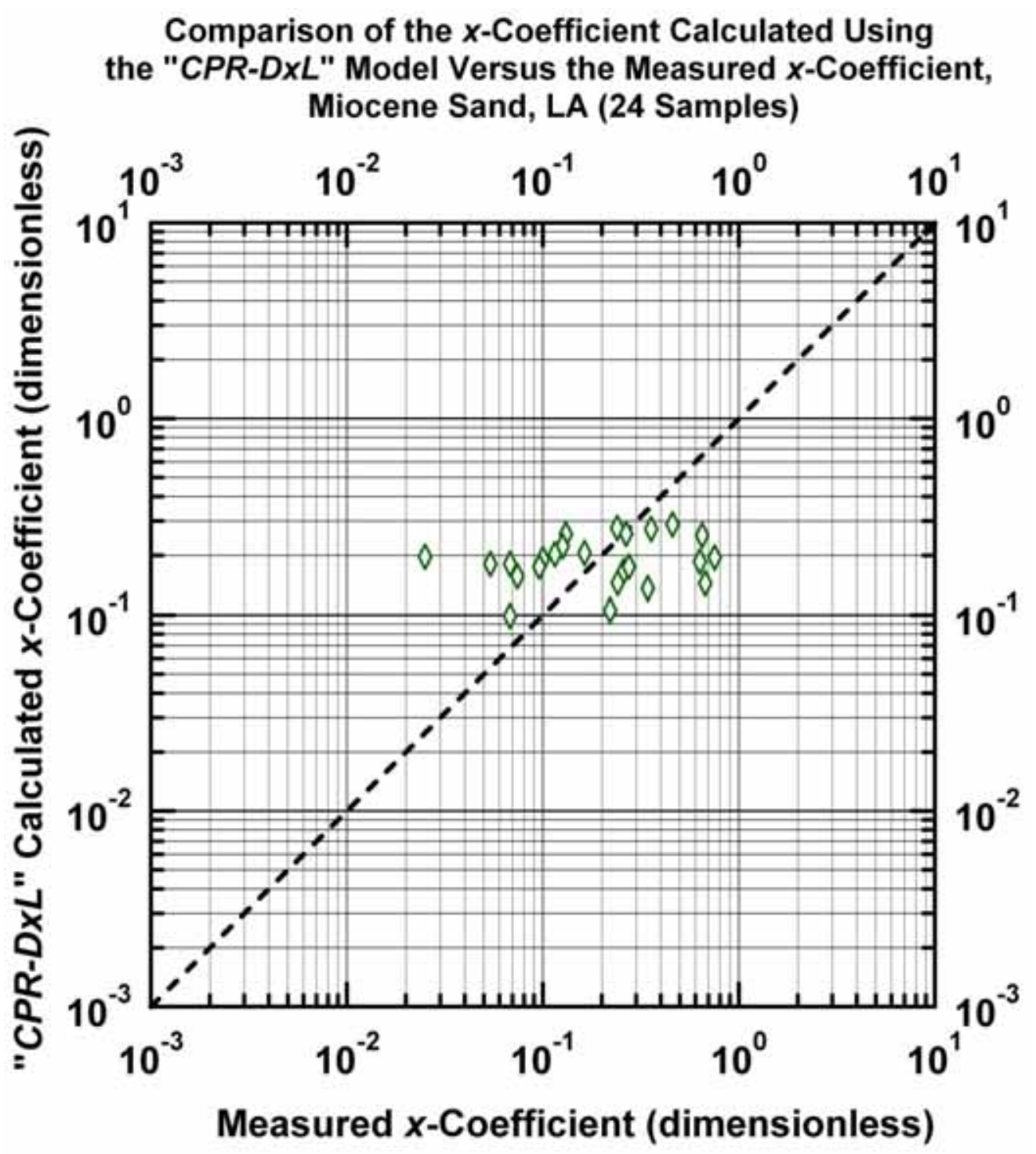

Figure C.8c - Model CPR-DxL: Calculated $x$-function weight values versus measured $x$ function weight values. [Case: Miocene Sand, S. LA (USA)] 
Plot of Depth (ft) Versus Measured and Calculated Permeability (md), Miocene Sand, LA (24 Samples)

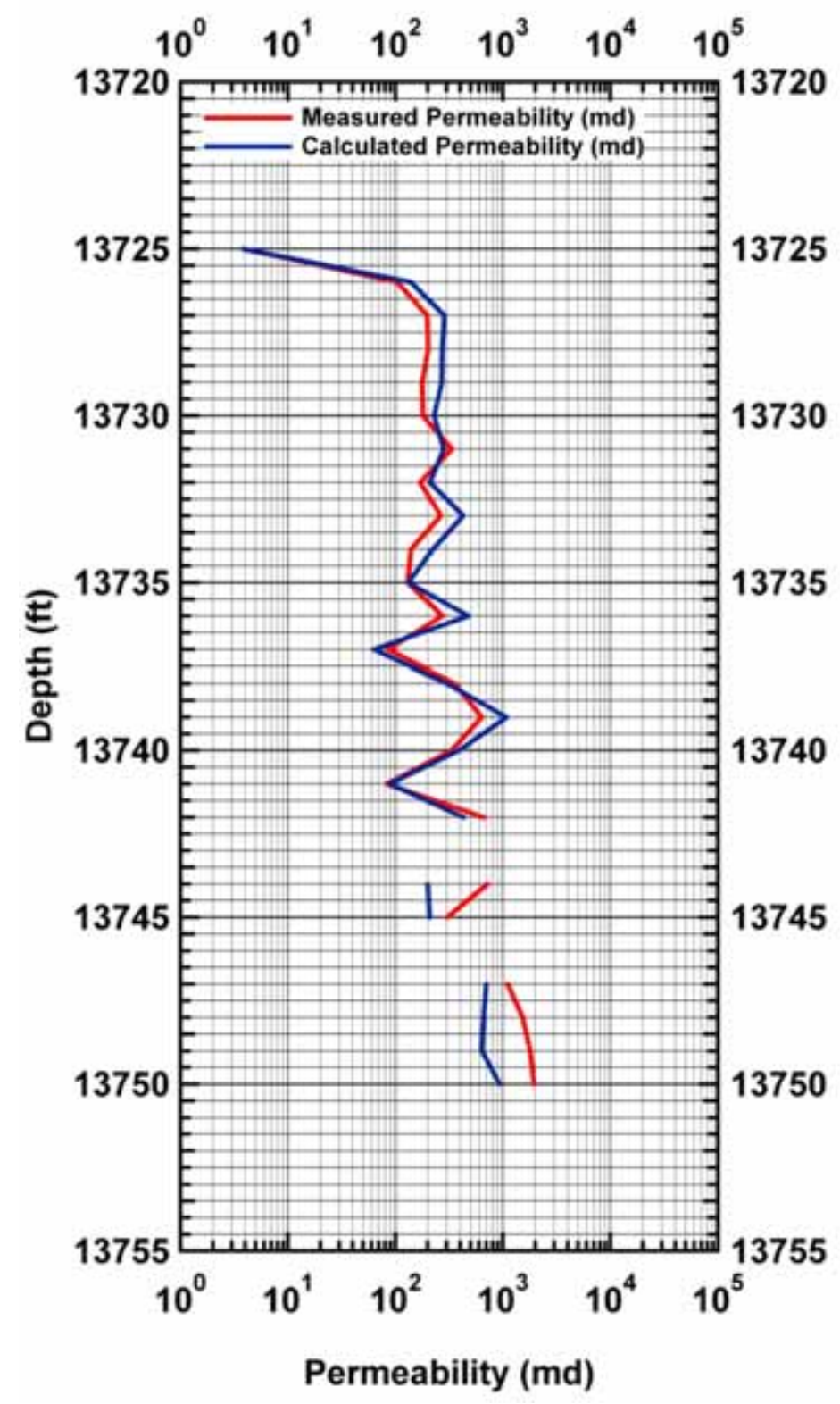

Figure C.8d - Model CPR-A: Calculated and measured permeability values versus depth. [Case: Miocene Sand, S. LA (USA)] 


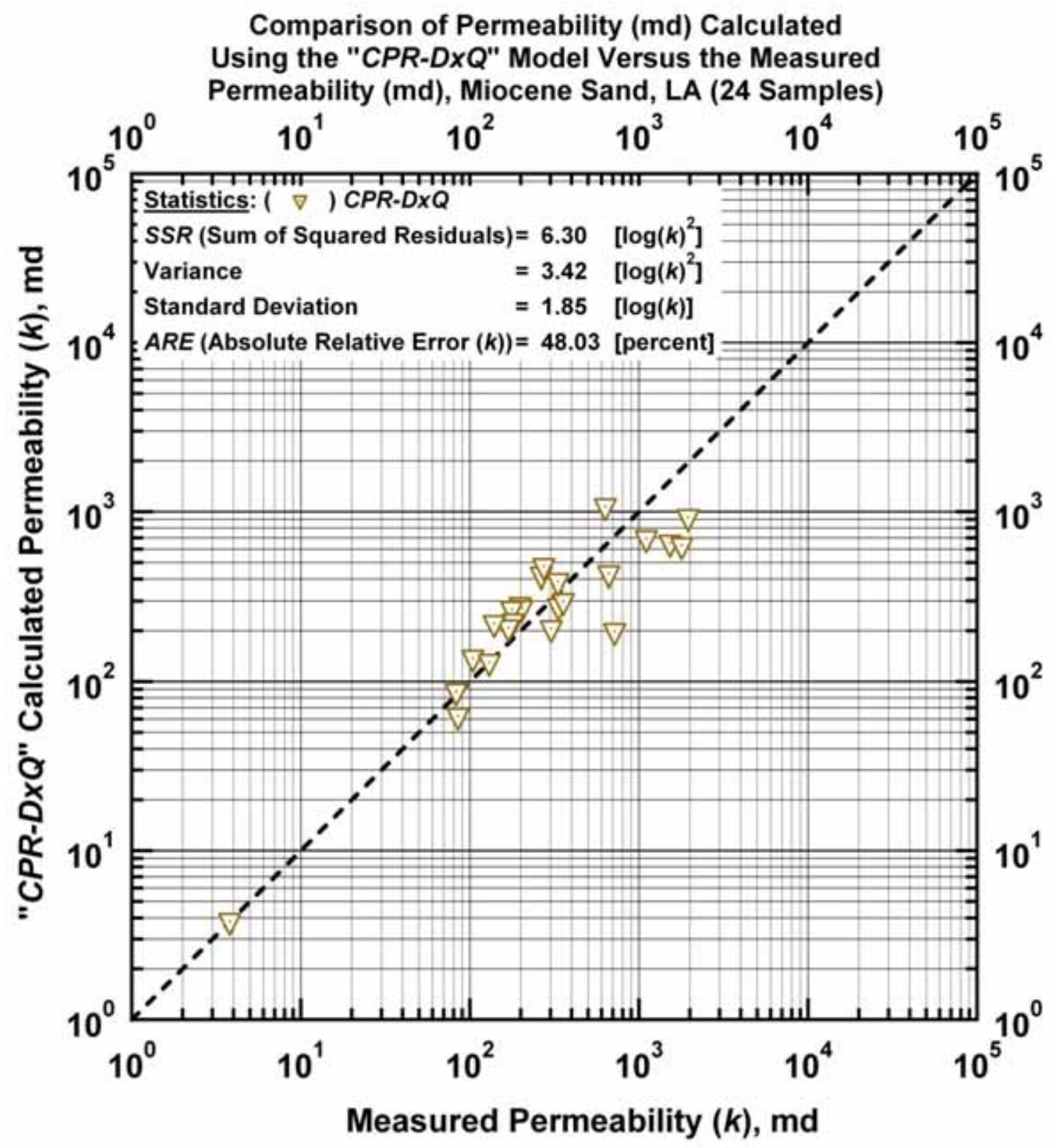

Figure C.9a - Model CPR-DxQ: Calculated versus measured permeability. [Case: Miocene Sand, S. LA (USA)] 


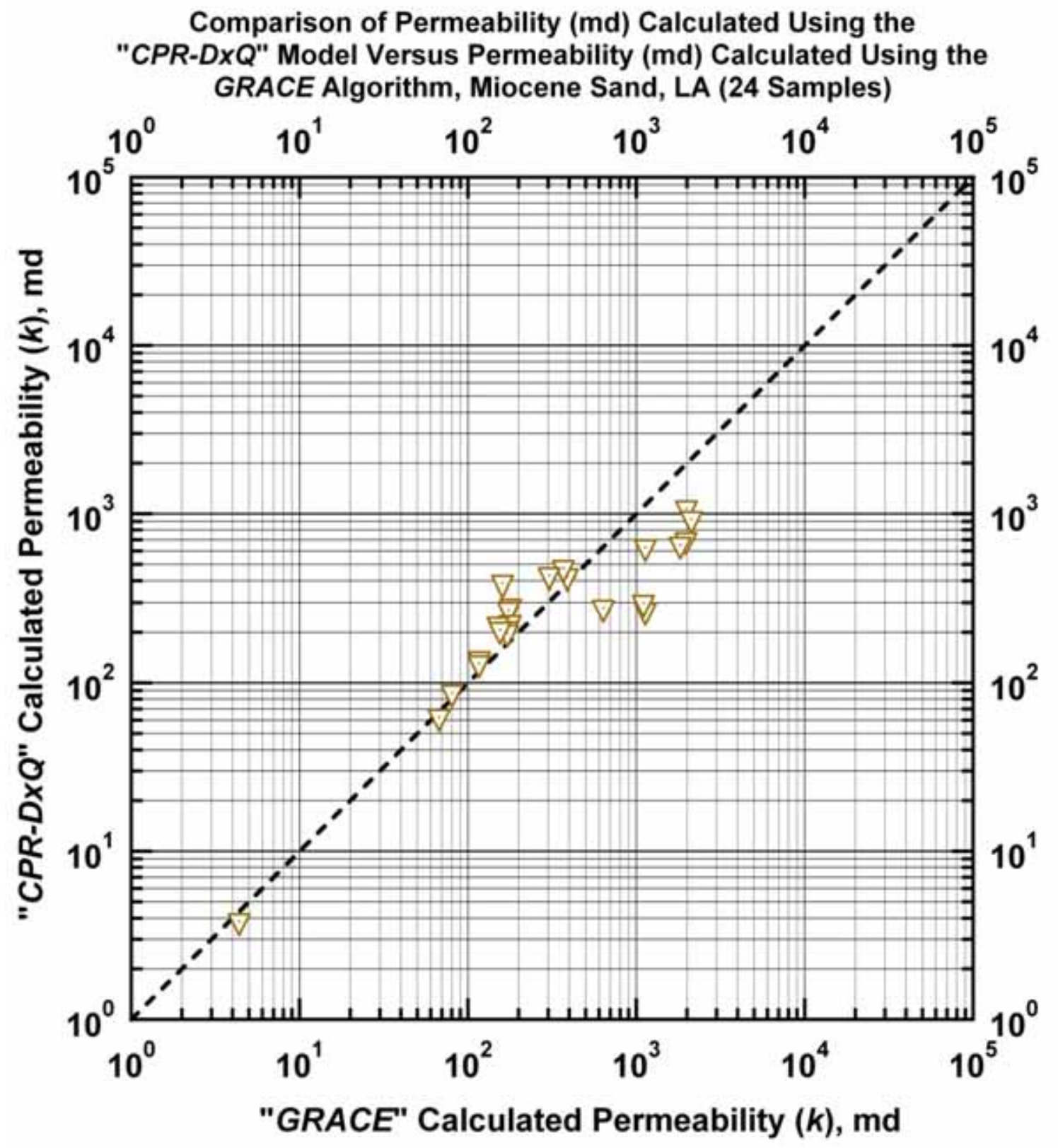

Figure C.9b - Model $C P R-D x Q$ : Permeability calculated using Model $C P R-C$ versus permeability calculated using the GRACE Algorithm. [Case: Miocene Sand, S. LA (USA)] 


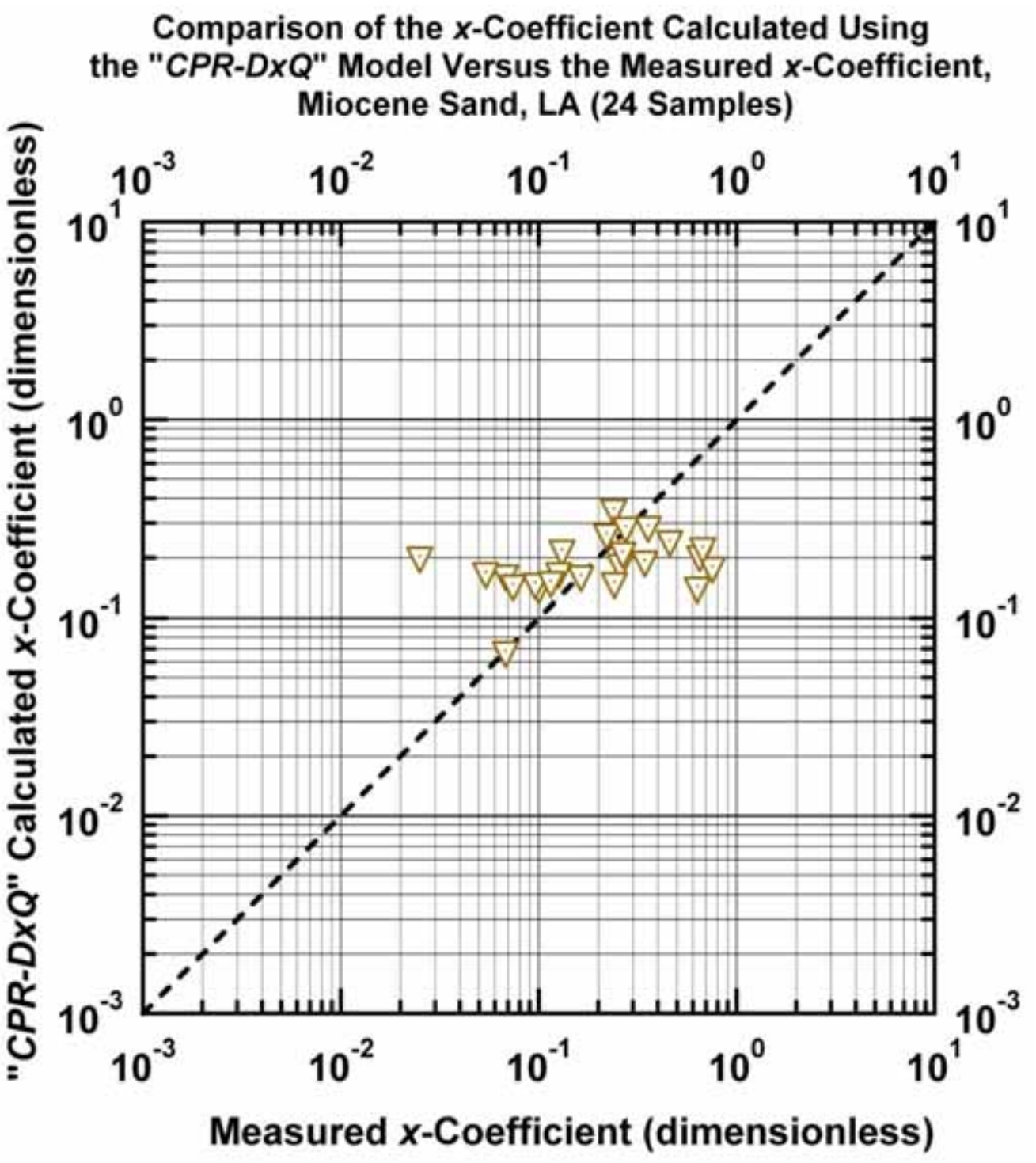

Figure C.9c - Model CPR-DxQ: Calculated $x$-function weight values versus measured $x$ function weight values. [Case: Miocene Sand, S. LA (USA)] 
Plot of Depth ( $\mathrm{ft}$ ) Versus Measured and Calculated Permeability (md), Miocene Sand, LA (24 Samples)

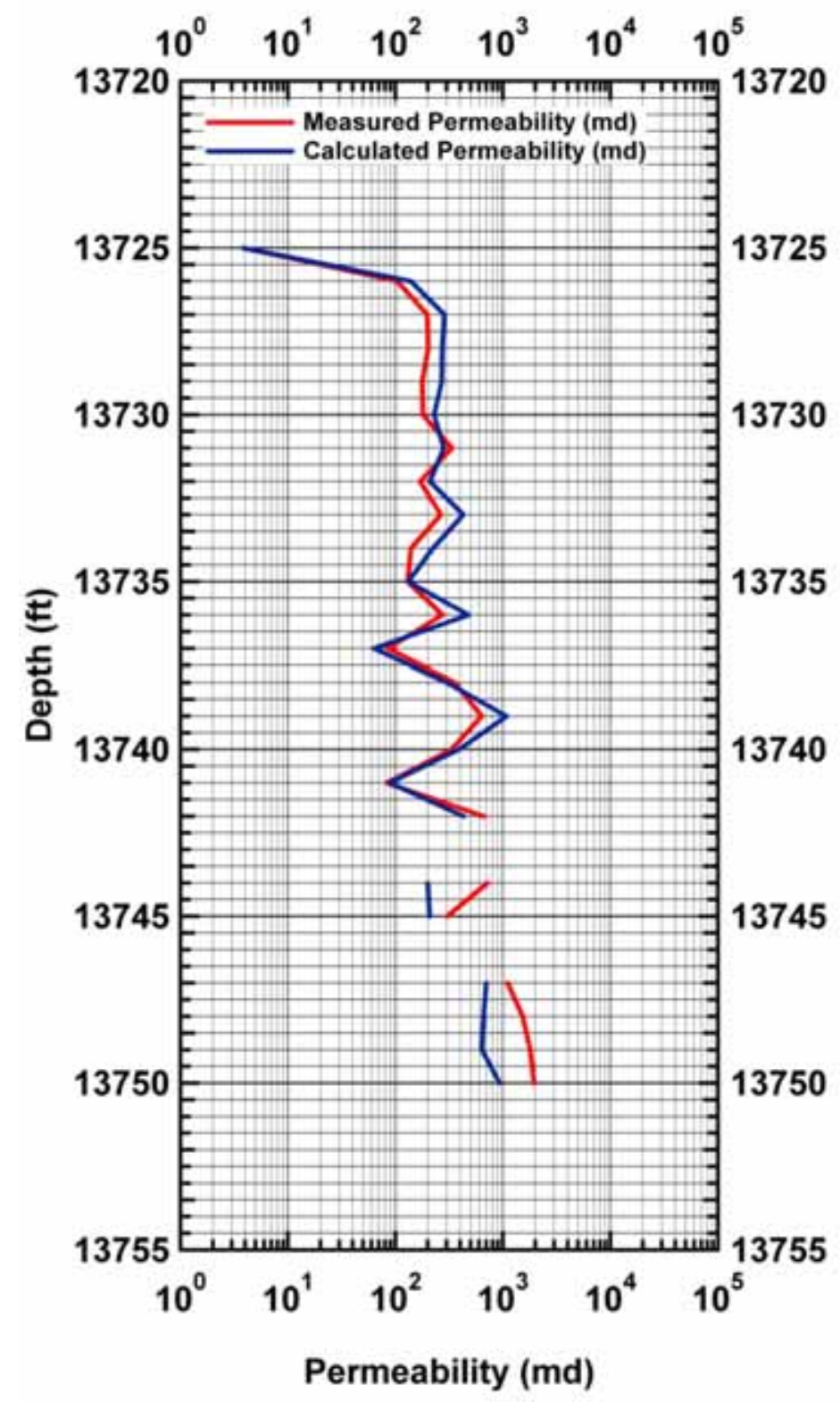

Figure C.9d - Model CPR-A: Calculated and measured permeability values versus depth. [Case: Miocene Sand, S. LA (USA)] 


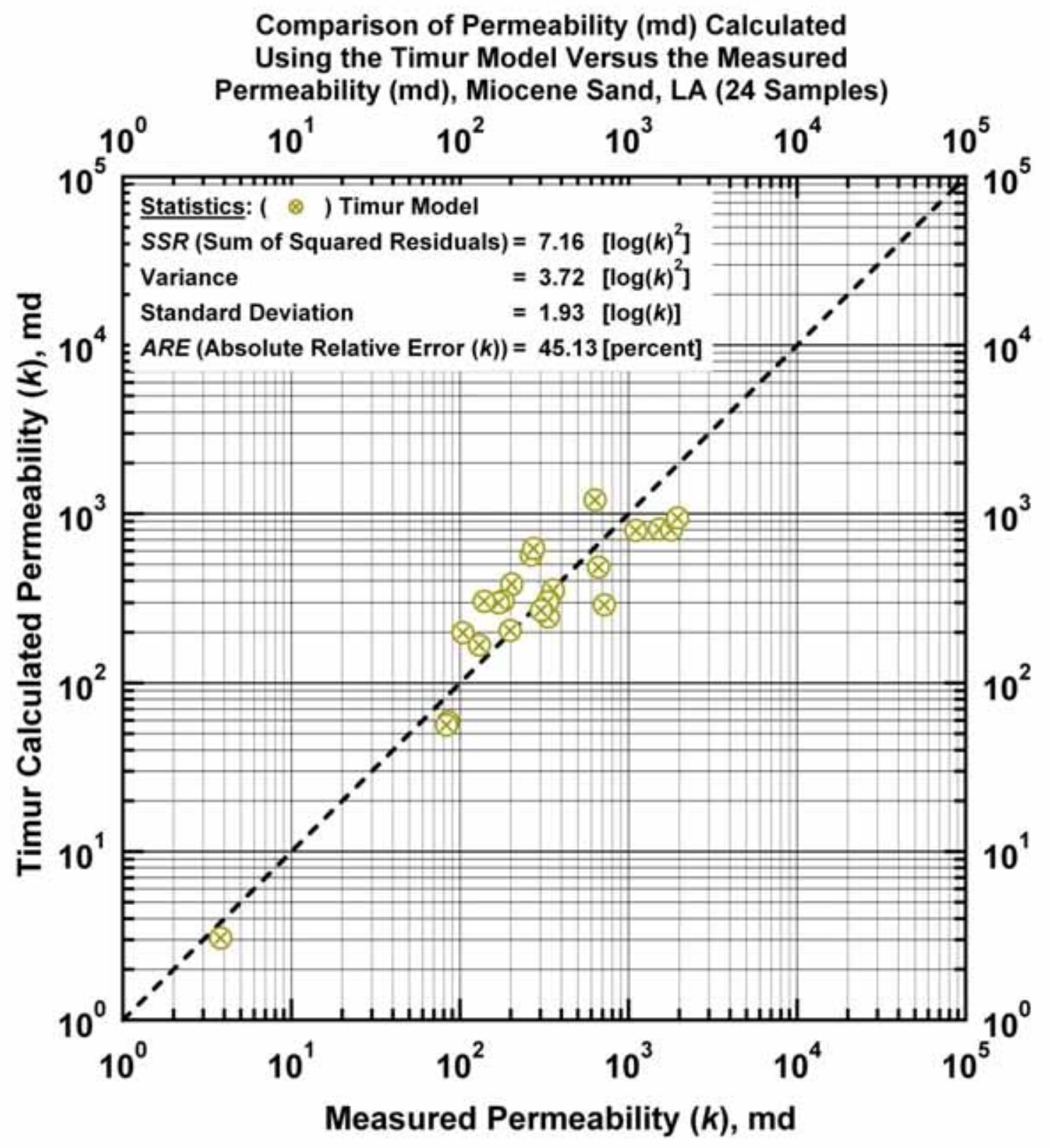

Figure C.10a - "Timur Model:" Calculated versus measured permeability. [Case: Miocene Sand, S. LA (USA)] 


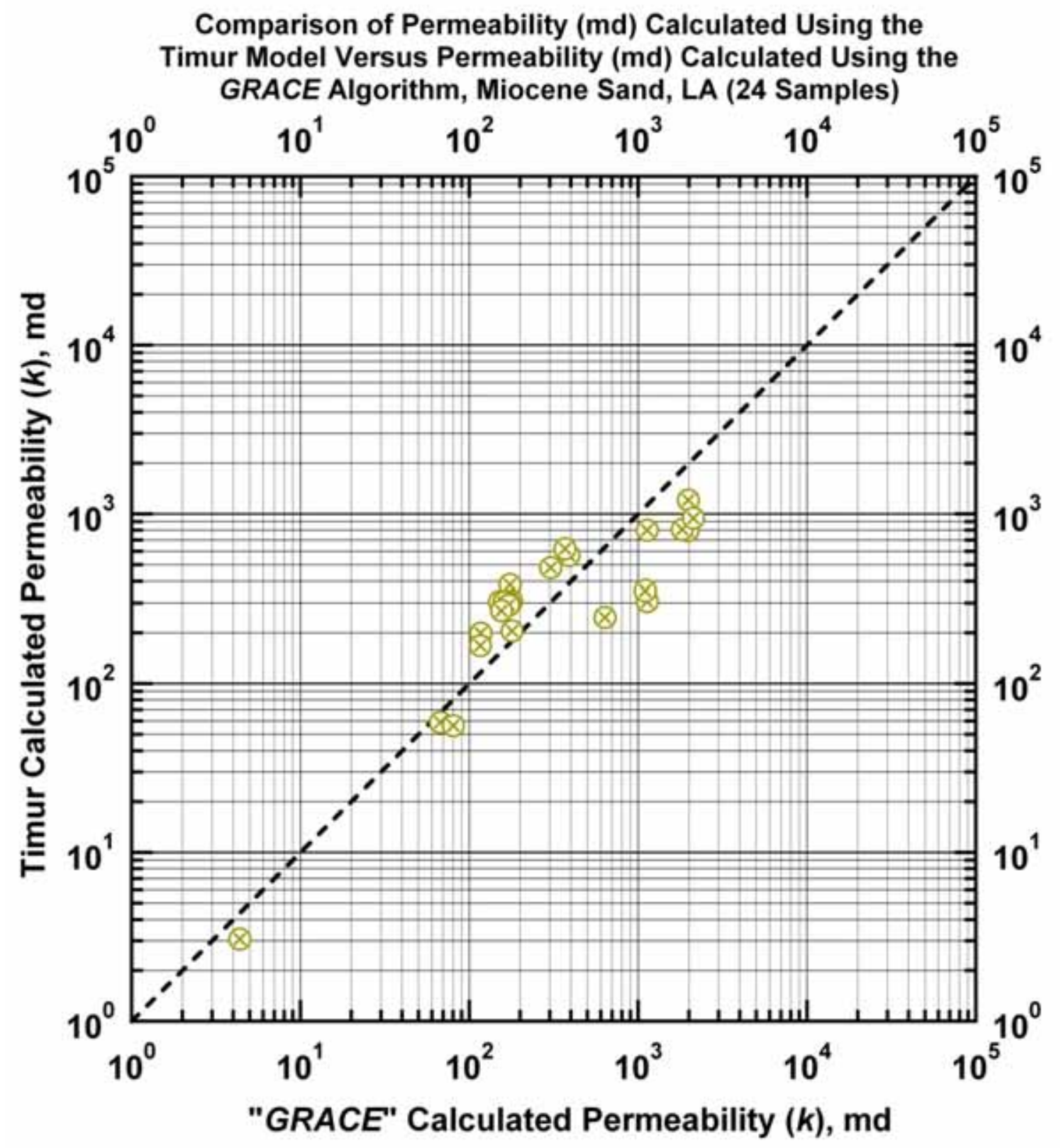

Figure C.10b — "Timur Model:" Permeability calculated using the "Timur Model" versus permeability calculated using the GRACE Algorithm. [Case: Miocene Sand, S. LA (USA)] 


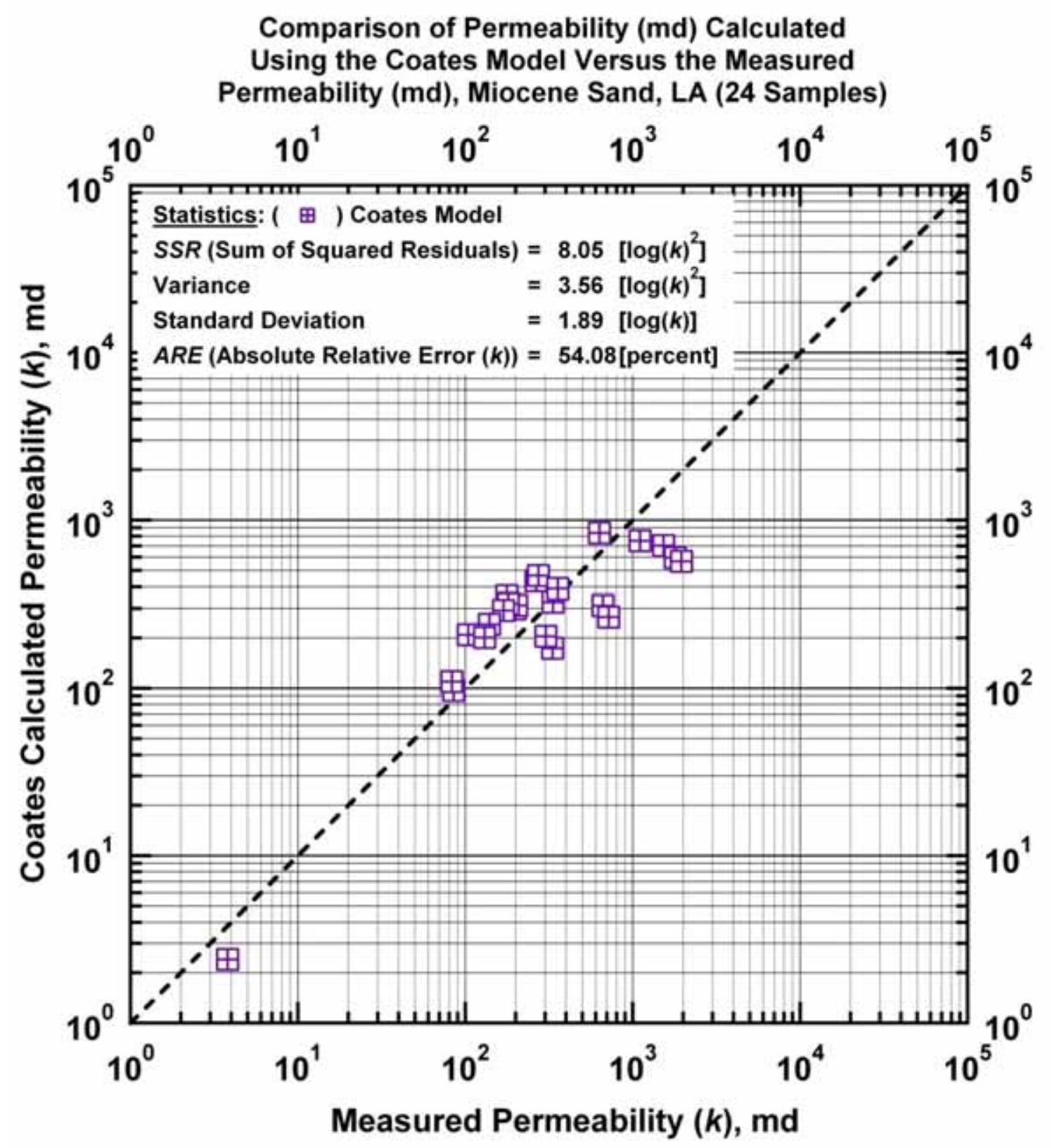

Figure C.11a - "Coates Model:" Calculated versus measured permeability. [Case: Miocene Sand, S. LA (USA)] 


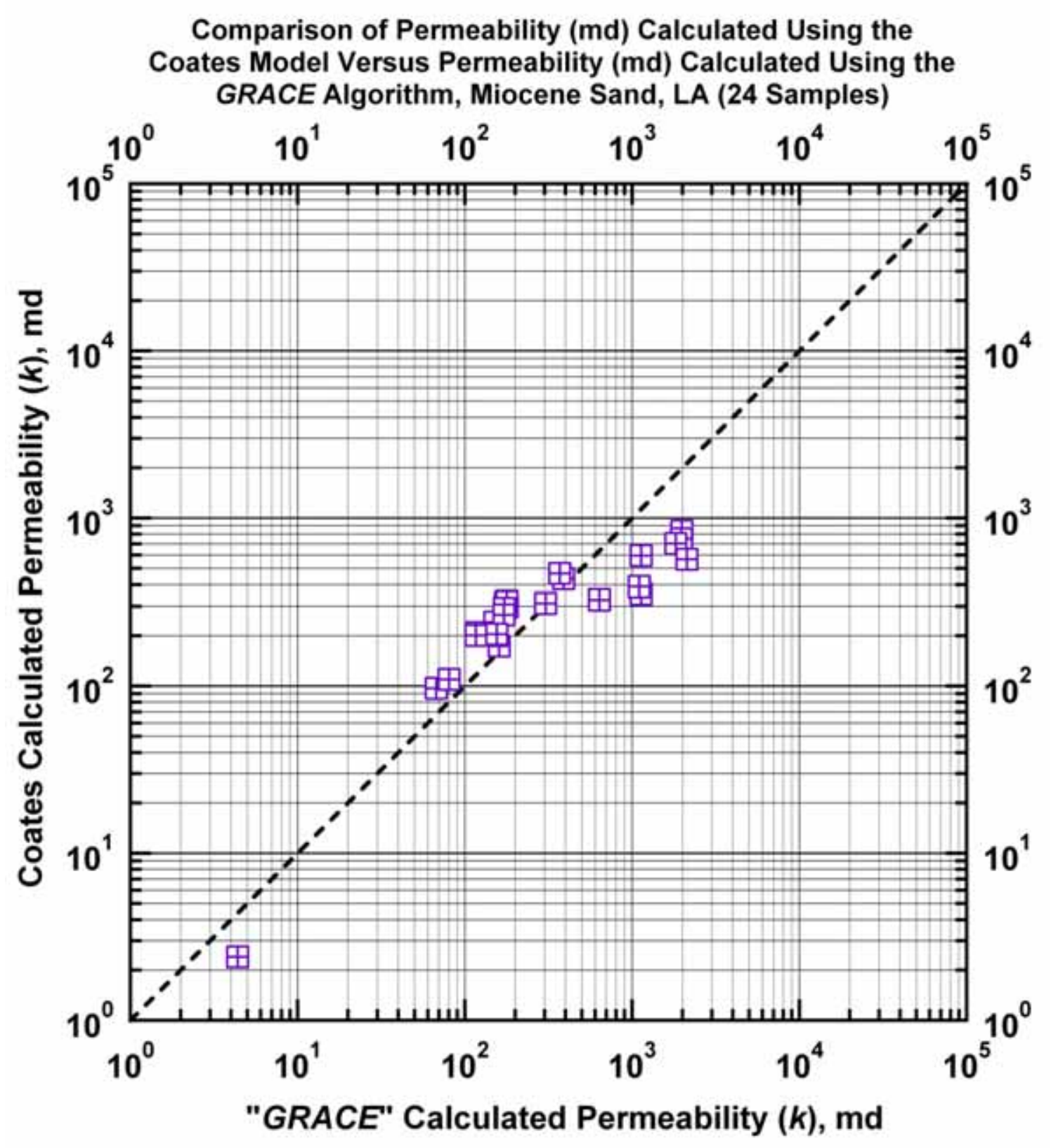

Figure C.11b - "Coates Model:" Permeability calculated using the "Coates Model" versus permeability calculated using the GRACE Algorithm. [Case: Miocene Sand, S. LA (USA)] 


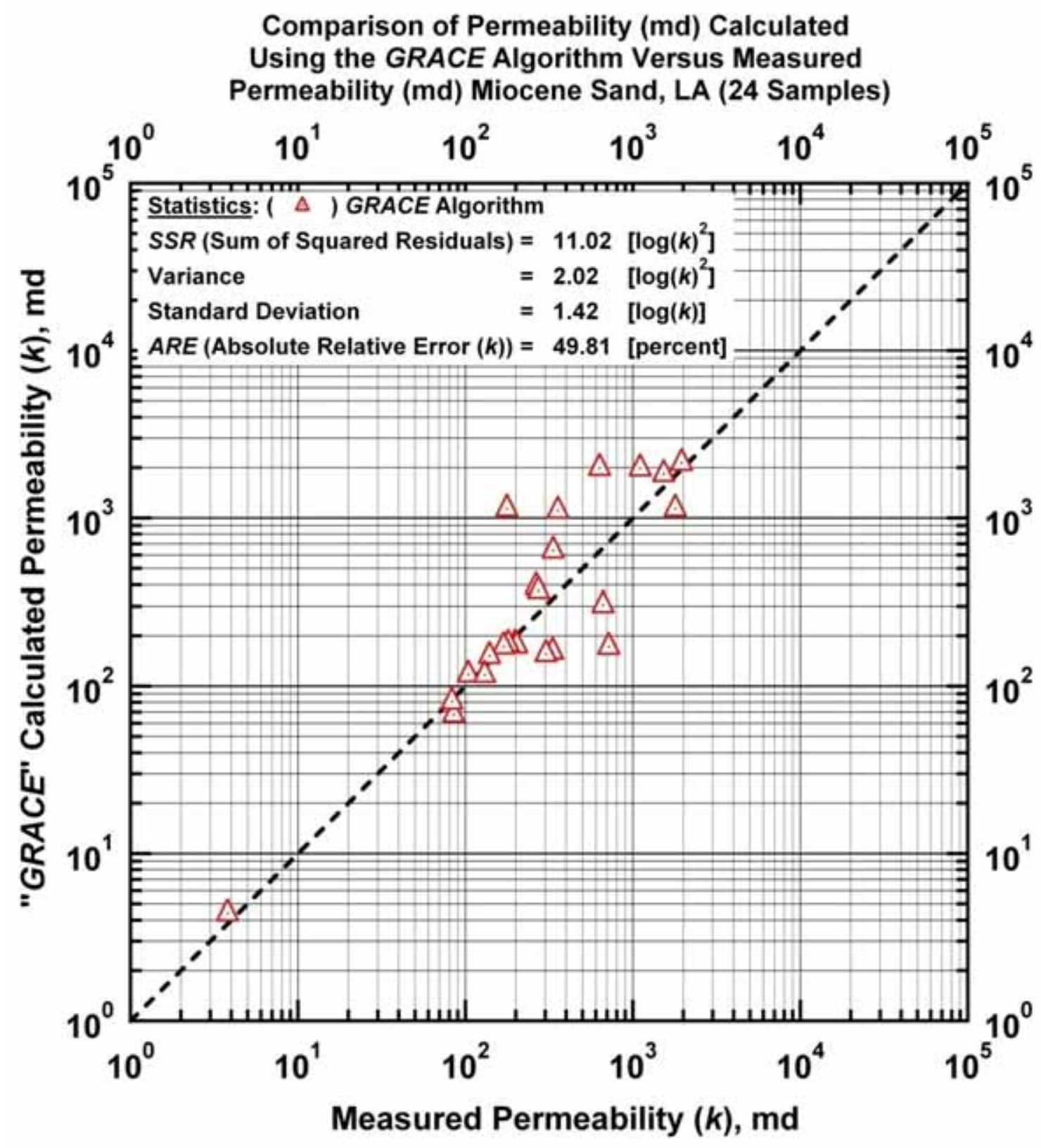

Figure C.12 - GRACE Model: Calculated versus measured permeability. [Case: Miocene Sand, S. LA (USA)] 


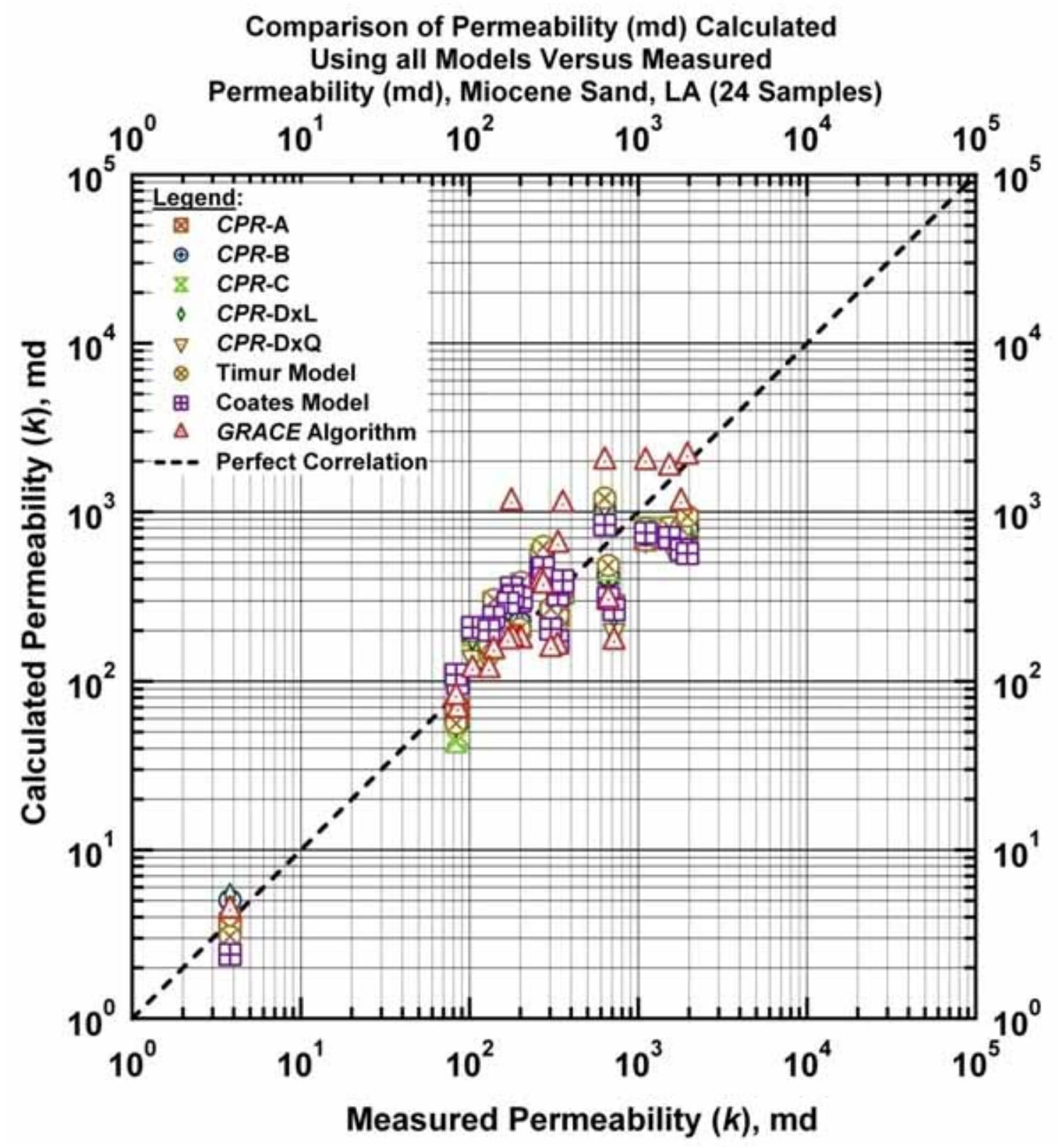

Figure C.13 - All Models: Calculated versus measured permeability. [Case: Miocene Sand, S. LA (USA)] 

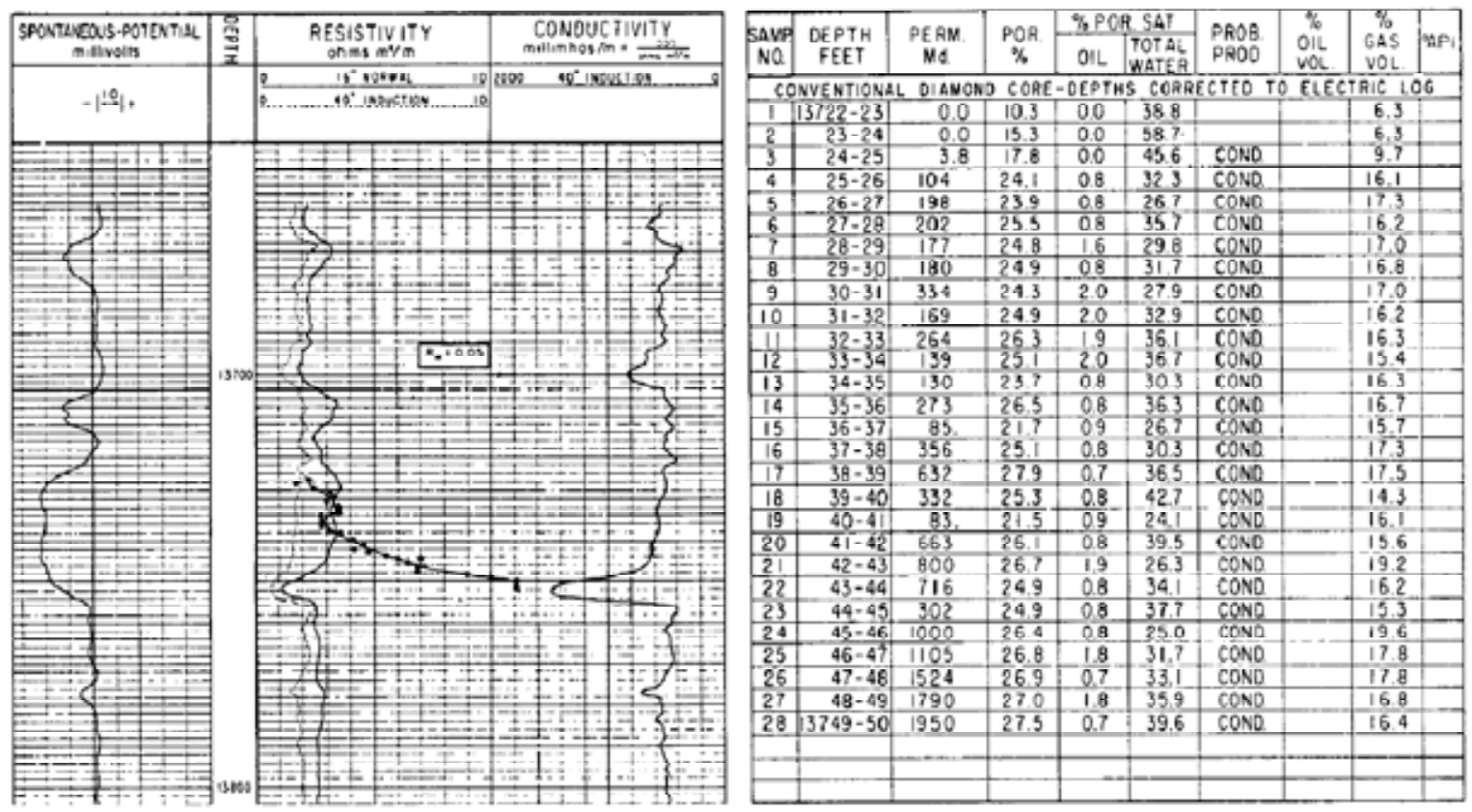

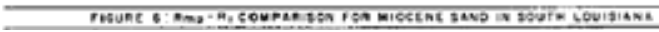

Figure C.14 - Original data from Granberry and Wilshusen (1962). [Case: Miocene Sand, S. LA (USA)] 


\section{APPENDIX D \\ RESULTS FOR VICKSBURG SAND \\ (S. TEXAS - USA)}

In this Appendix we present the Vicksburg Sand, located in S. Texas (USA), - this is a sandstone reservoir (Granberry and Wilshusen (1962)).

\section{Model CPR-A: (Appendix D)}

The "characteristic permeability relation" (or CPR) Model CPR-A (i.e., the modified Archie "Clean Sand" Model) is given as:

$$
k=a(\phi-c)^{b} \quad c=c_{\max } \exp \left[-c_{1} \phi^{c_{2}} S_{w}^{c_{3}}\right]
$$

Where a plot of $\log (k)$ versus $\log (\phi)$ is used to graphically calibrate Eq. D-1 in terms of the coefficients $a, b$, and $c_{\max }$. Fig. D.1 shows that the $C P R-A$ model performs reasonably well for this case. Figs. D.1a and $\mathbf{b}$ show the $\log -\log$ and the semilog format of permeability versus porosity, respectively. In Fig. D.2 we provide the "error" plots for this case, where the measured and computed data are compared systematically. In Fig. D.2a we note a good correlation of the results obtained using Model CPR-A versus the measured permeability data - we observe good agreement with the perfect correlation trend (i.e., the 45degree line). In Fig. D.2b we provide a comparison with the GRACE algorithm correlation (Xue et al. 1997)), and we note that the CPR-A model results correlate very well with the GRACE algorithm results for this case. Fig. D.2c we present the calculated and measured $c$-function - and again we note a reasonable correlation of the data about the perfect correlation trend. Lastly, in Fig. D.2d we present the measured and calculated permeability with depth.

\section{Model CPR-B: (Appendix D)}

The "characteristic permeability relation" (or CPR) Model CPR-B (i.e., the modified Archie "Dirty Sand" Model (power-law basis)) is given as:

$$
k=a(\phi+c)^{b} \quad c=c_{\max } \exp \left[-c_{1} \phi^{c_{2}} S_{w}^{c_{3}}\right]
$$

Where a plot of $\log (k)$ versus $\log (\phi)$ is used to graphically calibrate Eq. D-2 in terms of the coefficients $a, b$, and $c_{\max }$. The results for this case are presented in Figs. D.3 and 4. In Fig. D.3a (log-log format) we note that a very distinct "envelope" is formed by the CPR-B model, essentially all of the data on the right and left flanks are well-matched (i.e., the "dirty sand" power law relation (applied on the right portion of the data) and its correction function (applied on the left portion of the data). Similarly, in Fig. D.3b (semilog format), we observe an excellent distribution of the power law model across the body of the data. The error analyses for this case are shown in Fig. D.4. In Fig. D.4a we present the results obtained using 
Model $C P R-B$ versus the measured permeability data and in this case there is good agreement with the perfect correlation trend (i.e., the 45-degree line). We provide a comparison with the GRACE algorithm correlation in Fig. D.4b - the results using Model CPR-B and the GRACE algorithm compare very well for this case. We compare the computed and data-derived values of the $c$-function for this case in Fig. D.4c and we note a reasonably "tight" correlation, except for the very lowest values of the $c$-function. Finally, in Fig. D.4d we present the measured and calculated permeability with depth. In summary, we believe that Model $C P R-B$ is a very effective correlation model for this particular data case.

\section{Model CPR-C: (Appendix D)}

The "characteristic permeability relation" (or CPR) Model CPR-C (i.e., the modified Archie "Dirty Sand" Model (exponential basis)) is given as:

$$
k=c \exp [\beta \phi] \quad c=c_{\max } \exp \left[-c_{1} \phi^{c_{2}} S_{w}^{c_{3}}\right]
$$

Where a plot of $\log (k)$ versus $\phi$ is used to graphically calibrate Eq. D-3 in terms of the coefficients - $\beta$ and $c_{\max }$. Model $C P R-C$ is fundamentally different than Models $C P R-A$ and $C P R-B$, as the basis for Model $C P R-C$ is an exponential function, not a power law relation. In Figs. D.5 and 6 we present the results of applying Model $C P R-B$ for this case, and in general, we observe a very consistent performance of Model $C P R-B$. Fig. D.5b illustrates the excellent conformance of Model $C P R-C$ for this case - again recalling that this model has an exponential relationship with porosity as its basis.

In Figs. D.6a, D.6b, and D.6c we review the error analyses plots for this case, and we note good performance (visually) in terms of the correlation of the results using Model CPR-C. The correlation of the $c$-function (see Fig. D.6c) appears to be consistent. We believe that the $C P R$ - $C$ model has performed well for this case, and Fig. D.6 confirms the value of this model as a "characteristic relation" for permeability.

\section{Model CPR-D: (Appendix D)}

The "characteristic permeability relation" (or $C P R$ ) Model CPR-D (i.e., the weighted power law-exponential model) is given in its fundamental form as:

$$
\left.k=x a \phi^{b}+(1-x) \alpha \exp [\beta \phi] \quad[0 \leq x \leq 1]\right]
$$

For Eq. D-4 there are two proposed models for the $x$-function (i.e., the weighting function) - these are:

$$
\begin{aligned}
& x=\exp \left[-\ln \left(x_{0}\right)+x_{1} \ln (\phi)+x_{2} \ln \left(S_{w}\right)+x_{3} \ln (\phi) \ln \left(S_{w}\right)\right] \text { (Model CPR-DxL) } \\
& x=\exp \left[\begin{array}{l}
-\ln \left(x_{0}\right)+x_{1} \ln (\phi)+x_{2} \ln \left(S_{w}\right)+x_{3} \ln (\phi) \ln \left(S_{w}\right) \\
+x_{4} \ln (\phi)^{2}+x_{5} \ln \left(S_{w}\right)^{2}+x_{6} \ln (\phi)^{2} \ln \left(S_{w}\right) \\
+x_{7} \ln (\phi) \ln \left(S_{w}\right)^{2}+x_{8} \ln (\phi)^{2} \ln \left(S_{w}\right)^{2}
\end{array}\right] \text { (Model CPR-DxL)},
\end{aligned}
$$

As noted in Eq. D-4b, Model CPR-DxL utilizes a logarithmic linear model for the weights ( $x$-values) and as seen in Eq. D-4c, Model $C P R-D x Q$ utilizes a logarithmic quadratic model for the weights ( $x$-values). 
While these models (Eqs. D-4b and D-4c) are empirical, we have found generally good application of these relations for all cases. In this case, the base results using Models $C P R-D x L$ and $C P R-D x Q$ are presented in Fig. D.7 - where the power law and exponential basis functions are fitted to the appropriate portion of the data. The power law equation represents the "Archie clean sand" trend and is fitted to the leftmost data as shown in Fig. D.7a and in contrast, the exponential equation is thought to represent the "Archie dirty sand" trend and the exponential is fitted to the far rightmost portion of the data (see Fig. D.7b).

In Figs. D.8 and 9 we present the error analyses for this case, where we have employed the weighting functions (Eqs. D-4b and D-4c) as appropriate. In Fig. D.8a we find a good correlation of permeability. The comparison of the Model CPR-DxL results are compared to the results obtained from the GRACE algorithm in Fig. D.8b, we note the very good results obtained using Model CPR-DxL. The computed weight function ( $x$-values) shown for the $C P R-D x L$ model in Fig. D.8c does exhibit more scatter than expected, but the trend is (relatively) centered about the perfect correlation line. Fig. D.8d provide the measured and calculated permeability versus depth.

The results obtained using Model $C P R-D x Q$ are presented in Fig. D.9, and we note substantially improved behavior over that of Model CPR-DxL. In particular, the base correlation of computed and measured permeabilities (Fig. D.9a) for Model $C P R-D x Q$ does indicate a slightly better correlation than that of Model $C P R-D x L$. Also, the comparison of Model CPR-DxQ with the results from the GRACE algorithm (Fig. D.9b) exhibits better agreement than the results of Model CPR-DxL. Finally, the correlation of the $x$-function (Fig. D.9c) for Model CPR-DxQ is better than the correlation for Model CPR-DxL. These comparisons suggest that Model CPR-DxQ (i.e., the combination of Eqs. D-4a and D-4c) has provided a better correlation of data, for this case, than Model CPR-DxL. Fig. D.9d provides the measured and calculated permeability versus depth.

"Timur Model": (Appendix D)

The "Timur Model" [Timur (1968)] is given as:

$$
k_{\text {Timur }}=a \phi^{b} S_{w}^{c}
$$

In Fig. D.10 we present the results of the "Timur Model" (Eq. D-5) which is essentially just a generalized power-law function relation in terms of $k, \phi$, and $S_{w}$. As shown in Figs. D.10a and b, the "Timur Model" also provides a good correlation of the data for this case. The comparison of results using the "Timur Model" with the results of the GRACE algorithm (Fig. D.10b). 


\section{"Coates Model": (Appendix D)}

The "Coates Model" [referenced by Ahmed et al (1989)] is given as:

$$
k_{\text {Coates }}=\left[100 \phi^{2} \frac{\left(1-S_{w}\right)}{S_{w}}\right]^{2}
$$

In Fig. D.11 we present the results of the "Coates Model" (Eq. D-6) which is essentially just a modified power-law function relation in terms of $k, \phi$, and $S_{w}$. The results are shown in Figs. D.11a and $\mathbf{b}$ of the data for this case. The comparison of results using the "Coates Model" with the results of the GRACE algorithm is also shown in Fig. D.11b.

\section{GRACE Algorithm: (Appendix G)}

In Fig. D.12 we present the results predicted by the GRACE algorithm, which is a non-parametric regression approach (see Xue et al. (1997) for details). In theory, the GRACE algorithm should provide the most unbiased correlation of the data - i.e., the GRACE algorithm is designed not to "fit the errors" as other regression approaches may. It is our contention that the GRACE algorithm is the statistical standard — and any algorithm/approach/model which achieves better regression statistics than the GRACE algorithm is actually "fitting the errors" in the data. None of the cases have significantly better regression statistics than the GRACE algorithm - hence, none of the cases are "over-fitted" in a statistical sense.

\section{Comparison of All Models: (Appendix D)}

Fig. D.13, illustrates all of the models on a single plot of calculated versus measured permeability. We also present a table of all statistical results in Table D.1. Our last plot, Fig. D.14, illustrates the source of the original data set.

Table D.1 - Statistical Results for all Models (Vicksburg Sand, S. TX (USA),

\begin{tabular}{|c|c|c|c|c|}
\hline Model & $\begin{array}{c}\text { Sum of } \\
\text { Squared } \\
\text { Residuals } \\
{\left[\log (k)^{2}\right]}\end{array}$ & $\begin{array}{l}\text { Variance } \\
{\left[\log (k)^{2}\right]}\end{array}$ & $\begin{array}{c}\text { Standard } \\
\text { Deviation } \\
{[\log (k)]}\end{array}$ & $\begin{array}{l}\text { Absolute } \\
\text { Relative } \\
\text { Error } \\
\text { [percent] }\end{array}$ \\
\hline$C P R-A$ & 8.26 & 3.18 & 1.78 & 46.25 \\
\hline$C P R-B$ & 5.13 & 2.73 & 1.65 & 38.79 \\
\hline$C P R-C$ & 6.36 & 2.76 & 1.66 & 40.14 \\
\hline$C P R-D x L$ & 5.42 & 2.62 & 1.62 & 41.06 \\
\hline$C P R-D x Q$ & 4.10 & 2.76 & 1.66 & 31.17 \\
\hline Timur & 8.12 & 2.60 & 1.61 & 45.95 \\
\hline Coates & 5.32 & 2.72 & 1.65 & 35.51 \\
\hline GRACE Algorithm & 4.22 & 2.74 & 1.66 & 28.90 \\
\hline
\end{tabular}

The coefficients for the models used in this case are provided in Table D.2. 
Table D.2a - Model Coefficients (Vicksburg Sand, S. TX (USA), $n=19$ ).

\begin{tabular}{|c|c|c|c|c|c|c|}
\hline Model & $a$ or $\alpha$ & $b$ or $\beta$ & $c$ or $c_{\max }$ & $c_{1}$ & $c_{2}$ & $c_{3}$ \\
\hline CPR-A & $5.00 \times 10^{7}$ & 8.00 & 0.070 & 0.25 & 0.37 & -1.44 \\
\hline$C P R-B$ & $2.00 \times 10^{6}$ & 8.00 & 0.100 & 958.97 & 1.03 & 4.71 \\
\hline$C P R-C$ & $5.00 \times 10^{-8}$ & 88.00 & $1.00 \times 10^{-5}$ & 3116.21 & 3.67 & 0.96 \\
\hline Timur & $7.76 \times 10^{7}$ & 9.83 & 0.971 & (1) & - & - \\
\hline Coates & $6.90 \times 10^{1}$ & 2.36 & 2.656 & - & - & - \\
\hline
\end{tabular}

Table D.2b - Model Coefficients (Vicksburg Sand, S. TX (USA), $n=19$ ).

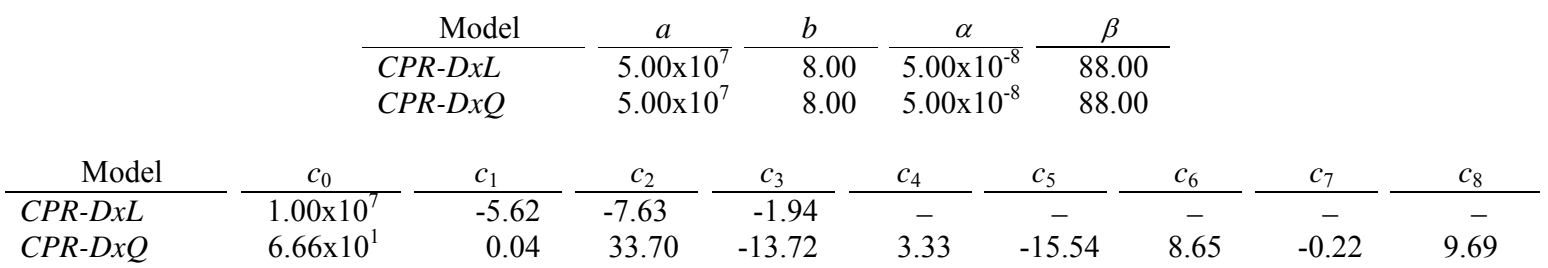

\section{Nomenclature: (Appendix D)}

$$
\begin{array}{ll}
S_{w} & =\text { Water Saturation (fraction) } \\
k & =\text { Formation Permeability, md (or any consistent units) } \\
\phi & =\text { Porosity, fraction }
\end{array}
$$

\section{References: (Appendix D)}

Ahmed, U., Crary, S.F., and Coates, D.R: "Permeability Estimation: The Various Sources and Their Interrelationship," paper SPE 19604 presented at the 1989 SPE Annual Technical Conference and Exhibition, San Antonio, TX, 8-11 Oct., 649-662.

Granberry, R.J., and Wilshusen, R.C.: "Improved Interpretation of Formation Productivity by Combined Use of Core Analysis and Electronic Log Data," Trans., Gulf Coast Association of Geological Societies (1962) 13, 213-221.

Timur, A.: "An Investigation of Permeability, Porosity, and Residual Water Saturation Relationships," Trans., SPWLA Symposium (1968) paper I.

Xue, D., Datta-Gupta, A., Valko, P., and Blasingame, T.A.: "Optimal Transformations for Multiple Regression: Application to Permeability Estimation from Well Logs," SPEFE (June 1997), 85-93. 


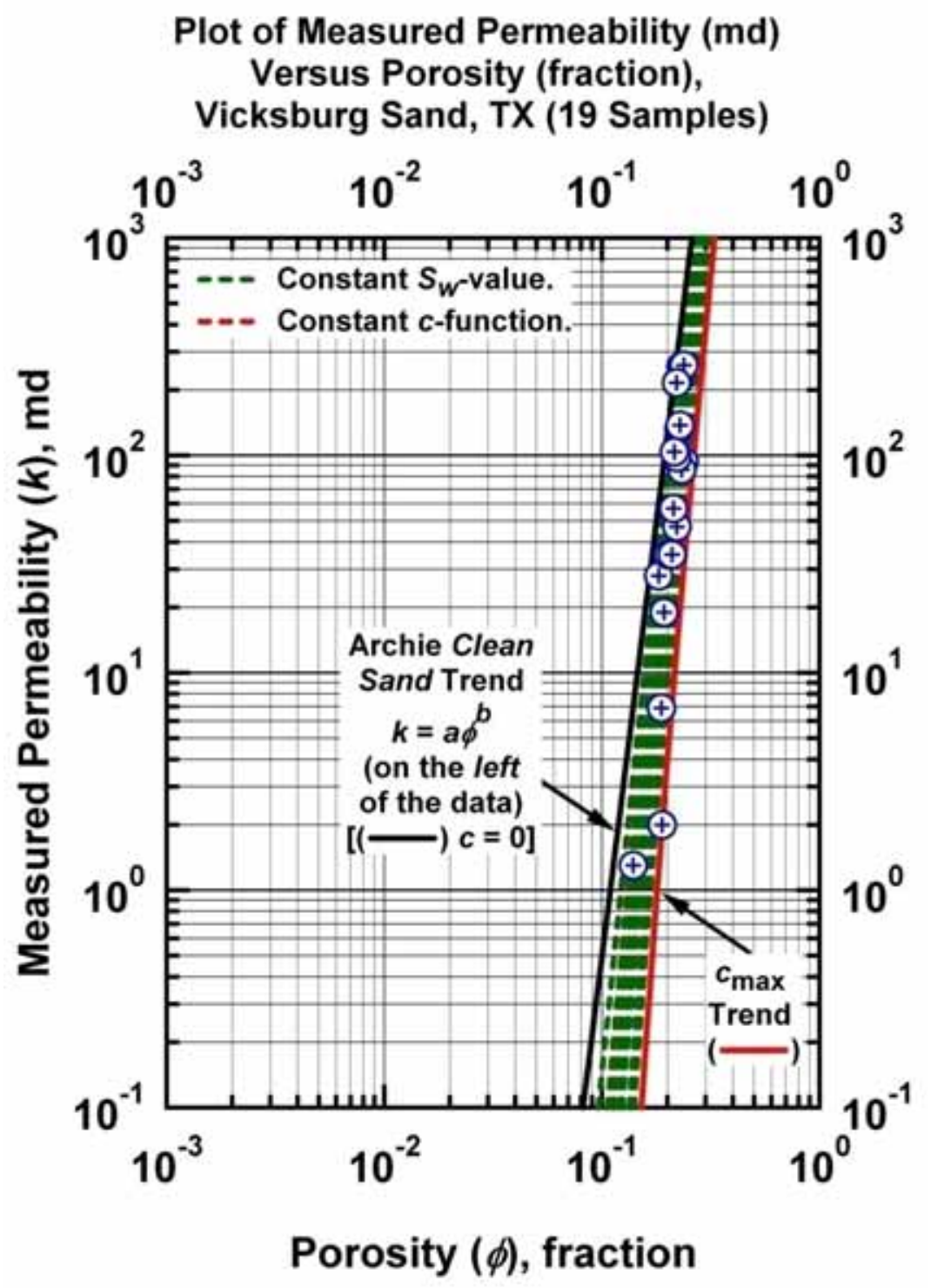

Figure D.1a - Model $C P R-A: \quad k=a(\phi-c)^{b} \quad c=c_{\max } \exp \left[-c_{1} \phi^{c_{2}} S_{w}^{c_{3}}\right] \quad$ - "Clean Sand" Plot (log-log format) - Archie "Clean Sand" trend is given by the straight-line trend at the far left of the data (power law model). [Case: Vicksburg Sand, S. TX (USA)] 


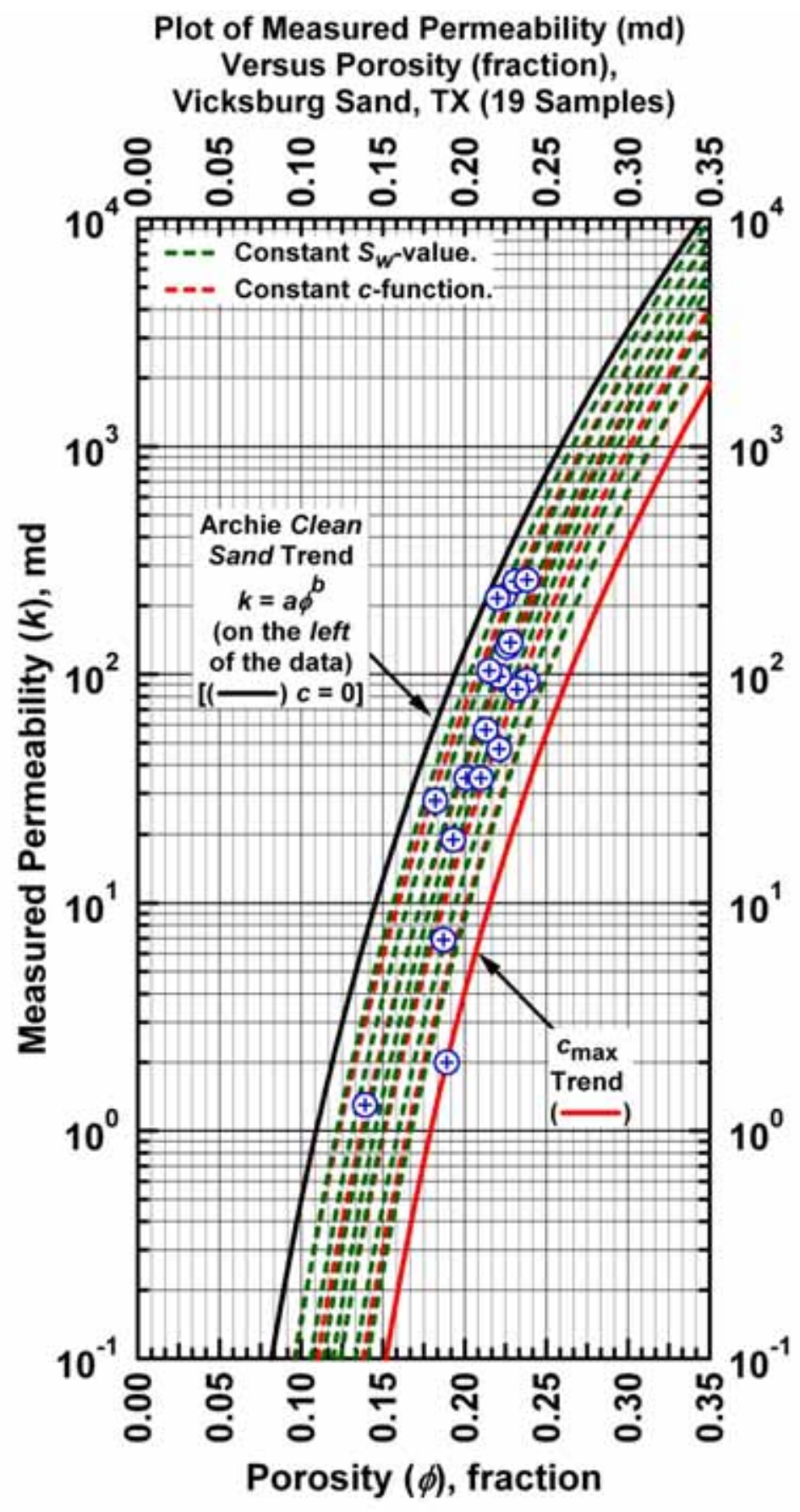

Figure D.1b - Model CPR-A: $k=a(\phi-c)^{b} \quad c=c_{\max } \exp \left[-c_{1} \phi^{c_{2}} S_{w}^{C_{3}}\right]$ - "Dirty Sand" Plot (semilog format). Archie "Clean Sand" trend is given by the curved trend at the far left of the data (power law model). [Case: Vicksburg Sand, S. TX (USA)] 


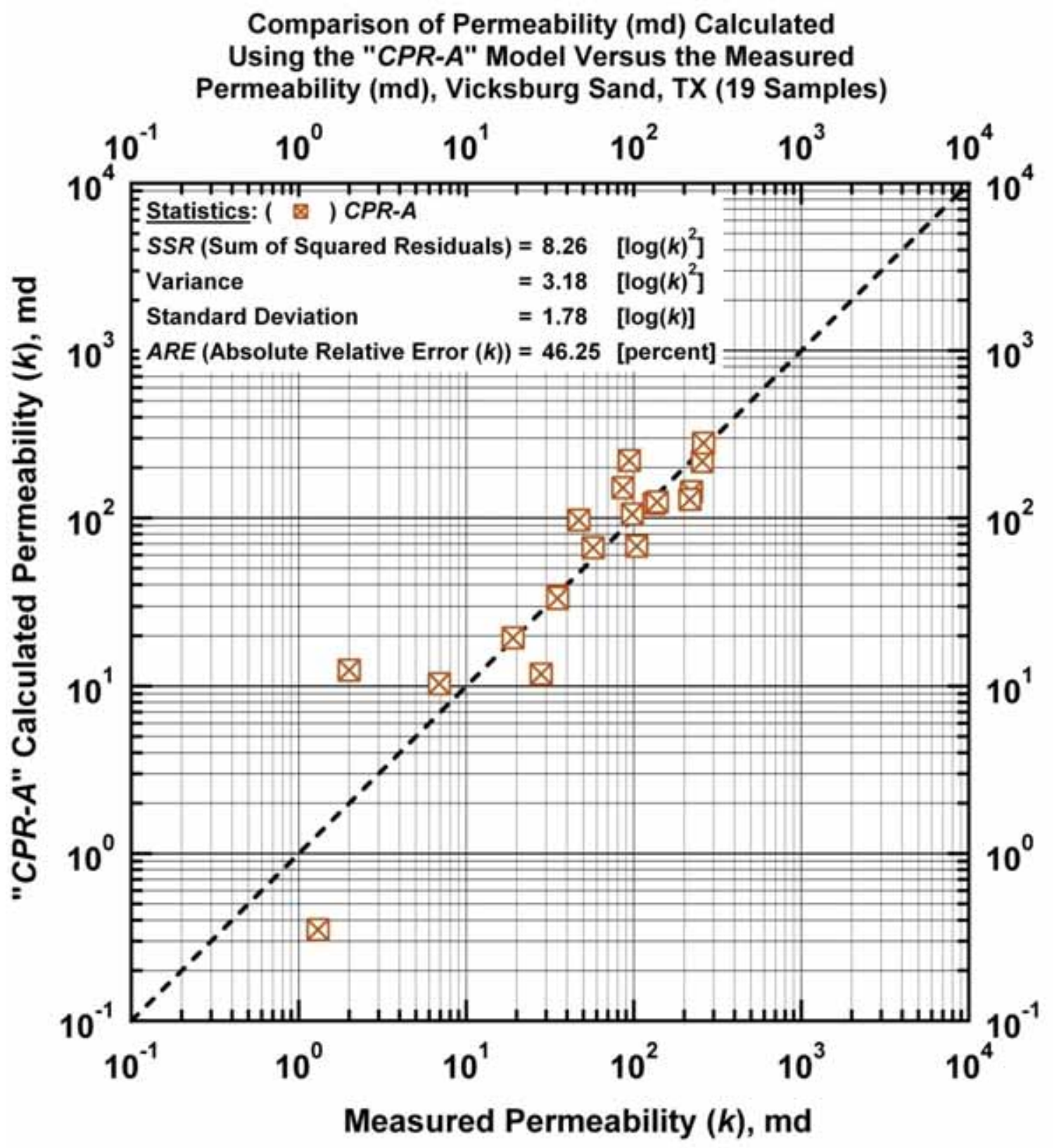

Figure D.2a - Model CPR-A: calculated versus measured permeability. [Case: Vicksburg Sand, S. TX (USA)] 


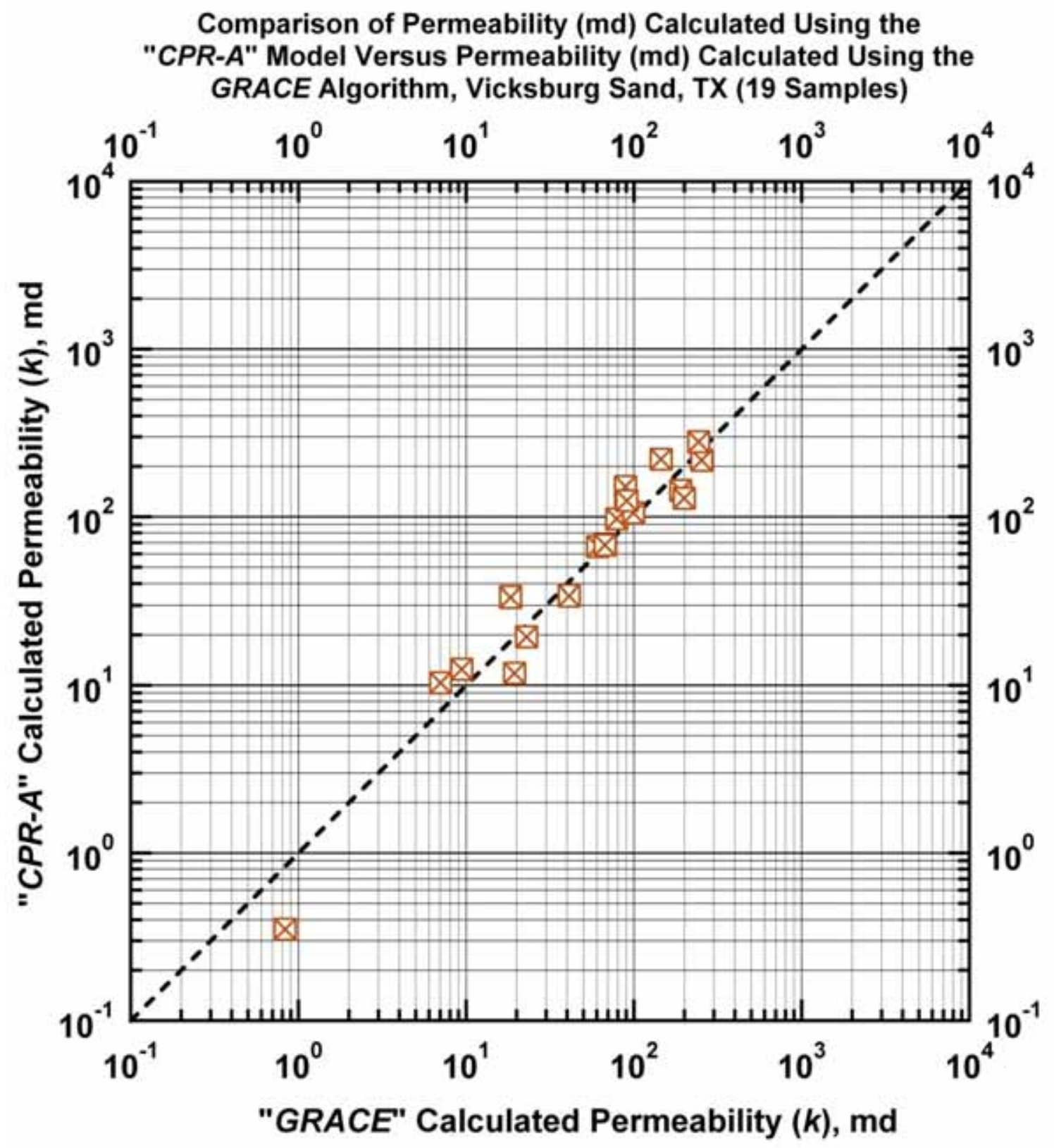

Figure D.2b - Model CPR-A: Permeability calculated using Model CPR-A versus permeability calculated using the GRACE Algorithm. [Case: Vicksburg Sand, S. TX (USA)] 
Comparison of the $c$-Coefficient Calculated Using the "CPR-A" Model Versus the Measured $c$-Coefficient, Vicksburg Sand, TX (19 Samples)

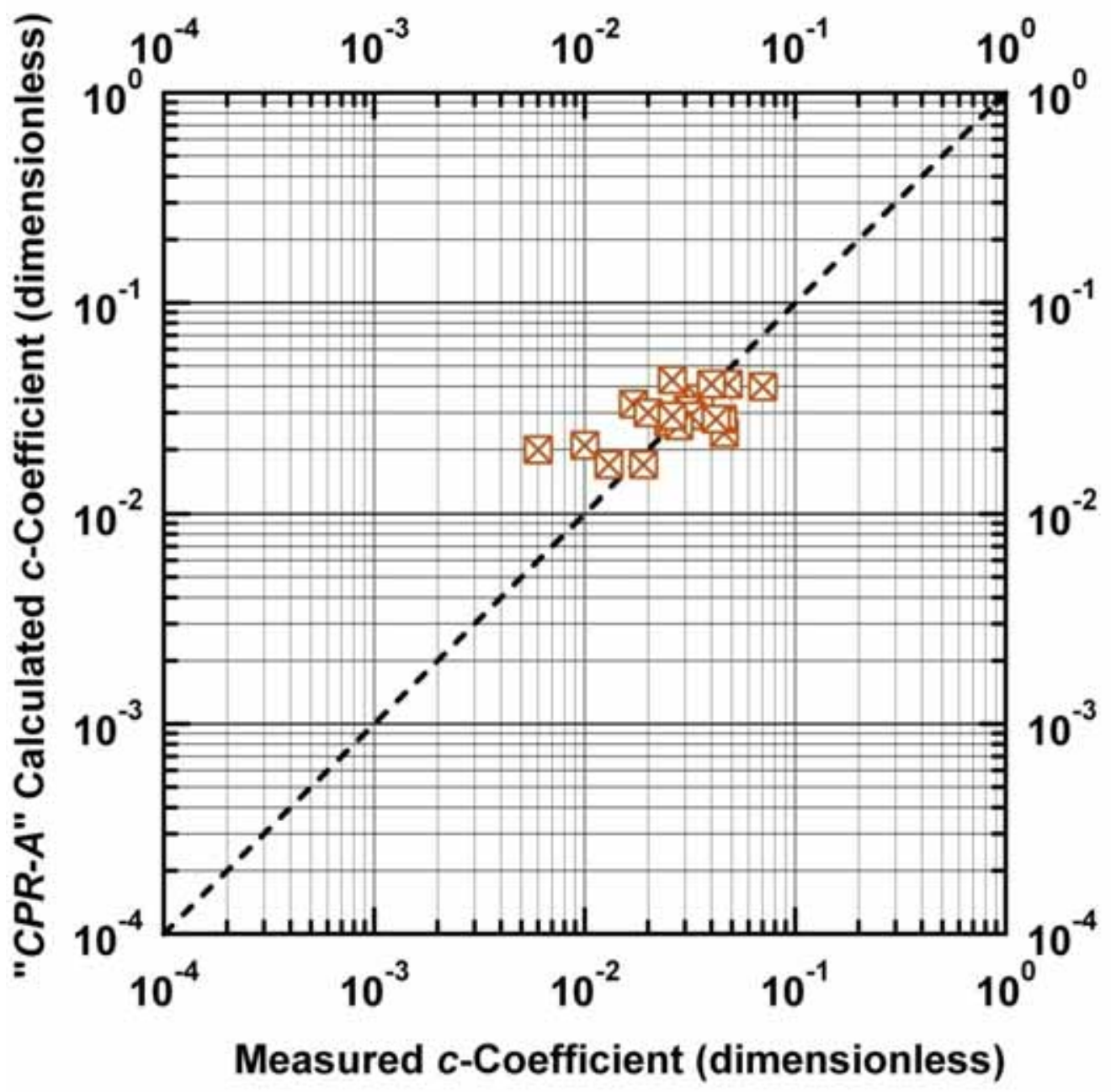

Figure D.2c - Model CPR-A: Calculated $c$-function values versus measured $c$-function values. [Case: Vicksburg Sand, S. TX (USA)] 
Plot of Depth (ft) Versus Measured and Calculated Permeability (md), Vicksburg Sand, TX (19 Samples)

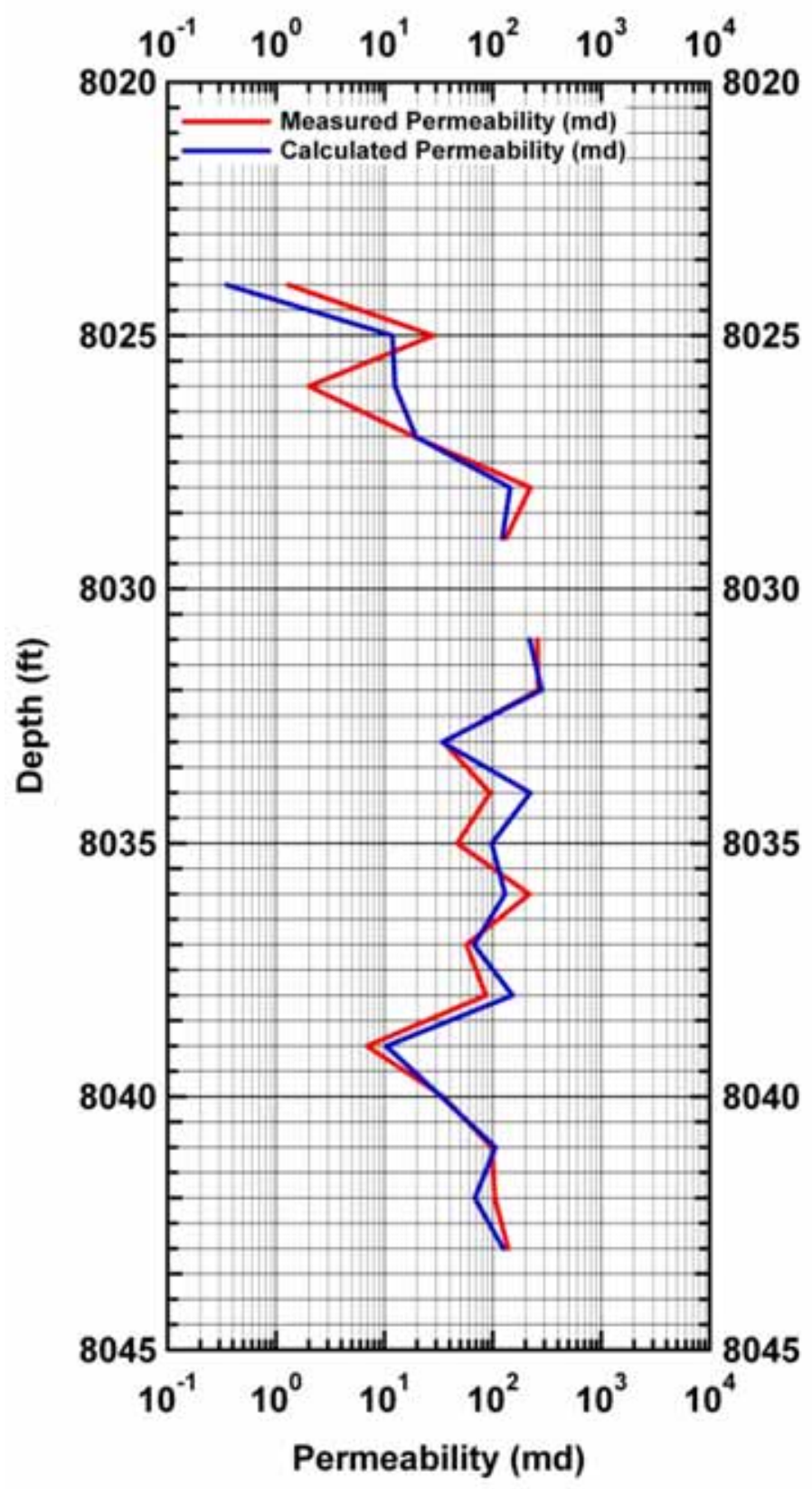

Figure D.2d - Model CPR-A: Calculated and measured permeability values versus depth. [Case: Vicksburg Sand, S. TX (USA)] 


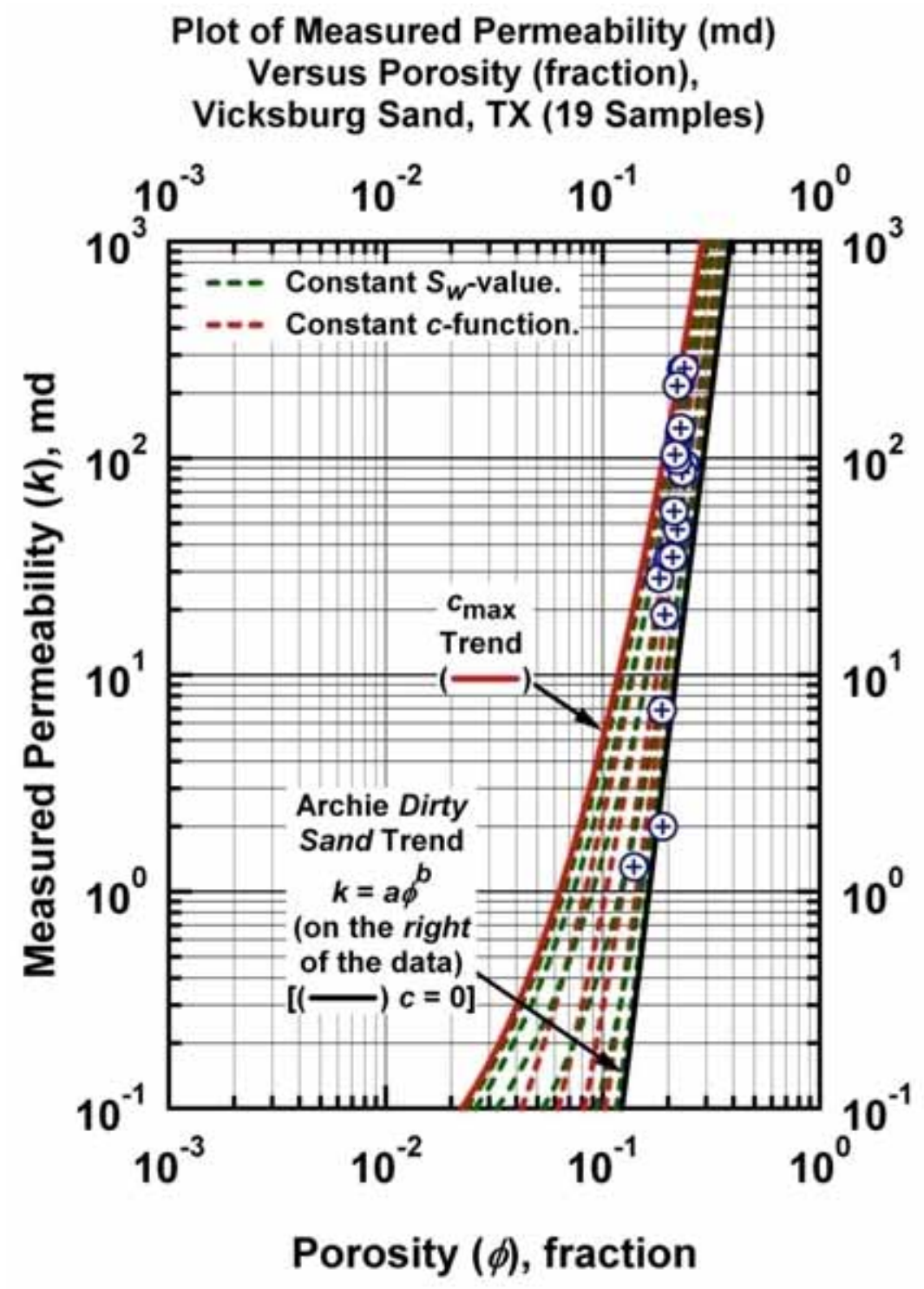

Figure D.3a - Model $C P R-B: \quad k=a(\phi+c)^{b} \quad c=c_{\max } \exp \left[-c_{1} \phi^{c_{2}} S_{w}^{c_{3}}\right]$ - "Clean Sand" Plot (log-log format) - Archie "Dirty Sand" trend is given by the straight-line trend at the far right of the data (power law model). [Case: Vicksburg Sand, S. TX (USA)] 


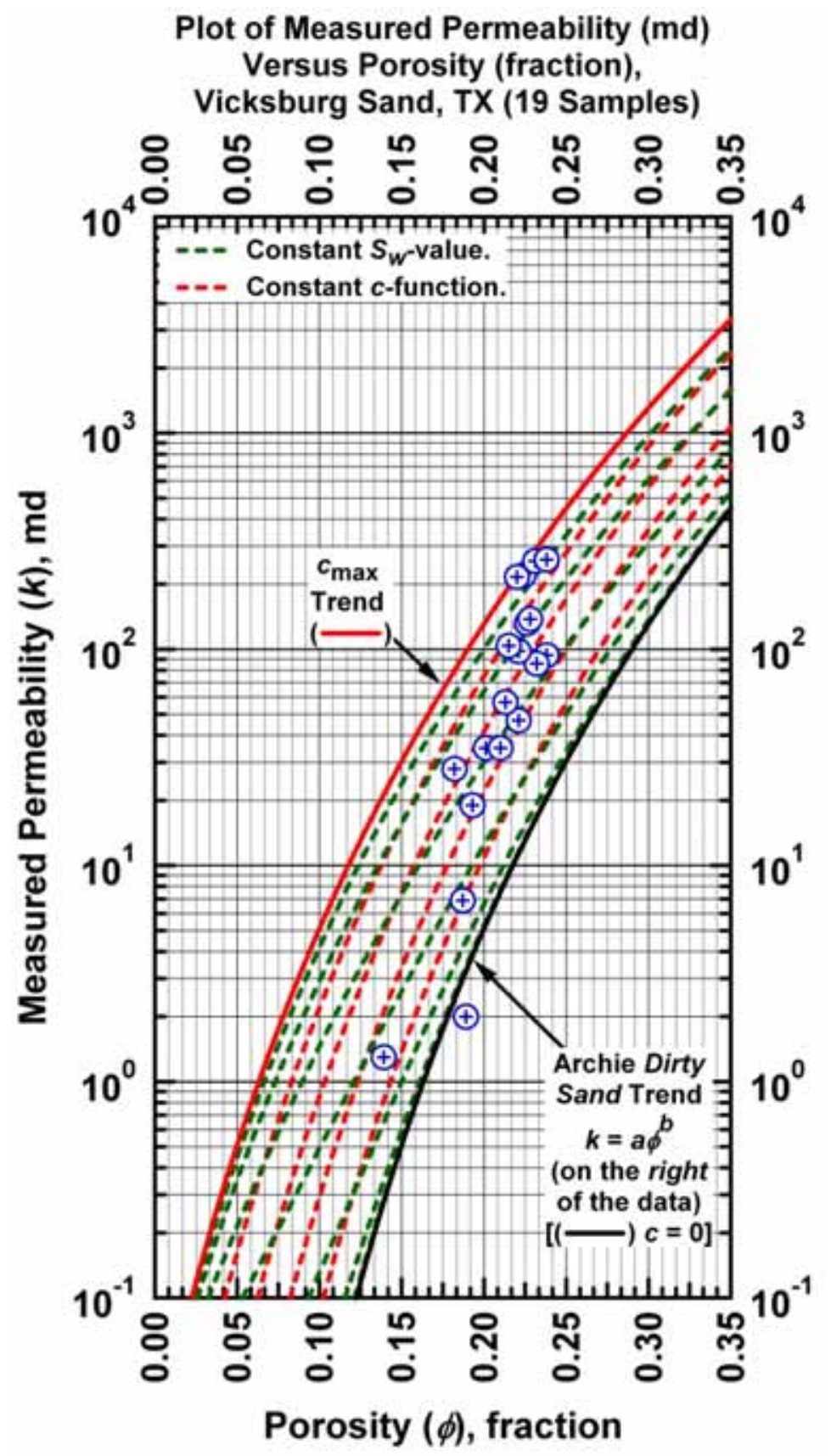

Figure D.3b - Model CPR-B: $k=a(\phi+c)^{b} \quad c=c_{\max } \exp \left[-c_{1} \phi^{c_{2}} S_{W}^{c_{3}}\right]$ - "Dirty Sand" Plot (semilog format) - Archie "Dirty Sand" trend is given by the curved trend at the far right of the data (power law model). [Case: Vicksburg Sand, S. TX (USA)] 


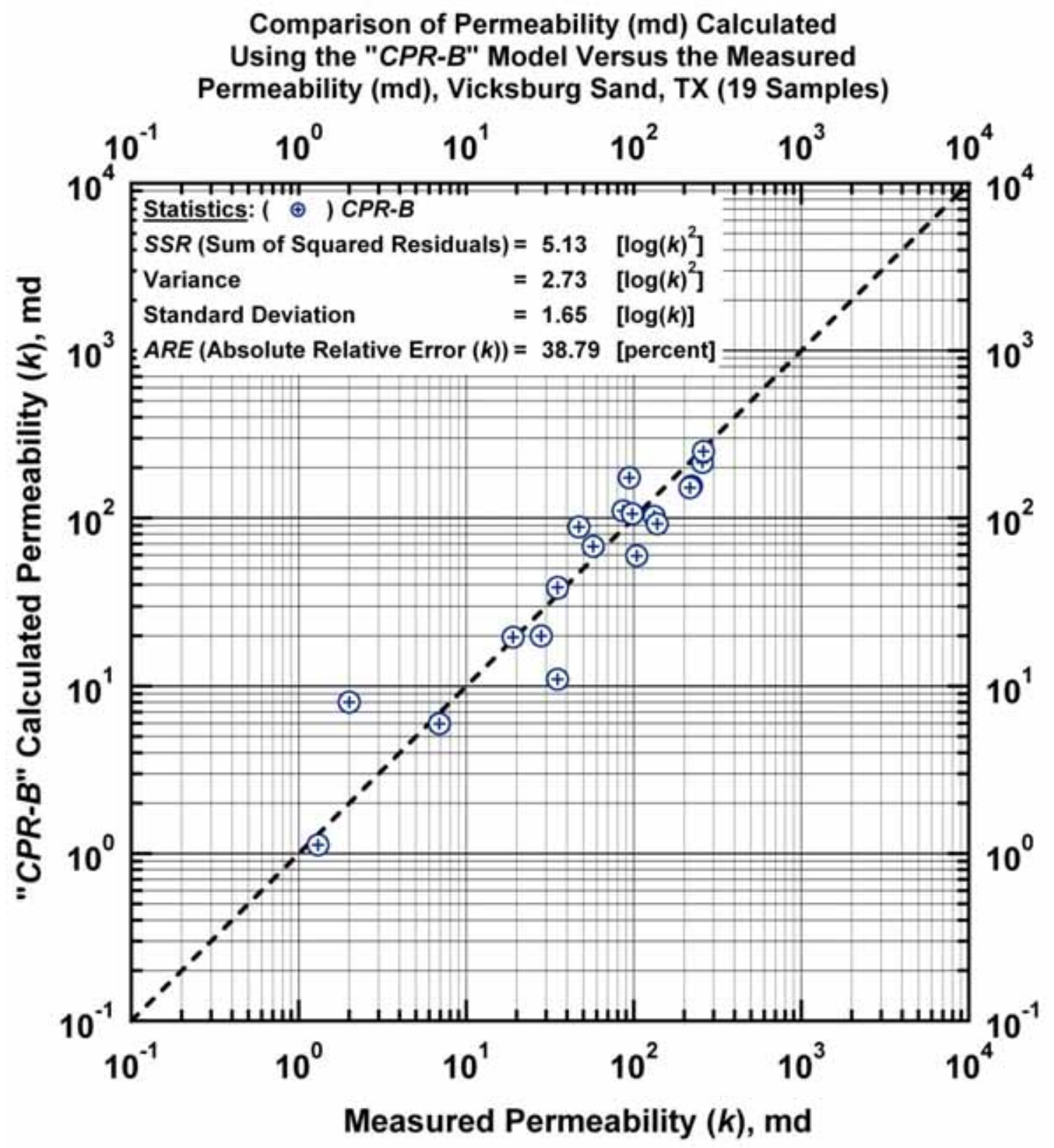

Figure D.4a - Model CPR-B: calculated versus measured permeability. [Case: Vicksburg Sand, S. TX (USA)] 


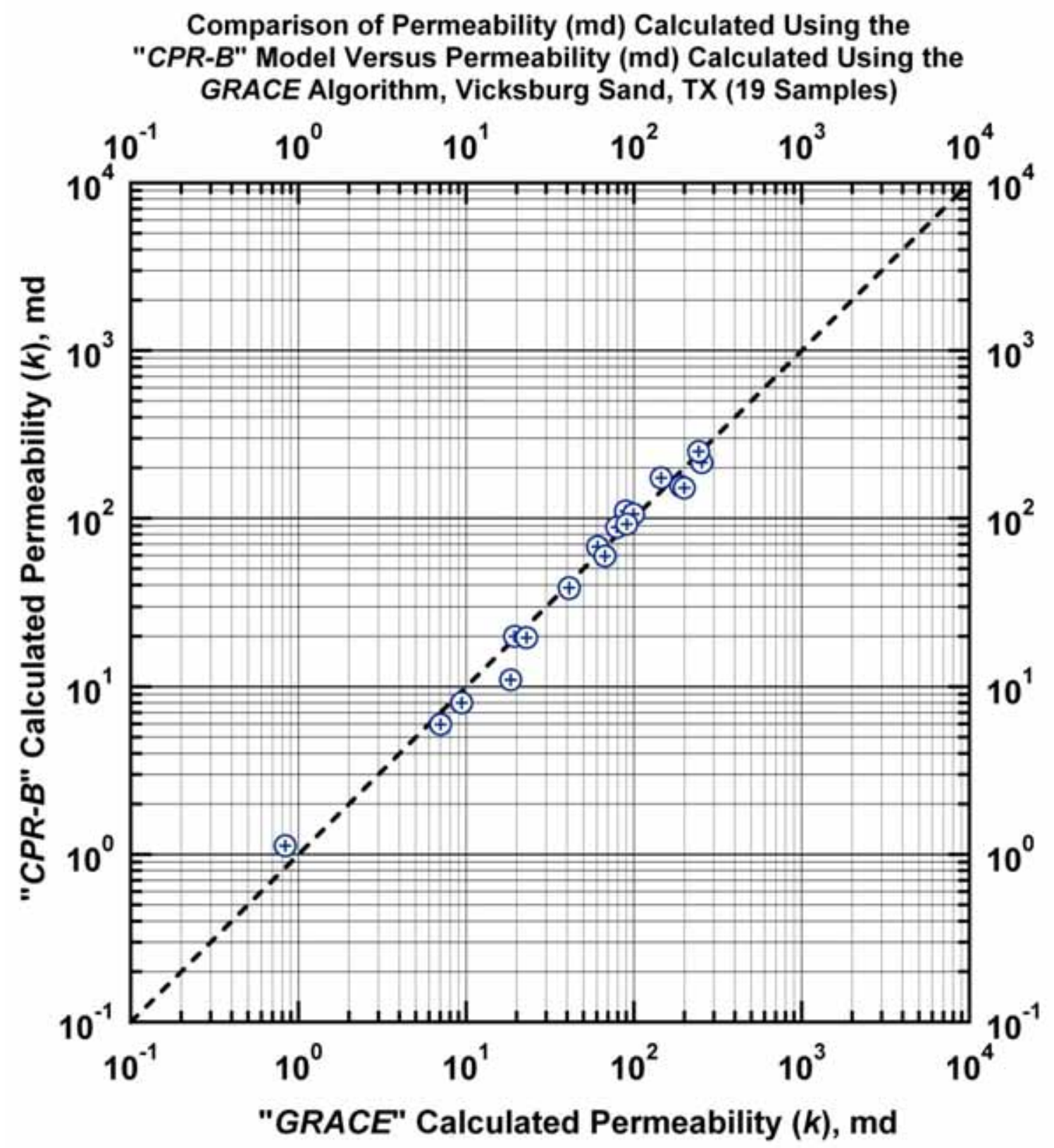

Figure D.4b - Model CPR-B: Permeability calculated using Model $C P R-B$ versus permeability calculated using the GRACE Algorithm. [Case: Vicksburg Sand, S. TX (USA)] 


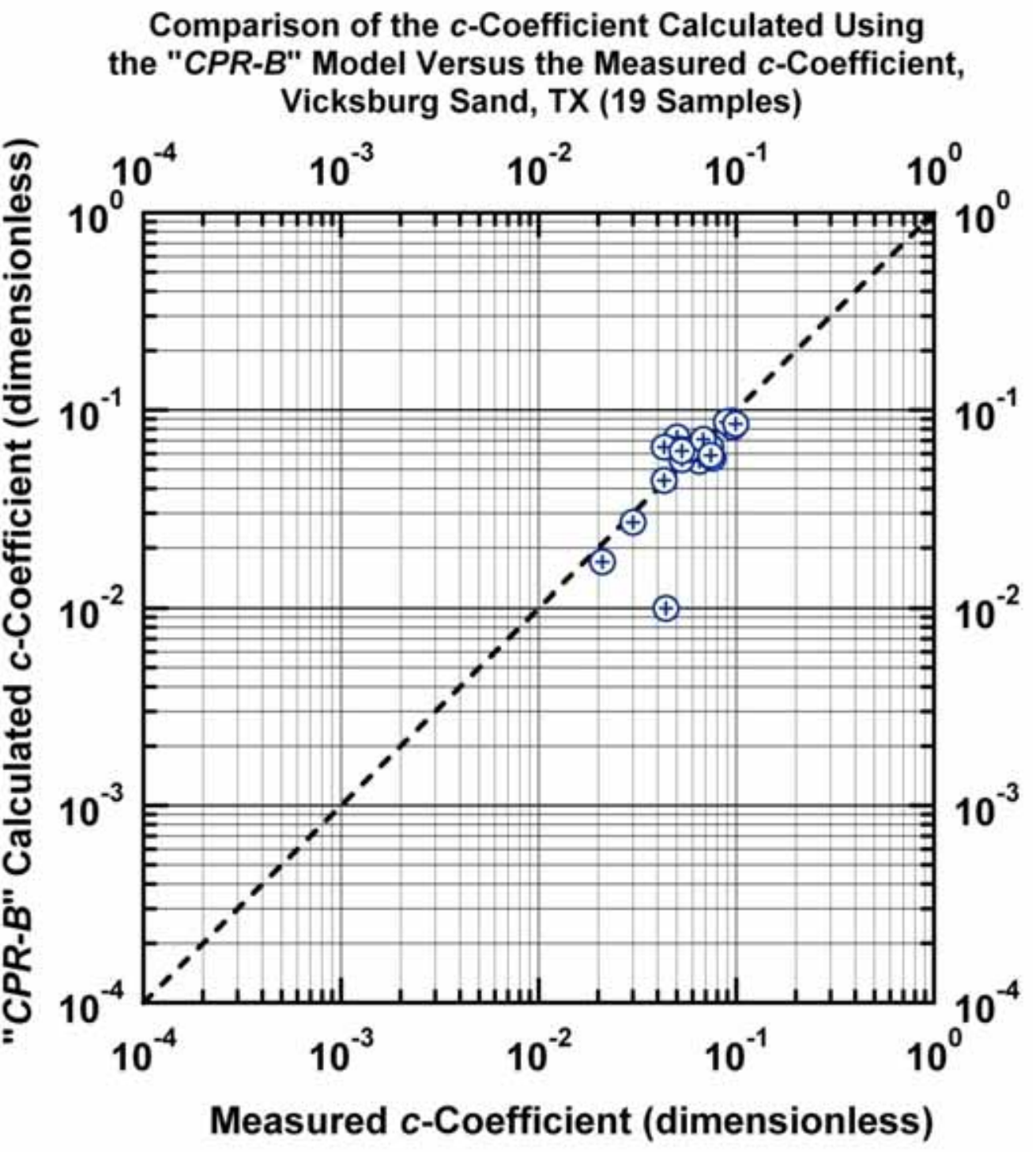

Figure D.4c - Model CPR-B: Calculated $c$-function values versus measured $c$-function values. [Case: Vicksburg Sand, S. TX (USA)] 
Plot of Depth ( $\mathrm{ft}$ ) Versus Measured and Calculated Permeability (md), Vicksburg Sand, TX (19 Samples)

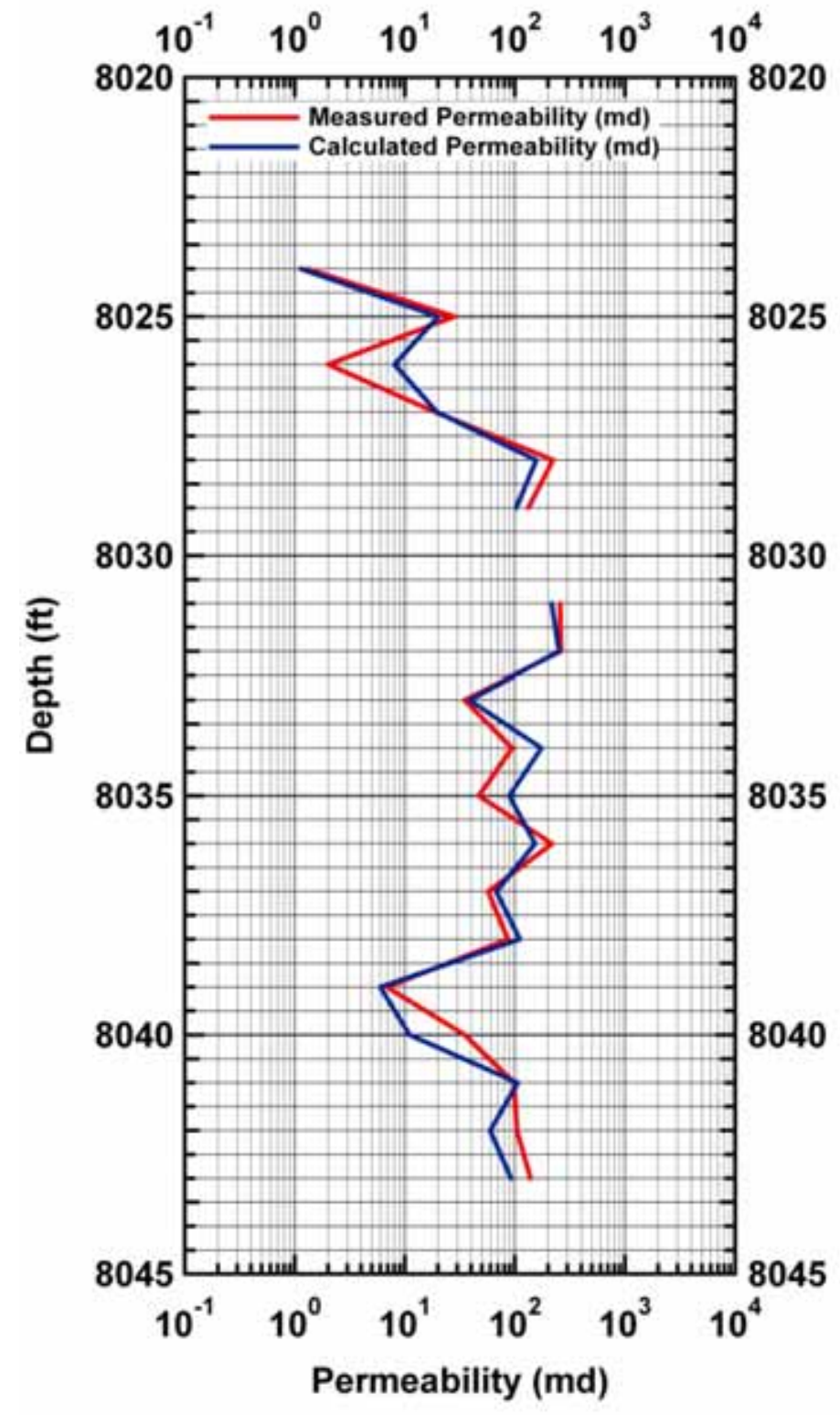

Figure D.4d - Model CPR-A: Calculated and measured permeability values versus depth. [Case: Vicksburg Sand, S. TX (USA)] 


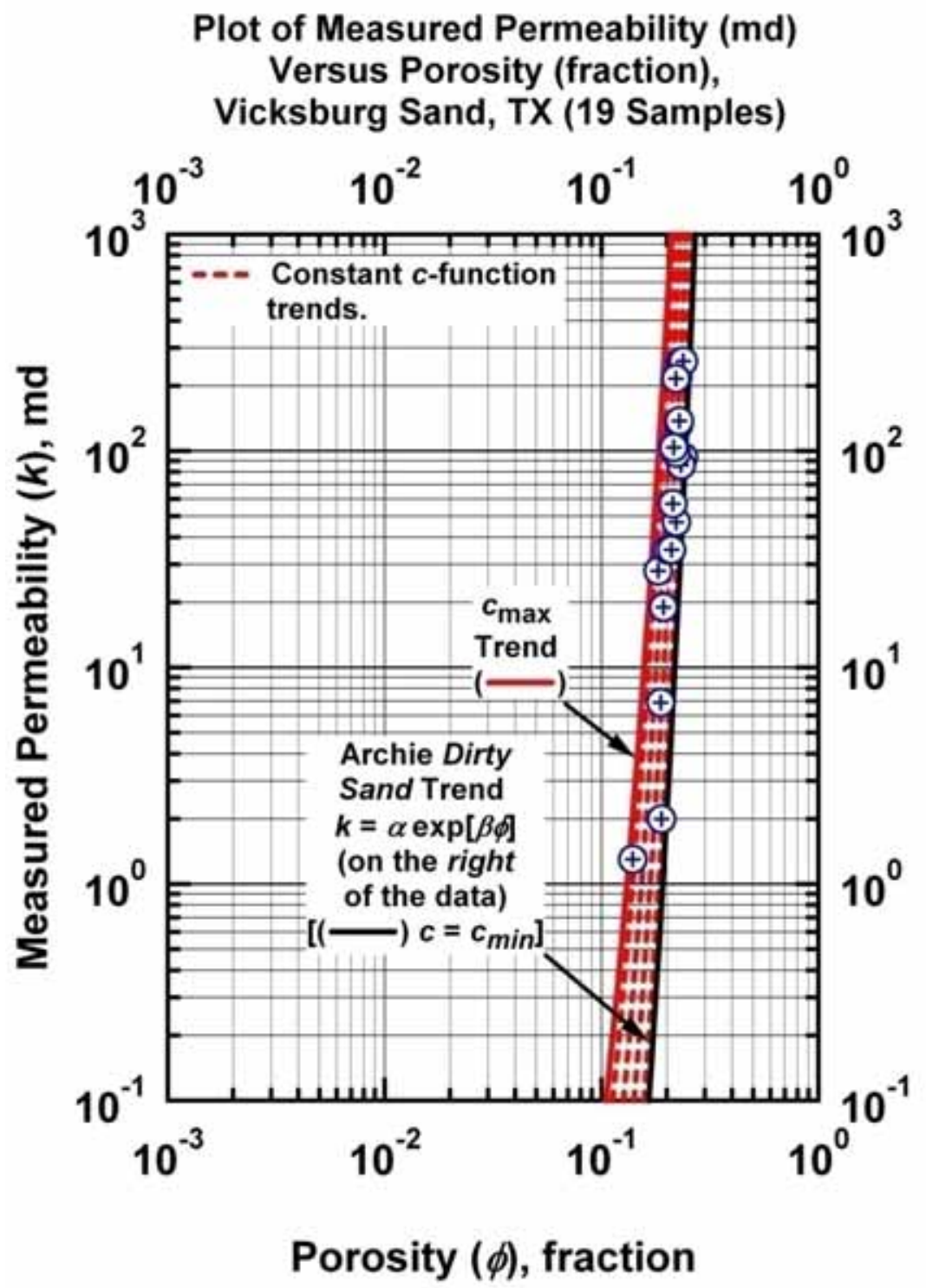

Figure D.5a - Model $C P R-C: \quad k=c \exp [\beta \phi] \quad c=c_{\max } \exp \left[-c_{1} \phi^{c_{2}} S_{w}^{c_{3}}\right]$ - "Clean Sand" Plot (log-log format) - Archie "Dirty Sand" trend is given by the curved trend at the far right of the data (exponential model). [Case: Vicksburg Sand, S. TX (USA)] 


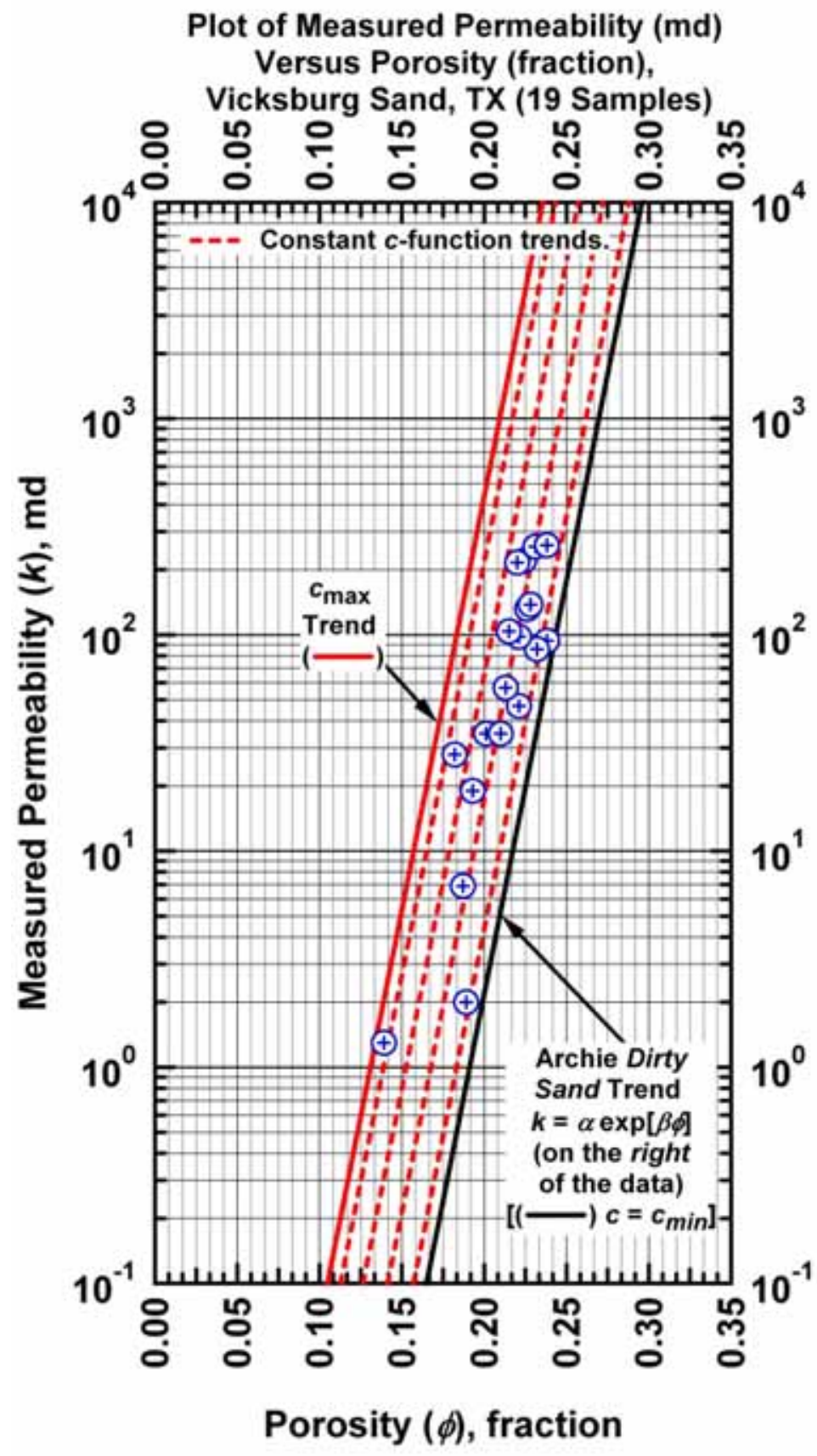

Figure D.5b - Model $C P R-C: k=c \exp [\beta \phi] \quad c=c_{\max } \exp \left[-c_{1} \phi^{c_{2}} S_{w}^{C_{3}}\right]$ - "Dirty Sand" Plot (semilog format) - Archie "Dirty Sand" trend is given by the straight-line trend at the far right of the data (exponential model). [Case: Vicksburg Sand, S. TX (USA)] 


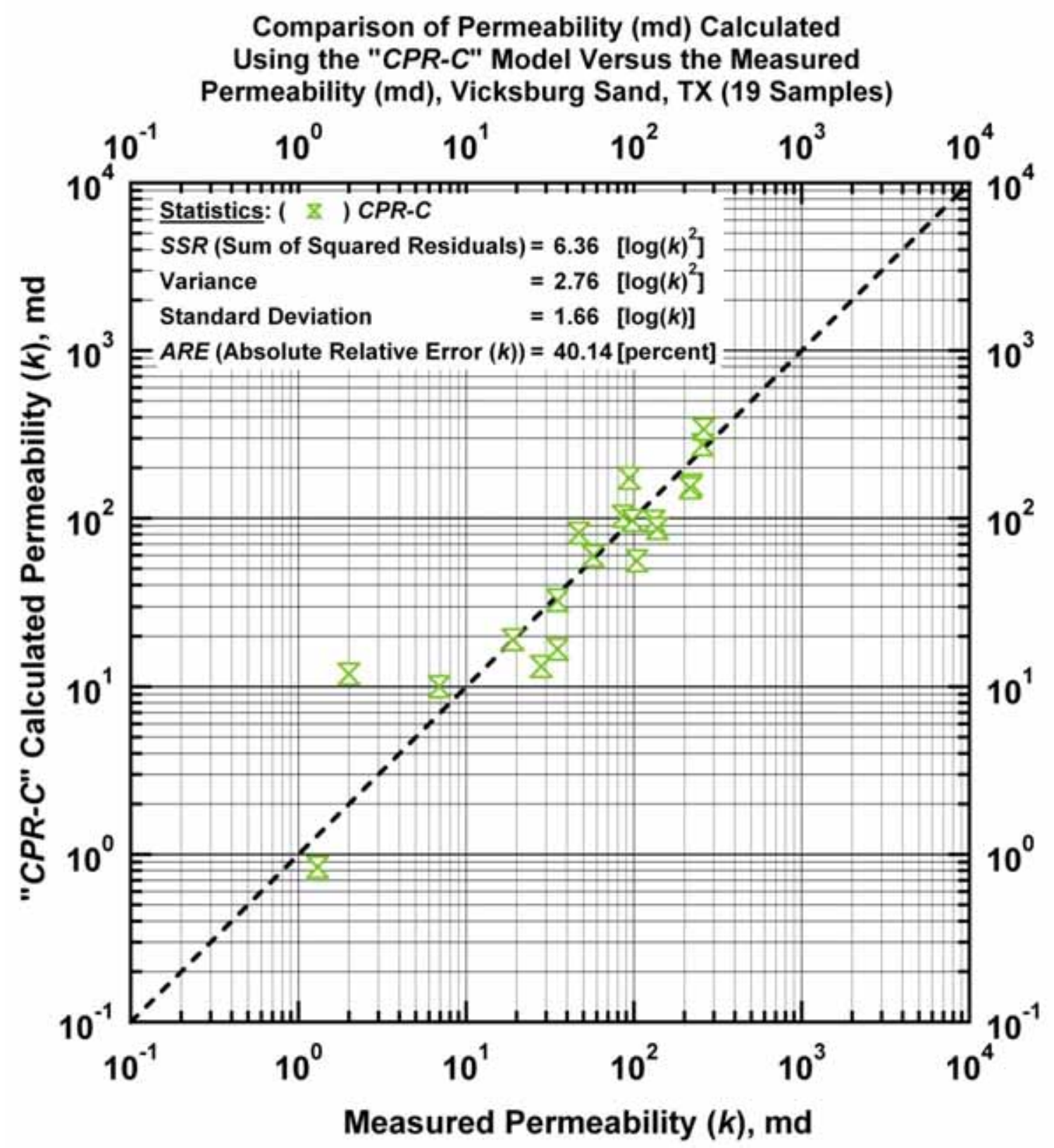

Figure D.6a - Model CPR-C: calculated versus measured permeability. [Case: Vicksburg Sand, S. TX (USA)] 


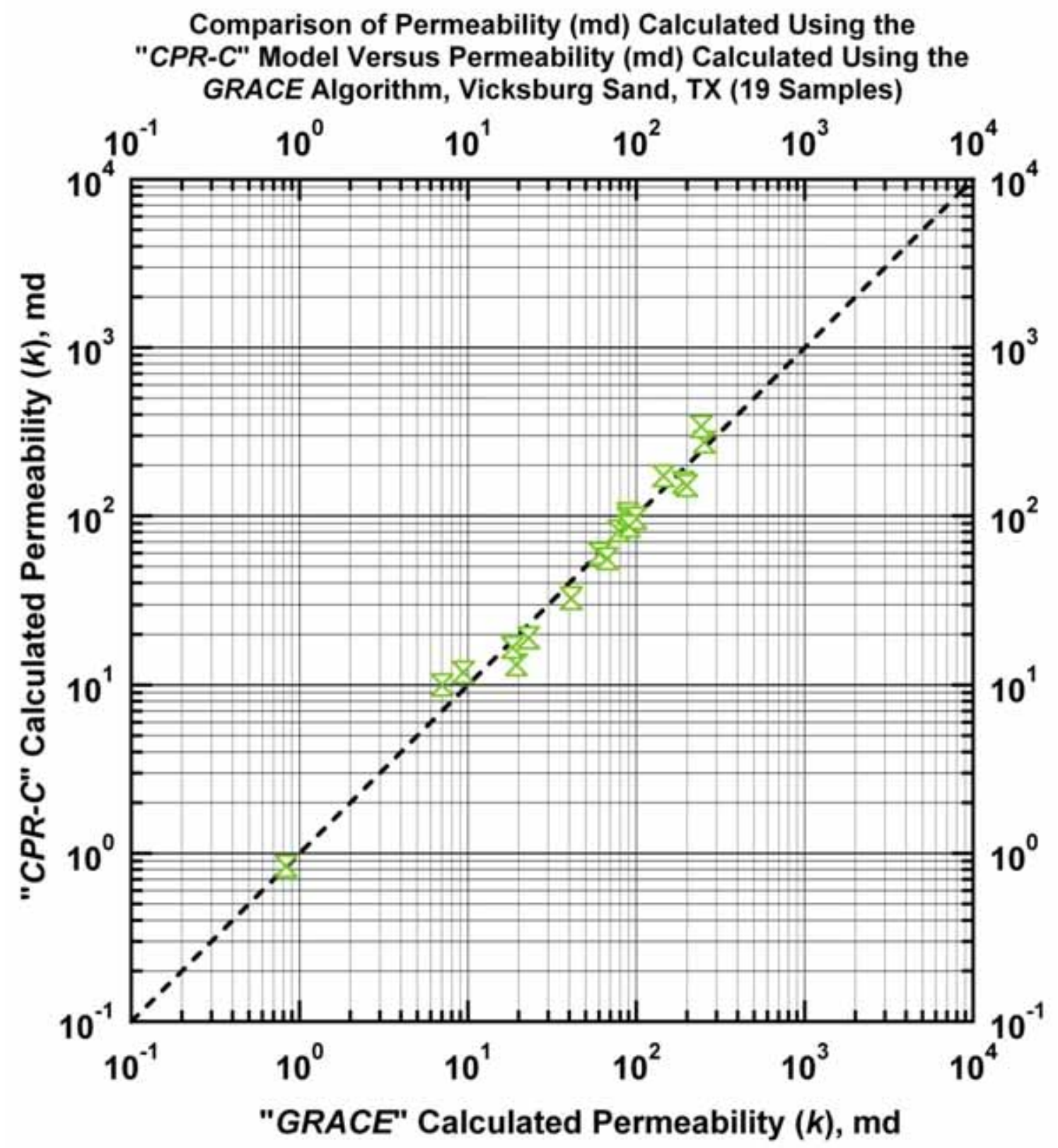

Figure D.6b - Model $C P R-C$ : Permeability calculated using Model $C P R-C$ versus permeability calculated using the GRACE Algorithm. [Case: Vicksburg Sand, S. TX (USA)] 


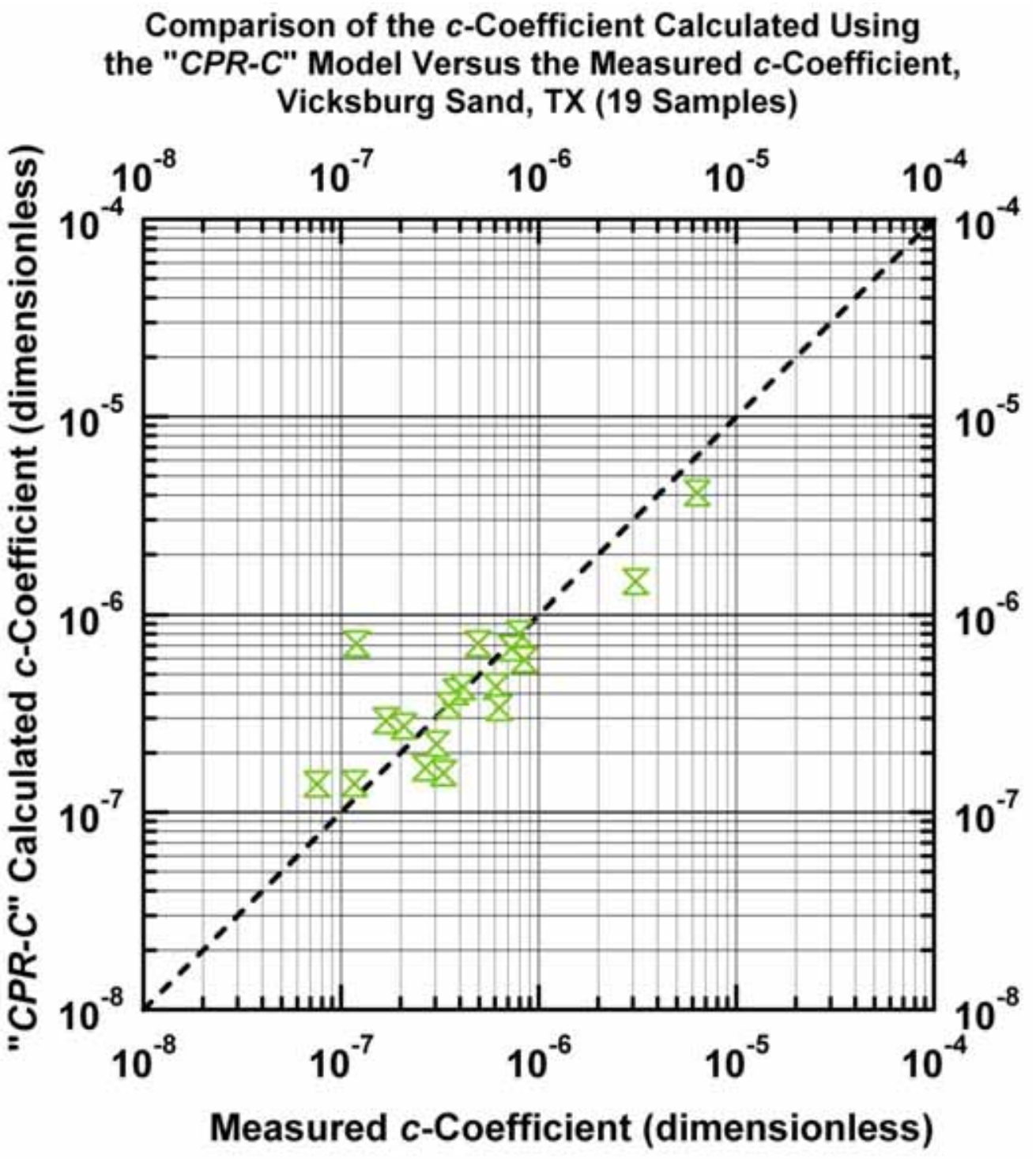

Figure D.6c - Model $C P R$-C: Calculated $c$-function values versus measured $c$-function values. [Case: Vicksburg Sand, S. TX (USA)] 
Plot of Depth (ft) Versus Measured and Calculated Permeability (md), Vicksburg Sand, TX (19 Samples)

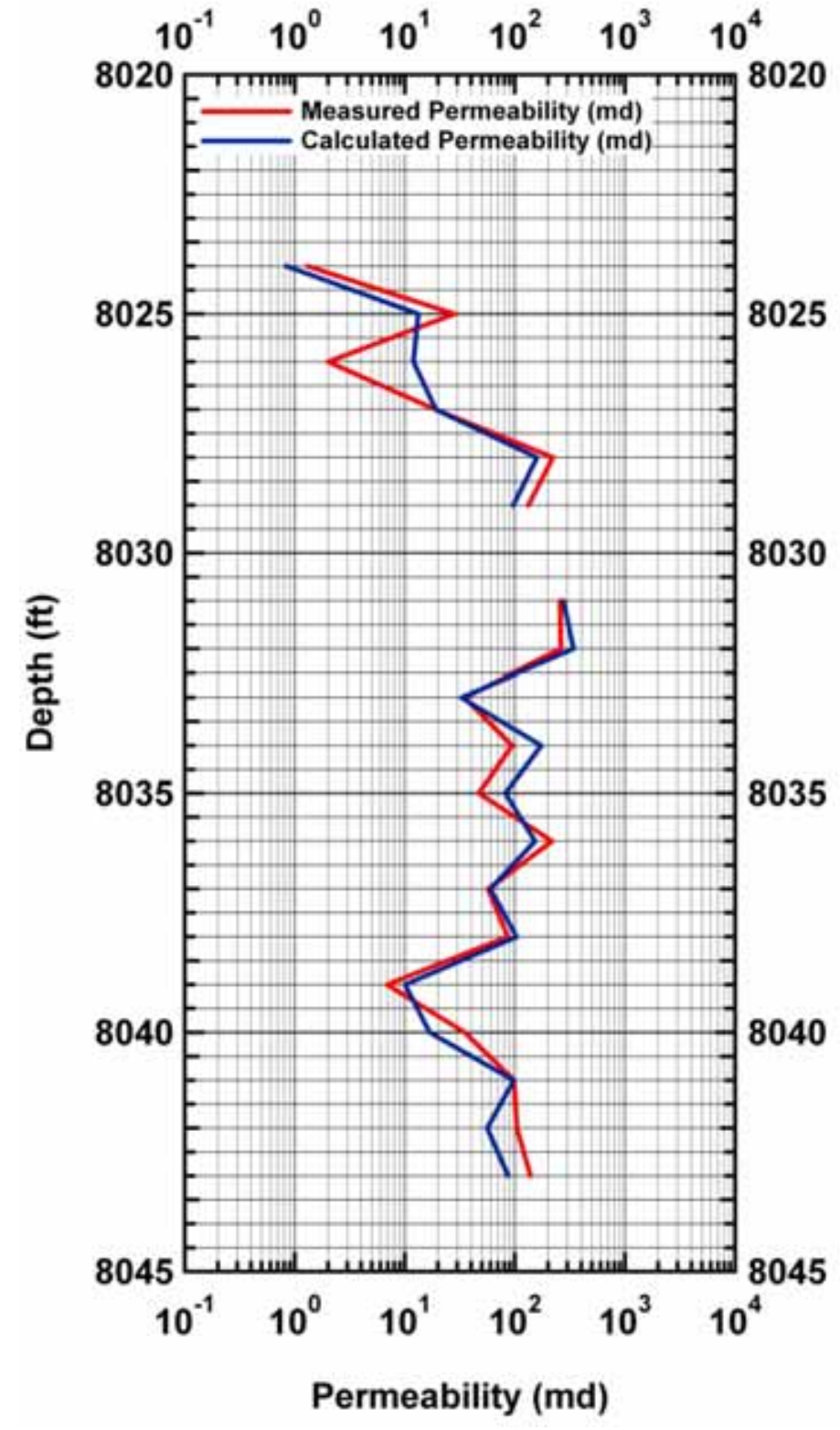

Figure D.6d - Model CPR-A: Calculated and measured permeability values versus depth. [Case: Vicksburg Sand, S. TX (USA)] 


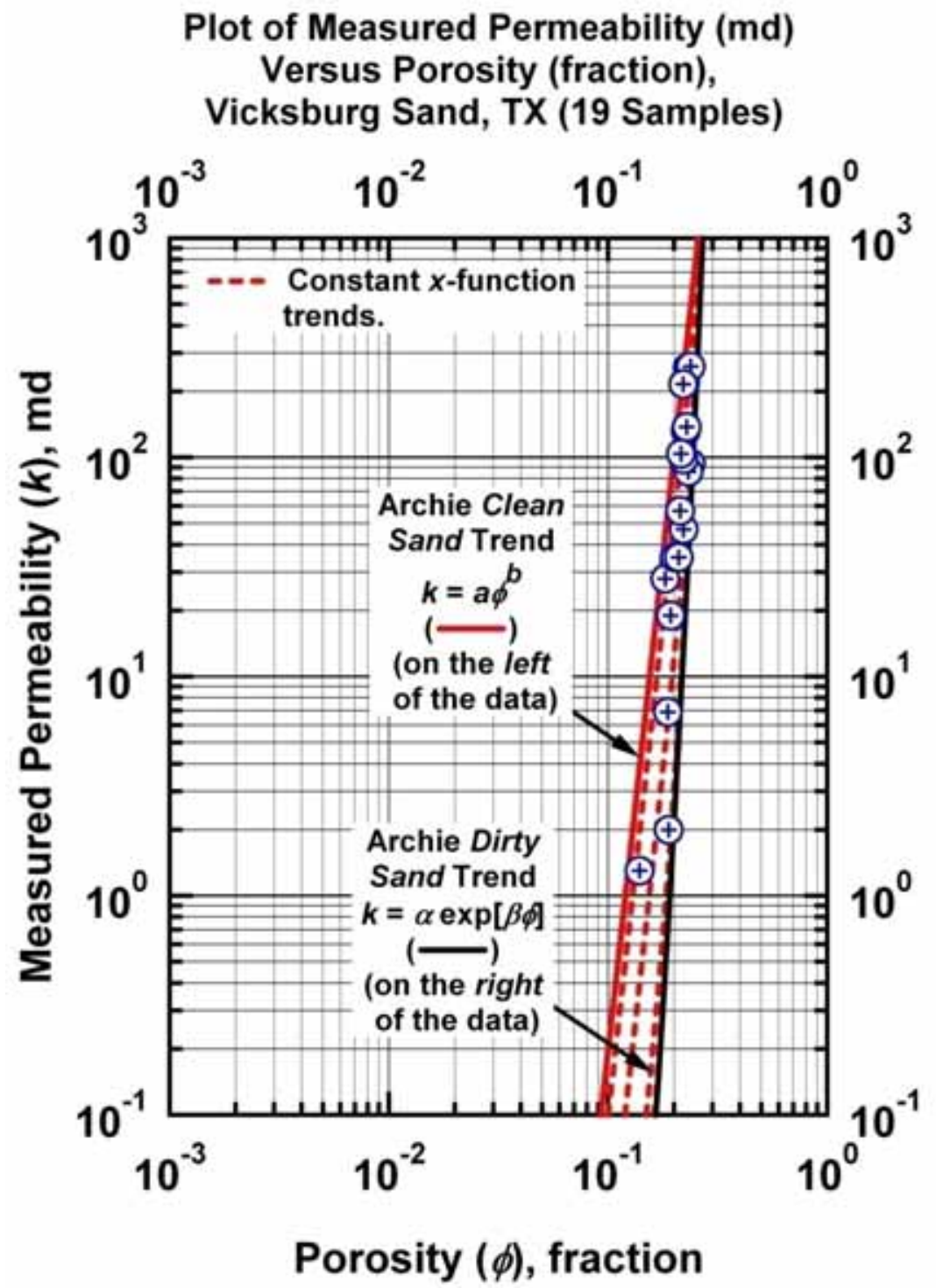

Figure D.7a - Model CPR-D: $k=x a \phi^{b}+(1-x) \alpha \exp [\beta \phi] \quad[0 \leq x \leq 1]$ - Weighted Power Law-Exponential Model used to correlate permeability $(k)$ and porosity $(\phi)$. "Clean Sand" Plot - Archie "Clean Sand" trend is given by the straight-line trend at the far left of the data (power law model). [Case: Vicksburg Sand, S. TX (USA)] 


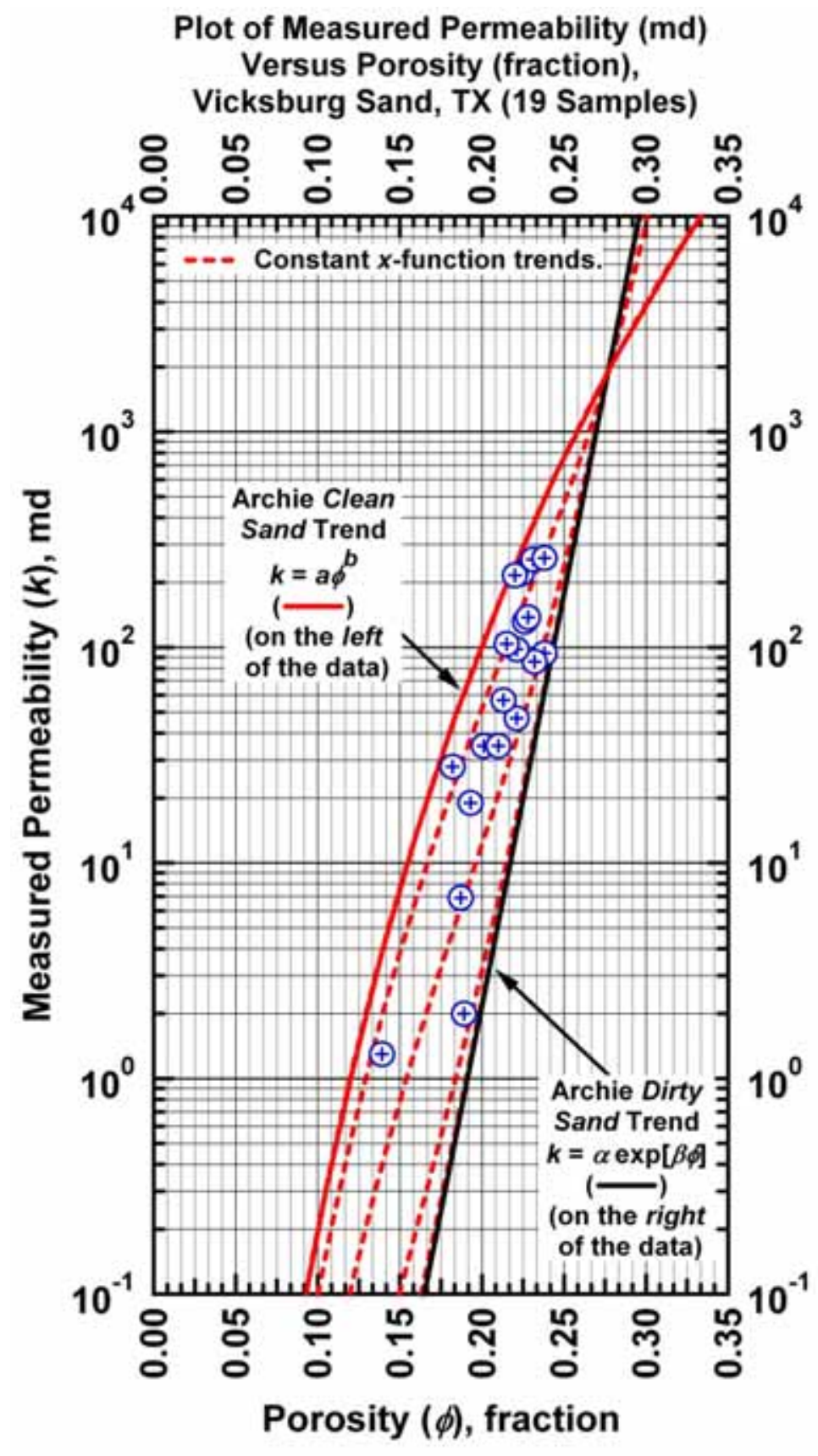

Figure D.7b - Model CPR-D: $k=x a \phi^{b}+(1-x) \alpha \exp [\beta \phi] \quad[0 \leq x \leq 1]$ - Weighted Power Law-Exponential Model used to correlate permeability $(k)$ and porosity $(\phi)$. "Dirty Sand" Plot - Archie "Dirty Sand" trend is given by the straight-line trend at the far right of the data (exponential model). [Case: Vicksburg Sand, S. TX (USA)] 


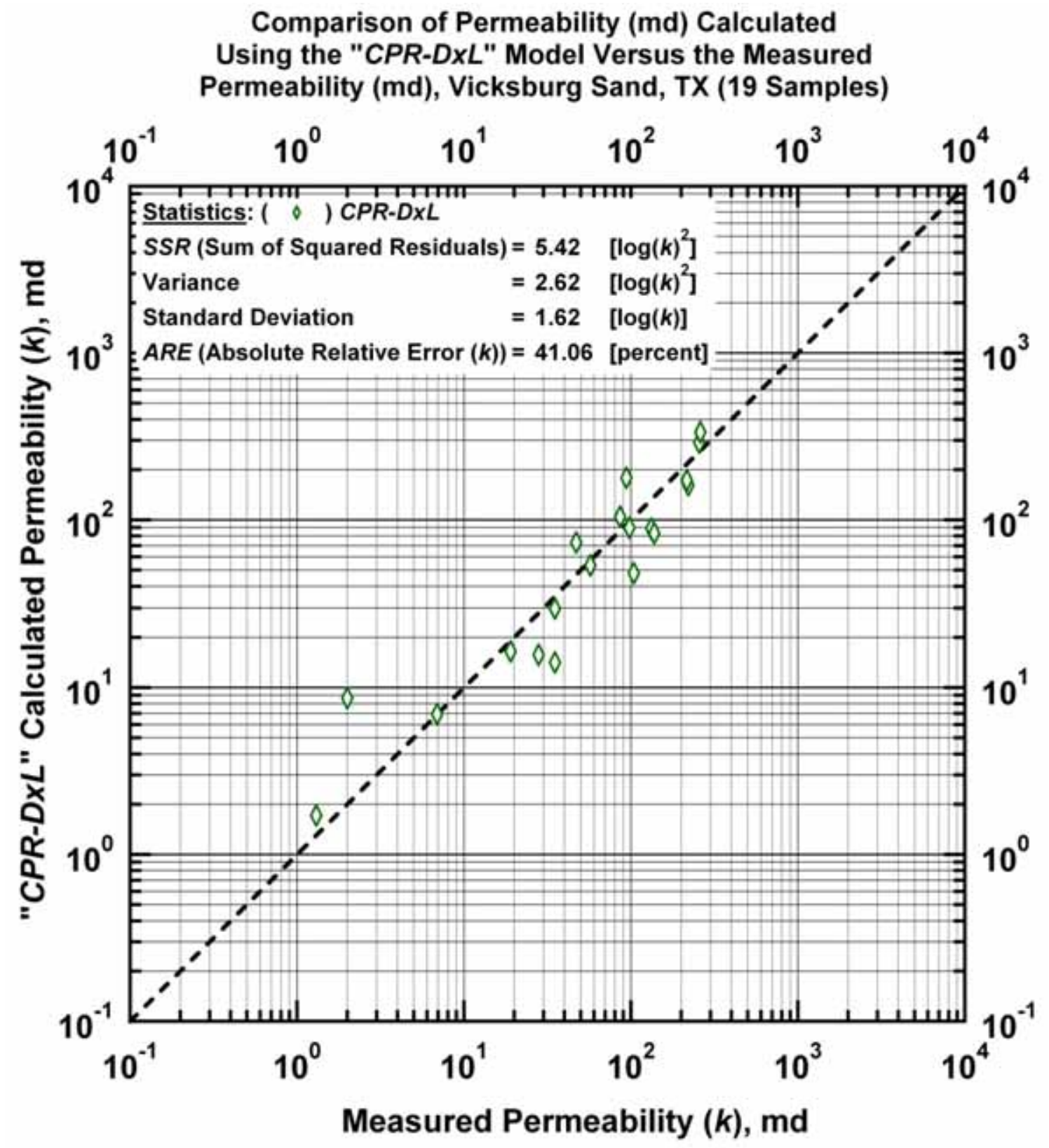

Figure D.8a - Model CPR-DxL: Calculated versus measured permeability. [Case: Vicksburg Sand, S. TX (USA)] 


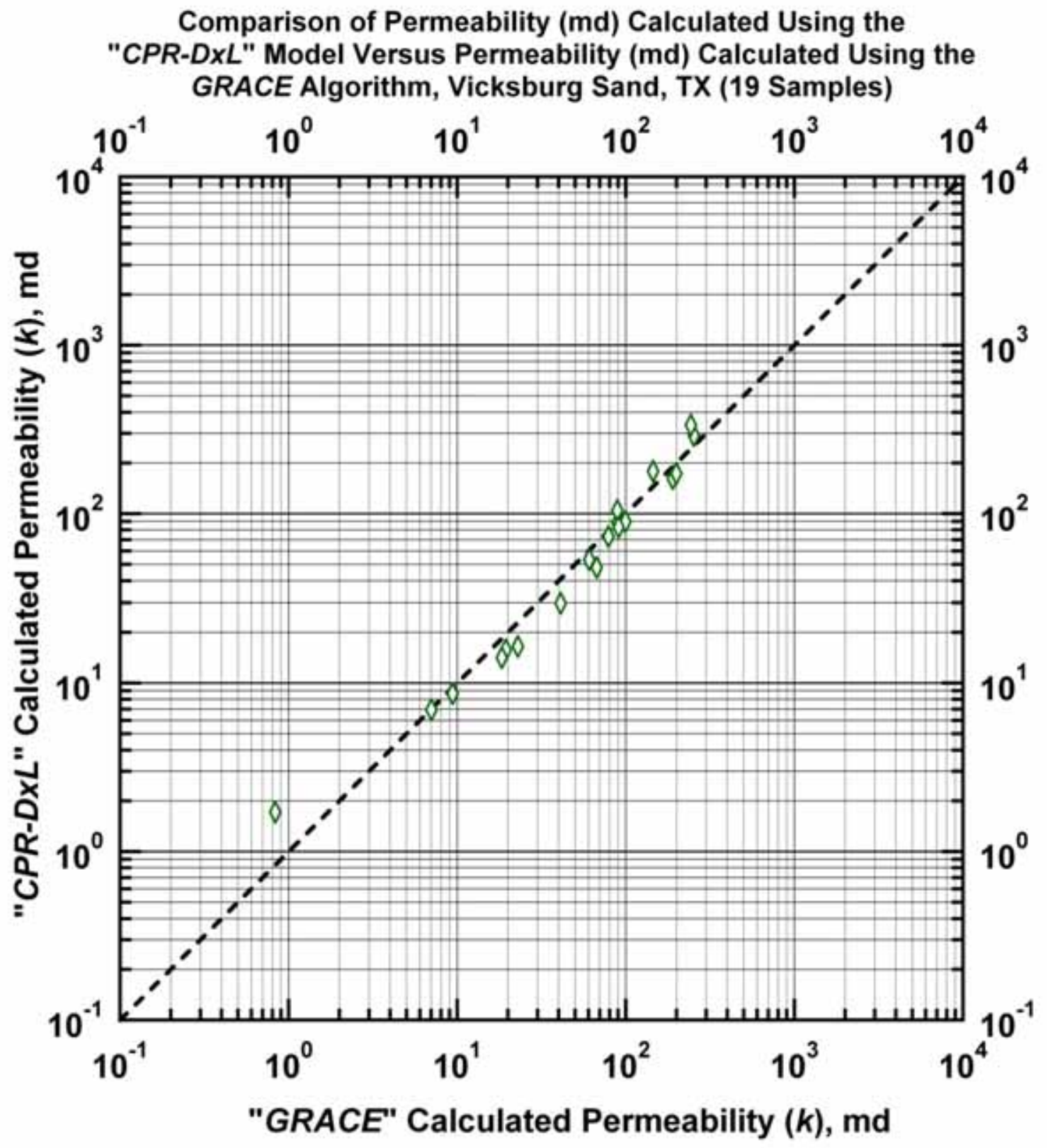

Figure D.8b - Model $C P R-D x L$ : Permeability calculated using Model $C P R-C$ versus permeability calculated using the GRACE Algorithm. [Case: Vicksburg Sand, S. TX (USA)] 


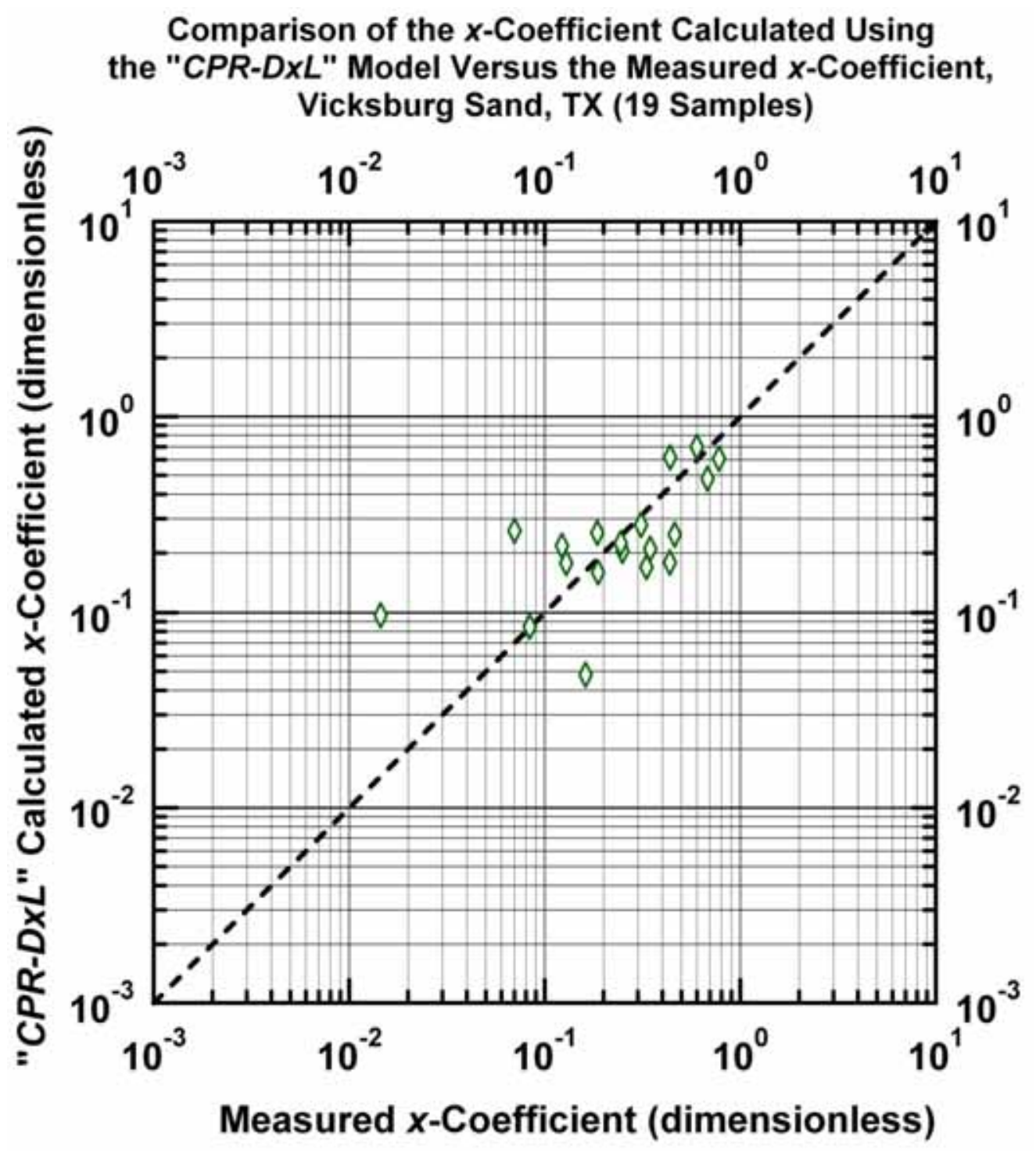

Figure D.8c - Model CPR-DxL: Calculated $x$-function weight values versus measured $x$ function weight values. [Case: Vicksburg Sand, S. TX (USA)] 
Plot of Depth (ft) Versus Measured and Calculated Permeability (md), Vicksburg Sand, TX (19 Samples)

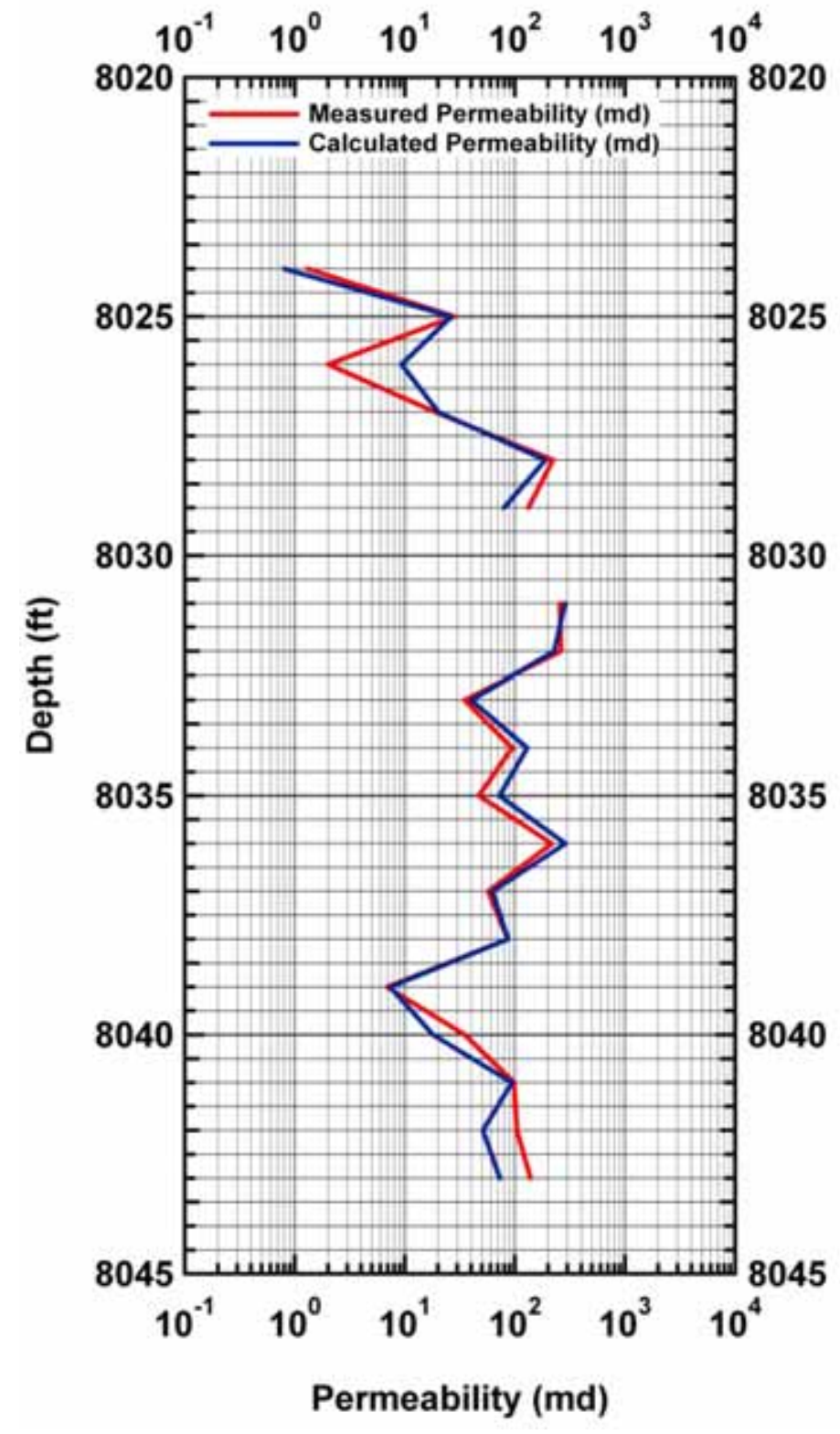

Figure D.8d - Model CPR-A: Calculated and measured permeability values versus depth. [Case: Vicksburg Sand, S. TX (USA)] 


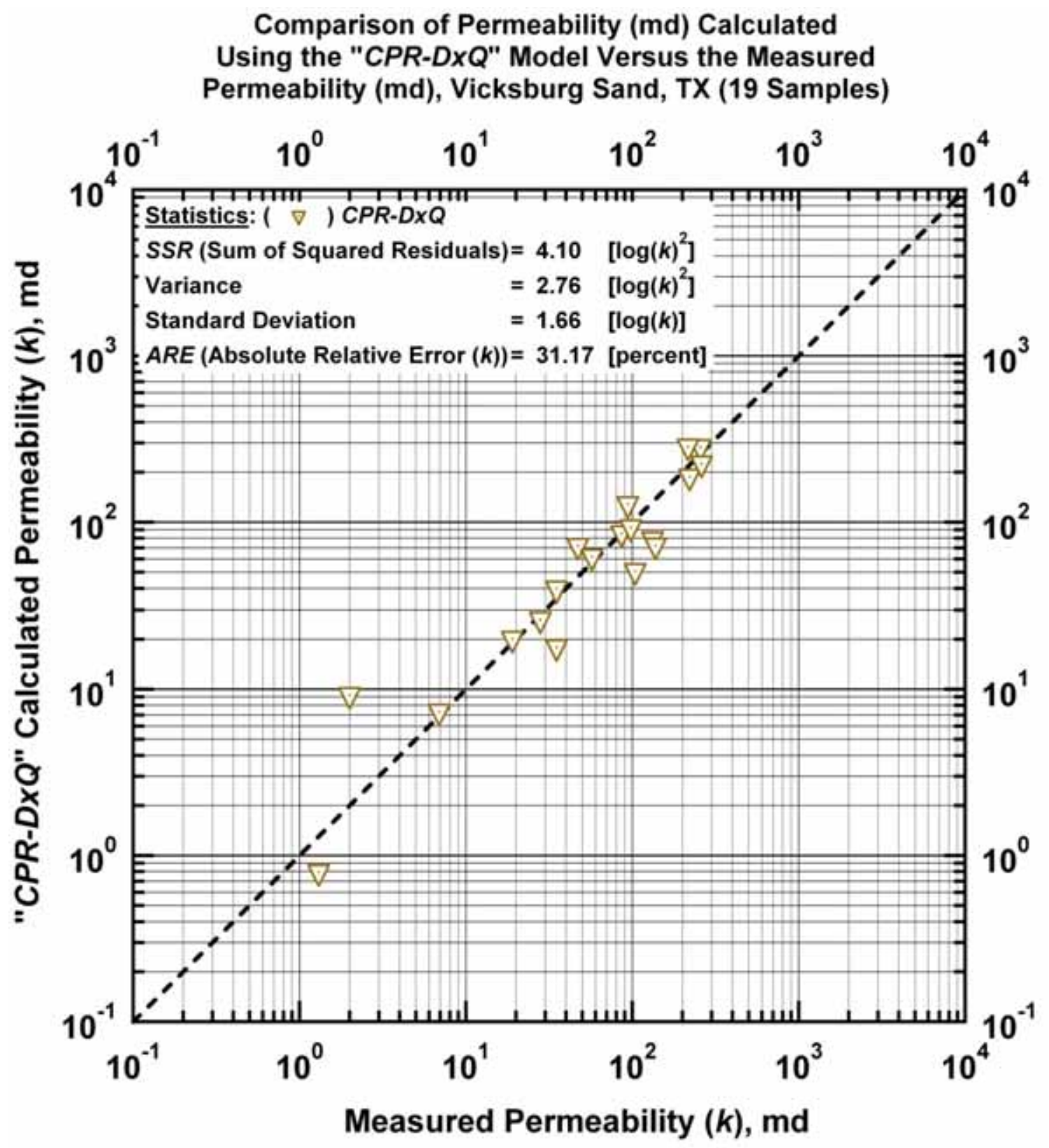

Figure D.9a - Model CPR-DxQ: Calculated versus measured permeability. [Case: Vicksburg Sand, S. TX (USA)] 


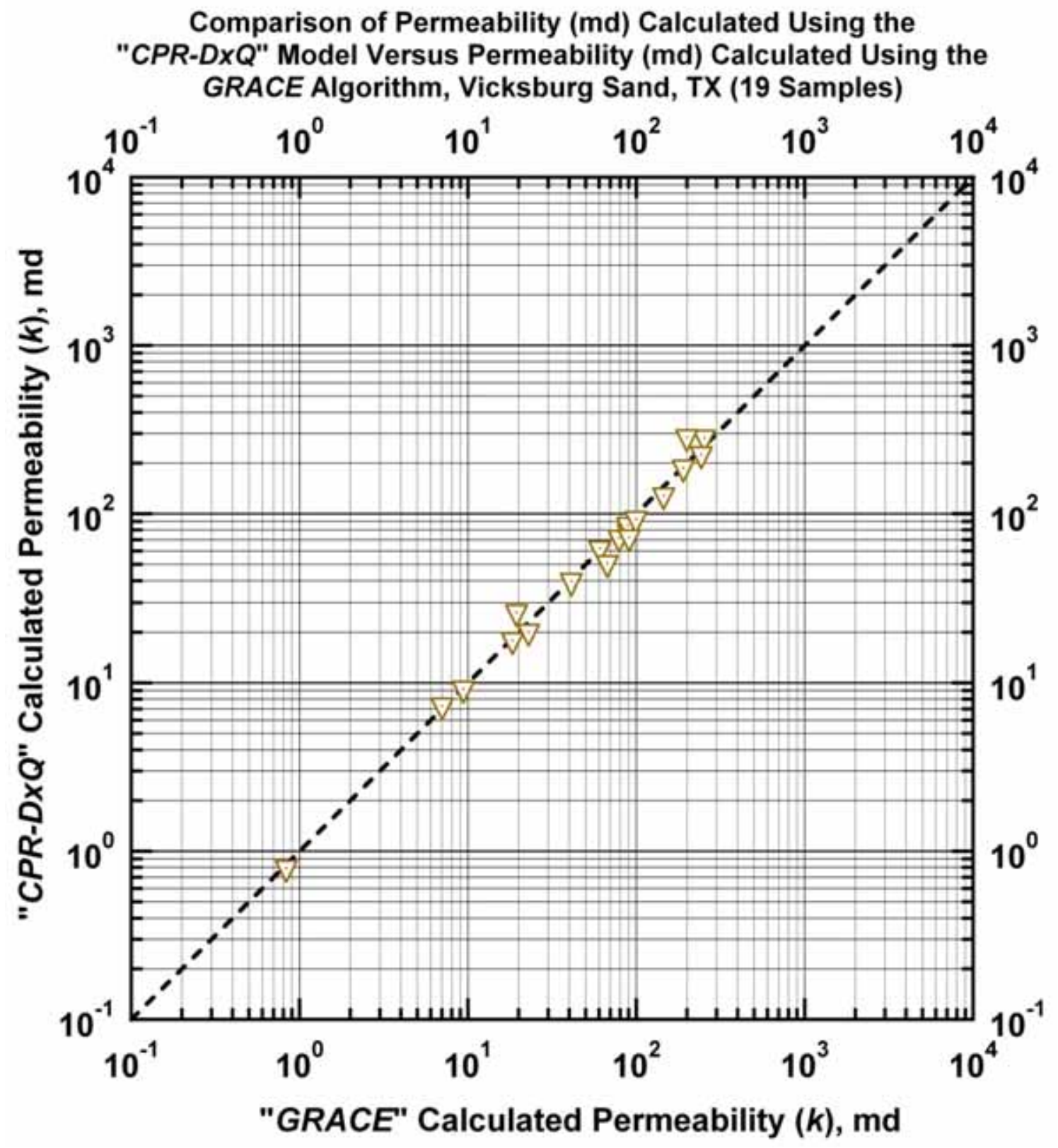

Figure D.9b - Model $C P R-D x Q$ : Permeability calculated using Model $C P R-C$ versus permeability calculated using the GRACE Algorithm. [Case: Vicksburg Sand, S. TX (USA)] 


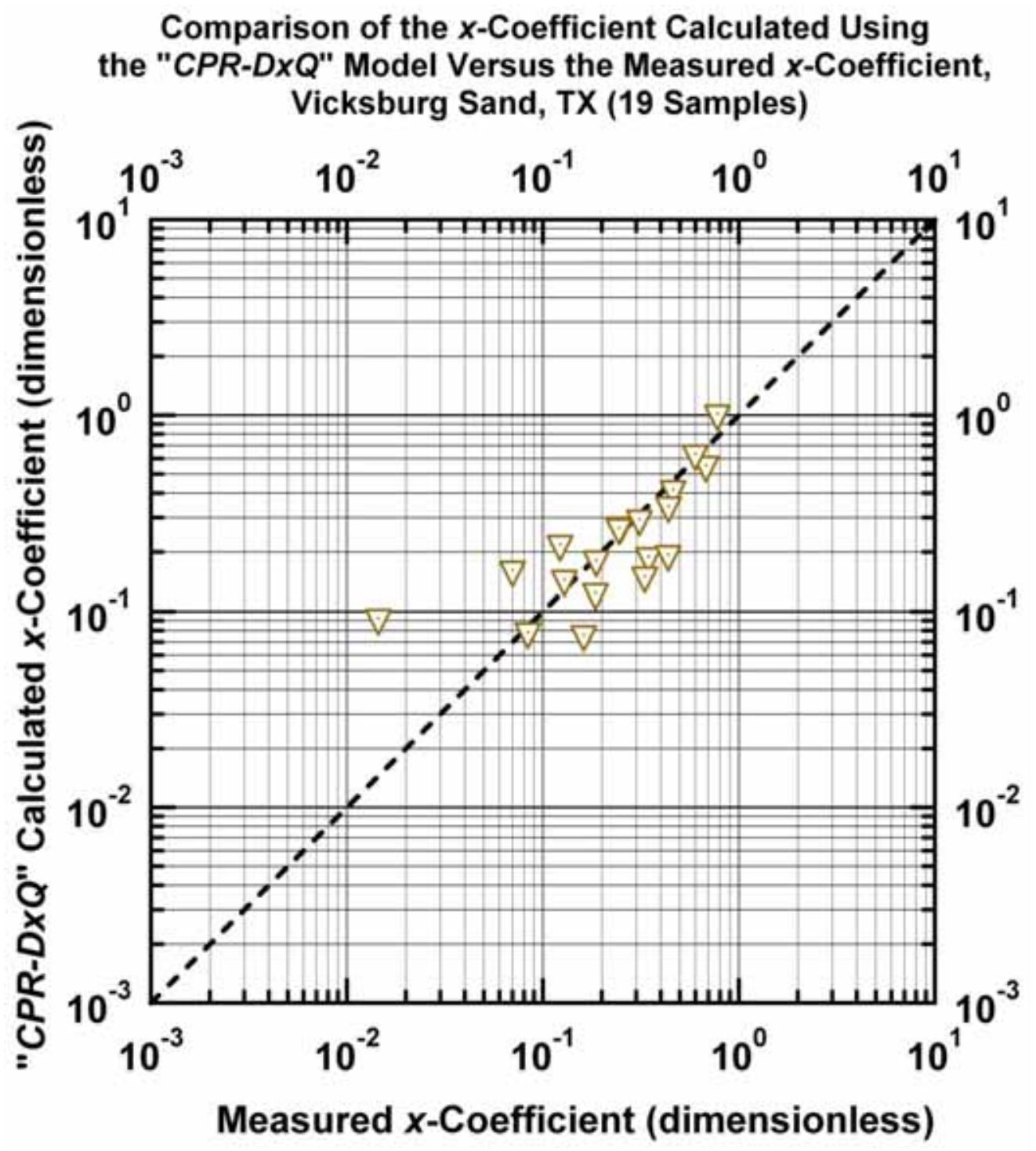

Figure D.9c - Model CPR-DxQ: Calculated $x$-function weight values versus measured $x$ function weight values. [Case: Vicksburg Sand, S. TX (USA)] 
Plot of Depth ( $\mathrm{ft}$ ) Versus Measured and Calculated Permeability (md), Vicksburg Sand, TX (19 Samples)

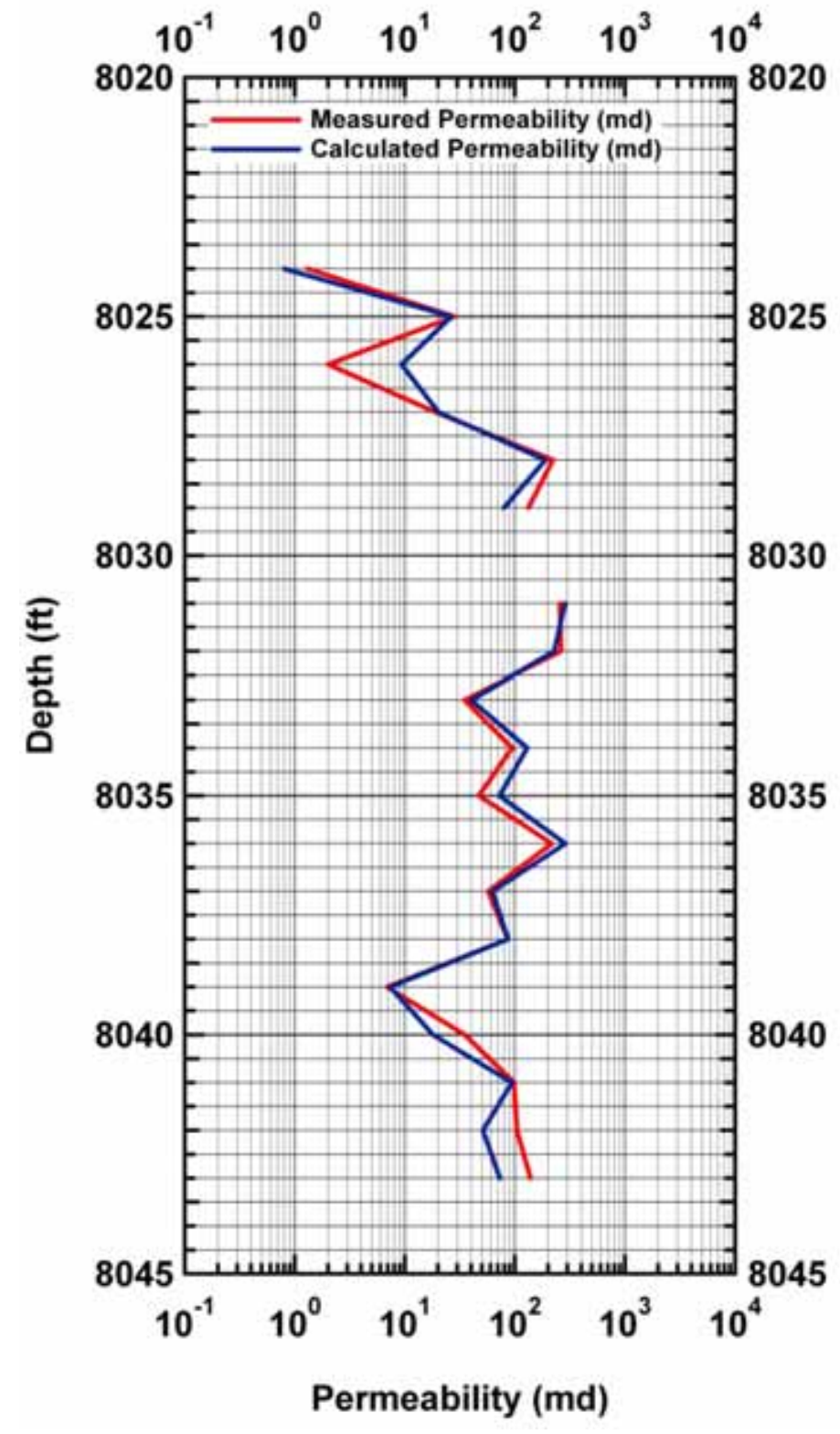

Figure D.9d - Model CPR-A: Calculated and measured permeability values versus depth. [Case: Vicksburg Sand, S. TX (USA)] 


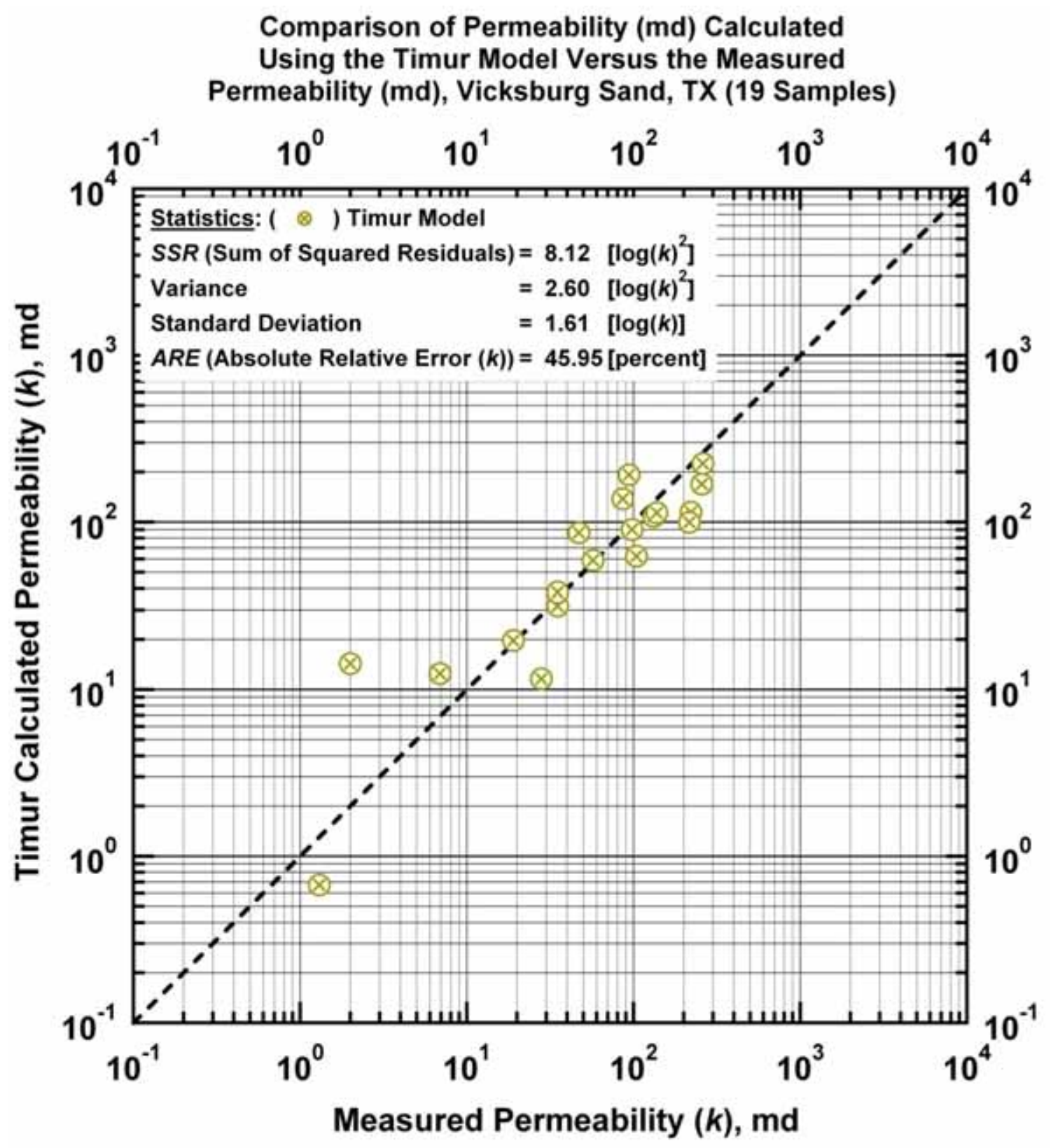

Figure D.10a — "Timur Model:" Calculated versus measured permeability. [Case: Vicksburg Sand, S. TX (USA)] 


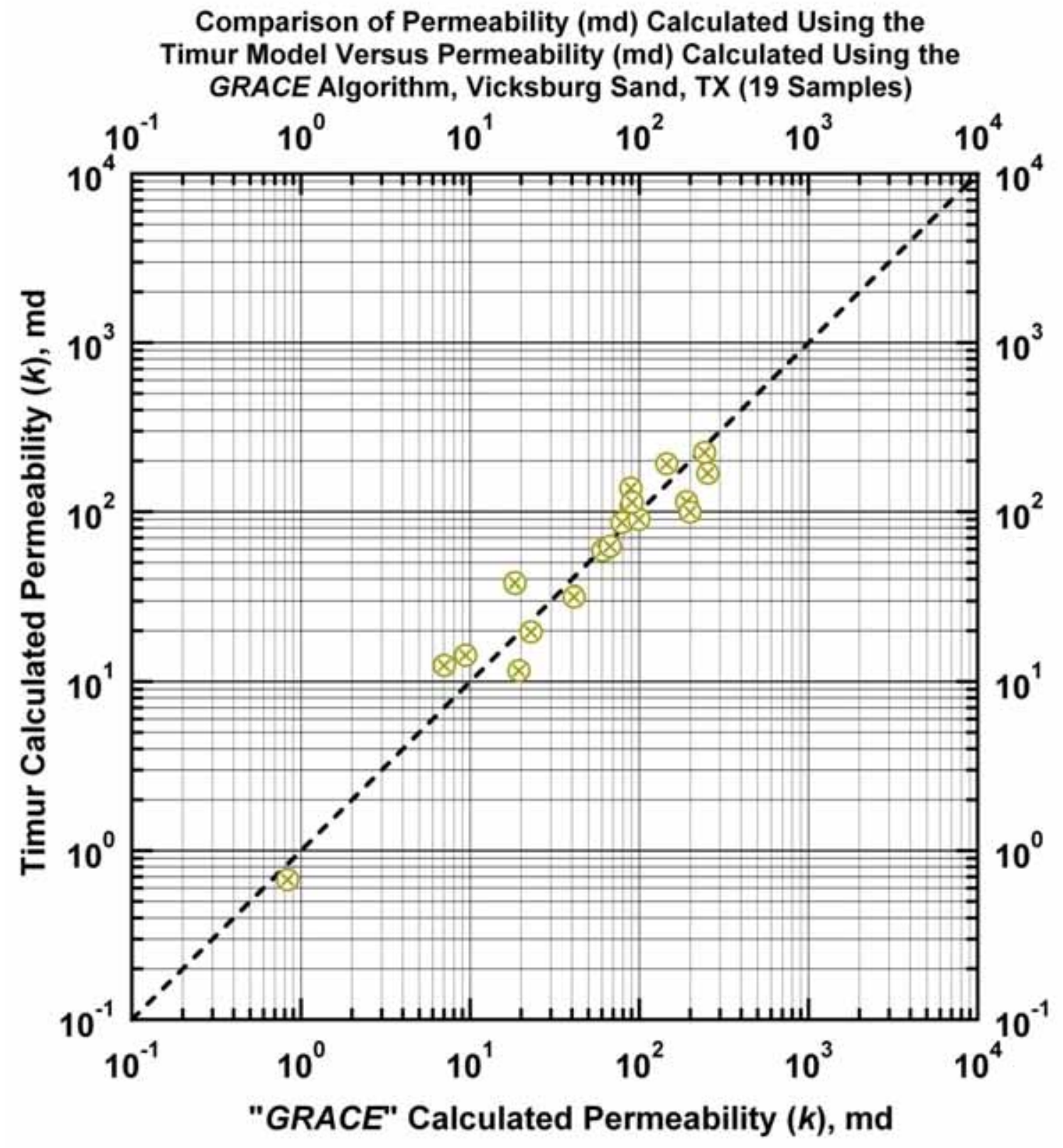

Figure D.10b — "Timur Model:" Permeability calculated using the "Timur Model" versus permeability calculated using the GRACE Algorithm. [Case: Vicksburg Sand, S. TX (USA)] 


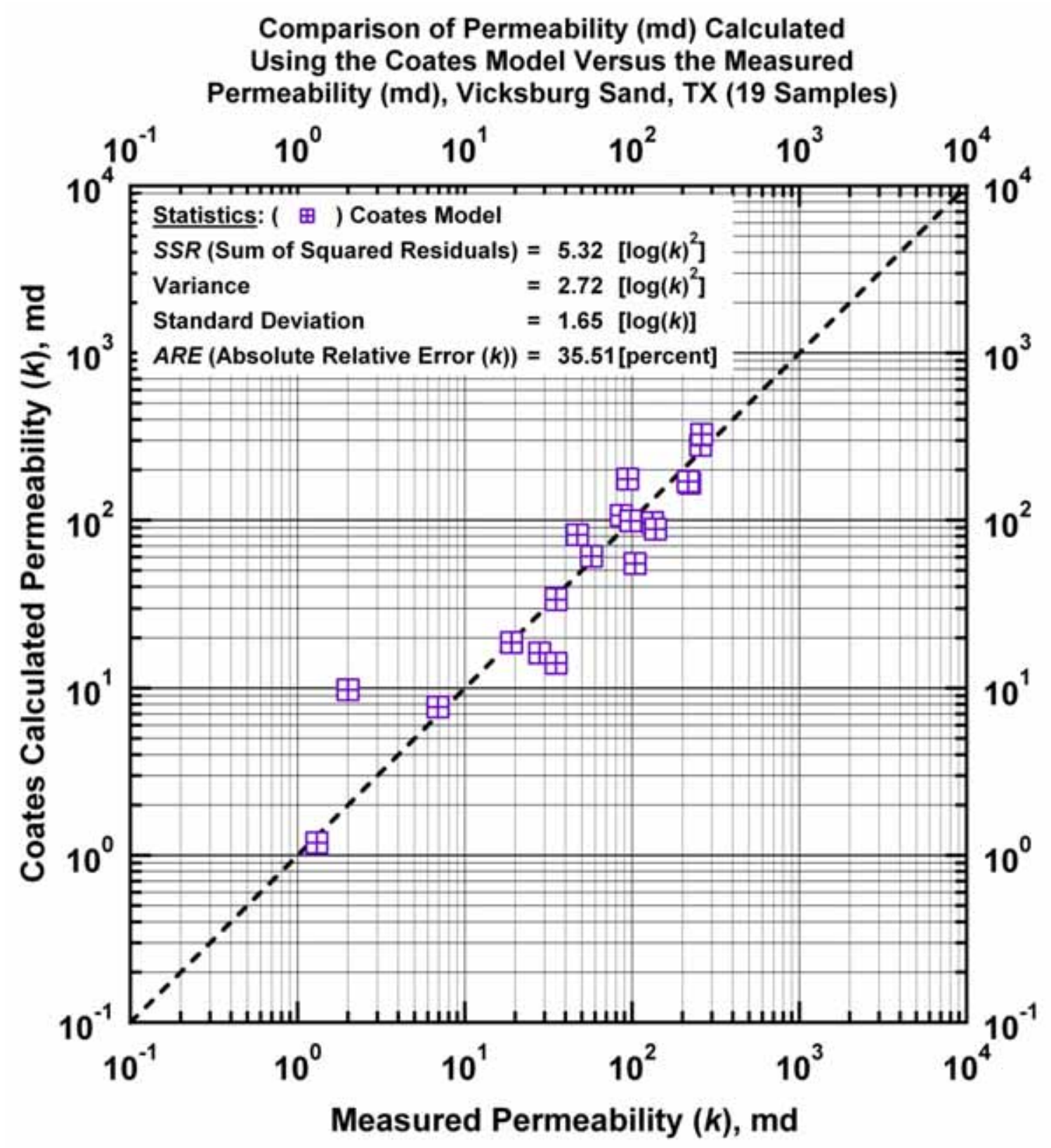

Figure D.11a - "Coates Model:" Calculated versus measured permeability. [Case: Vicksburg Sand, S. TX (USA)] 


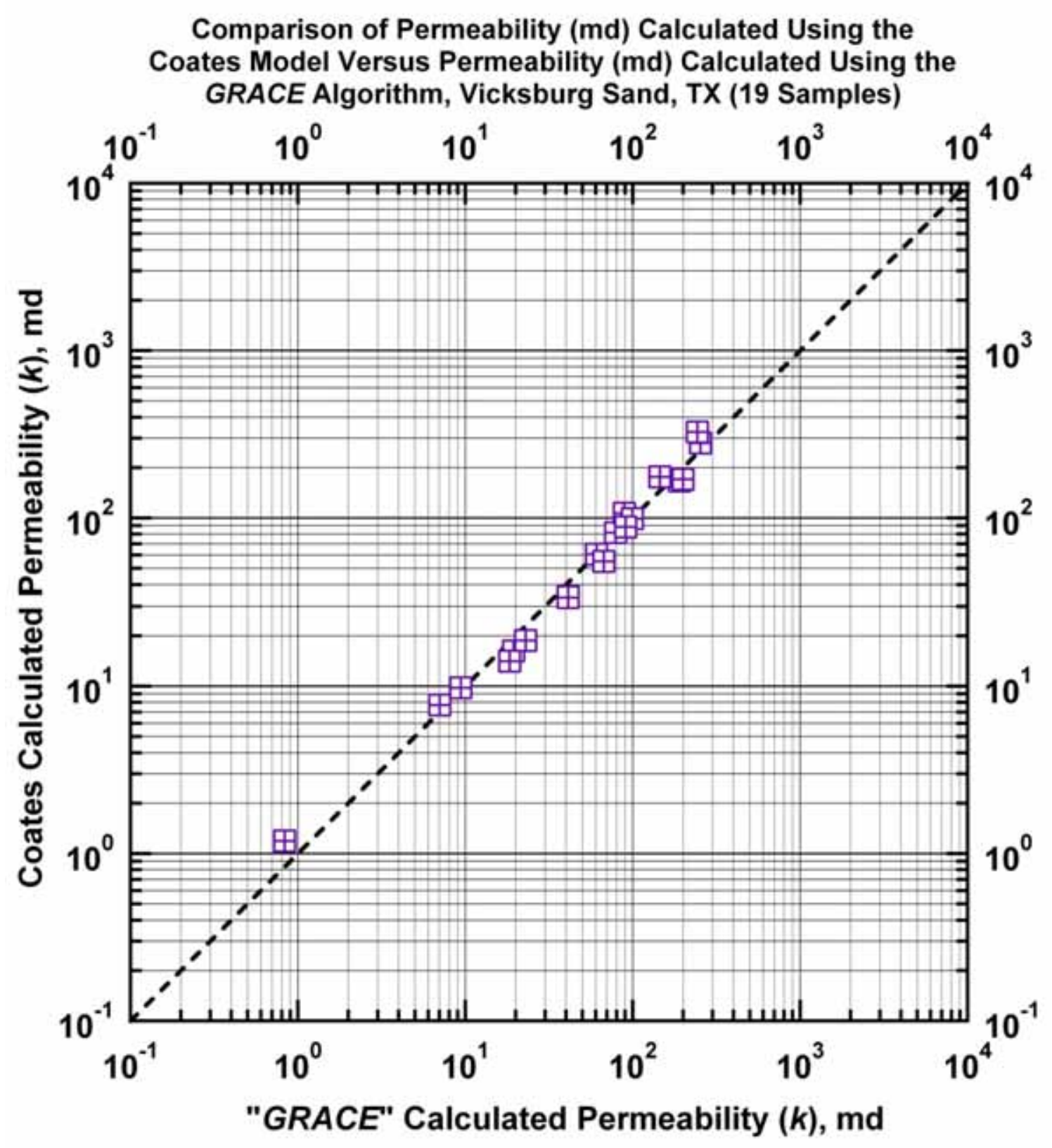

Figure D.11b — "Coates Model:" Permeability calculated using the "Coates Model" versus permeability calculated using the GRACE Algorithm. [Case: Vicksburg Sand, S. TX (USA)] 


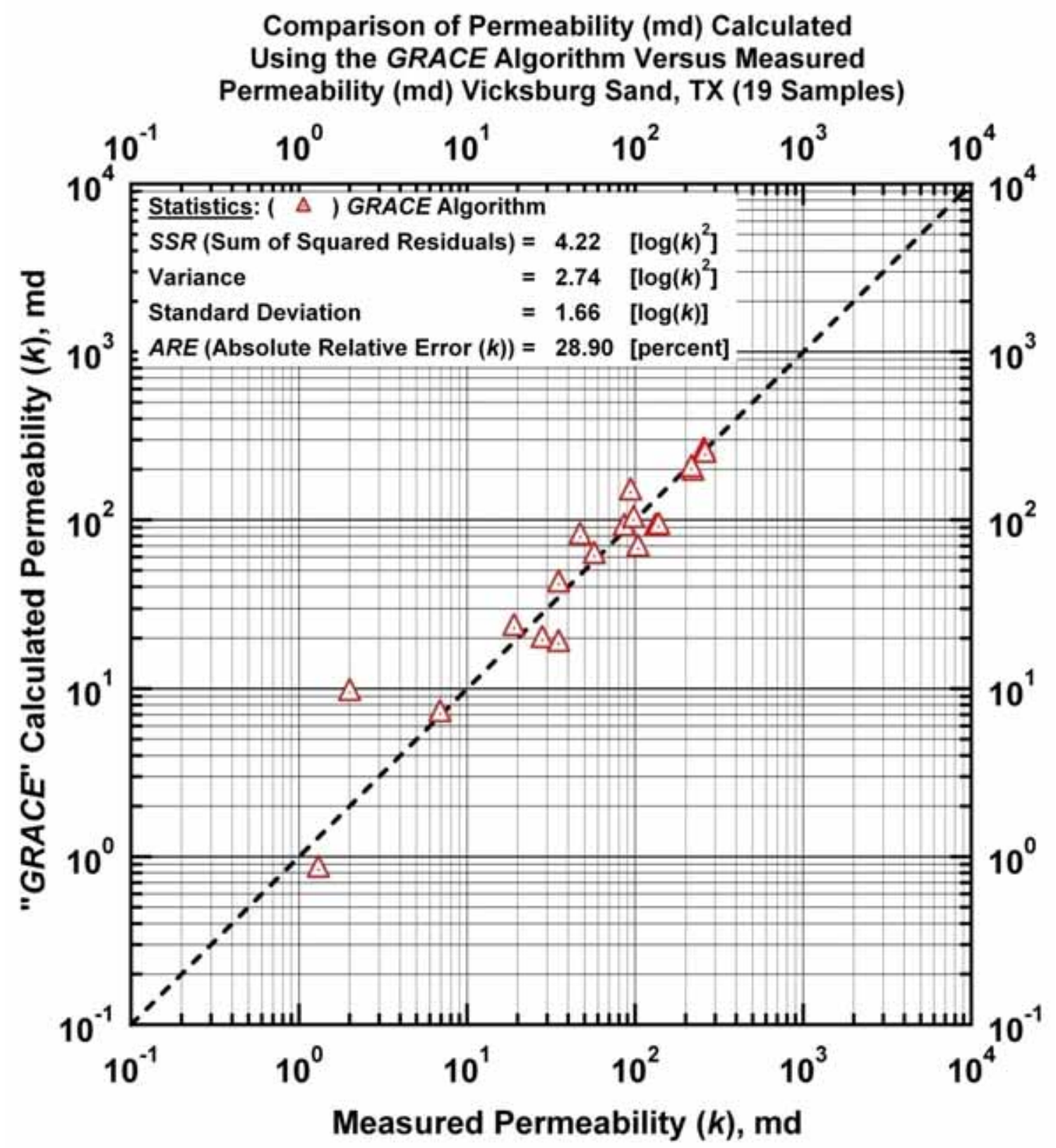

Figure D.12 - GRACE Model: Calculated versus measured permeability. [Case: Vicksburg Sand, S. TX (USA)] 


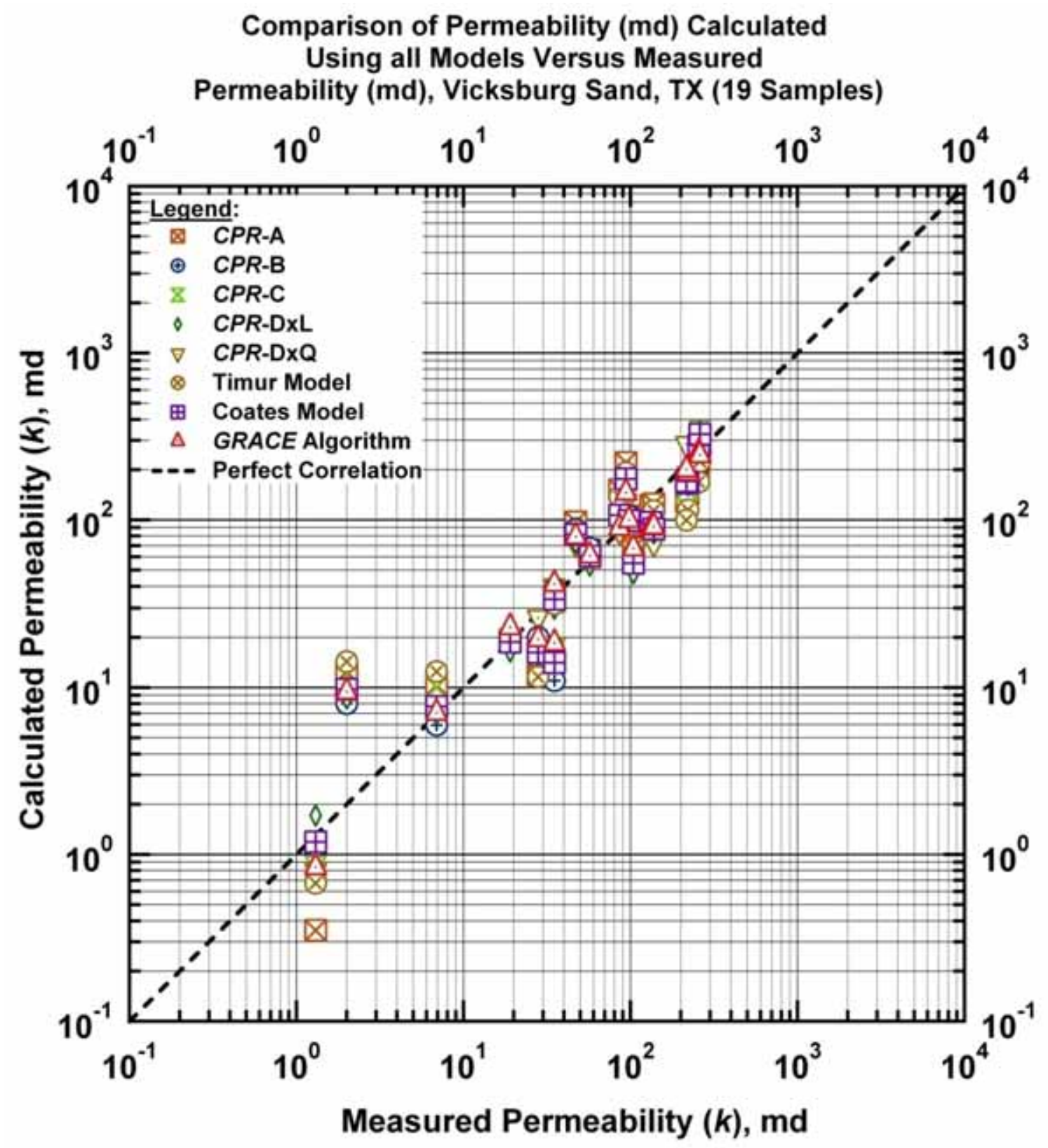

Figure D.13 - All Models: Calculated versus measured permeability. [Case: Vicksburg Sand, S. TX (USA)] 


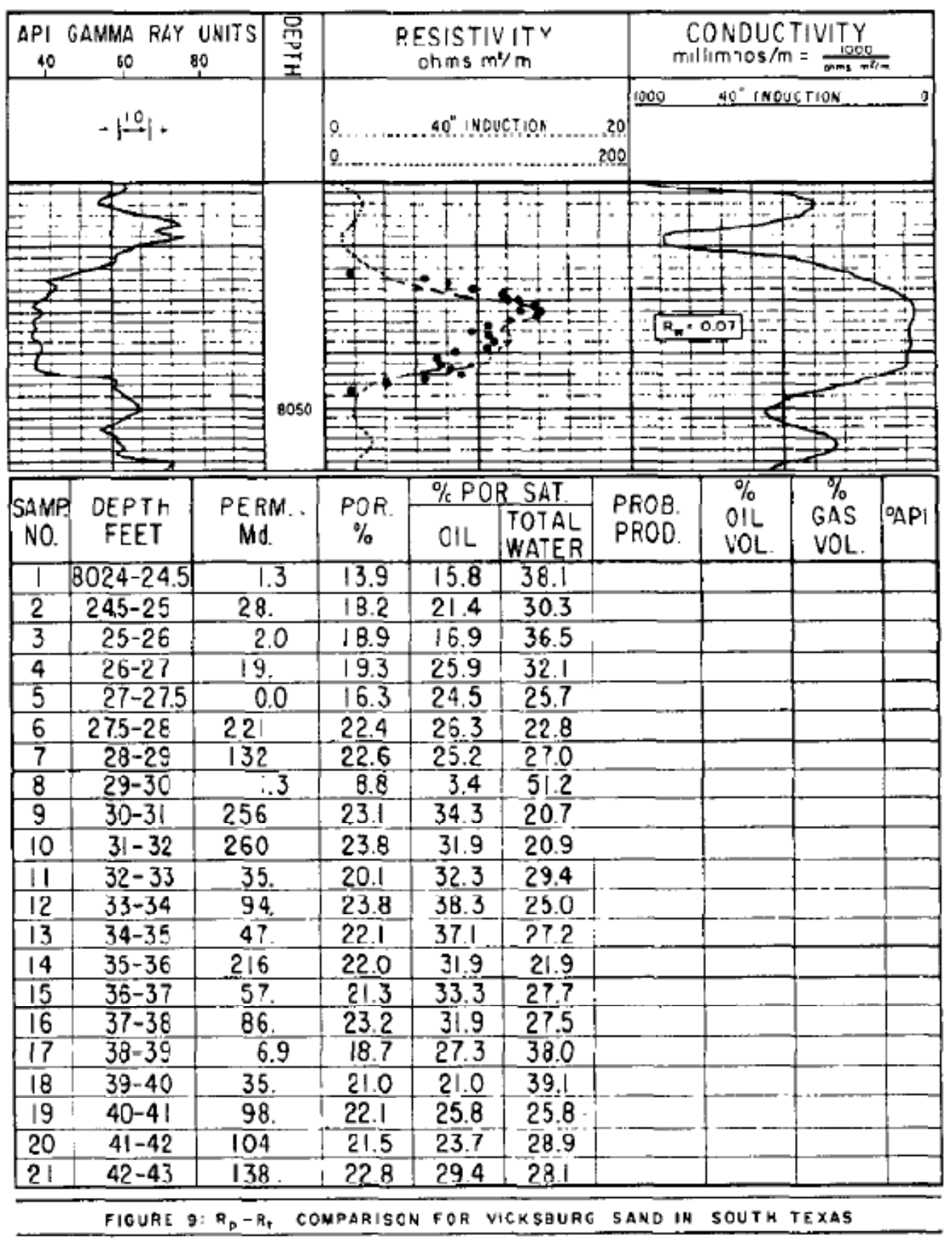

Figure D.14 - Original data from Granberry and Wilshusen (1962). [Case: Vicksburg Sand, S. TX (USA)] 


\section{APPENDIX E}

\section{RESULTS FOR WILCOX SAND \\ (S. TEXAS - USA)}

In this Appendix we present the Wilcox Sand, located in S. Texas (USA), — this is a sandstone reservoir (Granberry and Wilshusen (1962)).

\section{Model CPR-A: (Appendix E)}

The "characteristic permeability relation" (or CPR) Model CPR-A (i.e., the modified Archie "Clean Sand" Model) is given as:

$$
k=a(\phi-c)^{b} \quad c=c_{\max } \exp \left[-c_{1} \phi^{c_{2}} S_{w}^{c_{3}}\right]
$$

Where a plot of $\log (k)$ versus $\log (\phi)$ is used to graphically calibrate Eq. E-1 in terms of the coefficients $a, b$, and $c_{\max }$. Fig. E.1 shows that the CPR-A model performs quite well for this case. Figs. E.1a and b show the log-log and the semilog format of permeability versus porosity, respectively. In Fig. E.2 we provide the "error" plots for this case, where the measured and computed data are compared systematically. In Fig. E.2a we note a good correlation of the results obtained using Model CPR-A versus the measured permeability data - Good agreement with the perfect correlation trend (i.e., the 45-degree line) is observed. In Fig. E.2b we provide a comparison with the GRACE algorithm correlation (Xue et al. 1997)), and we note that the CPR-A model results correlate very well with the GRACE algorithm results for this case. Fig. E.2c we present the calculated and measure $c$-function - and again we note a reasonable correlation of the data about the perfect correlation trend. Lastly, in Fig. E.2d we present the measured and calculated permeability with depth.

\section{Model CPR-B: (Appendix E)}

The "characteristic permeability relation" (or CPR) Model CPR-B (i.e., the modified Archie "Dirty Sand" Model (power-law basis)) is given as:

$$
k=a(\phi+c)^{b} \quad c=c_{\max } \exp \left[-c_{1} \phi^{c_{2}} S_{w}^{c_{3}}\right]
$$

Where a plot of $\log (k)$ versus $\log (\phi)$ is used to graphically calibrate Eq. E-2 in terms of the coefficients $a, b$, and $c_{\max }$. The results for this case are presented in Figs. E.3 and 4. In Fig. E.3a (log-log format) we note that a very distinct "envelope" is formed by the CPR-B model, essentially all of the data on the right and left flanks are well-matched (i.e., the "dirty sand" power law relation (applied on the right portion of the data) and its correction function (applied on the left portion of the data). Similarly, in Fig. E.3b (semilog format), we observe an excellent distribution of the power law model across the body of the data. The error analyses for this case are shown in Fig. E.4. In Fig. E.4a we present the results obtained using 
Model $C P R-B$ versus the measured permeability data and in this case there is good agreement with the perfect correlation trend (i.e., the 45-degree line). We provide comparison with the GRACE algorithm correlation in Fig. E.4b - the results using Model CPR-B and the GRACE algorithm compare very well for this case. We compare the computed and data-derived values of the $c$-function for this case in Fig. E.4c and we note a reasonably "tight" correlation, except for the very lowest values of the $c$-function. Lastly, in Fig. E.4d we present the measured and calculated permeability with depth. In summary, we believe that Model $C P R-B$ is an efficient correlation model for this particular data case.

\section{Model CPR-C: (Appendix E)}

The "characteristic permeability relation" (or CPR) Model CPR-C (i.e., the modified Archie "Dirty Sand" Model (exponential basis)) is given as:

$$
k=c \exp [\beta \phi] \quad c=c_{\max } \exp \left[-c_{1} \phi^{c_{2}} S_{w}^{c_{3}}\right]
$$

Where a plot of $\log (k)$ versus $\phi$ is used to graphically calibrate Eq. E-3 in terms of the coefficients $-\beta$ and $C_{\max }$. Model $C P R-C$ is fundamentally different than Models $C P R-A$ and $C P R-B$, as the basis for Model $C P R-C$ is an exponential function, not a power law relation. In Figs. E.5 and 6 we present the results of applying Model $C P R-B$ for this case, and in general, we observe a very consistent performance of Model $C P R-B$. Fig. E.5b illustrates the excellent conformance of Model $C P R-C$ for this case - again recalling that this model has an exponential relationship with porosity as its basis.

In Figs. E.6a, E.6b, and E.6c we review the error analyses plots for this case, and we note good performance (visually) in terms of the correlation of the results using Model CPR-C. The correlation of the $c$-function (see Fig. E.6c) appears to be very consistent. We believe that the $C P R-C$ model has performed well for this case, and Fig. E.6 confirms the value of this model as a "characteristic relation" for permeability.

\section{Model CPR-D: (Appendix E)}

The "characteristic permeability relation" (or $C P R$ ) Model CPR-D (i.e., the weighted power law-exponential model) is given in its fundamental form as:

$$
\left.k=x a \phi^{b}+(1-x) \alpha \exp [\beta \phi] \quad[0 \leq x \leq 1]\right]
$$

For Eq. E-4 there are two proposed models for the $x$-function (i.e., the weighting function) - these are:

$$
\begin{aligned}
& x=\exp \left[-\ln \left(x_{0}\right)+x_{1} \ln (\phi)+x_{2} \ln \left(S_{w}\right)+x_{3} \ln (\phi) \ln \left(S_{w}\right)\right] \text { (Model CPR-DxL). } \\
& x=\exp \left[\begin{array}{l}
-\ln \left(x_{0}\right)+x_{1} \ln (\phi)+x_{2} \ln \left(S_{w}\right)+x_{3} \ln (\phi) \ln \left(S_{w}\right) \\
+x_{4} \ln (\phi)^{2}+x_{5} \ln \left(S_{w}\right)^{2}+x_{6} \ln (\phi)^{2} \ln \left(S_{w}\right) \\
+x_{7} \ln (\phi) \ln \left(S_{w}\right)^{2}+x_{8} \ln (\phi)^{2} \ln \left(S_{w}\right)^{2}
\end{array}\right] \text { (Model CPR-DxL)......... }
\end{aligned}
$$

As noted in Eq. E-4b, Model CPR-DxL utilizes a logarithmic linear model for the weights ( $x$-values) and as seen in Eq. E-4c, Model CPR-DxQ utilizes a logarithmic quadratic model for the weights ( $x$-values). 
While these models (Eqs. E-4b and E-4c) are empirical, we have found generally good application of these relations for all cases. In this case, the base results using Models $C P R-D x L$ and $C P R-D x Q$ are presented in Fig. E.7 - where the power law and exponential basis functions are fitted to the appropriate portion of the data. The power law equation represents the "Archie clean sand" trend and is fitted to the leftmost data as shown in Fig. E.7a and in contrast, the exponential equation is thought to represent the "Archie Dirty Sand" trend and the exponential is fitted to the far rightmost portion of the data (see Fig. E.7b).

In Figs. E.8 and 9 we present the error analyses for this case, where we have employed the weighting functions (Eqs. E-4b and E-4c) as appropriate. In Fig. E.8a, we find a good correlation of permeability. The comparison of the Model CPR-DxL results are compared to the results obtained from the GRACE algorithm in Fig. E.8b, we note the very good results obtained using Model CPR-DxL. The computed weight function ( $x$-values) shown for the CPR-DxL model in Fig. E.8c does exhibit more scatter than expected, but the trend is (relatively) centered about the perfect correlation line. Fig. E.8d provides the measured and calculated permeability versus depth.

The results obtained using Model $C P R-D x Q$ are presented in Fig. E.9, and we note substantially improved behavior over that of Model $C P R-D x L$. In particular, the base correlation of computed and measured permeabilities (Fig. E.9a) for Model CPR-DxQ does indicate a slightly better correlation than that of Model $C P R-D x L$. Also, the comparison of Model CPR-DxQ with the results from the GRACE algorithm (Fig. E.9b) exhibits better agreement than the results of Model CPR-DxL. Finally, the correlation of the $x$ function (Fig. E.9c) for Model CPR-DxQ is better than the correlation for Model CPR-DxL. These comparisons suggest that Model CPR-DxQ (i.e., the combination of Eqs. E-4a and E-4c) provides a better correlation of data, for this case, than Model CPR-DxL. Fig. E.9d provides the measured and calculated permeability versus depth.

"Timur Model": (Appendix E)

The "Timur Model" [Timur (1968)] is given as:

$$
k_{\text {Timur }}=a \phi^{b} S_{w}^{c}
$$

In Fig. E.10 we present the results of the "Timur Model" (Eq. E-5) which is essentially just a generalized power law function relation in terms of $k, \phi$, and $S_{w}$. As shown in Figs. E.10a and b, the "Timur Model" also provides a good correlation of the data for this case. The comparison of results using the "Timur Model" with the results of the GRACE algorithm (Fig. E.10b). 


\section{"Coates Model": (Appendix E)}

The "Coates Model" [referenced by Ahmed et al. (1989)] is given as:

$$
k_{\text {Coates }}=\left[100 \phi^{2} \frac{\left(1-S_{w}\right)}{S_{w}}\right]^{2}
$$

In Fig. E.11 we present the results of the "Coates Model" (Eq. E-6) which is essentially just a modified power function relation in terms of $k, \phi$, and $S_{w}$. The results are shown in Figs. E.11a and $\mathbf{b}$ of the data for this case. The comparison of results using the "Coates Model" with the results of the GRACE algorithm (Fig. E.11b) is also shown.

\section{GRACE Algorithm: (Appendix E)}

In Fig. E.12 we present the results predicted by the GRACE algorithm, which is a non-parametric regression approach (see Xue et al. (1997) for details). In theory, the GRACE algorithm should provide the most unbiased correlation of the data - i.e., the GRACE algorithm is designed not "fit the errors" as other regression approaches may. It is our contention that the GRACE algorithm is the statistical standard - and any algorithm/approach/model which achieves better regression statistics than the GRACE algorithm is actually "fitting the errors" in the data. None of the cases have significantly better regression statistics than the GRACE algorithm — hence, none of the cases are "over-fitted" in a statistical sense.

\section{Comparison of All Models: (Appendix E)}

Fig. E.13, illustrates all of the models on a single plot of calculated versus measured permeability. We also present a table of all statistical results in Table E.1. Our last plot, Fig. E.14, illustrates the source of the original data set.

Table E.1 - Statistical Results for all Models (Wilcox Sand, S. TX (USA),

\begin{tabular}{|c|c|c|c|c|}
\hline Model & $\begin{array}{c}\text { Sum of } \\
\text { Squared } \\
\text { Residuals } \\
{\left[\log (k)^{2}\right]}\end{array}$ & $\begin{array}{l}\text { Variance } \\
{\left[\log (k)^{2}\right]}\end{array}$ & $\begin{array}{c}\text { Standard } \\
\text { Deviation } \\
{[\log (k)]}\end{array}$ & $\begin{array}{c}\text { Absolute } \\
\text { Relative } \\
\text { Error } \\
\text { [percent] }\end{array}$ \\
\hline CPR-A & 8.31 & 2.14 & 1.46 & 43.45 \\
\hline$C P R-B$ & 7.92 & 2.21 & 1.49 & 42.18 \\
\hline$C P R-C$ & 7.83 & 2.36 & 1.54 & 40.82 \\
\hline$C P R-D x L$ & 7.15 & 2.03 & 1.42 & 44.53 \\
\hline$C P R-D x Q$ & 6.87 & 2.04 & 1.43 & 41.50 \\
\hline Timur & 7.74 & 2.19 & 1.48 & 41.11 \\
\hline Coates & 7.66 & 2.19 & 1.48 & 40.74 \\
\hline GRACE Algorithm & 5.21 & 2.18 & 1.48 & 34.10 \\
\hline
\end{tabular}


The coefficients for the models used in this case are provided in Table E.2.

Table E.2a - Model Coefficients (Wilcox Sand, S. TX (USA), $n=27$ ).

\begin{tabular}{|c|c|c|c|c|c|c|}
\hline Model & $a$ or $\alpha$ & $b$ or $\beta$ & $C$ or $C_{\max }$ & $c_{1}$ & $c_{2}$ & $c_{3}$ \\
\hline$C P R-A$ & $1.00 \times 10^{8}$ & 8.00 & 0.050 & 0.03 & -0.22 & -2.73 \\
\hline$C P R-B$ & $5.00 \times 10^{6}$ & 8.00 & 0.070 & 0.09 & -2.68 & 3.00 \\
\hline$C P R-C$ & $5.00 \times 10^{-8}$ & 110.00 & $3.00 \times 10^{-6}$ & 1109.49 & 2.68 & 1.19 \\
\hline Timur & $2.65 \times 10^{7}$ & 9.66 & 2.999 & - & - & - \\
\hline Coates & $2.94 \times 10^{4}$ & 5.20 & 1.835 & - & - & - \\
\hline
\end{tabular}

Table E.2b - Model Coefficients (Wilcox Sand, S. TX (USA), $n=27$ ).

\begin{tabular}{|c|c|c|c|c|}
\hline Model & $a$ & $b$ & $\alpha$ & $\beta$ \\
\hline$C P R-D x L$ & $1.00 \times 10^{8}$ & 8.00 & $5.00 \times 10^{-8}$ & 110.00 \\
\hline$C P R-D x Q$ & $1.00 \times 10^{8}$ & 8.00 & $5.00 \times 10^{-8}$ & 110.00 \\
\hline
\end{tabular}

\begin{tabular}{|c|c|c|c|c|c|c|c|c|c|}
\hline Model & $c_{0}$ & $c_{1}$ & $c_{2}$ & $c_{3}$ & $C_{4}$ & $c_{5}$ & $c_{6}$ & $c_{7}$ & $c_{8}$ \\
\hline$C P R-D x L$ & $9.54 \times 10^{8}$ & -7.61 & -16.93 & -6.60 & - & - & - & - & - \\
\hline$C P R-D x Q$ & $1.76 \times 10^{4}$ & 3.25 & 14.42 & 16.70 & 1.78 & 8.42 & 1.52 & -0.35 & -3.63 \\
\hline
\end{tabular}

\section{Nomenclature: (Appendix E)}

$$
\begin{array}{ll}
S_{w} & =\text { Water Saturation (fraction) } \\
k & =\text { Formation Permeability, md (or any consistent units) } \\
\phi & =\text { Porosity, fraction }
\end{array}
$$

\section{References: (Appendix E)}

Ahmed, U., Crary, S.F., and Coates, G.R: "Permeability Estimation: The Various Sources and Their Interrelationship," paper SPE 19604 presented at the 1989 SPE Annual Technical Conference and Exhibition, San Antonio, TX, 8-11 Oct., 649-662.

Granberry, R.J., and Wilshusen, R.C.: "Improved Interpretation of Formation Productivity by Combined Use of Core Analysis and Electronic Log Data," Trans., Gulf Coast Association of Geological Societies (1962) 13, 213-221.

Timur, A.: "An Investigation of Permeability, Porosity, and Residual Water Saturation Relationships," Trans., SPWLA Symposium (1968) paper I.

Xue, G., Datta-Gupta, A., Valko, P., and Blasingame, T.A.: "Optimal Transformations for Multiple Regression: Application to Permeability Estimation from Well Logs," SPEFE (June 1997), 85-93. 


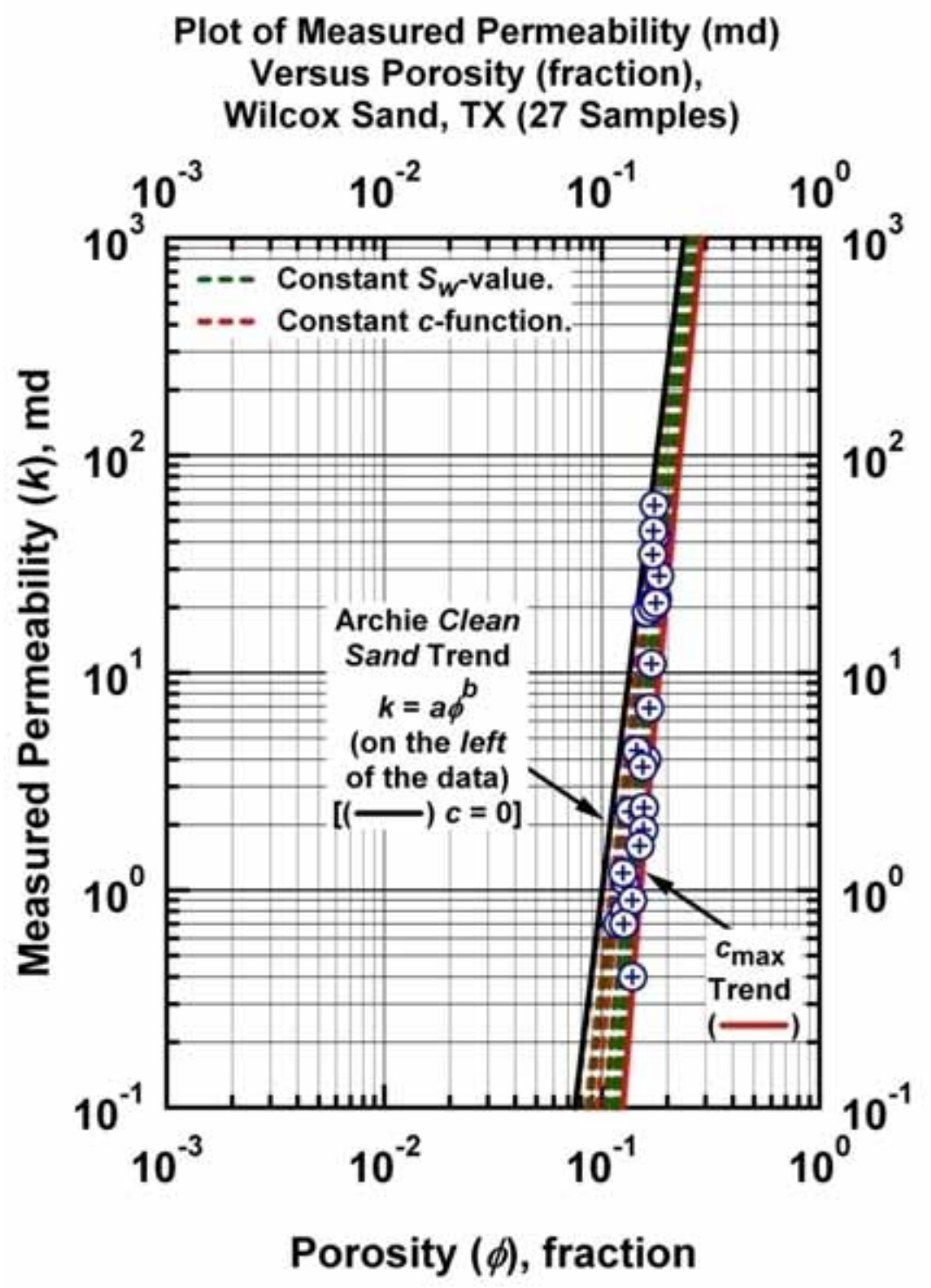

Figure E.1a - Model CPR-A: $\quad k=a(\phi-c)^{b} \quad c=c_{\max } \exp \left[-c_{1} \phi^{c_{2}} S_{w}^{c_{3}}\right] \quad$ - "Clean Sand" Plot (log-log format) - Archie "Clean Sand" trend is given by the straight-line trend at the far left of the data (power law model). [Case: Wilcox Sand, S. TX (USA)] 


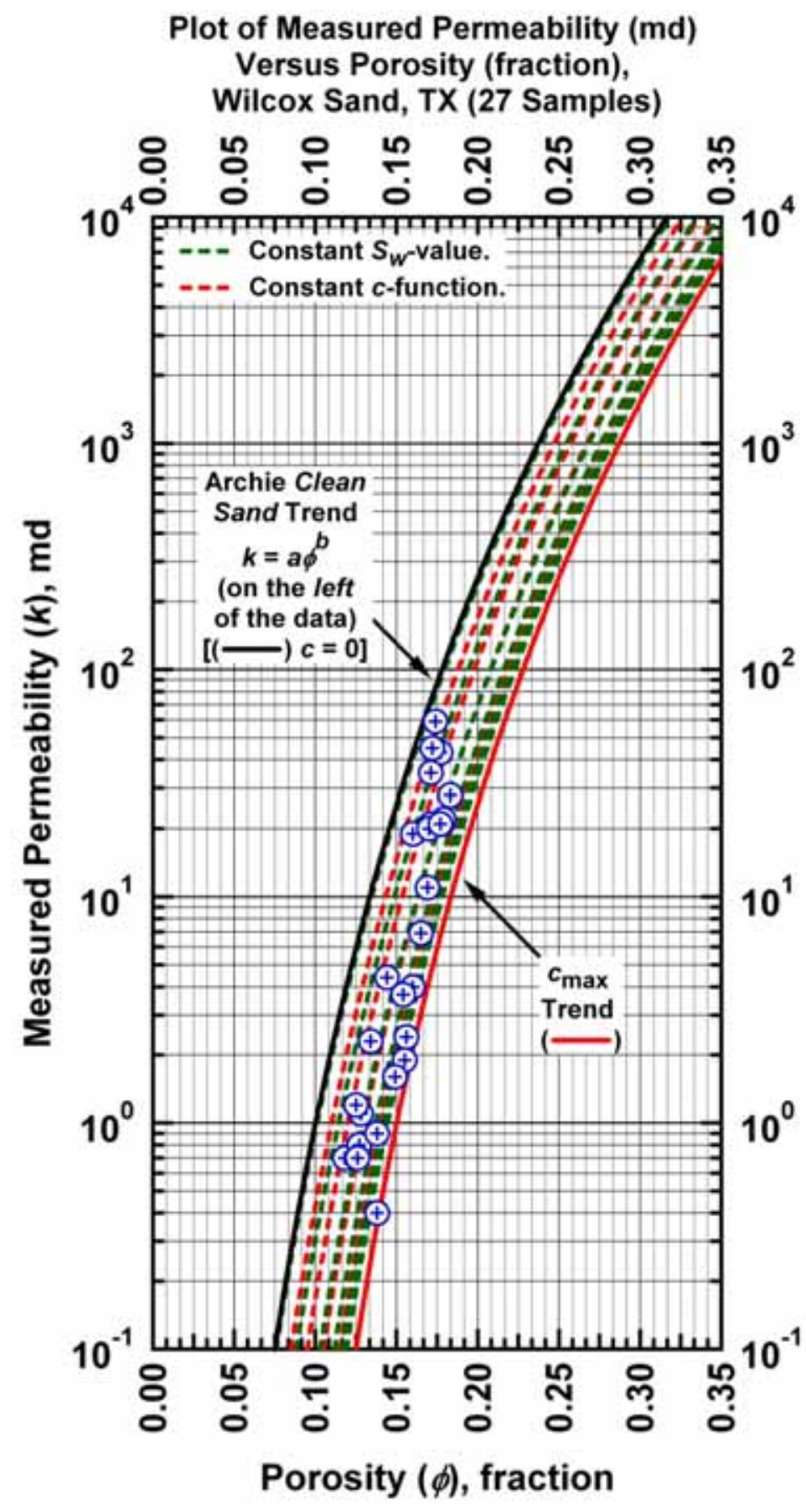

Figure E.1b - Model CPR-A: $k=a(\phi-c)^{b} \quad c=c_{\max } \exp \left[-c_{1} \phi^{c_{2}} S_{w}^{c_{3}}\right]$ - "Dirty Sand" Plot (semilog format). Archie "Clean Sand" trend is given by the curved trend at the far left of the data (power law model). [Case: Wilcox Sand, S. TX (USA)] 
Comparison of Permeability (md) Calculated Using the "CPR-A" Model Versus the Measured Permeability (md), Wilcox Sand, TX (27 Samples)

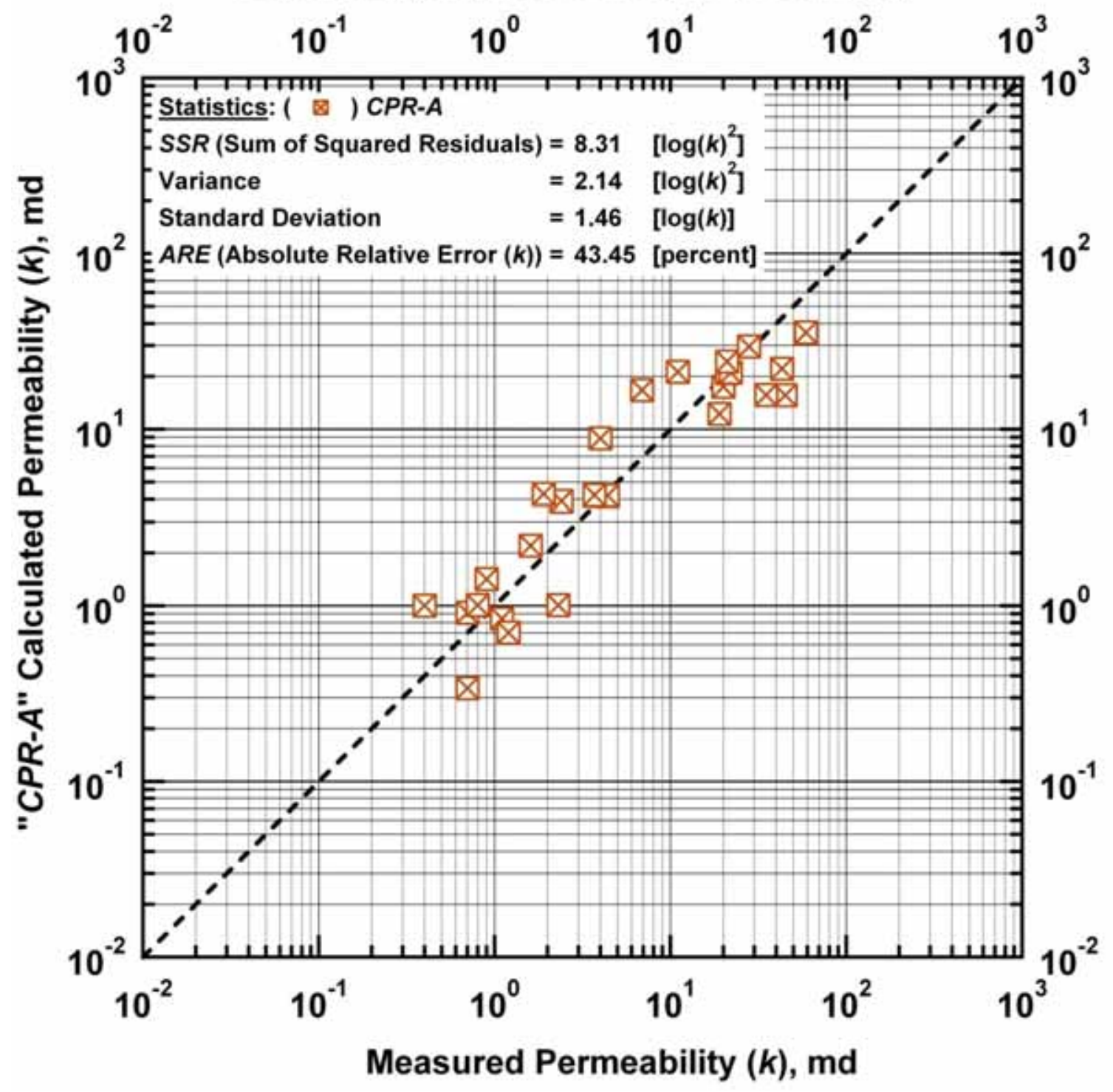

Figure E.2a - Model CPR-A: calculated versus measured permeability. [Case: Wilcox Sand, S. TX (USA)] 


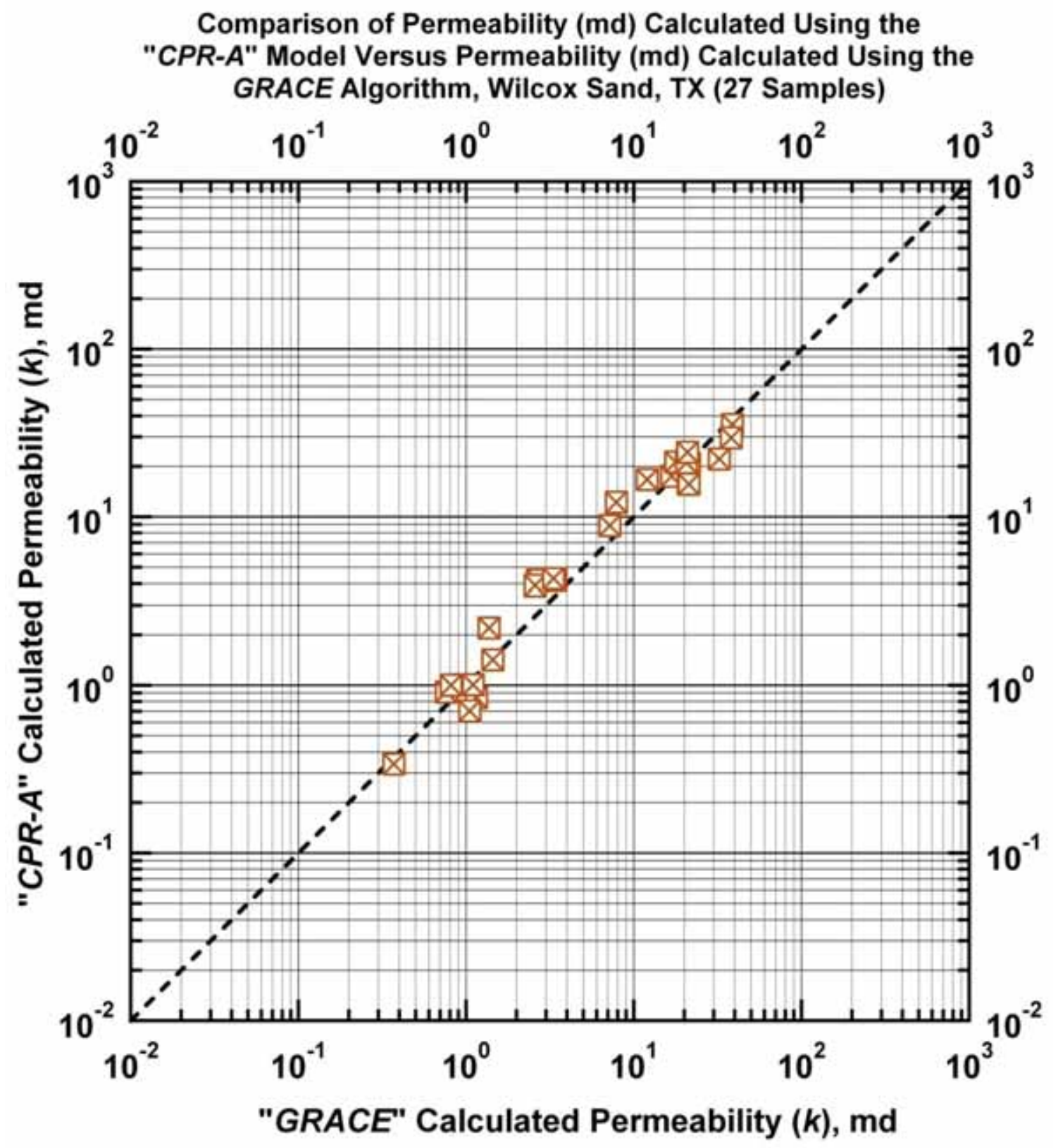

Figure E.2b - Model CPR-A: Permeability calculated using Model CPR-A versus permeability calculated using the GRACE Algorithm. [Case: Wilcox Sand, S. TX (USA)] 


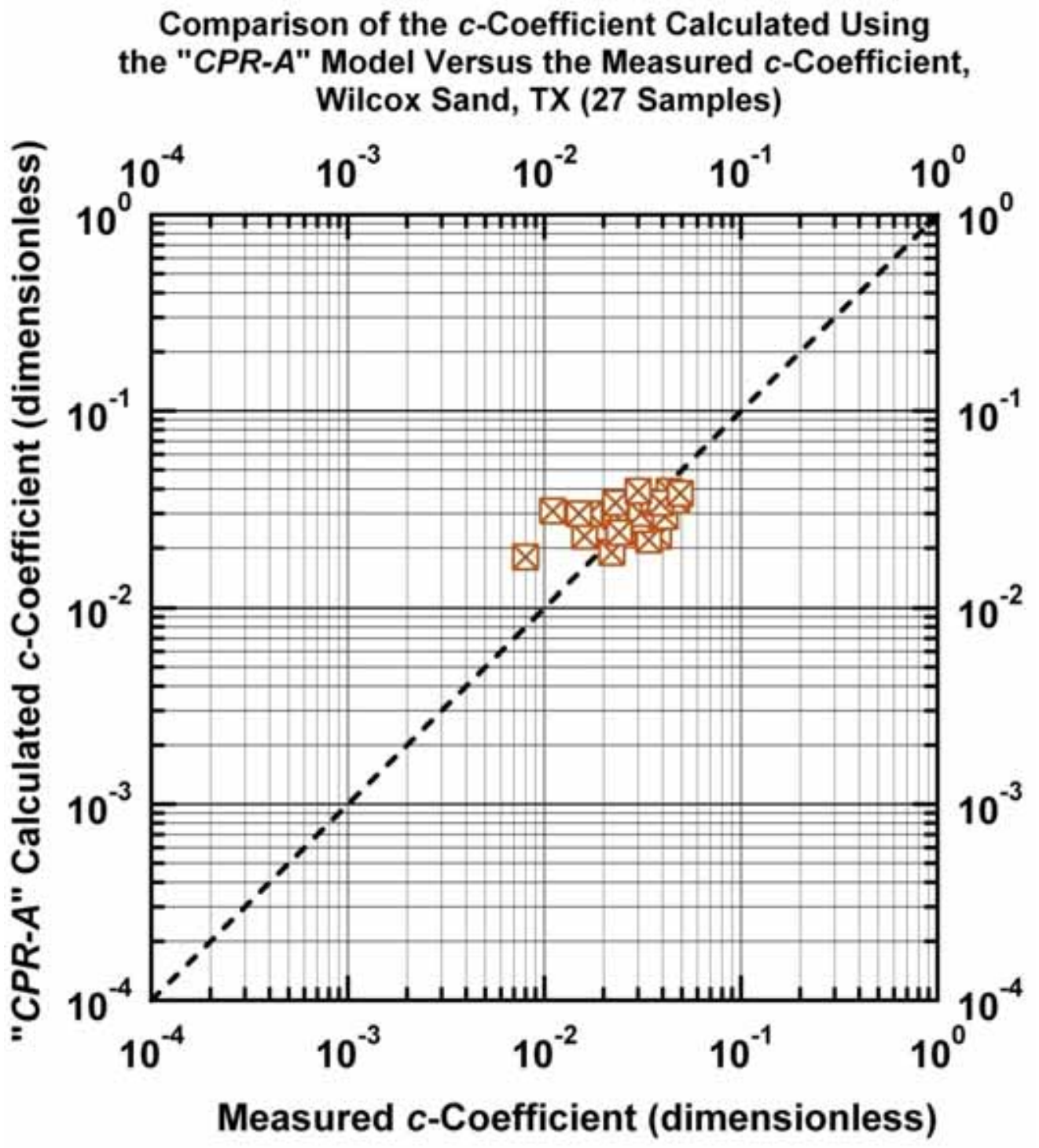

Figure E.2c - Model $C P R$ - $A$ : Calculated $c$-function values versus measured $c$-function values. [Case: Wilcox Sand, S. TX (USA)] 


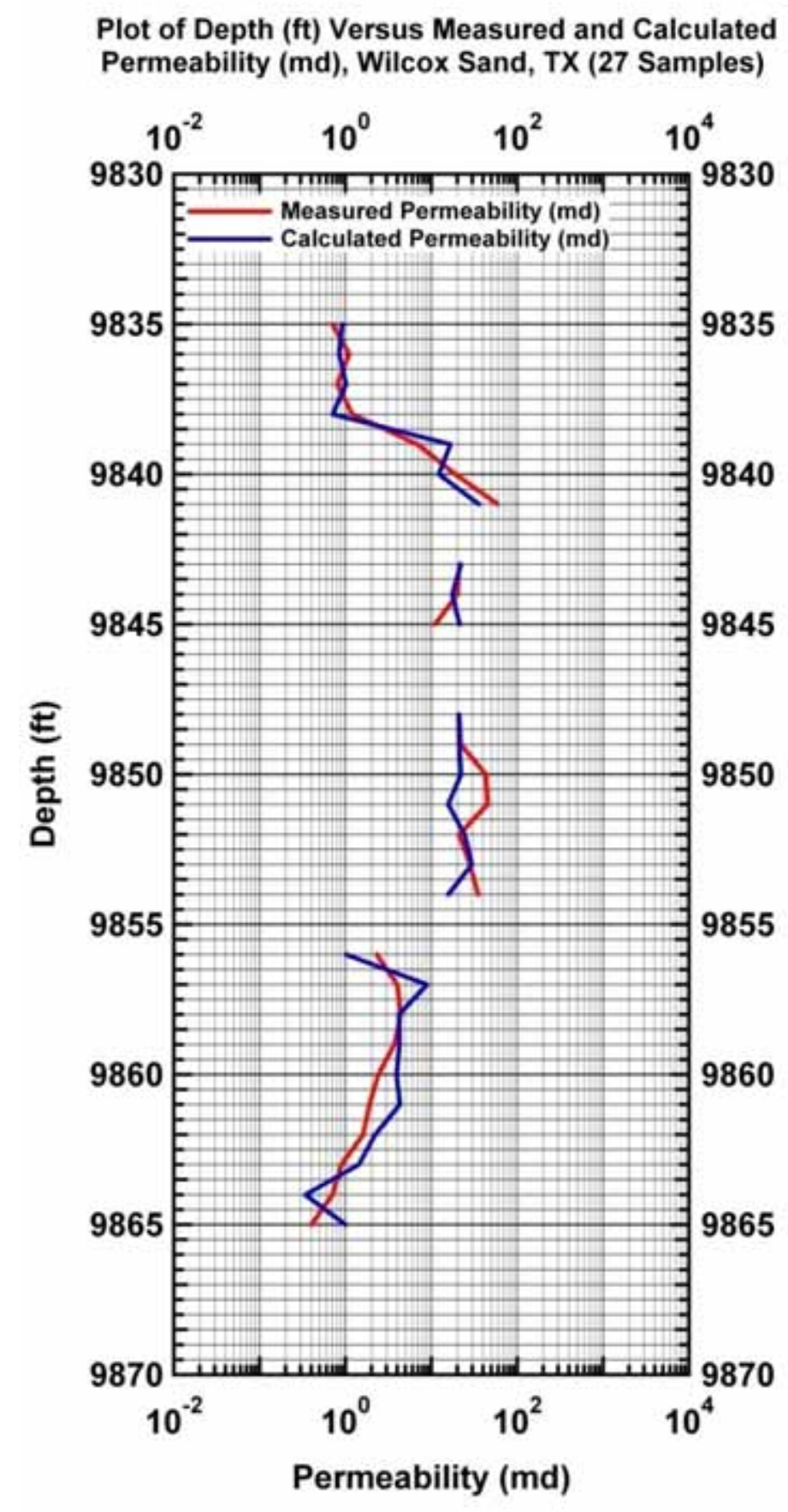

Figure E.2d - Model CPR-A: Calculated and measured permeability values versus deptE. [Case: Wilcox Sand, S. TX (USA)] 


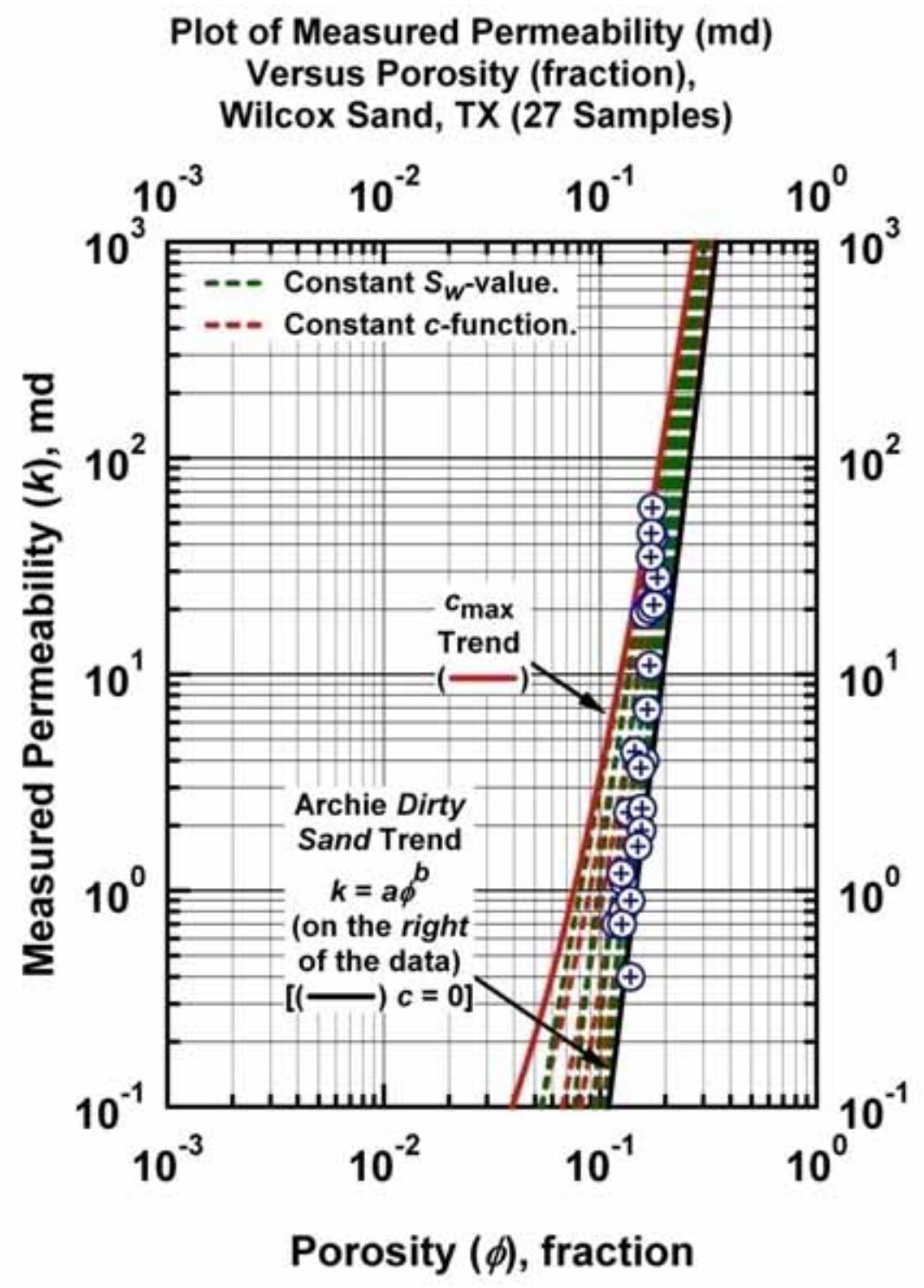

Figure E.3a - Model $C P R-B: \quad k=a(\phi+c)^{b} \quad c=c_{\max } \exp \left[-c_{1} \phi^{c_{2}} S_{w}^{c_{3}}\right]$ - "Clean Sand" Plot (log-log format) - Archie "Dirty Sand" trend is given by the straight-line trend at the far right of the data (power law model). [Case: Wilcox Sand, S. TX (USA)] 


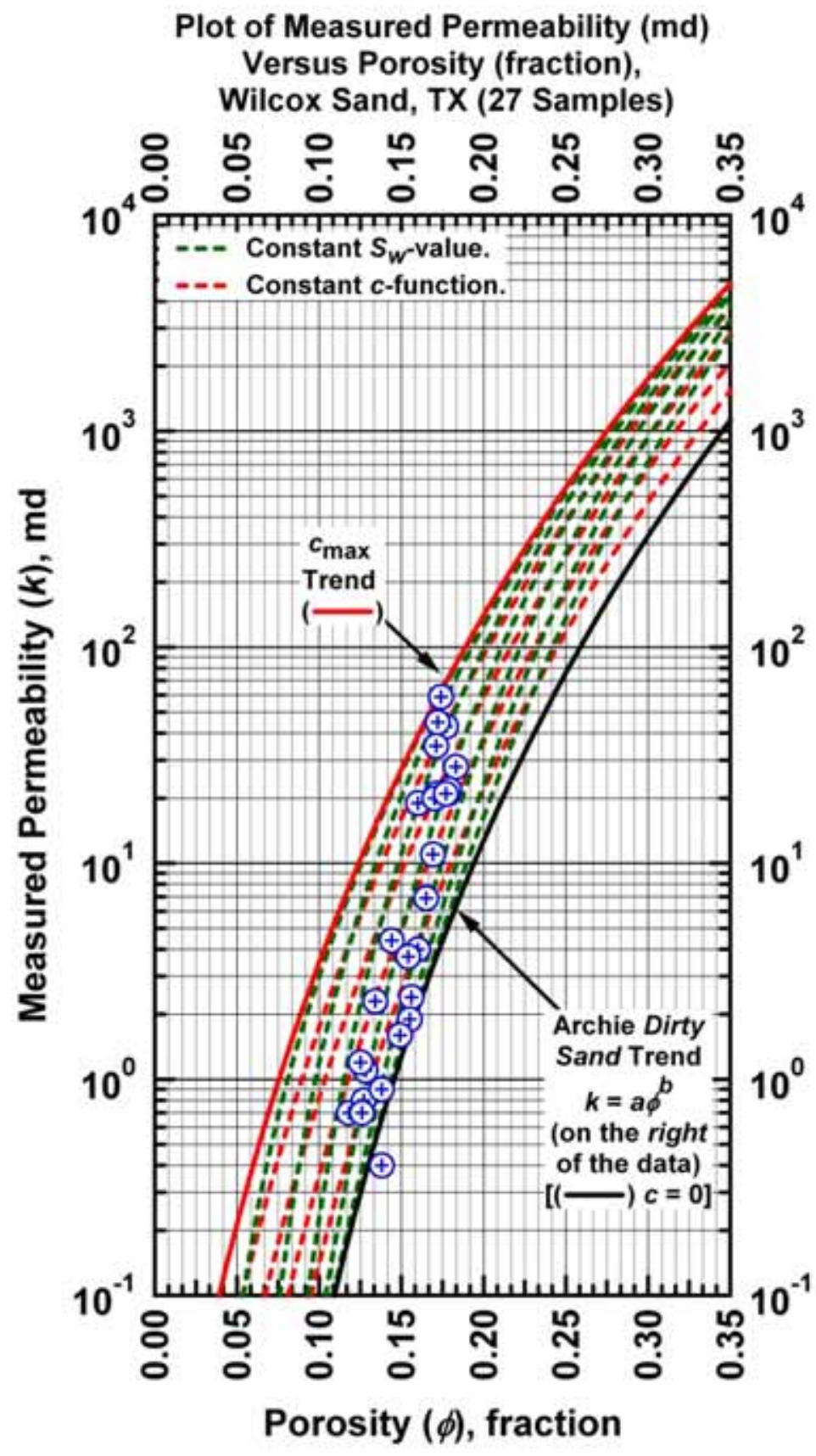

Figure E.3b - Model CPR-B: $k=a(\phi+c)^{b} \quad c=c_{\max } \exp \left[-c_{1} \phi^{c_{2}} S_{w}^{c_{3}}\right]$ - "Dirty Sand" Plot (semilog format) - Archie "Dirty Sand" trend is given by the curved trend at the far right of the data (power law model). [Case: Wilcox Sand, S. TX (USA)] 


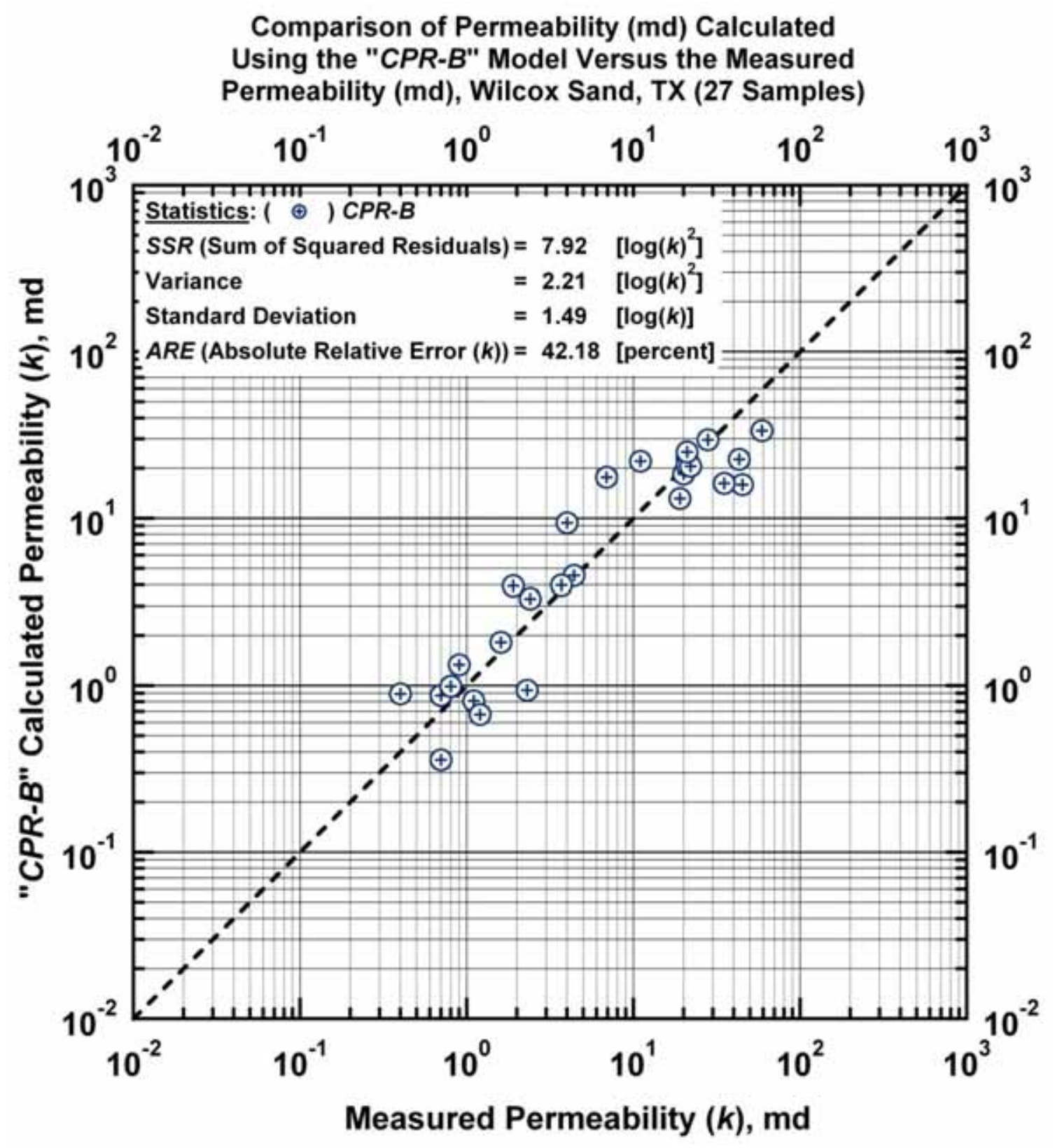

Figure E.4a - Model CPR-B: calculated versus measured permeability. [Case: Wilcox Sand, S. TX (USA)] 


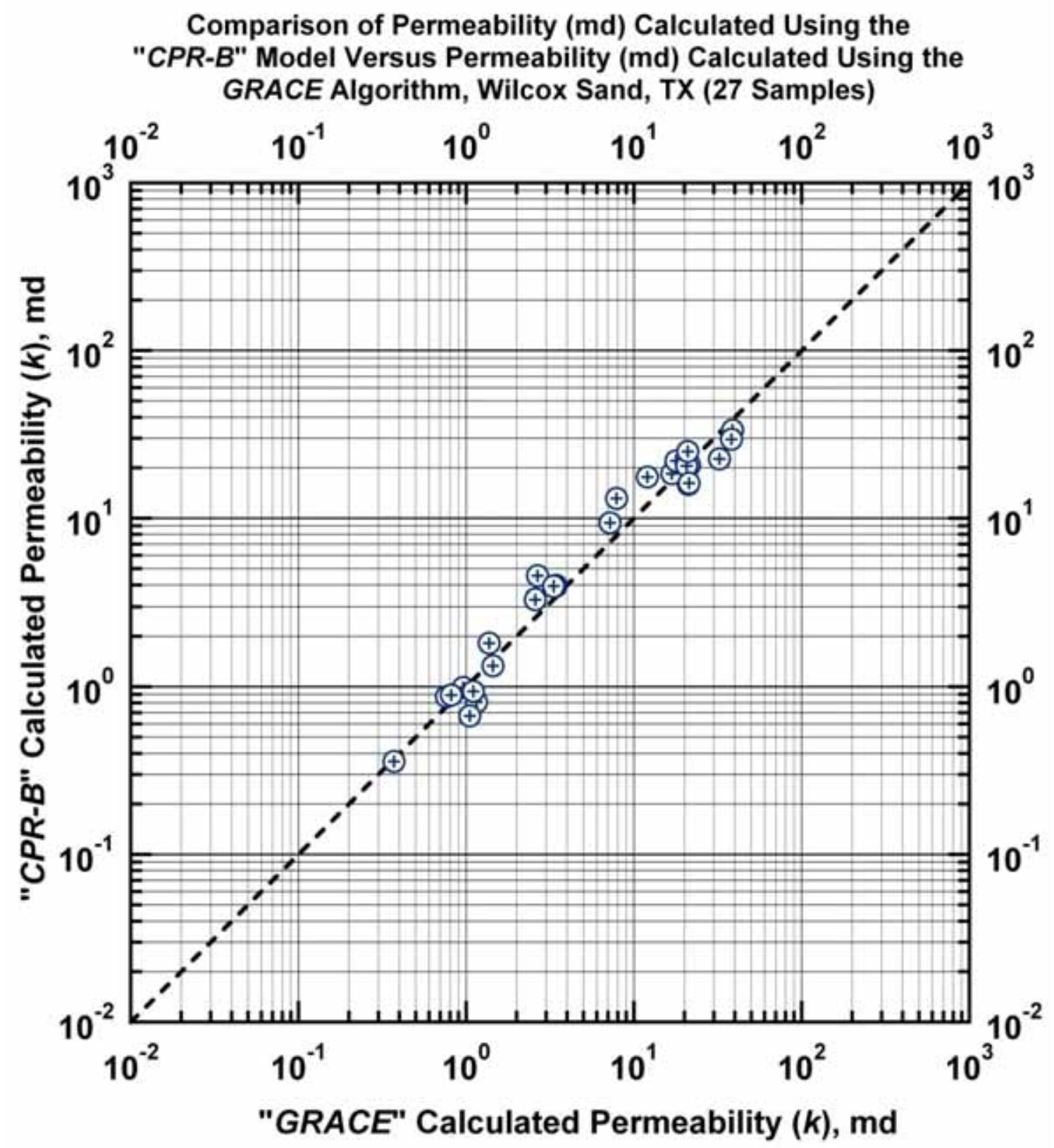

Figure E.4b - Model CPR-B: Permeability calculated using Model $C P R-B$ versus permeability calculated using the GRACE Algorithm. [Case: Wilcox Sand, S. TX (USA)] 


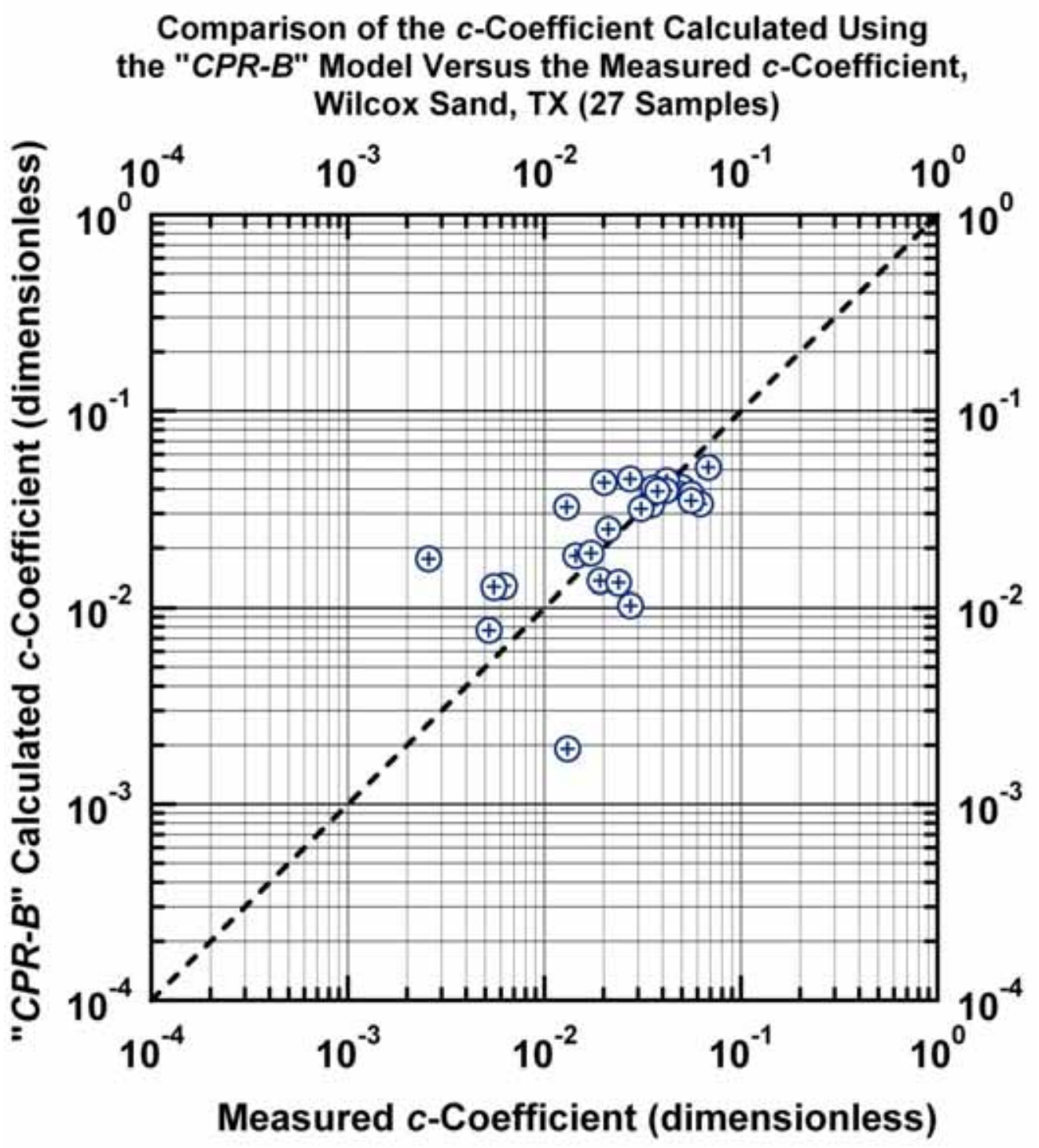

Figure E.4c - Model CPR-B: Calculated $c$-function values versus measured $c$-function values. [Case: Wilcox Sand, S. TX (USA)] 
Plot of Depth ( $\mathrm{ft}$ ) Versus Measured and Calculated Permeability (md), Wilcox Sand, TX (27 Samples)

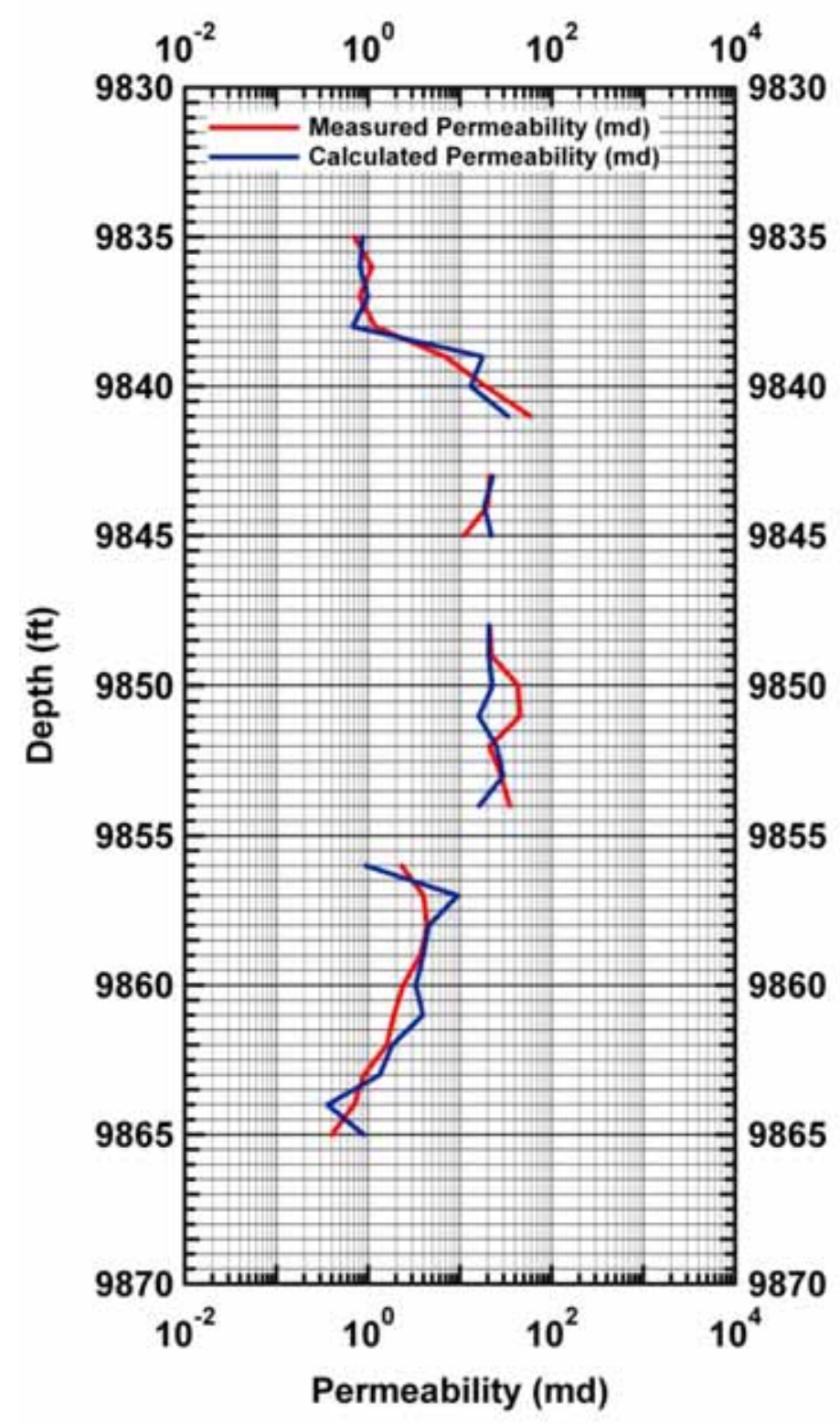

Figure E.4d - Model CPR-A: Calculated and measured permeability values versus depth. [Case: Wilcox Sand, S. TX (USA)] 


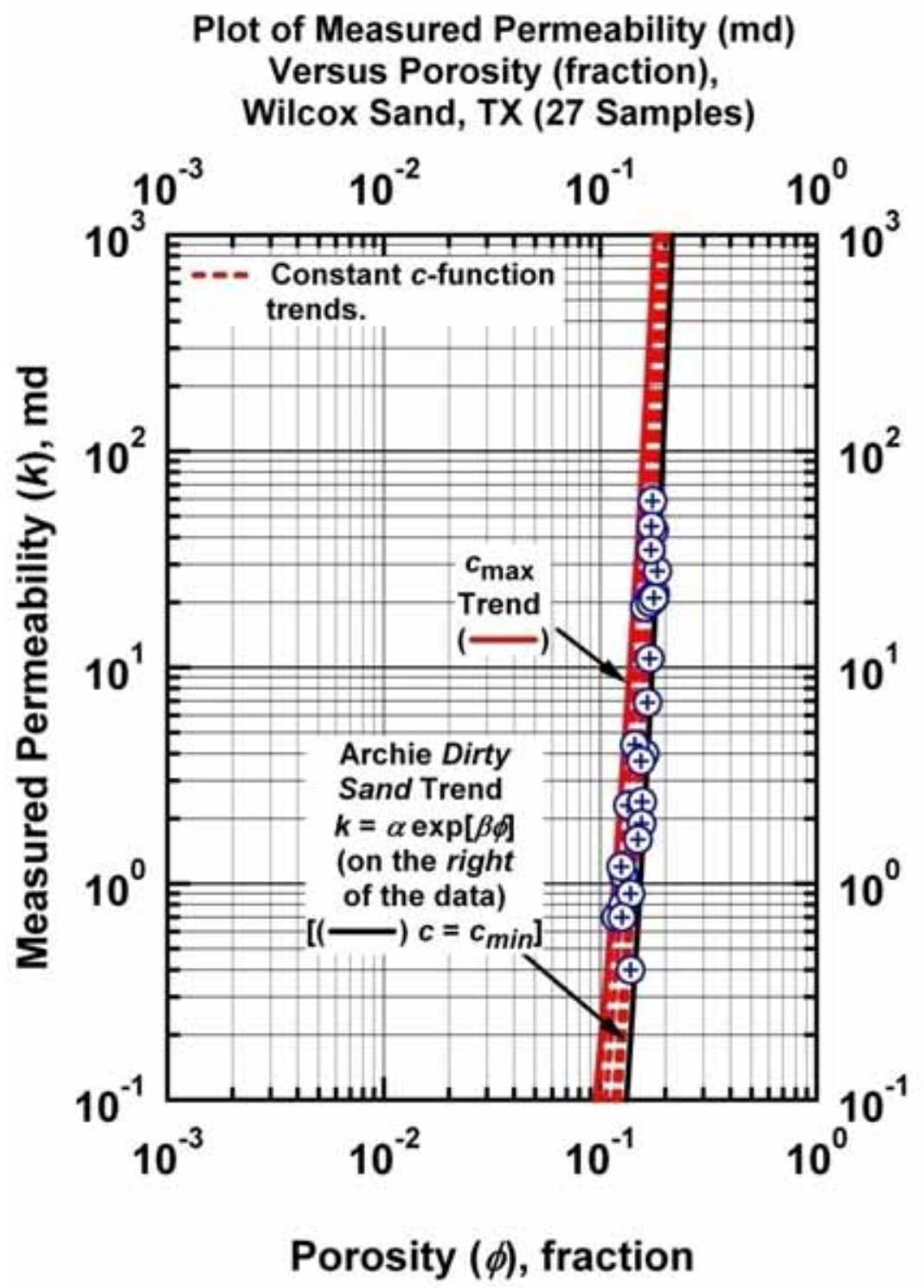

Figure E.5a - Model $C P R-C: \quad k=c \exp [\beta \phi] \quad c=c_{\max } \exp \left[-c_{1} \phi^{c_{2}} S_{w}^{c_{3}}\right]$ - "Clean Sand" Plot (log-log format) - Archie "Dirty Sand" trend is given by the curved trend at the far right of the data (exponential model). [Case: Wilcox Sand, S. TX (USA)] 


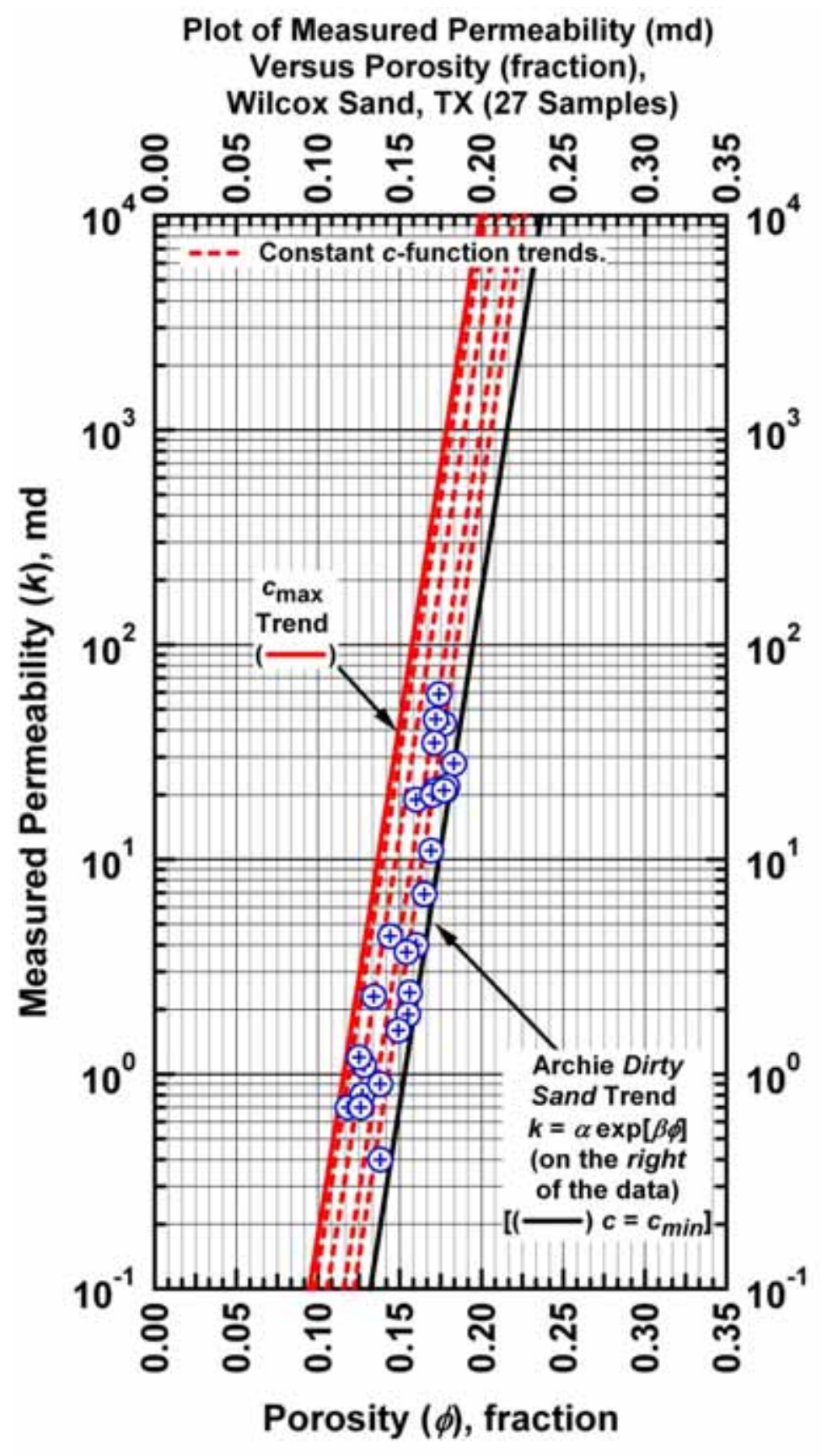

Figure E.5b - Model CPR-C: $k=c \exp [\beta \phi] \quad c=c_{\max } \exp \left[-c_{1} \phi^{C_{2}} S_{w}^{C_{3}}\right]$ - "Dirty Sand" Plot (semilog format) - Archie "Dirty Sand" trend is given by the straight-line trend at the far right of the data (exponential model). [Case: Wilcox Sand, S. TX (USA)] 
Comparison of Permeability (md) Calculated Using the "CPR-C" Model Versus the Measured Permeability (md), Wilcox Sand, TX (27 Samples)

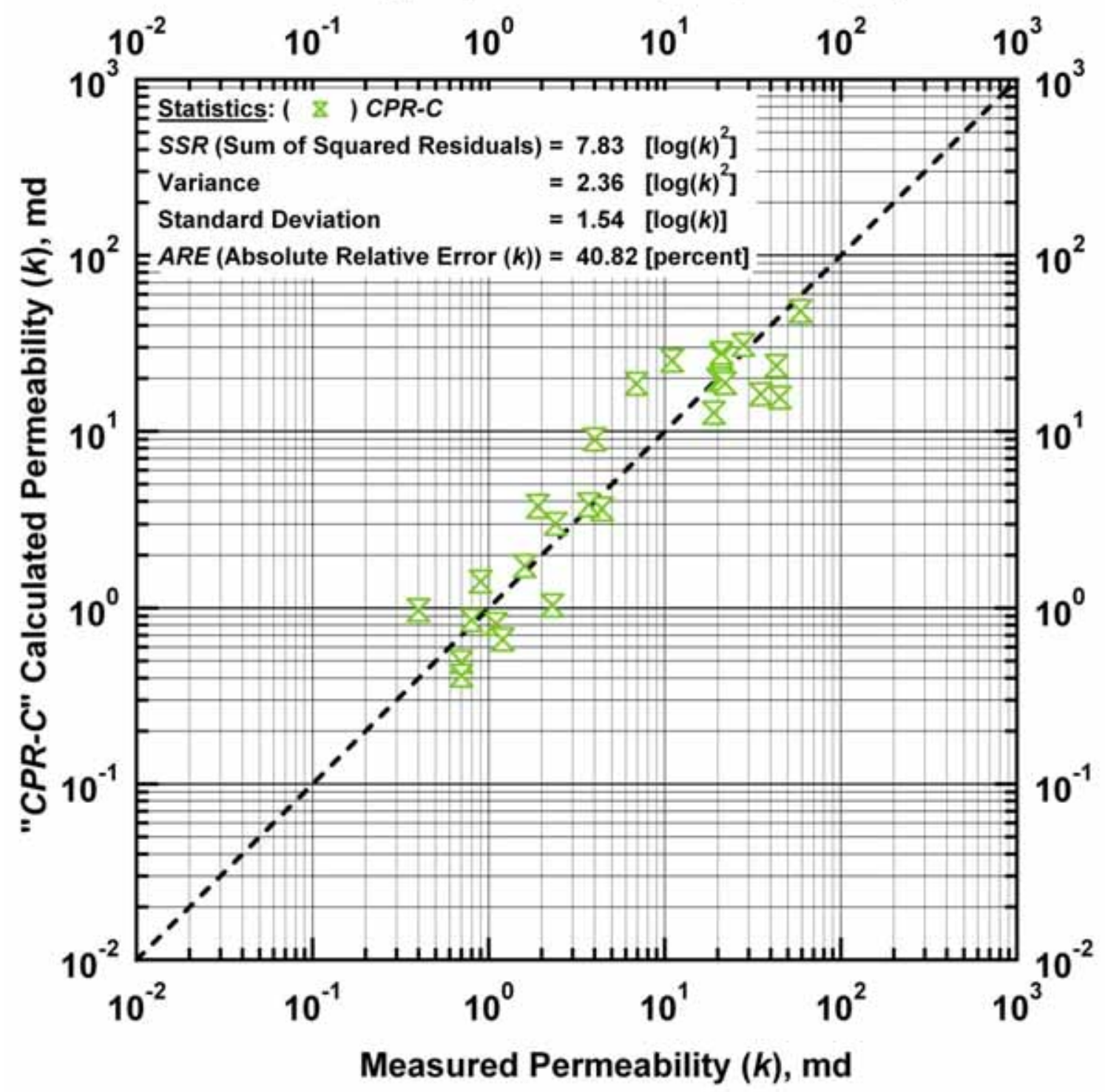

Figure E.6a - Model CPR-C: calculated versus measured permeability. [Case: Wilcox Sand, S. TX (USA)] 


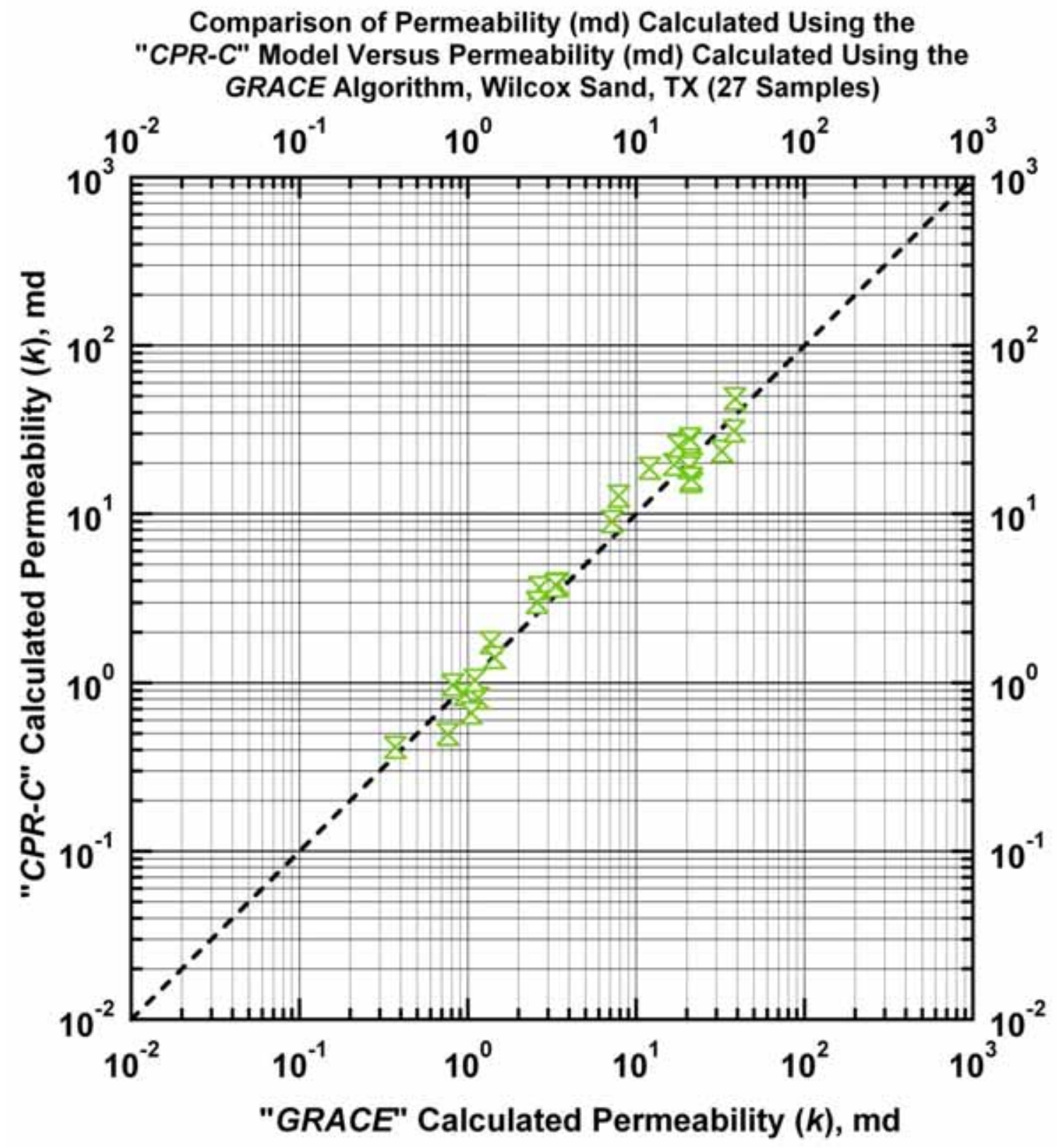

Figure E.6b - Model $C P R-C$ : Permeability calculated using Model $C P R-C$ versus permeability calculated using the GRACE Algorithm. [Case: Wilcox Sand, S. TX (USA)] 


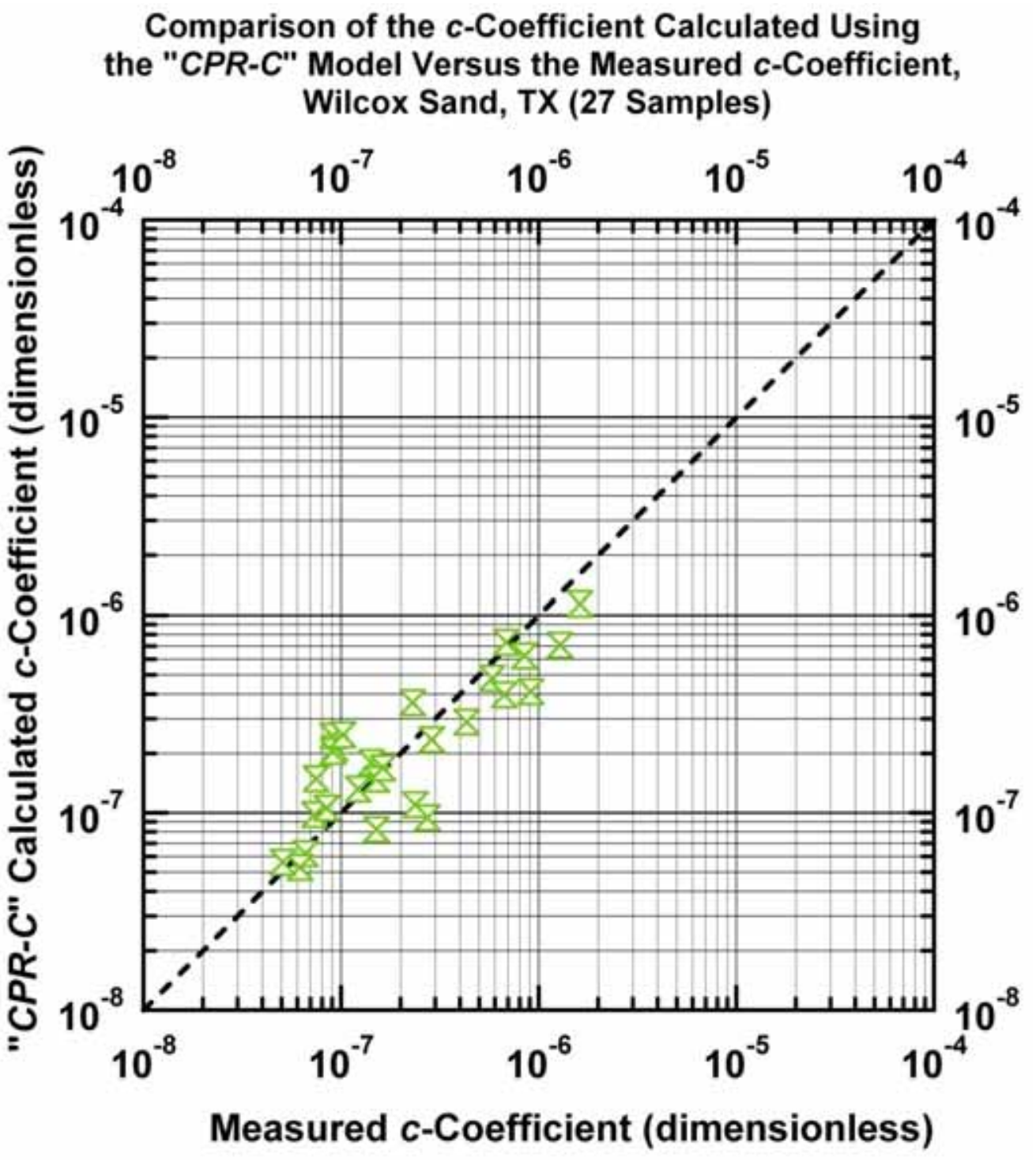

Figure E.6c - Model $C P R-C$ : Calculated $c$-function values versus measured $c$-function values. [Case: Wilcox Sand, S. TX (USA)] 


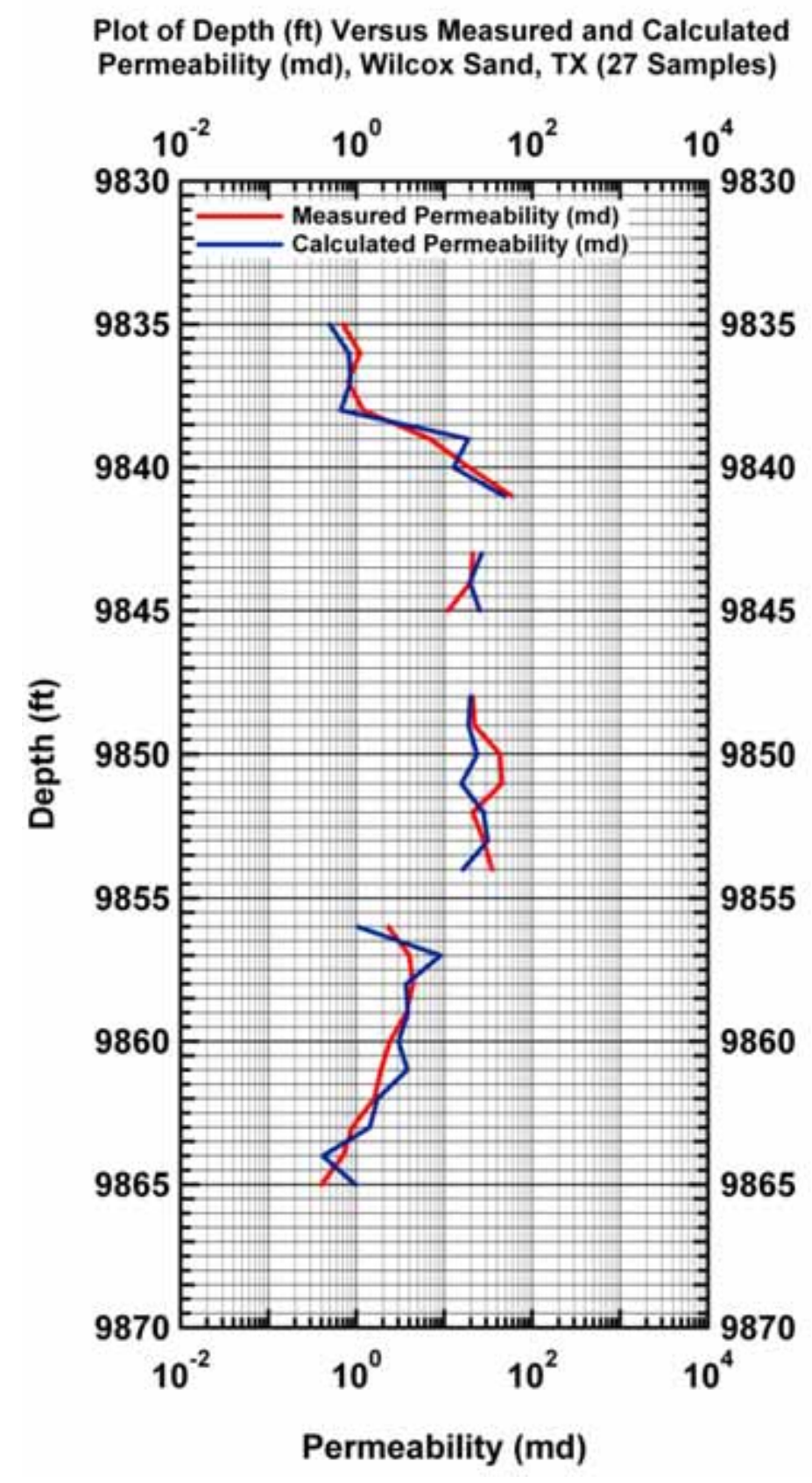

Figure E.6d - Model CPR-A: Calculated and measured permeability values versus depth. [Case: Wilcox Sand, S. TX (USA)] 


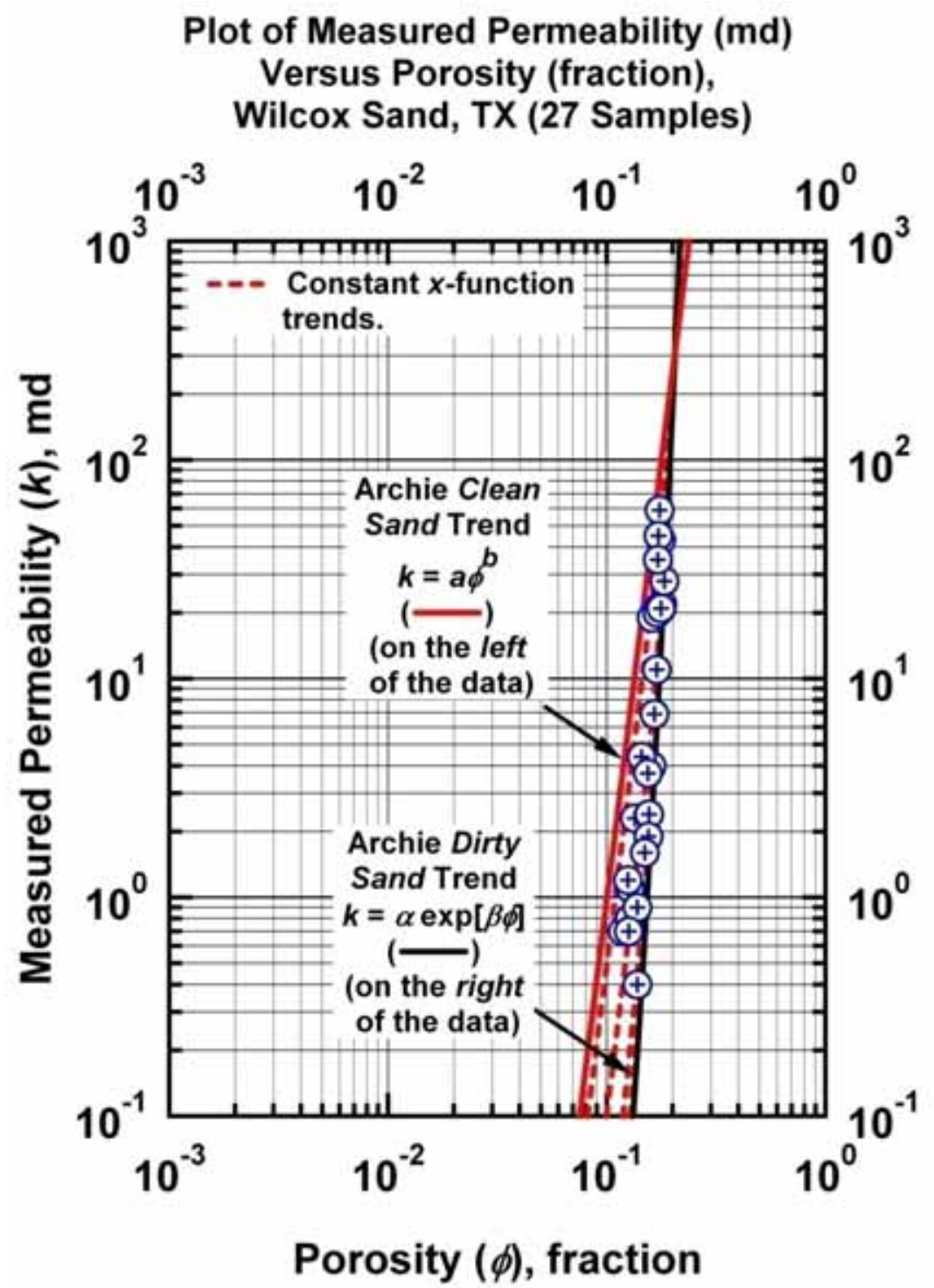

Figure E.7a - Model CPR-D: $k=x a \phi^{b}+(1-x) \alpha \exp [\beta \phi] \quad[0 \leq x \leq 1]$ - Weighted Power Law-Exponential Model used to correlate permeability $(k)$ and porosity $(\phi)$. "Clean Sand" Plot - Archie "Clean Sand" trend is given by the straight-line trend at the far left of the data (power law model). [Case: Wilcox Sand, S. TX (USA)] 


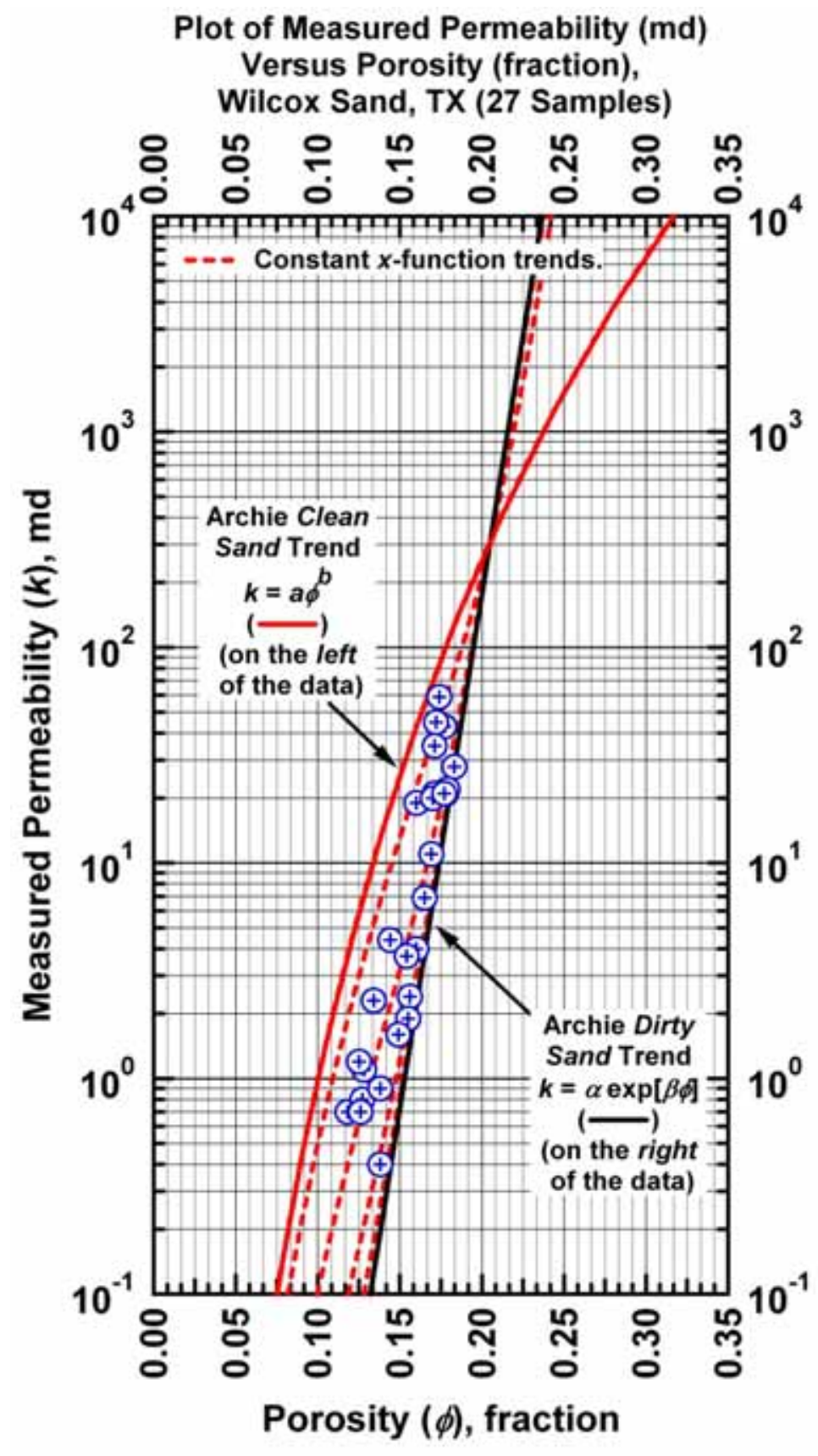

Figure E.7b - Model CPR-D: $k=x a \phi^{b}+(1-x) \alpha \exp [\beta \phi] \quad[0 \leq x \leq 1]$ - Weighted Power Law-Exponential Model used to correlate permeability $(k)$ and porosity $(\phi)$. "Dirty Sand" Plot - Archie "Dirty Sand" trend is given by the straight-line trend at the far right of the data (exponential model). [Case: Wilcox Sand, S. TX (USA)] 


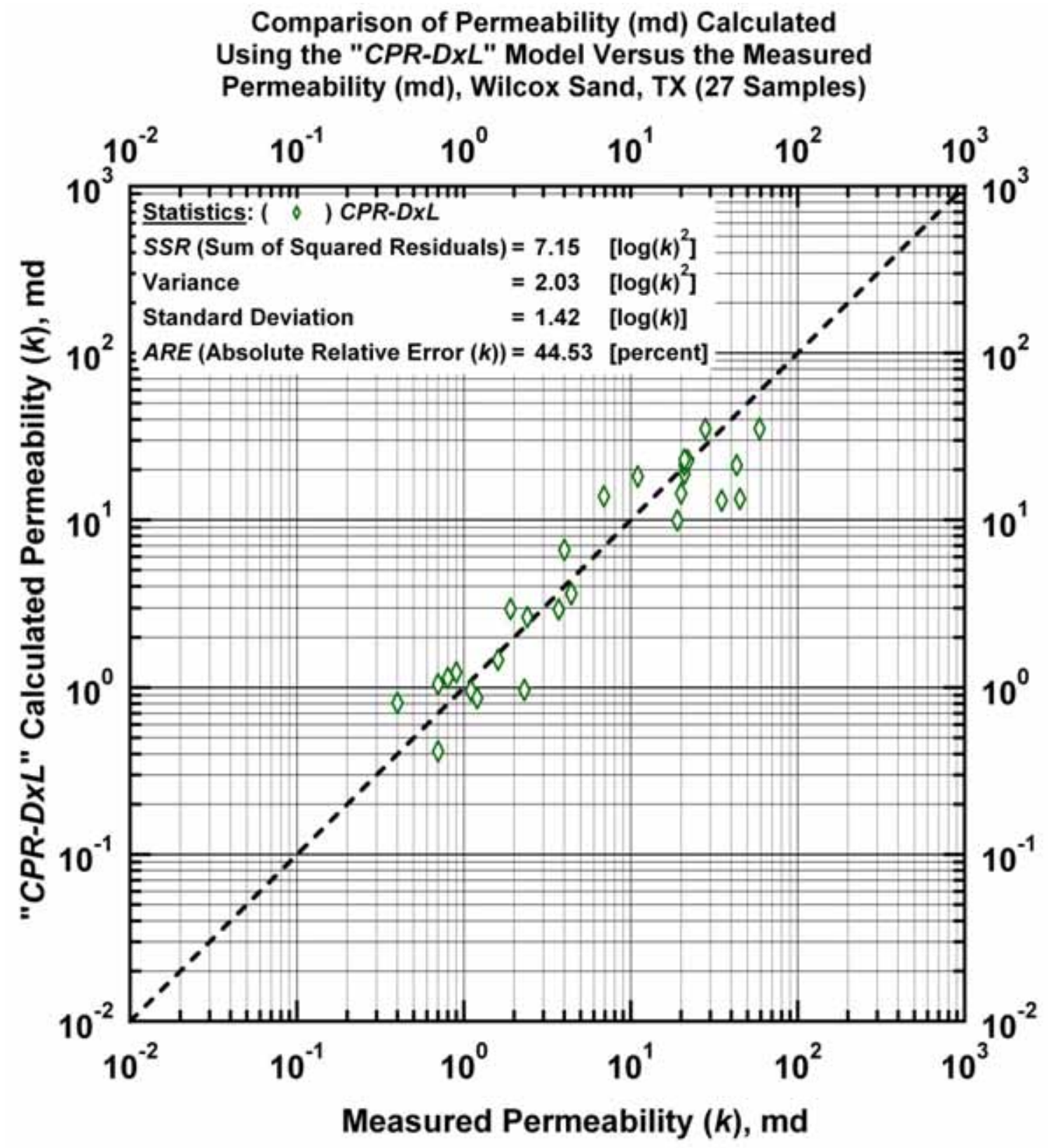

Figure E.8a - Model CPR-DxL: Calculated versus measured permeability. [Case: Wilcox Sand, S. TX (USA)] 


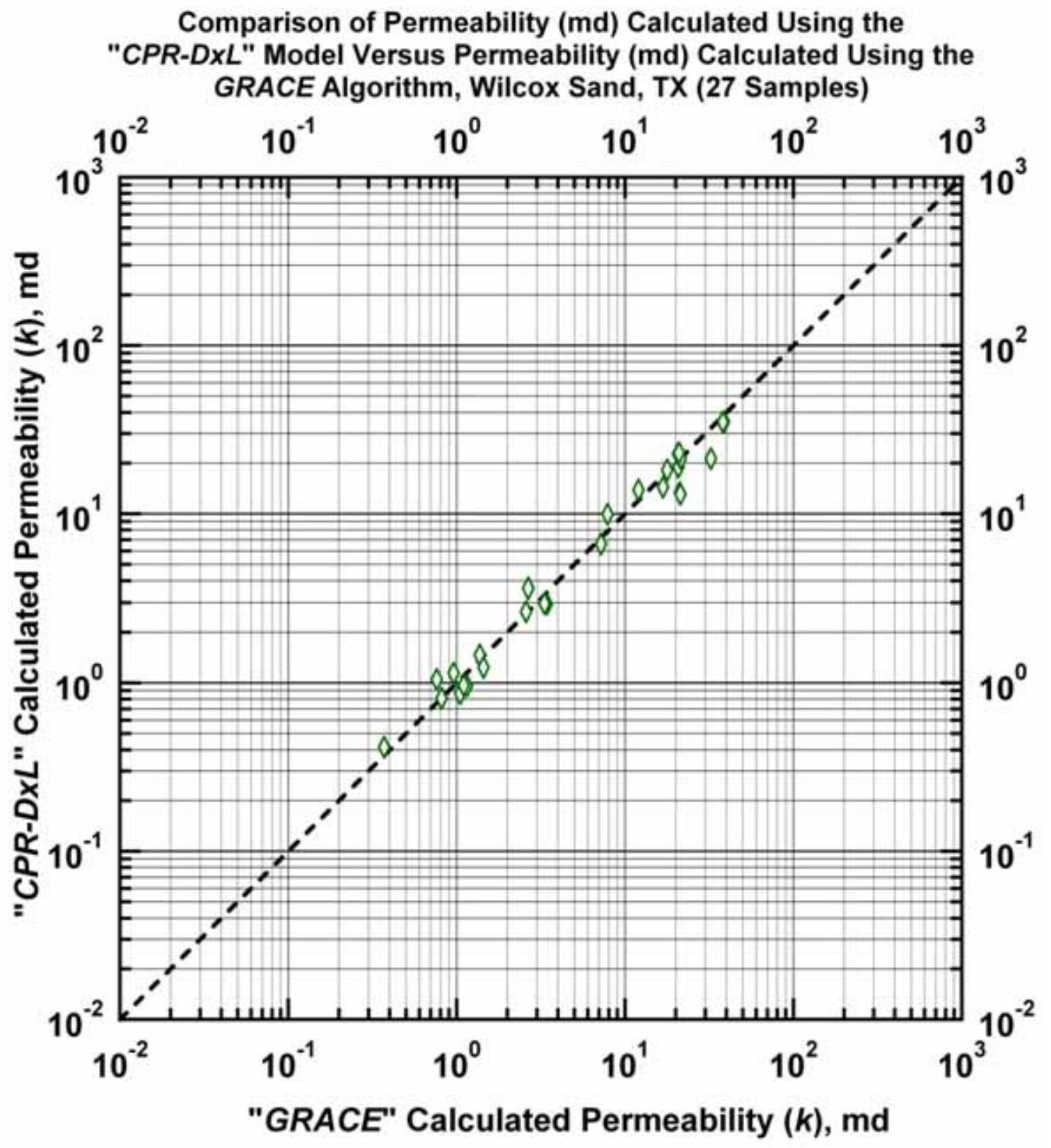

Figure E.8b - Model $C P R-D x L$ : Permeability calculated using Model $C P R-C$ versus permeability calculated using the GRACE Algorithm. [Case: Wilcox Sand, S. TX (USA)] 


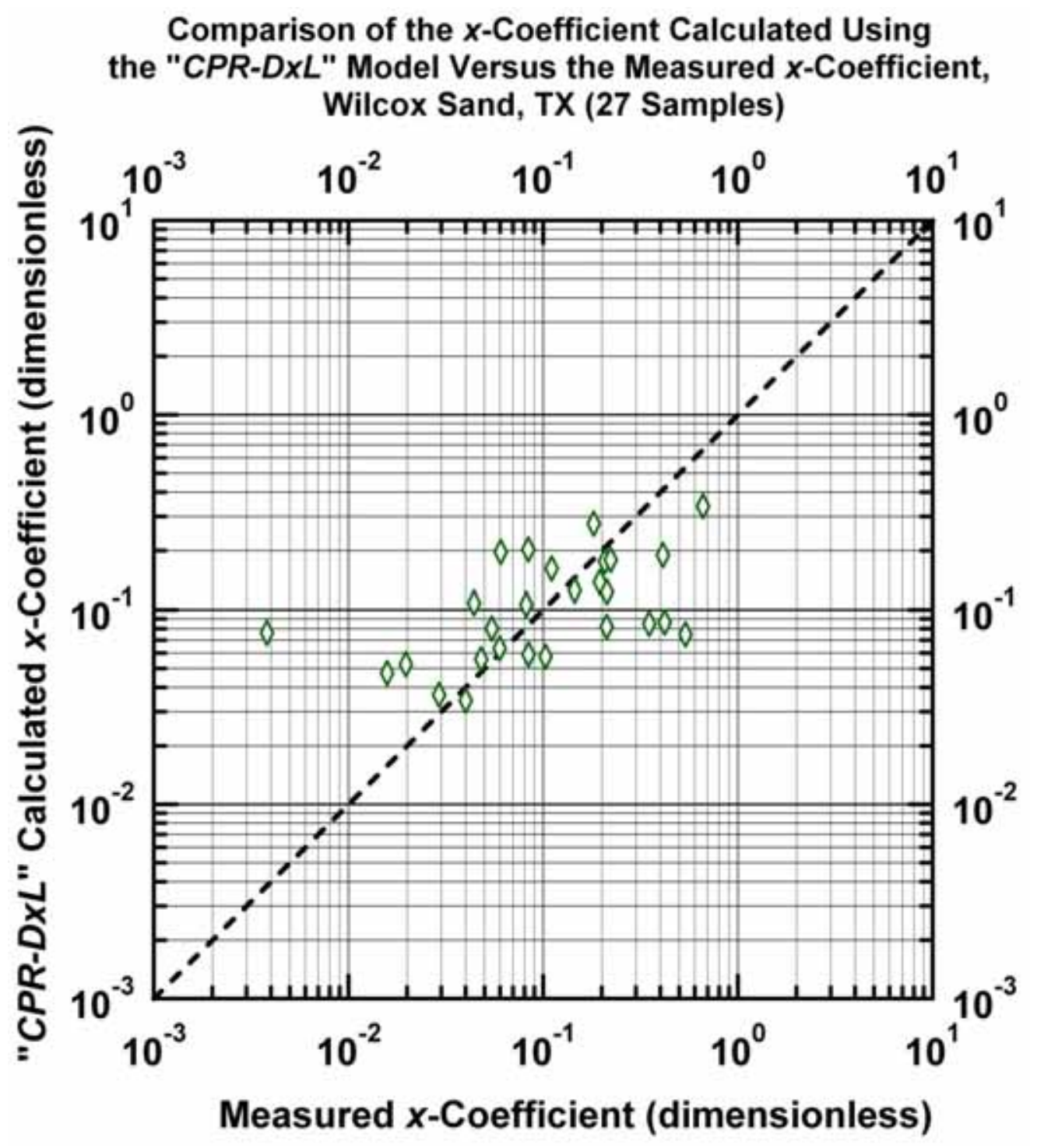

Figure E.8c - Model CPR-DxL: Calculated $x$-function weight values versus measured $x$ function weight values. [Case: Wilcox Sand, S. TX (USA)] 


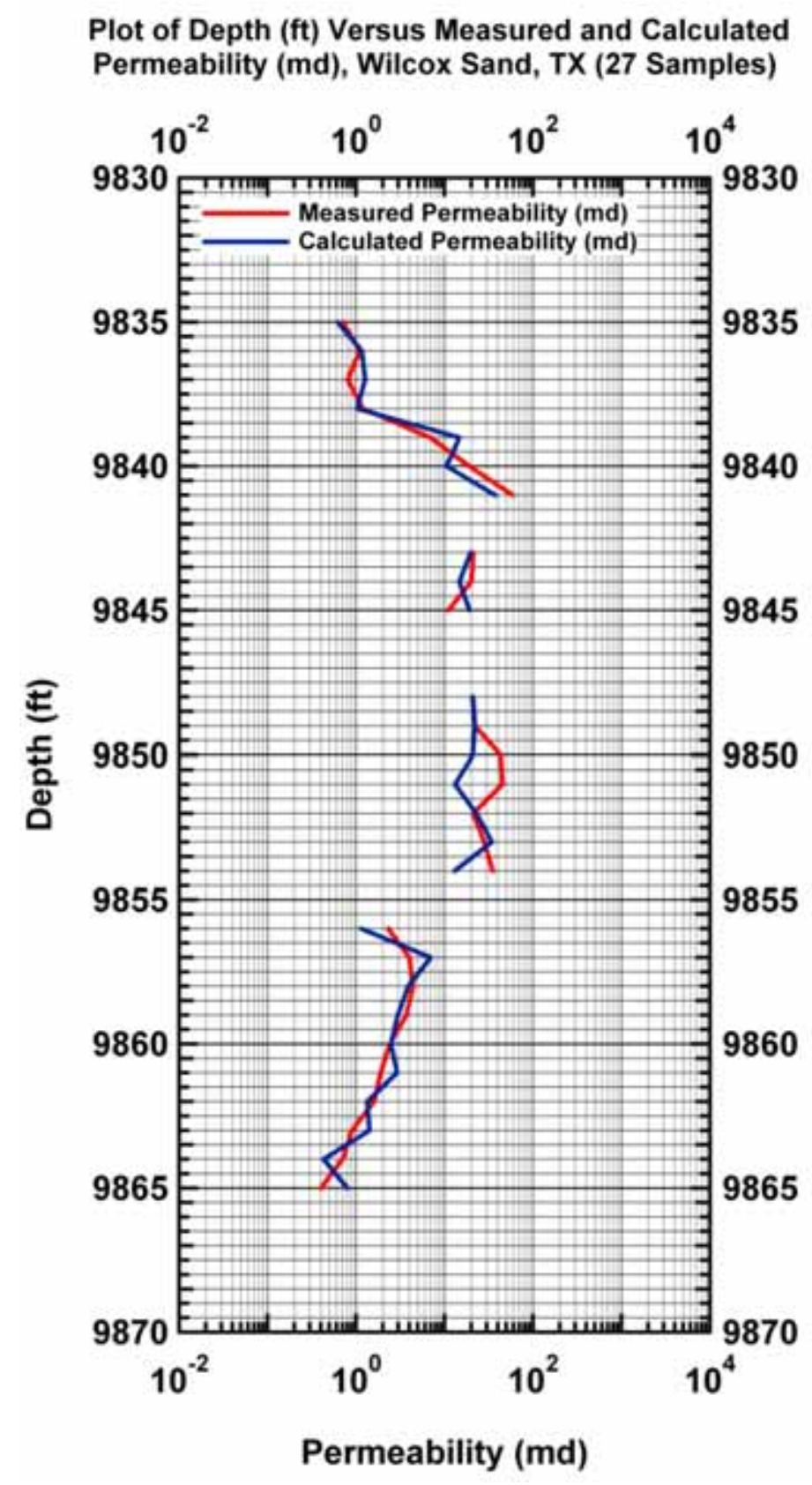

Figure E.8d - Model CPR-A: Calculated and measured permeability values versus depth. [Case: Wilcox Sand, S. TX (USA)] 


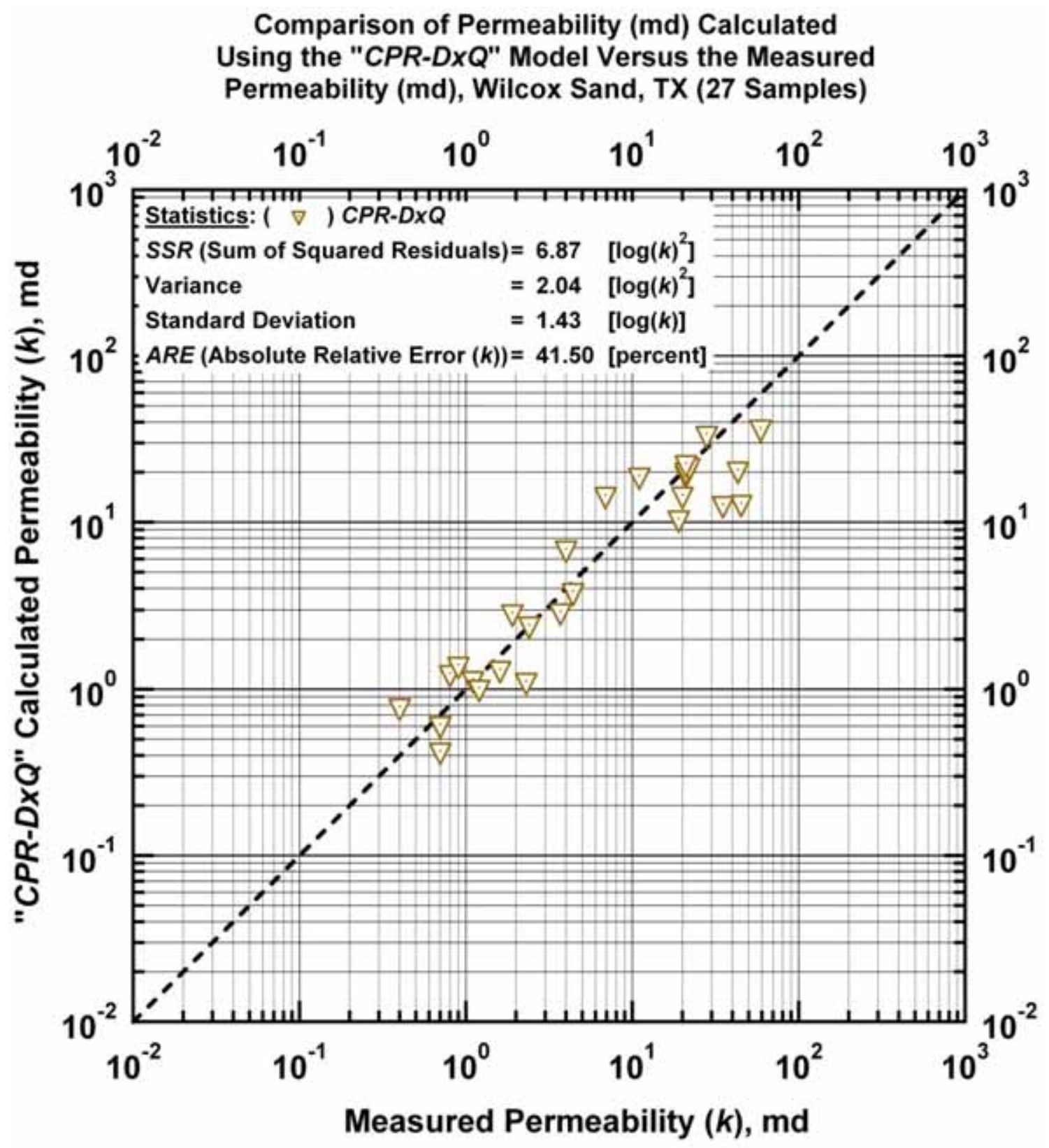

Figure E.9a - Model CPR-DxQ: Calculated versus measured permeability. [Case: Wilcox Sand, S. TX (USA)] 


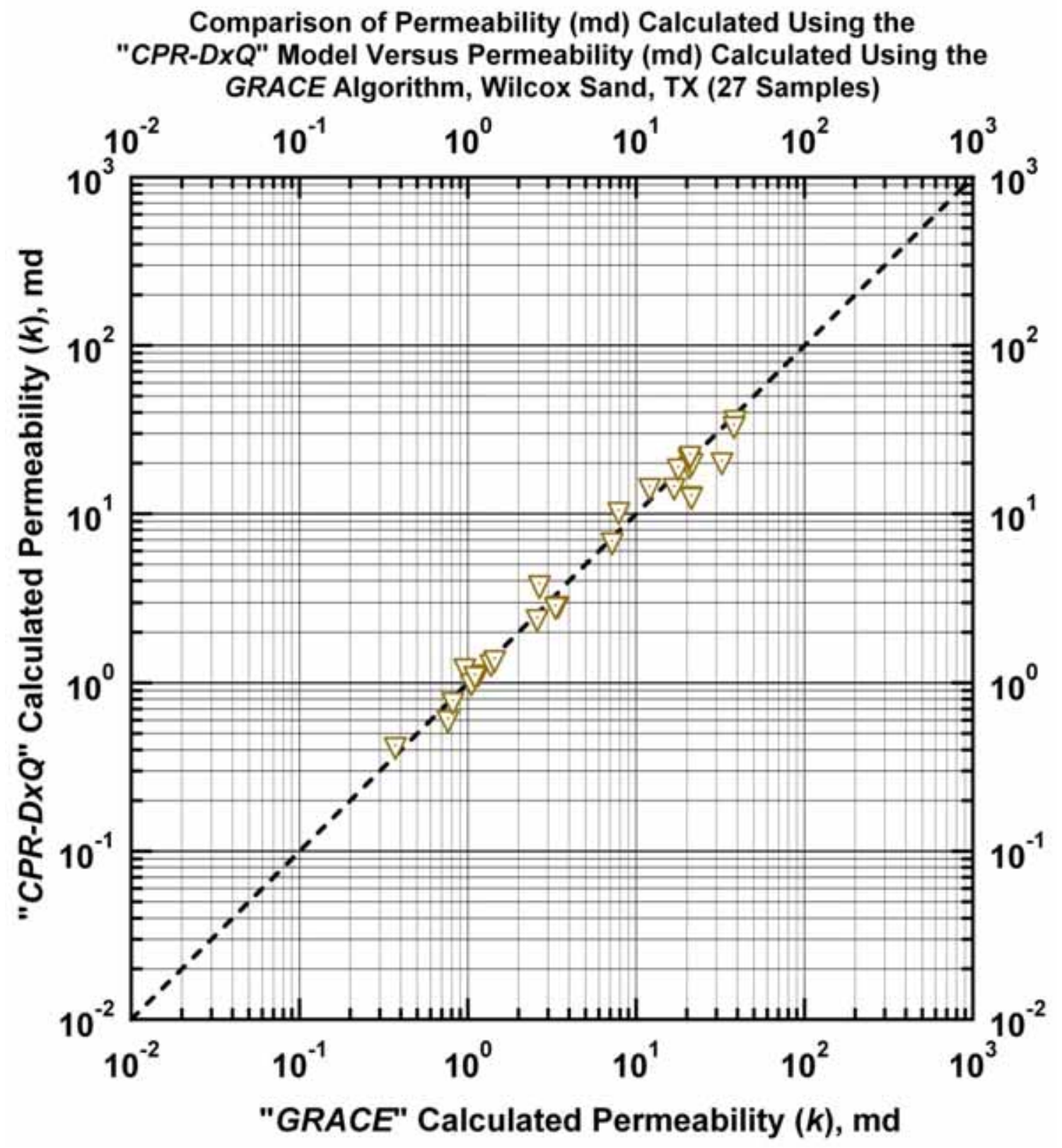

Figure E.9b - Model $C P R-D x Q$ : Permeability calculated using Model $C P R-C$ versus permeability calculated using the GRACE Algorithm. [Case: Wilcox Sand, S. TX (USA)] 


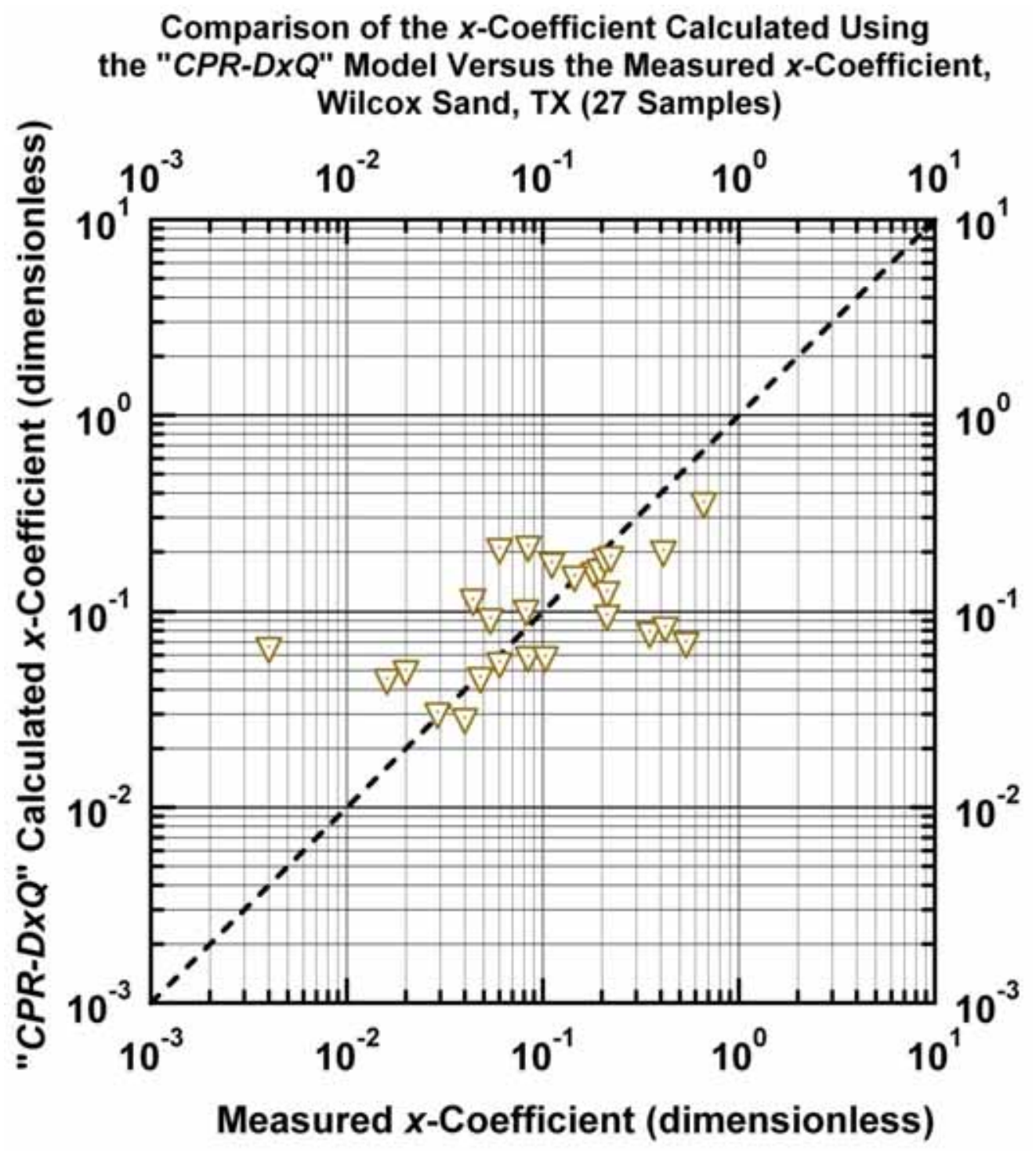

Figure E.9c - Model CPR-DxQ: Calculated $x$-function weight values versus measured $x$ function weight values. [Case: Wilcox Sand, S. TX (USA)] 


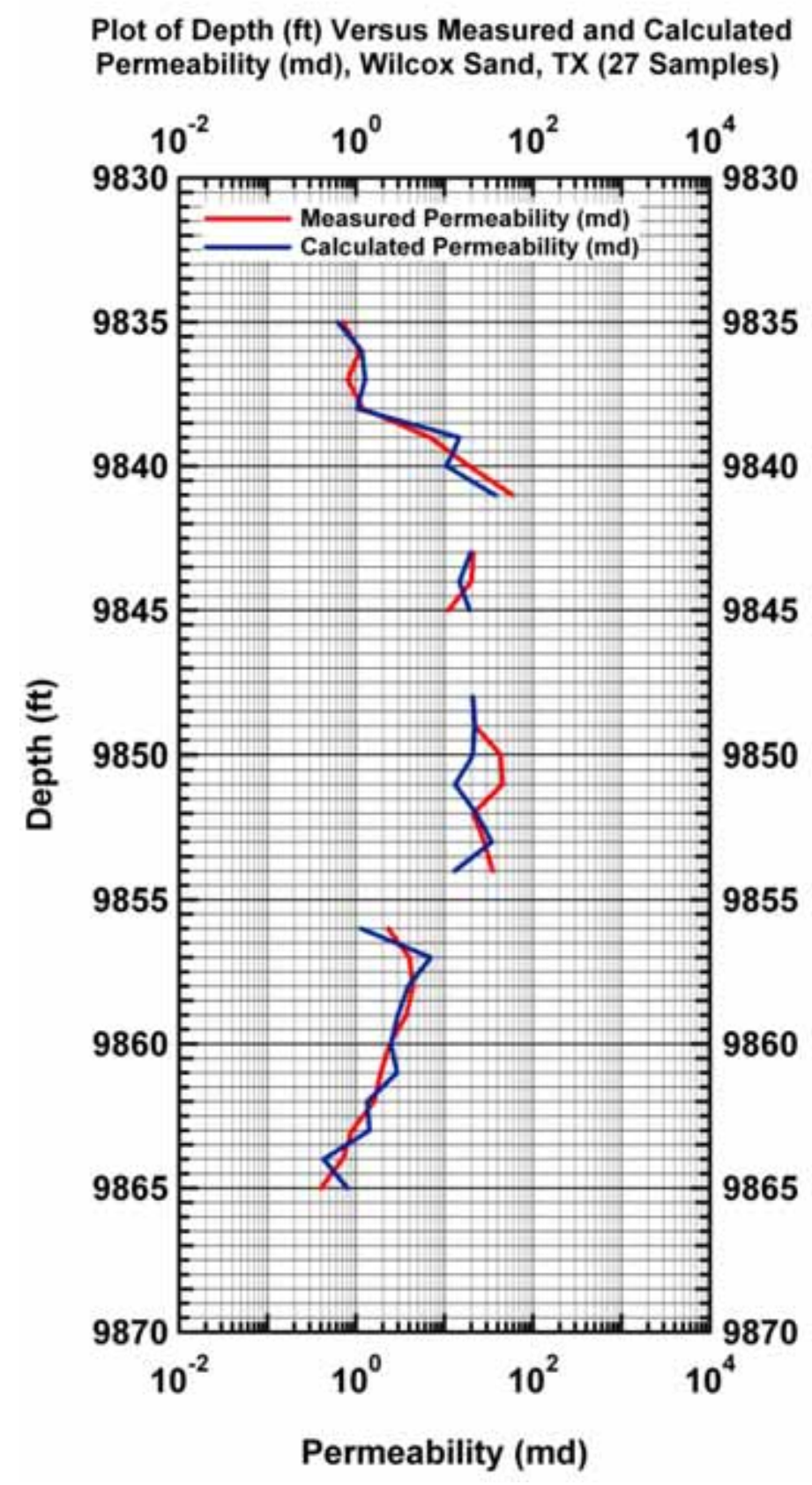

Figure E.9d - Model CPR-A: Calculated and measured permeability values versus depth. [Case: Wilcox Sand, S. TX (USA)] 


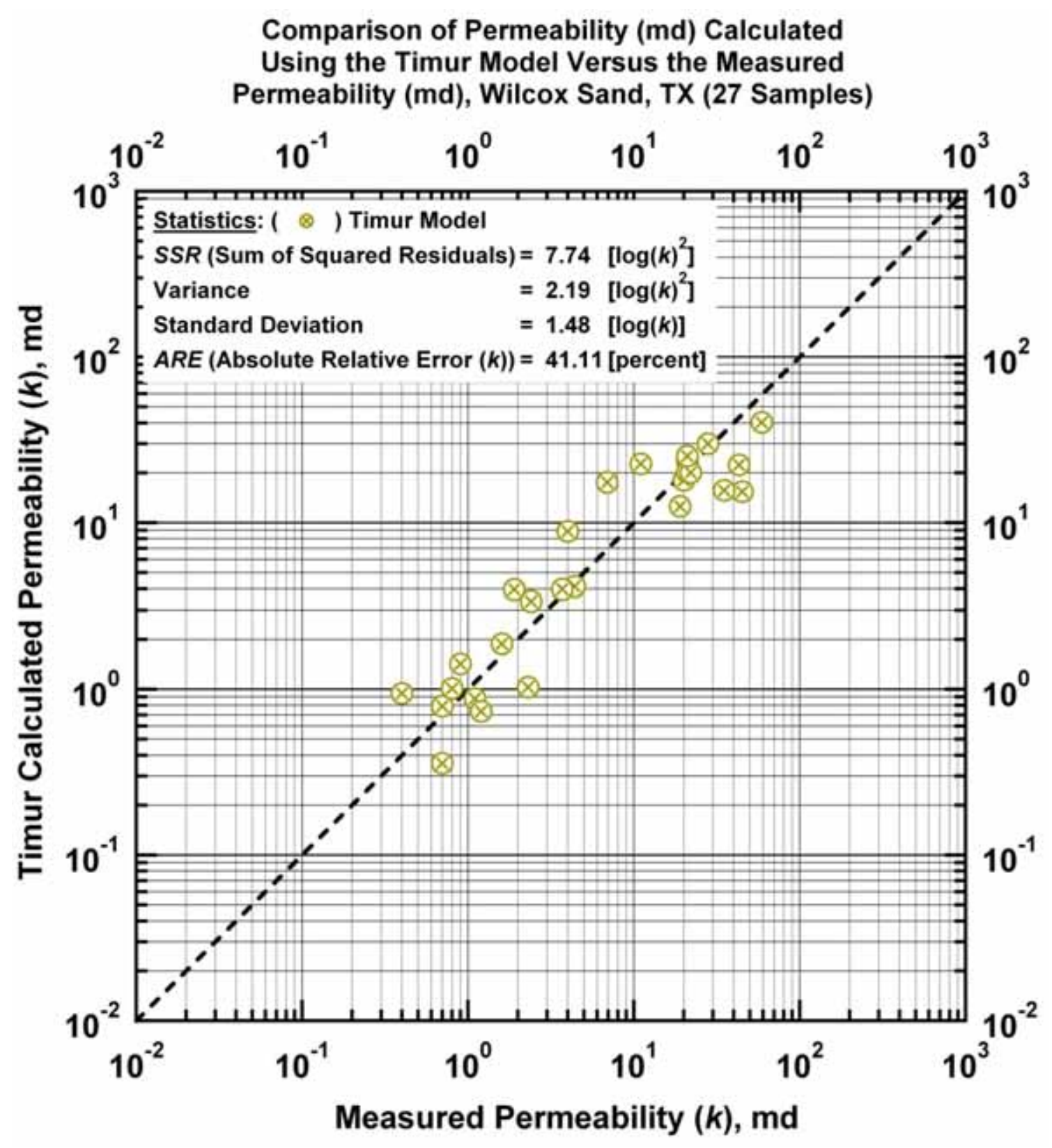

Figure E.10a - "Timur Model:" Calculated versus measured permeability. [Case: Wilcox Sand, S. TX (USA)] 


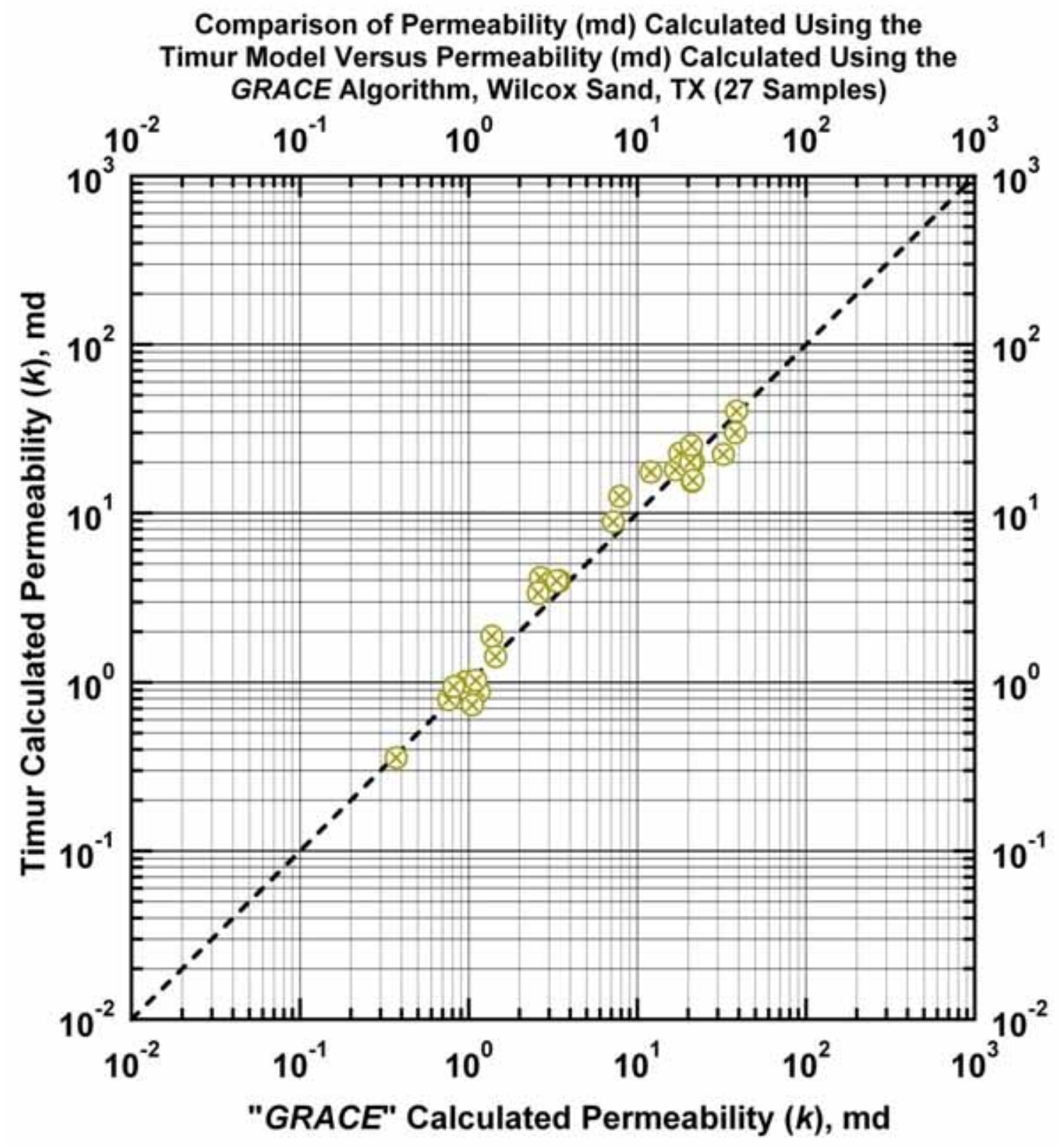

Figure E.10b — "Timur Model:" Permeability calculated using the "Timur Model" versus permeability calculated using the GRACE Algorithm. [Case: Wilcox Sand, S. TX (USA)] 


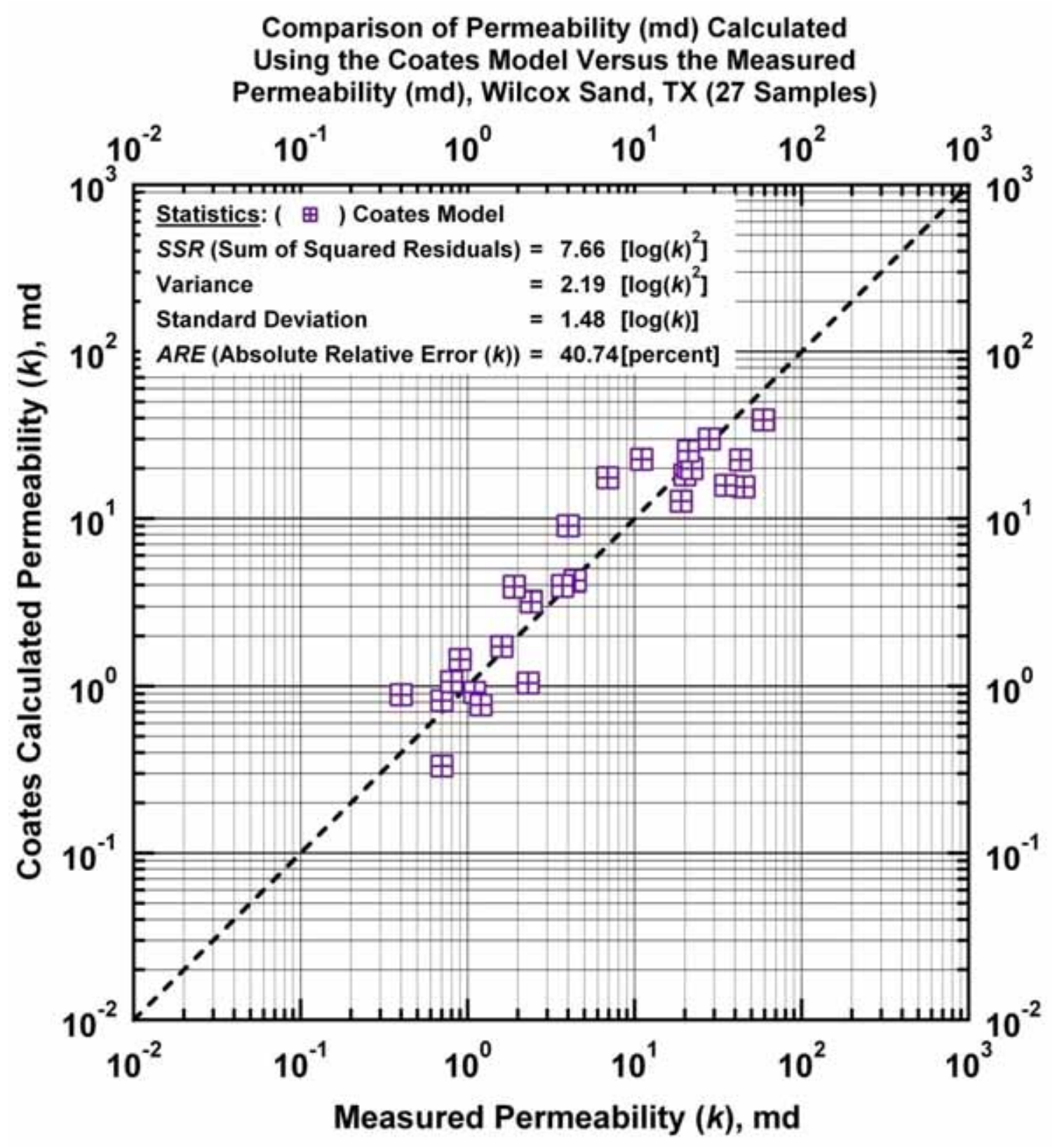

Figure E.11a - "Coates Model:" Calculated versus measured permeability. [Case: Wilcox Sand, S. TX (USA)] 


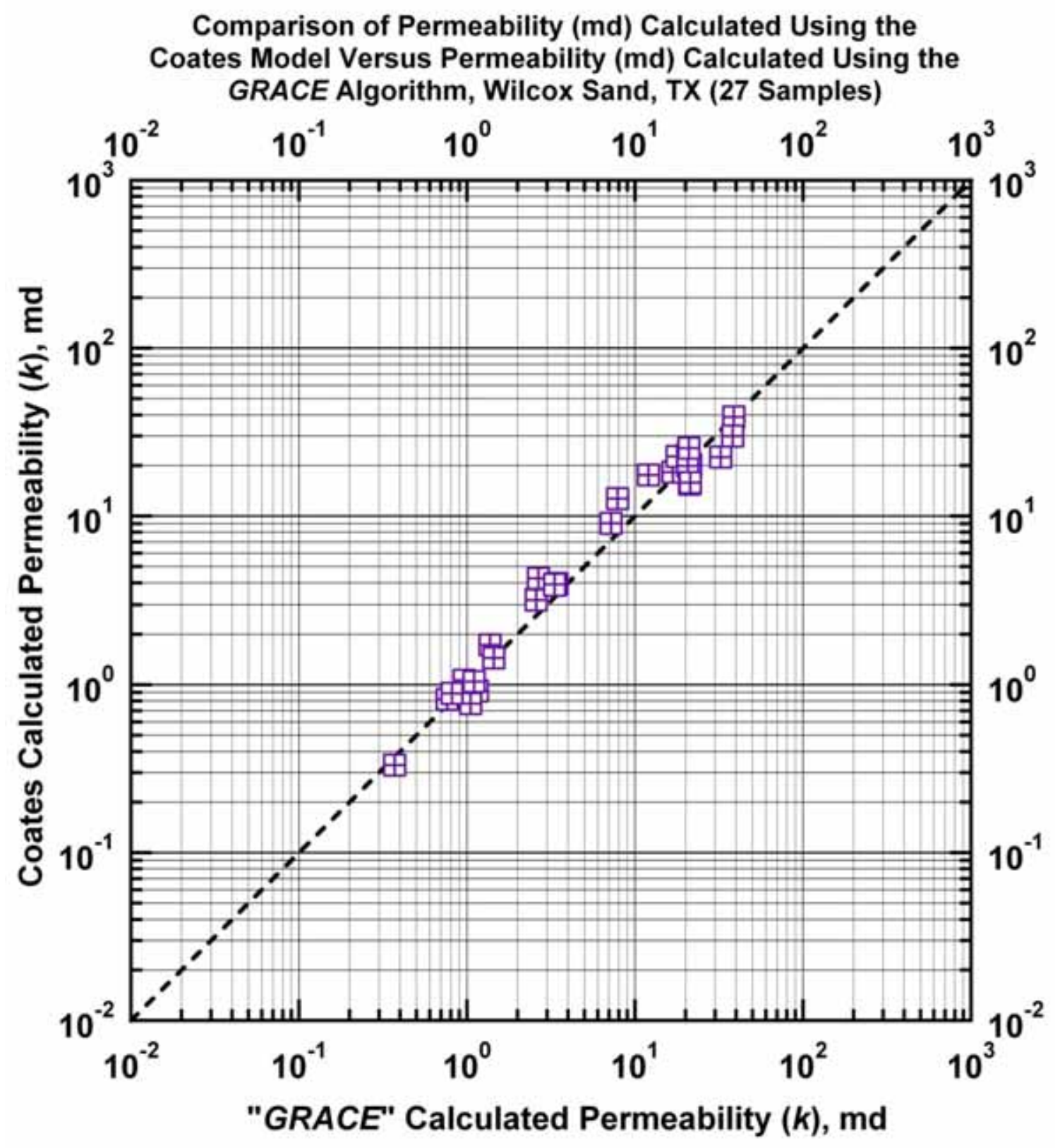

Figure E.11b - "Coates Model:" Permeability calculated using the "Coates Model" versus permeability calculated using the GRACE Algorithm. [Case: Wilcox Sand, S. TX (USA)] 


\section{Comparison of Permeability (md) Calculated} Using the GRACE Algorithm Versus Measured Permeability (md) Wilcox Sand, TX (27 Samples)

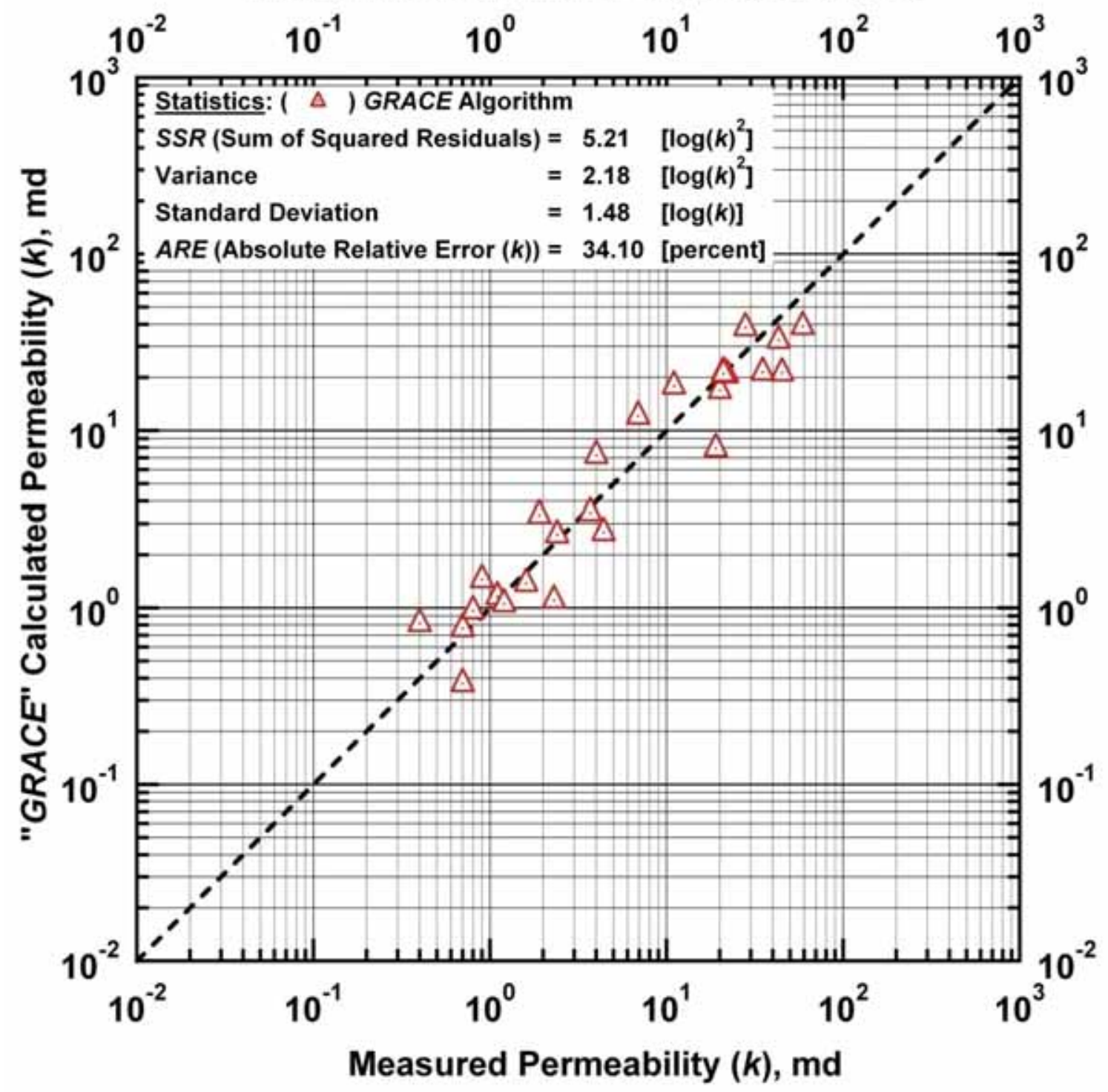

Figure E.12 - GRACE Model: Calculated versus measured permeability. [Case: Wilcox Sand, S. TX (USA)] 


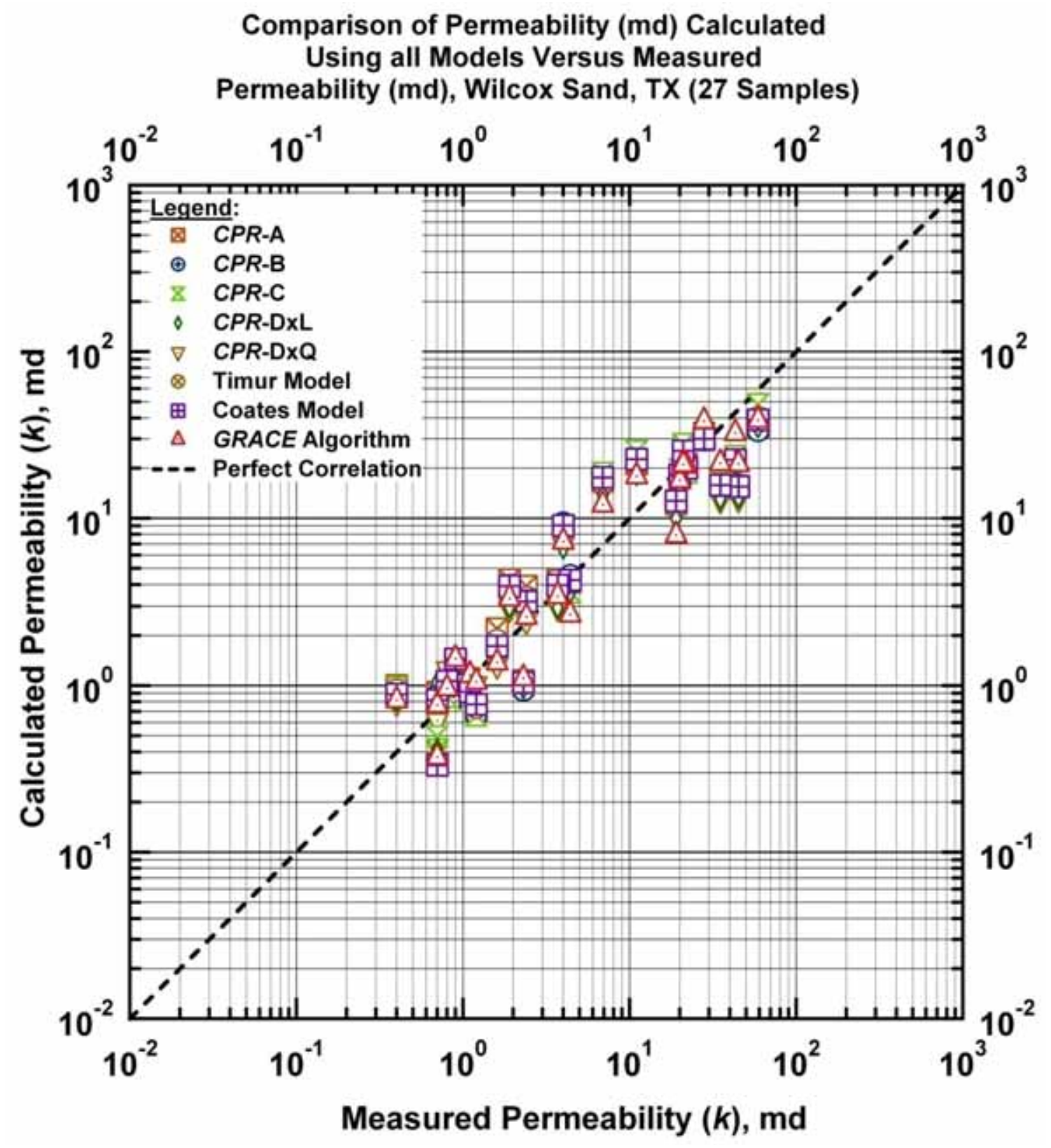

Figure E.13 - All Models: Calculated versus measured permeability. [Case: Wilcox Sand, S. TX (USA)] 

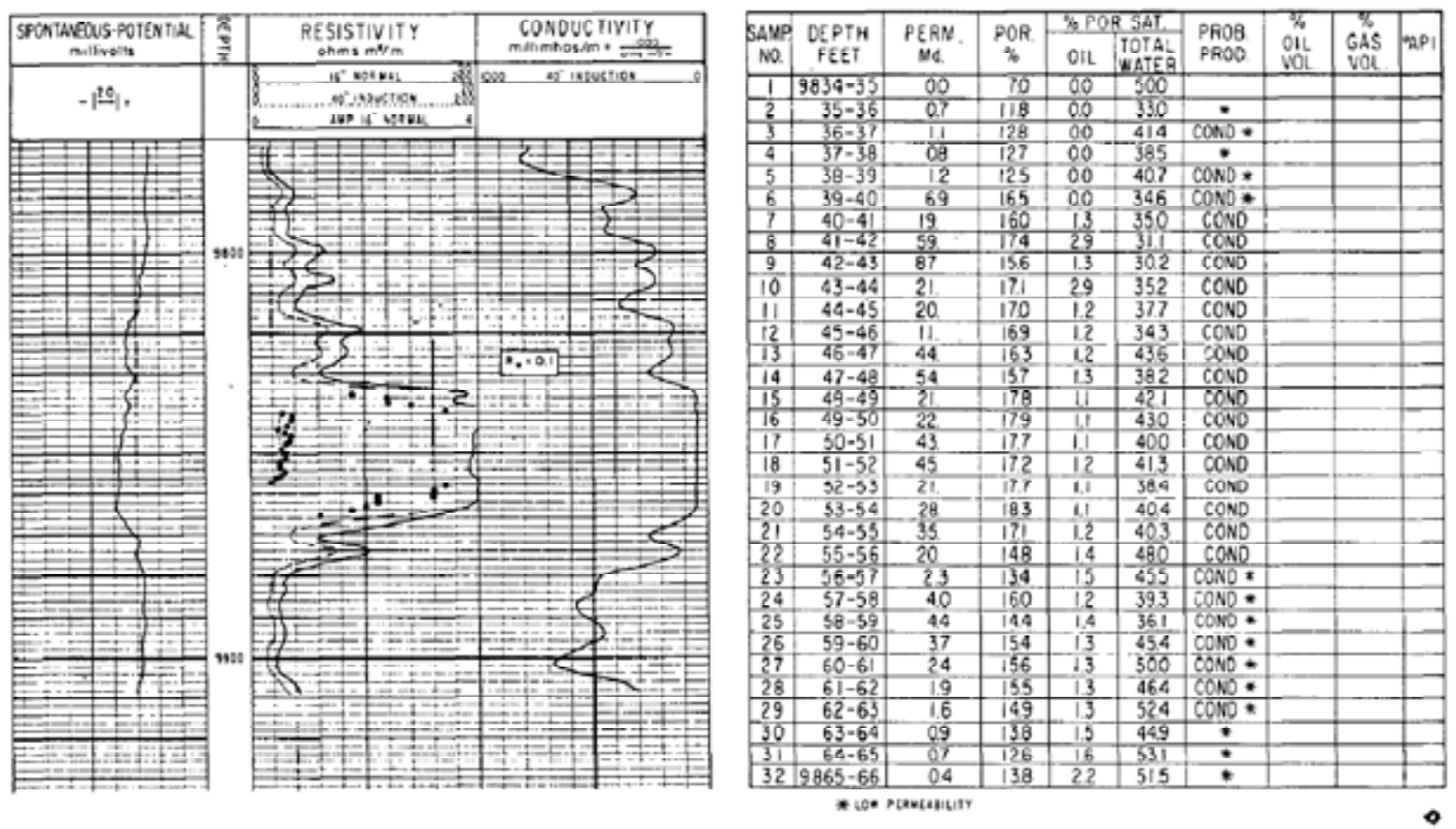

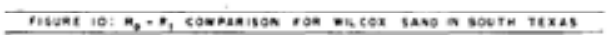

Figure E.14 - Original data from Granberry and Wilshusen (1962). [Case: Wilcox Sand, S. TX (USA)] 


\section{APPENDIX F \\ RESULTS FOR HAZLETT WELL 103}

In this Appendix we present the "Hazlett" Well 103, (Hazlett, 1989).

\section{Model CPR-A: (Appendix F)}

The "characteristic permeability relation" (or CPR) Model CPR-A (i.e., the modified Archie "Clean Sand" Model) is given as:

$$
k=a(\phi-c)^{b} \quad c=c_{\max } \exp \left[-c_{1} \phi^{c 2} F^{c 3}\right]
$$

Where a plot of $\log (k)$ versus $\log (\phi)$ is used to graphically calibrate Eq. F-1 in terms of the coefficients $a, b$, and $c_{\max }$. Fig. F.1 shows that the $C P R-A$ model performs quite well for this case. Figs. F.1a and $\mathbf{b}$ show the $\log -\log$ and the semilog format of permeability versus porosity, respectively. In Fig. F.2 we provide the "error" plots for this case, where the measured and computed data are compared systematically. In Fig. F.2a we note a good correlation of the results obtained using Model CPR-A versus the measured permeability data - there is good agreement with the perfect correlation trend (i.e., the 45degree line). In Fig. F.2b we provide a comparison with the GRACE algorithm correlation (Xue et al. 1997)), and we note that the CPR-A model results correlate very well with the GRACE algorithm results for this case. Lastly, in Fig. F.2c we present the calculated and measured $c$-function - and again we note a reasonable correlation of the data about the perfect correlation trend.

\section{Model CPR-B: (Appendix F)}

The "characteristic permeability relation" (or CPR) Model CPR-B (i.e., the modified Archie "Dirty Sand" Model (power-law basis)) is given as:

$$
k=a(\phi+c)^{b} \quad c=c_{\max } \exp \left[-c_{1} \phi^{c_{2}} F^{c_{3}}\right]
$$

Where a plot of $\log (k)$ versus $\log (\phi)$ is used to graphically calibrate Eq. F-2 in terms of the coefficients $a, b$, and $c_{\max }$. The results for this case are presented in Figs. F.3 and 4. In Fig. F.3a (log-log format) we note that a very distinct "envelope" is formed by the CPR-B model, essentially all of the data on the right and left flanks are well-matched (i.e., the "dirty sand" power law relation (applied on the right portion of the data) and its correction function (applied on the left portion of the data). Similarly, in Fig. F.3b (semilog format), we observe an excellent distribution of the power law model across the body of the data. The error analyses for this case are shown in Fig. F.4. In Fig. F.4a we present the results obtained using Model $C P R-B$ versus the measured permeability data and in this case there is good agreement with the perfect correlation trend (i.e., the 45-degree line). We provide a comparison with the GRACE algorithm correlation in Fig. F.4b - the results using Model CPR-B and the GRACE algorithm compare very well 
for this case. We compare the computed and data-derived values of the $c$-function for this case in Fig. F.4c and we note the computed $c$-function does not change much form the data-derived values of the $c$ function. In summary, we believe that Model $C P R-B$ works well for this particular data case.

\section{Model CPR-C: (Appendix F)}

The "characteristic permeability relation" (or CPR) Model CPR-C (i.e., the modified Archie "Dirty Sand" Model (exponential basis)) is given as:

$$
k=c \exp [\beta \phi] \quad c=c_{\max } \exp \left[-c_{1} \phi^{c 2} F^{c 3}\right]
$$

Where a plot of $\log (k)$ versus $\phi$ is used to graphically calibrate Eq. F-3 in terms of the coefficients $-\beta$ and $C_{\max }$. Model $C P R-C$ is fundamentally different than Models $C P R-A$ and $C P R-B$, as the basis for Model $C P R-C$ is an exponential function, not a power law relation. In Figs. F.5 and 6 we present the results of applying Model $C P R-B$ for this case, and in general, we observe a very consistent performance of Model $C P R-B$. Fig. F.5b illustrates the excellent conformance of Model $C P R-C$ for this case - again recalling that this model has an exponential relationship with porosity as its basis.

In Figs. F.6a, F.6b, and F.6c we review the error analyses plots for this case, and we note good performance (visually) in terms of the correlation of the results using Model CPR-C. The correlation of the $c$-function (see Fig. F.6c) appears to be consistent - but scatter and a slight off-center trend is evident. However, as we are approaching this work from the perspective that the primary value of the work is the characteristic model, not the statistical "best fit" correlation, we believe that the CPR-C model has performed well for this case, and the plots in Fig. F.6 confirms the value of this model as a "characteristic relation" for permeability.

\section{Model CPR-D: (Appendix F)}

The "characteristic permeability relation" (or $C P R$ ) Model CPR-D (i.e., the weighted power law-exponential model) is given in its fundamental form as:

$$
\left.k=x a \phi^{b}+(1-x) \alpha \exp [\beta \phi] \quad[0 \leq x \leq 1]\right] .
$$

For Eq. F-4 there are two proposed models for the $x$-function (i.e., the weighting function) - these are:

$$
\begin{aligned}
& x=\exp \left[-\ln \left(x_{0}\right)+x_{1} \ln (\phi)+x_{2} \ln (F)+x_{3} \ln (\phi) \ln (F)\right] \text { (Model CPR-DxL)... } \\
& x=\exp \left[\begin{array}{l}
-\ln \left(x_{0}\right)+x_{1} \ln (\phi)+x_{2} \ln (F)+x_{3} \ln (\phi) \ln (F) \\
+x_{4} \ln (\phi)^{2}+x_{5} \ln (F)^{2}+x_{6} \ln (\phi)^{2} \ln (F) \\
+x_{7} \ln (\phi) \ln (F)^{2}+x_{8} \ln (\phi)^{2} \ln (F)^{2}
\end{array}\right] \text { (Model CPR-DxL).... }
\end{aligned}
$$

As noted in Eq. F-4b, Model CPR-DxL utilizes a logarithmic linear model for the weights ( $x$-values) and as seen in Eq. F-4c, Model CPR-DxQ utilizes a logarithmic quadratic model for the weights ( $x$-values). While these models (Eqs. F-4b and F-4c) are empirical, we have found generally good application of these relations for all cases. In this case, the base results using Models $C P R-D x L$ and $C P R-D x Q$ are presented in 
Fig. F.7 - where the power law and exponential basis functions are fitted to the appropriate portion of the data. The power law equation represents the "Archie Clean Sand" trend and is fitted to the leftmost data as shown in Fig. F.7a and in contrast, the exponential equation is thought to represent the "Archie Dirty Sand" trend and the exponential is fitted to the far rightmost portion of the data (see Fig. F.7b).

In Figs. F.8 and 9 we present the error analyses for this case, where we have employed the weighting functions (Eqs. F-4b and F-4c) as appropriate. In Fig. F.8a we observe a good correlation of permeability (with the out-lying points noted in the middle of the trend). The comparison of the Model CPR-DxL results are compared to the results obtained from the GRACE algorithm in Fig. F.8b. The computed weight function ( $x$-values) shown for the $C P R-D x L$ model in Fig. F.8c does exhibit more scatter than expected, but the trend is (relatively) centered about the perfect correlation line.

The results obtained using Model $C P R-D x Q$ are presented in Fig. F.9, and we note substantially improved behavior over that of Model $C P R-D x L$. In particular, the base correlation of computed and measured permeabilities (Fig. F.9a) for Model $C P R-D x Q$ does indicate a slightly better correlation than that of Model CPR-DxL. Also, the comparison of Model CPR-DxQ with the results from the GRACE algorithm (Fig. F.9b) exhibits better agreement than the results of Model CPR-DxL. Finally, the correlation of the $x$ function (Fig. F.9c) for Model $C P R-D x Q$ is better than the correlation for Model CPR-DxL. These comparisons suggest that Model $C P R-D x Q$ (i.e., the combination of Eqs. F-4a and F-4c) provides a better correlation of data, for this case, than Model CPR-DxL.

"Modified Timur Model": (Appendix F)

The "Modified Timur Model" is given as:

$$
k_{\text {Timur }}=a \phi^{b} F^{c}
$$

In Fig. F.10 we present the results of the "Modified Timur Model" (Eq. F-5) which is essentially just a generalized power-law function relation in terms of $k$, $\phi$, and $F$. Figs. F.10a and b, provide the "Modified Timur Model" results of the data for this case.

\section{GRACE Algorithm: (Appendix F)}

In Fig. F.11 we present the results predicted by the GRACE algorithm, which is a noF-parametric regression approach (see Xue et al. (1997) for details). In theory, the GRACE algorithm should provide the most unbiased correlation of the data - i.e., the GRACE algorithm is designed not to "fit the errors" as other regression approaches may. It is our contention that the GRACE algorithm is the statistical standard - and any algorithm/approach/model which achieves better regression statistics than the GRACE algorithm is actually "fitting the errors" in the data. None of the cases have significantly better regression statistics than the GRACE algorithm - hence, none of the cases are "over-fitted" in a statistical sense. 


\section{Comparison of All Models: (Appendix F)}

Our final graphic, Fig. F.12, illustrates all of the models on a single plot of calculated versus measured permeability. We also present a table of all statistical results in Table F.1.

Table F.1 Statistical Results for all Models (Hazlett Well 103 Case, $n=34$ ).

\begin{tabular}{|c|c|c|c|c|}
\hline Model & $\begin{array}{c}\text { Sum of } \\
\text { Squared } \\
\text { Residuals } \\
{\left[\log (k)^{2}\right]}\end{array}$ & $\begin{array}{c}\text { Variance } \\
{\left[\log (k)^{2}\right]}\end{array}$ & $\begin{array}{c}\text { Standard } \\
\text { Deviation } \\
{[\log (k)]}\end{array}$ & $\begin{array}{c}\text { Absolute } \\
\text { Relative } \\
\text { Error } \\
\text { [percent] }\end{array}$ \\
\hline$C P R-A$ & 20.00 & 4.38 & 2.09 & 70.78 \\
\hline$C P R-B$ & 25.76 & 3.76 & 1.94 & 79.06 \\
\hline$C P R-C$ & 21.69 & 4.38 & 2.09 & 72.39 \\
\hline$C P R-D x L$ & 25.18 & 4.05 & 2.01 & 75.42 \\
\hline$C P R-D \times Q$ & 18.56 & 4.34 & 2.08 & 69.46 \\
\hline Modified Timur & 28.11 & 4.13 & 2.03 & 83.89 \\
\hline GRACE Algorithm & 15.21 & 4.45 & 2.11 & 55.57 \\
\hline
\end{tabular}

The coefficients for the models used in this case are provided in Table F.2.

Table F.2a - Model Coefficients (Hazlett Well 103 Case, $n=34$ ).

\begin{tabular}{|c|c|c|c|c|c|c|}
\hline Model & $a$ or $\alpha$ & $b$ or $\beta$ & $C$ or $c_{\max }$ & $c_{1}$ & $C_{2}$ & $c_{3}$ \\
\hline$C P R-A$ & $8.00 \times 10^{8}$ & 8.00 & 0.050 & $1.33 \times 10^{-3}$ & -1.88 & 0.35 \\
\hline$C P R-B$ & $1.00 \times 10^{7}$ & 8.00 & 0.050 & 1.09 & -0.32 & -0.26 \\
\hline$C P R-C$ & $1.00 \times 10^{-6}$ & 100.00 & $5.00 \times 10^{-4}$ & 24.49 & 0.66 & -0.11 \\
\hline Modified Timur & $1.54 \times 10^{6}$ & 6.87 & 0.246 & - & - & - \\
\hline
\end{tabular}

Table F.2b - Model Coefficients (Hazlett Well 103 Case, $n=34$ ).

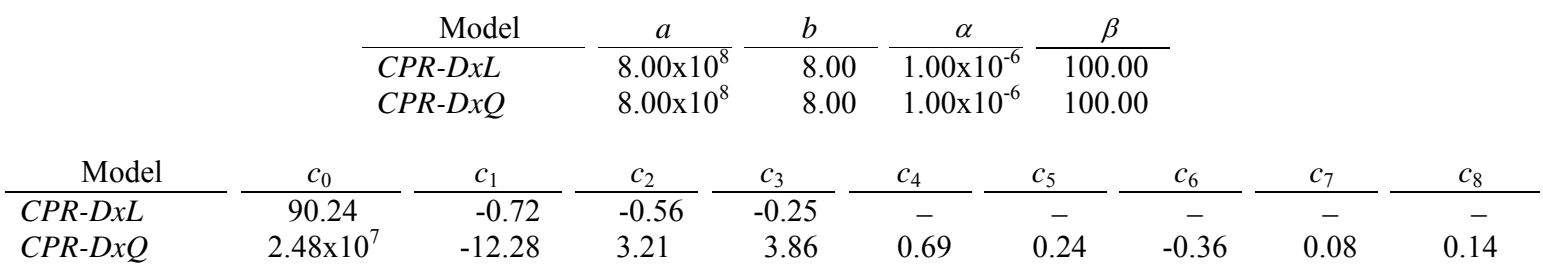

Nomenclature: (Appendix F)

$F=$ Archie Formation Factor (dimensionless)

$k=$ Formation Permeability, md (or any consistent units)

$\phi \quad=$ Porosity, fraction

\section{References: (Appendix F)}

Hazlett, W.G.: "Correlation of Reservoir Rock Properties in Porous Media." PhD dissertation, Texas A\&M U., College Station, TX (1989).

Xue, G., Datta-Gupta, A., Valko, P., and Blasingame, T.A.: "Optimal Transformations for Multiple Regression: Application to Permeability Estimation from Well Logs," SPEFE (June 1997), 85-93. 


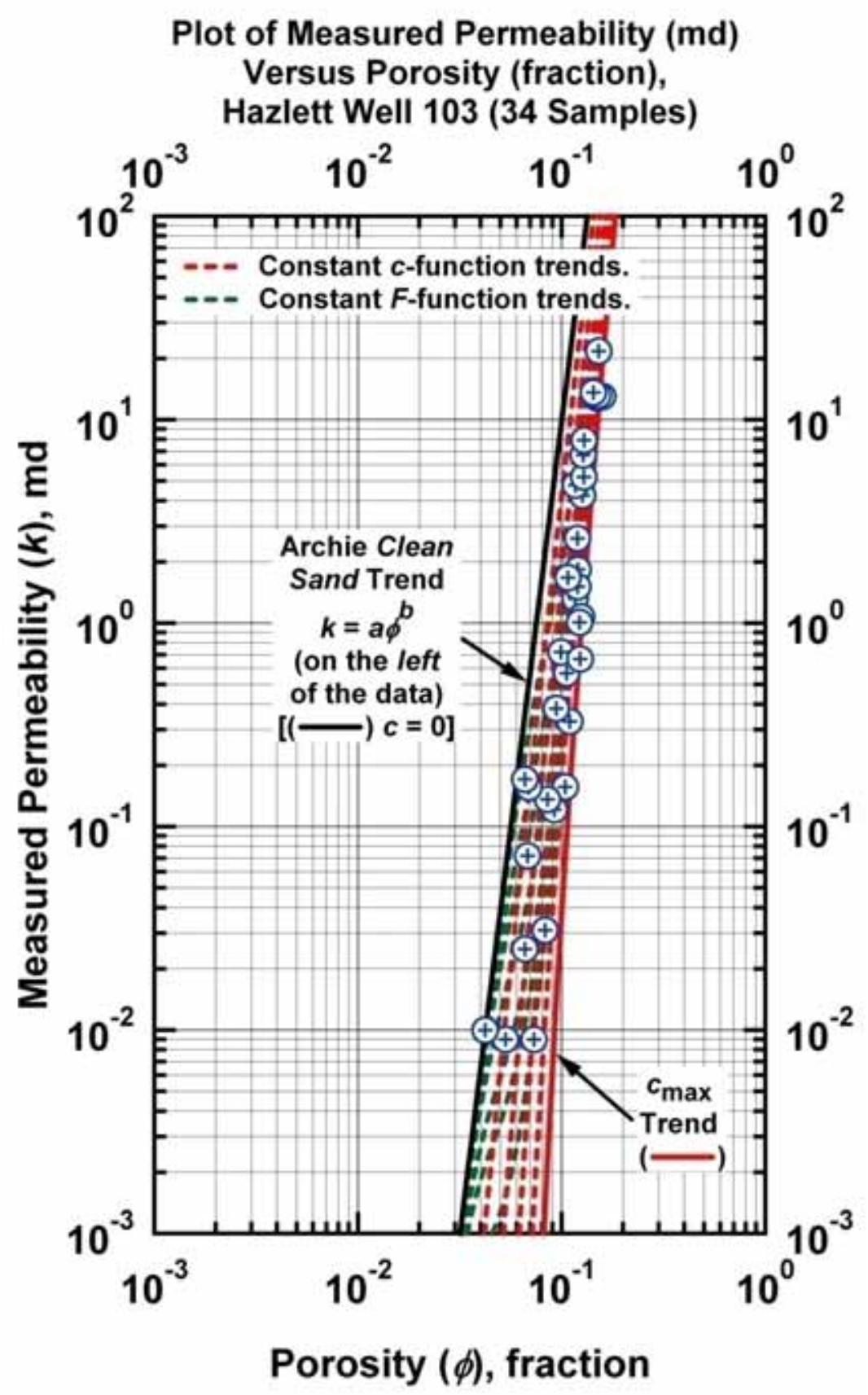

Figure F.1a - Model $C P R-A: \quad k=a(\phi-c)^{b}\left(c=c_{\max } \exp \left[-c_{1} \phi^{c_{2}} F^{c_{3}}\right]\right)$ - "Clean Sand" Plot (log-log format) - Archie "Clean Sand" trend is given by the straight-line trend at the far left of the data (power law model). [Case: Hazlett Well 103] 


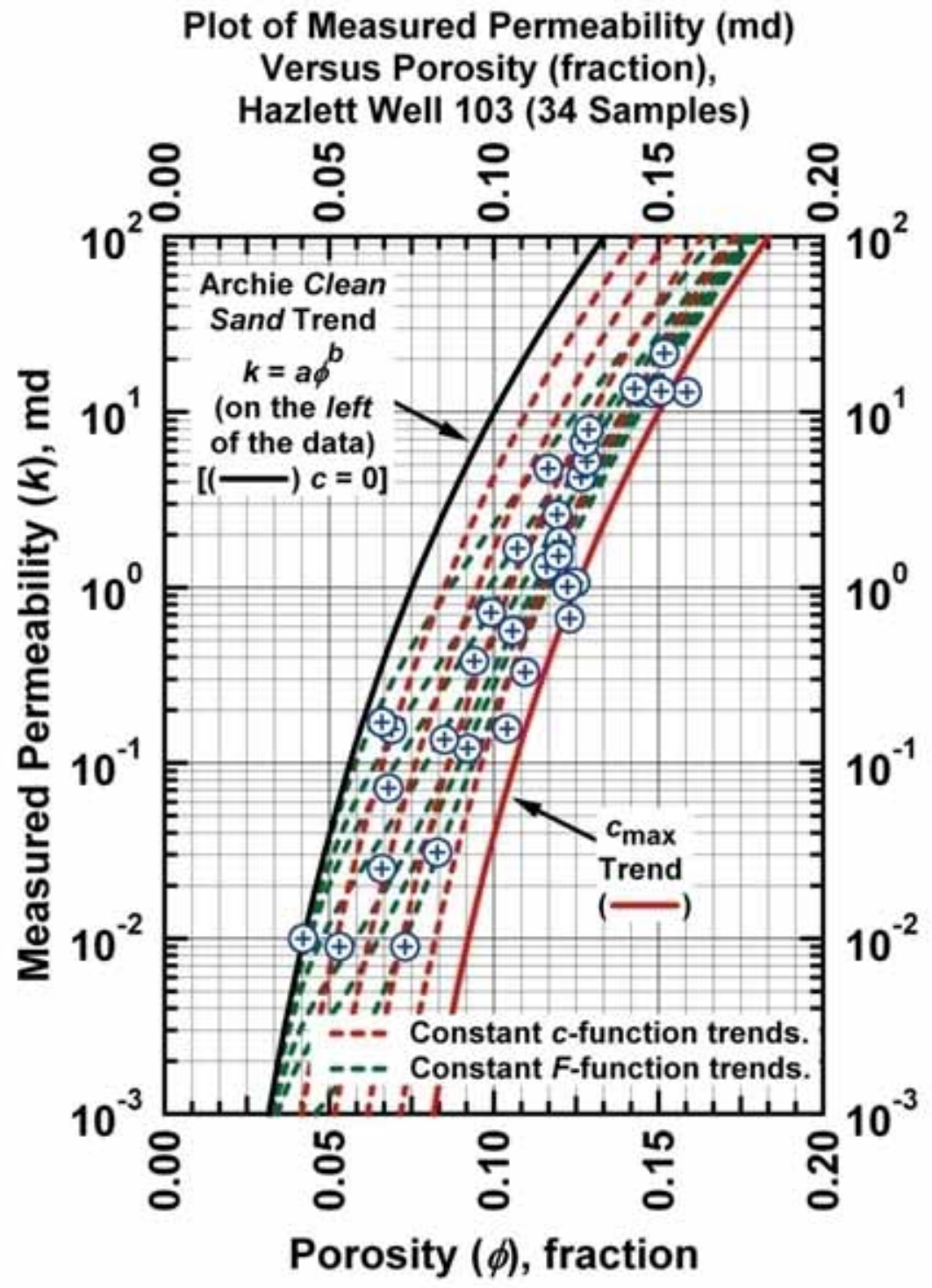

Figure F.1b - Model $C P R-A: \quad k=a(\phi-c)^{b}\left(c=c_{\max } \exp \left[-c_{1} \phi^{c_{2}} F^{c_{3}}\right]\right)$ - "Dirty Sand" Plot (semilog format). Archie "Clean Sand" trend is given by the curved trend at the far left of the data (power law model). [Case: Hazlett Well 103] 
Comparison of Permeability (md) Calculated Using the "CPR-A" Model Versus the Measured Permeability (md), Hazlett Well 103 (34 Samples)

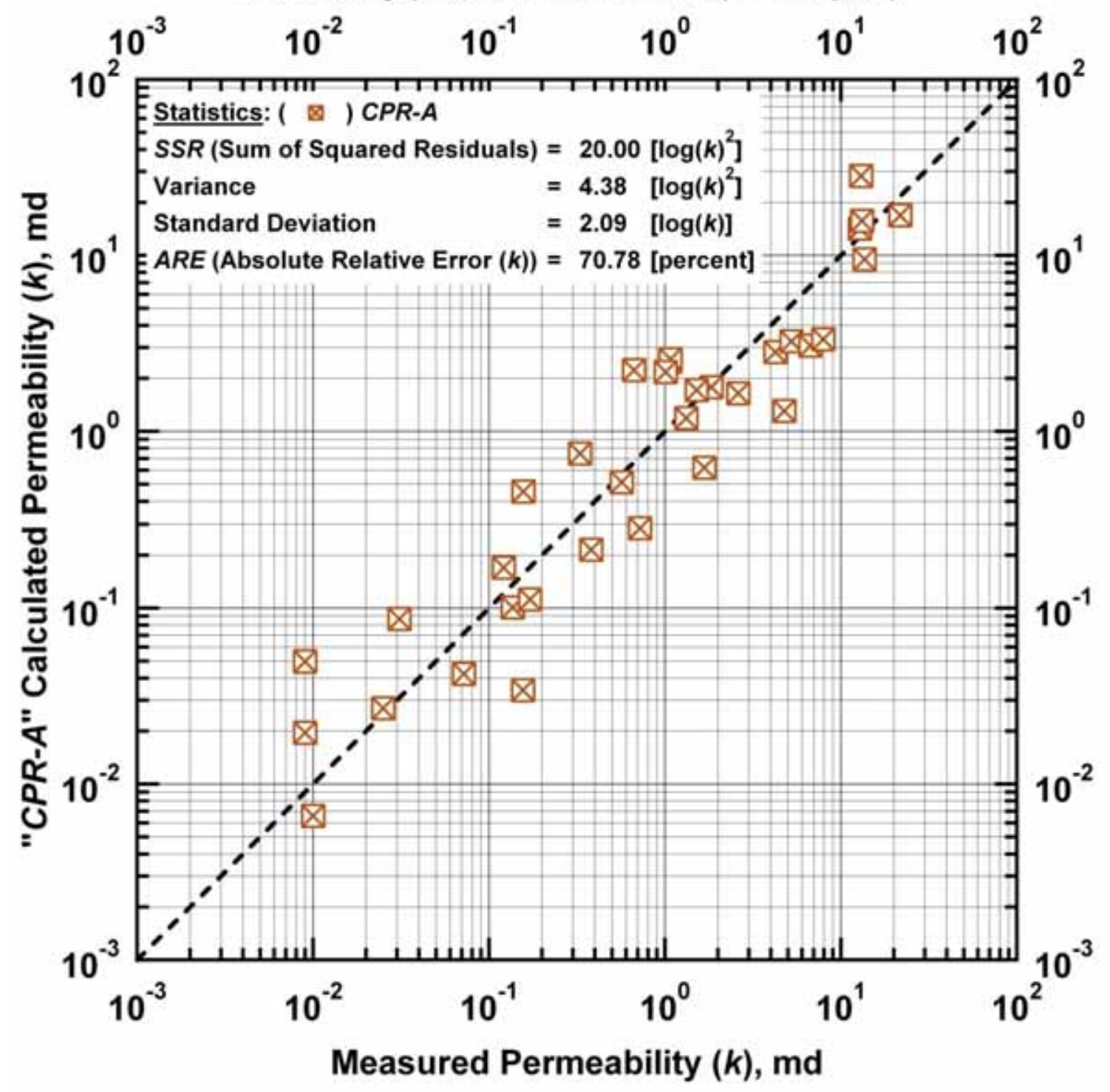

Figure F.2a $\quad$ Model CPR-A: calculated versus measured permeability. [Case: Hazlett Well 103] 


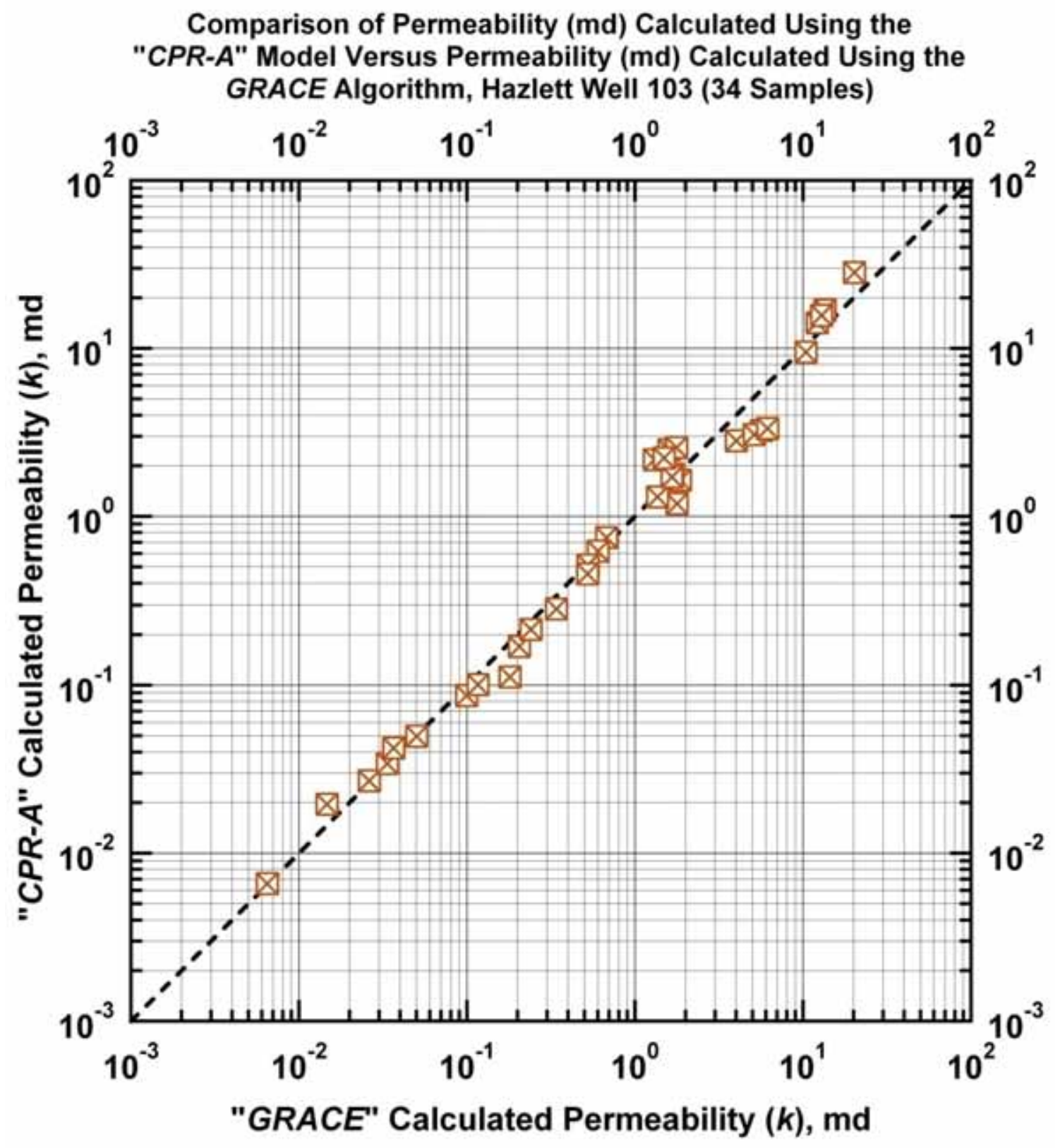

Figure F.2b - Model CPR-A: Permeability calculated using Model CPR-A versus permeability calculated using the GRACE Algorithm. [Case: Hazlett Well 103] 


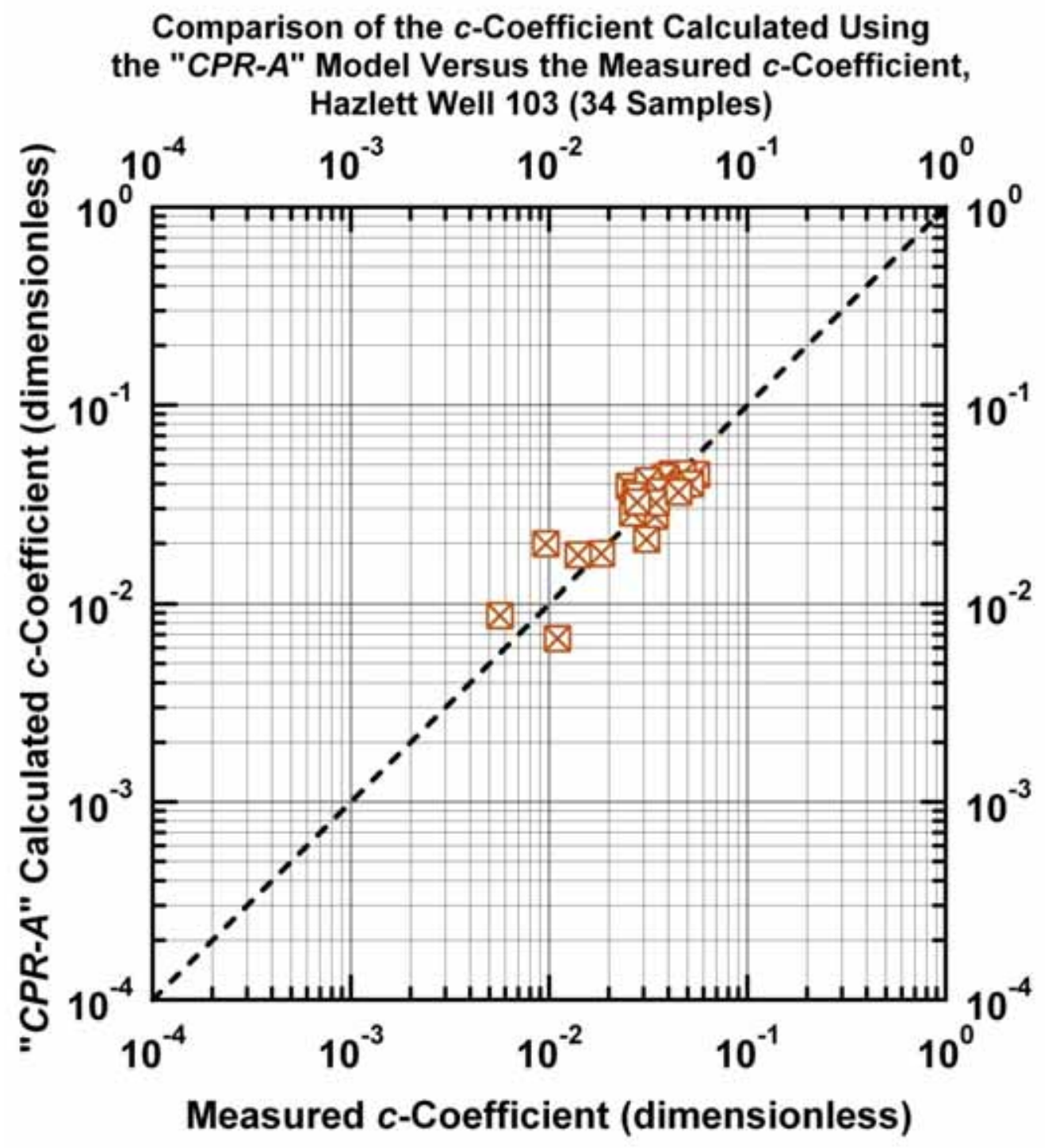

Figure F.2c - Model $C P R$ - $A$ : Calculated $c$-function values versus measured $c$-function values. [Case: Hazlett Well 103] 


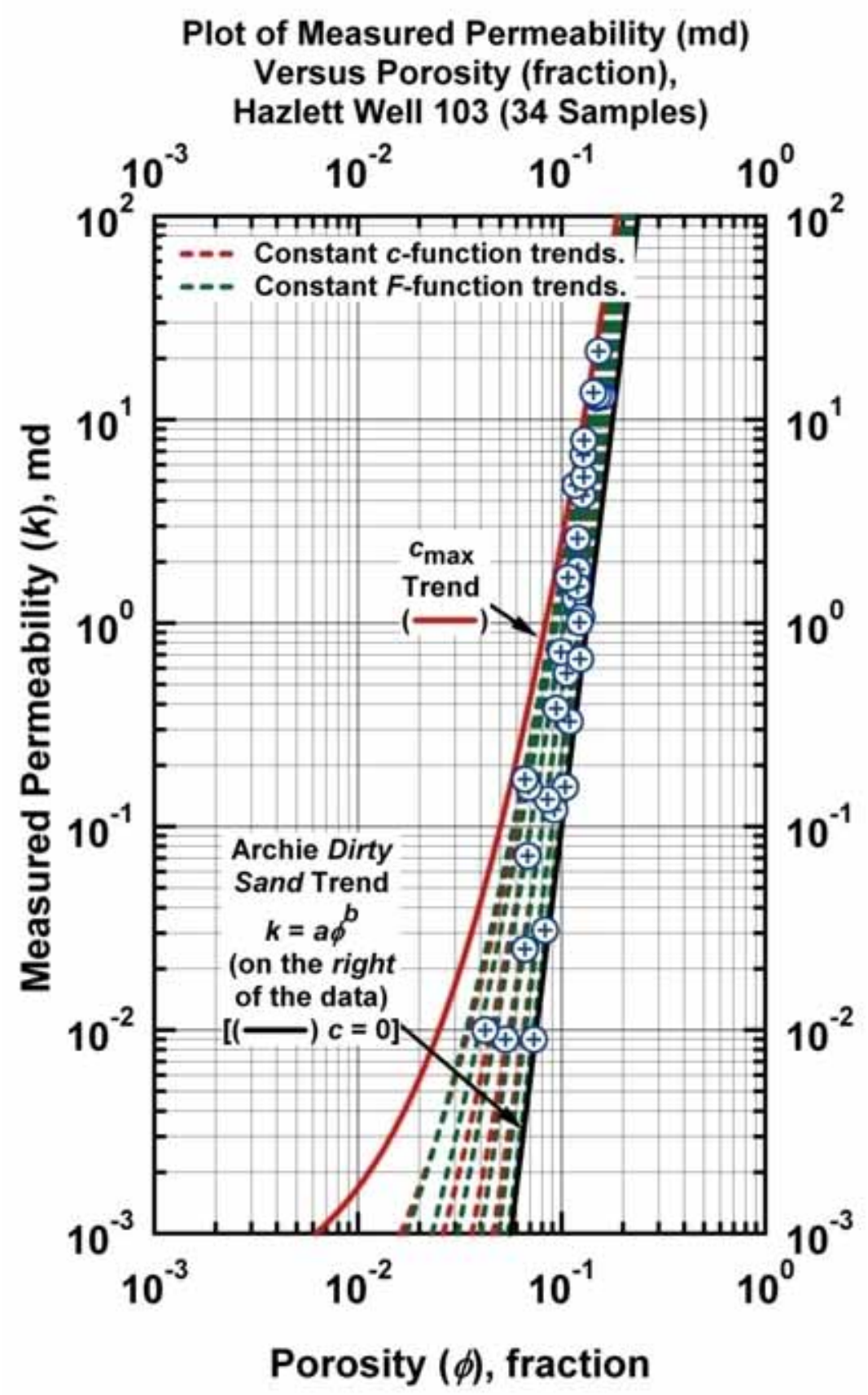

Figure F.3a - Model CPR-B: $k=a(\phi+c)^{b} \quad c=c_{\max } \exp \left[-c_{1} \phi^{c_{2}} F^{c_{3}}\right]$ - "Clean Sand" Plot (log-log format) - Archie "Dirty Sand" trend is given by the straight-line trend at the far right of the data (power law model). [Case: Hazlett Well 103] 


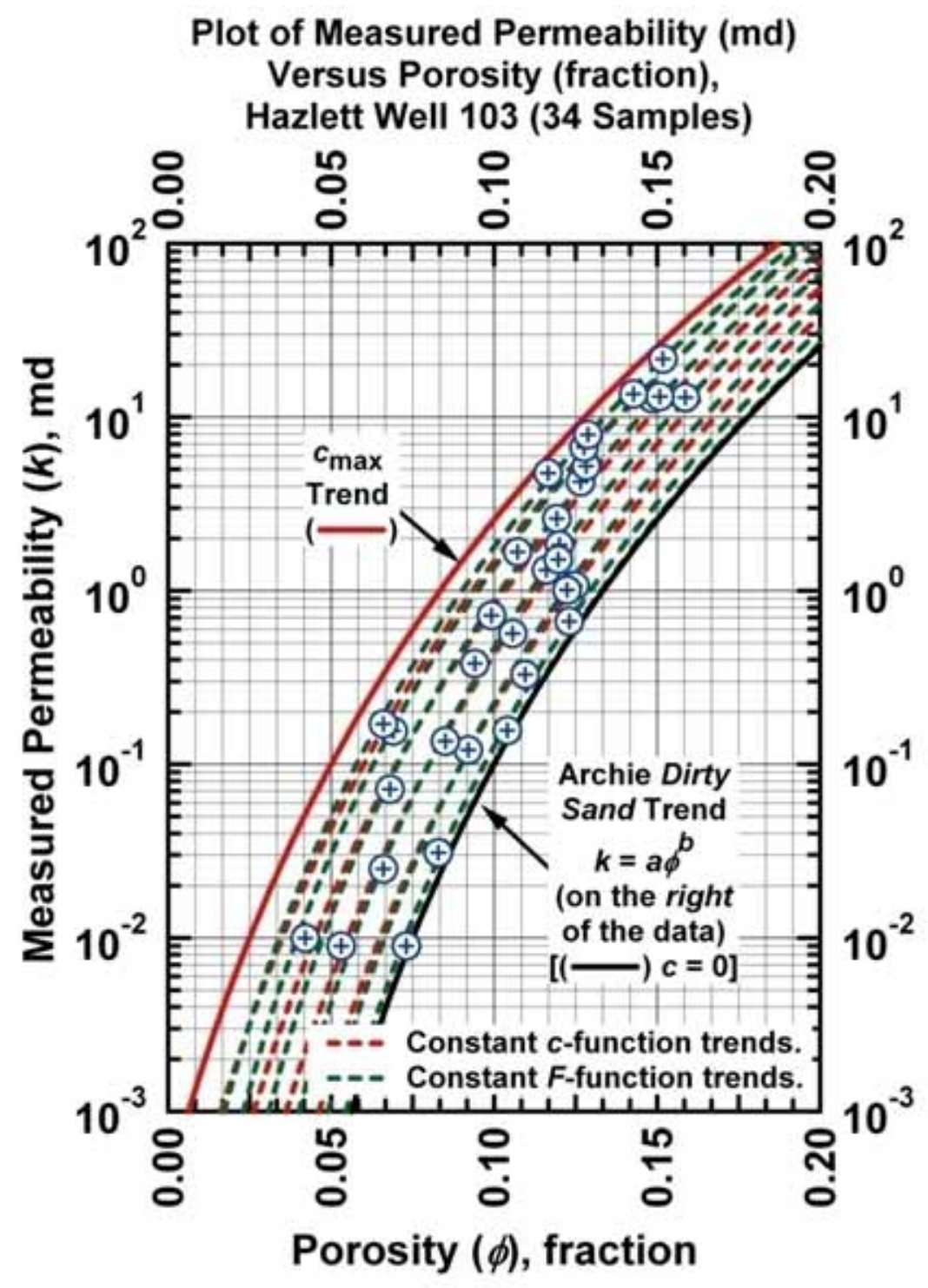

Figure F.3b - Model $C P R-B: k=a(\phi+c)^{b} \quad c=c_{\max } \exp \left[-c_{1} \phi^{c 2} F^{c 3}\right]$ — "Dirty Sand" Plot (semilog format) - Archie "Dirty Sand" trend is given by the curved trend at the far right of the data (power law model). [Case: Hazlett Well 103] 
Comparison of Permeability (md) Calculated Using the "CPR-B" Model Versus the Measured Permeability (md), Hazlett Well 103 (34 Samples)

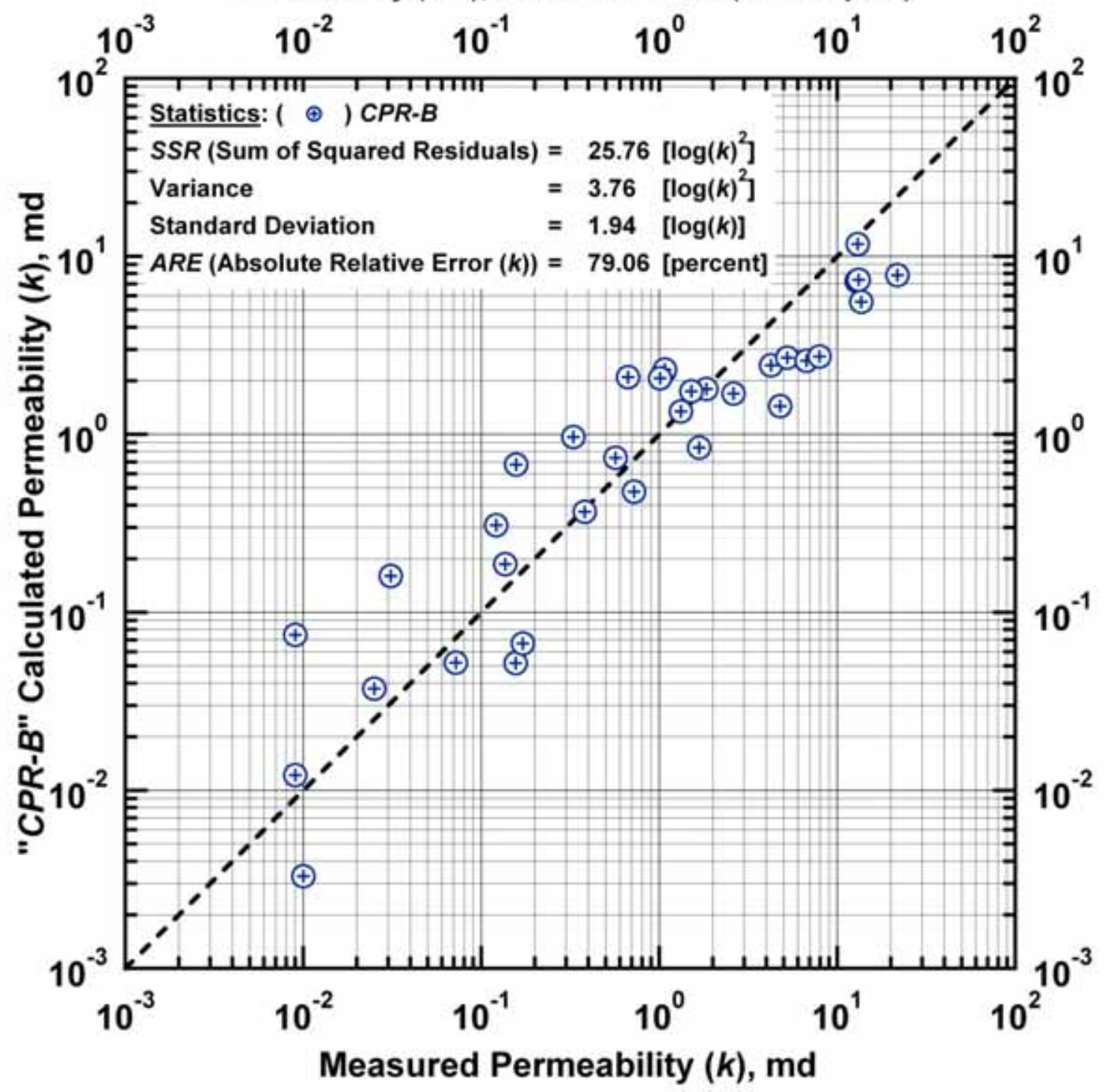

Figure F.4a - Model CPR-B: calculated versus measured permeability. [Case: Hazlett Well 103] 


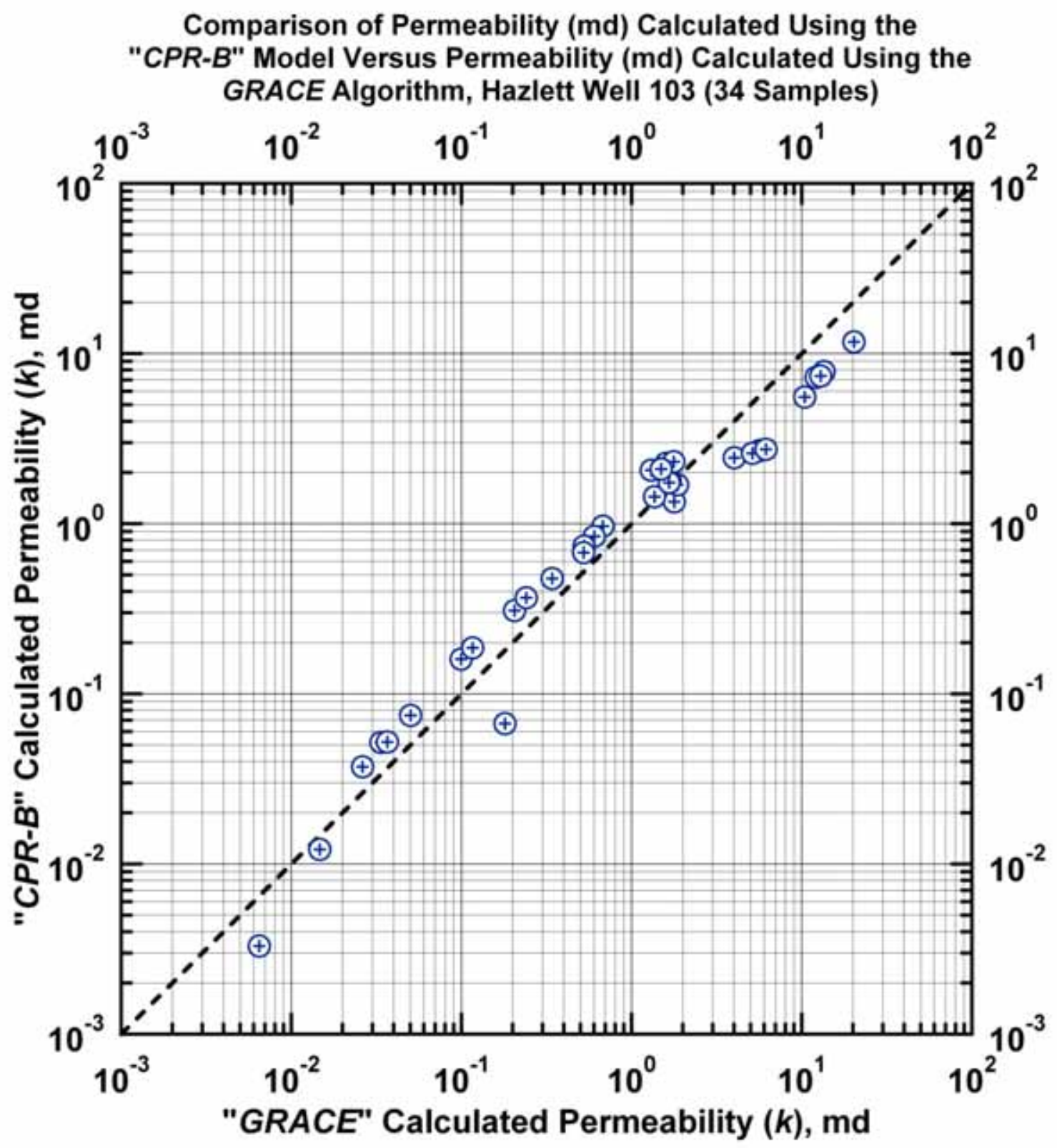

Figure F.4b - Model $C P R$ - $B$ : Permeability calculated using Model $C P R-B$ versus permeability calculated using the GRACE Algorithm. [Case: Hazlett Well 103] 


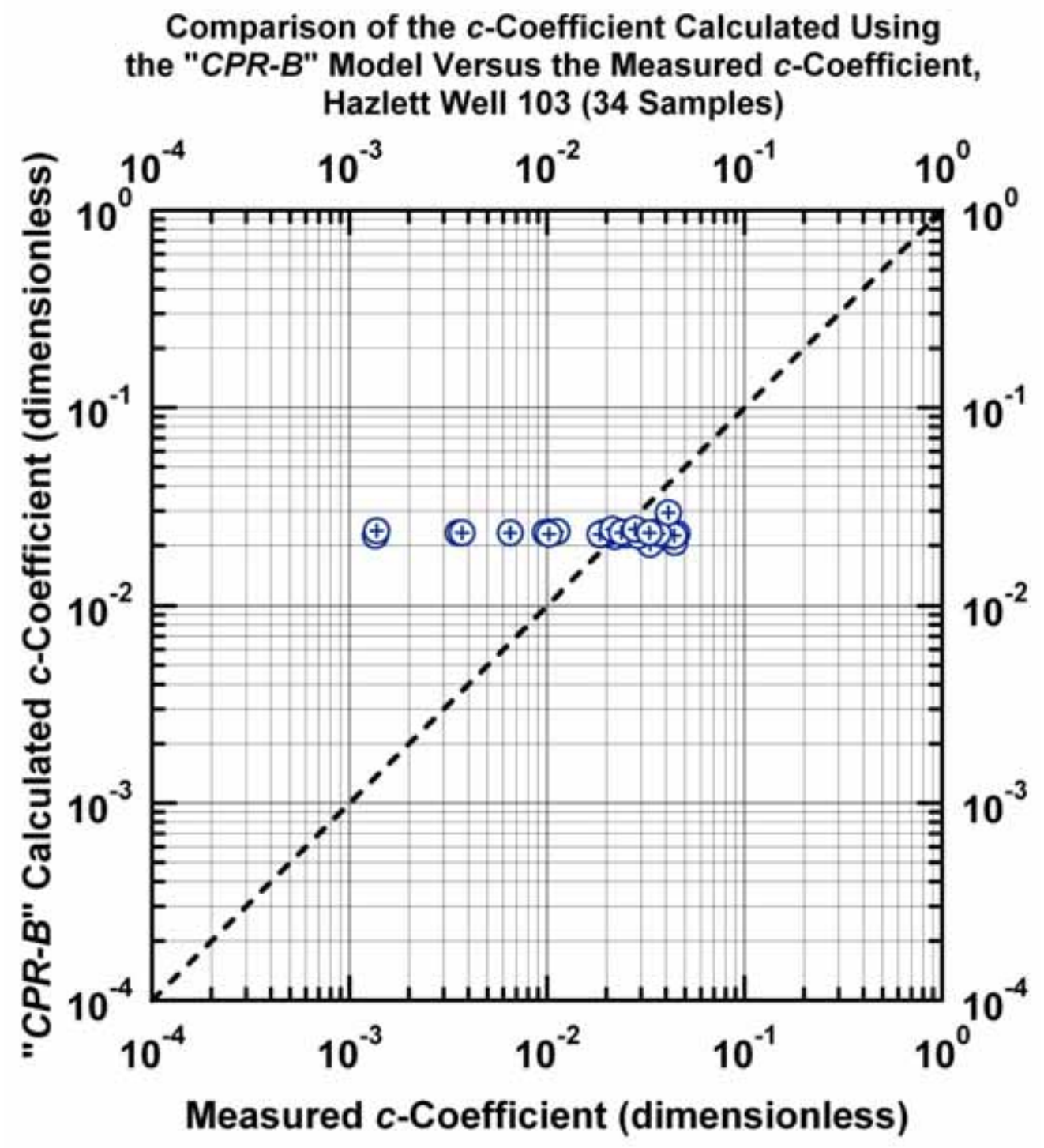

Figure F.4c - Model $C P R$ - $B$ : Calculated $c$-function values versus measured $c$-function values. [Case: Hazlett Well 103] 


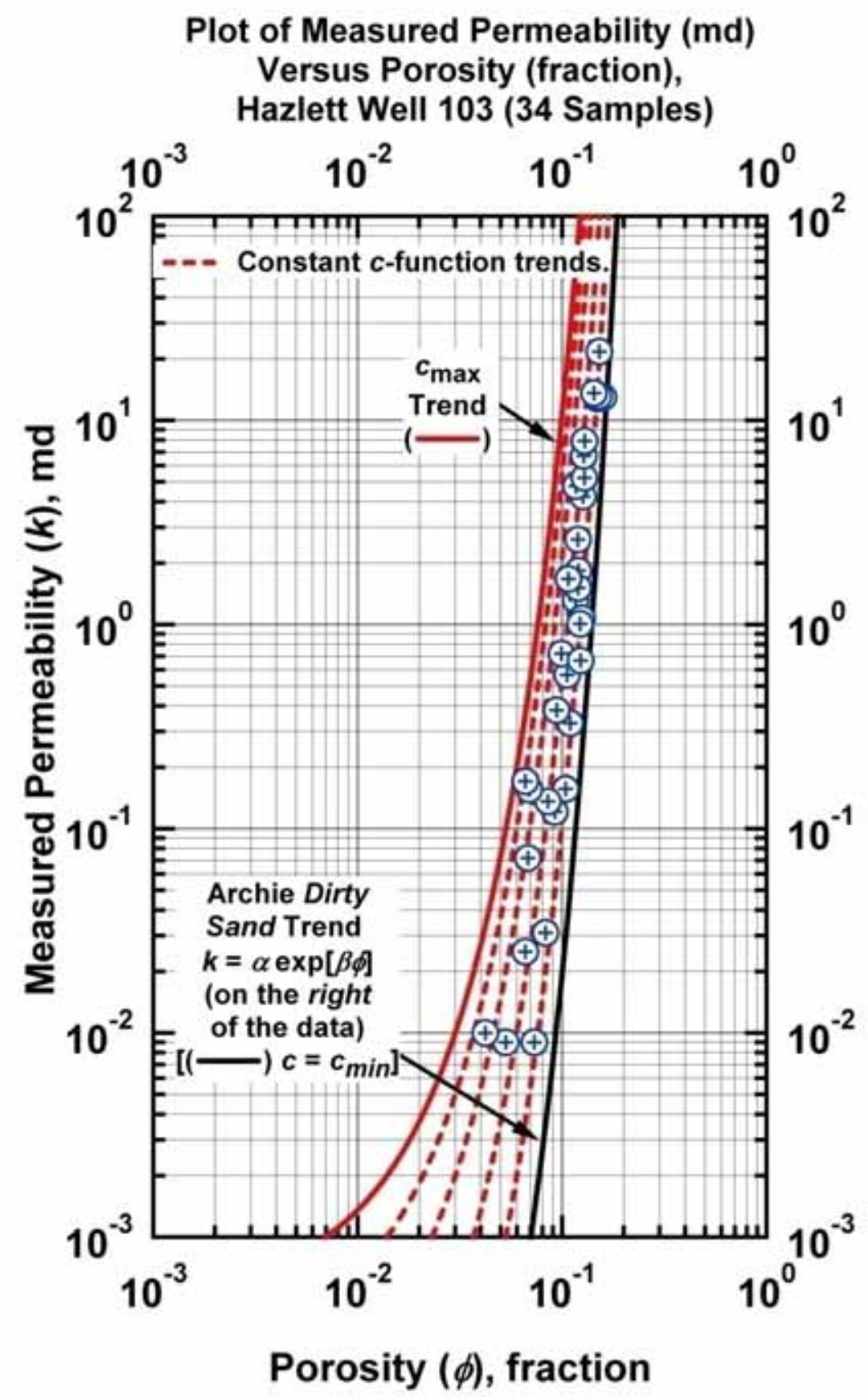

Figure F.5a - Model $C P R-C: \quad k=c \exp [\beta \phi] \quad c=c_{\max } \exp \left[-c_{1} \phi^{c 2} F^{c 3}\right]$ - "Clean Sand" Plot (log-log format) - Archie "Dirty Sand" trend is given by the curved trend at the far right of the data (exponential model). [Case: Hazlett Well 103] 


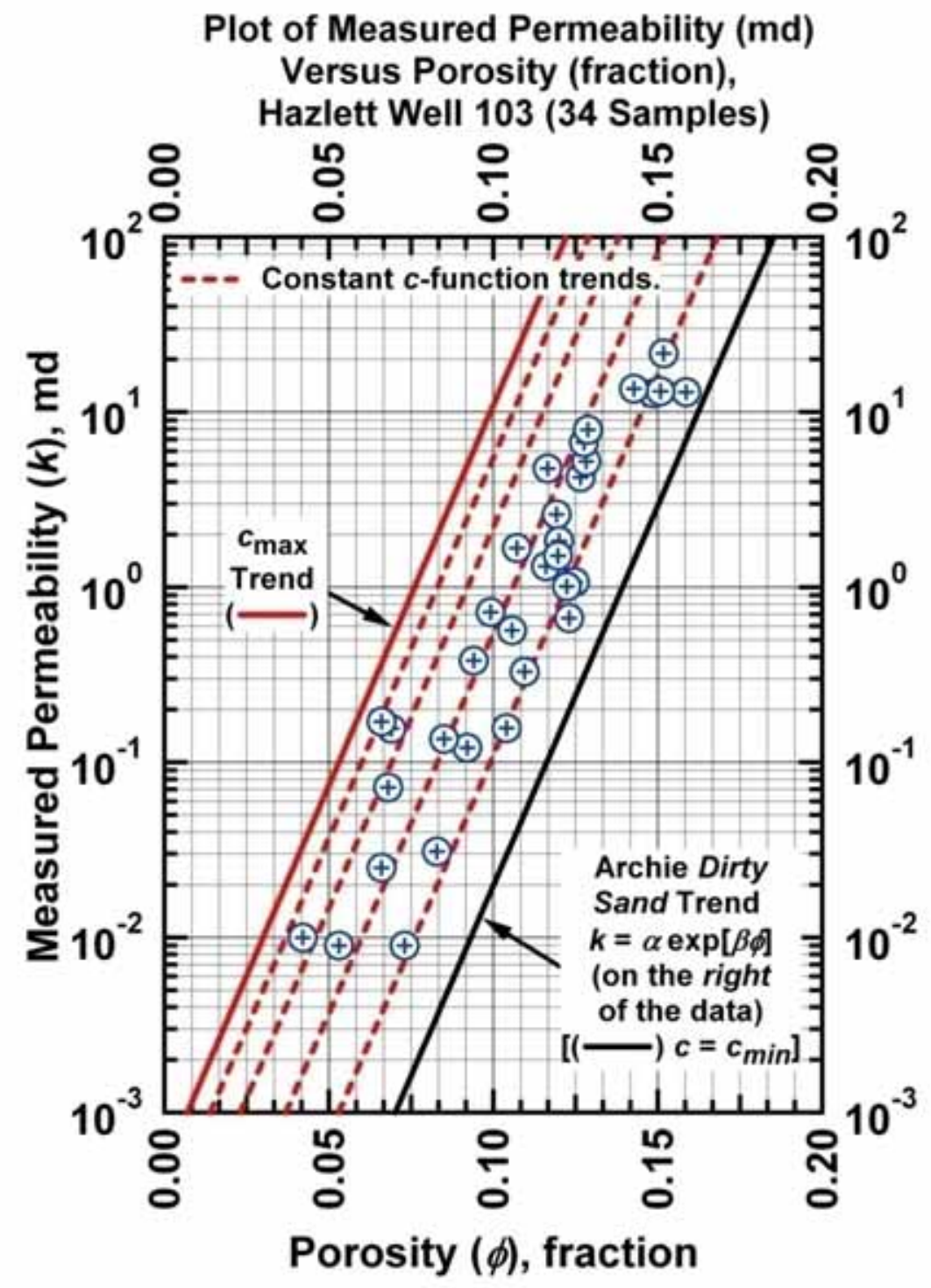

Figure F.5b - Model $C P R-C: \quad k=c \exp [\beta \phi] \quad c=c_{\max } \exp \left[-c_{1} \phi^{c_{2}} F^{c_{3}}\right]$ - "Dirty Sand" Plot (semilog format) - Archie "Dirty Sand" trend is given by the straight-line trend at the far right of the data (exponential model). [Case: Hazlett Well 103] 


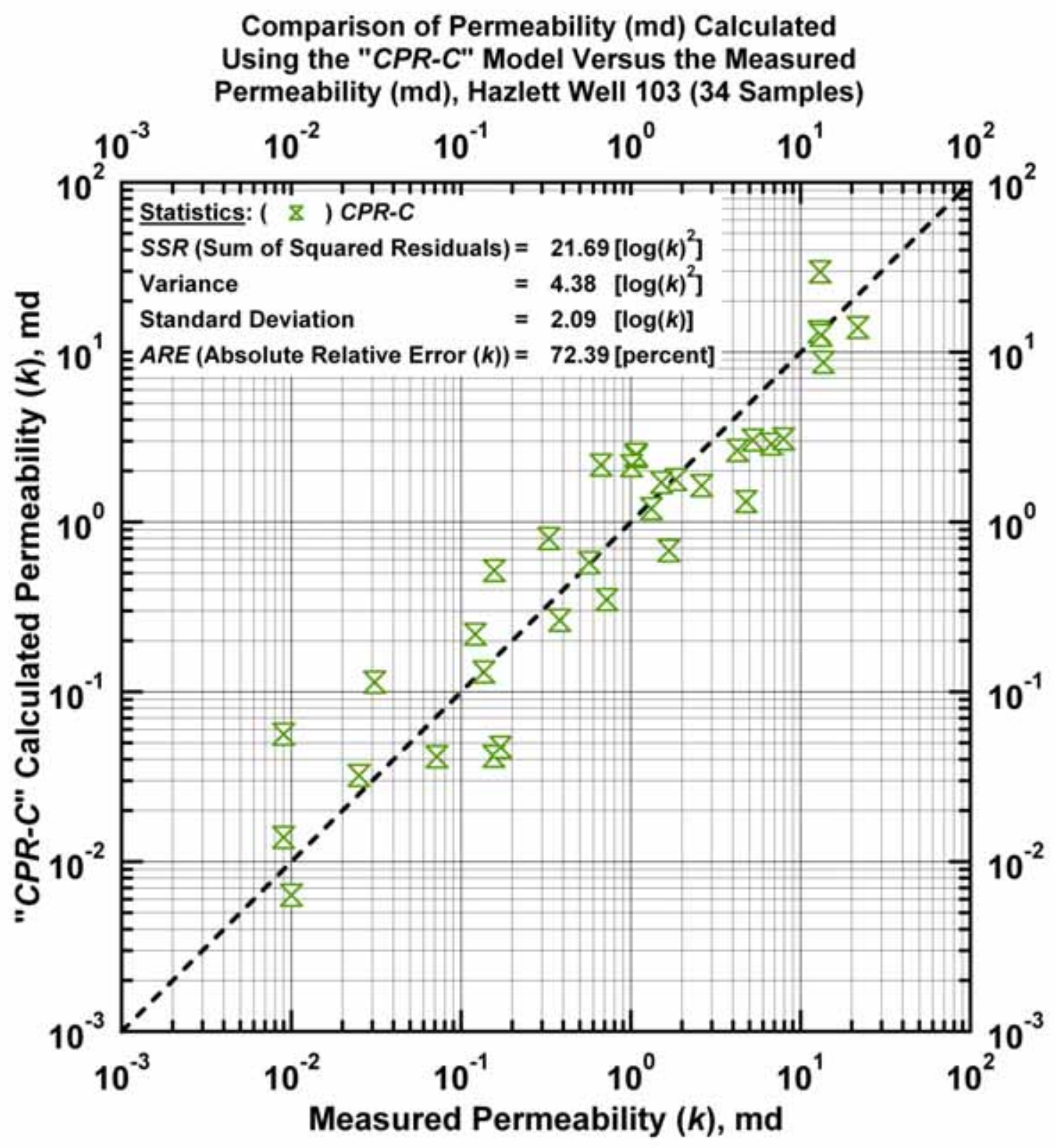

Figure F.6a - Model $C P R-C$ : calculated versus measured permeability. [Case: Hazlett Well 103] 


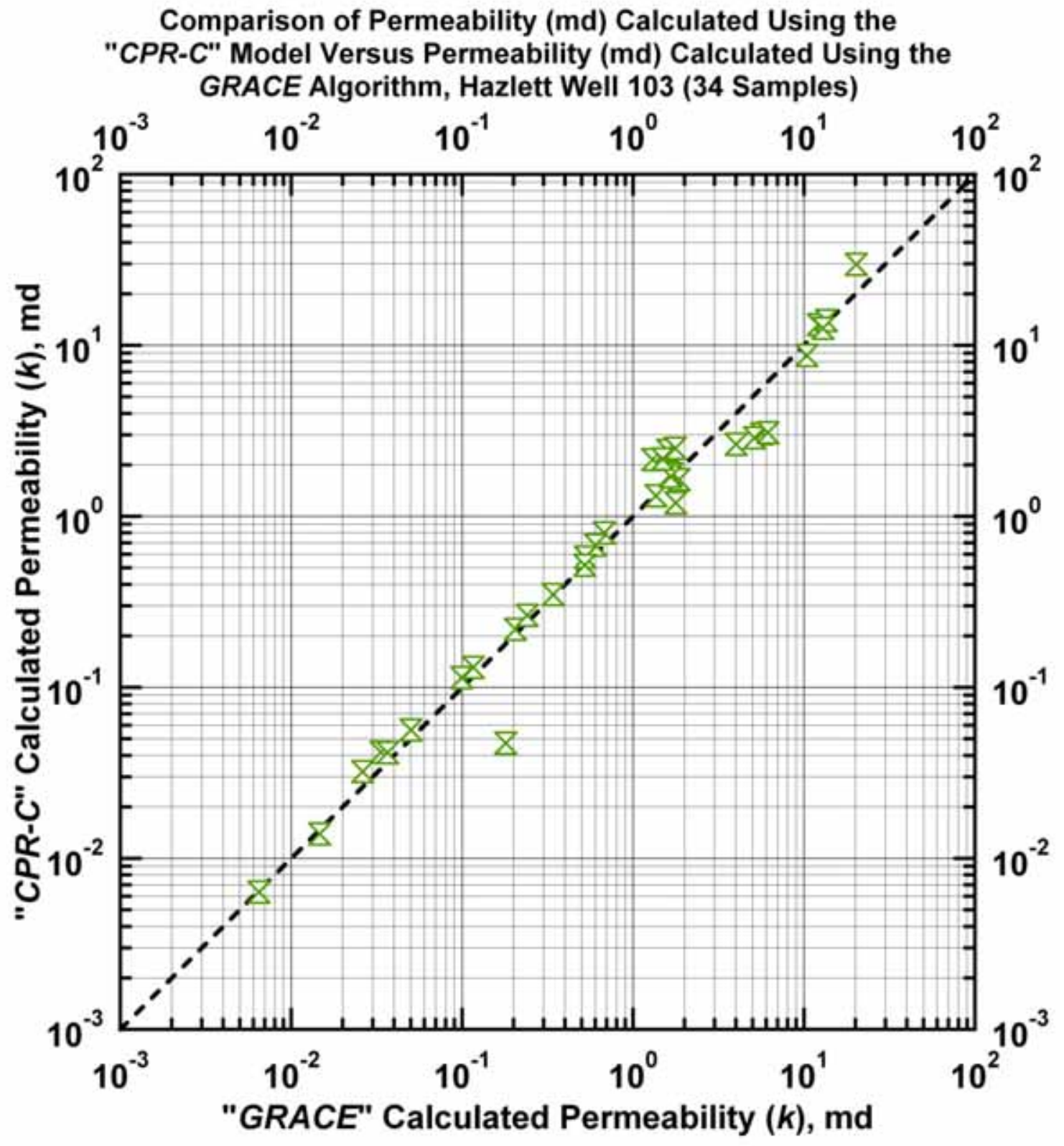

Figure F.6b - Model $C P R-C$ : Permeability calculated using Model $C P R-C$ versus permeability calculated using the GRACE Algorithm. [Case: Hazlett Well 103] 


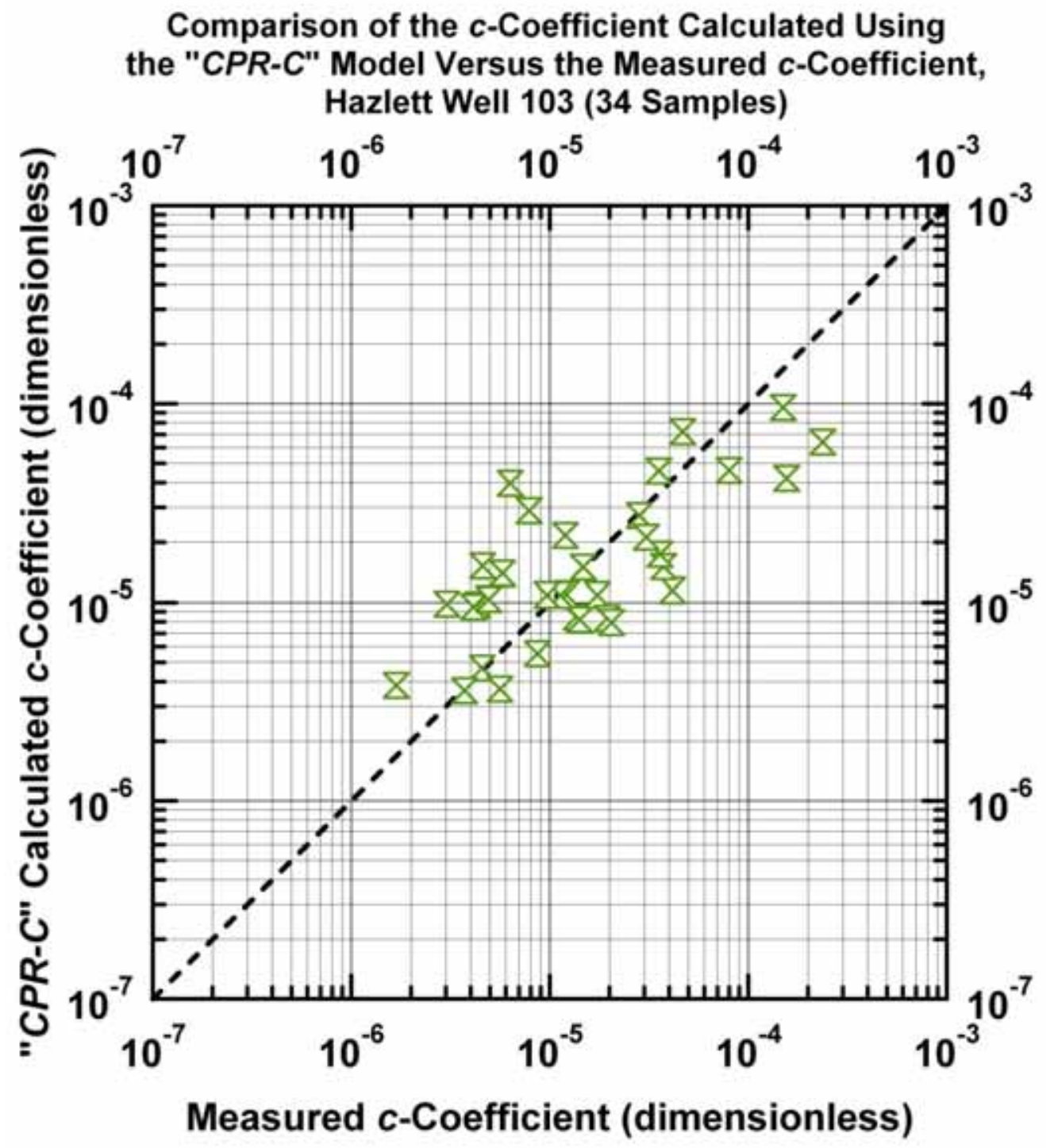

Figure F.6c - Model $C P R$-C: Calculated $c$-function values versus measured $c$-function values. [Case: Hazlett Well 103] 


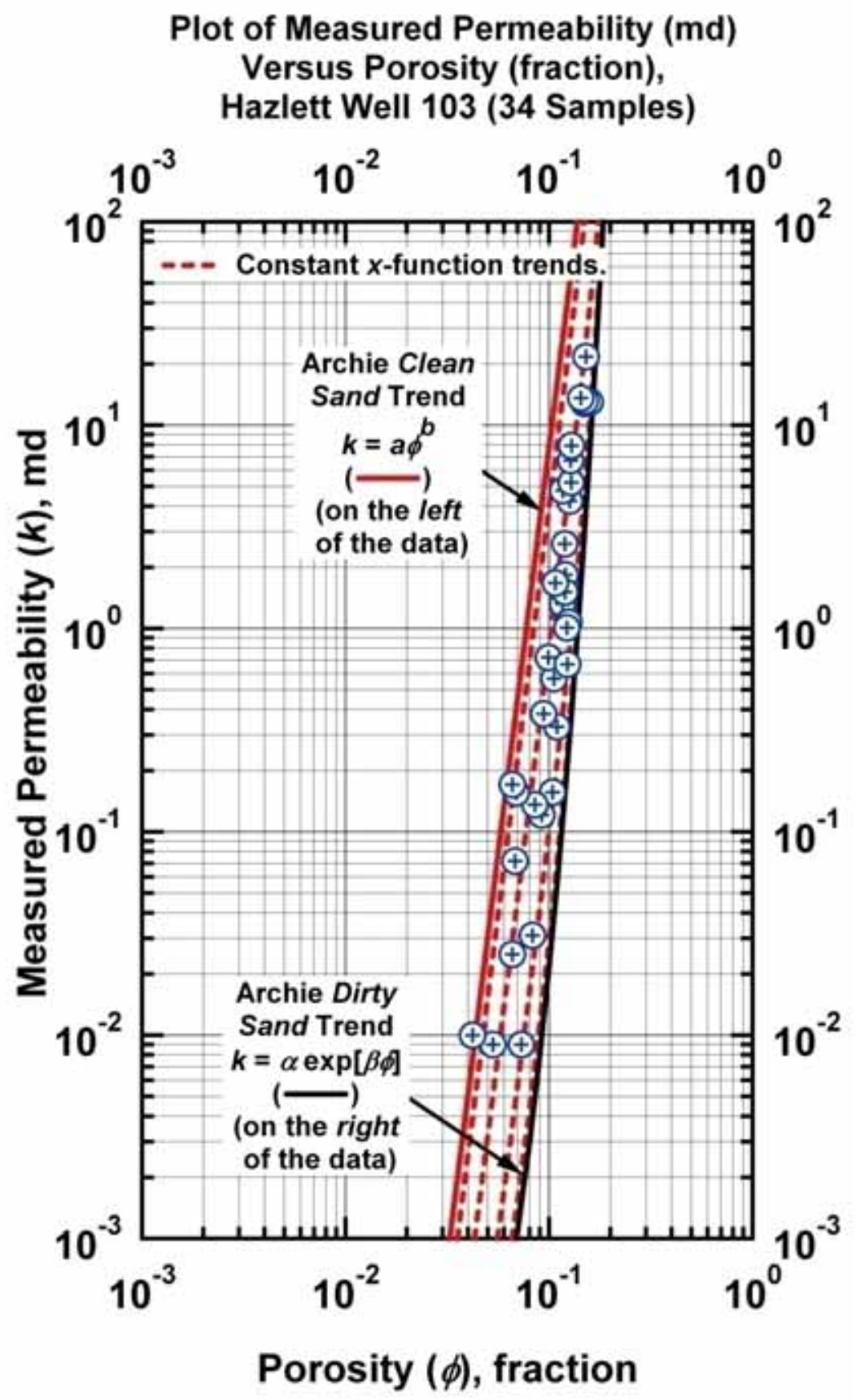

Figure F.7a - Model CPR-D: $k=x a \phi^{b}+(1-x) \alpha \exp [\beta \phi] \quad[0 \leq x \leq 1]$ - Weighted Power Law-Exponential Model used to correlate permeability $(k)$ and porosity $(\phi)$. "Clean Sand" Plot - Archie "Clean Sand" trend is given by the straight-line trend at the far left of the data (power law model). [Case: Hazlett Well 103] 


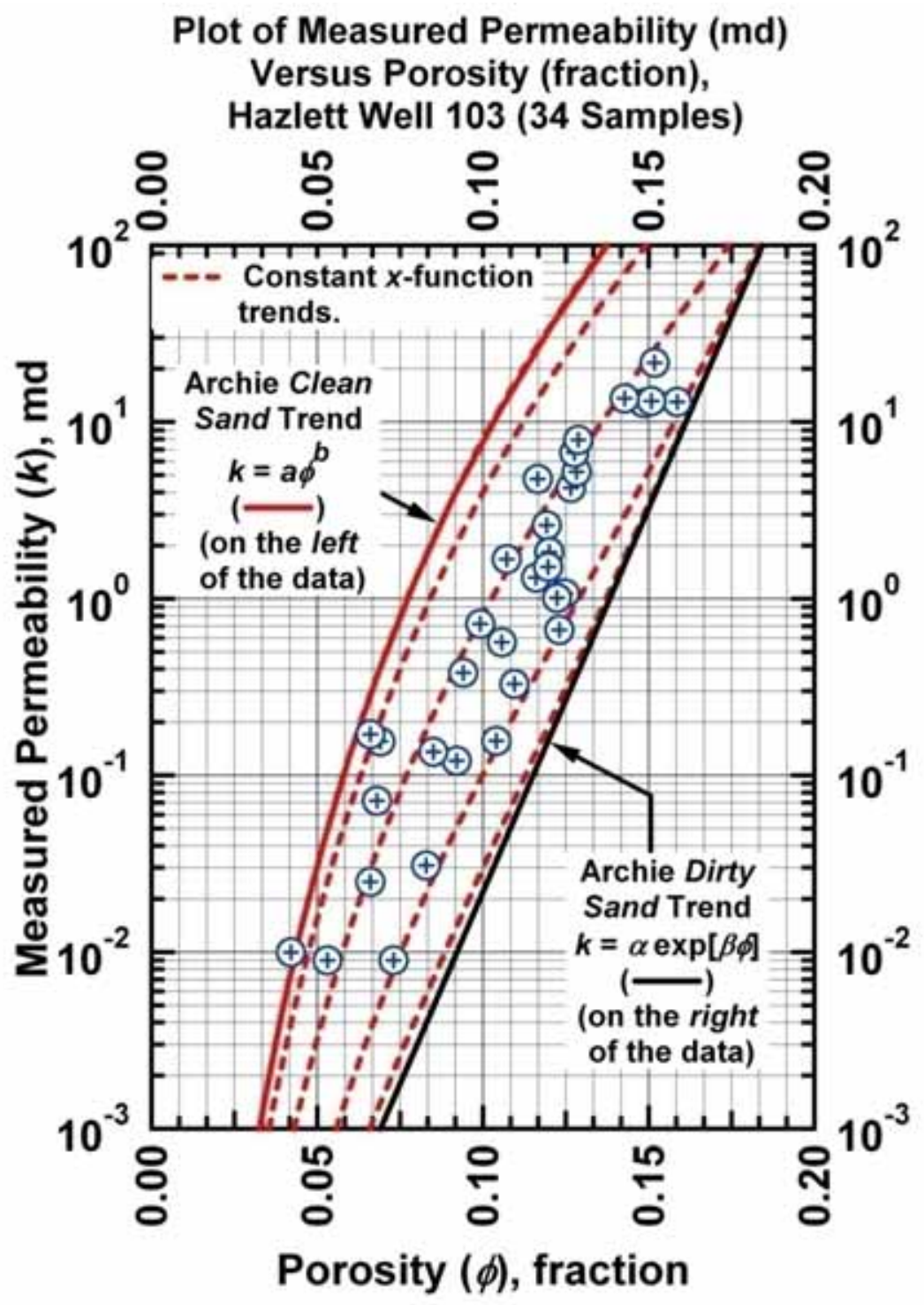

Figure F.7b - Model CPR-D: $k=x a \phi^{b}+(1-x) \alpha \exp [\beta \phi] \quad[0 \leq x \leq 1]$ - Weighted Power Law-Exponential Model used to correlate permeability $(k)$ and porosity $(\phi)$. "Dirty Sand" Plot - Archie "Dirty Sand" trend is given by the straight-line trend at the far right of the data (exponential model). [Case: Hazlett Well 103] 


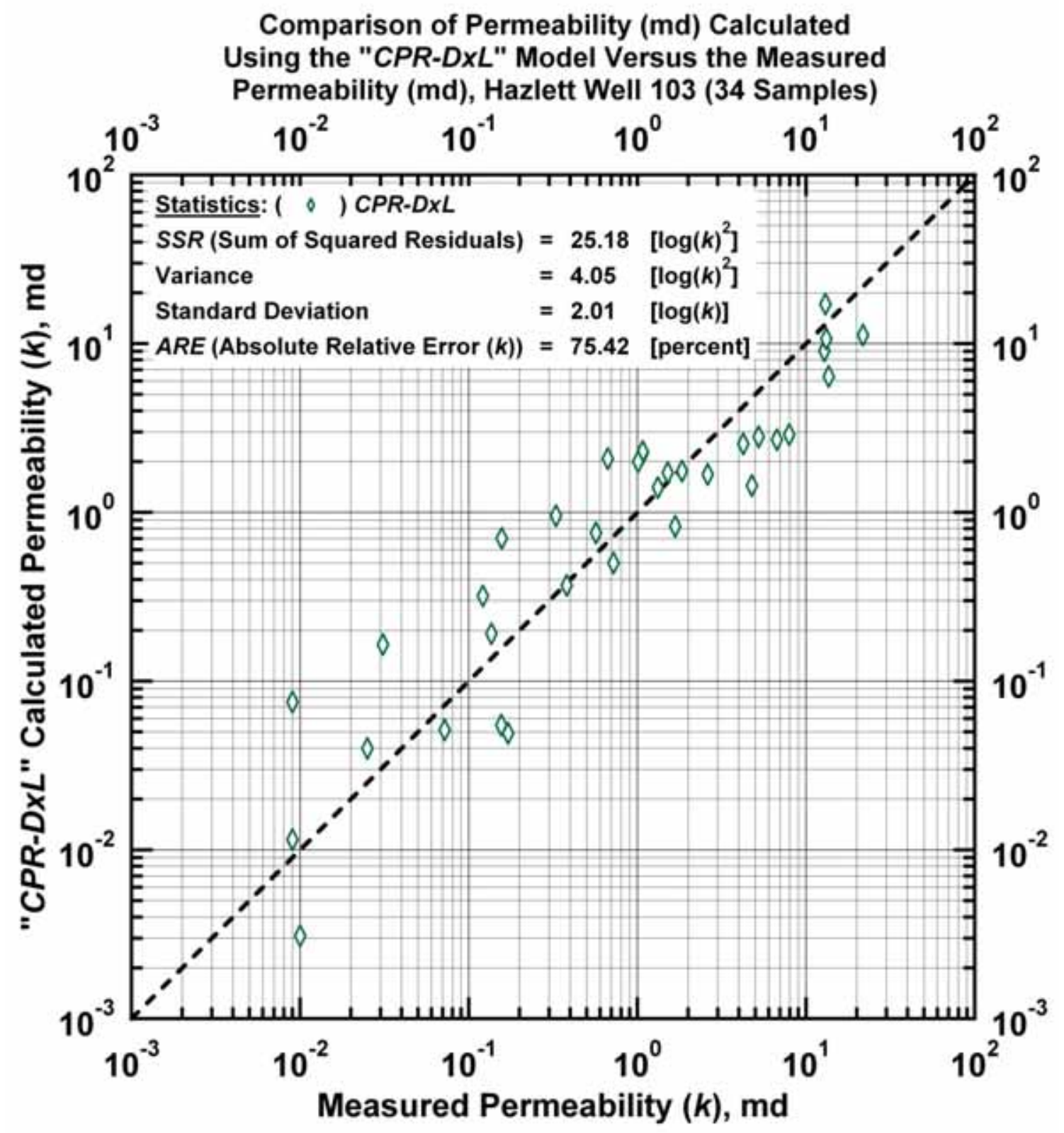

Figure F.8a - Model CPR-DxL: Calculated versus measured permeability. [Case: Hazlett Well 103] 


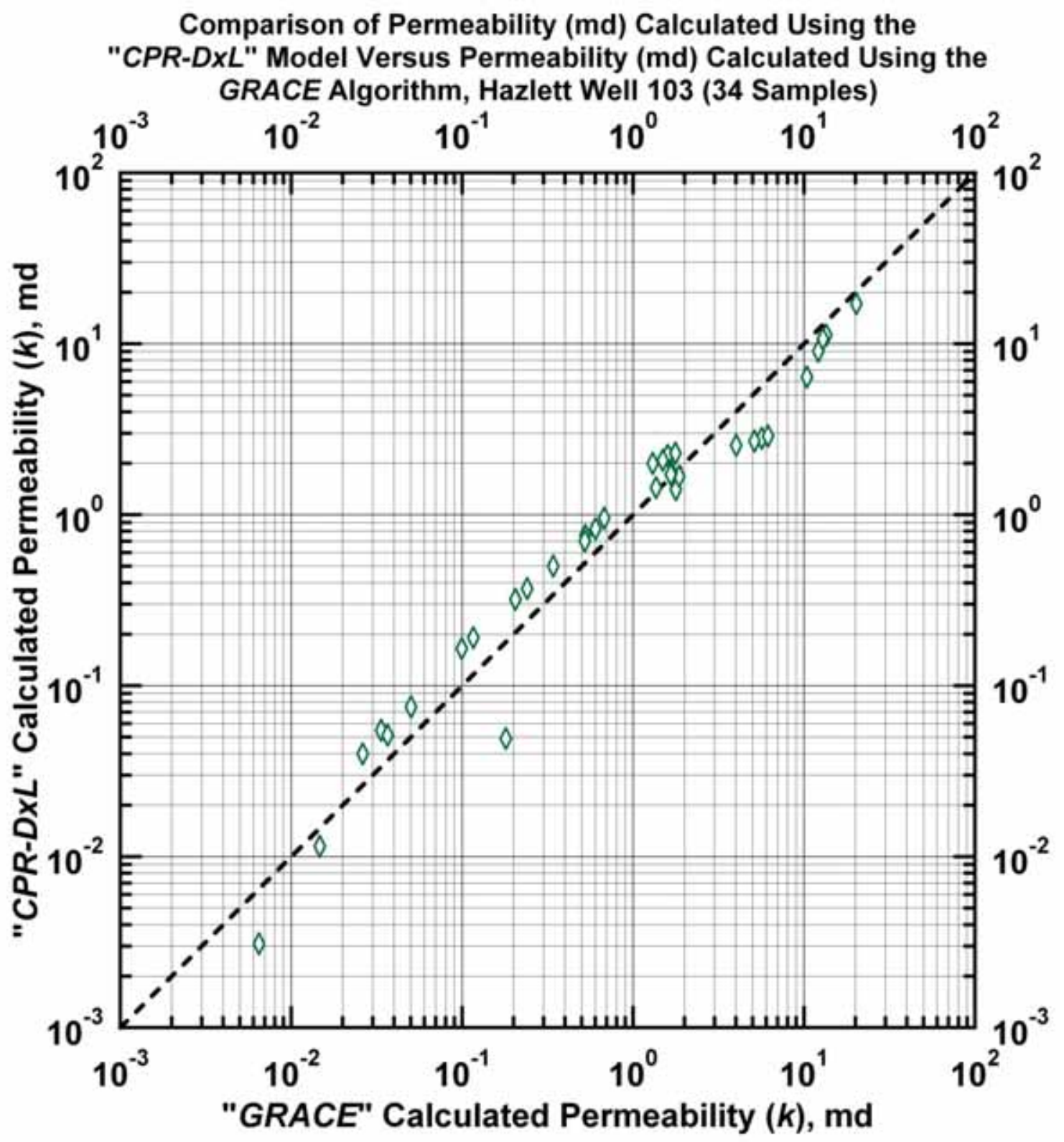

Figure F.8b - Model $C P R-D x L$ : Permeability calculated using Model $C P R-C$ versus permeability calculated using the GRACE Algorithm. [Case: Hazlett Well 103] 


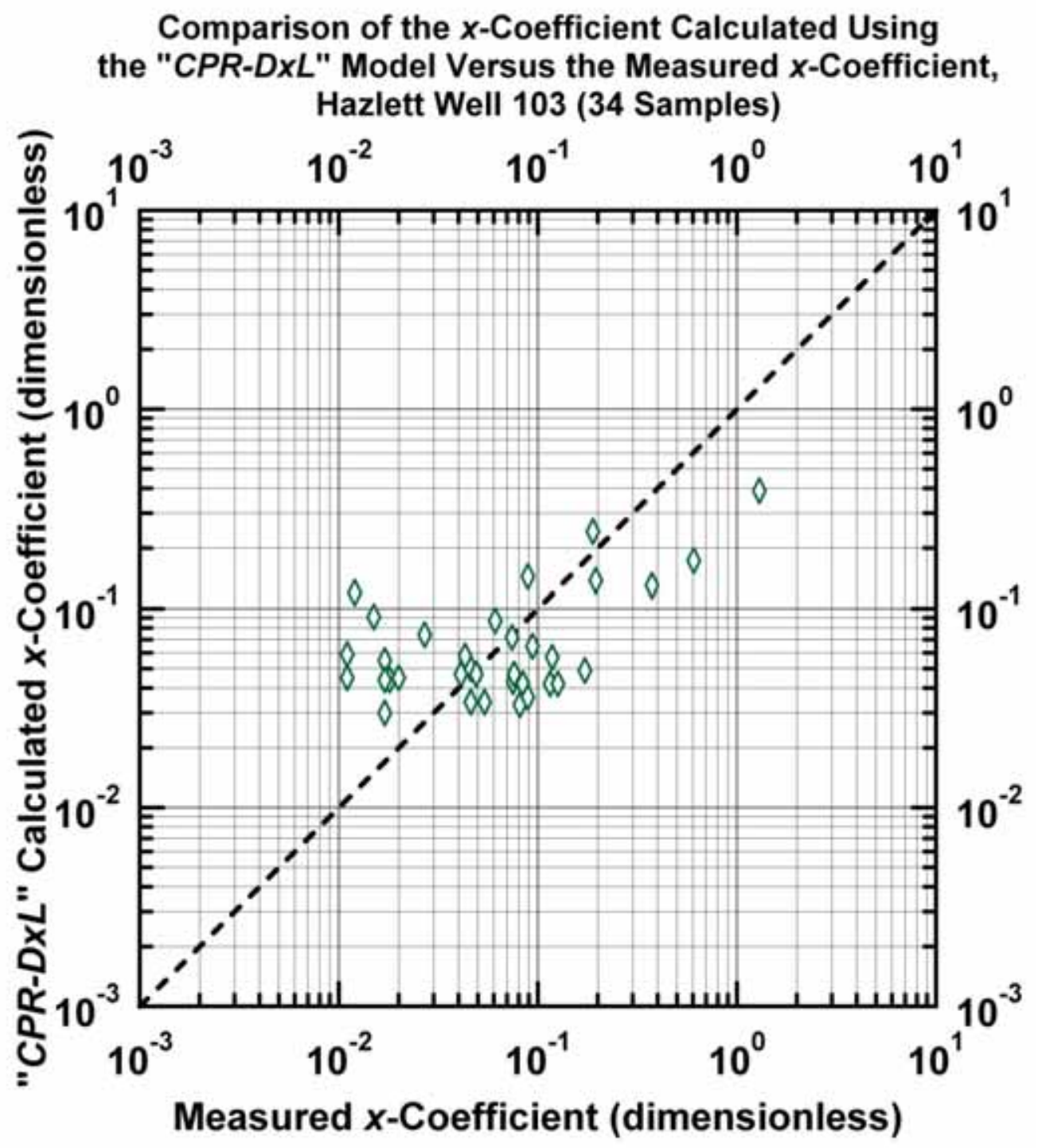

Figure F.8c - Model $C P R-D x L$ : Calculated $x$-function weight values versus measured $x$ function weight values. [Case: Hazlett Well 103] 
Comparison of Permeability (md) Calculated Using the "CPR-DXQ" Model Versus the Measured Permeability (md), Hazlett Well 103 (34 Samples)

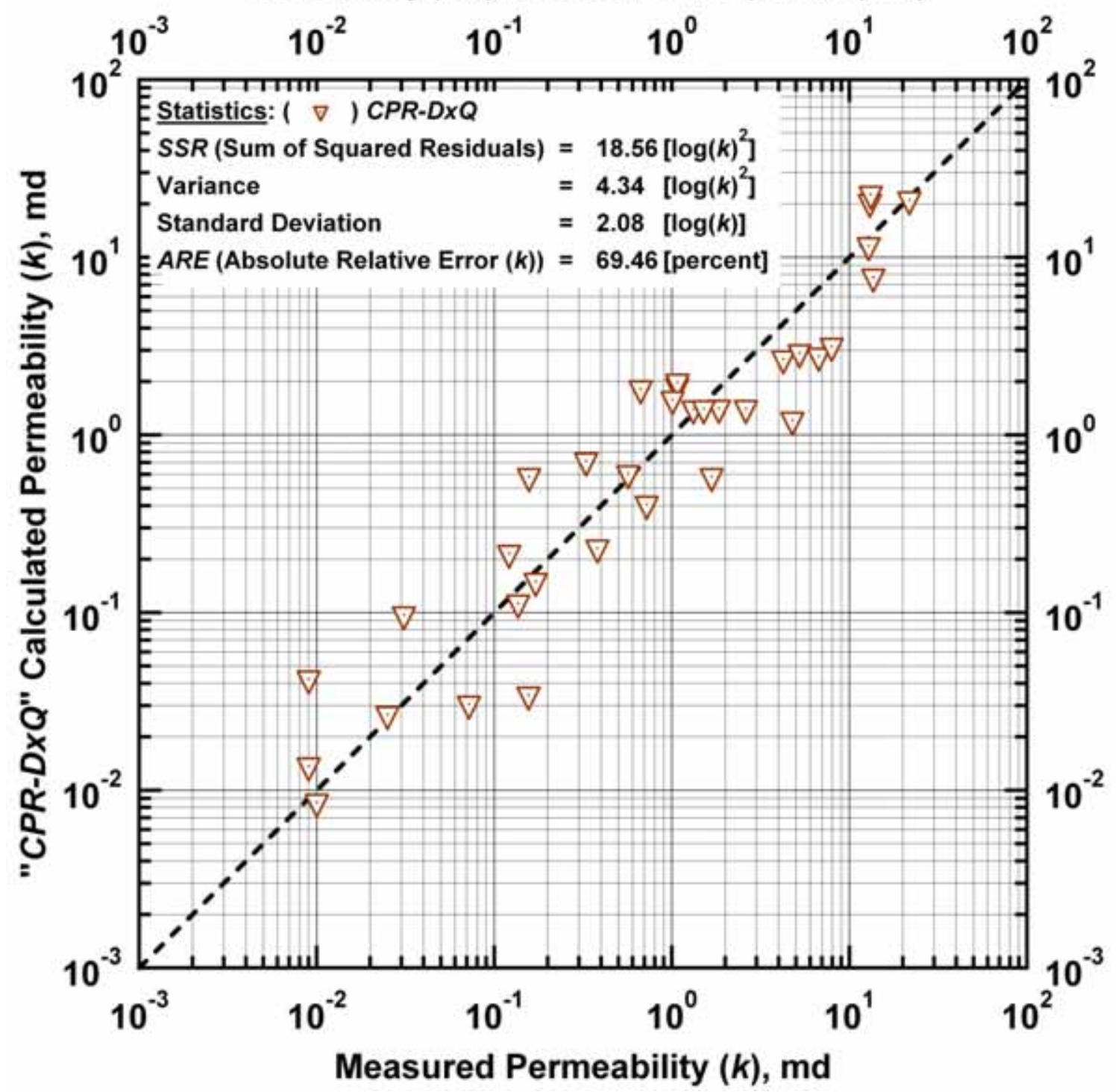

Figure F.9a - Model CPR-DxQ: Calculated versus measured permeability. [Case: Hazlett Well 103] 


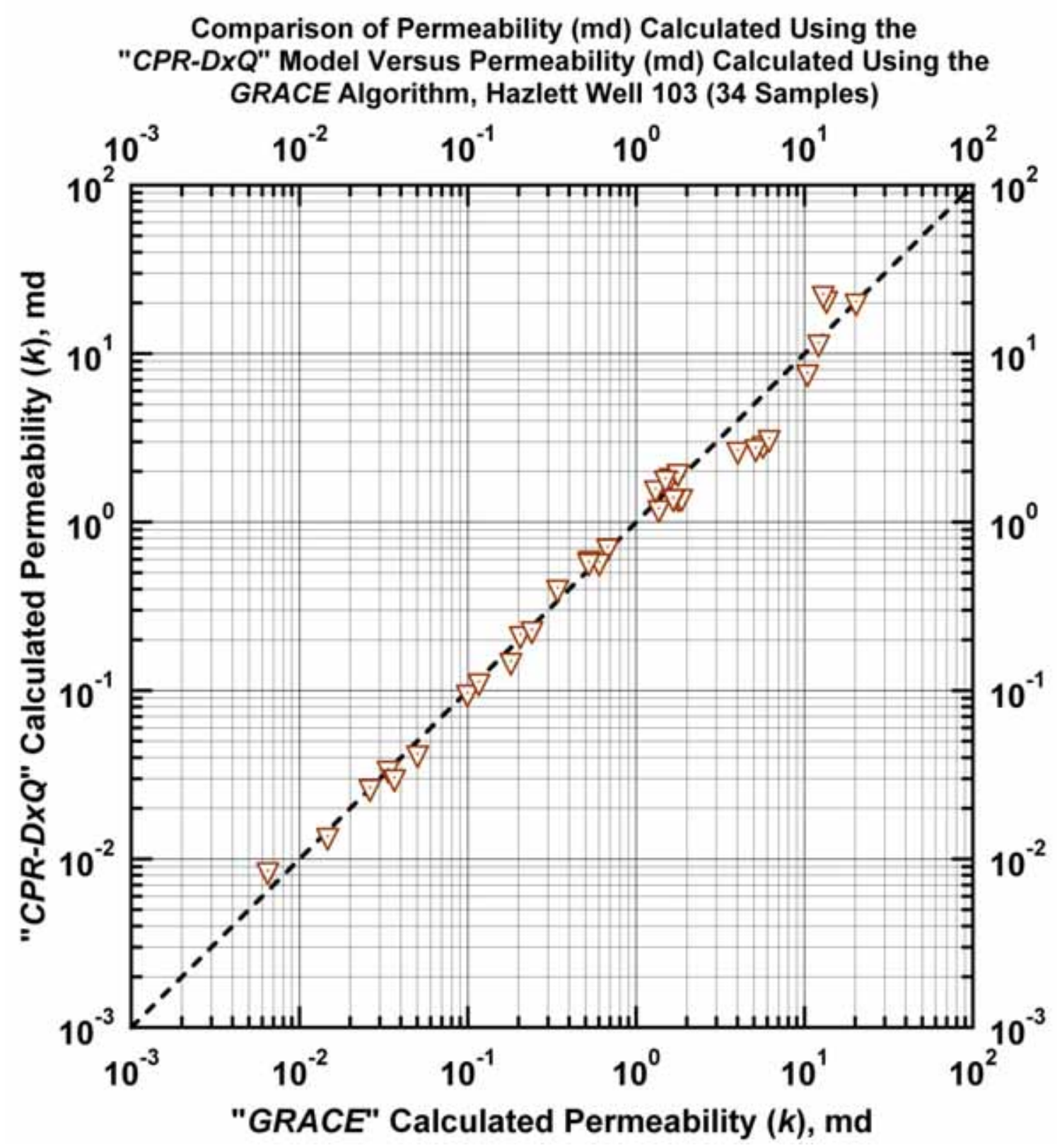

Figure F.9b - Model $C P R-D x Q$ : Permeability calculated using Model $C P R-C$ versus permeability calculated using the GRACE Algorithm. [Case: Hazlett Well 103] 


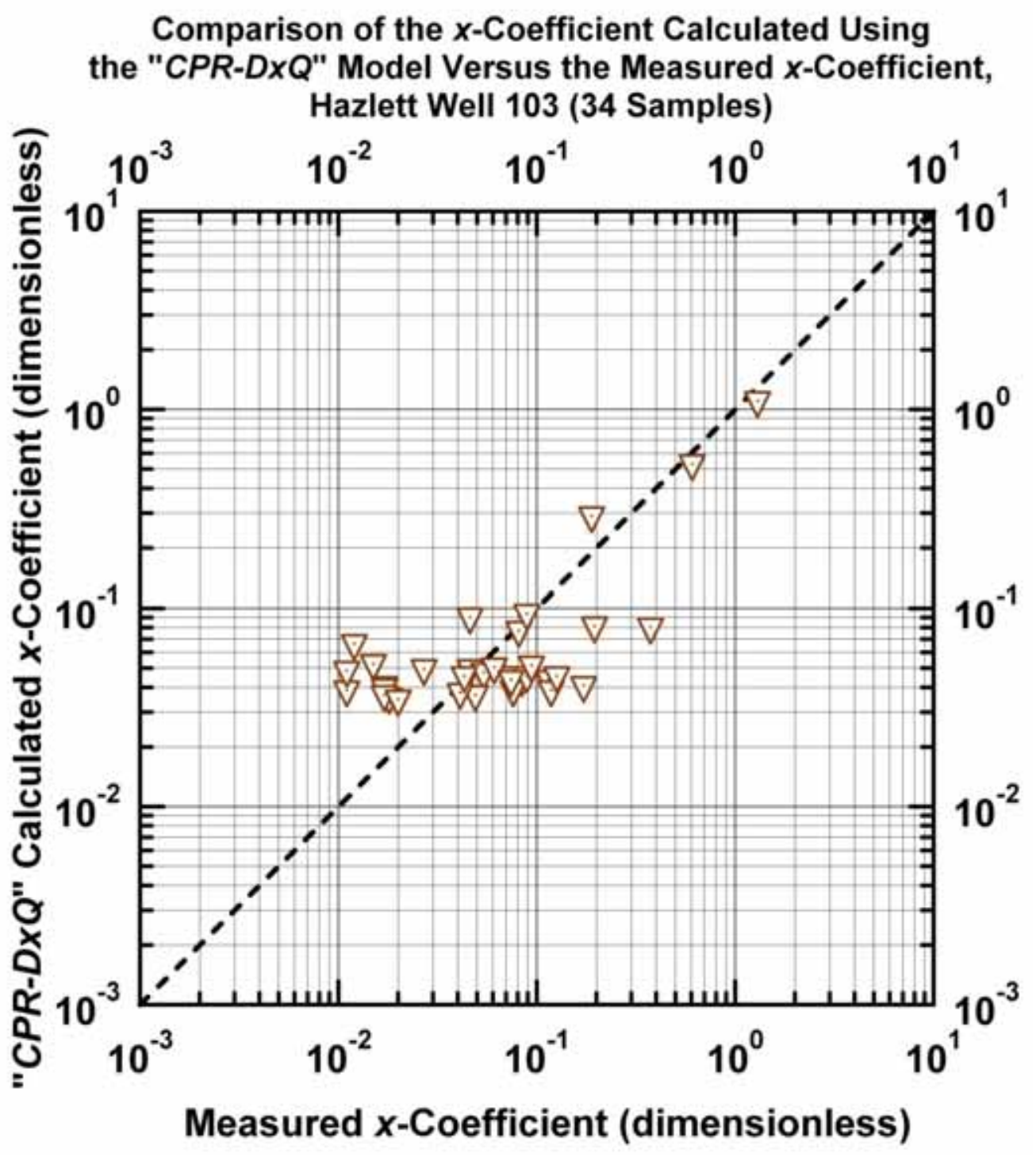

Figure F.9c - Model CPR-DxQ: Calculated $x$-function weight values versus measured $x$ function weight values. [Case: Hazlett Well 103] 
Comparison of Permeability (md) Calculated Using the "Modified Timur" Model Versus the Measured Permeability (md), Hazlett Well 103 (34 Samples)

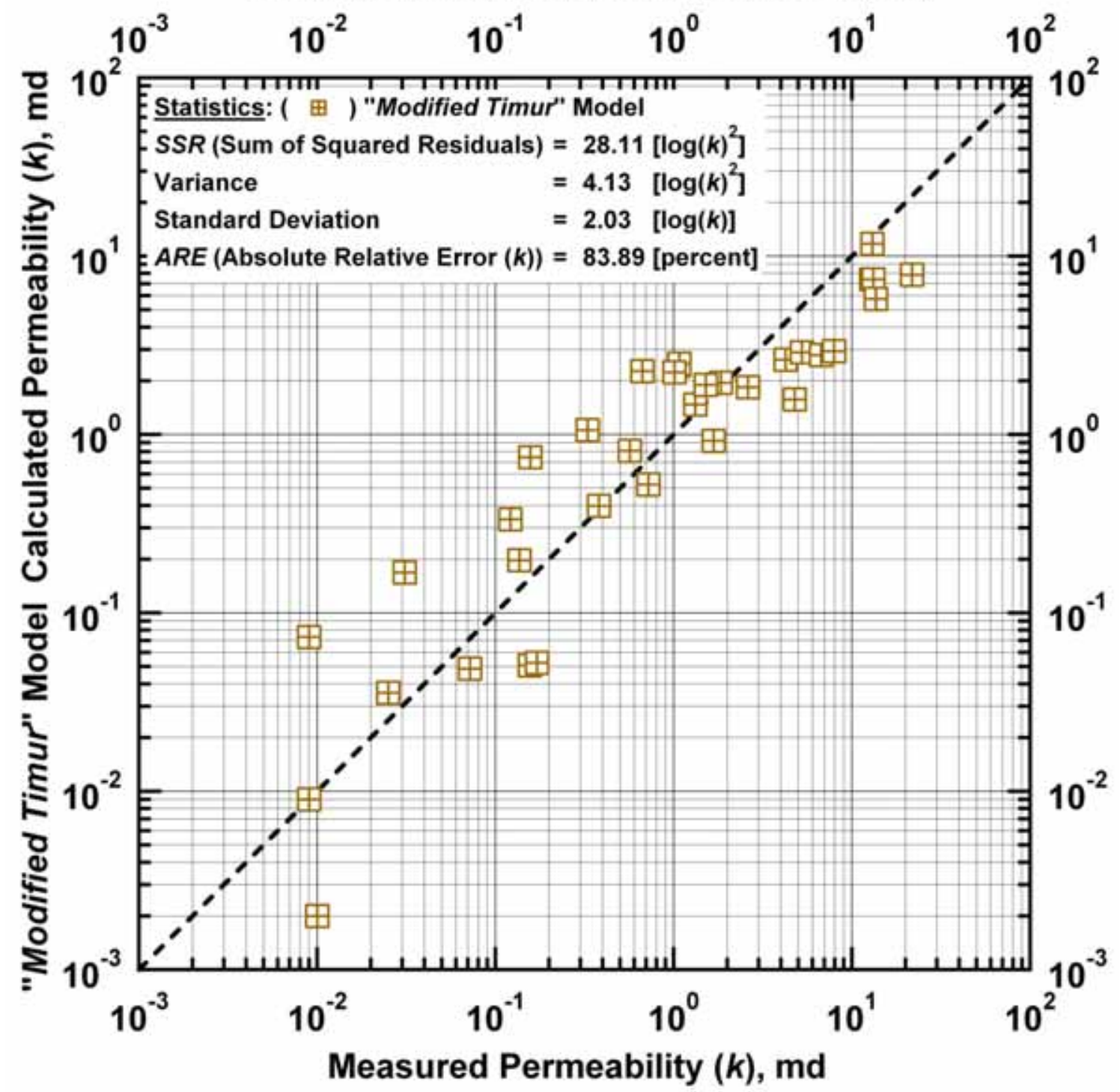

Figure F.10a - "Modified Timur Model:" Calculated versus measured permeability. [Case: Hazlett Well 103] 


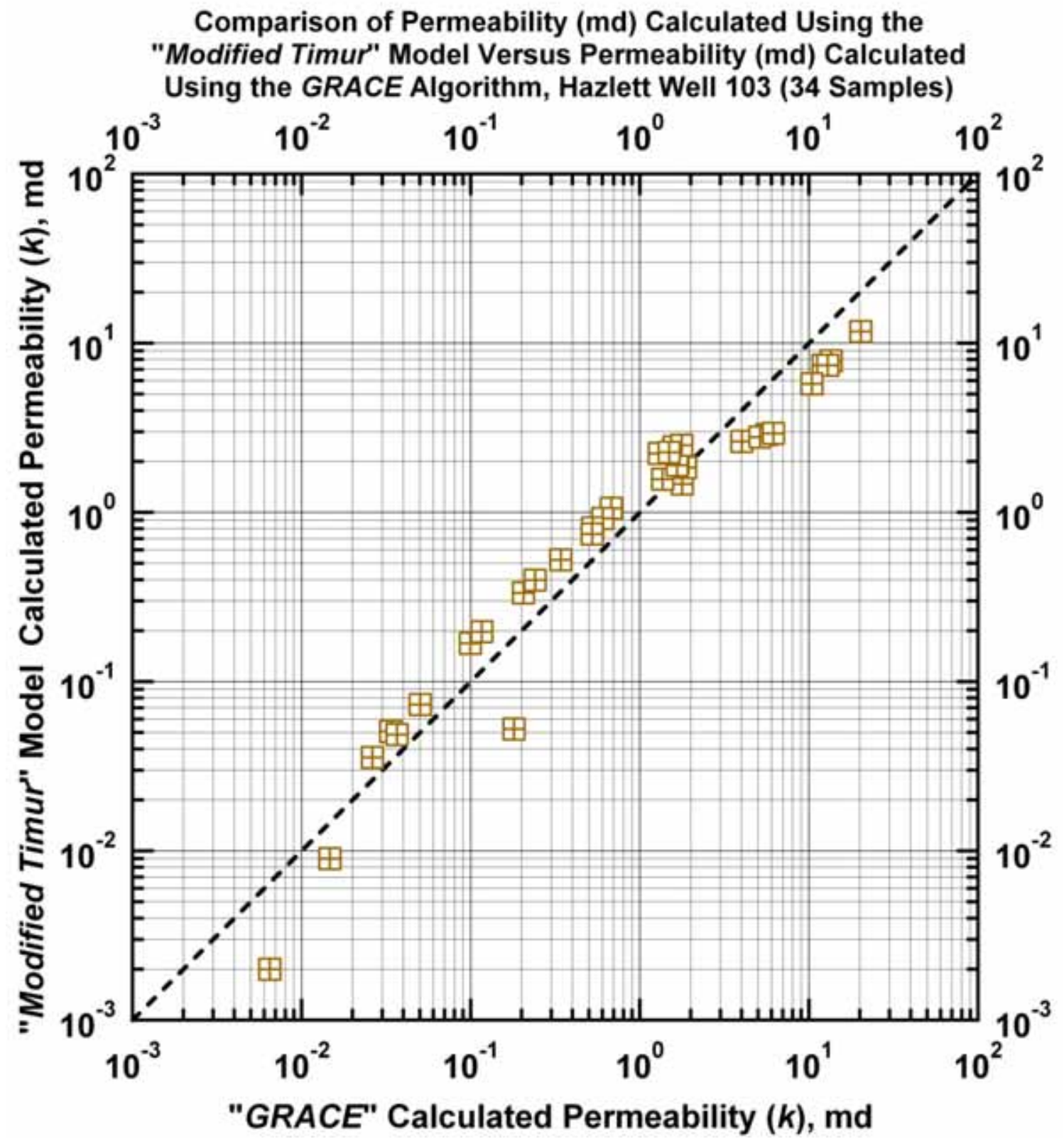

Figure F.10b - "Modified Timur Model:" Permeability calculated using the "Modified Timur Model" versus permeability calculated using the GRACE Algorithm. [Case: Hazlett Well 103] 


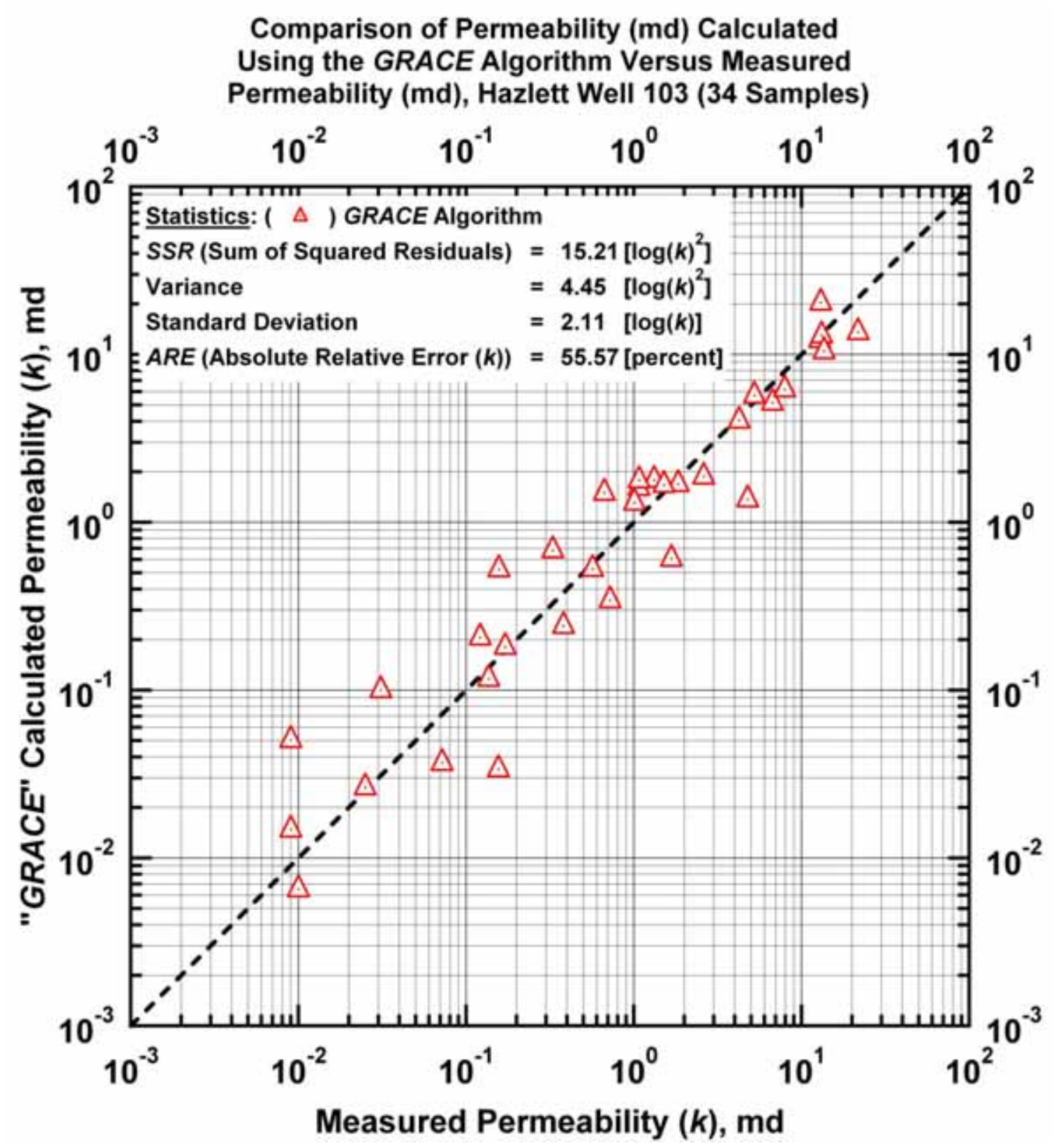

Figure F.11 - GRACE Model: Calculated versus measured permeability. [Case: Hazlett Well 103] 


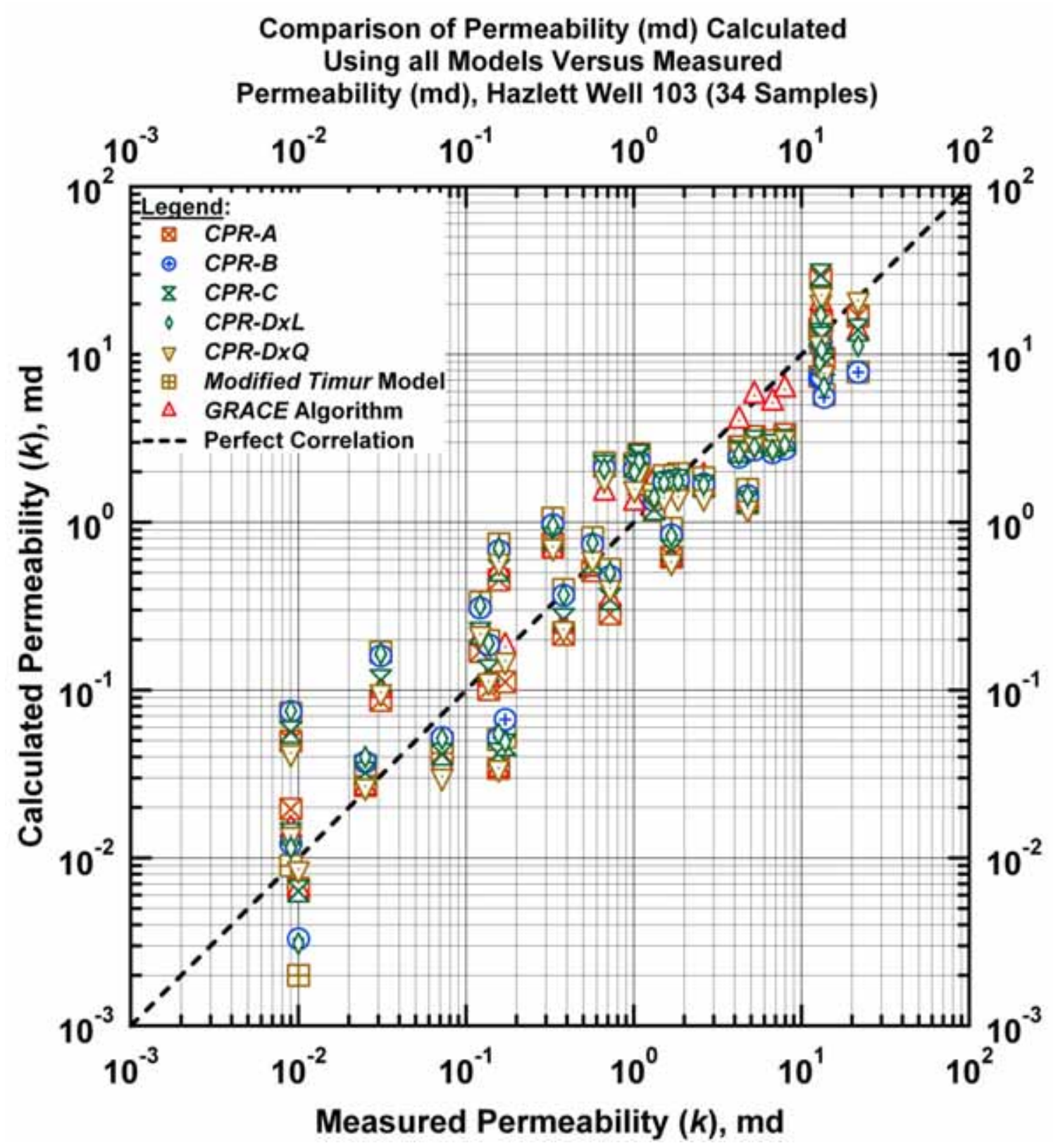

Figure F.12 - All Models: Calculated versus measured permeability. [Case: Hazlett Well 103] 


\section{APPENDIX G \\ RESULTS FOR DEVONIAN LIMESTONE \\ (TEXAS - USA)}

In this Appendix we present the "Devonian Limestone" Well, located in Texas (USA — this is a carbonate reservoir system (Ellis, 1987).

\section{Model CPR-A: (Appendix G)}

The "characteristic permeability relation" (or $C P R$ ) Model CPR-A (i.e., the modified Archie "Clean Sand" Model) is given as:

$$
k=a(\phi-c)^{b} \quad c=c_{\max } \exp \left[-c_{1} \phi^{c 2} F^{c_{3}}\right]
$$

Where a plot of $\log (k)$ versus $\log (\phi)$ is used to graphically calibrate Eq. G-1 in terms of the coefficients $a, b$, and $c_{\max }$. Fig. G.1 shows that the $C P R-A$ model performs quite well for this case. Figs. G.1a and $\mathbf{b}$ show the log-log and the semilog format of permeability versus porosity, respectively. In Fig. G.2 we provide the "error" plots for this case, where the measured and computed data are compared systematically. In Fig. G.2a we note a good correlation of the results obtained using Model CPR-A versus the measured permeability data - there is good agreement with the perfect correlation trend (i.e., the 45degree line). In Fig. G.2b we provide a comparison with the GRACE algorithm correlation (Xue et al. 1997)), and we note that the CPR-A model results correlate very well with the GRACE algorithm results for this case. Lastly, in Fig. G.2c we present the calculated and measured $c$-function — and again we note a reasonable correlation of the data about the perfect correlation trend.

\section{Model CPR-B: (Appendix G)}

The "characteristic permeability relation" (or CPR) Model CPR-B (i.e., the modified Archie "Dirty Sand" Model (power-law basis)) is given as:

$$
k=a(\phi+c)^{b} \quad c=c_{\max } \exp \left[-c_{1} \phi^{c_{2}} F^{c_{3}}\right]
$$

Where a plot of $\log (k)$ versus $\log (\phi)$ is used to graphically calibrate Eq. G-2 in terms of the coefficients $a, b$, and $c_{\max }$. The results for this case are presented in Figs. G.3 and 4. In Fig. G.3a (log-log format) we note that a very distinct "envelope" is formed by the CPR-B model, essentially all of the data on the right and left flanks are well-matched (i.e., the "dirty sand" power law relation (applied on the right portion of the data) and its correction function (applied on the left portion of the data). Similarly, in Fig. G.3b (semilog format), we observe an excellent distribution of the power law model across the body of the data. The error analyses for this case are shown in Fig. G.4. In Fig. G.4a we present the results obtained using Model $C P R-B$ versus the measured permeability data and in this case there is good agreement with the 
perfect correlation trend (i.e., the 45-degree line). We provide a comparison with the GRACE algorithm correlation in Fig. G.4b - the results using Model CPR-B and the GRACE algorithm compare very well for this case. We compare the computed and data-derived values of the $c$-function for this case in Fig. G.4c and we note a "tight" correlation. In summary, we believe that Model $C P R-B$ is a very effective correlation model for this particular data case.

\section{Model CPR-C: (Appendix G)}

The "characteristic permeability relation" (or $C P R$ ) Model CPR-C (i.e., the modified Archie "Dirty Sand" Model (exponential basis)) is given as:

$$
k=c \exp [\beta \phi] \quad c=c_{\max } \exp \left[-c_{1} \phi^{c_{2}} F^{c_{3}}\right] .
$$

Where a plot of $\log (k)$ versus $\phi$ is used to graphically calibrate Eq. G-3 in terms of the coefficients - $\beta$ and $c_{\max }$. Model $C P R-C$ is fundamentally different than Models $C P R-A$ and $C P R-B$, as the basis for Model $C P R-C$ is an exponential function, not a power law relation. In Figs. G.5 and 6 we present the results of applying Model $C P R-C$ for this case, and in general, we observe a very consistent performance of Model $C P R-C$. Fig. G.5b illustrates the excellent conformance of Model $C P R-C$ for this case - again recalling that this model has an exponential relationship with porosity as its basis.

In Figs. G.6a, G.6b, and G.6c we review the error analyses plots for this case, and we note good performance (visually) in terms of the correlation of the results using Model CPR-C. In this case, the $c$ function (in Eq. G-3) is not a correction term, but rather the instantaneous intercept for the exponential basis. The correlation of the $c$-function (see Fig. G.6c) appears to be consistent - but scatter and a slight off-center trend is evident. We believe that the $C P R-C$ model has performed well for this case, and the Fig. G.6 confirms the value of this model as a "characteristic relation" for permeability.

\section{Model CPR-D: (Appendix G)}

The "characteristic permeability relation" (or $C P R$ ) Model CPR-D (i.e., the weighted power law-exponential model) is given in its fundamental form as:

$$
\left.k=x a \phi^{b}+(1-x) \alpha \exp [\beta \phi] \quad[0 \leq x \leq 1]\right]
$$

For Eq. G-4 there are two proposed models for the $x$-function (i.e., the weighting function) - these are:

$$
\begin{aligned}
& \left.x=\exp \left[-\ln \left(x_{0}\right)+x_{1} \ln (\phi)+x_{2} \ln (F)+x_{3} \ln (\phi) \ln (F)\right] \text { (Model CPR-D } x L\right) . \\
& x=\exp \left[\begin{array}{l}
-\ln \left(x_{0}\right)+x_{1} \ln (\phi)+x_{2} \ln (F)+x_{3} \ln (\phi) \ln (F) \\
+x_{4} \ln (\phi)^{2}+x_{5} \ln (F)^{2}+x_{6} \ln (\phi)^{2} \ln (F) \\
+x_{7} \ln (\phi) \ln (F)^{2}+x_{8} \ln (\phi)^{2} \ln (F)^{2}
\end{array}\right] \text { (Model CPR-DxL).... }
\end{aligned}
$$

As noted in Eq. G-4b, Model CPR-DxL utilizes a logarithmic linear model for the weights ( $x$-values) and as seen in Eq. G-4c, Model CPR-DxQ utilizes a logarithmic quadratic model for the weights ( $x$-values). While these models (Eqs. G-4b and G-4c) are empirical, we have found generally good application of 
these relations for all cases. In this case, the base results using Models CPR-DxL and CPR-DxQ are presented in Fig. G.7 - where the power law and exponential basis functions are fitted to the appropriate portion of the data. The power law equation represents the "Archie Clean Sand" trend and is fitted to the leftmost data as shown in Fig. G.7a and in contrast, the exponential equation is thought to represent the "Archie Dirty Sand" trend and the exponential is fitted to the far rightmost portion of the data (see Fig. G.7b).

In Figs. G.8 and 9 we present the error analyses for this case, where we have employed the weighting functions (Eqs. G-4b and G-4c) as appropriate. In Fig. G.8a we find a good correlation of permeability. The comparison of the Model CPR-DxL results are compared to the results obtained from the GRACE algorithm in Fig. G.8b, we note that the very good results obtained using Model CPR-DxL. The computed weight function ( $x$-values) shown for the CPR-DxL model in Fig. G.8c does exhibit more scatter than expected, but the trend is (relatively) centered about the perfect correlation line.

The results obtained using Model $C P R-D x Q$ are presented in Fig. G.9, and we note substantially improved behavior over that of Model $C P R-D x L$. In particular, the base correlation of computed and measured permeabilities (Fig. G.9a) for Model CPR-DxQ does indicate a slightly better correlation than that of Model $C P R-D x L$. Also, the comparison of Model $C P R-D x Q$ with the results from the GRACE algorithm (Fig. G.9b) exhibits better agreement than the results of Model CPR-DxL. Finally, the correlation of the $x$-function (Fig. G.9c) for Model CPR-DxQ is better than the correlation for Model CPR-DxL. These comparisons suggest that Model CPR-DxQ (i.e., the combination of Eqs. G-4a and G-4c) has provided a better correlation of data, for this case, than Model CPR-DxL.

\section{"Modified Timur Model": (Appendix G)}

The "Modified Timur Model" is given as:

$$
k_{\text {Timur }}=a \phi^{b} F^{c}
$$

In Fig. G.10 we present the results of the "Modified Timur Model" (Eq. G-5) which is essentially just a generalized power-law function relation in terms of $k, \phi$, and F. Figs. G.10a and b, provide the "Modified Timur Model" results of the data for this case.

\section{GRACE Algorithm: (Appendix G)}

In Fig. G.11 we present the results predicted by the GRACE algorithm, which is a non-parametric regression approach (see Xue et al. (1997) for details). In theory, the GRACE algorithm should provide the most unbiased correlation of the data - i.e., the GRACE algorithm is designed not to "fit the errors" as other regression approaches may. It is our contention that the GRACE algorithm is the statistical standard - and any algorithm/approach/model which achieves better regression statistics than the GRACE algorithm is actually "fitting the errors" in the data. None of the cases have significantly better regression

statistics than the GRACE algorithm - hence, none of the cases are "over-fitted" in a statistical sense. 


\section{Comparison of All Models: (Appendix G)}

Our final plot, Fig. G.12, illustrates all of the models on a single plot of calculated versus measured permeability. We also present a table of all statistical results in Table G.1.

Table G.1 Statistical Results for all Models (Devonian Limestone Case, $n=58$ ).

\begin{tabular}{|c|c|c|c|c|}
\hline Model & $\begin{array}{c}\text { Sum of } \\
\text { Squared } \\
\text { Residuals } \\
{\left[\log (k)^{2}\right]}\end{array}$ & $\begin{array}{l}\text { Variance } \\
{\left[\log (k)^{2}\right]}\end{array}$ & $\begin{array}{c}\text { Standard } \\
\text { Deviation } \\
{[\log (k)]}\end{array}$ & $\begin{array}{c}\text { Absolute } \\
\text { Relative } \\
\text { Error } \\
\text { [percent] }\end{array}$ \\
\hline$C P R-A$ & 8.24 & 1.86 & 1.36 & 29.92 \\
\hline$C P R-B$ & 9.45 & 1.83 & 1.35 & 32.85 \\
\hline$C P R-C$ & 8.91 & 1.65 & 1.29 & 30.87 \\
\hline$C P R-D x L$ & 9.03 & 1.80 & 1.34 & 33.01 \\
\hline$C P R-D x Q$ & 8.99 & 1.87 & 1.37 & 33.21 \\
\hline Modified Timur & 8.50 & 1.82 & 1.35 & 31.35 \\
\hline GRACE Algorithm & 8.25 & 1.85 & 1.36 & 29.12 \\
\hline
\end{tabular}

The coefficients for the models used in this case are provided in Table G.2.

Table G.2a - Model Coefficients (Devonian Limestone Case, $n=58$ ).

\begin{tabular}{|c|c|c|c|c|c|c|}
\hline Model & $a$ or $\alpha$ & $b$ or $\beta$ & $c$ or $C_{\max }$ & $c_{1}$ & $C_{2}$ & $c_{3}$ \\
\hline$C P R-A$ & $4.00 \times 10^{3}$ & 4.00 & 0.110 & 0.15 & -2.79 & -0.89 \\
\hline$C P R-B$ & $1.02 \times 10^{5}$ & 8.00 & 0.110 & 10.79 & 3.61 & 0.95 \\
\hline$C P R-C$ & $1.00 \times 10^{-2}$ & 18.00 & 0.080 & $2.85 \times 10^{-2}$ & 1.33 & 1.43 \\
\hline Modified Timur & $1.10 \times 10^{3}$ & 1.55 & -1.185 & - & - & - \\
\hline
\end{tabular}

Table G.2b - Model Coefficients (Devonian Limestone Case, $n=58$ ).

\begin{tabular}{|c|c|c|c|c|c|c|c|c|c|}
\hline & \multicolumn{2}{|c|}{ Model } & $a$ & $b$ & $\alpha$ & $\beta$ & \multirow[b]{4}{*}{$c_{6}$} & \multirow[b]{4}{*}{$c_{7}$} & \multirow[b]{4}{*}{$C_{8}$} \\
\hline & & & $4.00 \times 10^{3}$ & 4.00 & $1.00 \times 10^{-2}$ & 18.00 & & & \\
\hline & & & $4.00 \times 10^{3}$ & 4.00 & $1.00 \times 10^{-2}$ & 18.00 & & & \\
\hline Model & $C_{0}$ & $c_{1}$ & $c_{2}$ & $c_{3}$ & $C_{4}$ & $C_{5}$ & & & \\
\hline$C P R-D x L$ & 61.66 & -5.60 & -1.12 & -0.50 & - & - & - & - & - \\
\hline$C P R-D x Q$ & $6.28 \times 10^{5}$ & -6.83 & -5.88 & 3.98 & 1.02 & -0.28 & 0.38 & 0.11 & 0.07 \\
\hline
\end{tabular}

\section{Nomenclature: (Appendix G)}

$F=$ Archie Formation Factor (dimensionless)

$k=$ Formation Permeability, md (or any consistent units)

$\phi \quad=$ Porosity, fraction

\section{References: (Appendix G)}

Ellis, K.W.: "Extended Correlations of Porosity, Permeability, and Formation Resistivity Factor," MS thesis, Texas A\&M U., College Station, TX (1987).

Xue, G., Datta-Gupta, A., Valko, P., and Blasingame, T.A.: "Optimal Transformations for Multiple Regression: Application to Permeability Estimation from Well Logs," SPEFE (June 1997), 85-93. 


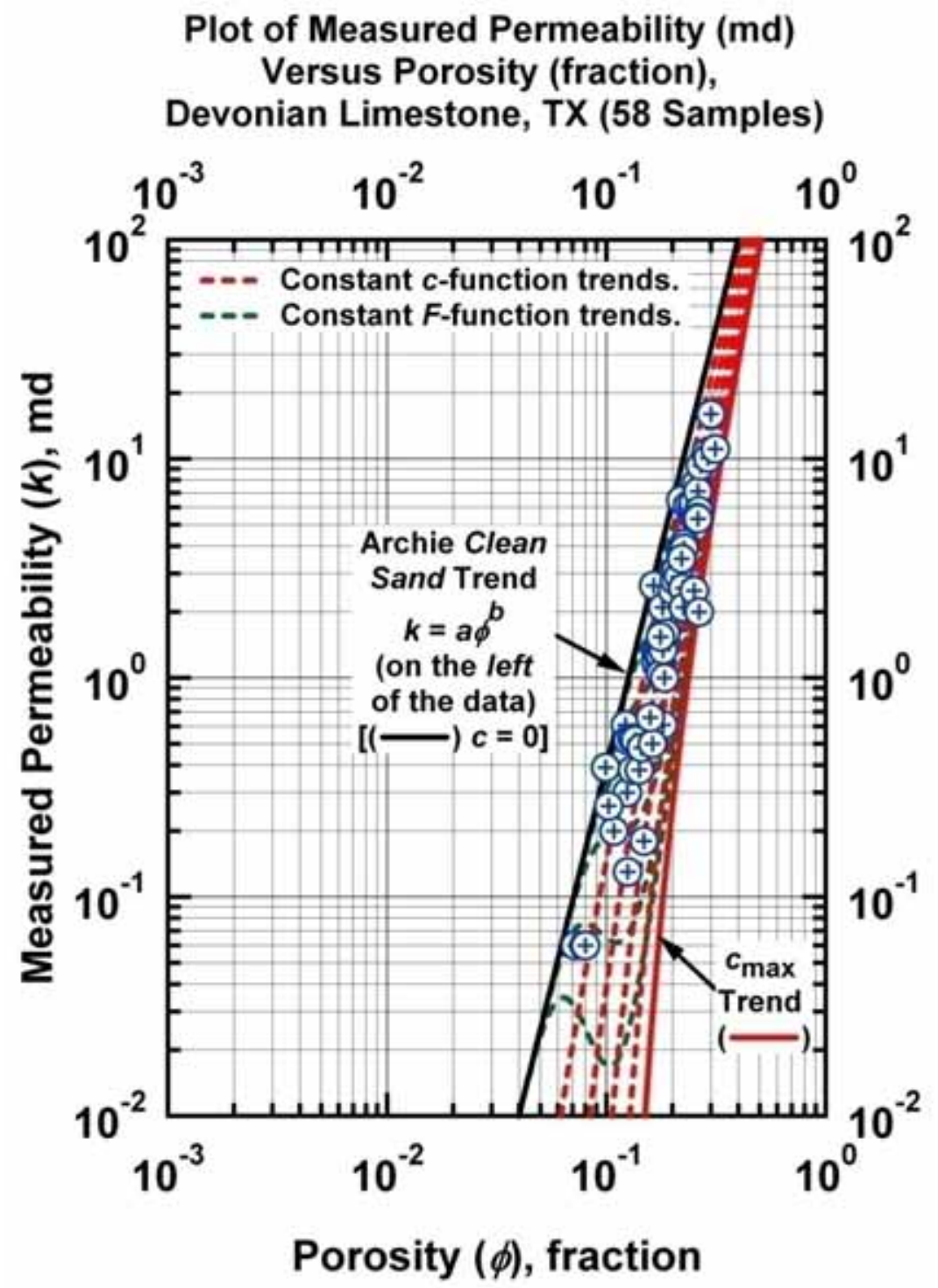

Figure G.1a - Model CPR-A: $k=a(\phi-c)^{b}\left(c=c_{\max } \exp \left[-c_{1} \phi^{c_{2}} F^{c_{3}}\right]\right)$ - "Clean Sand" Plot (log-log format) - Archie "Clean Sand" trend is given by the straight-line trend at the far left of the data (power law model). [Case: Devonian Limestone TX (USA)] 


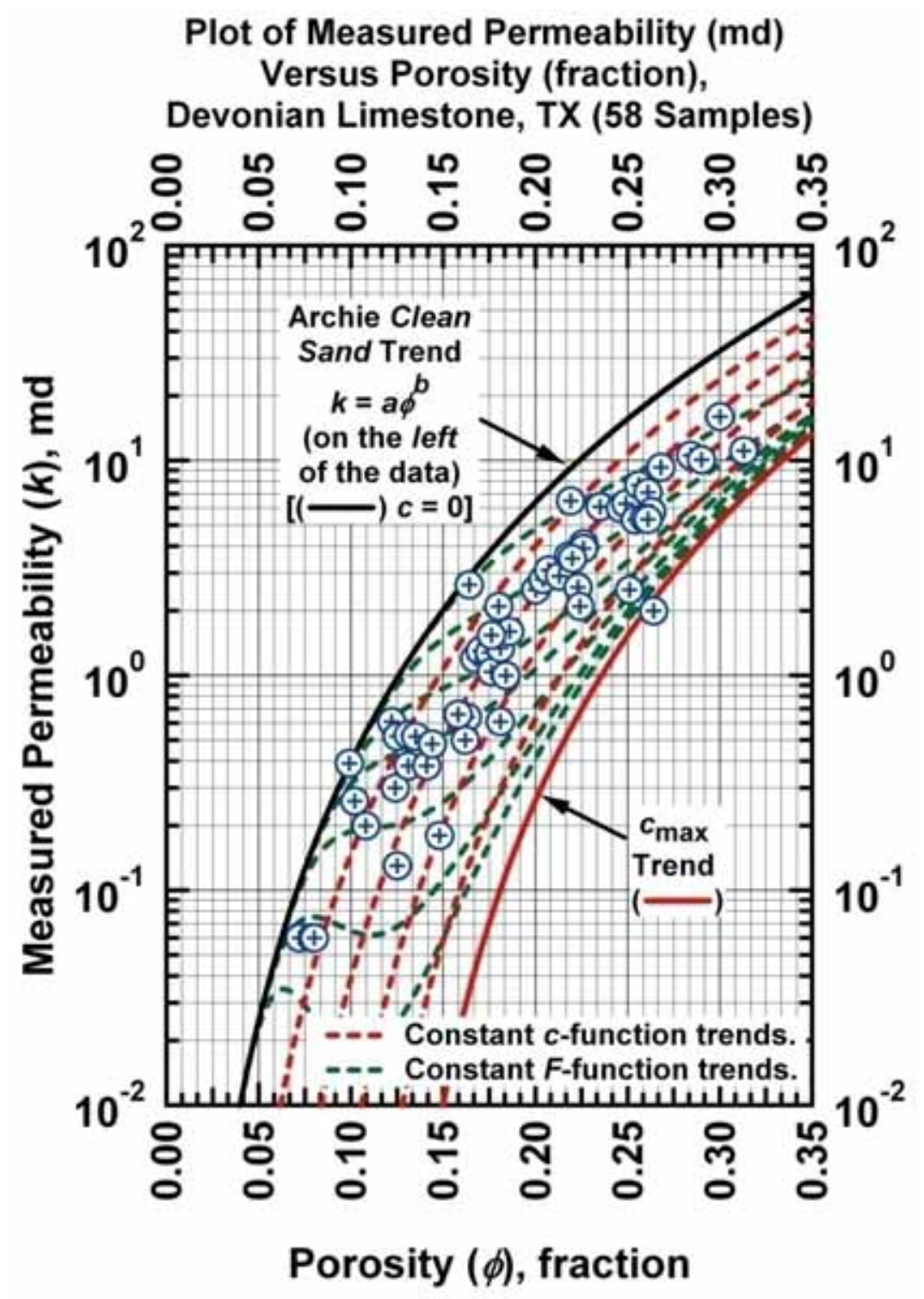

Figure G.1b - Model $C P R-A: \quad k=a(\phi-c)^{b}\left(c=c_{\max } \exp \left[-c_{1} \phi^{c_{2}} F^{c_{3}}\right]\right)$ — "Dirty Sand" Plot (semilog format). Archie "Clean Sand" trend is given by the curved trend at the far left of the data (power law model). [Case: Devonian Limestone TX (USA)] 


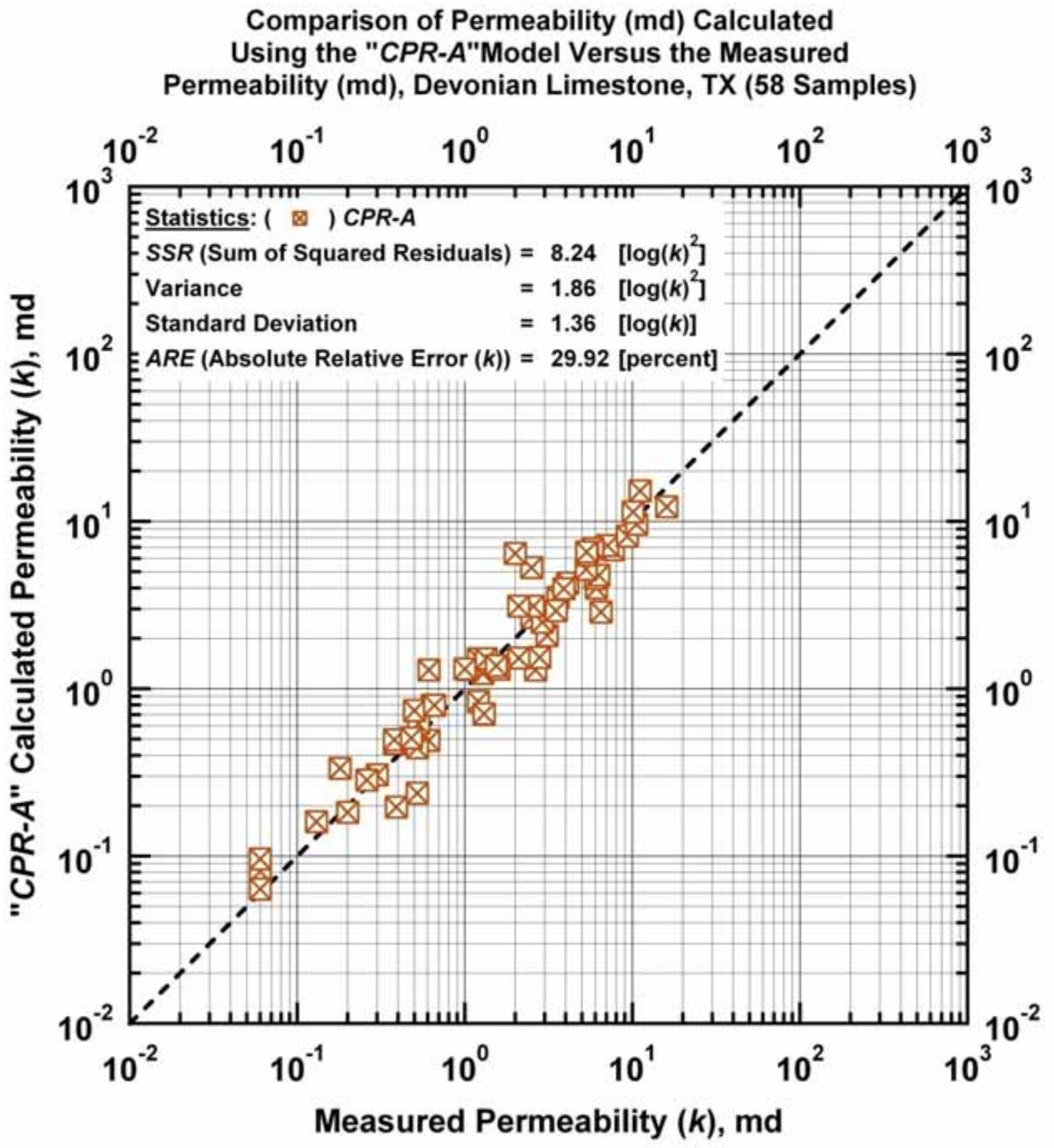

Figure G.2a - Model CPR-A: calculated versus measured permeability. [Case: Devonian Limestone TX (USA)] 


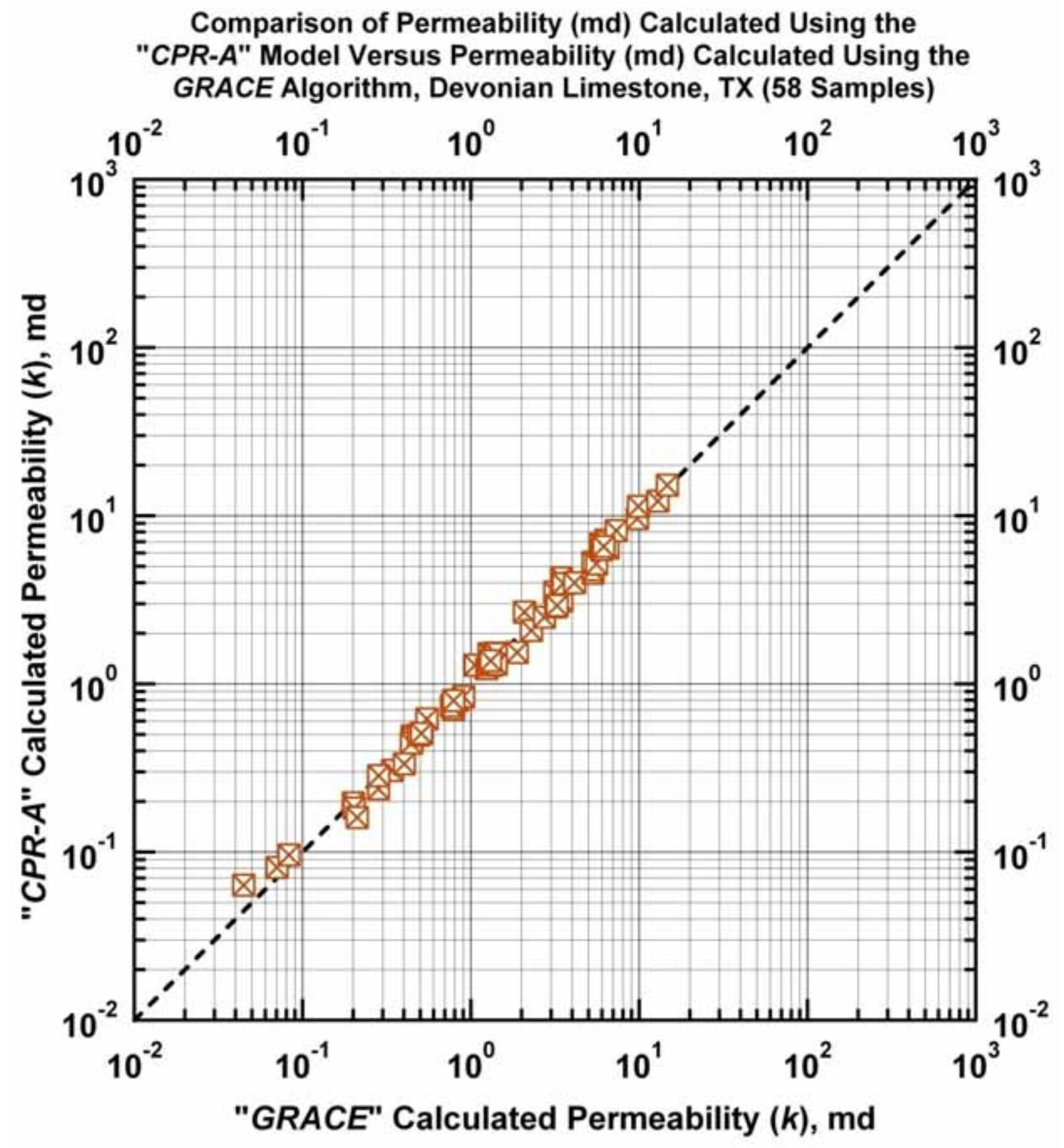

Figure G.2b - Model CPR-A: Permeability calculated using Model CPR-A versus permeability calculated using the GRACE Algorithm. [Case: Devonian Limestone TX (USA)] 


\section{Comparison of the $c$-Coefficient Calculated Using} the "CPR-A" Model Versus the Measured $c$-Coefficient, Devonian Limestone, TX (58 Samples)

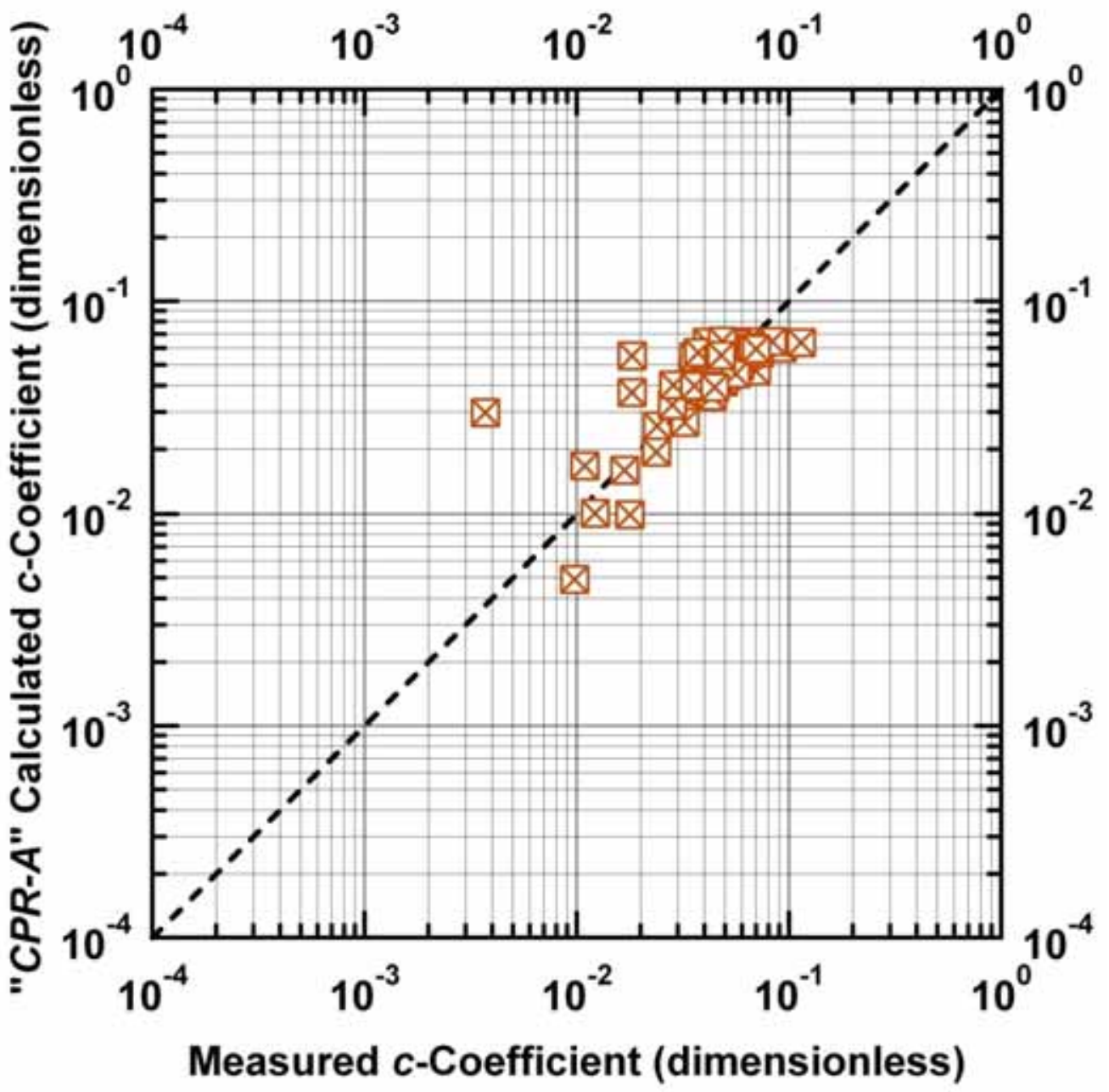

Figure G.2c - Model CPR-A: Calculated $c$-function values versus measured $c$-function values. [Case: Devonian Limestone TX (USA)] 


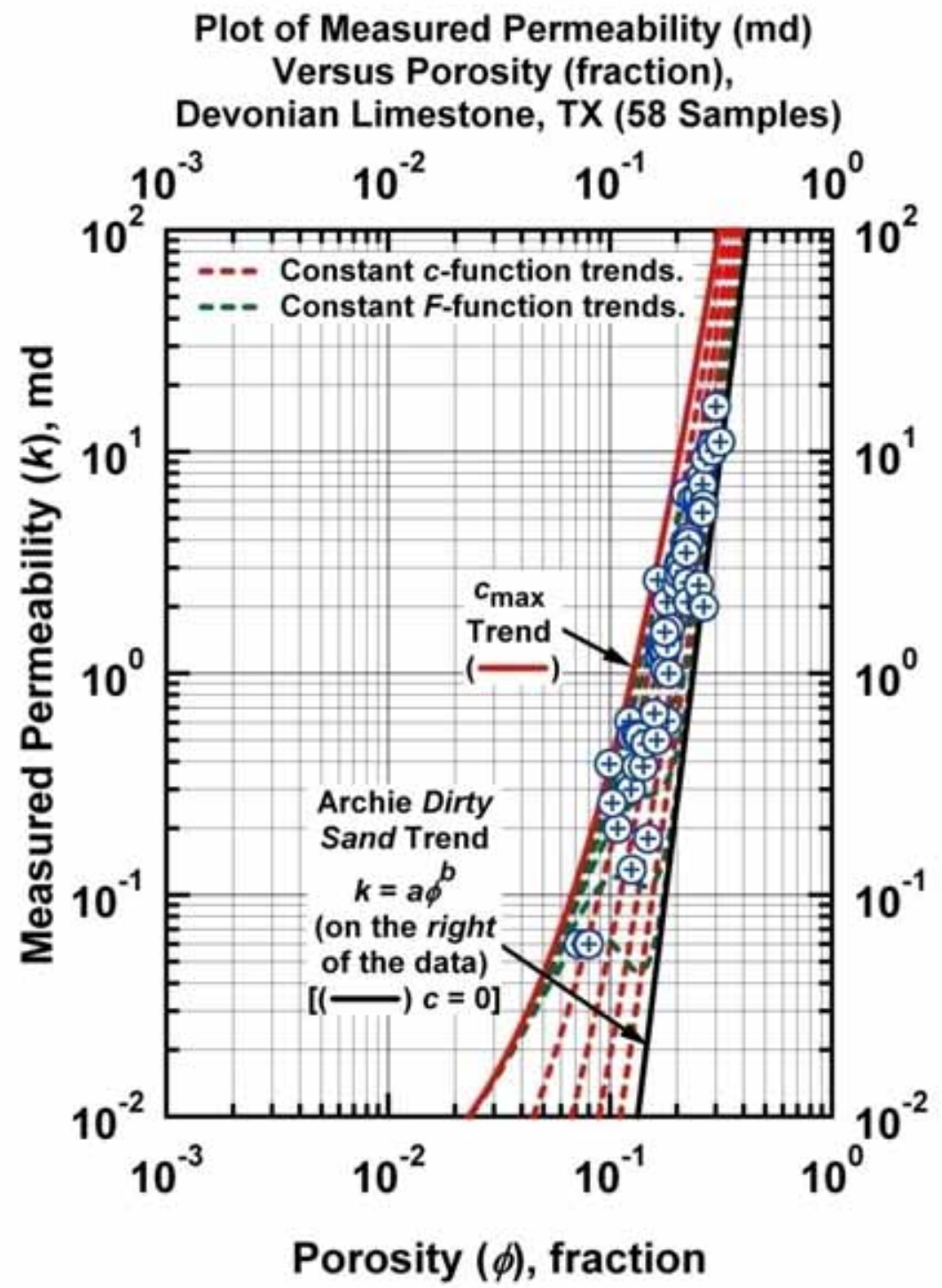

Figure G.3a - Model $C P R-B: \quad k=a(\phi+c)^{b} \quad c=c_{\max } \exp \left[-c_{1} \phi^{c^{2}} F^{c_{3}}\right]$ - "Clean Sand" Plot (log-log format) - Archie "Dirty Sand" trend is given by the straight-line trend at the far right of the data (power law model). [Case: Devonian Limestone TX (USA)] 


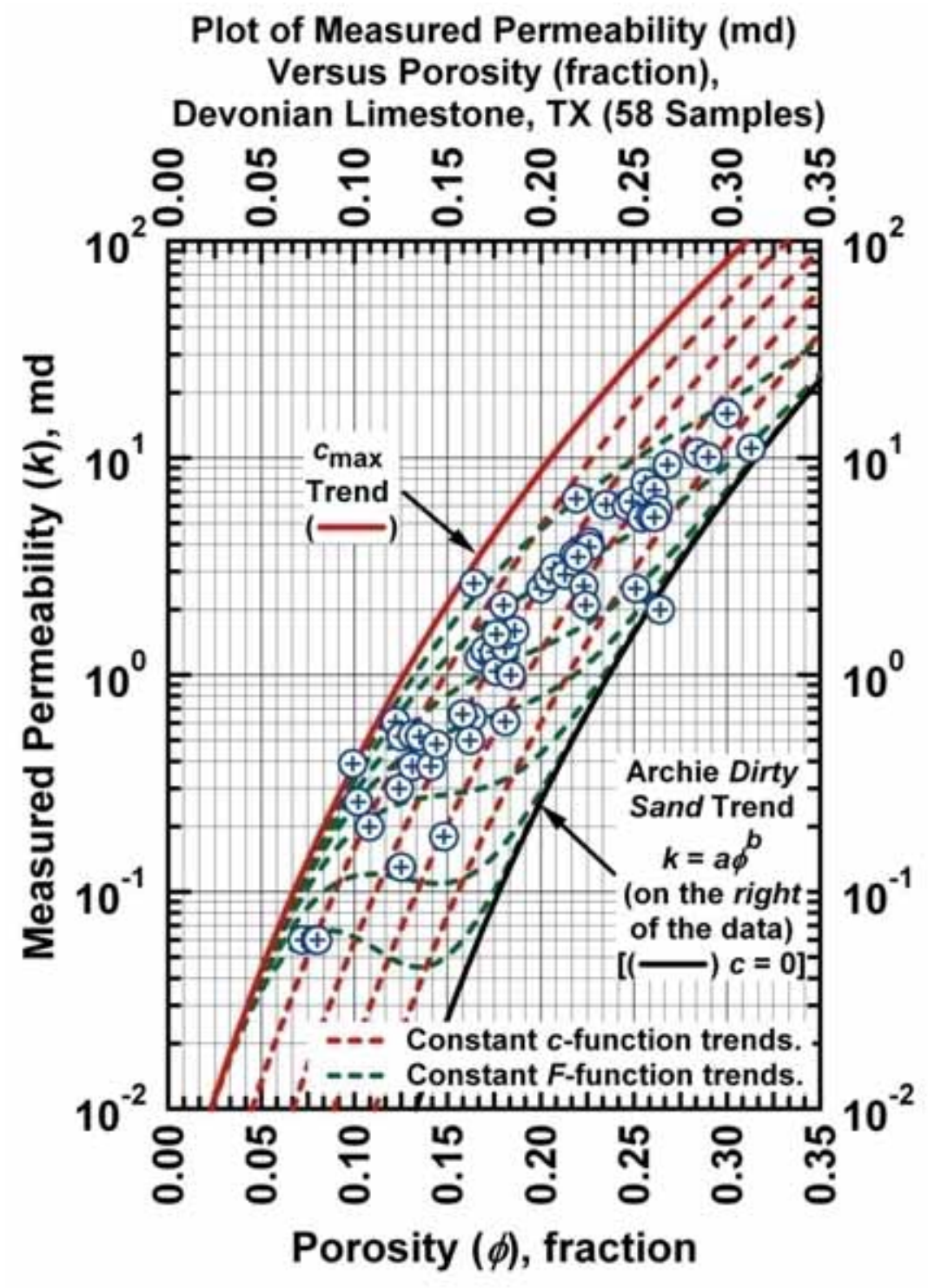

Figure G.3b - Model $C P R-B: k=a(\phi+c)^{b} \quad c=c_{\max } \exp \left[-c_{1} \phi^{c 2} F^{c 3}\right]$ — "Dirty Sand" Plot (semilog format) - Archie "Dirty Sand" trend is given by the curved trend at the far right of the data (power law model). [Case: Devonian Limestone TX (USA)] 


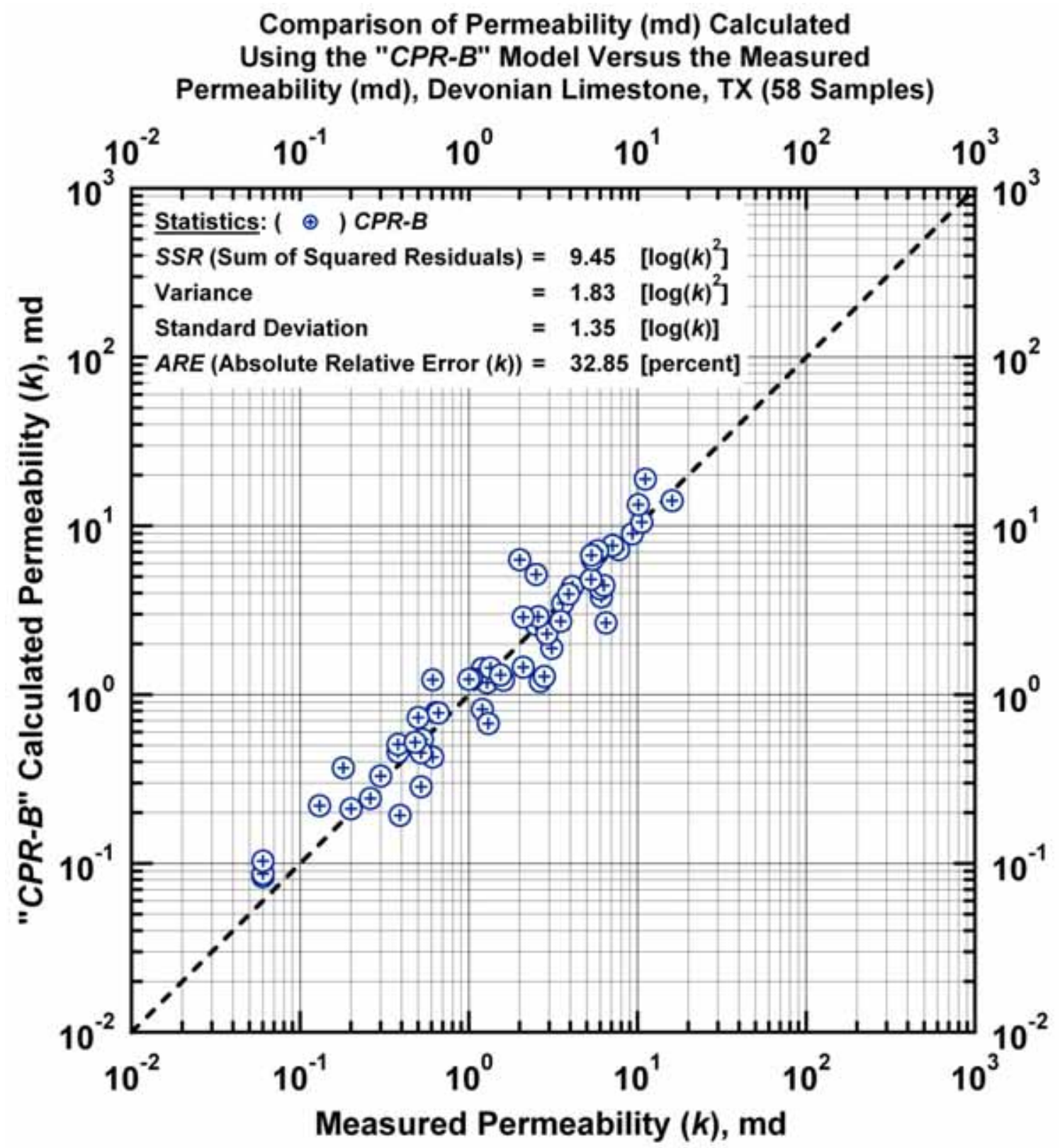

Figure G.4a - Model CPR-B: calculated versus measured permeability. [Case: Devonian Limestone TX (USA)] 


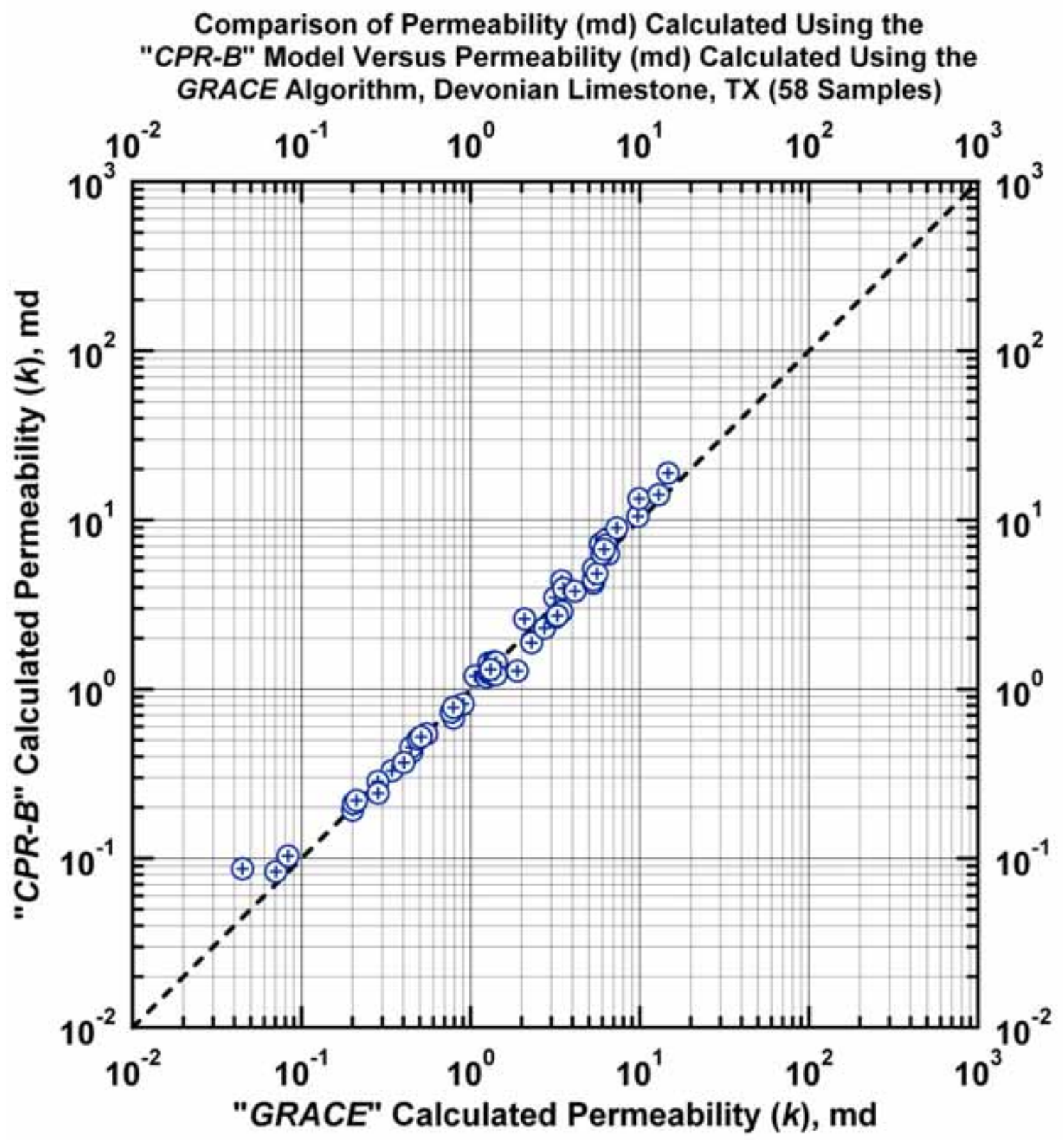

Figure G.4b - Model CPR-B: Permeability calculated using Model $C P R$ - $B$ versus permeability calculated using the GRACE Algorithm. [Case: Devonian Limestone TX (USA)] 


\section{Comparison of the $c$-Coefficient Calculated Using} the "CPR-B" Model Versus the Measured $c$-Coefficient, Devonian Limestone, TX (58 Samples)

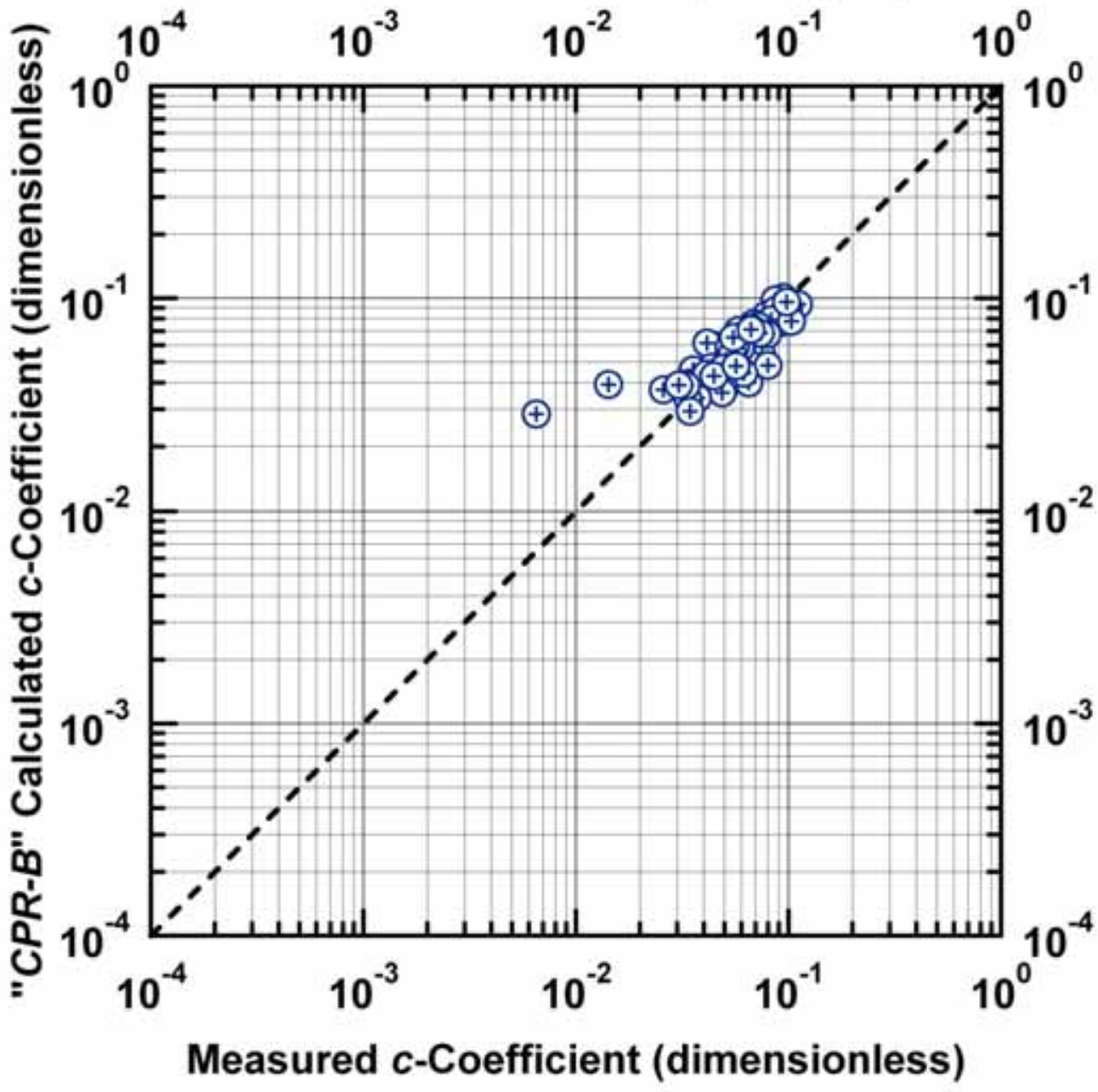

Figure G.4c - Model $C P R$ - $B$ : Calculated $c$-function values versus measured $c$-function values. [Case: Devonian Limestone TX (USA)] 


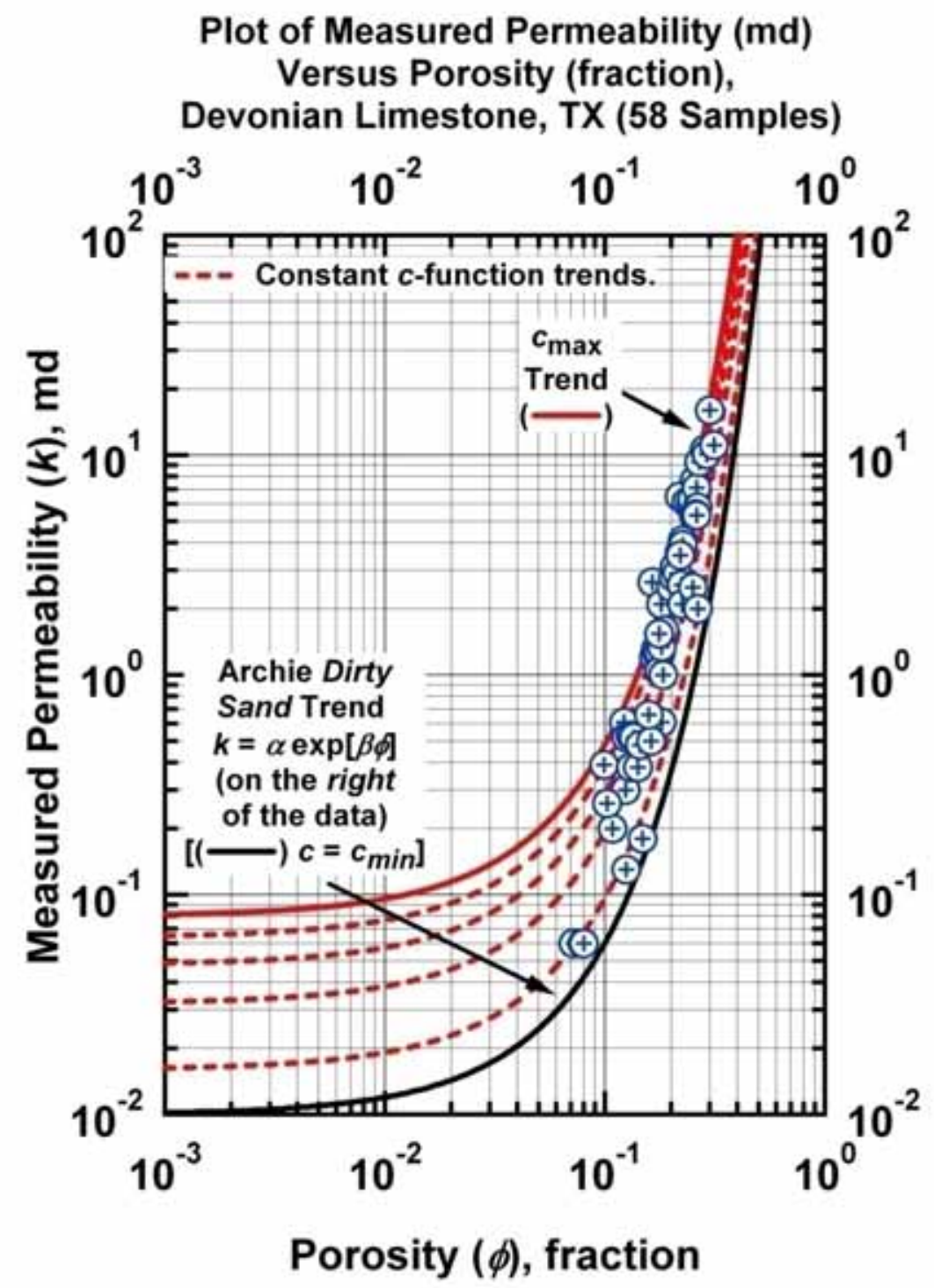

Figure G.5a - Model $C P R-C: \quad k=c \exp [\beta \phi] \quad c=c_{\max } \exp \left[-c_{1} \phi^{c_{2}} F^{c_{3}}\right]$ — "Clean Sand" Plot (log-log format) - Archie "Dirty Sand" trend is given by the curved trend at the far right of the data (exponential model). [Case: Devonian Limestone TX (USA)] 


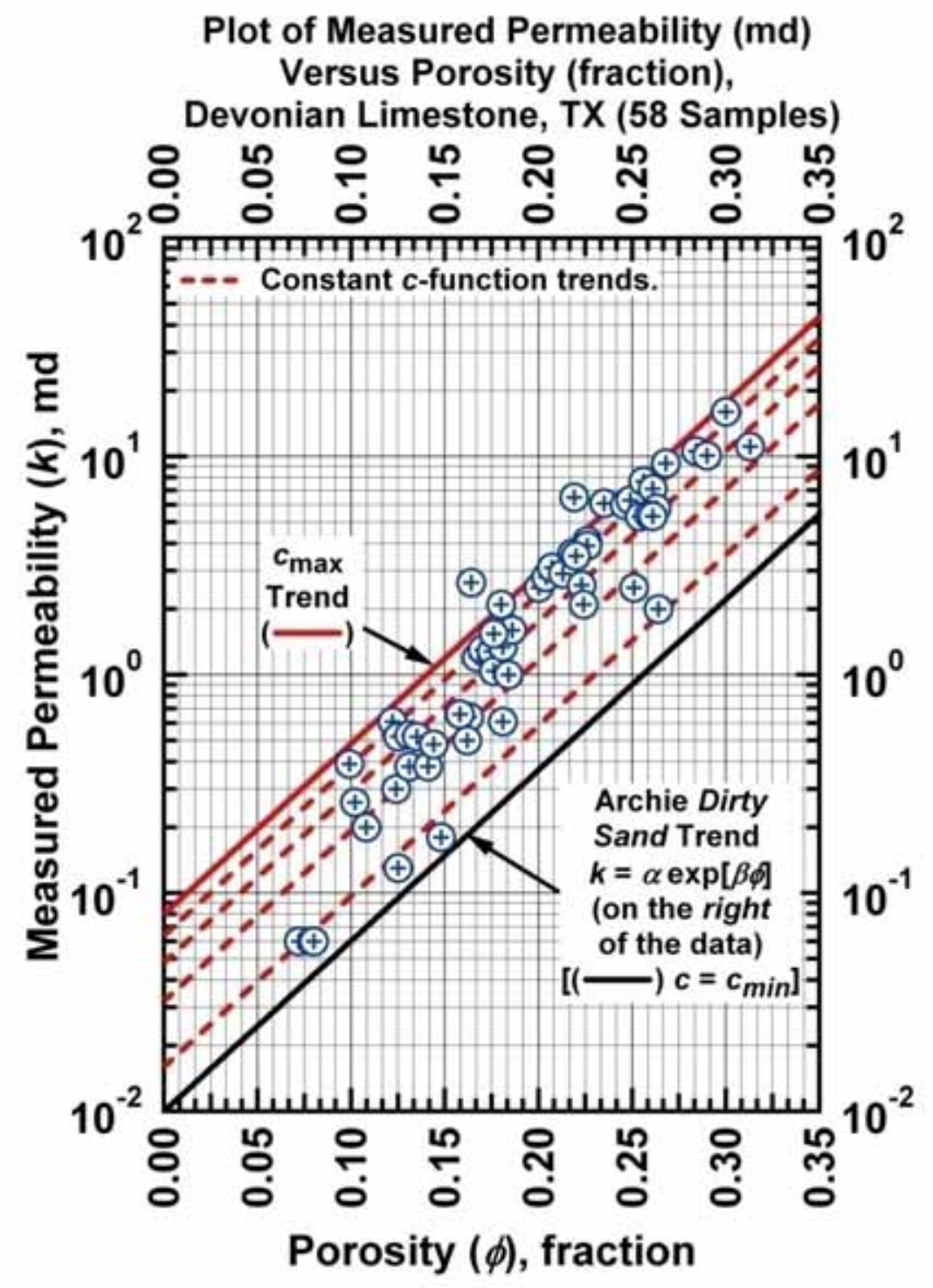

Figure G.5b - Model $C P R-C: \quad k=c \exp [\beta \phi] \quad c=c_{\max } \exp \left[-c_{1} \phi^{c_{2}} F^{c_{3}}\right]$ - "Dirty Sand" Plot (semilog format) - Archie "Dirty Sand" trend is given by the straight-line trend at the far right of the data (exponential model). [Case: Devonian
[Cong Limestone TX (USA)] 


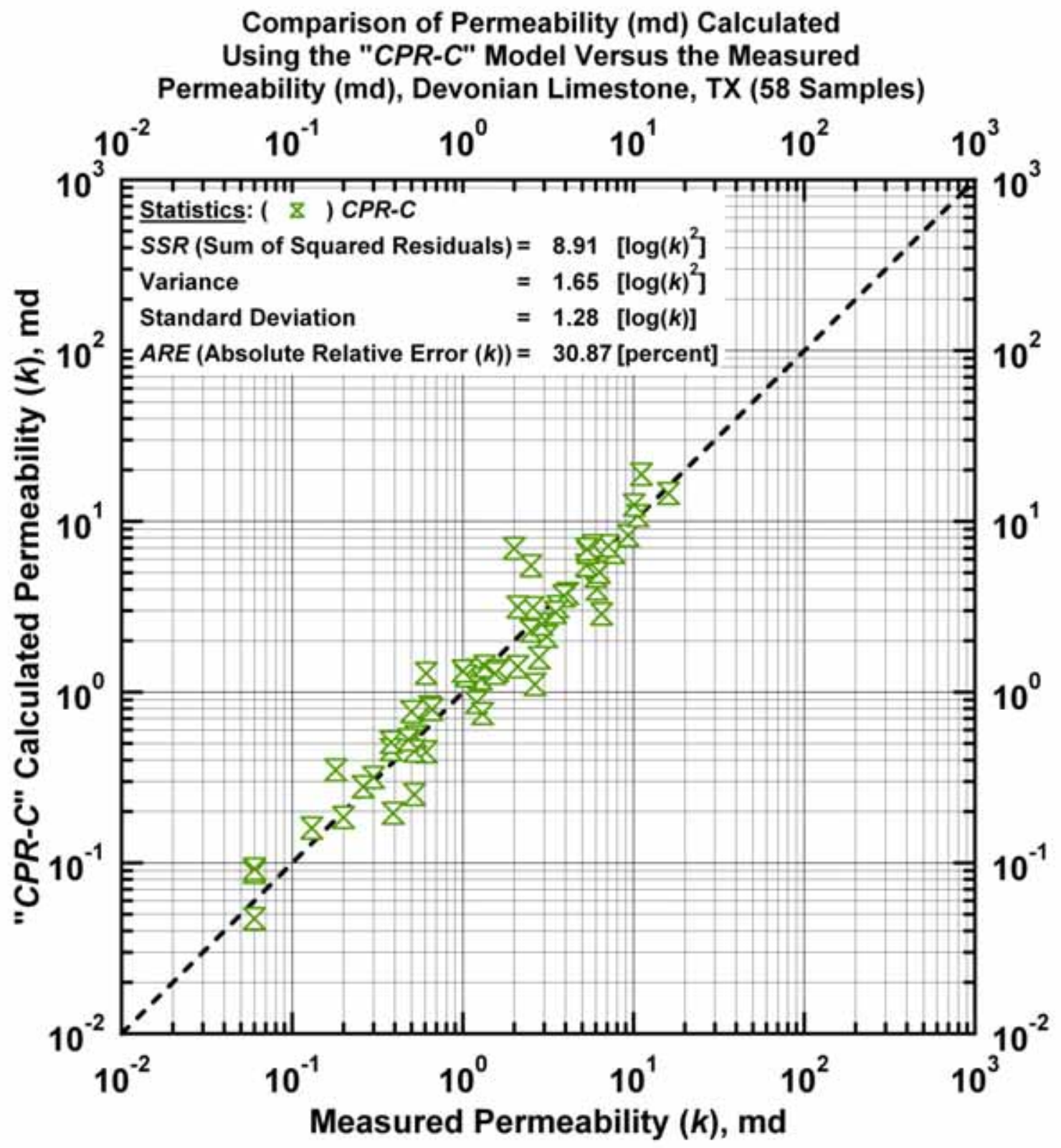

Figure G.6a - Model $C P R-C$ : calculated versus measured permeability. [Case: Devonian Limestone TX (USA)] 


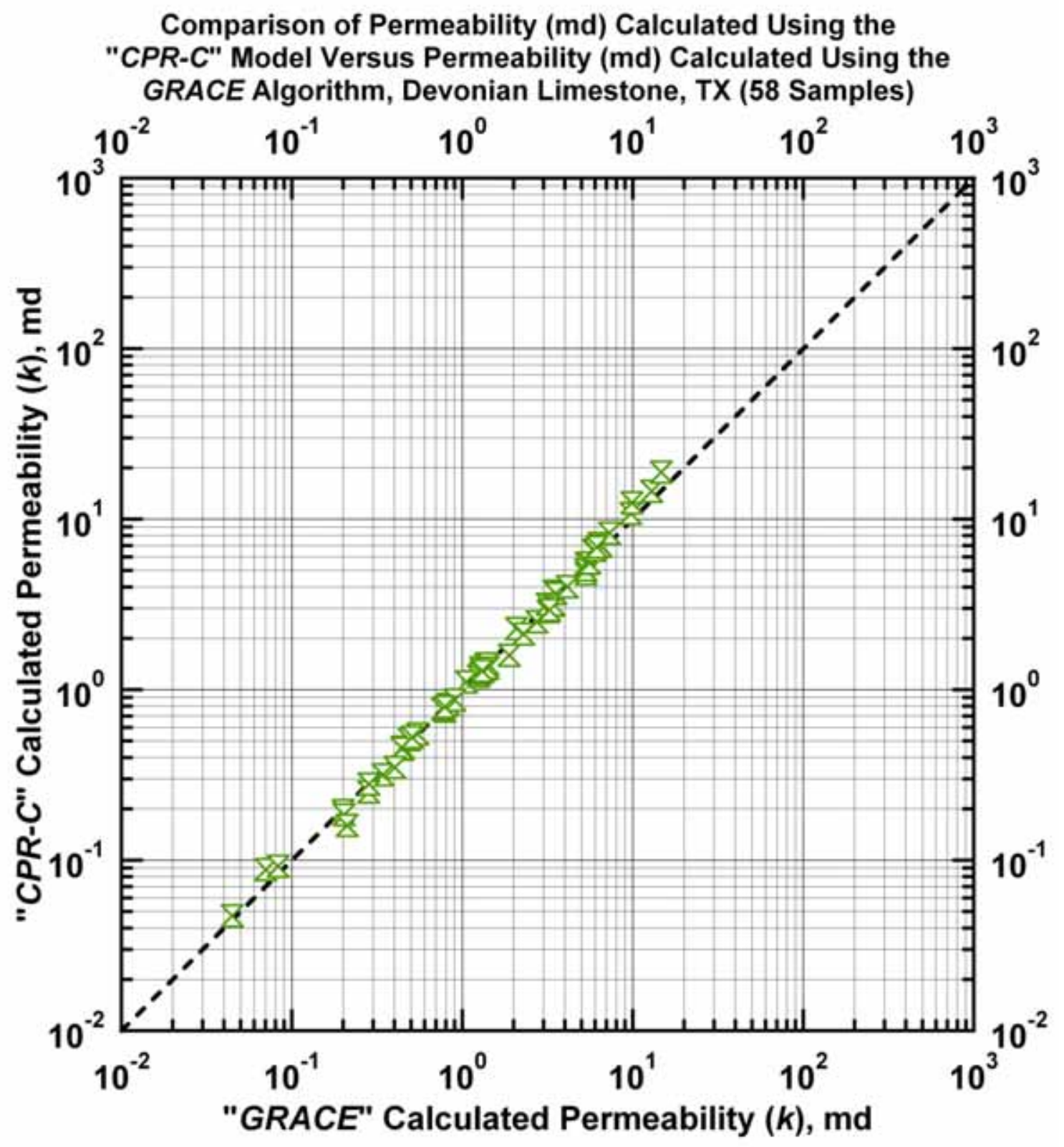

Figure G.6b - Model $C P R$ - $C$ : Permeability calculated using Model $C P R-C$ versus permeability calculated using the GRACE Algorithm. [Case: Devonian Limestone TX (USA)] 
Comparison of the $c$-Coefficient Calculated Using the "CPR-C" Model Versus the Measured $c$-Coefficient, Devonian Limestone, TX (58 Samples)

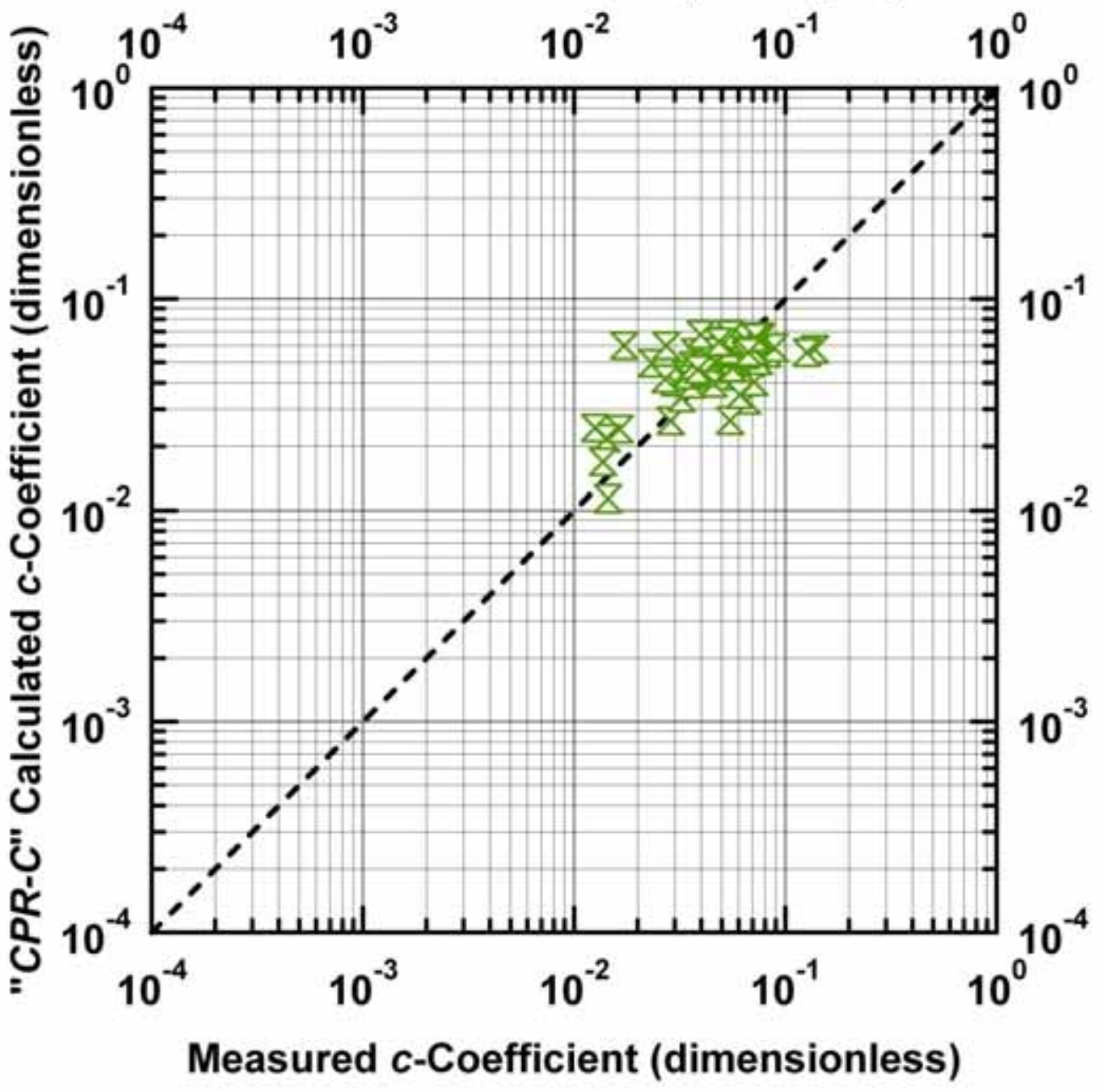

Figure G.6c - Model $C P R$-C: Calculated $c$-function values versus measured $c$-function values. [Case: Devonian Limestone TX (USA)] 


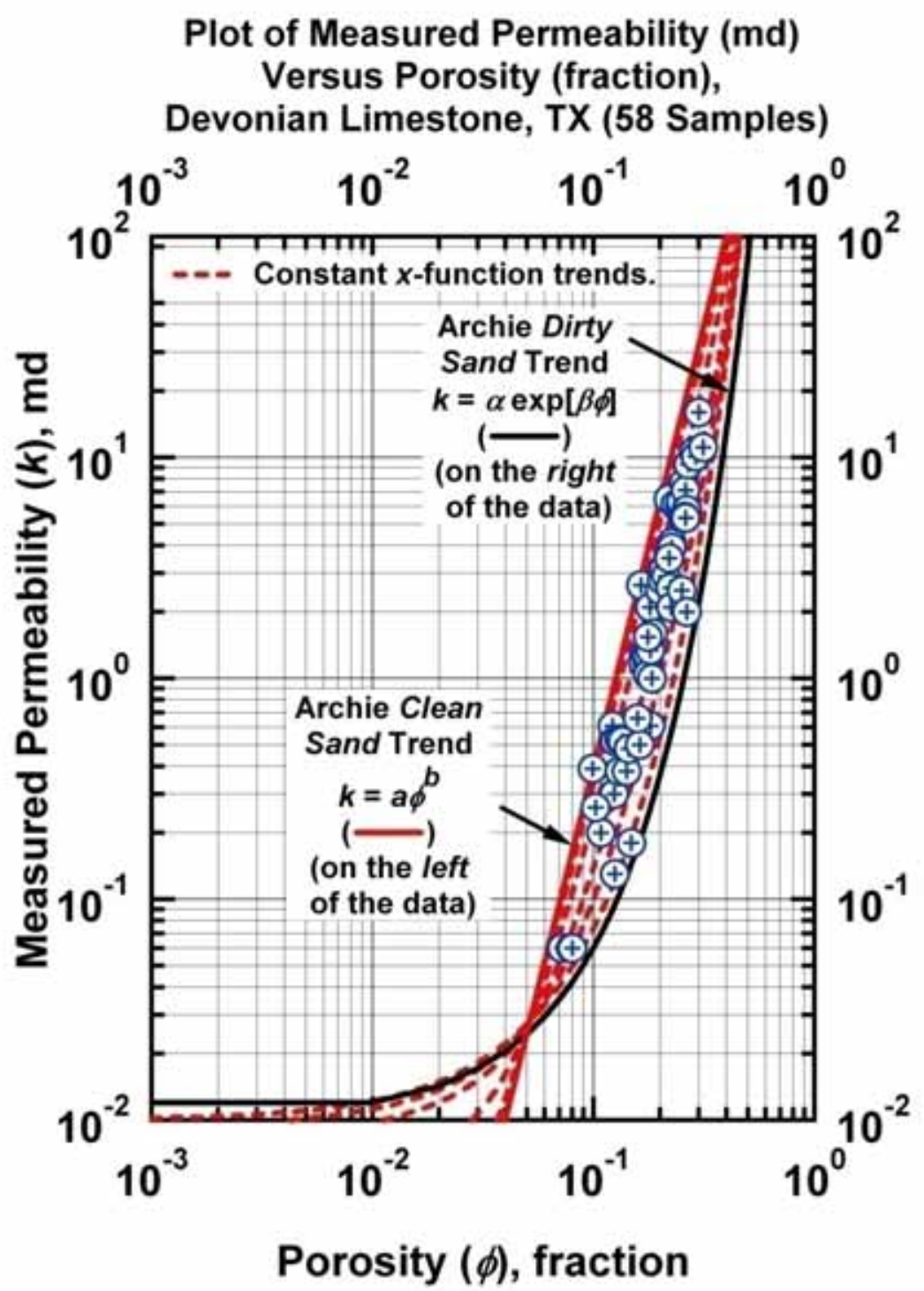

Figure G.7a - Model CPR-D: $k=x a \phi^{b}+(1-x) \alpha \exp [\beta \phi] \quad[0 \leq x \leq 1]$ - Weighted Power Law-Exponential Model used to correlate permeability $(k)$ and porosity $(\phi)$. "Clean Sand" Plot - Archie "Clean Sand" trend is given by the straight-line trend at the far left of the data (power law model). [Case: Devonian Limestone TX (USA)] 


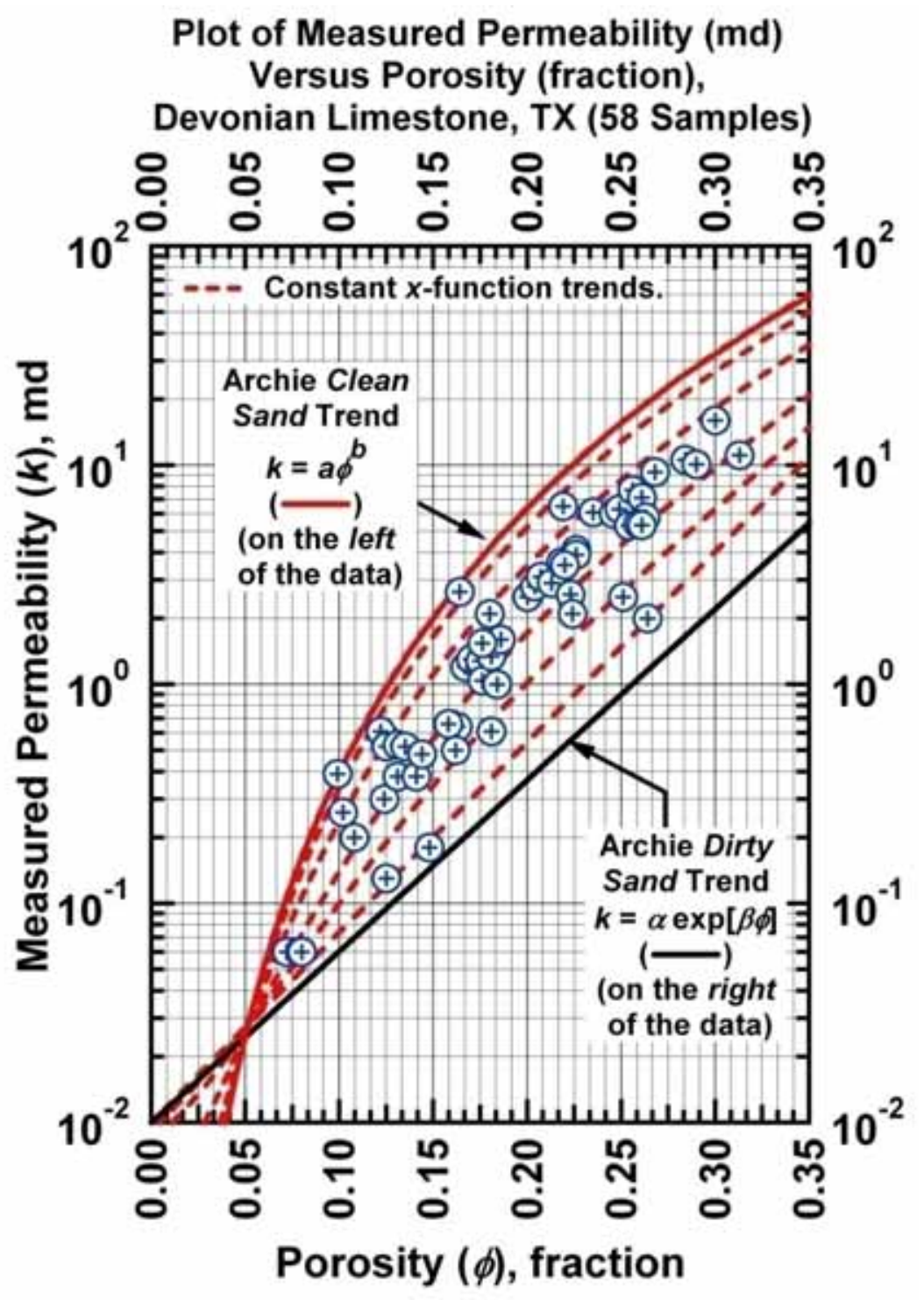

Figure G.7b - Model CPR-D: $k=x a \phi^{b}+(1-x) \alpha \exp [\beta \phi] \quad[0 \leq x \leq 1]$ - Weighted Power Law-Exponential Model used to correlate permeability $(k)$ and porosity $(\phi)$. "Dirty Sand" Plot - Archie "Dirty Sand" trend is given by the straight-line trend at the far right of the data (exponential model). [Case: Devonian Limestone TX (USA)] 


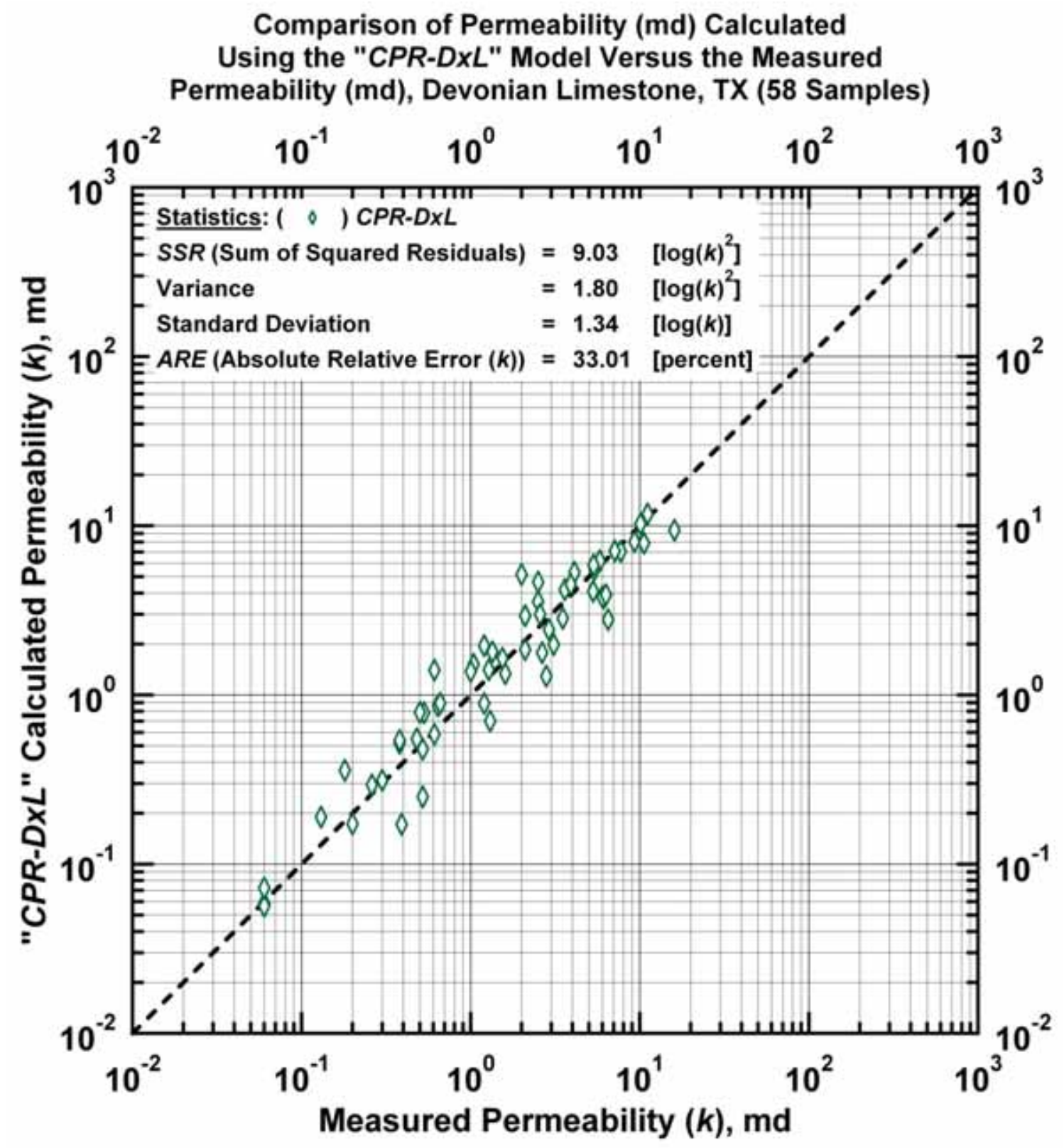

Figure G.8a - Model CPR-DxL: Calculated versus measured permeability. [Case: Devonian Limestone TX (USA)] 


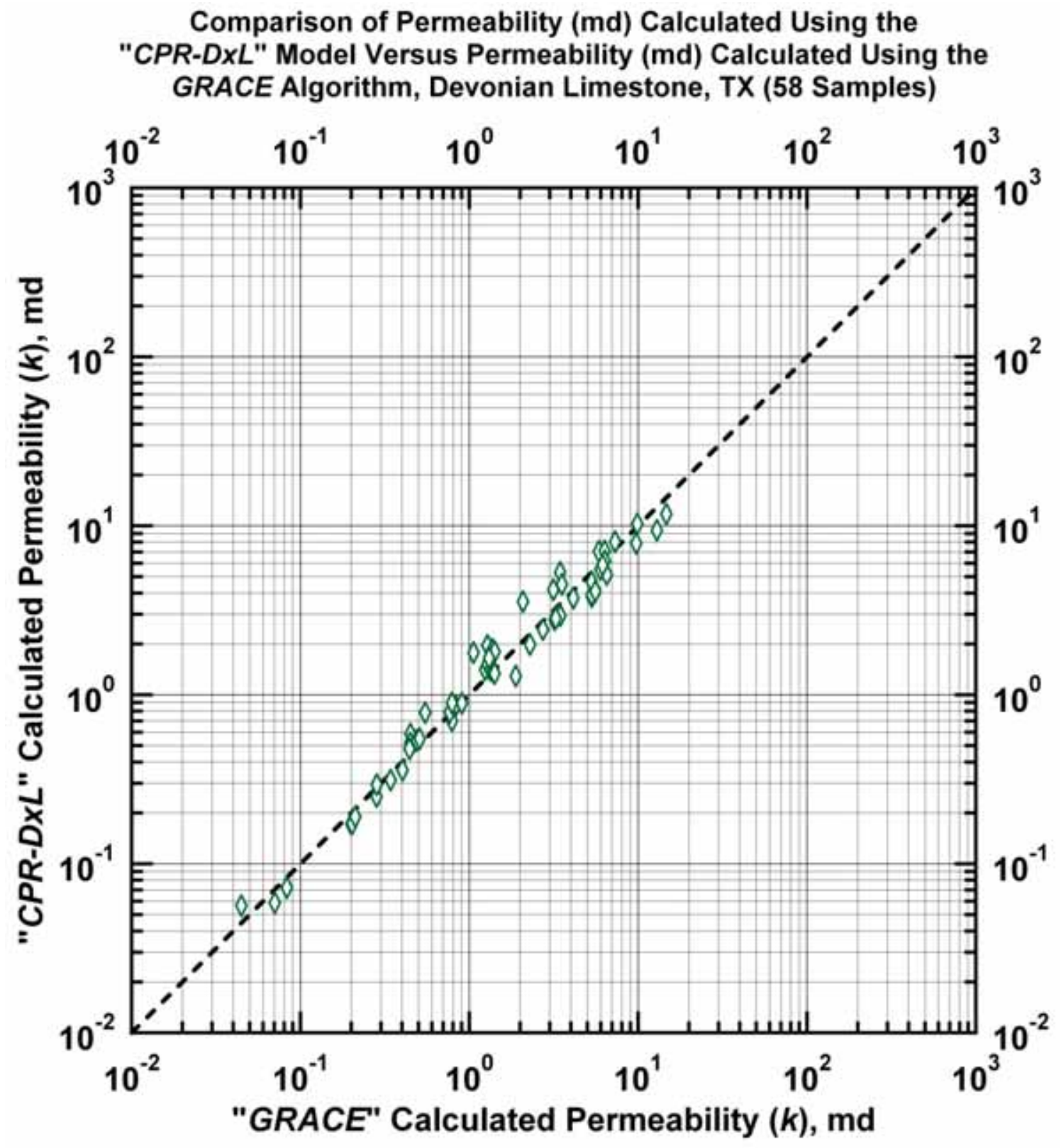

Figure G.8b - Model $C P R-D x L$ : Permeability calculated using Model $C P R-C$ versus permeability calculated using the GRACE Algorithm. [Case: Devonian Limestone TX (USA)] 


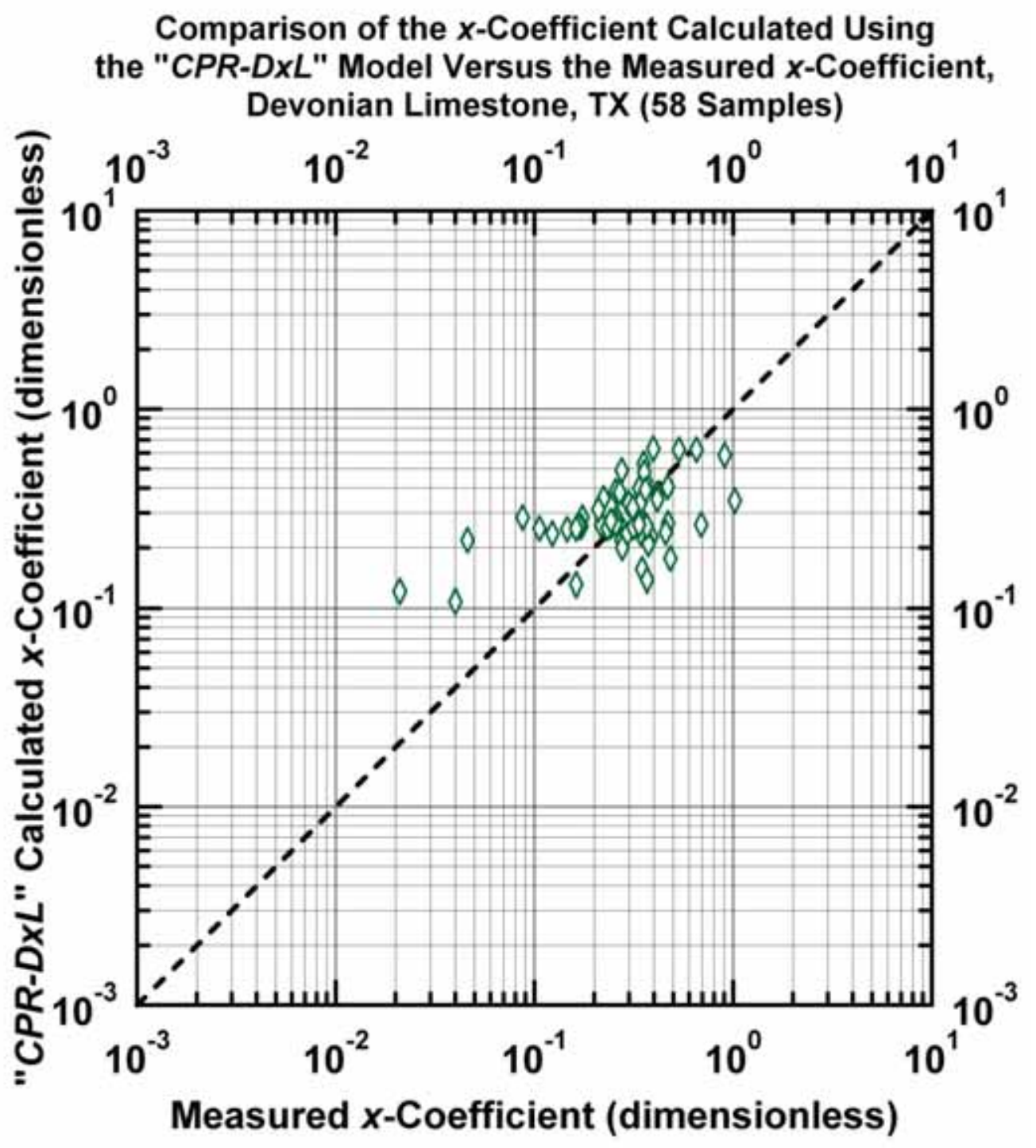

Figure G.8c - Model $C P R$ - $D x L$ : Calculated $x$-function weight values versus measured $x$ function weight values. [Case: Devonian Limestone TX (USA)] 


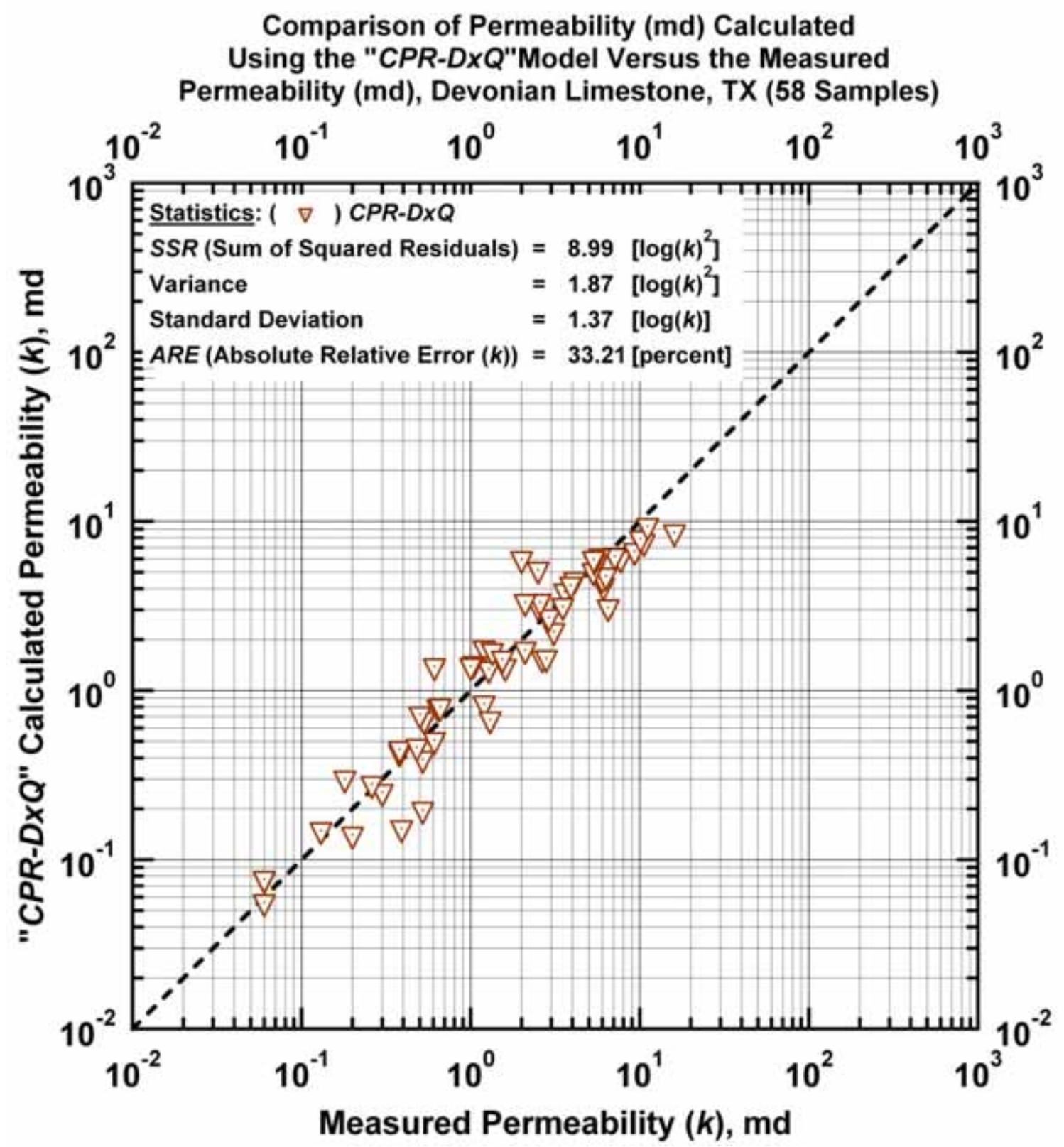

Figure G.9a - Model CPR-DxO: Calculated versus measured permeability. [Case: Devonian Limestone TX (USA)] 


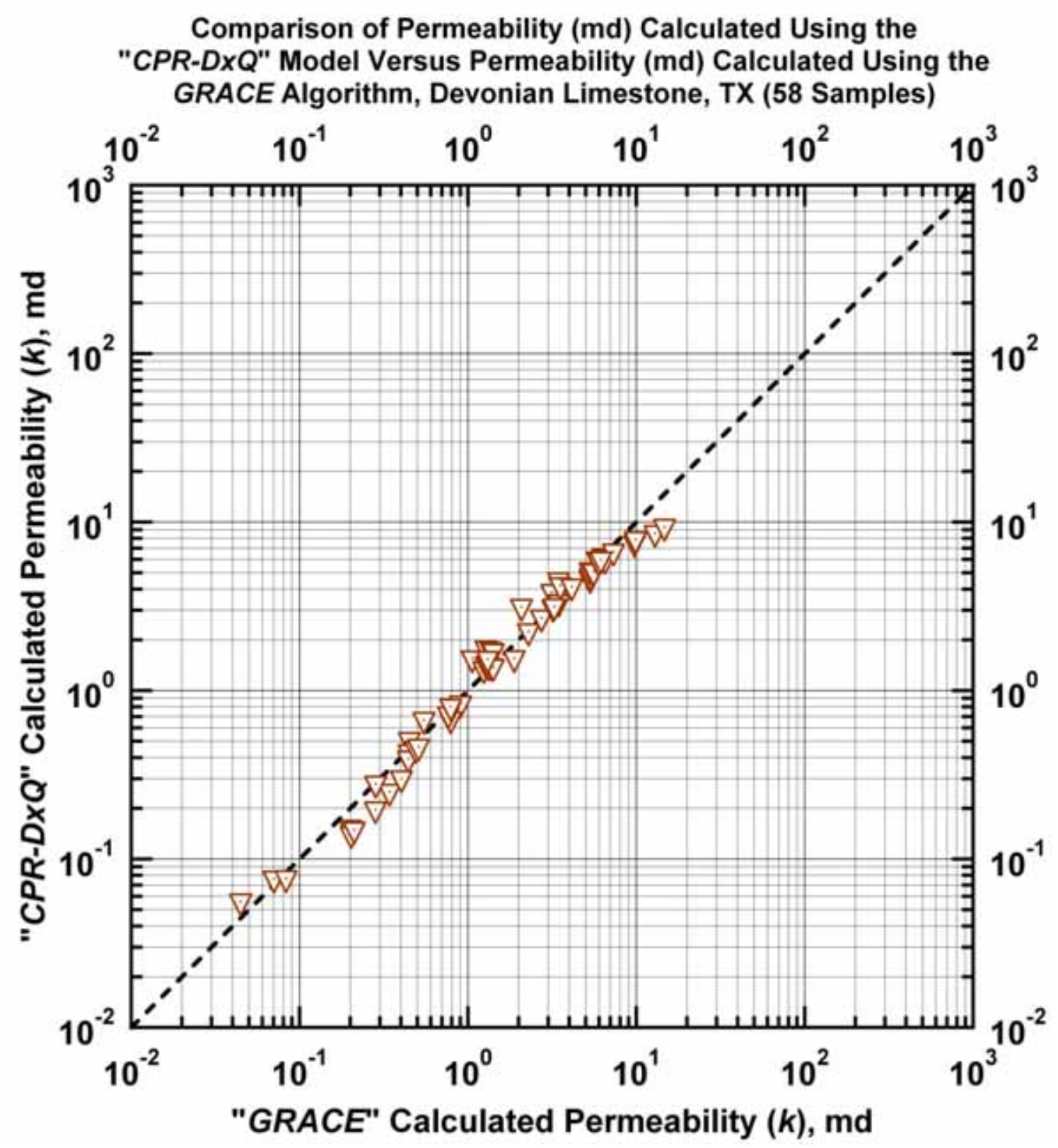

Figure G.9b - Model CPR-DxQ: Permeability calculated using Model $C P R-C$ versus permeability calculated using the GRACE Algorithm. [Case: Devonian Limestone TX (USA)] 


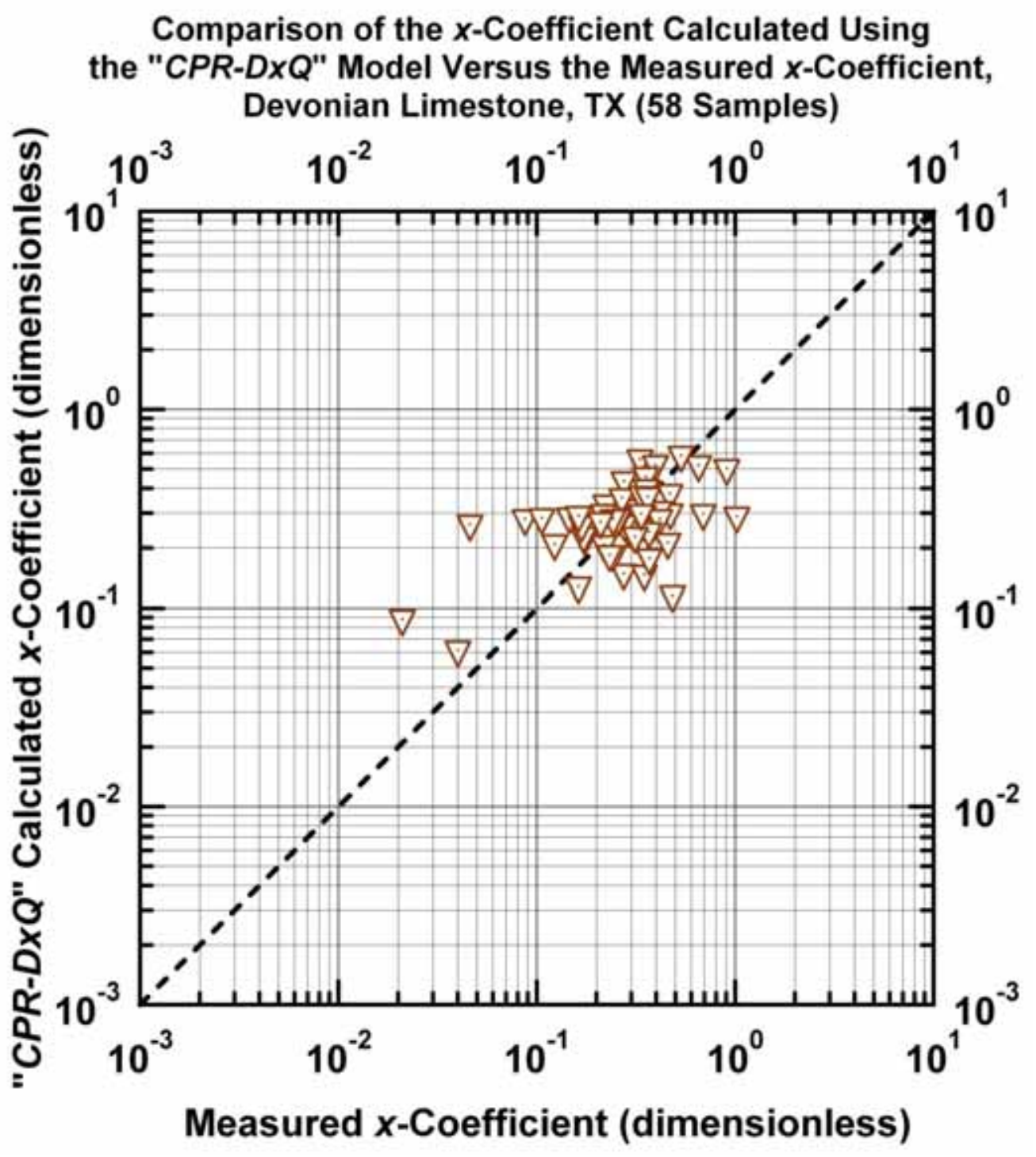

Figure G.9c - Model CPR-DxQ: Calculated $x$-function weight values versus measured $x$ function weight values. [Case: Devonian Limestone TX (USA)] 


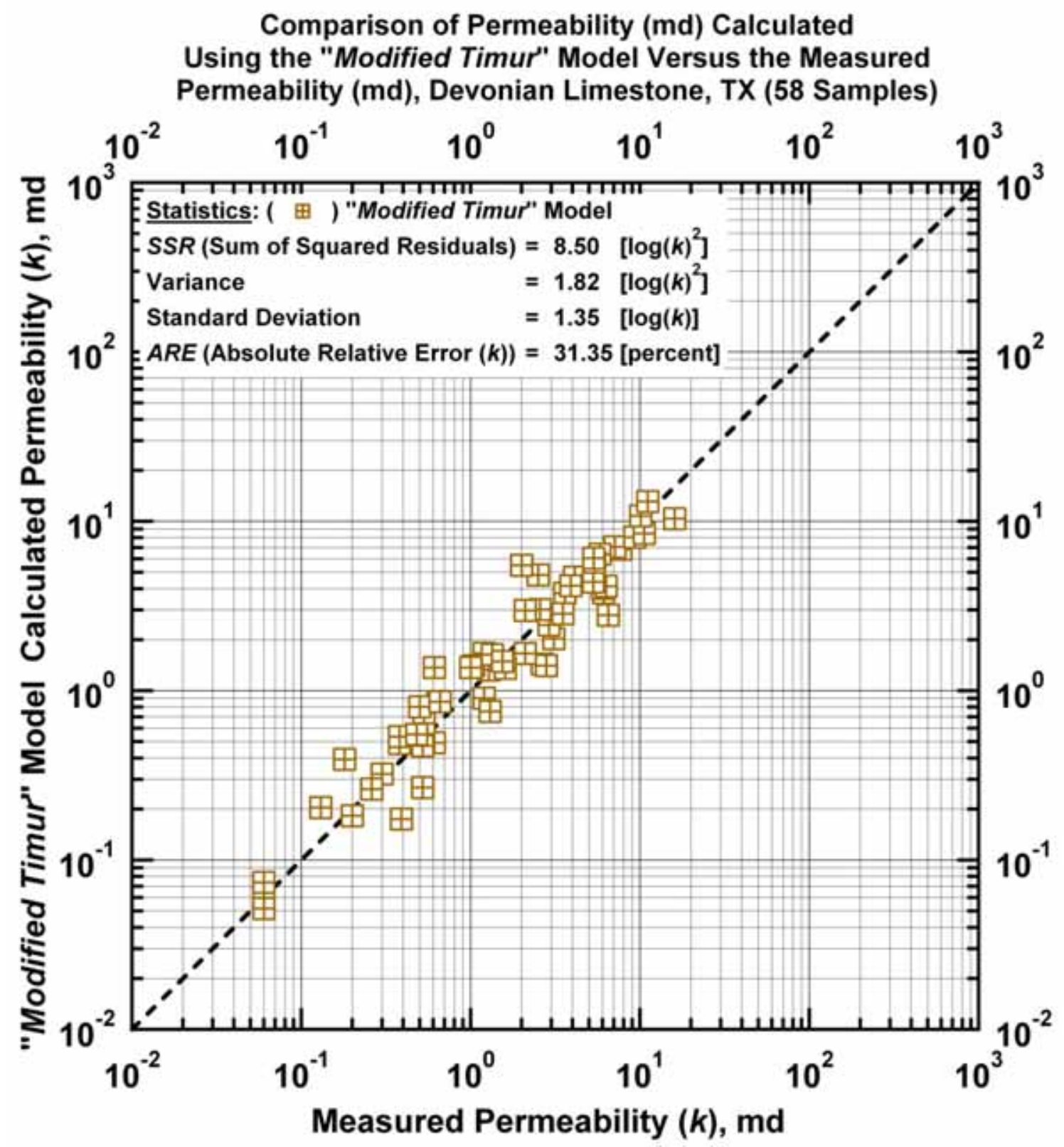

Figure G.10a — "Modified Timur Model:" Calculated versus measured permeability. [Case: Devonian Limestone TX (USA)] 


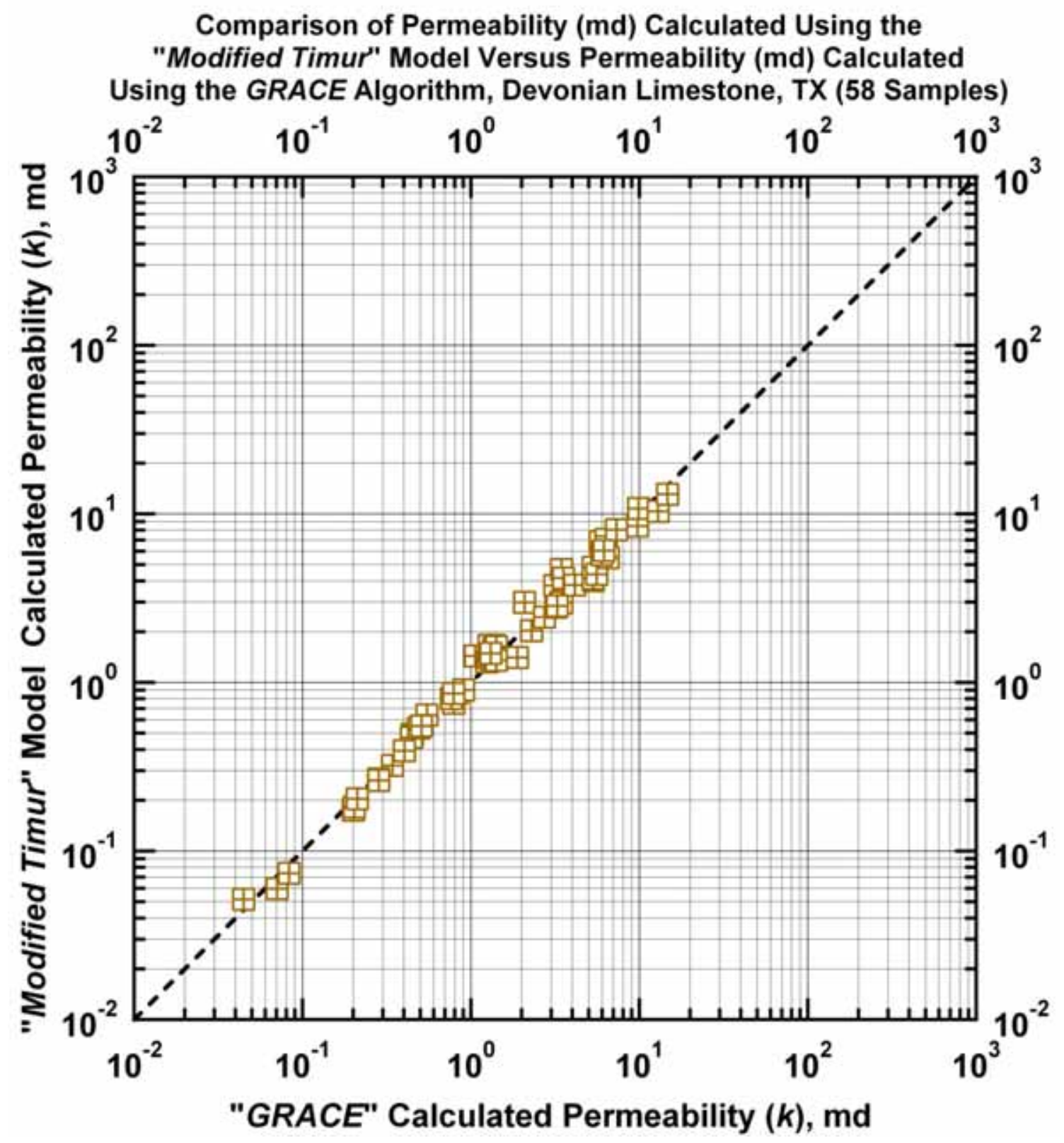

Figure G.10b — "Modified Timur Model:" Permeability calculated using the "Modified Timur Model" versus permeability calculated using the GRACE Algorithm. [Case: Devonian Limestone TX (USA)] 


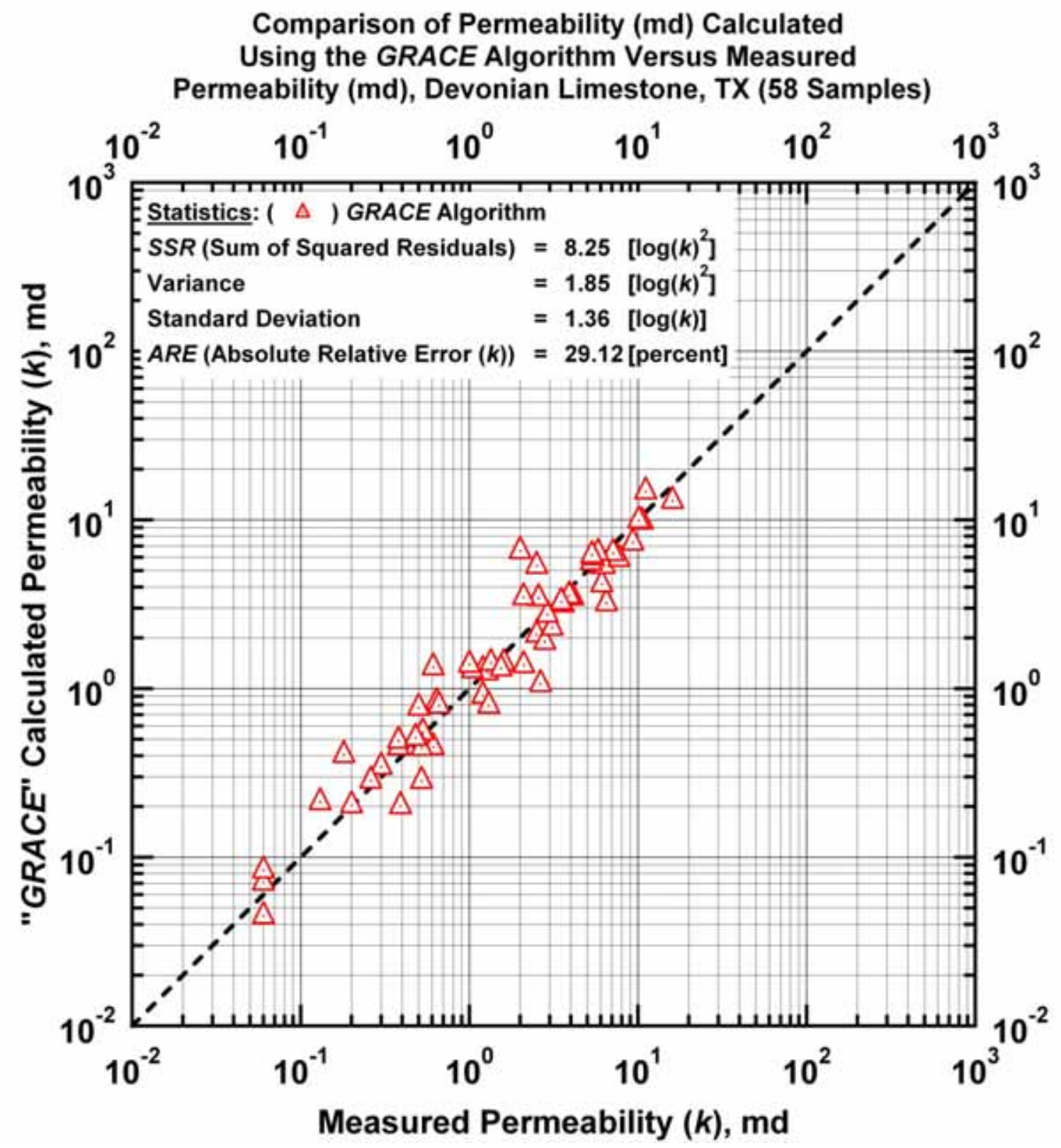

Figure G.11 - GRACE Model: Calculated versus measured permeability. [Case: Devonian Limestone TX (USA)] 


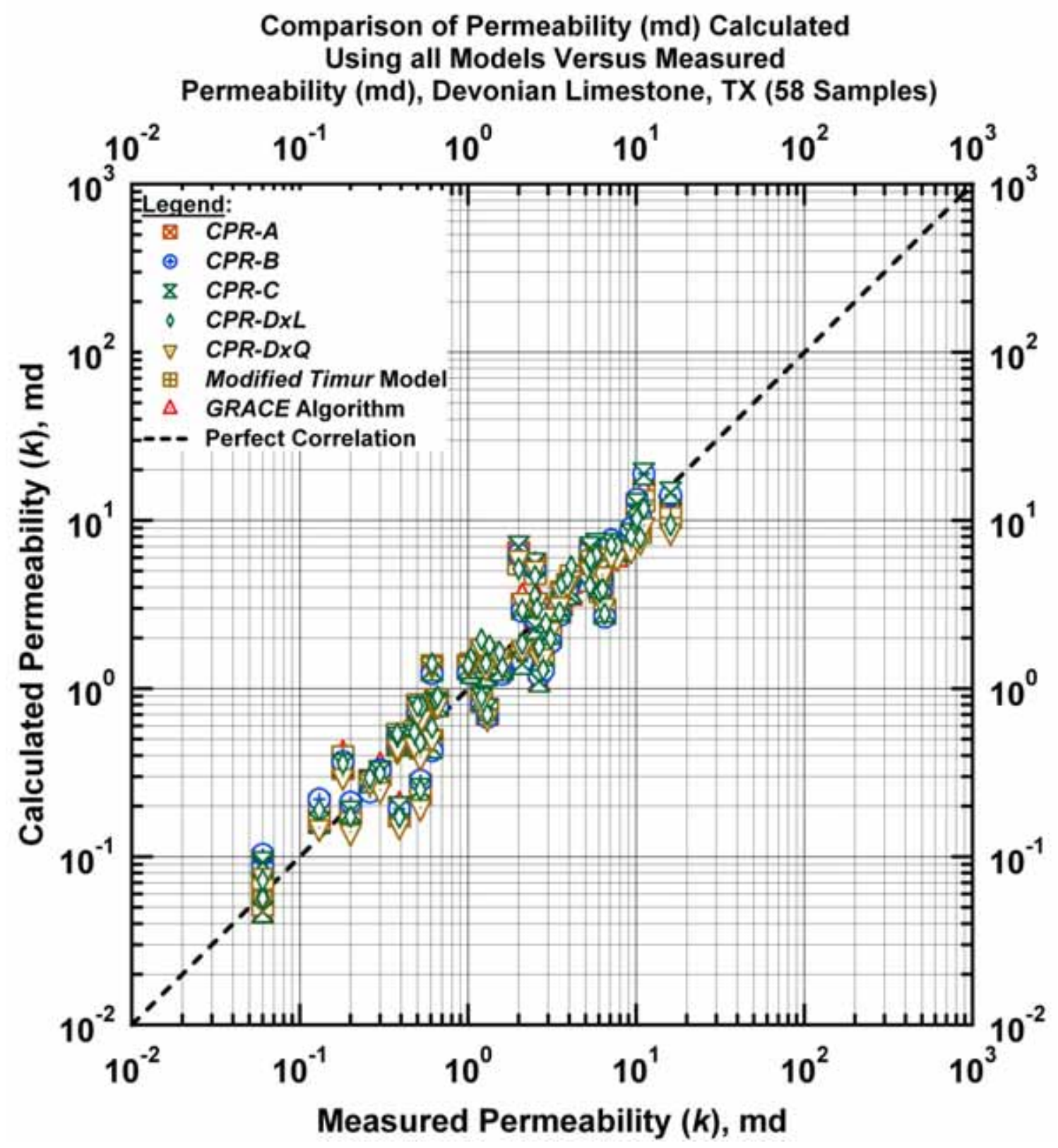

Figure G.12 - All Models: Calculated versus measured permeability. [Case: Devonian Limestone TX (USA)] 


\section{APPENDIX H \\ RESULTS FOR FRIO SHALY SANDSTONE \\ (TEXAS - USA)}

In this Appendix we present the "Frio" Well, located in Texas (USA) — this is a shaly sandstone reservoir (Ellis, 1987).

\section{Model CPR-A: (Appendix H)}

The "characteristic permeability relation" (or CPR) Model CPR-A (i.e., the modified Archie "Clean Sand" Model) is given as:

$$
k=a(\phi-c)^{b} \quad c=c_{\max } \exp \left[-c_{1} \phi^{c 2} F^{c 3}\right]
$$

Where a plot of $\log (k)$ versus $\log (\phi)$ is used to graphically calibrate Eq. H-1 in terms of the coefficients $a, b$, and $c_{\max }$. In this case we had to remove some of the points from the original data set in order to better fit this equation. Fig. H.1 shows that the $C P R-A$ model performs quite well for this case. Figs. H.1a and b show the log-log and the semilog format of permeability versus porosity, respectively. In Fig. H.2 we provide the "error" plots for this case, where the measured and computed data are compared systematically. In Fig. H.2a we note a good correlation of the results obtained using Model $C P R-A$ versus the measured permeability data - we observe good agreement with the perfect correlation trend (i.e., the 45degree line). In Fig. H.2b we provide a comparison with the GRACE algorithm correlation (Xue et al. 1997)), and we note that the CPR-A model results correlate very well with the GRACE algorithm results for this case. Finally, in Fig. H.2c we present the calculated and measured $c$-function - and again we note a reasonable correlation of the data about the perfect correlation trend.

\section{Model CPR-B: (Appendix H)}

The "characteristic permeability relation" (or CPR) Model CPR-B (i.e., the modified Archie "Dirty Sand" Model (power-law basis)) is given as:

$$
k=a(\phi+c)^{b} \quad c=c_{\max } \exp \left[-c_{1} \phi^{c_{2}} F^{c_{3}}\right]
$$

Where a plot of $\log (k)$ versus $\log (\phi)$ is used to graphically calibrate Eq. H-2 in terms of the coefficients $a, b$, and $c_{\max }$. The results for this case are presented in Figs. H.3 and 4. In Fig. H.3a (log-log format) we note that a very distinct "envelope" is formed by the $C P R-B$ model, essentially all of the data on the right and left flanks are well-matched (i.e., the "dirty sand" power law relation (applied on the right portion of the data) and its correction function (applied on the left portion of the data). Similarly, in Fig. H.3b (semilog format), we observe an excellent distribution of the power law model across the body of the data. The error analyses for this case are shown in Fig. H.4. In Fig. H.4a we present the results obtained using 
Model $C P R-B$ versus the measured permeability data and in this case there is good agreement with the perfect correlation trend (i.e., the 45-degree line) - a few data points are out-lying, mostly in the middle of the trend. We provide a comparison with the GRACE algorithm correlation in Fig. H.4b - the results using Model $C P R-B$ and the GRACE algorithm compare very well for this case. We compare the computed and data-derived values of the $c$-function for this case in Fig. H.4c and we note a reasonably "tight" correlation, except for the very lowest values of the $c$-function. In summary, we believe that Model $C P R-B$ is a very effective correlation model for this particular data case.

\section{Model CPR-C: (Appendix H)}

The "characteristic permeability relation" (or CPR) Model CPR-C (i.e., the modified Archie "Dirty Sand" Model (exponential basis)) is given as:

$$
k=c \exp [\beta \phi] \quad c=c_{\max } \exp \left[-c_{1} \phi^{c 2} F^{c_{3}}\right]
$$

Where a plot of $\log (k)$ versus $\phi$ is used to graphically calibrate Eq. H-3 in terms of the coefficients $-\beta$ and $c_{\max }$. Model $C P R-C$ is fundamentally different than Models $C P R-A$ and $C P R-B$, as the basis for Model $C P R-C$ is an exponential function, not a power law relation. In Figs. H.5 and 6, we present the results of applying Model $C P R-C$ for this case, and in general, we observe a very consistent performance of Model $C P R$-C. Fig. H.5b illustrates the excellent conformance of Model $C P R-C$ for this case - again recalling that this model has an exponential relationship with porosity as its basis.

In Figs. H.6a, H.6b, and H.6c we review the error analyses plots for this case, and we note good performance (visually) in terms of the correlation of the results using Model CPR-C. In this case, the $c$ function (in Eq. H-3) is not a correction term, but rather the instantaneous intercept for the exponential basis. The correlation of the $c$-function (see Fig. H.6c) appears to be consistent - but scatter and a slight off-center trend is evident. We believe that the $C P R-C$ model has performed well for this case, and the Fig. H.6 confirms the value of this model as a "characteristic relation" for permeability.

\section{Model CPR-D: (Appendix H)}

The "characteristic permeability relation" (or $C P R$ ) Model CPR-D (i.e., the weighted power law-exponential model) is given in its fundamental form as:

$$
\left.k=x a \phi^{b}+(1-x) \alpha \exp [\beta \phi] \quad[0 \leq x \leq 1]\right] .
$$

For Eq. H-4 there are two proposed models for the $x$-function (i.e., the weighting function) - these are:

$$
\begin{aligned}
& x=\exp \left[-\ln \left(x_{0}\right)+x_{1} \ln (\phi)+x_{2} \ln (F)+x_{3} \ln (\phi) \ln (F)\right](\text { Model CPR-D } x L) \\
& x=\exp \left[\begin{array}{l}
-\ln \left(x_{0}\right)+x_{1} \ln (\phi)+x_{2} \ln (F)+x_{3} \ln (\phi) \ln (F) \\
+x_{4} \ln (\phi)^{2}+x_{5} \ln (F)^{2}+x_{6} \ln (\phi)^{2} \ln (F) \\
+x_{7} \ln (\phi) \ln (F)^{2}+x_{8} \ln (\phi)^{2} \ln (F)^{2}
\end{array}\right] \text { (Model CPR-DxL).... }
\end{aligned}
$$


As noted in Eq. H-4b, Model CPR-DxL utilizes a logarithmic linear model for the weights ( $x$-values) and as seen in Eq. H-4c, Model $C P R-D x Q$ utilizes a logarithmic quadratic model for the weights ( $x$-values). While these models (Eqs. H-4b and $\mathrm{H}-4 \mathrm{c}$ ) are empirical, we have found generally good application of these relations for all cases. In this case, the base results using Models $C P R-D x L$ and $C P R-D x Q$ are presented in Fig. H.7 — where the power law and exponential basis functions are fitted to the appropriate portion of the data. The power law equation represents the "Archie clean sand" trend and is fitted to the leftmost data as shown in Fig. H.7a and in contrast, the exponential equation is thought to represent the "Archie dirty sand" trend and the exponential is fitted to the far rightmost portion of the data (see Fig. H.7b).

In Figs. H.8 and 9 we present the error analyses for this case, where we have employed the weighting functions (Eqs. H-4b and H-4c) as appropriate. In Fig. H.8a we find a good correlation of permeability (with the out-lying points noted in the middle of the trend). The comparison of the Model CPR-DxL results are compared to the results obtained from the GRACE algorithm in Fig. H.8b, we note the good results obtained using Model CPR-DxL. The computed weight function ( $x$-values) shown for the CPR$D x L$ model in Fig. H.8c does exhibit more scatter than expected, but the trend is (relatively) centered about the perfect correlation line.

The results obtained using Model $C P R-D x Q$ are presented in Fig. H.9, and we note substantially improved behavior over that of Model $C P R-D x L$. In particular, the base correlation of computed and measured permeabilities (Fig. H.9a) for Model CPR-DxQ does indicate a slightly better correlation than that of Model $C P R-D x L$. Also, the comparison of Model CPR-DxQ with the results from the GRACE algorithm (Fig. H.9b) exhibits better agreement than the results of Model CPR-DxL. Finally, the correlation of the $x$-function (Fig. H.9c) for Model CPR-DxQ is better than the correlation for Model CPR-DxL. These comparisons suggest that Model CPR-DxQ (i.e., the combination of Eqs. $\mathrm{H}-4 \mathrm{a}$ and $\mathrm{H}-4 \mathrm{c}$ ) provides a better correlation of data, for this case, than Model CPR-DxL.

\section{"Modified Timur Model": (Appendix H)}

The "Modified Timur Model" is given as:

$$
k_{\text {Timur }}=a \phi^{b} F^{c}
$$

In Fig. H.10 we present the results of the "Modified Timur Model" (Eq. H-5) which is essentially just a generalized power-law function relation in terms of $k, \phi$, and $F$. Figs. H.10a and b, provide the "Modified Timur Model" results of the data for this case.

\section{GRACE Algorithm: (Appendix H)}

In Fig. H.11 we present the results predicted by the GRACE algorithm, which is a non-parametric regression approach (see Xue et al. (1997) for details). In theory, the GRACE algorithm should provide the most unbiased correlation of the data - i.e., the GRACE algorithm is designed not to "fit the errors" as 
other regression approaches may. It is our contention that the GRACE algorithm is the statistical standard — and any algorithm/approach/model which achieves better regression statistics than the GRACE algorithm is actually "fitting the errors" in the data. The only case which has significantly better regression statistics than the GRACE algorithm is the CPR-A — but we must remember that for this case we used fewer number of points.

\section{Comparison of All Models: (Appendix H)}

Our final graphic, Fig. H.12, illustrates all of the models on a single plot of calculated versus measured permeability. We also present a table of all statistical results in Table H.1.

Table H.1 - $\quad$ Statistical Results for all Models (Frio, TX (USA), $n=64)$

\begin{tabular}{|c|c|c|c|c|}
\hline Model & $\begin{array}{c}\text { Sum of } \\
\text { Squared } \\
\text { Residuals } \\
{\left[\log (k)^{2}\right]}\end{array}$ & $\begin{array}{l}\text { Variance } \\
{\left[\log (k)^{2}\right]}\end{array}$ & $\begin{array}{c}\text { Standard } \\
\text { Deviation } \\
{[\log (k)]}\end{array}$ & $\begin{array}{c}\text { Absolute } \\
\text { Relative } \\
\text { Error } \\
\text { [percent] }\end{array}$ \\
\hline$C P R-A$ & 59.49 & 4.57 & 2.14 & 78.38 \\
\hline$C P R-B$ & 67.78 & 5.76 & 2.40 & 99.21 \\
\hline$C P R-C$ & 57.11 & 5.59 & 2.36 & 100.09 \\
\hline$C P R-D x L$ & 58.80 & 5.31 & 2.30 & 103.74 \\
\hline$C P R-D x Q$ & 54.49 & 5.52 & 2.35 & 88.71 \\
\hline Modified Timur & 71.00 & 6.12 & 2.47 & 134.64 \\
\hline GRACE Algorithm & 51.94 & 5.50 & 2.35 & 88.99 \\
\hline
\end{tabular}

The coefficients for the models used in this case are provided in Table H.2.

Table H.2a - Model Coefficients (Frio, TX (USA), $n=64$ ).

\begin{tabular}{|c|c|c|c|c|c|c|}
\hline Model & $a$ or $\alpha$ & $b$ or $\beta$ & $c$ or $c_{\max }$ & $c_{1}$ & $c_{2}$ & $c_{3}$ \\
\hline$C P R-A$ & $5.00 \times 10^{11}$ & 12.00 & 0.100 & $4.14 \times 10^{-3}$ & -4.89 & -0.97 \\
\hline$C P R-B$ & $1.00 \times 10^{14}$ & 20.00 & 0.135 & 28.25 & 2.67 & 0.40 \\
\hline$C P R-C$ & $7.00 \times 10^{-5}$ & 50.00 & $5.00 \times 10^{-3}$ & 11.969 & 4.32 & 1.48 \\
\hline Modified Timur & $1.00 \times 10^{7}$ & 0.17 & -3.8271 & - & - & - \\
\hline
\end{tabular}

Table H.2b - Model Coefficients (Frio, TX (USA), $n=64$ ).

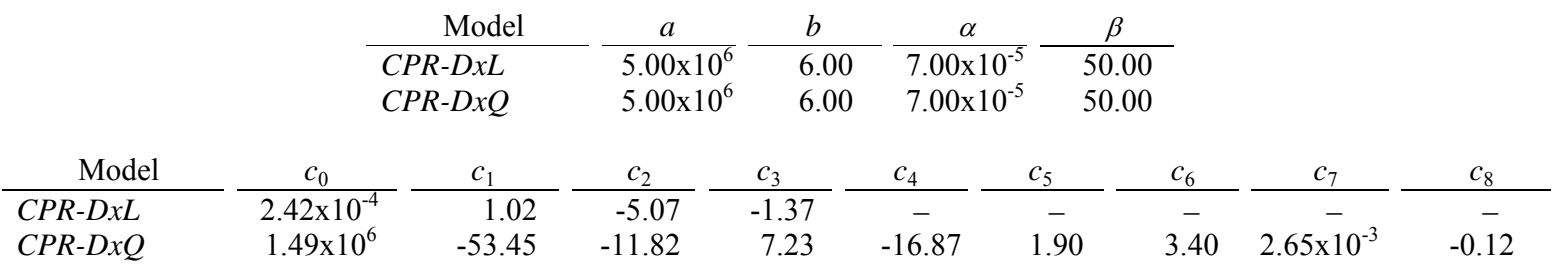

\section{Nomenclature: (Appendix H)}

$$
\begin{aligned}
F & =\text { Archie Formation Factor (dimensionless) } \\
k & =\text { Formation Permeability, md (or any consistent units) } \\
\phi & =\text { Porosity, fraction }
\end{aligned}
$$




\section{References: (Appendix H)}

Ellis, K.W.: "Extended Correlations of Porosity, Permeability, and Formation Resistivity Factor," MS thesis, Texas A\&M U., College Station, TX (1987).

Xue, G., Datta-Gupta, A., Valko, H., and Blasingame, T.A.: "Optimal Transformations for Multiple Regression: Application to Permeability Estimation from Well Logs," SPEFE (June 1997), 85-93. 


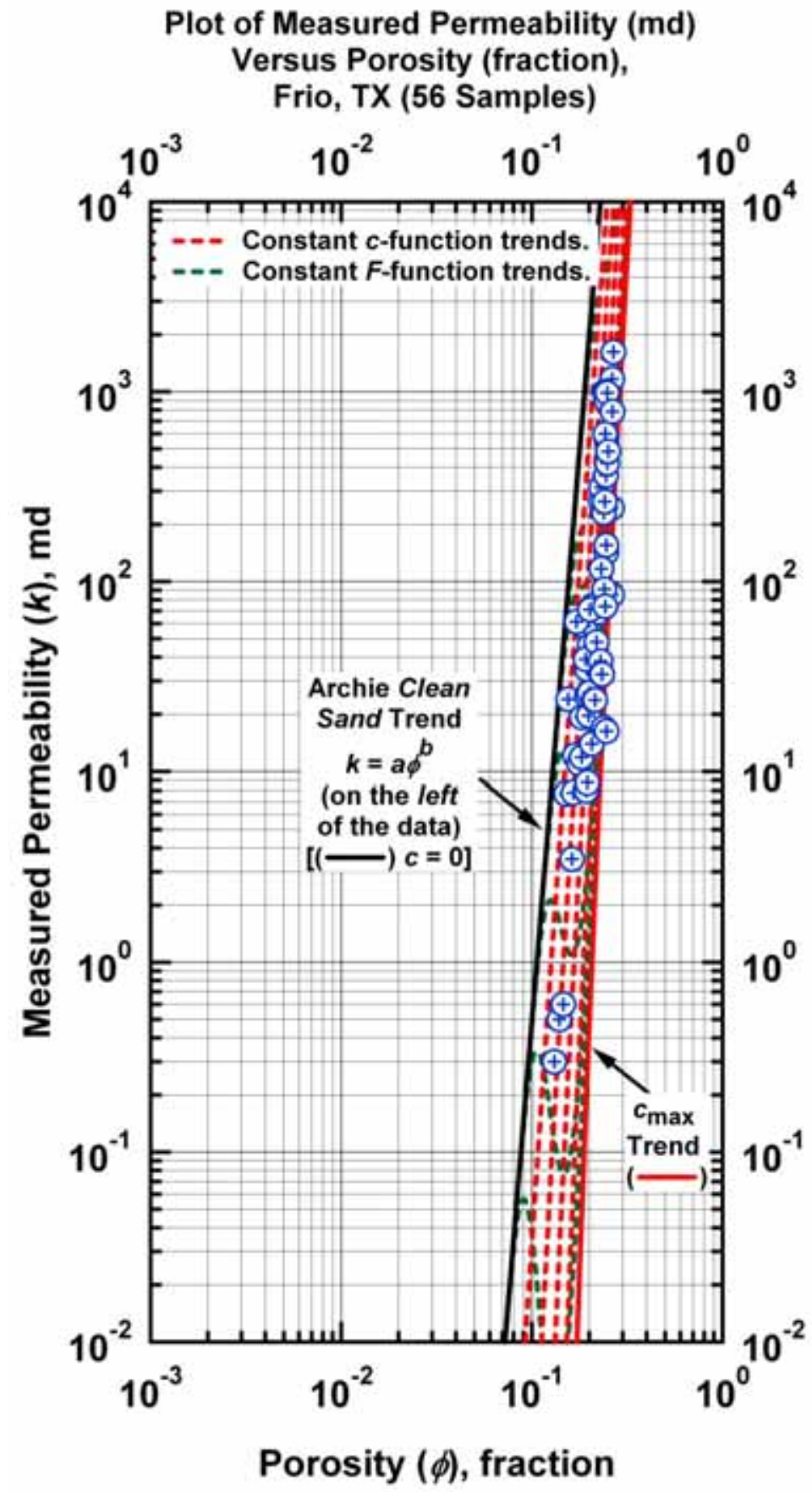

Figure H.1a - Model CPR-A: $k=a(\phi-c)^{b}\left(c=c_{\max } \exp \left[-c_{1} \phi^{c_{2}} F^{c 3}\right]\right)$ - "Clean Sand" Plot (log-log format) - Archie "Clean Sand" trend is given by the straight-line trend at the far left of the data (power law model). [Case: Frio, TX (USA)] 


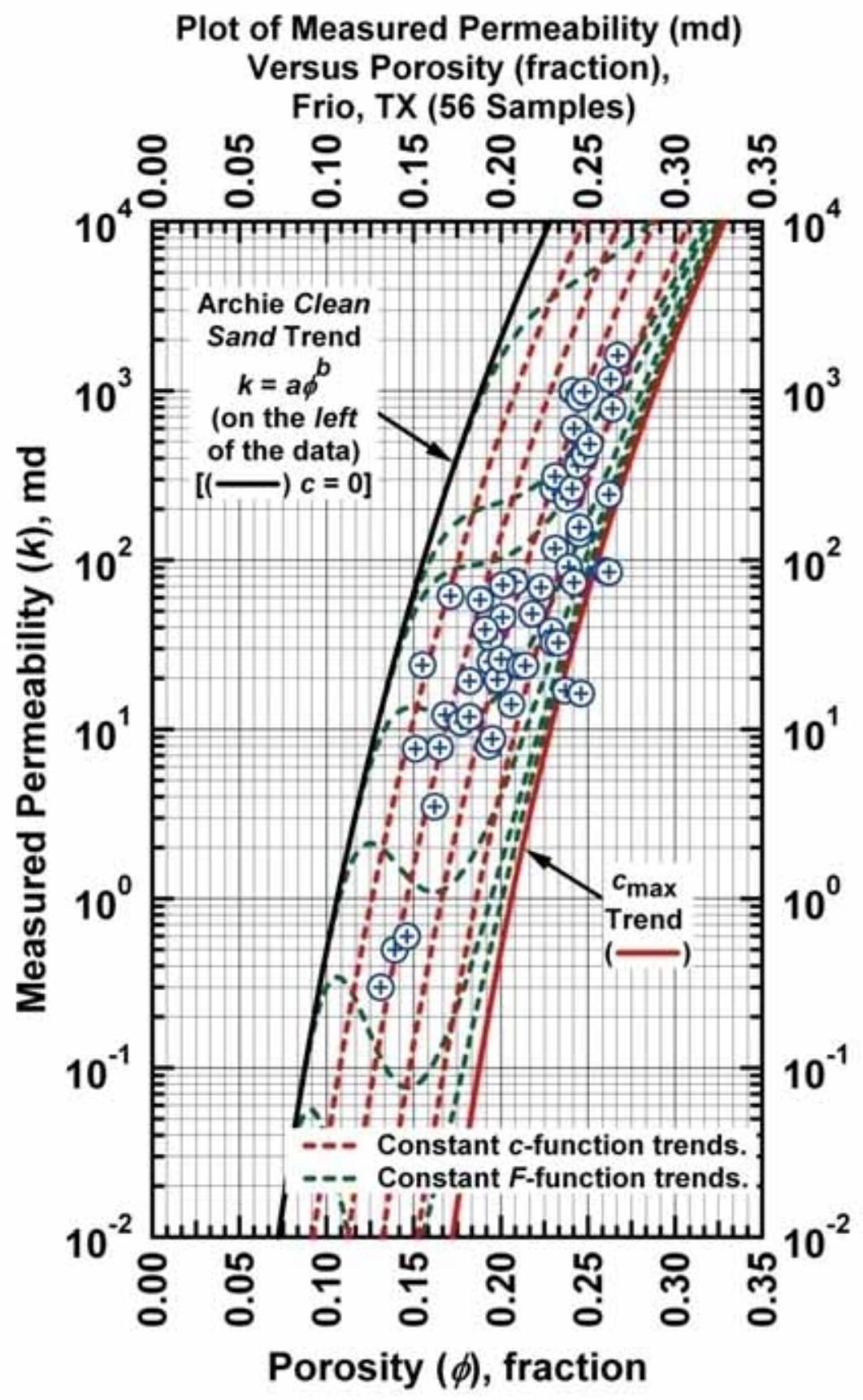

Figure H.1b - Model CPR-A: $k=a(\phi-c)^{b}\left(c=c_{\max } \exp \left[-c_{1} \phi^{c 2} F^{c 3}\right]\right)$ - "Dirty Sand" Plot (semilog format). Archie "Clean Sand" trend is given by the curved trend at the far left of the data (power law model). [Case: Frio, TX (USA)] 


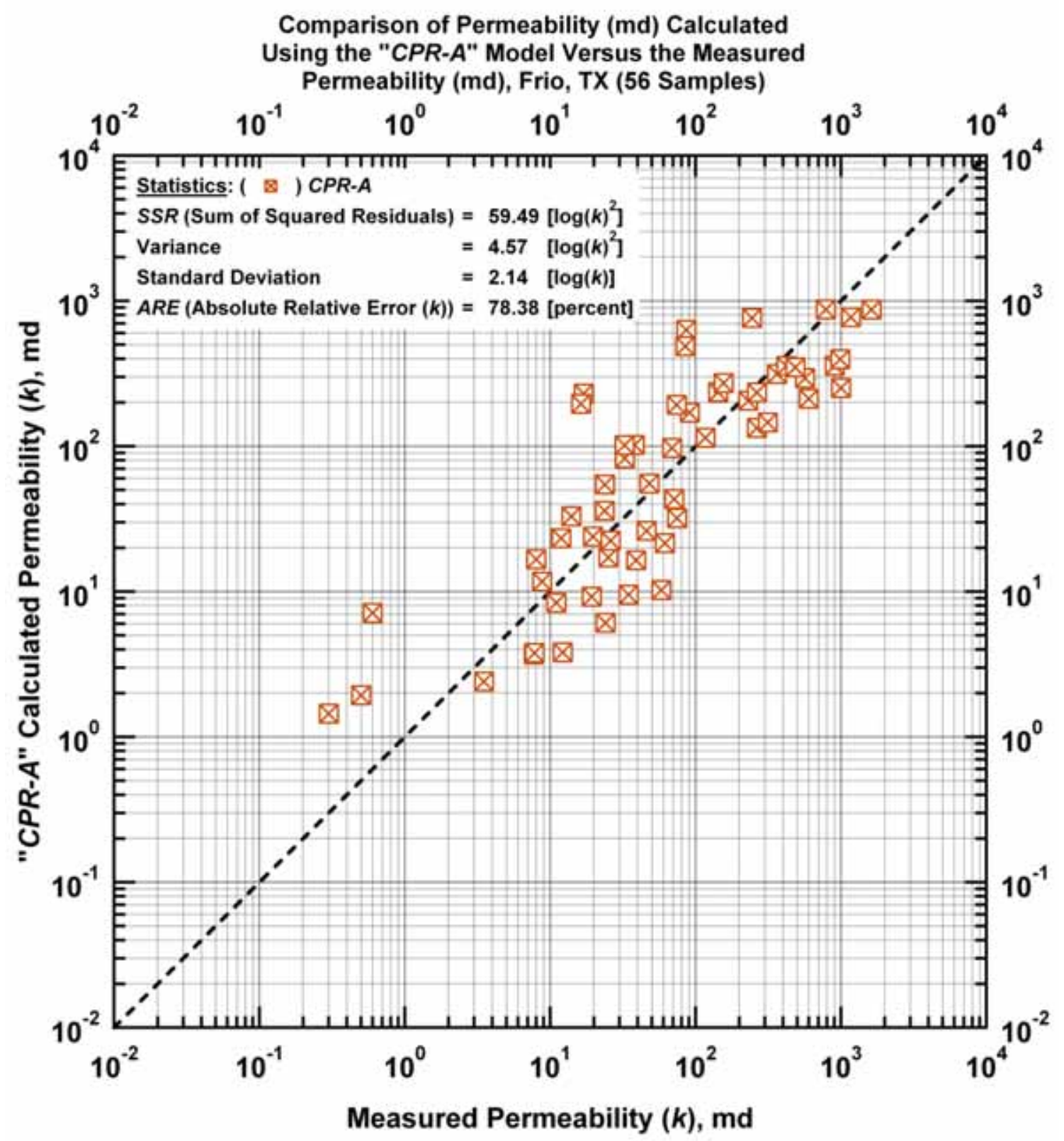

Figure H.2a - Model CPR-A: calculated versus measured permeability. [Case: Frio, TX (USA)] 


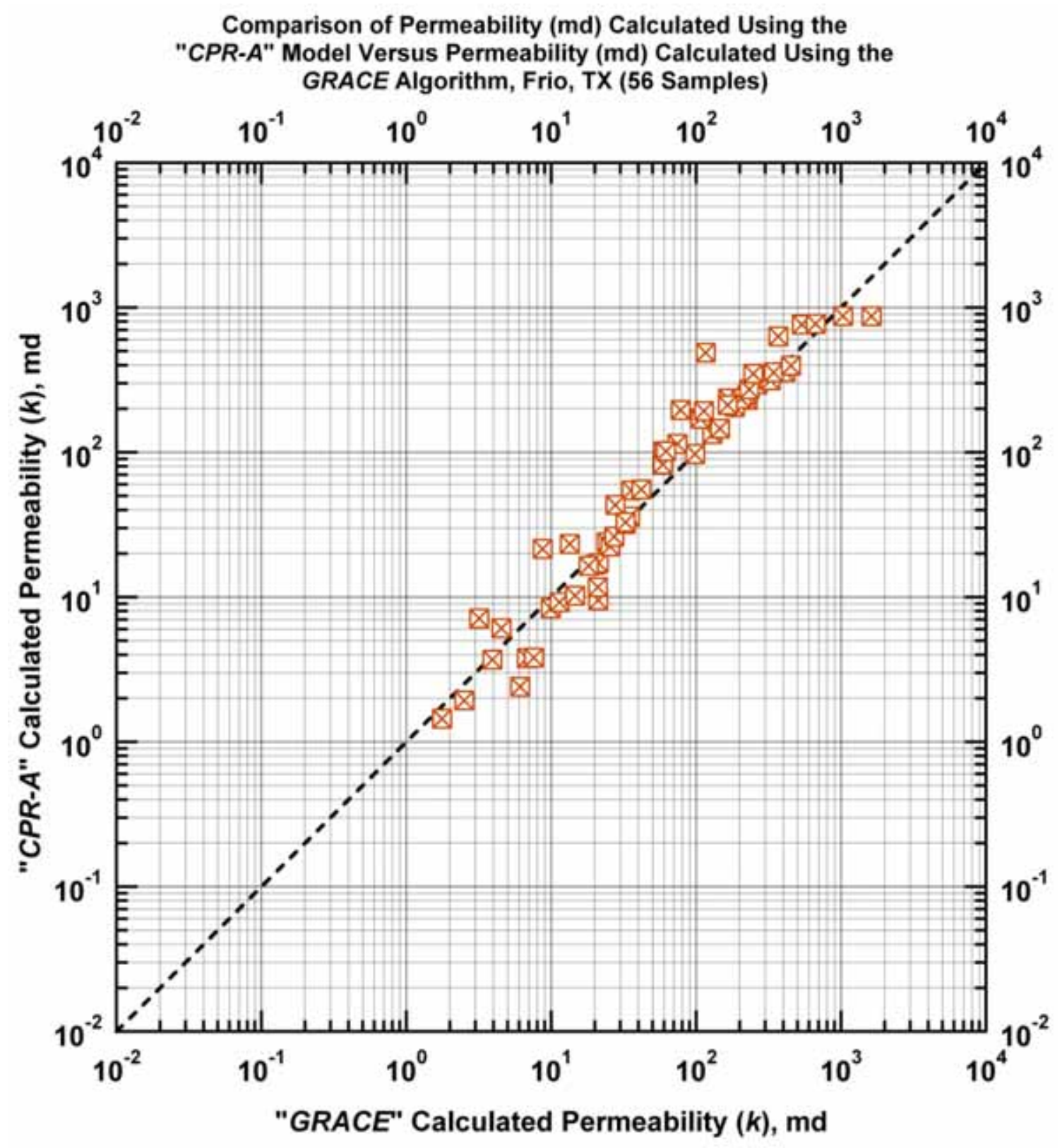

Figure H.2b - Model CPR-A: Permeability calculated using Model CPR-A versus permeability calculated using the GRACE Algorithm. [Case: Frio, TX (USA)] 


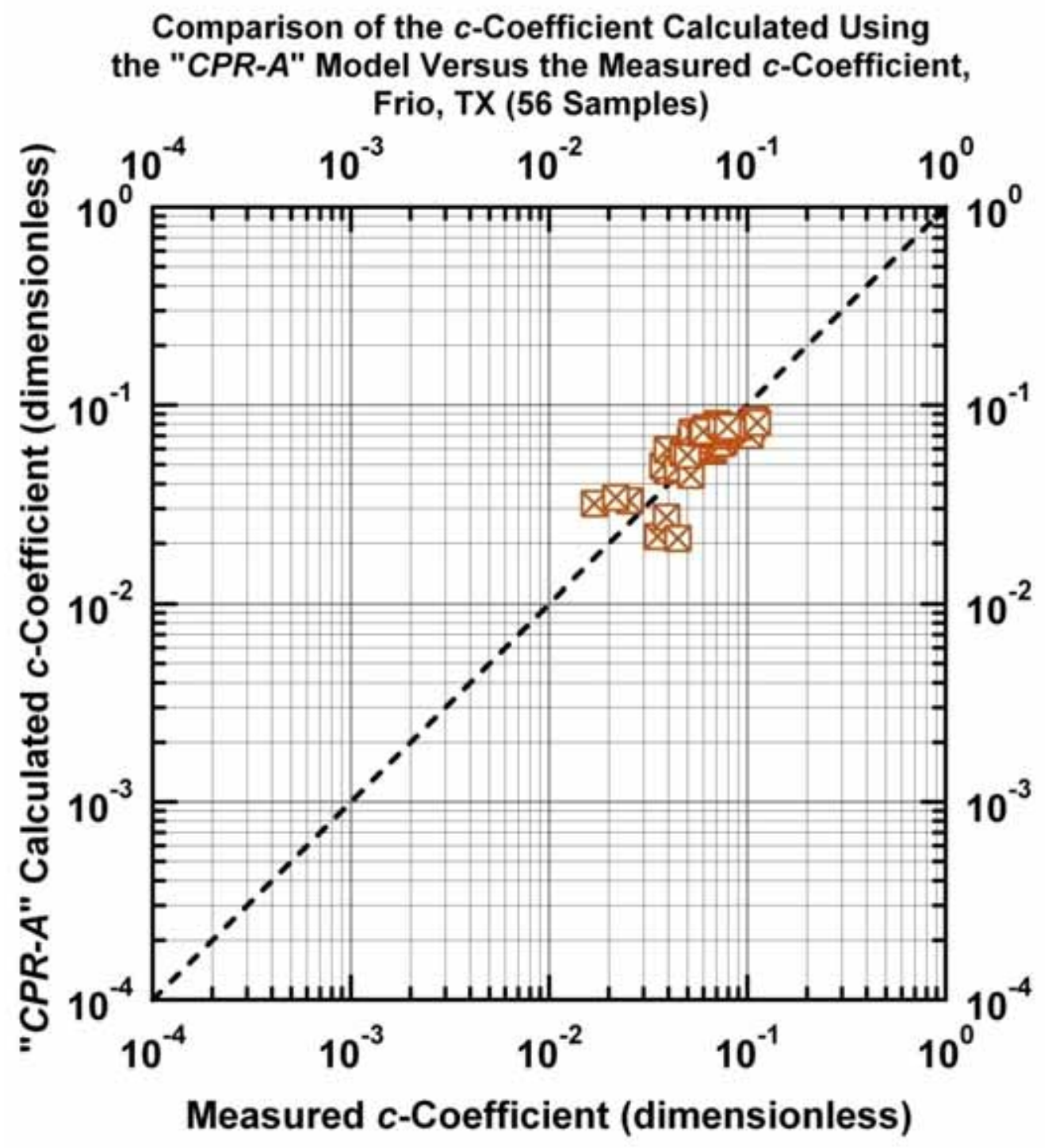

Figure H.2c - Model $C P R$ - $A$ : Calculated $c$-function values versus measured $c$-function values. [Case: Frio, TX (USA)] 


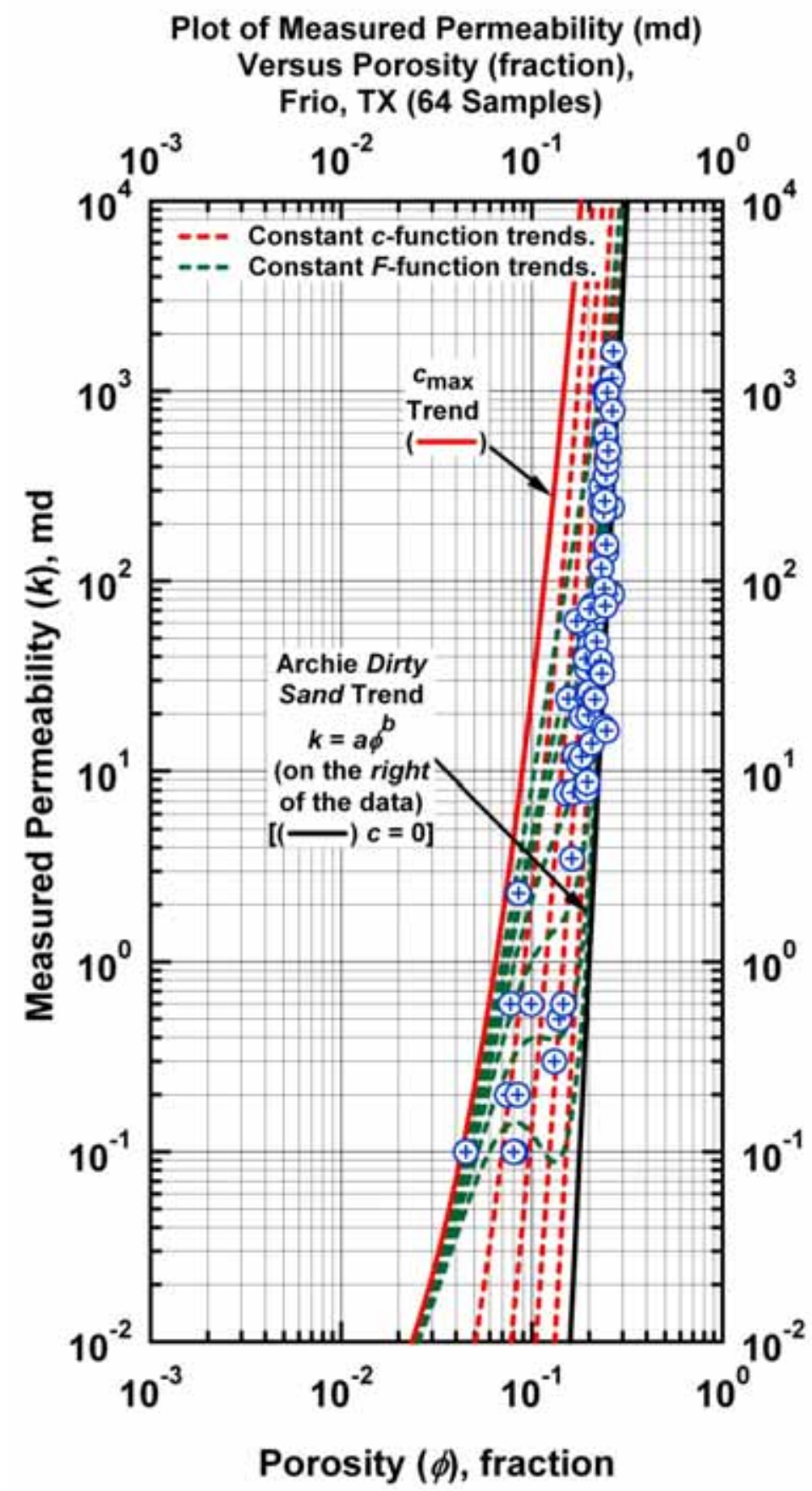

Figure H.3a - Model CPR-B: $\quad k=a(\phi+c)^{b} \quad c=c_{\max } \exp \left[-c_{1} \phi^{c_{2}} F^{c 3}\right]$ - "Clean Sand" Plot (log-log format) - Archie "Dirty Sand" trend is given by the straight-line trend at the far right of the data (power law model). [Case: Frio, TX (USA)] 


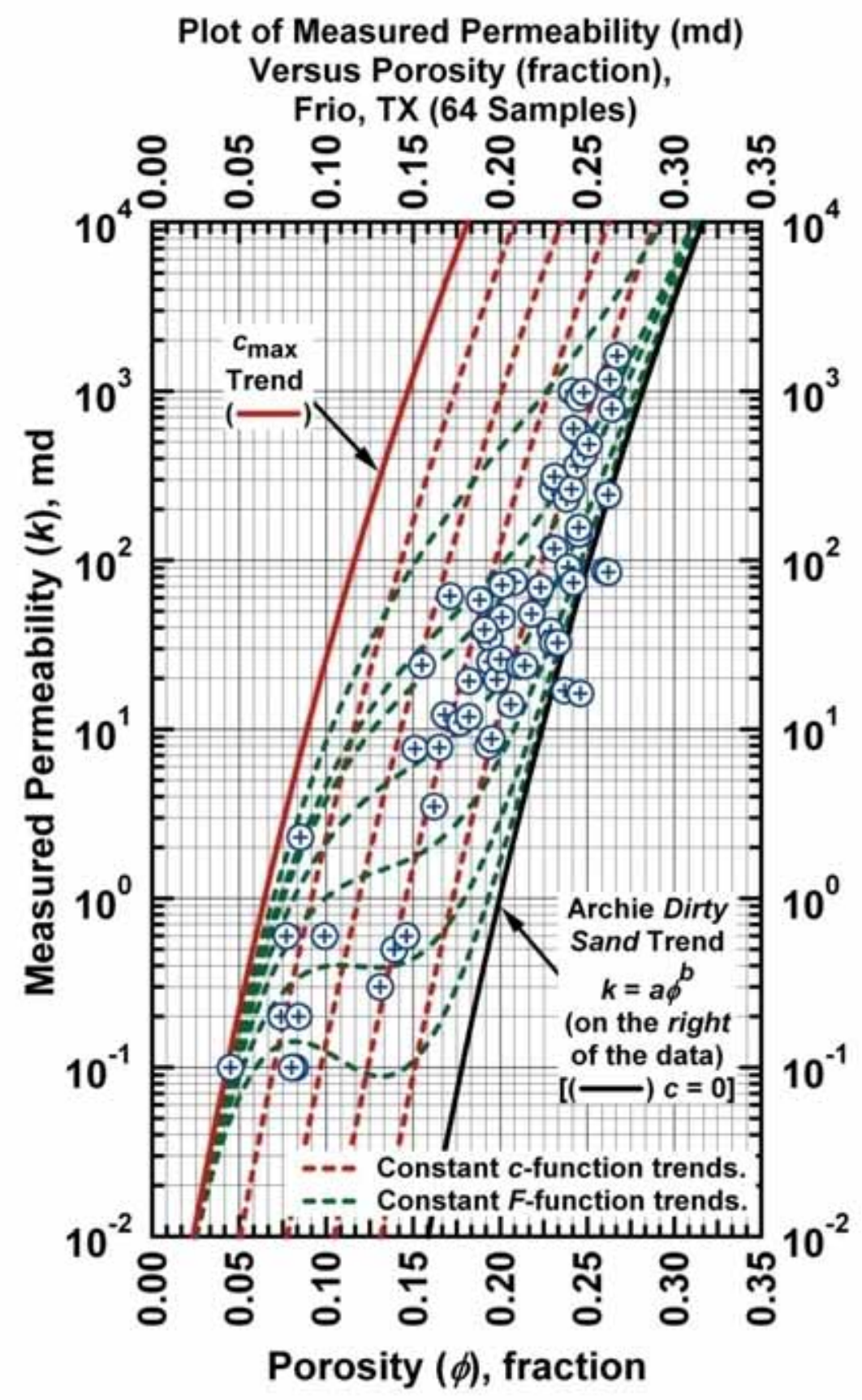

Figure H.3b - Model CPR-B: $k=a(\phi+c)^{b} \quad c=c_{\max } \exp \left[-c_{1} \phi^{c 2} F^{c_{3}}\right]$ — "Dirty Sand" Plot (semilog format) - Archie "Dirty Sand" trend is given by the curved trend at the far right of the data (power law model). [Case: Frio, TX (USA)] 


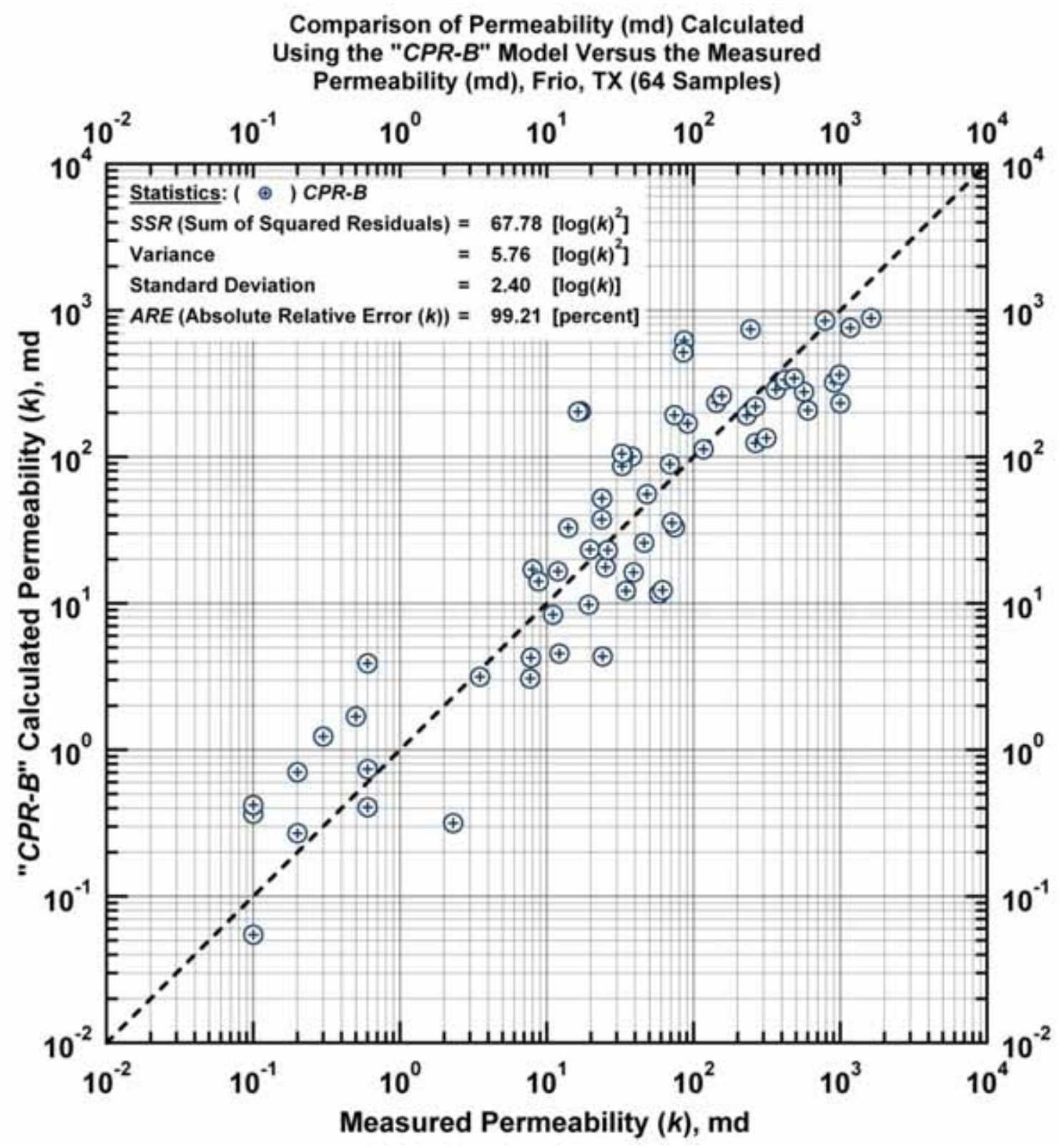

Figure H.4a - Model CPR-B: calculated versus measured permeability. [Case: Frio, TX (USA)] 


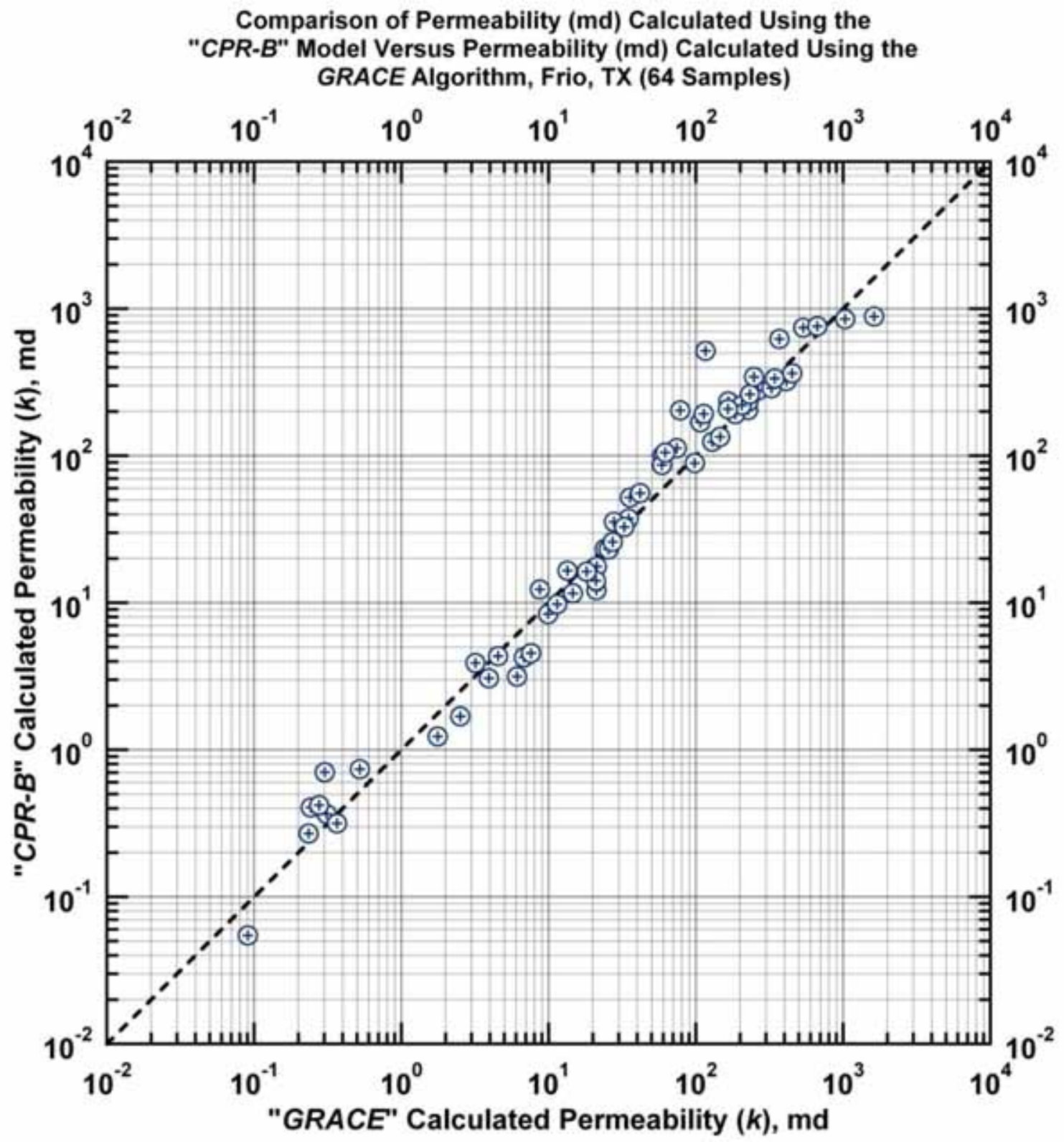

Figure H.4b - Model CPR-B: Permeability calculated using Model CPR-B versus permeability calculated using the GRACE Algorithm. [Case: Frio, TX (USA)] 


\section{Comparison of the $c$-Coefficient Calculated Using} the "CPR-B" Model Versus the Measured $c$-Coefficient, Frio, TX (64 Samples)

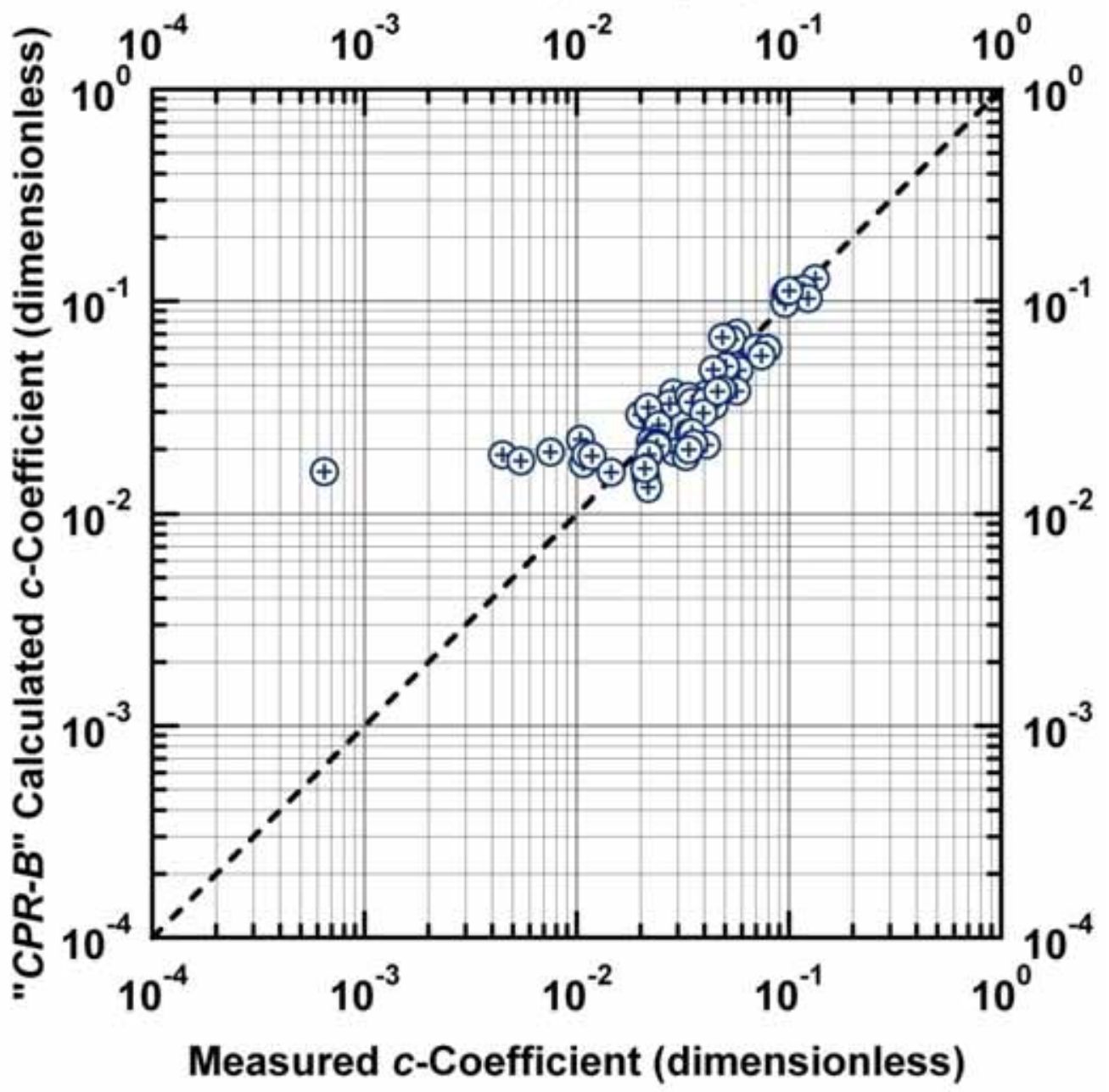

Figure H.4c - Model $C P R$-B: Calculated $c$-function values versus measured $c$-function values. [Case: Frio, TX (USA)] 


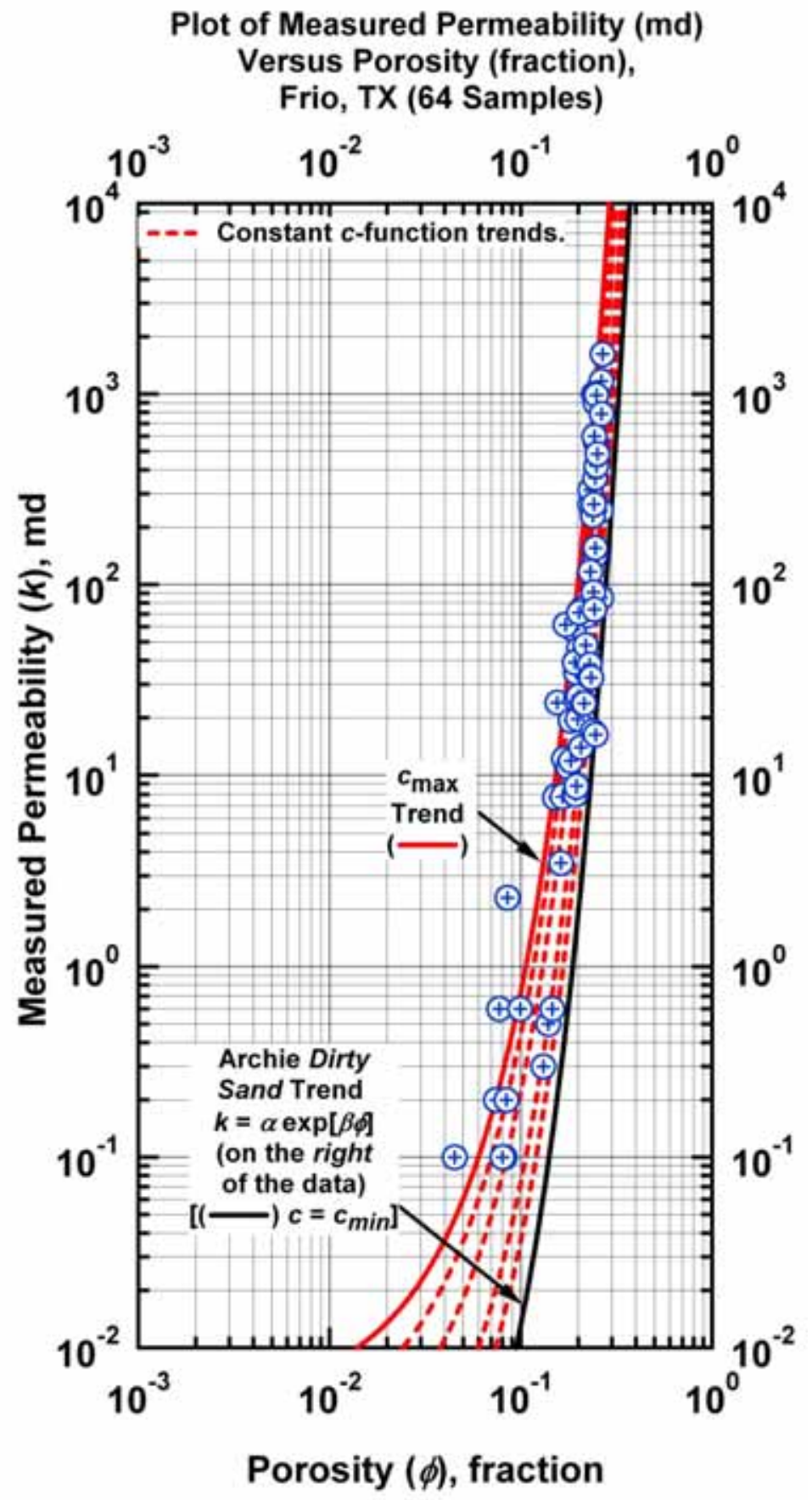

Figure H.5a - Model $C P R-C: \quad k=c \exp [\beta \phi] \quad c=c_{\max } \exp \left[-c_{1} \phi^{c 2} F^{c 3}\right]$ - "Clean Sand" Plot (log-log format) - Archie "Dirty Sand" trend is given by the curved trend at the far right of the data (exponential model). [Case: Frio, TX (USA)] 


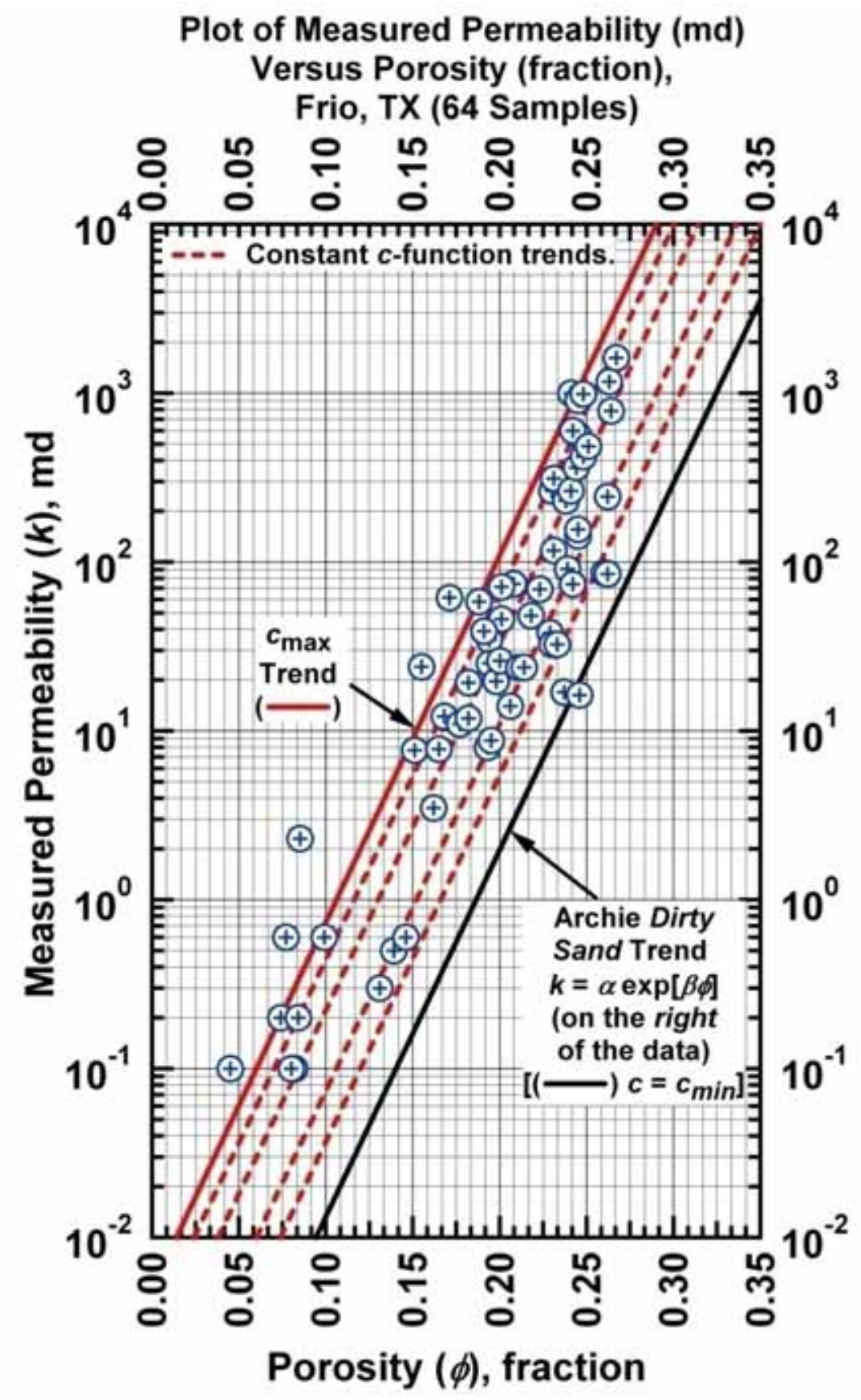

Figure H.5b - Model $C P R-C: \quad k=c \exp [\beta \phi] \quad c=c_{\max } \exp \left[-c_{1} \phi^{c_{2}} F^{c_{3}}\right]$ - "Dirty Sand" Plot (semilog format) - Archie "Dirty Sand" trend is given by the straight-line trend at the far right of the data (exponential model). [Case: Frio, TX (USA)] 


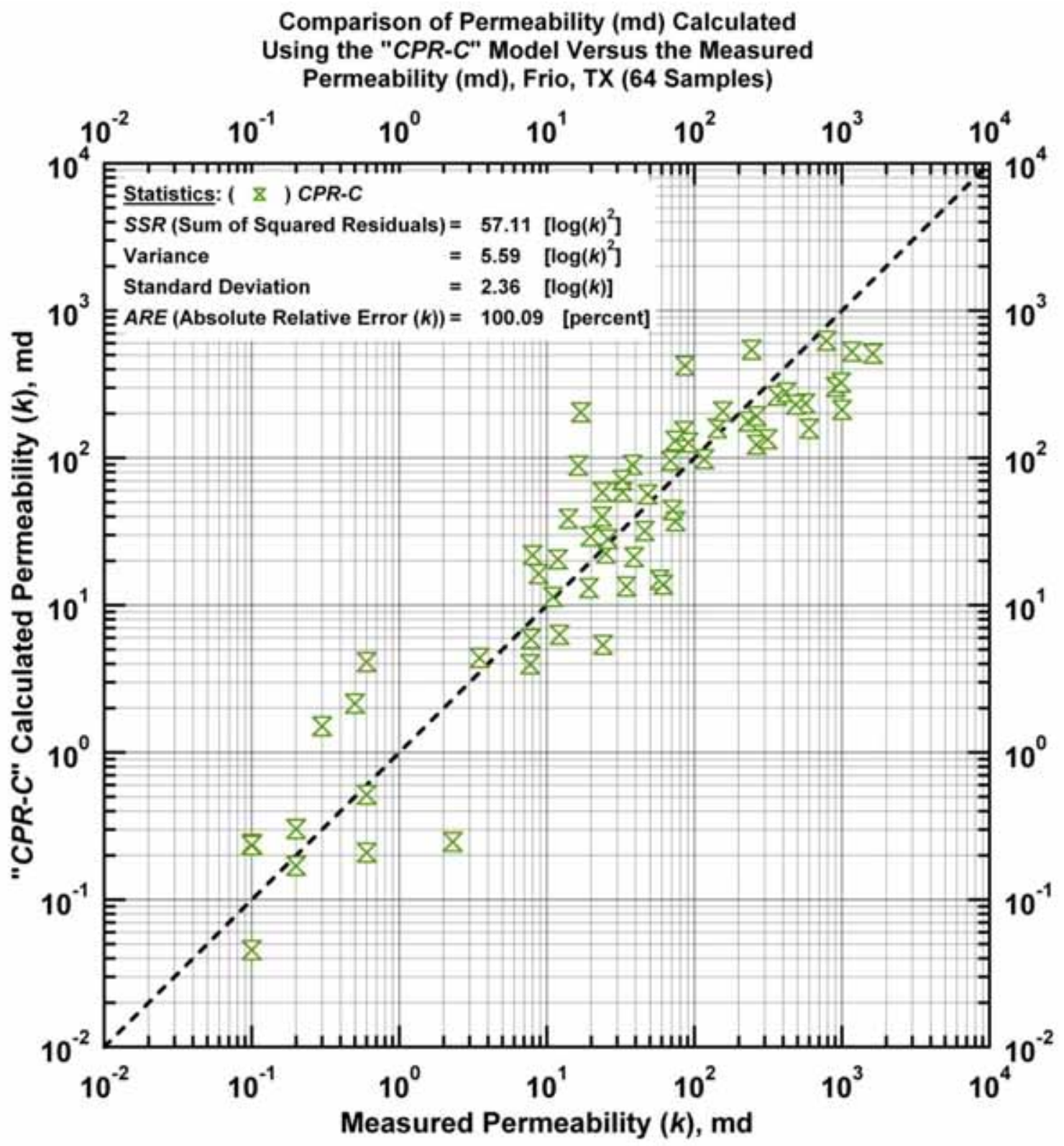

Figure H.6a - Model CPR-C: calculated versus measured permeability. [Case: Frio, TX (USA)] 


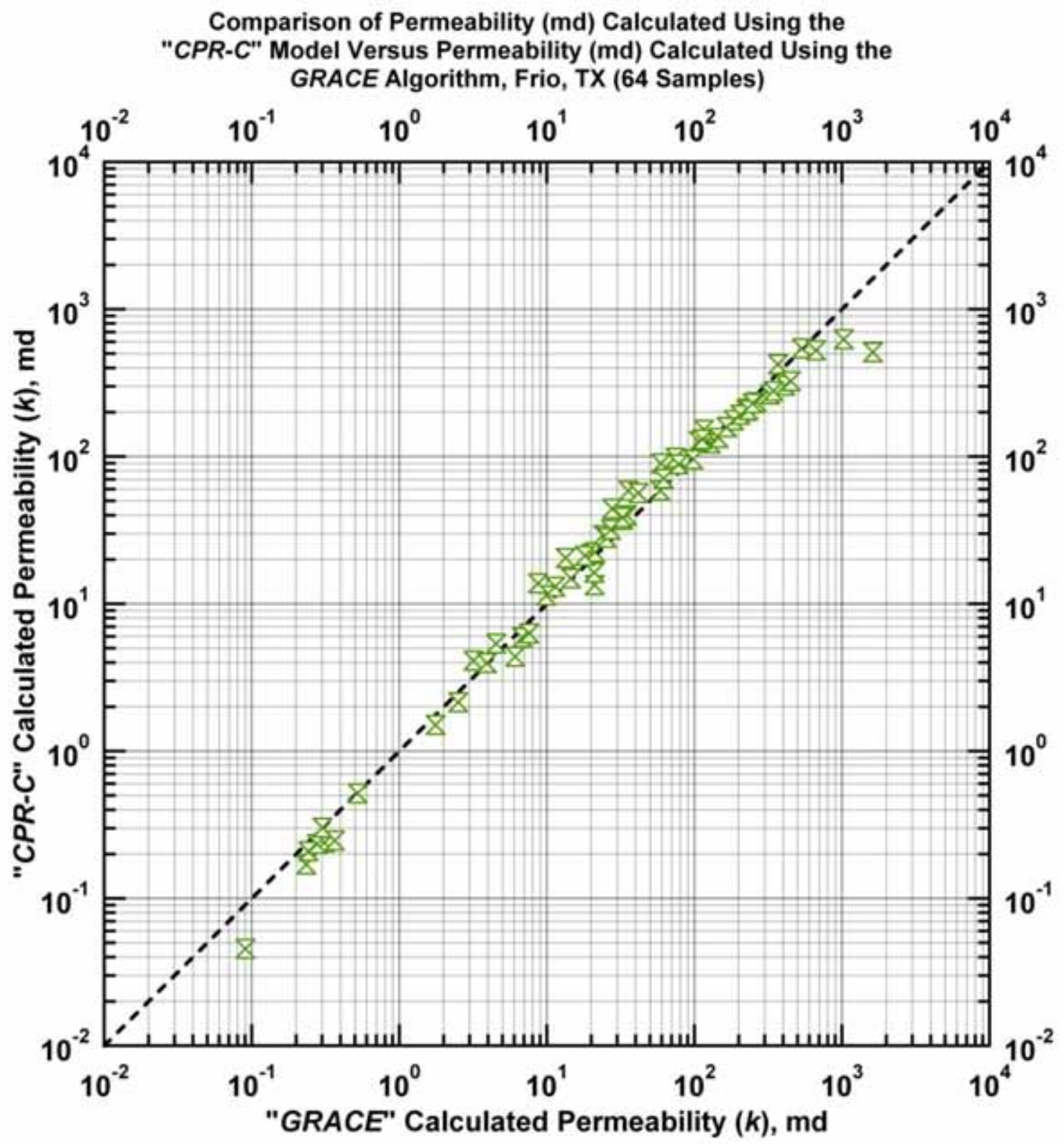

Figure H.6b - Model $C P R-C$ : Permeability calculated using Model $C P R-C$ versus permeability calculated using the GRACE Algorithm. [Case: Frio, TX (USA)] 


\section{Comparison of the $c$-Coefficient Calculated Using} the "CPR-C" Model Versus the Measured $c$-Coefficient, Frio, TX (64 Samples)

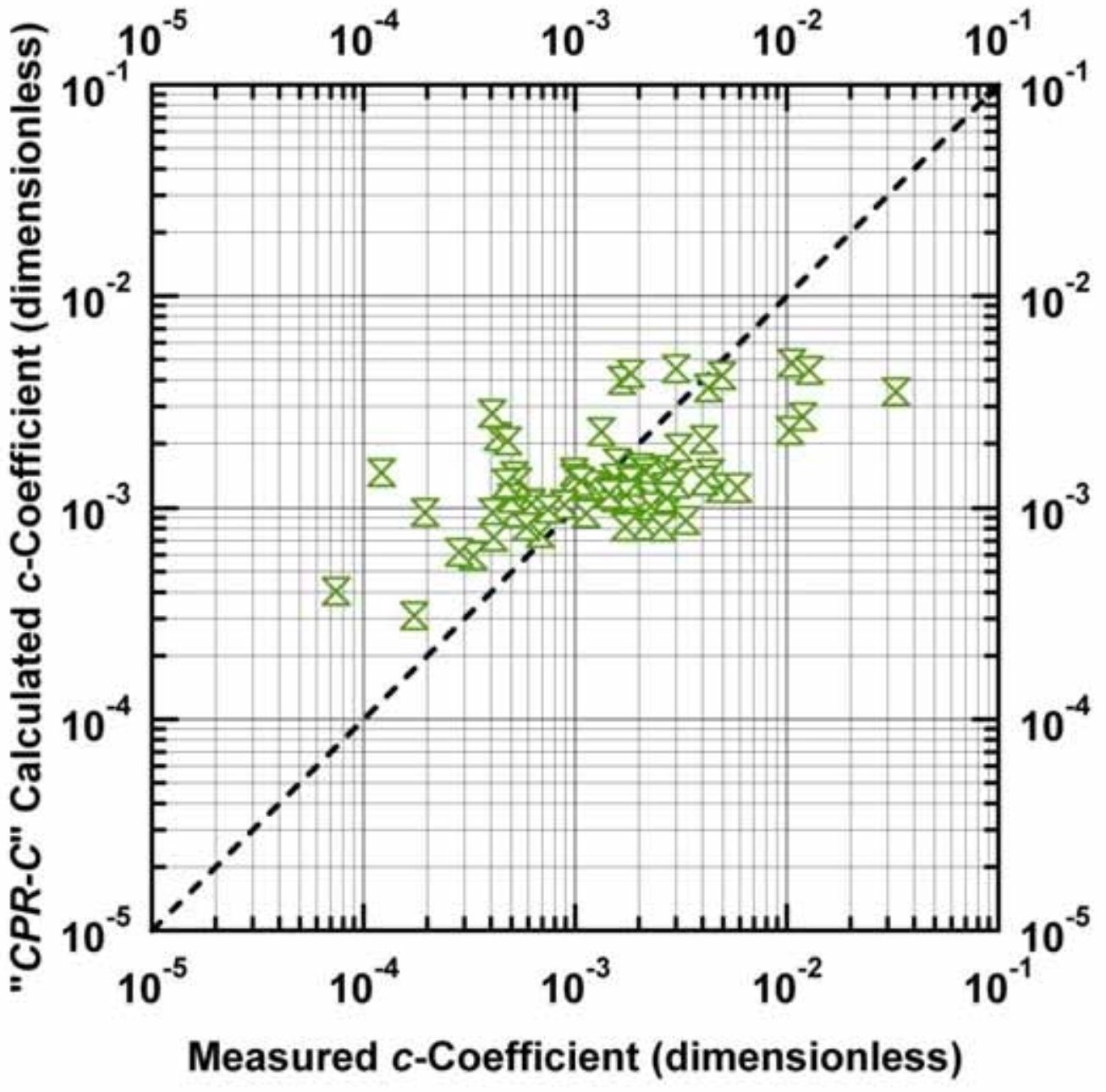

Figure H.6c - Model $C P R$ - $C$ : Calculated $c$-function values versus measured $c$-function values. [Case: Frio, TX (USA)] 


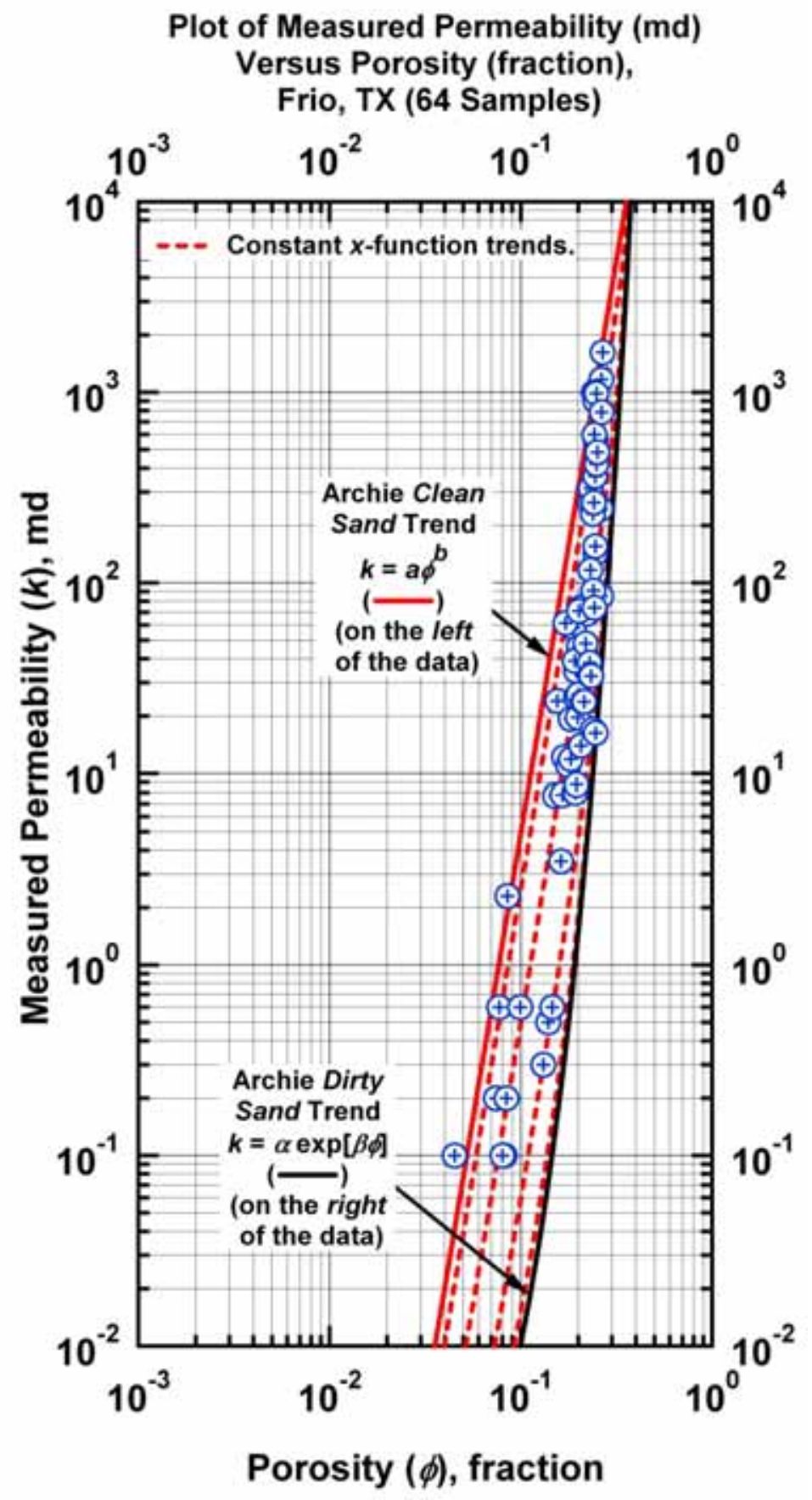

Figure H.7a - Model CPR-D: $k=x a \phi^{b}+(1-x) \alpha \exp [\beta \phi] \quad[0 \leq x \leq 1] \quad$ - Weighted Power Law-Exponential Model used to correlate permeability $(k)$ and porosity $(\phi)$. "Clean Sand" Plot - Archie "Clean Sand" trend is given by the straight-line trend at the far left of the data (power law model). [Case: Frio, TX (USA)] 


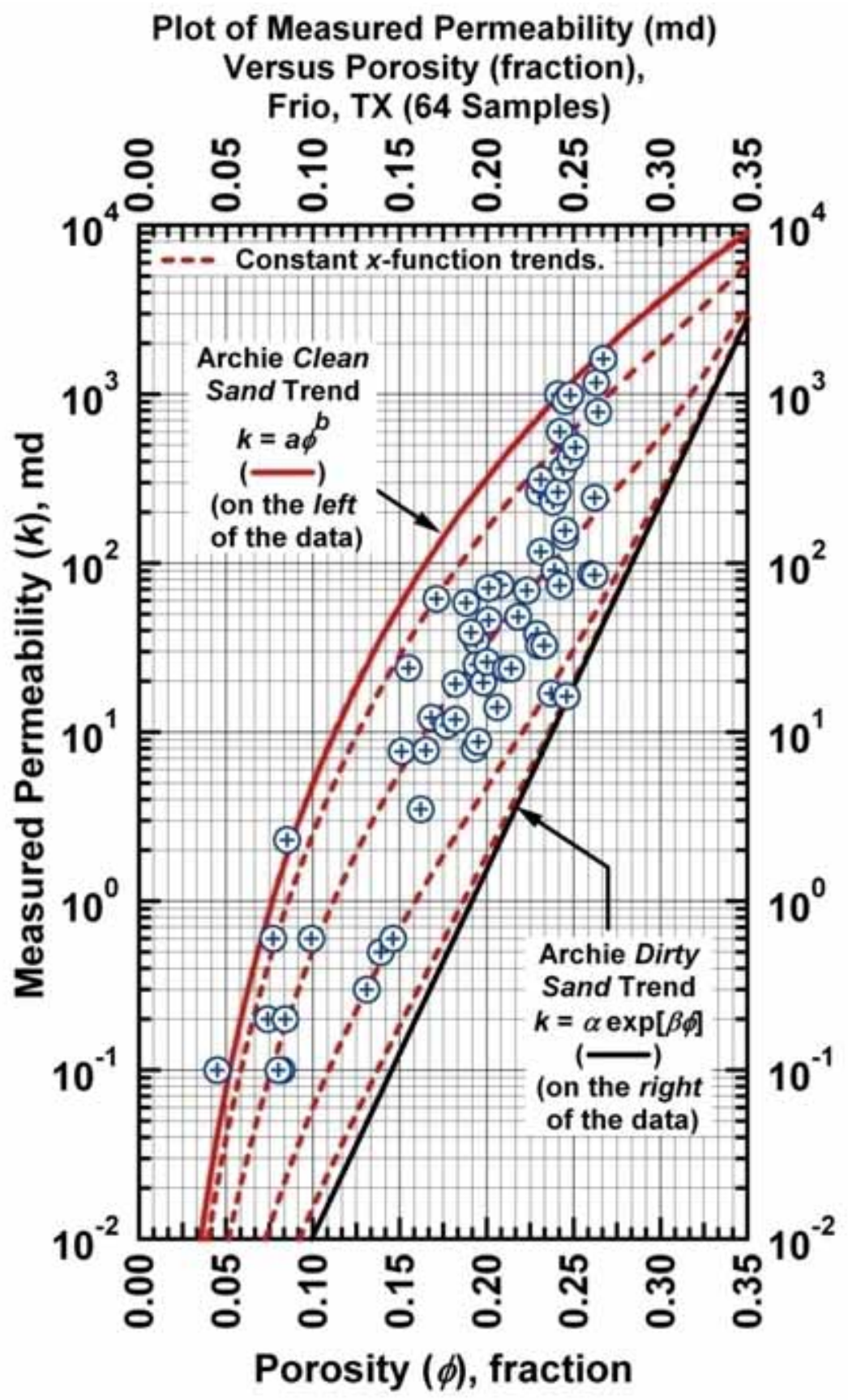

Figure H.7b - Model CPR-D: $k=x a \phi^{b}+(1-x) \alpha \exp [\beta \phi] \quad[0 \leq x \leq 1]$ - Weighted Power Law-Exponential Model used to correlate permeability $(k)$ and porosity $(\phi)$. "Dirty Sand" Plot - Archie "Dirty Sand" trend is given by the straight-line trend at the far right of the data (exponential model). [Case: Frio, TX (USA)] 


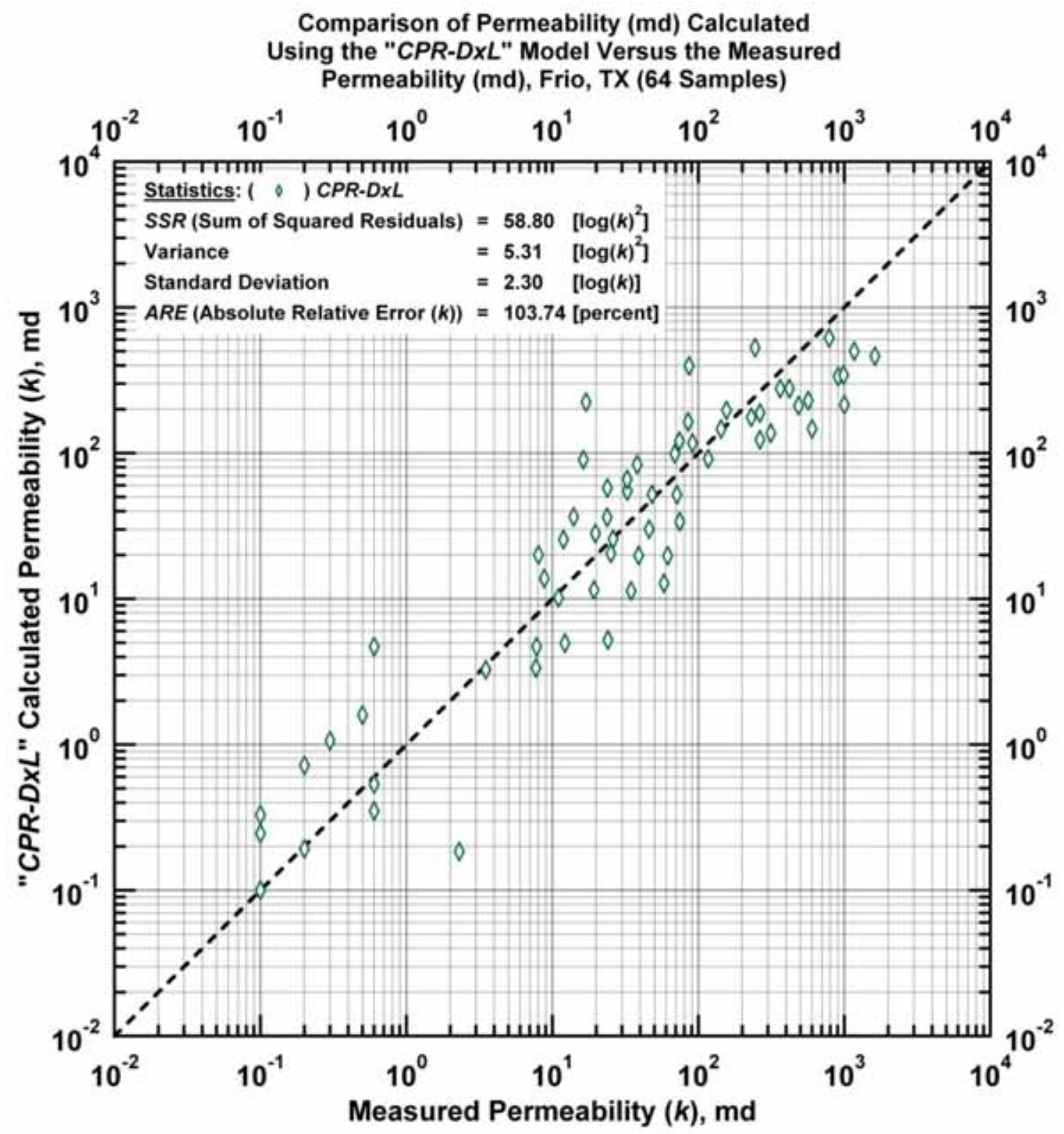

Figure H.8a - Model CPR-DxL: Calculated versus measured permeability. [Case: Frio, TX (USA)] 


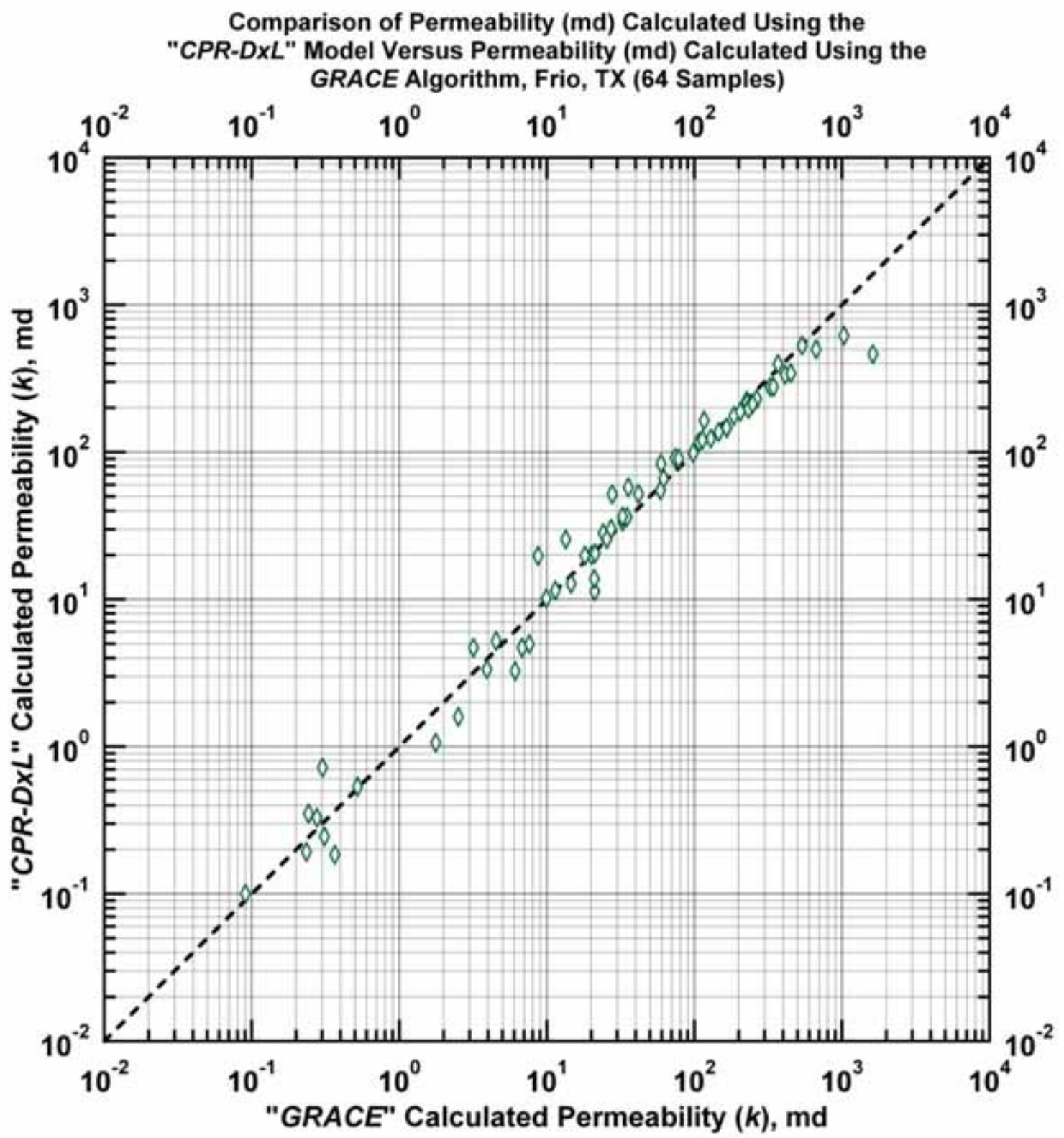

Figure H.8b - Model $C P R-D x L$ : Permeability calculated using Model $C P R-C$ versus permeability calculated using the GRACE Algorithm. [Case: Frio, TX (USA)] 


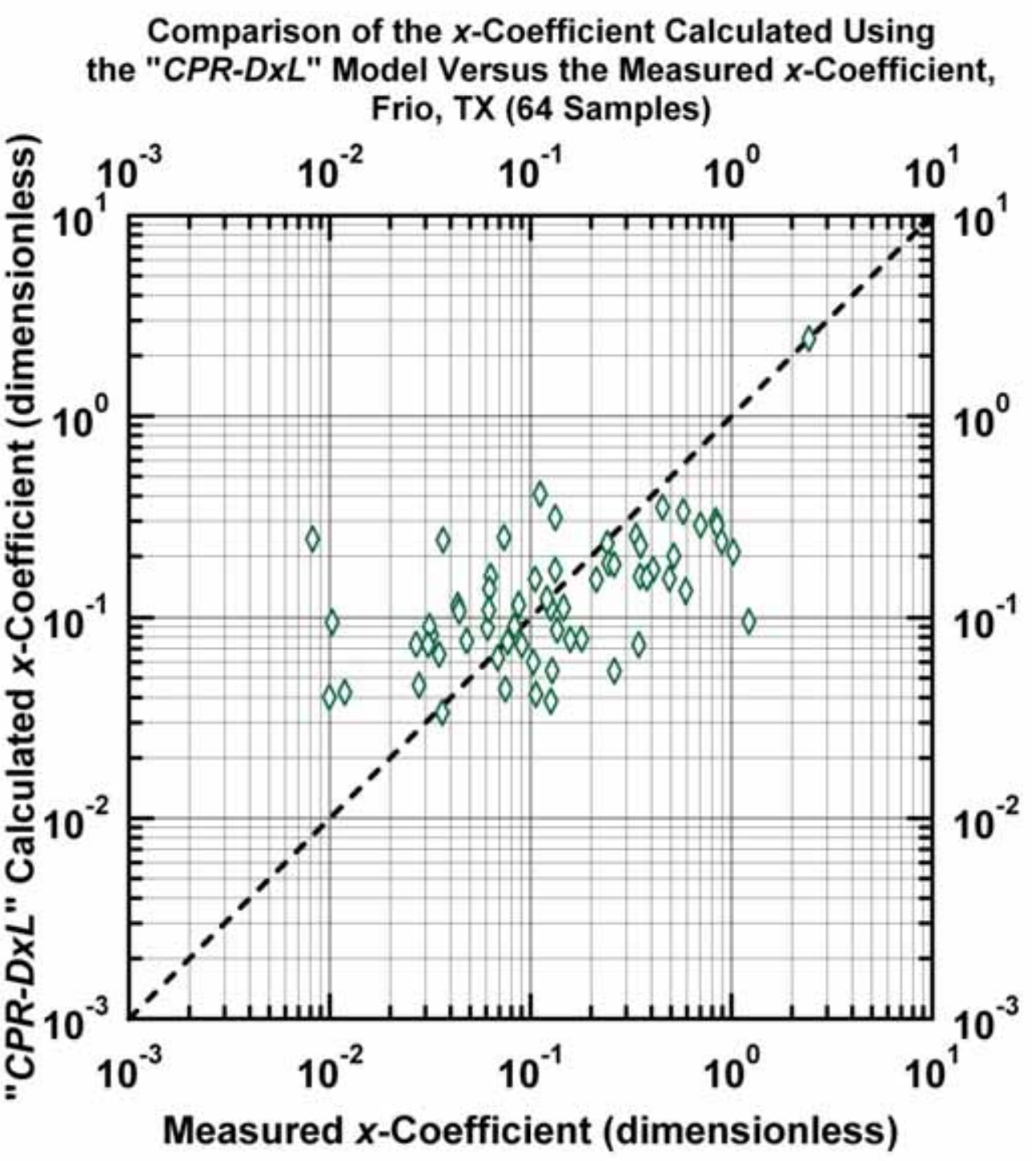

Figure H.8c - Model $C P R$ - $D x L$ : Calculated $x$-function weight values versus measured $x$ function weight values. [Case: Frio, TX (USA)] 


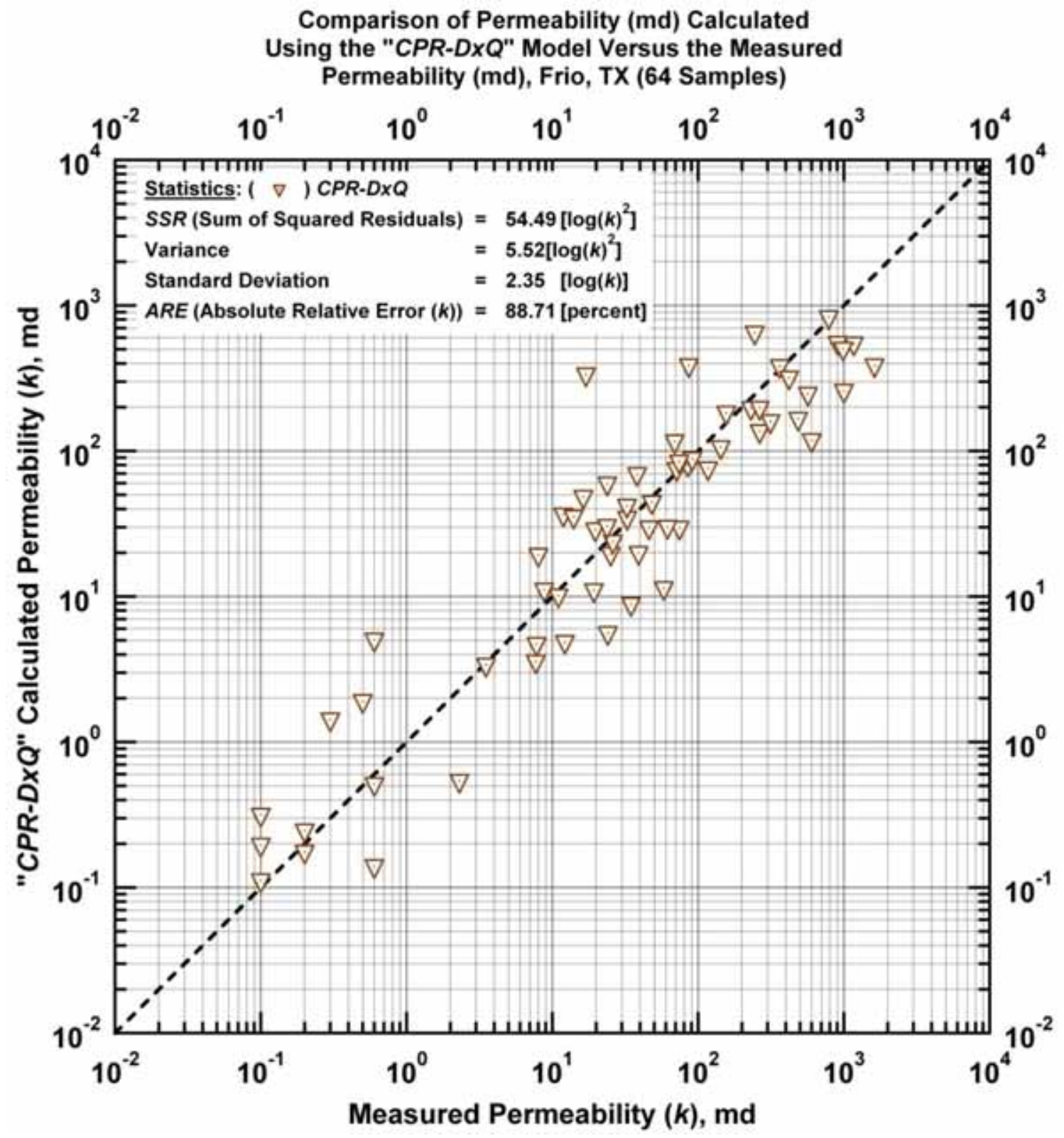

Figure H.9a - Model CPR-DxQ: Calculated versus measured permeability. [Case: Frio, TX (USA)] 


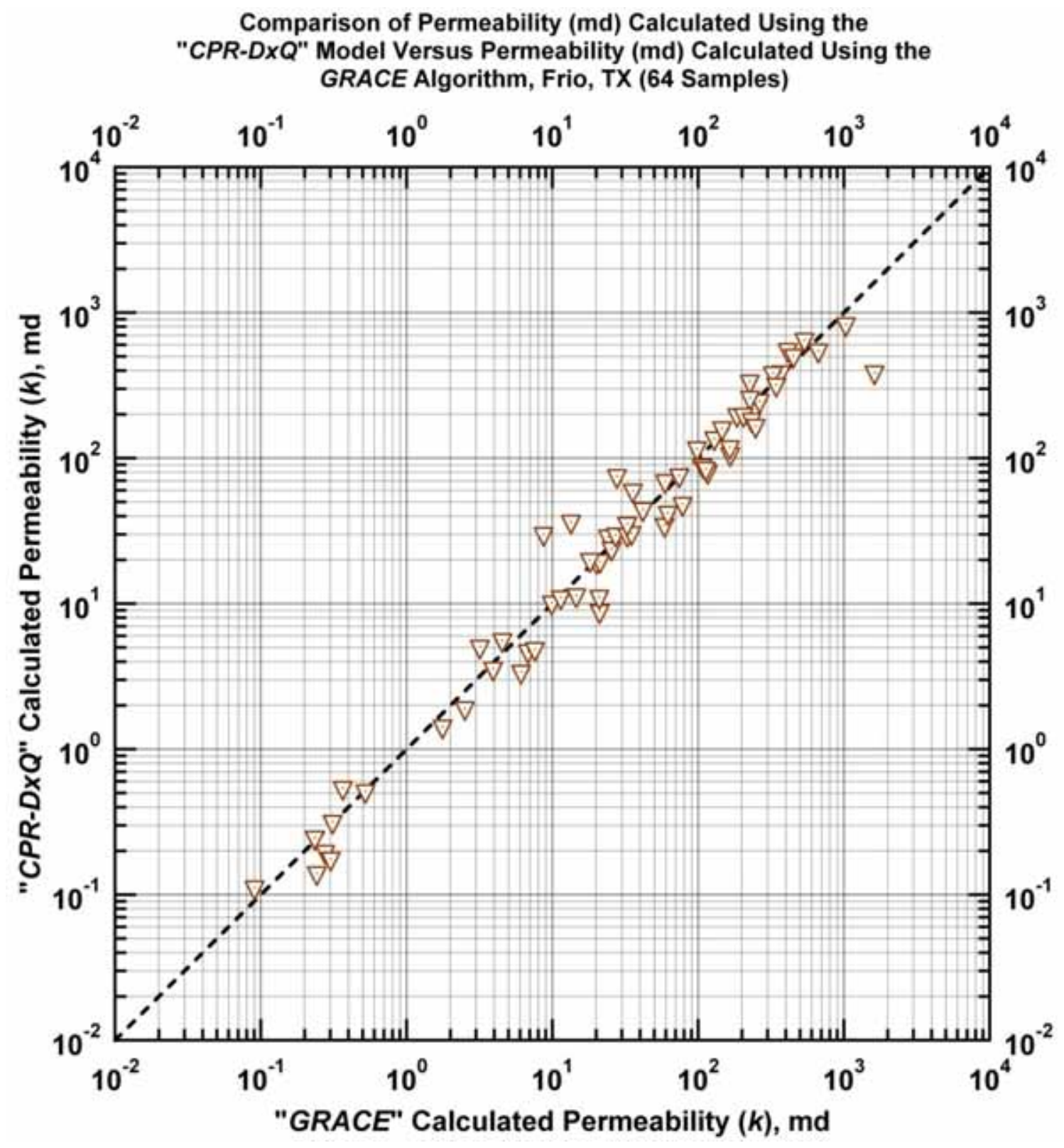

Figure H.9b - Model $C P R-D x Q$ : Permeability calculated using Model $C P R-C$ versus permeability calculated using the GRACE Algorithm. [Case: Frio, TX (USA)] 


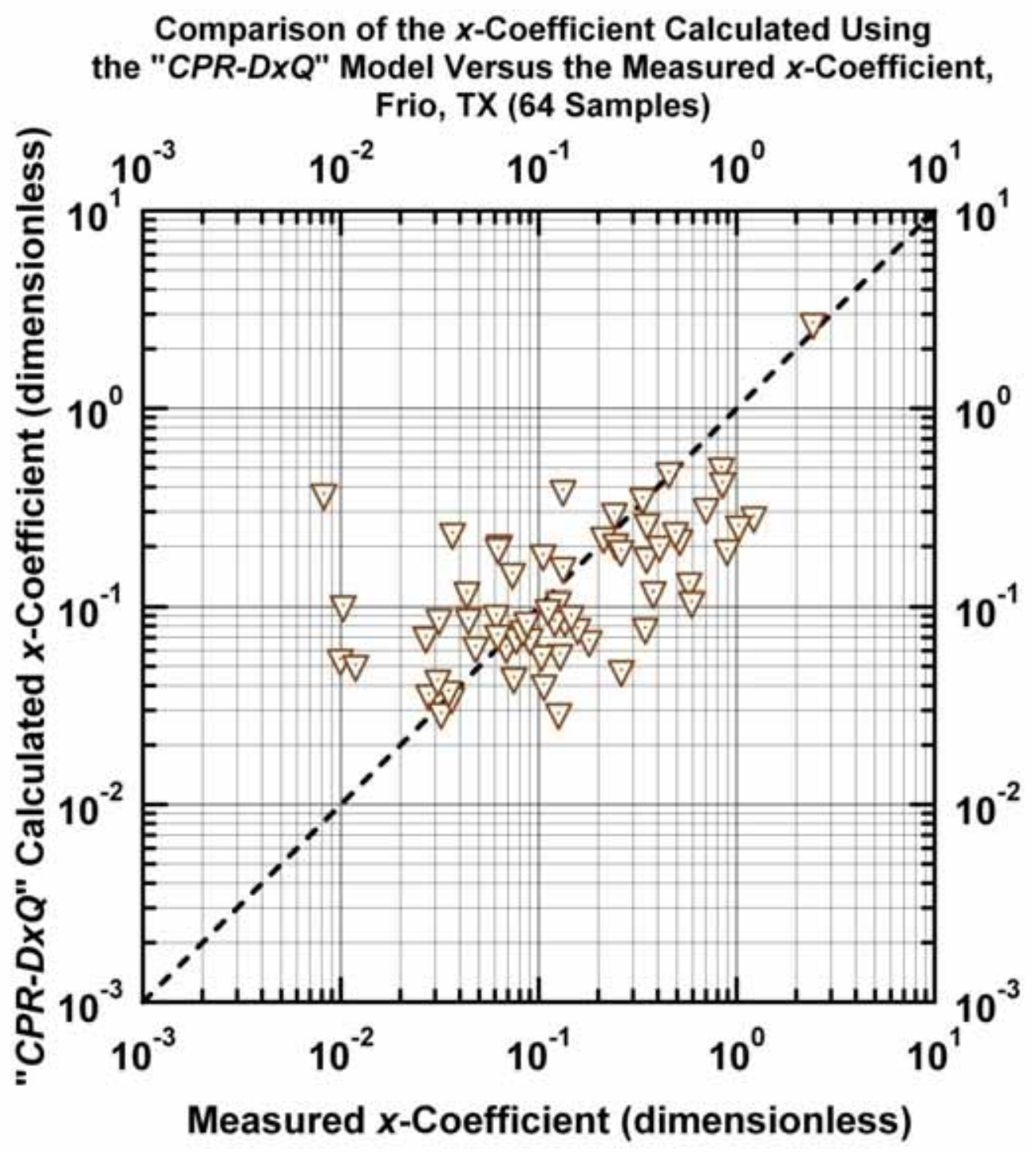

Figure H.9c - Model CPR-DxQ: Calculated $x$-function weight values versus measured $x$ function weight values. [Case: Frio, TX (USA)] 


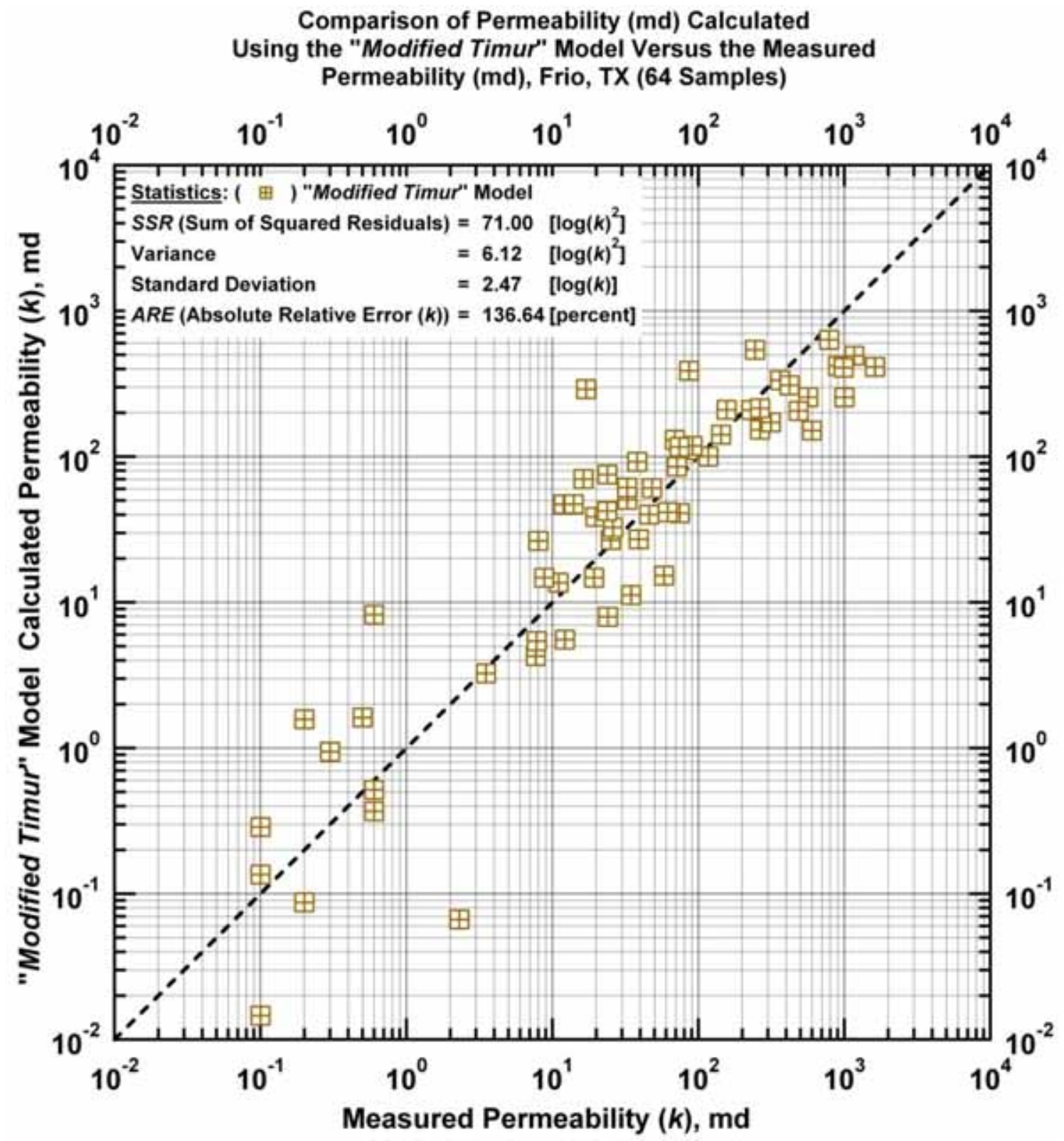

Figure H.10a - "Modified Timur Model:" Calculated versus measured permeability. [Case: Frio, TX (USA)] 


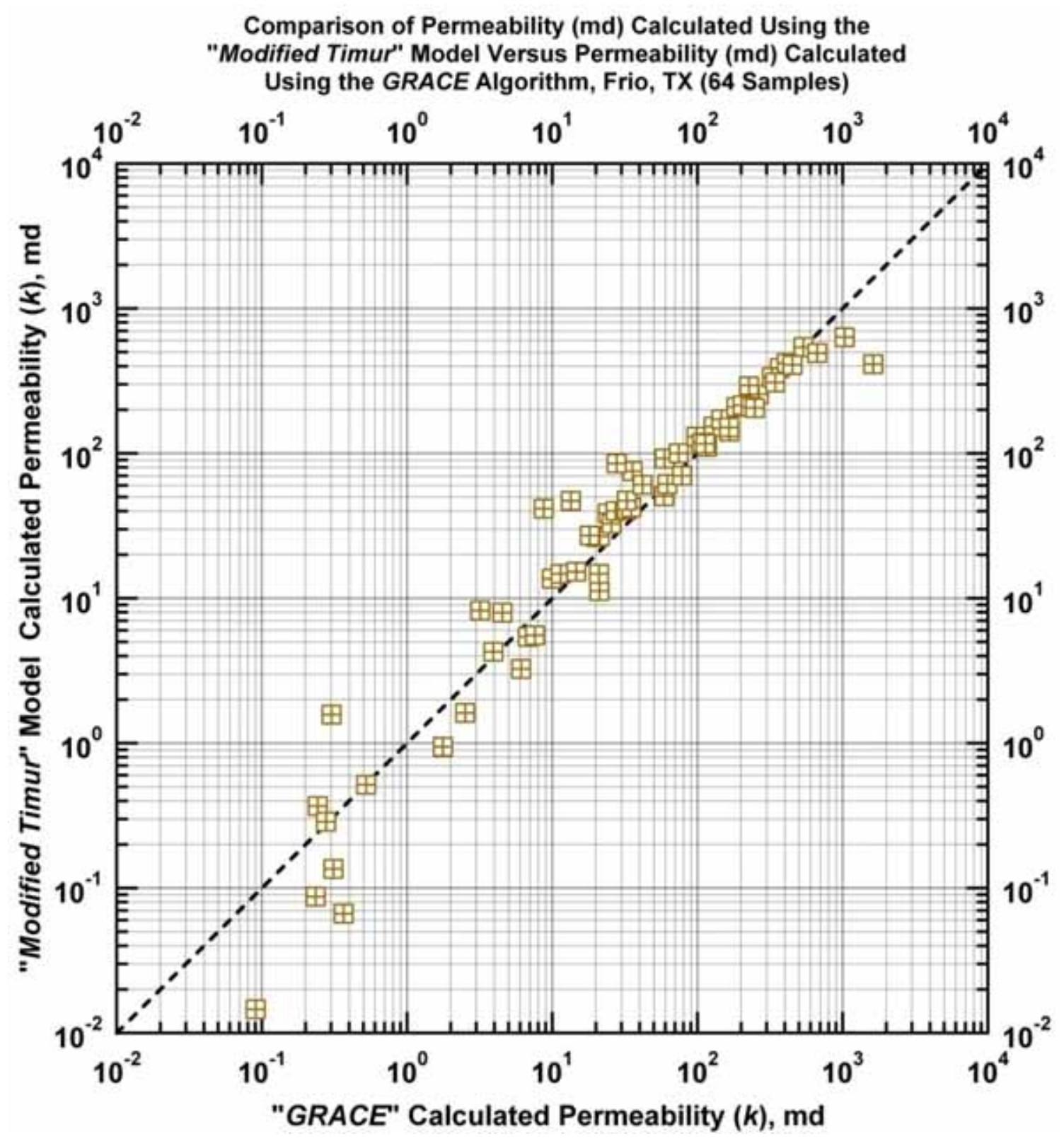

Figure H.10b - "Modified Timur Model:" Permeability calculated using the "Modified Timur Model" versus permeability calculated using the GRACE Algorithm. [Case: Frio, TX (USA)] 


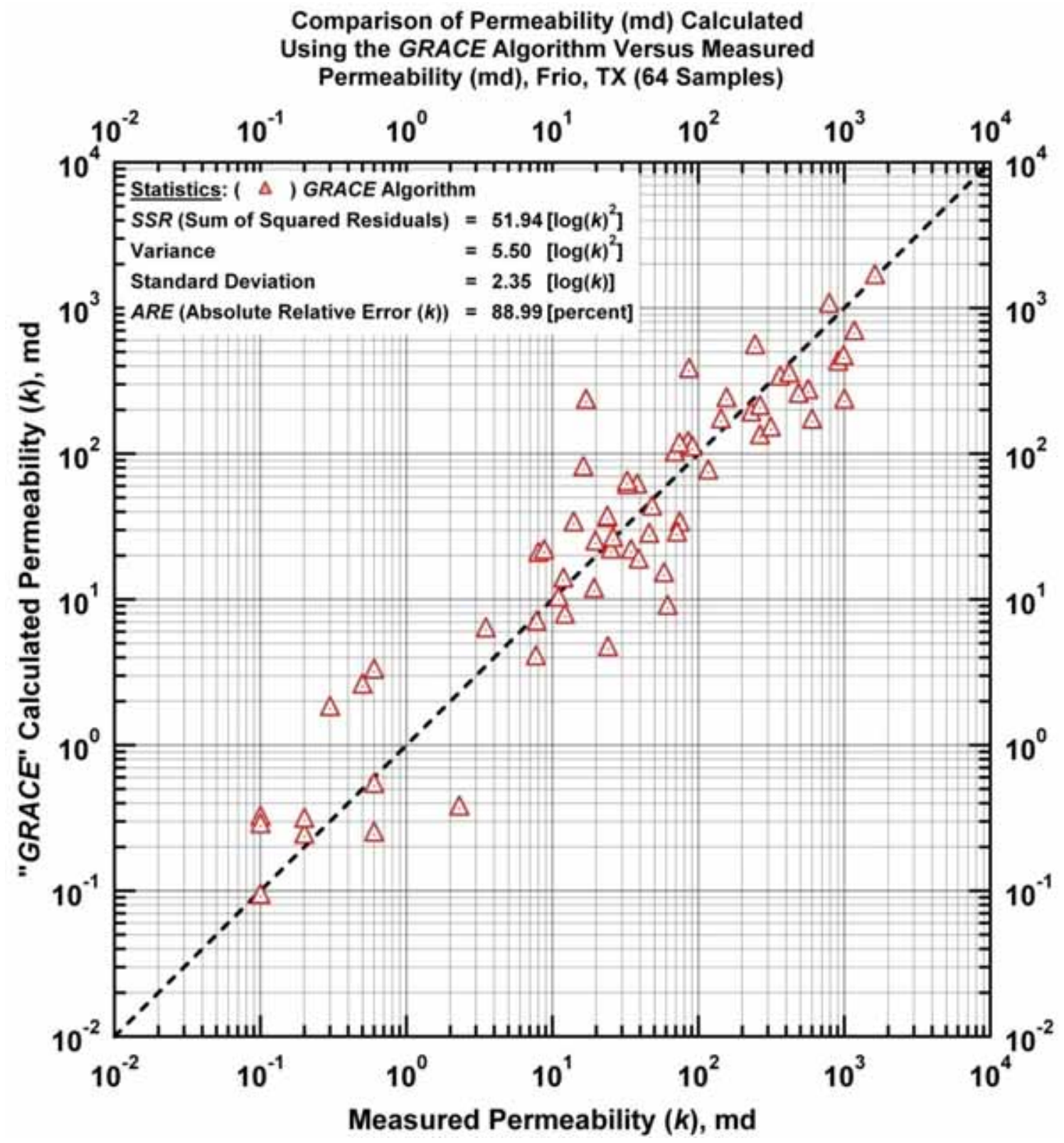

Figure H.11 - GRACE Model: Calculated versus measured permeability. [Case: Frio, TX (USA)] 


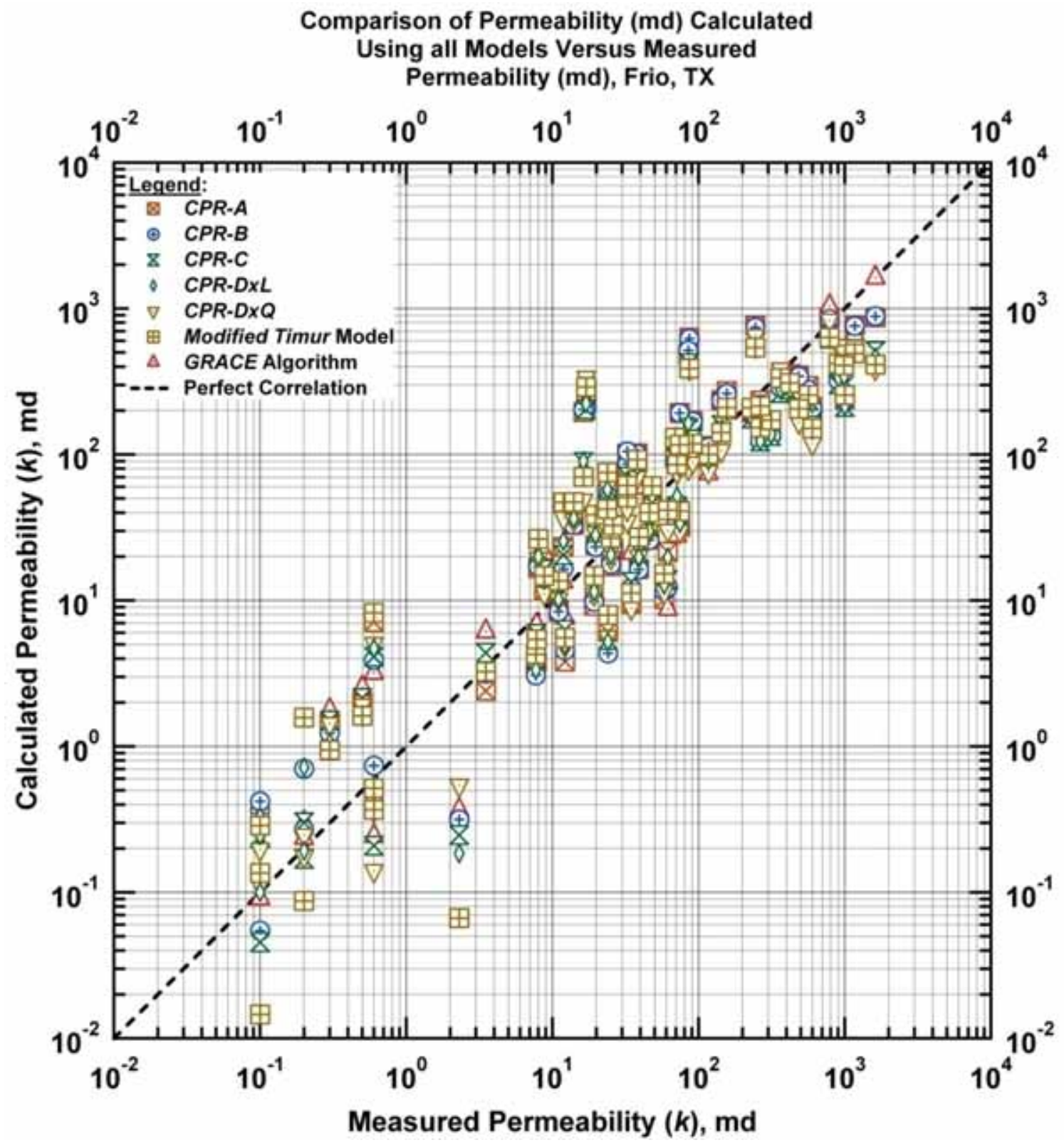

Figure H.12 - All Models: Calculated versus measured permeability. [Case: Frio, TX (USA)] 


\section{APPENDIX I \\ RESULTS FOR LOWER WILCOX SHALY SANDSTONE \\ (TEXAS - USA)}

In this Appendix we present the "Lower Wilcox" Well, located in South Texas (USA) - this is a tight gas reservoir system comprised of shaly (clastic) sediments (Ellis, 1987).

\section{Model CPR-A: (Appendix I)}

The "characteristic permeability relation" (or CPR) Model CPR-A (i.e., the modified Archie "Clean Sand" Model) is given as:

$$
k=a(\phi-c)^{b} \quad c=c_{\max } \exp \left[-c_{1} \phi^{c 2} F^{c_{3}}\right]
$$

Where a plot of $\log (k)$ versus $\log (\phi)$ is used to graphically calibrate EI. I-1 in terms of the coefficients $a, b$, and $c_{\max }$. Fig. I.1 shows that the $C P R-A$ model performs quite well for this case. Figs. I.1a and $\mathbf{b}$ show the log-log and the semilog format of permeability versus porosity, respectively. In Fig. I.2 we provide the "error" plots for this case, where the measured and computed data are compared systematically. In Fig. I.1a we note a good correlation of the results obtained using Model $C P R-A$ versus the measured permeability data - we observe good agreement with the perfect correlation trend (i.e., the 45degree line). In Fig. I.2b we provide a comparison with the GRACE algorithm correlation (Xue et al. 1997)), and we note that the CPR-A model results correlate very well with the GRACE algorithm results for this case. Finally, in Fig. I.2c we present the calculated and measured $c$-function - and again we note a reasonable correlation of the data about the perfect correlation trend.

\section{Model CPR-B: (Appendix I)}

The "characteristic permeability relation" (or CPR) Model CPR-B (i.e., the modified Archie "Dirty Sand" Model (power-law basis)) is given as:

$$
k=a(\phi+c)^{b} \quad c=c_{\max } \exp \left[-c_{1} \phi^{c_{2}} F^{c_{3}}\right]
$$

Where a plot of $\log (k)$ versus $\log (\phi)$ is used to graphically calibrate Eq. I-2 in terms of the coefficients $a, b$, and $c_{\max }$. The results for this case are presented in Figs. I.3 and 4. In Fig. I.3a (log-log format) we note that a very distinct "envelope" is formed by the CPR-B model, essentially all of the data on the right and left flanks are well-matched (i.e., the "dirty sand" power law relation (applied on the right portion of the data) and its correction function (applied on the left portion of the data). Similarly, in Fig. I.3b (semilog format), we observe an excellent distribution of the power law model across the body of the data. The error analyses for this case are shown in Fig. I.4. In Fig. I.4a we present the results obtained using Model $C P R-B$ versus the measured permeability data and in this case there is good agreement with the 
perfect correlation trend (i.e., the 45-degree line) — a few data points are out-lying, mostly in the middle of the trend. We provide a comparison with the GRACE algorithm correlation in Fig. I.4b - the results using Model CPR-B and the GRACE algorithm compare very well for this case. We compare the computed and data-derived values of the $c$-function for this case in Fig. I.4c and we note a reasonably "tight" correlation, except for the very lowest values of the $c$-function. In summary, we believe that Model $C P R-B$ is a very effective correlation model for this particular data case.

\section{Model CPR-C: (Appendix I)}

The "characteristic permeability relation" (or CPR) Model CPR-B (i.e., the modified Archie "Dirty Sand" Model (exponential basis)) is given as:

$$
k=c \exp [\beta \phi] \quad c=c_{\max } \exp \left[-c_{1} \phi^{c 2} F^{c 3}\right]
$$

Where a plot of $\log (k)$ versus $\phi$ is used to graphically calibrate Eq. I-3 in terms of the coefficients $-\beta$ and $C_{\text {max }}$. Model $C P R-C$ is fundamentally different than Models $C P R-A$ and $C P R-B$, as the basis for Model $C P R-C$ is an exponential function, not a power law relation. In Figs. I.5 and 6 we present the results of applying Model $C P R-B$ for this case, and in general, we observe a very consistent performance of Model $C P R-B$. Fig. I.5b illustrates the excellent conformance of Model $C P R-C$ for this case - again recalling that this model has an exponential relationship with porosity as its basis.

In Figs. I.6a, I.6b, and I.6c we review the error analyses plots for this case, and we note good performance (visually) in terms of the correlation of the results using Model CPR-C. We do note that in this case, the overall (statistical) error is somewhat high, but we have to remember that we are proposing "characteristic models" in this work, and as such, this case appears (again, based on visual inspections) to have good clustering of the correlated results in terms of permeability (Figs. I.6a and I.6b). In this case, the $c$-function (in Eq. I-3) is not a correction term, but rather the instantaneous intercept for the exponential basis. The correlation of the $c$-function (see Fig. I.6c) appears to be consistent - but scatter and a slight off-center trend is evident. However, as we are approaching this work from the perspective that the primary value of the work is the characteristic model, not the statistical "best fit" correlation, we believe that the $C P R-C$ model has performed well for this case, and Fig. I.6 confirms the value of this model as a "characteristic relation" for permeability.

\section{Model CPR-D: (Appendix I)}

The "characteristic permeability relation" (or $C P R$ ) Model CPR-D (i.e., the weighted power law-exponential model) is given in its fundamental form as:

$$
\left.k=x a \phi^{b}+(1-x) \alpha \exp [\beta \phi] \quad[0 \leq x \leq 1]\right] .
$$

For Eq. I-4 there are two proposed models for the $x$-function (i.e., the weighting function) - these are:

$$
x=\exp \left[-\ln \left(x_{0}\right)+x_{1} \ln (\phi)+x_{2} \ln (F)+x_{3} \ln (\phi) \ln (F)\right](\text { Model CPR-DxL) . }
$$




$$
x=\exp \left[\begin{array}{l}
-\ln \left(x_{0}\right)+x_{1} \ln (\phi)+x_{2} \ln (F)+x_{3} \ln (\phi) \ln (F) \\
+x_{4} \ln (\phi)^{2}+x_{5} \ln (F)^{2}+x_{6} \ln (\phi)^{2} \ln (F) \\
+x_{7} \ln (\phi) \ln (F)^{2}+x_{8} \ln (\phi)^{2} \ln (F)^{2}
\end{array}\right] \text { (Model CPR-DxL) }
$$

As noted in Eq. I-4b, Model CPR-DxL utilizes a logarithmic linear model for the weights ( $x$-values) and as seen in Eq. I-4c, Model CPR-DxQ utilizes a logarithmic quadratic model for the weights ( $x$-values). While these models (Eqs. I-4c and I-4c) are empirical, we have found generally good application of these relations for all cases. In this case, the base results using Models $C P R-D x L$ and $C P R-D x Q$ are presented in Fig. I.7 - where the power law and exponential basis functions are fitted to the appropriate portion of the data. The power law equation represents the "Archie clean sand" trend and is fitted to the leftmost data as shown in Fig. I.7a and in contrast, the exponential equation is thought to represent the "Archie Dirty Sand" trend and the exponential is fitted to the far rightmost portion of the data (see Fig. I.7b).

In Figs. I.8 and 9 we present the error analyses for this case, where we have employed the weighting functions (Eqs. I-4c and I-4c) as appropriate. In Fig. I.8a we find a good correlation of permeability (with the out-lying points noted in the middle of the trend). The comparison of the Model CPR-DxL results are compared to the results obtained from the GRACE algorithm in Fig. I.8b, we note that the results obtained using Model CPR-DxL does "drift" slightly from the GRACE solution at lower values of permeability, indicating some inconsistency. The computed weight function ( $x$-values) shown for the $C P R-D x L$ model in Fig. I.8c does exhibit more scatter than expected, but the trend is (relatively) centered about the perfect correlation line.

The results obtained using Model $C P R-D x Q$ are presented in Fig. I.9, and we note substantially improved behavior over that of Model $C P R-D x L$. In particular, the base correlation of computed and measured permeabilities (Fig. I.9a) for Model $C P R-D x Q$ does indicate a slightly better correlation than that of Model $C P R-D x L$. Also, the comparison of Model CPR-DxQ with the results from the GRACE algorithm (Fig. I.9b) exhibits better agreement than the results of Model CPR-DxL. Finally, the correlation of the $x$ function (Fig. I.9c) for Model CPR-DxQ is better than the correlation for Model CPR-DxL. These comparisons suggest that Model CPR-DxQ (i.e., the combination of Eqs. I-4a and I-4c) has provided a better correlation of data, for this case, than Model CPR-DxL. 
"Modified Timur Model": (Appendix I)

The "Modified Timur Model" is given as:

$$
k_{\text {Timur }}=a \phi^{b} F^{c}
$$

In Fig. I.10 we present the results of the "Modified Timur Model" (EI. I-5) which is essentially just a generalized power-law function relation in terms of $k, \phi$, and $F$. Somewhat surprisingly, as shown in Figs. I.10a and $\mathbf{b}$, the "Modified Timur Model" provides the best correlation (in a statistical sense) of the data for this case. The comparison of results using the "Modified Timur Model" with the results of the GRACE algorithm (Fig. I.10b) is outstanding — a near perfect comparison with the GRACE algorithm.

\section{GRACE Algorithm: (Appendix I)}

In Fig. I.11 we present the results predicted by the GRACE algorithm, which is a non-parametric regression approach (see Xue et al. (1997) for details). In theory, the GRACE algorithm should provide the most unbiased correlation of the data - i.e., the GRACE algorithm is designed not to "fit the errors" as other regression approaches may. It is our contention that the GRACE algorithm is the statistical standard — and any algorithm/approach/model which achieves better regression statistics than the GRACE algorithm is actually "fitting the errors" in the data. The only case which has significantly better regression statistics than the GRACE algorithm is the "Modified Timur Model" - hence, we must label this case as "over-fitted" in a statistical sense.

\section{Comparison of All Models: (Appendix I)}

Our final plot, Fig. I.12, illustrates all of the models on a single plot of calculated versus measured permeability. We also present a table of all statistical results in Table I.1.

Table I.1 - Statistical Results for all Models (Lower Wilcox, S. TX (USA),

\begin{tabular}{|c|c|c|c|c|}
\hline Model & $\begin{array}{c}\text { Sum of } \\
\text { Squared } \\
\text { Residuals } \\
{\left[\log (k)^{2}\right]}\end{array}$ & $\begin{array}{l}\text { Variance } \\
{\left[\log (k)^{2}\right]}\end{array}$ & $\begin{array}{c}\text { Standard } \\
\text { Deviation } \\
{[\log (k)]}\end{array}$ & $\begin{array}{c}\text { Absolute } \\
\text { Relative } \\
\text { Error } \\
\text { [percent] }\end{array}$ \\
\hline$C P R-A$ & 48.89 & 2.08 & 1.44 & 86.39 \\
\hline$C P R-B$ & 48.78 & 2.11 & 1.45 & 90.10 \\
\hline$C P R-C$ & 57.32 & 2.20 & 1.48 & 99.03 \\
\hline$C P R-D x L$ & 51.74 & 2.36 & 1.54 & 102.77 \\
\hline$C P R-D x Q$ & 49.10 & 2.15 & 1.46 & 93.05 \\
\hline Modified Timur & 47.83 & 2.11 & 1.45 & 84.41 \\
\hline GRACE Algorithm & 50.47 & 2.17 & 1.47 & 88.14 \\
\hline
\end{tabular}


The coefficients for the models used in this case are provided in Table I.2.

Table I.2a - Model Coefficients (Lower Wilcox, S. TX (USA), $n=62$ ).

\begin{tabular}{|c|c|c|c|c|c|c|}
\hline Model & $a$ or $\alpha$ & $b$ or $\beta$ & $C$ or $C_{\max }$ & $c_{1}$ & $c_{2}$ & $c_{3}$ \\
\hline$C P R-A$ & $1.00 \times 10^{8}$ & 8.00 & 0.090 & 0.03 & -5.71 & -2.06 \\
\hline$C P R-B$ & $4.00 \times 10^{5}$ & 8.00 & 0.150 & $9.16 \times 10^{-2}$ & 3.49 & 2.38 \\
\hline$C P R-C$ & $9.00 \times 10^{-9}$ & 100.00 & $5.00 \times 10^{-5}$ & $1.49 \times 10^{2}$ & 3.14 & 0.65 \\
\hline Modified Timur & $1.89 \times 10^{6}$ & -0.92 & -4.207 & - & - & - \\
\hline
\end{tabular}

Table I.2b - Model Coefficients (Lower Wilcox, S. TX (USA), $n=62$ ).

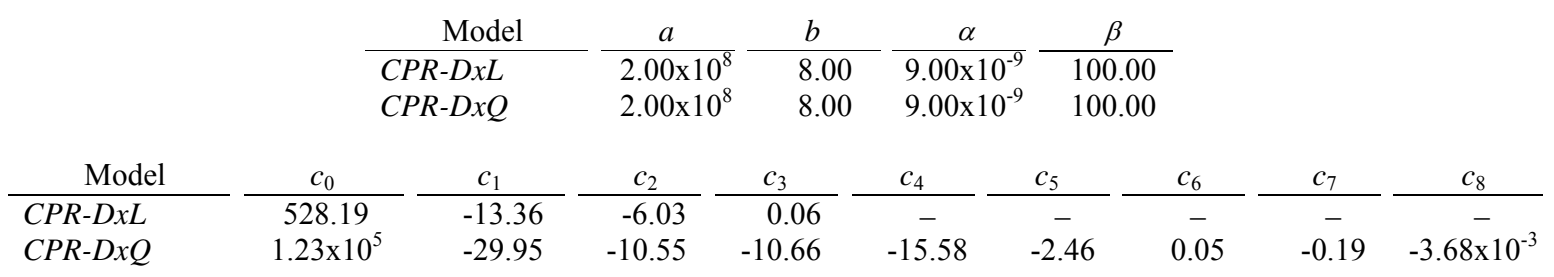

\section{Nomenclature: (Appendix I)}

$F=$ Archie Formation Factor (dimensionless)

$k=$ Formation Permeability, md (or any consistent units)

$\phi \quad=$ Porosity, fraction

\section{References: (Appendix I)}

Ellis, K.W.: "Extended Correlations of Porosity, Permeability, and Formation Resistivity Factor," MS thesis, Texas A\&M U., College Station, TX (1987).

Xue, G., Datta-Gupta, A., Valko, P., and Blasingame, T.A.: "Optimal Transformations for Multiple Regression: Application to Permeability Estimation from Well Logs," SPEFE (June 1997), 85-93. 


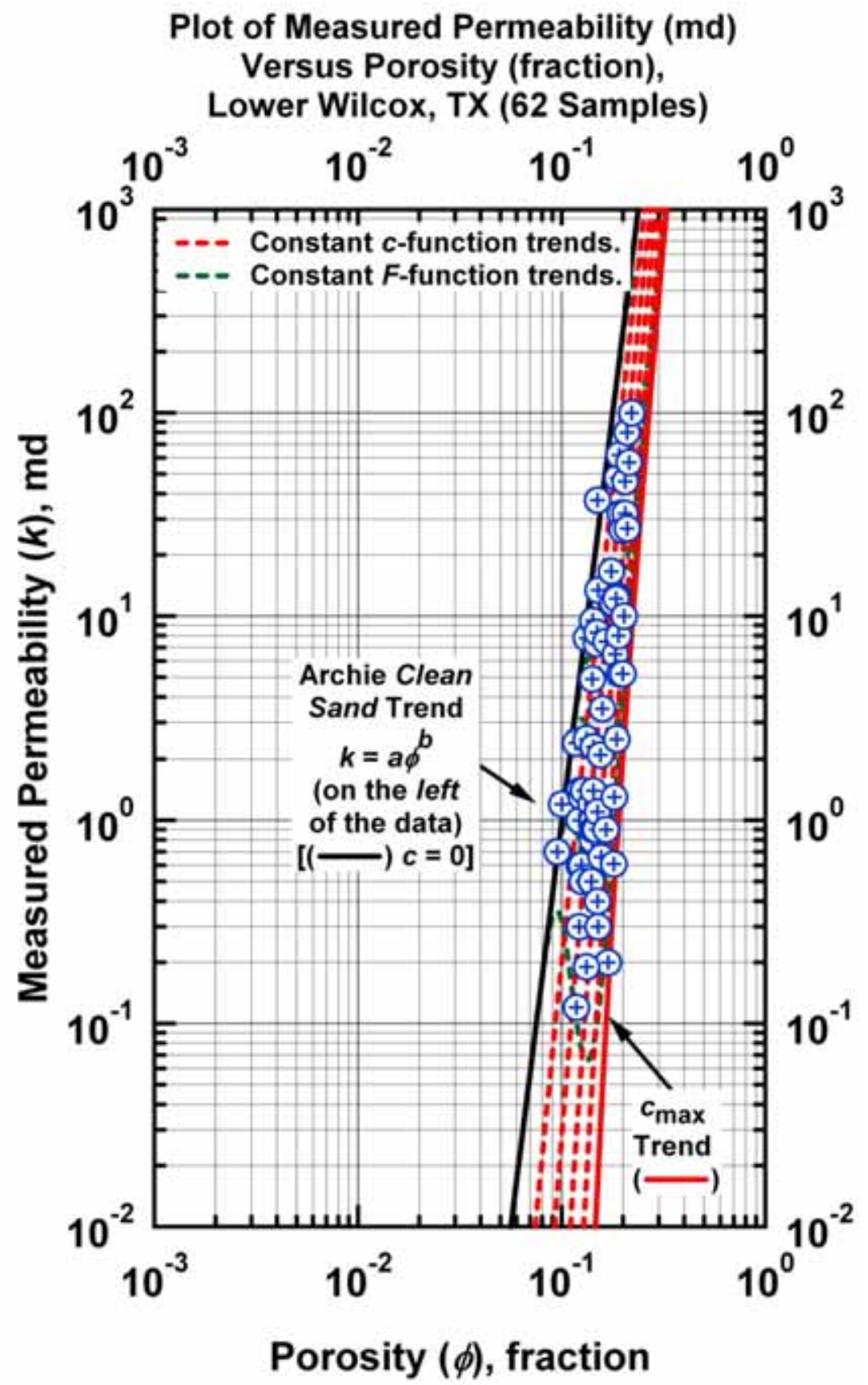

Figure I.1a - Model CPR-A: $k=a(\phi-c)^{b}\left(c=c_{\max } \exp \left[-c_{1} \phi^{c_{2}} F^{c 3}\right]\right)$ - "Clean Sand" Plot (log-log format) - Archie "Clean Sand" trend is given by the straight-line trend at the far left of the data (power law model). [Case: Lower Wilcox S. TX (USA)] 


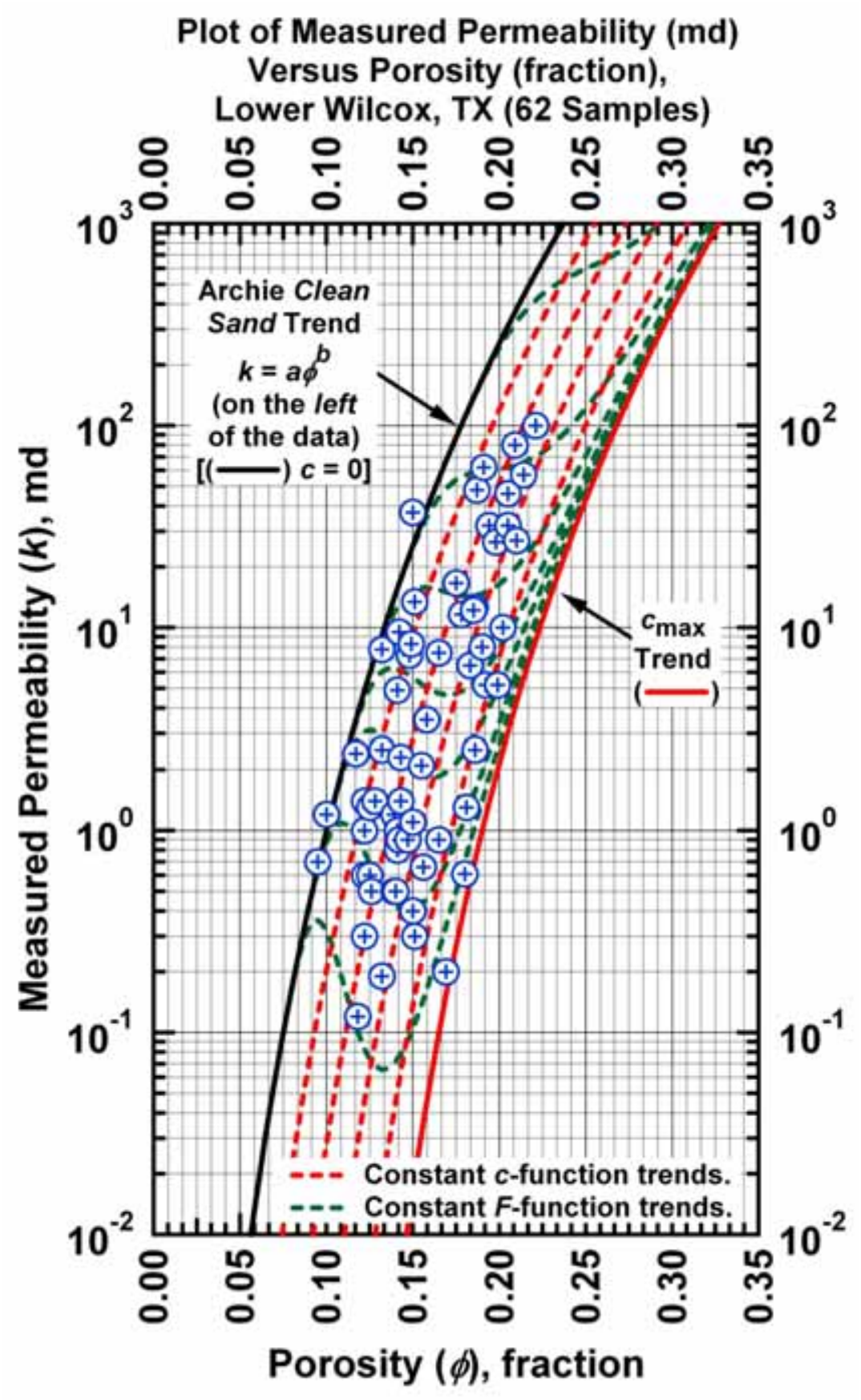

Figure I.1b - Model $C P R-A: \quad k=a(\phi-c)^{b}\left(c=c_{\max } \exp \left[-c_{1} \phi^{c_{2}} F^{c_{3}}\right]\right)$ — "Dirty Sand" Plot (semilog format). Archie "Clean Sand" trend is given by the curved trend at the far left of the data (power law model). [Case: Lower Wilcox S. TX (USA)] 
Comparison of Permeability (md) Calculated Using the "CPR-A" Model Versus the Measured Permeability (md), Lower Wilcox, TX (62 Samples)

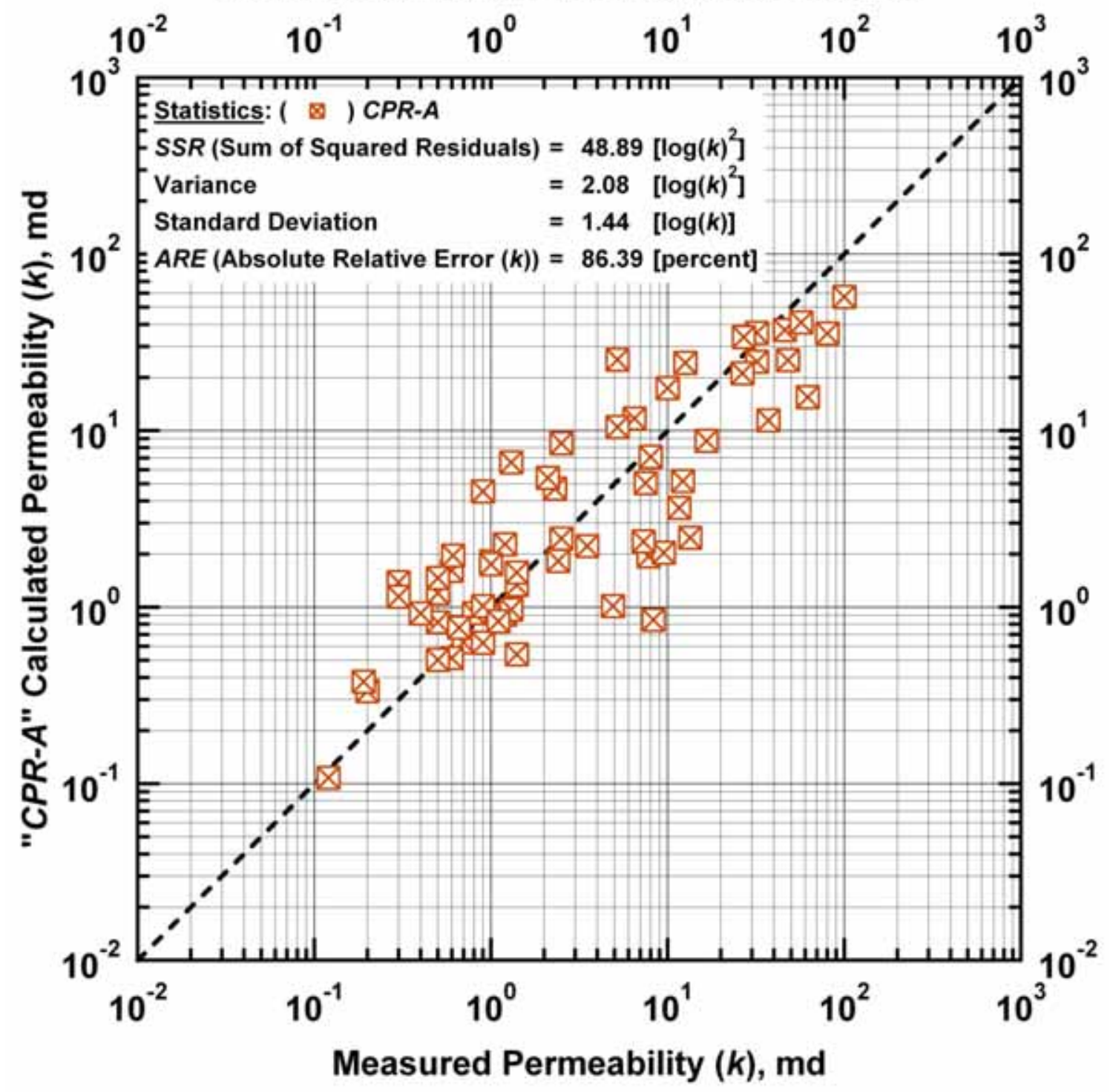

Figure I.2a - Model CPR-A: calculated versus measured permeability. [Case: Lower Wilcox S. TX (USA)] 


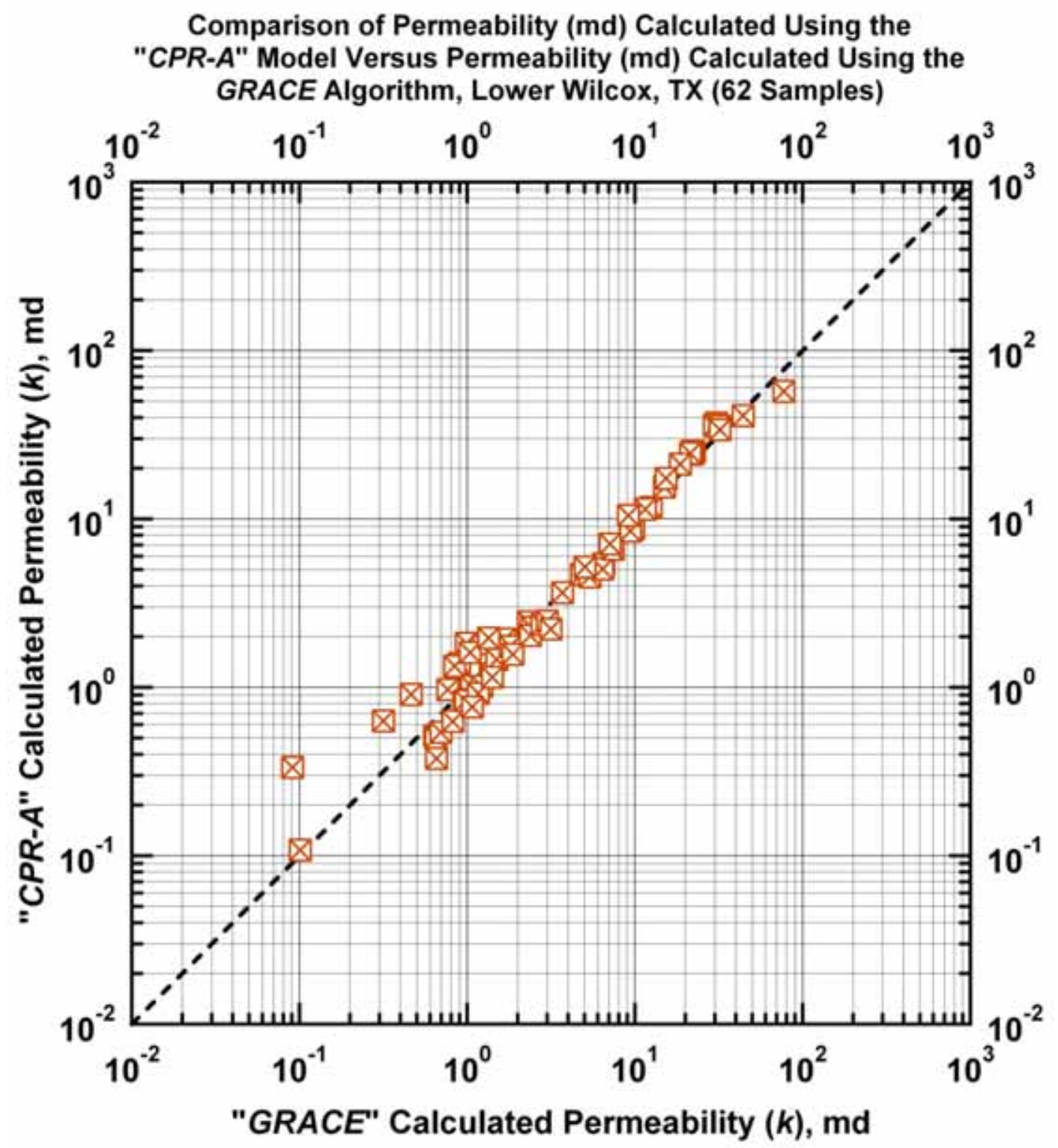

Figure I.2b - Model CPR-A: Permeability calculated using Model $C P R-A$ versus permeability calculated using the GRACE Algorithm. [Case: Lower Wilcox S. TX (USA)] 


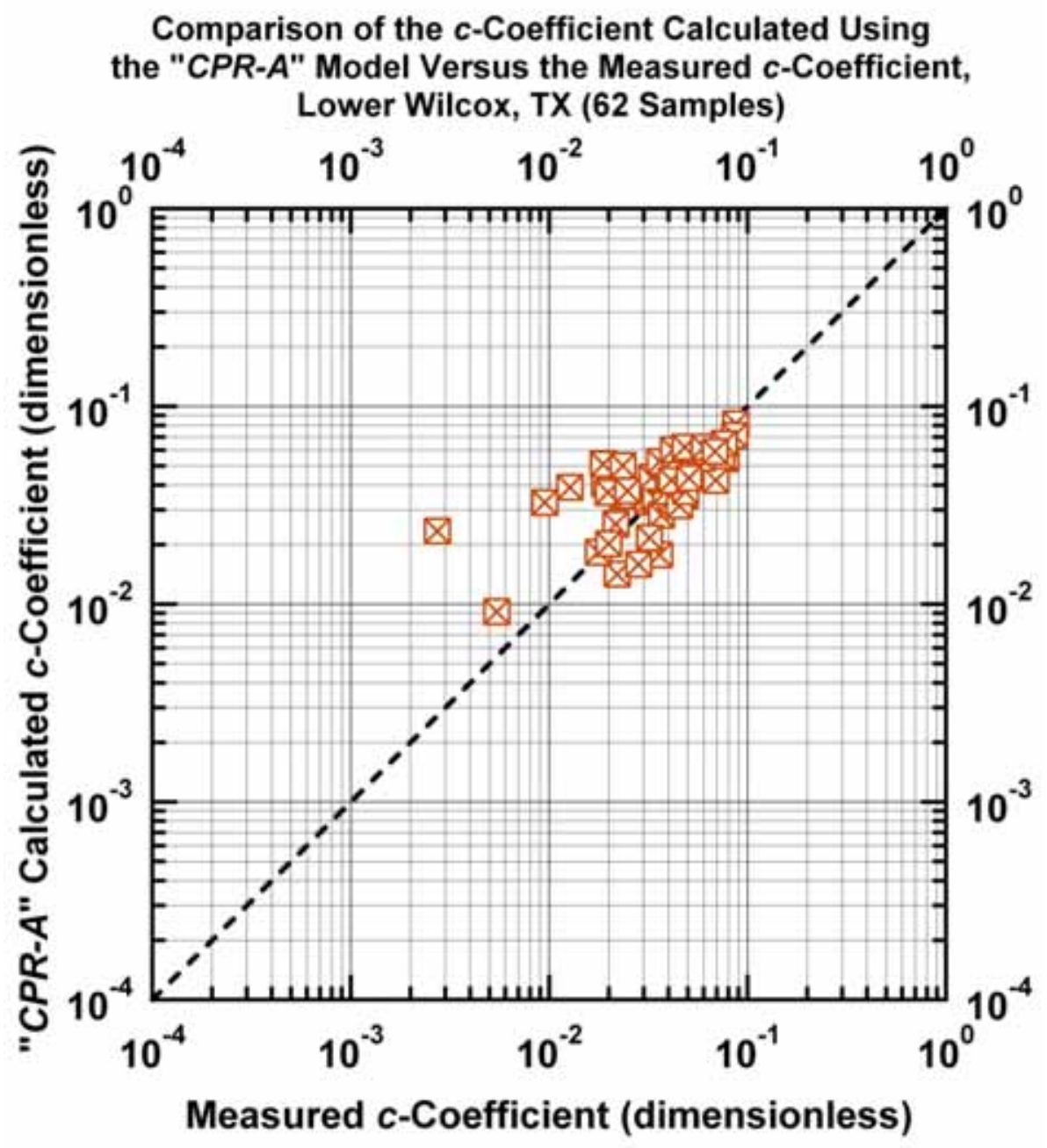

Figure I.2c - Model $C P R$ - $A$ : Calculated $c$-function values versus measured $c$-function values. [Case: Lower Wilcox S. TX (USA)] 


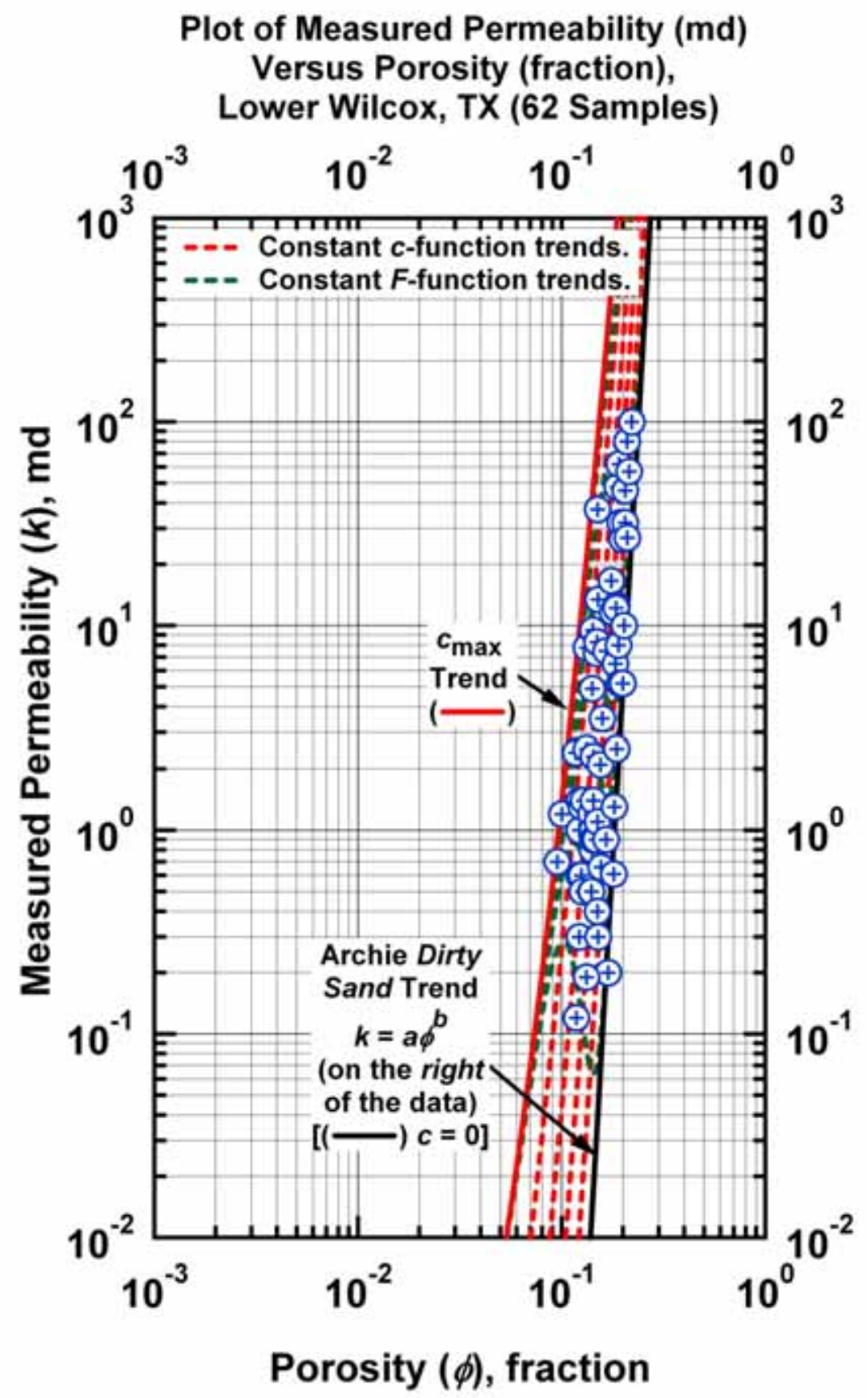

Figure I.3a - Model $C P R-B: \quad k=a(\phi+c)^{b} \quad c=c_{\max } \exp \left[-c_{1} \phi^{c^{2}} F^{c_{3}}\right]$ - "Clean Sand" Plot (log-log format) - Archie "Dirty Sand" trend is given by the straight-line trend at the far right of the data (power law model). [Case: Lower Wilcox S. TX (USA)] 


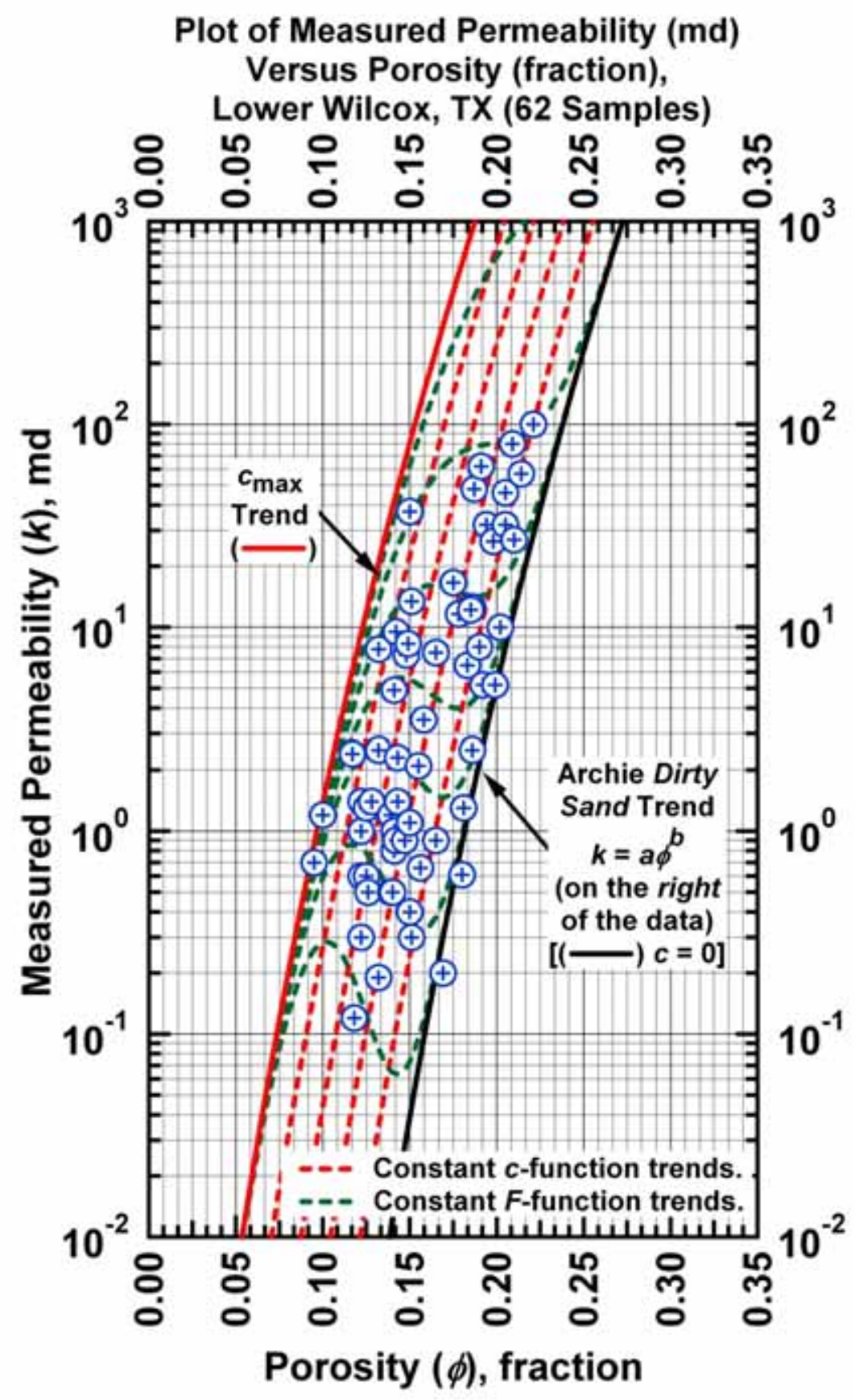

Figure I.3b - Model CPR-B: $k=a(\phi+c)^{b} \quad c=c_{\max } \exp \left[-c_{1} \phi^{c_{2}} F^{c_{3}}\right]$ — "Dirty Sand" Plot (semilog format) - Archie "Dirty Sand" trend is given by the curved trend at the far right of the data (power law model). [Case: Lower Wilcox S. TX (USA)] 


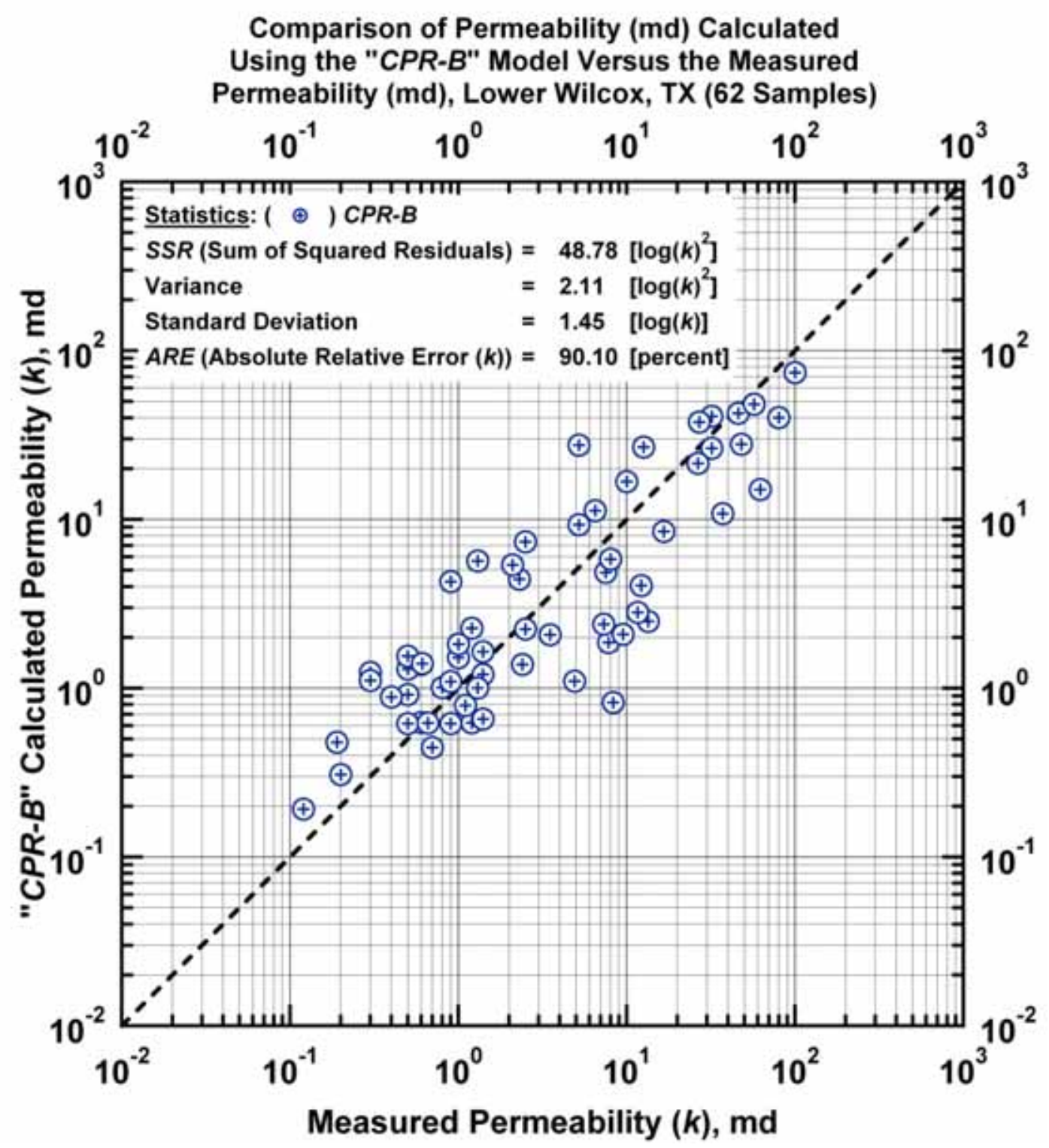

Figure I.4a - Model CPR-B: calculated versus measured permeability. [Case: Lower Wilcox S. TX (USA)] 


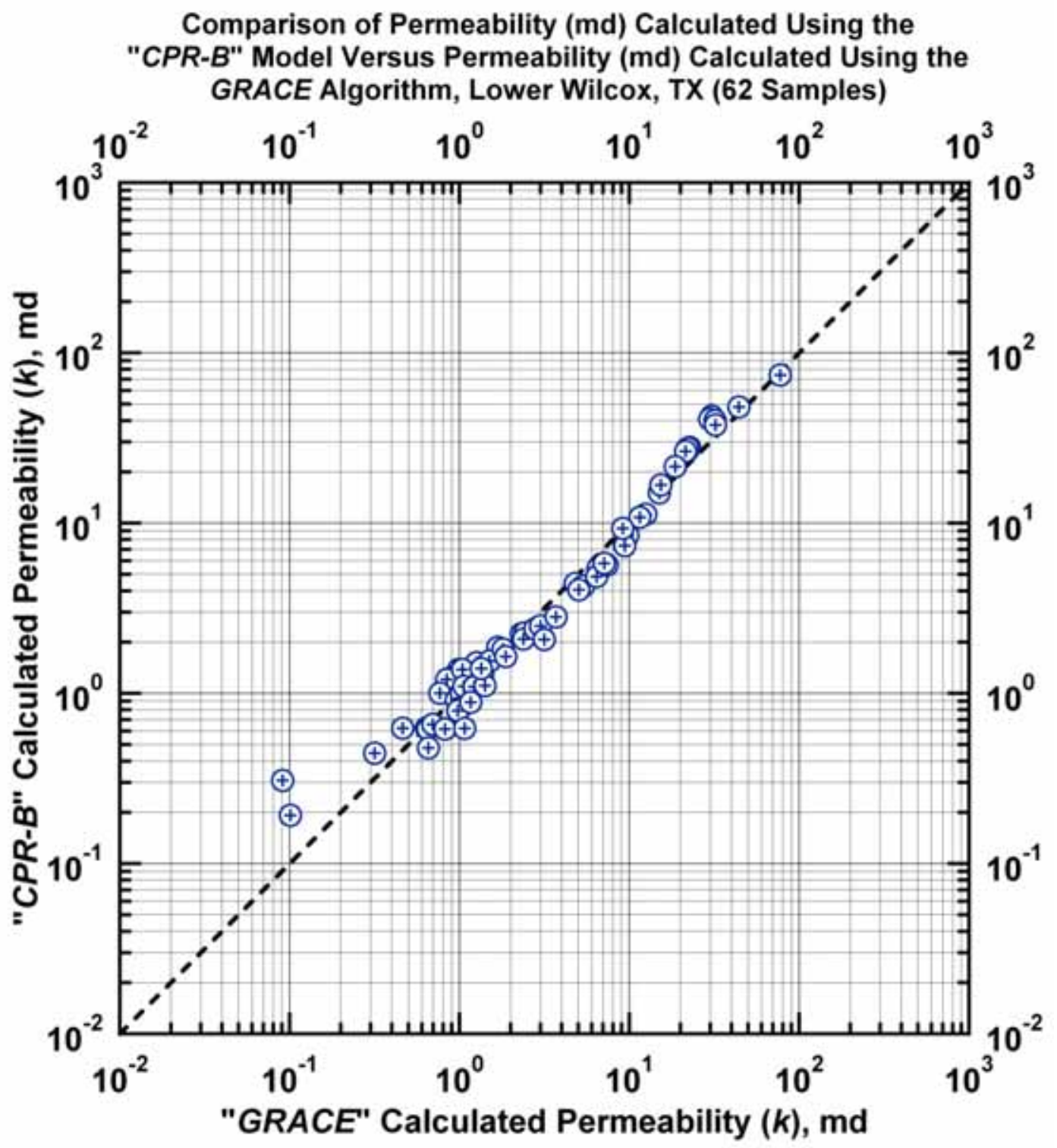

Figure I.4b - Model $C P R$ - $B$ : Permeability calculated using Model $C P R-B$ versus permeability calculated using the GRACE Algorithm. [Case: Lower Wilcox S. TX (USA)] 


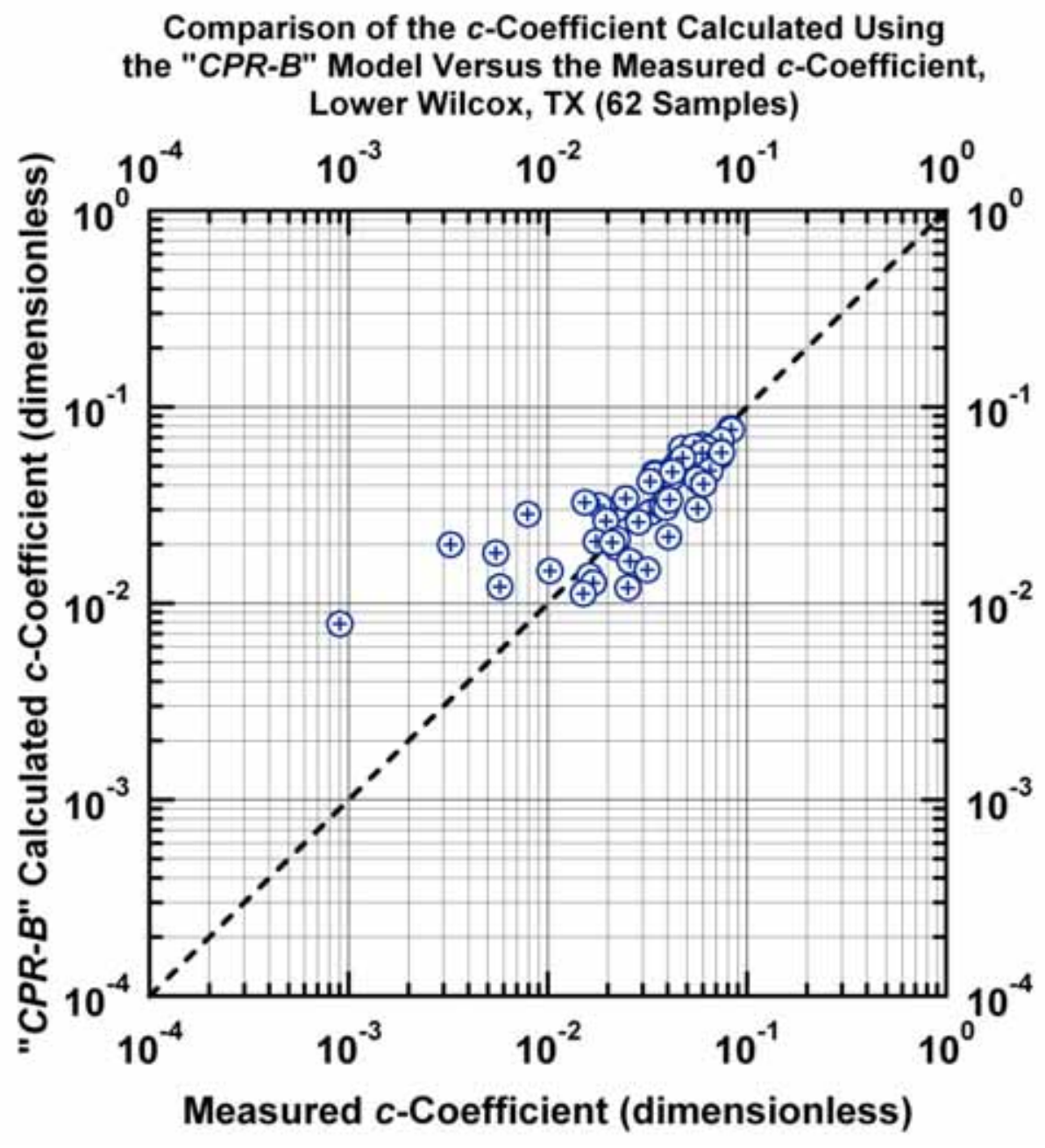

Figure I.4c - Model $C P R$ - $B$ : Calculated $c$-function values versus measured $c$-function values. [Case: Lower Wilcox S. TX (USA)] 


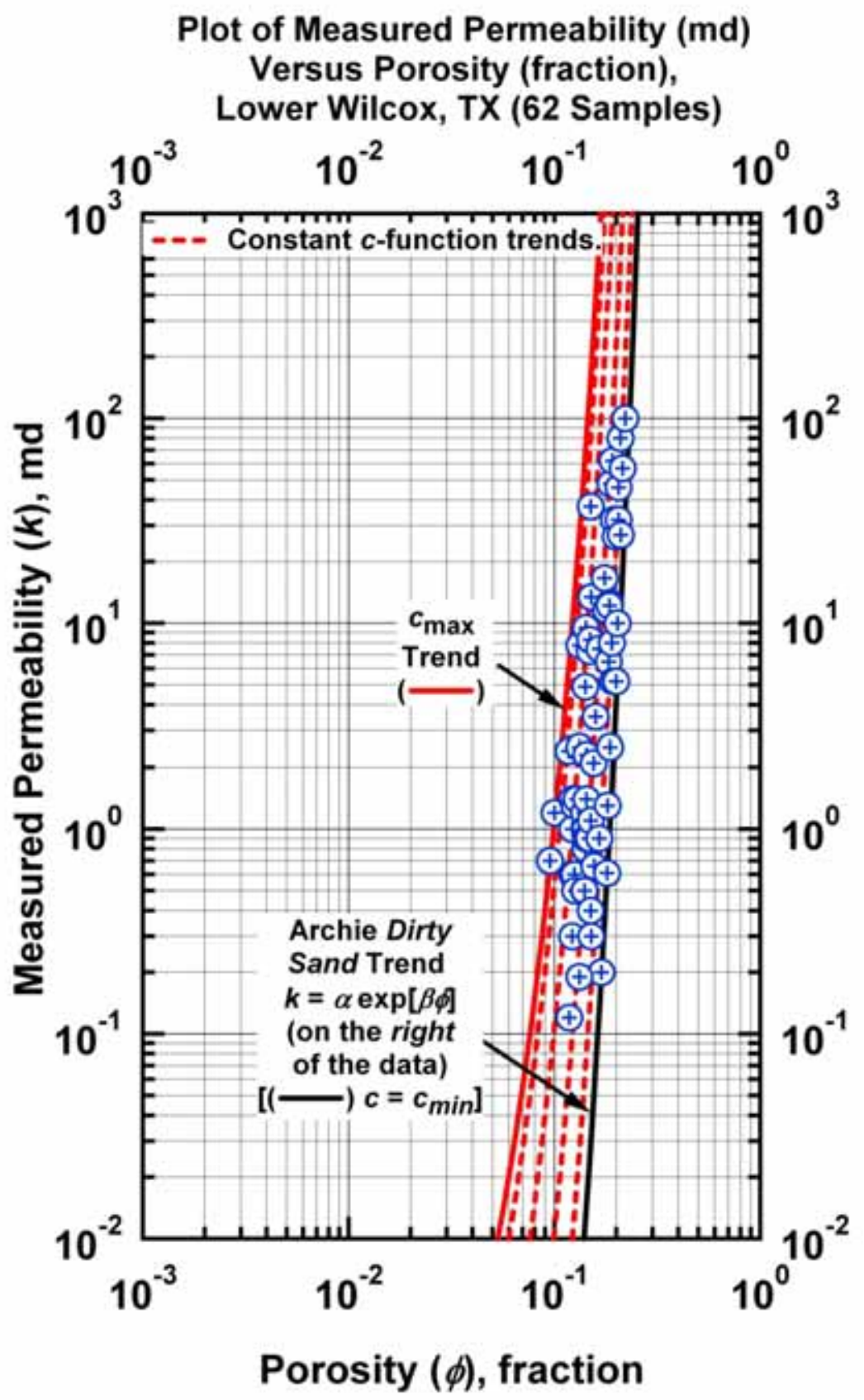

Figure I.5a - Model $C P R-C: \quad k=c \exp [\beta \phi] \quad c=c_{\max } \exp \left[-c_{1} \phi^{c_{2}} F^{c 3}\right]$ - "Clean Sand" Plot (log-log format) - Archie "Dirty Sand" trend is given by the curved trend at the far right of the data (exponential model). [Case: Lower Wilcox S. TX (USA)] 


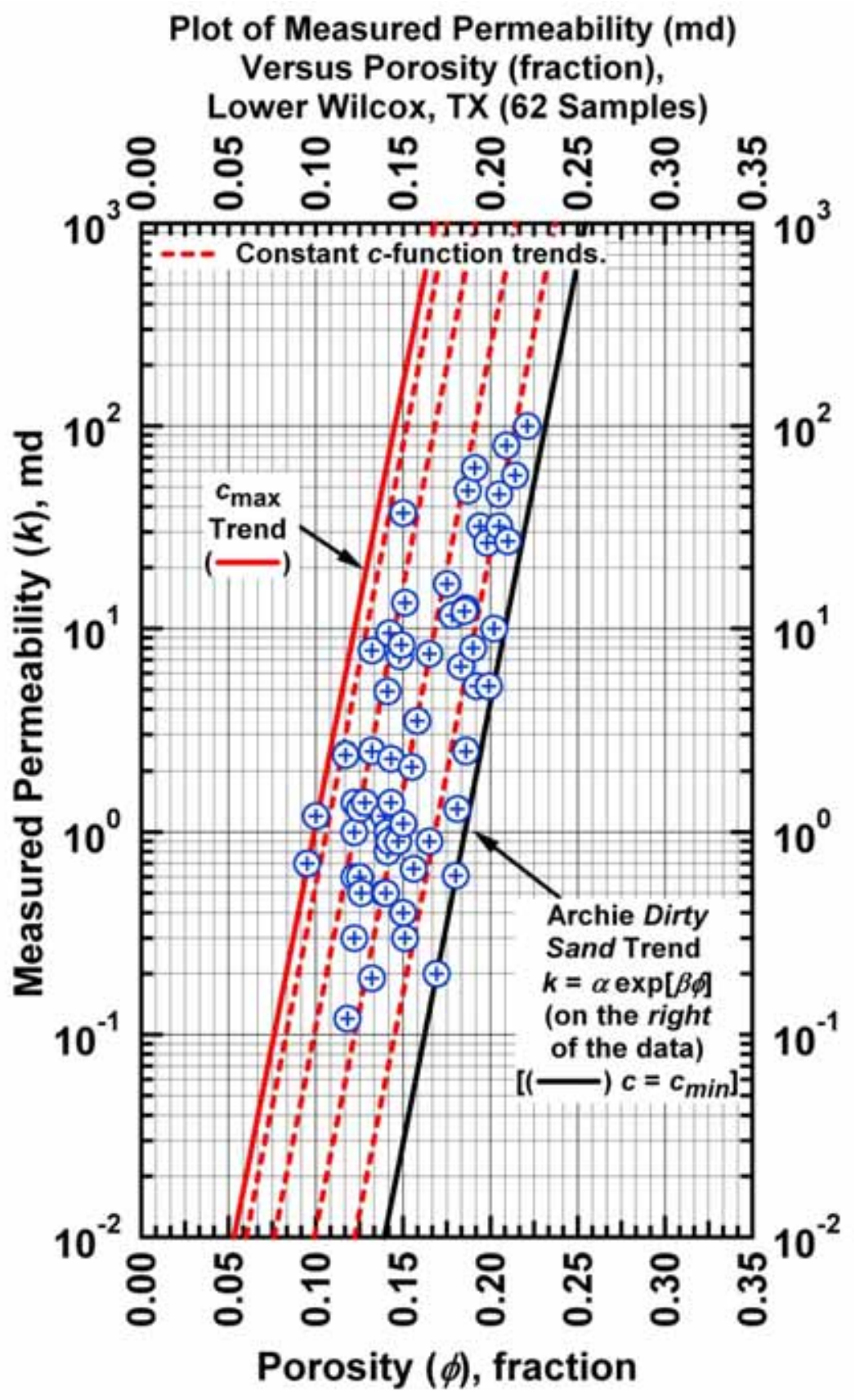

Figure I.5b - Model $C P R-C: \quad k=c \exp [\beta \phi] \quad c=c_{\max } \exp \left[-c_{1} \phi^{c_{2}} F^{c_{3}}\right]$ - "Dirty Sand" Plot (semilog format) - Archie "Dirty Sand" trend is given by the straight-line trend at the far right of the data (exponential model). [Case: Lower Wilcox S. TX (USA)] 


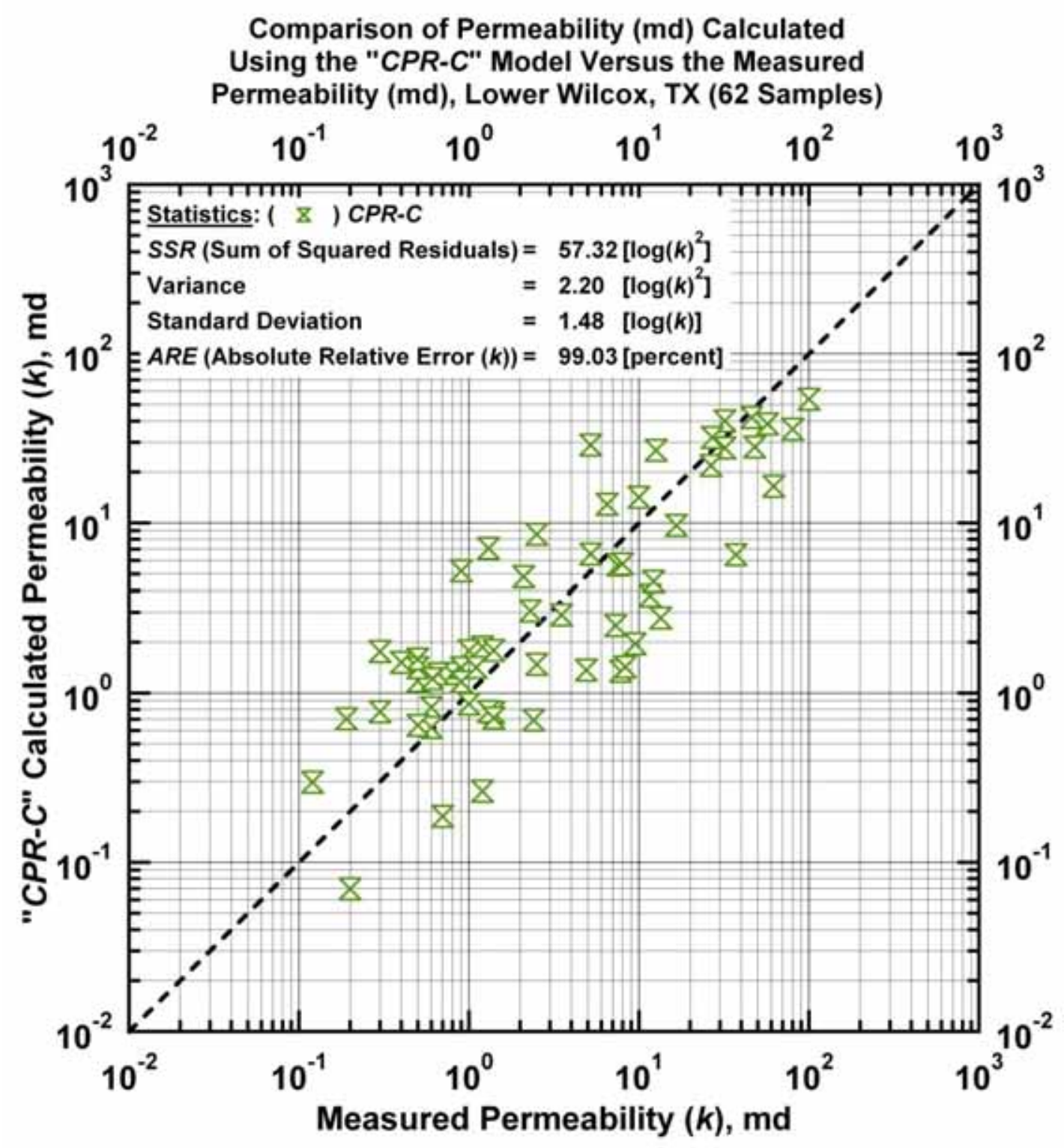

Figure I.6a - Model $C P R-C$ : calculated versus measured permeability. [Case: Lower Wilcox S. TX (USA)] 


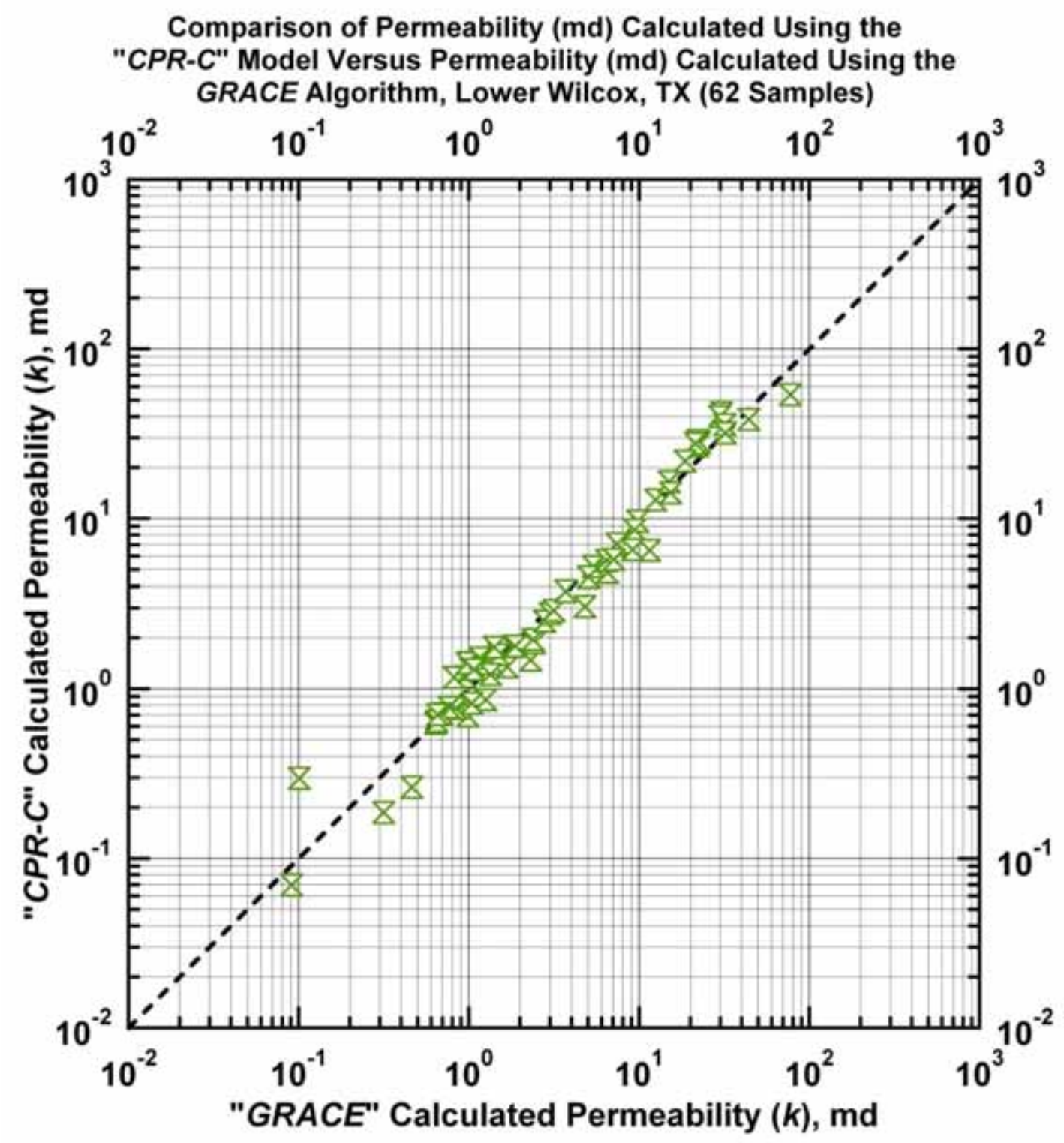

Figure I.6b - Model $C P R-C$ : Permeability calculated using Model $C P R-C$ versus permeability calculated using the GRACE Algorithm. [Case: Lower Wilcox S. TX (USA)] 


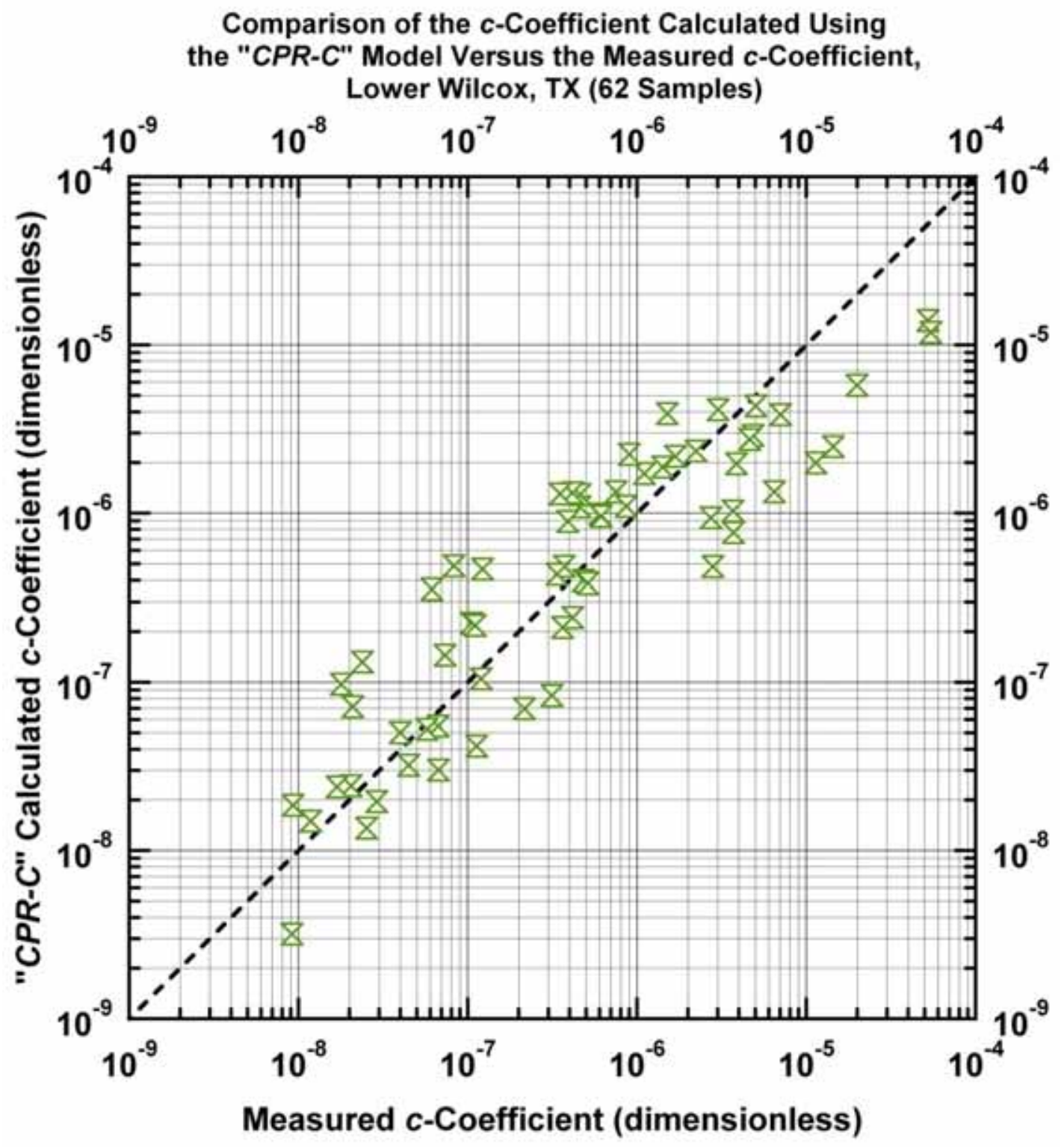

Figure I.6c - Model $C P R$-C: Calculated $c$-function values versus measured $c$-function values. [Case: Lower Wilcox S. TX (USA)] 


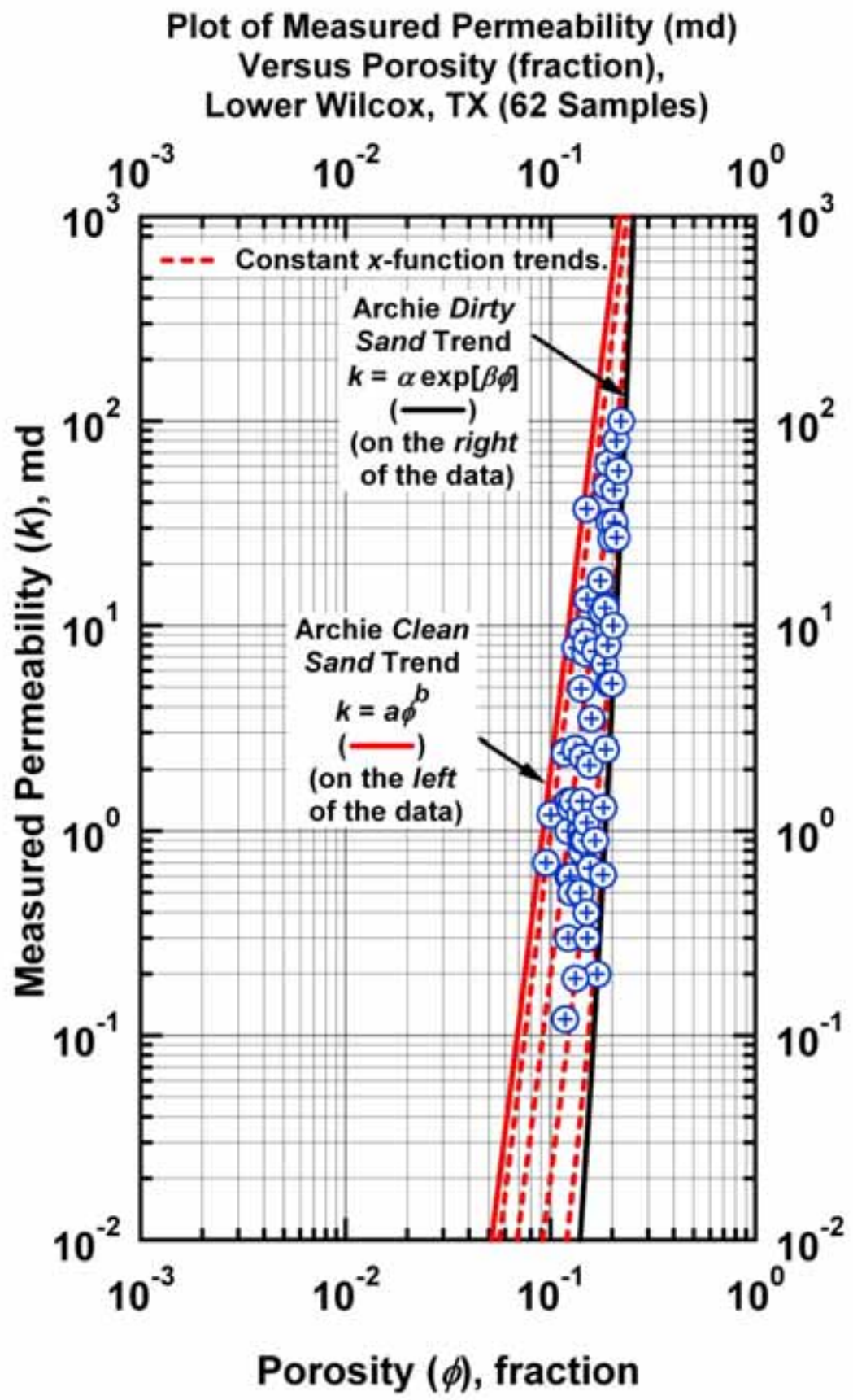

Figure I.7a - Model CPR-D: $k=x a \phi^{b}+(1-x) \alpha \exp [\beta \phi] \quad[0 \leq x \leq 1]$ - Weighted Power Law-Exponential Model used to correlate permeability $(k)$ and porosity $(\phi)$. "Clean Sand" Plot - Archie "Clean Sand" trend is given by the straight-line trend at the far left of the data (power law model). [Case: Lower Wilcox S. TX (USA)] 


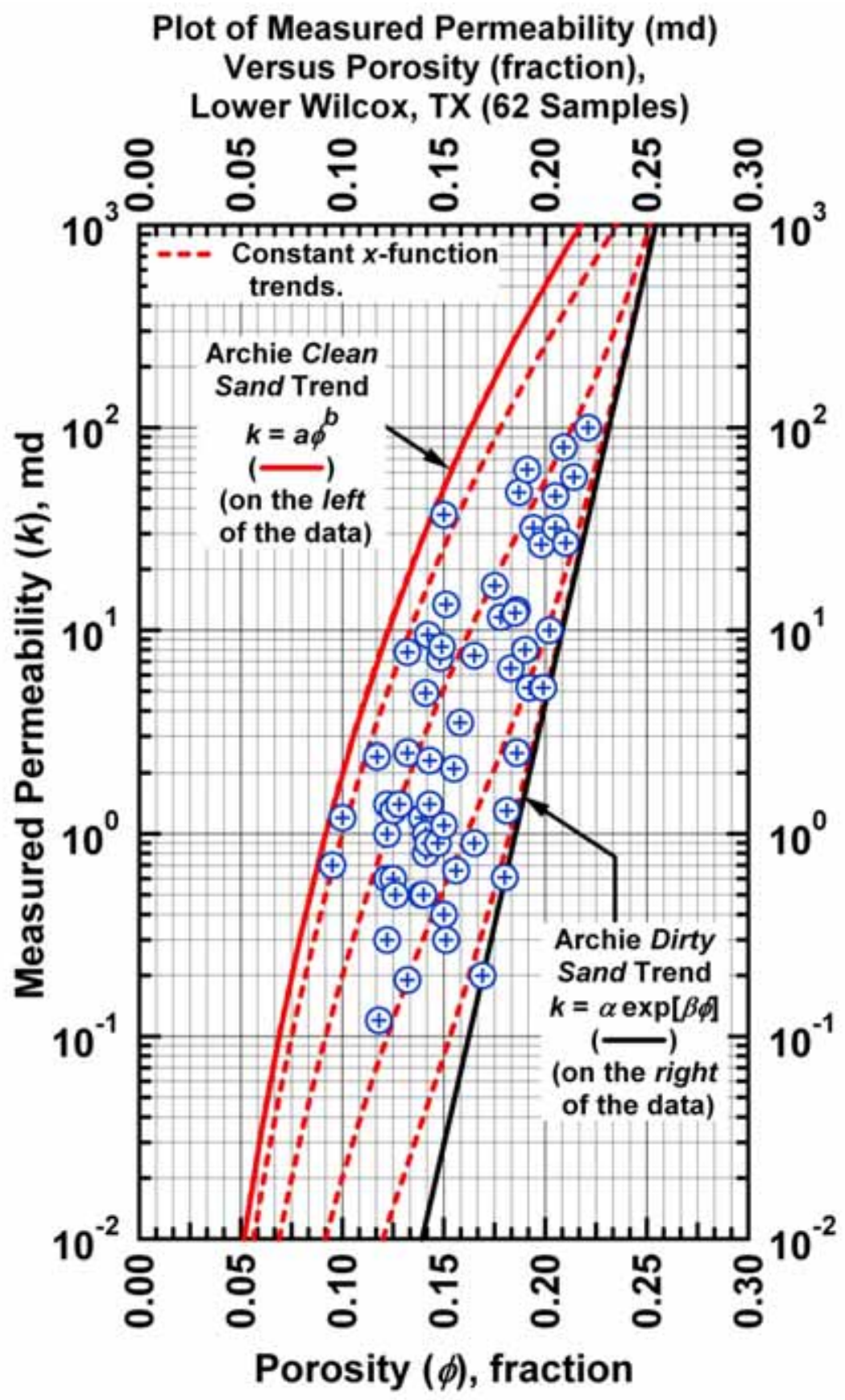

Figure I.7b - Model $C P R-D: k=x a \phi^{b}+(1-x) \alpha \exp [\beta \phi] \quad[0 \leq x \leq 1]$ - Weighted Power Law-Exponential Model used to correlate permeability $(k)$ and porosity $(\phi)$. "Dirty Sand" Plot - Archie "Dirty Sand" trend is given by the straight-line trend at the far right of the data (exponential model). [Case: Lower Wilcox S. TX (USA)] 


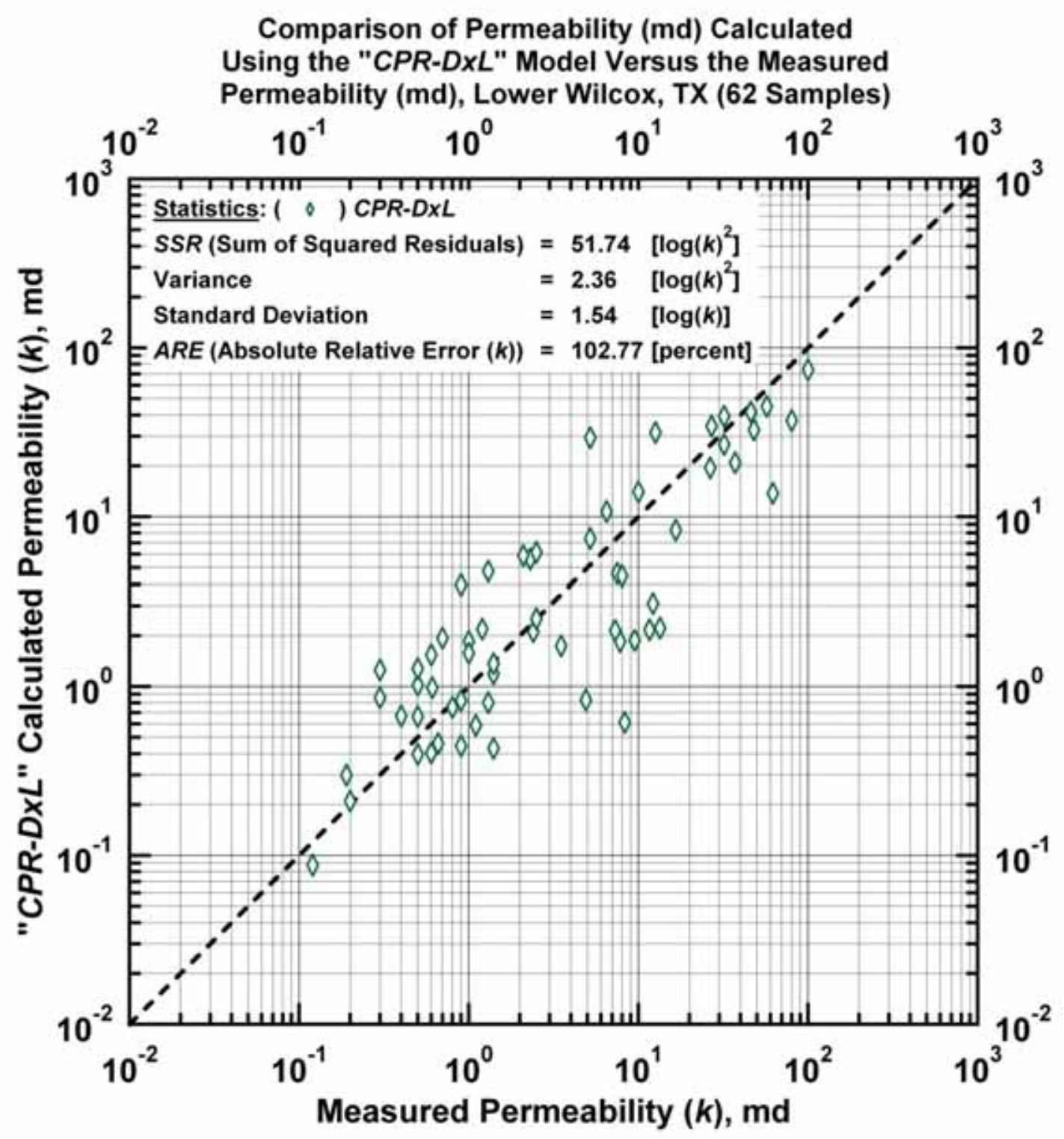

Figure I.8a - Model CPR-DxL: Calculated versus measured permeability. [Case: Lower Wilcox S. TX (USA)] 


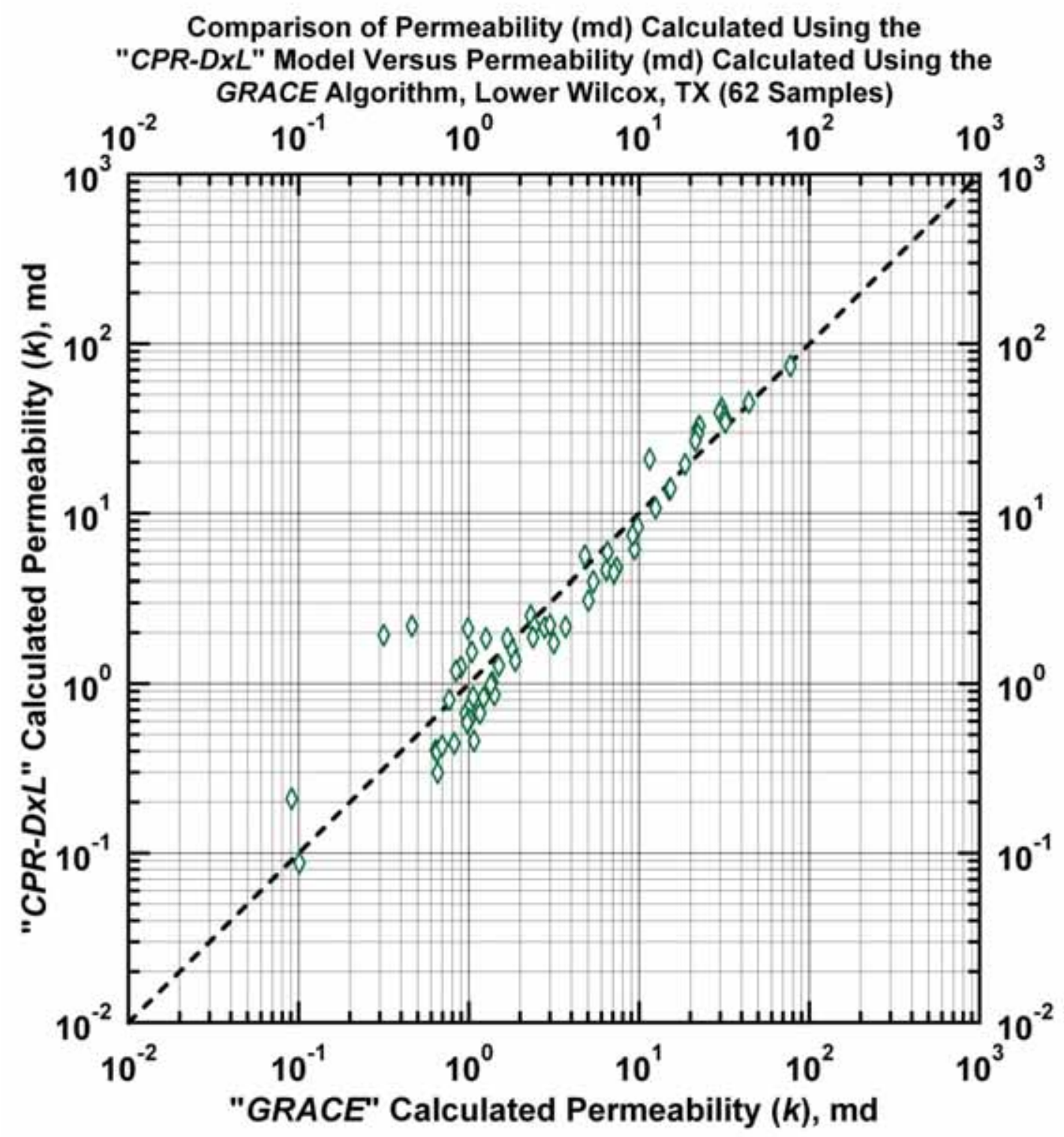

Figure I.8b - Model $C P R-D x L$ : Permeability calculated using Model $C P R-C$ versus permeability calculated using the GRACE Algorithm. [Case: Lower Wilcox S. TX (USA)] 


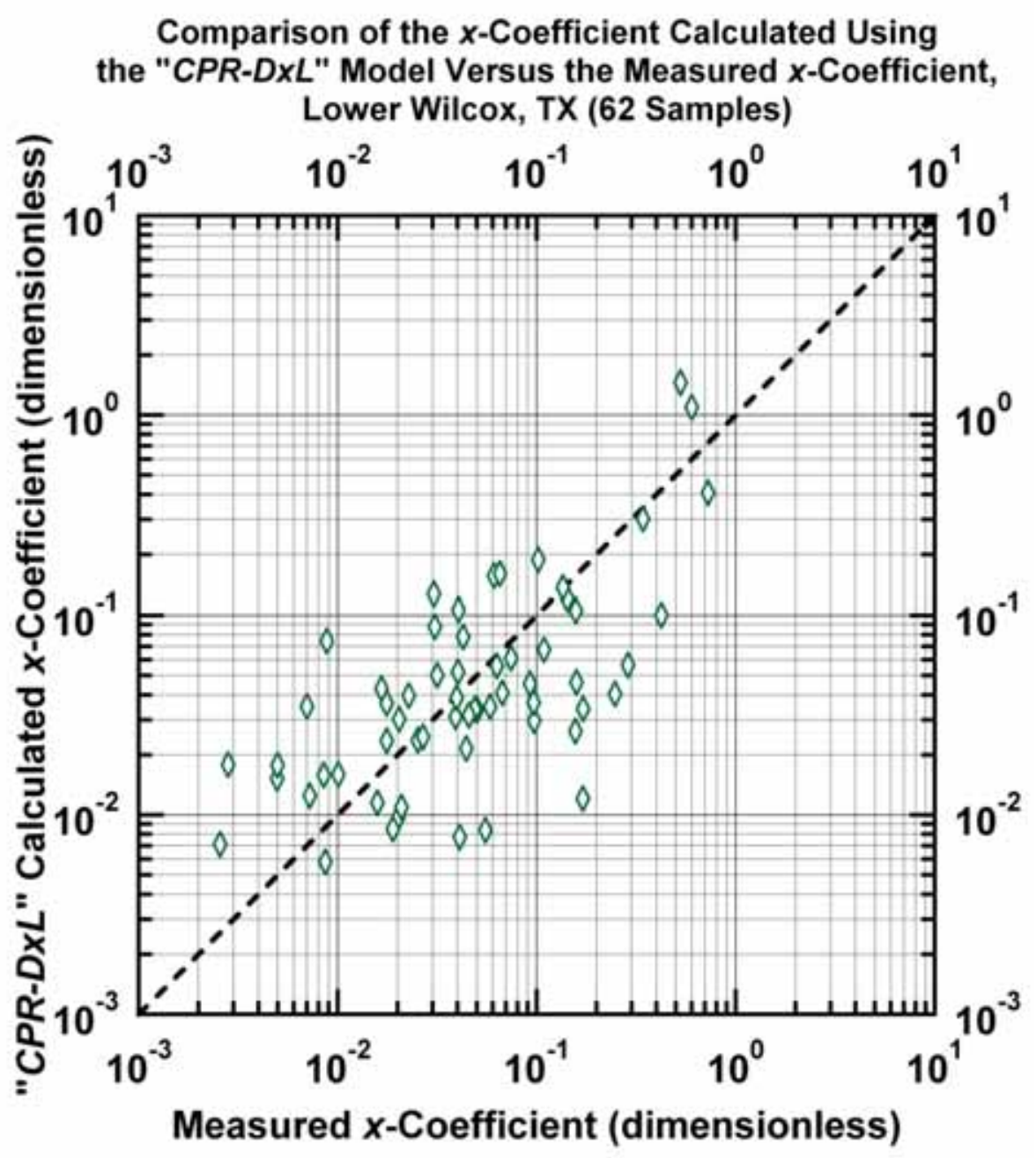

Figure I.8c - Model $C P R$ - $D x L$ : Calculated $x$-function weight values versus measured $x$ function weight values. [Case: Lower Wilcox S. TX (USA)] 


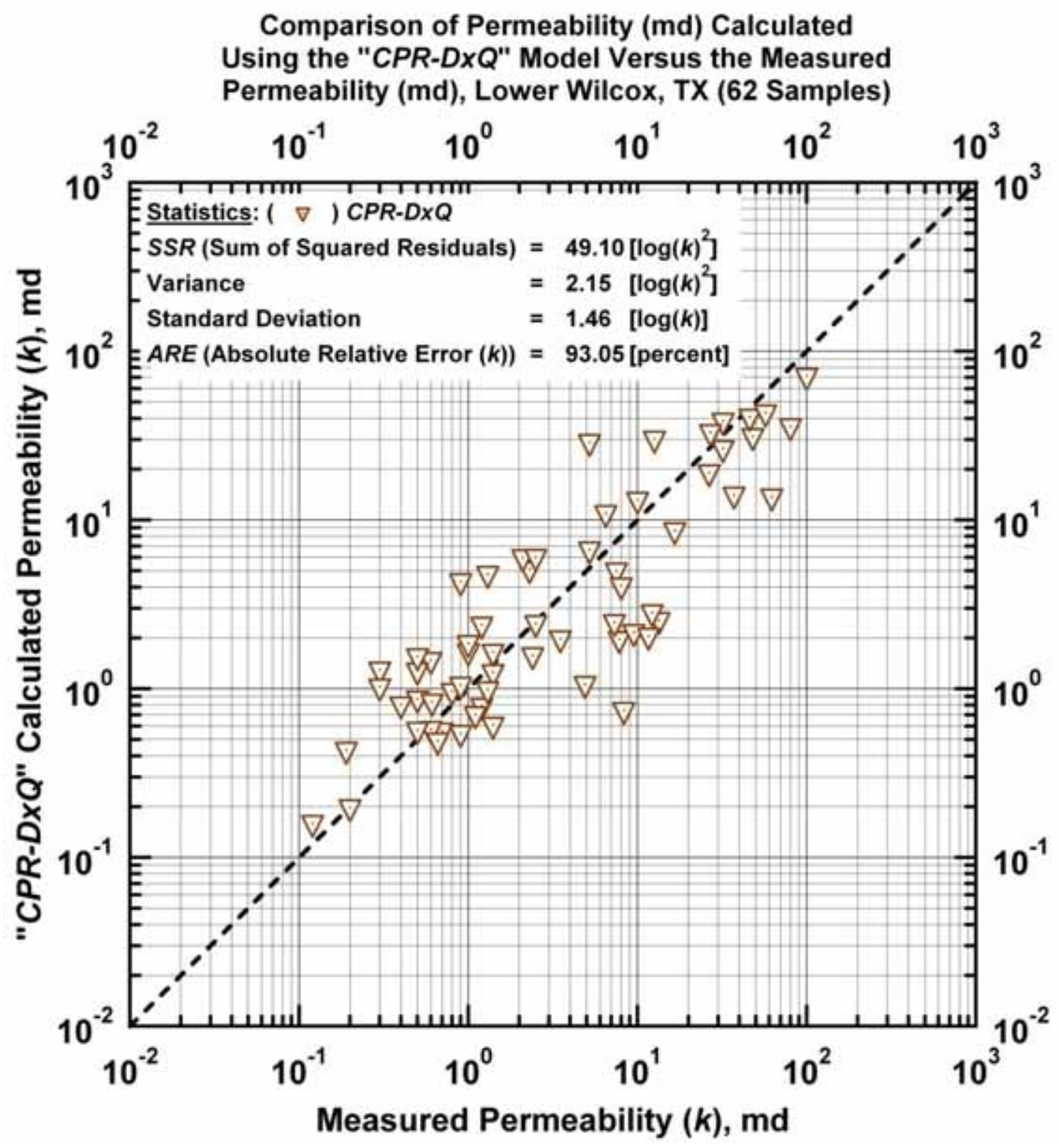

Figure I.9a - Model CPR-DxQ: Calculated versus measured permeability. [Case: Lower Wilcox S. TX (USA)] 


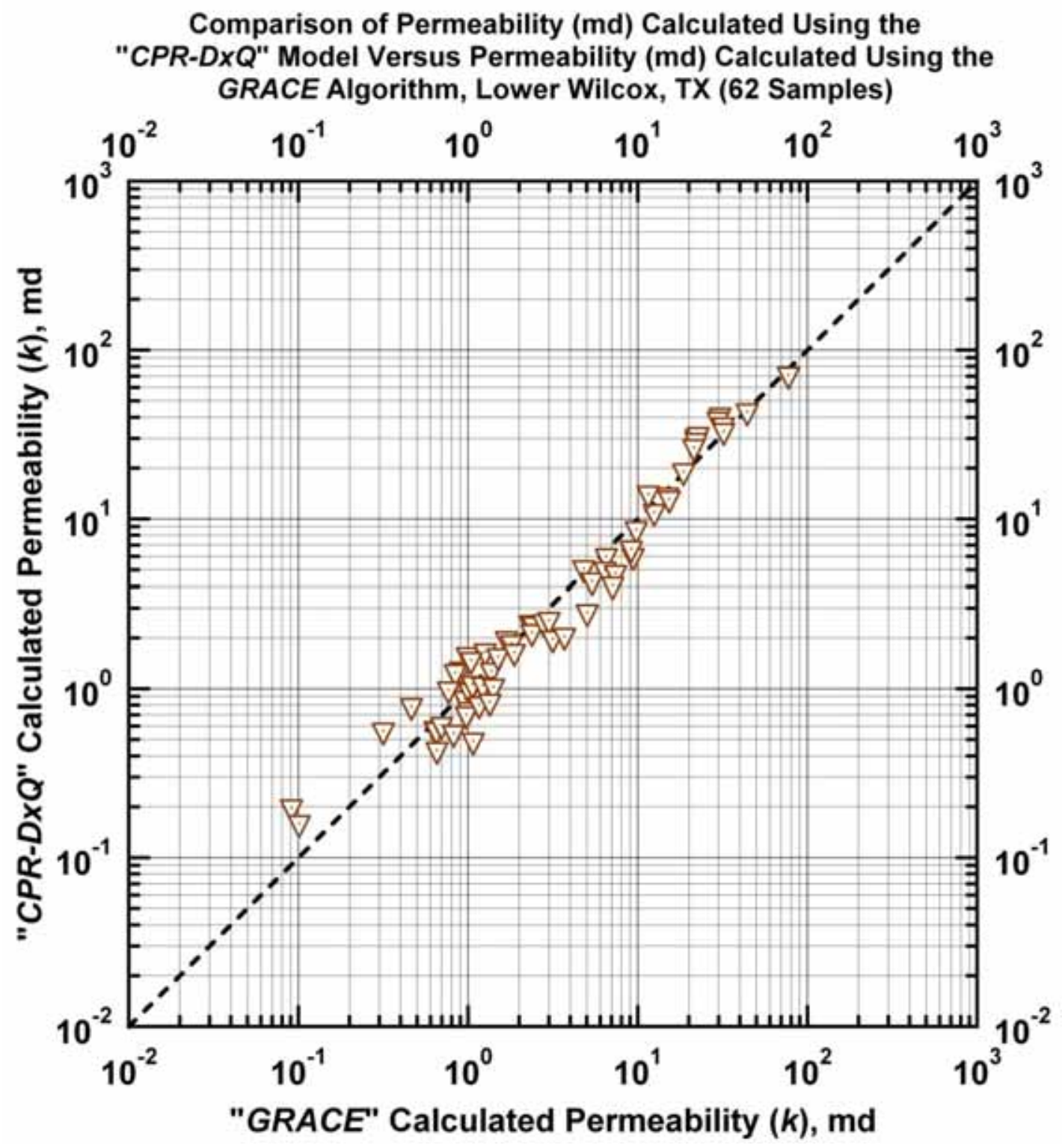

Figure I.9b - Model $C P R-D x Q$ : Permeability calculated using Model $C P R-C$ versus permeability calculated using the GRACE Algorithm. [Case: Lower Wilcox S. TX (USA)] 


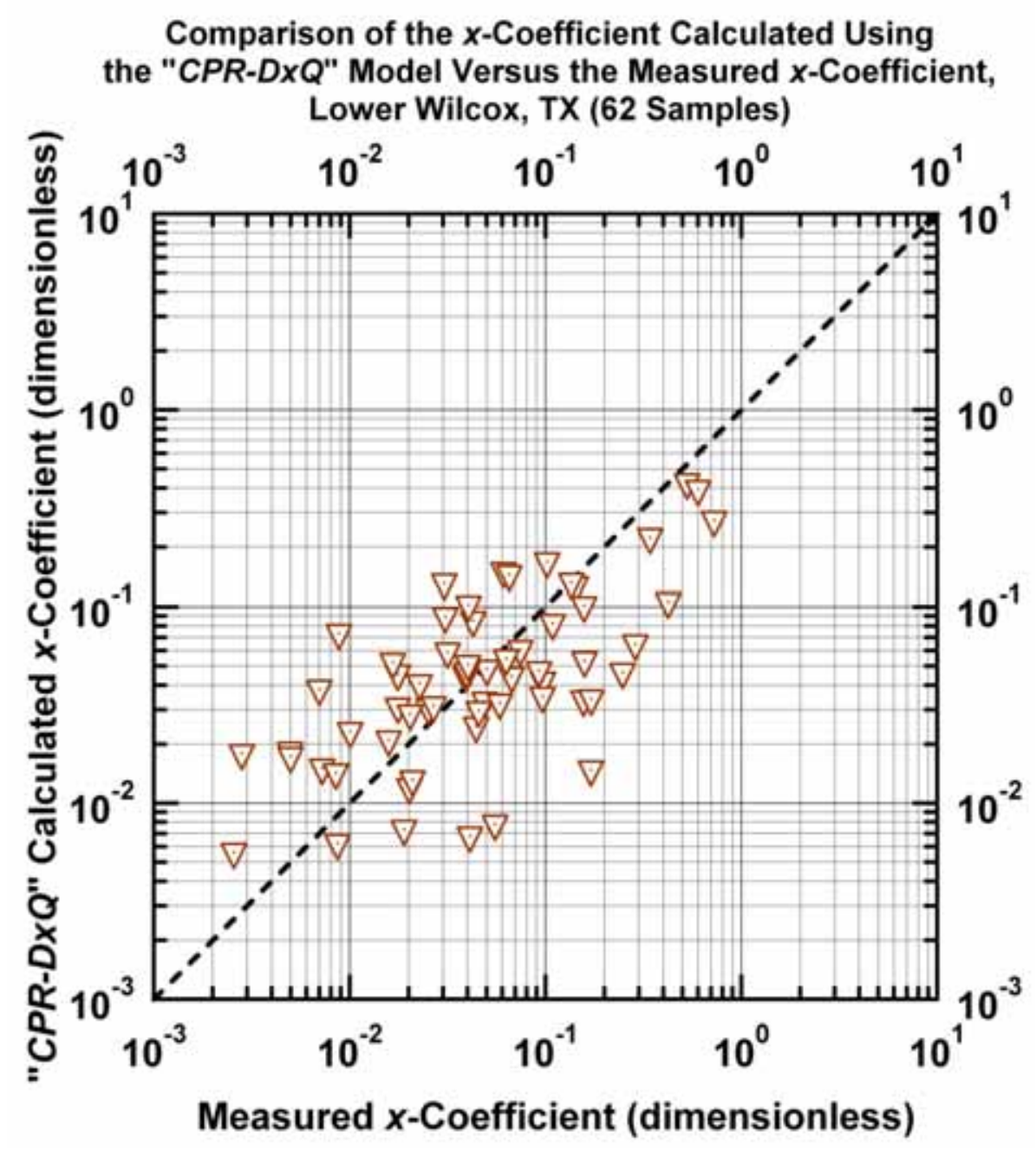

Figure I.9c - Model $C P R$ - $D x Q$ : Calculated $x$-function weight values versus measured $x$ function weight values. [Case: Lower Wilcox S. TX (USA)] 


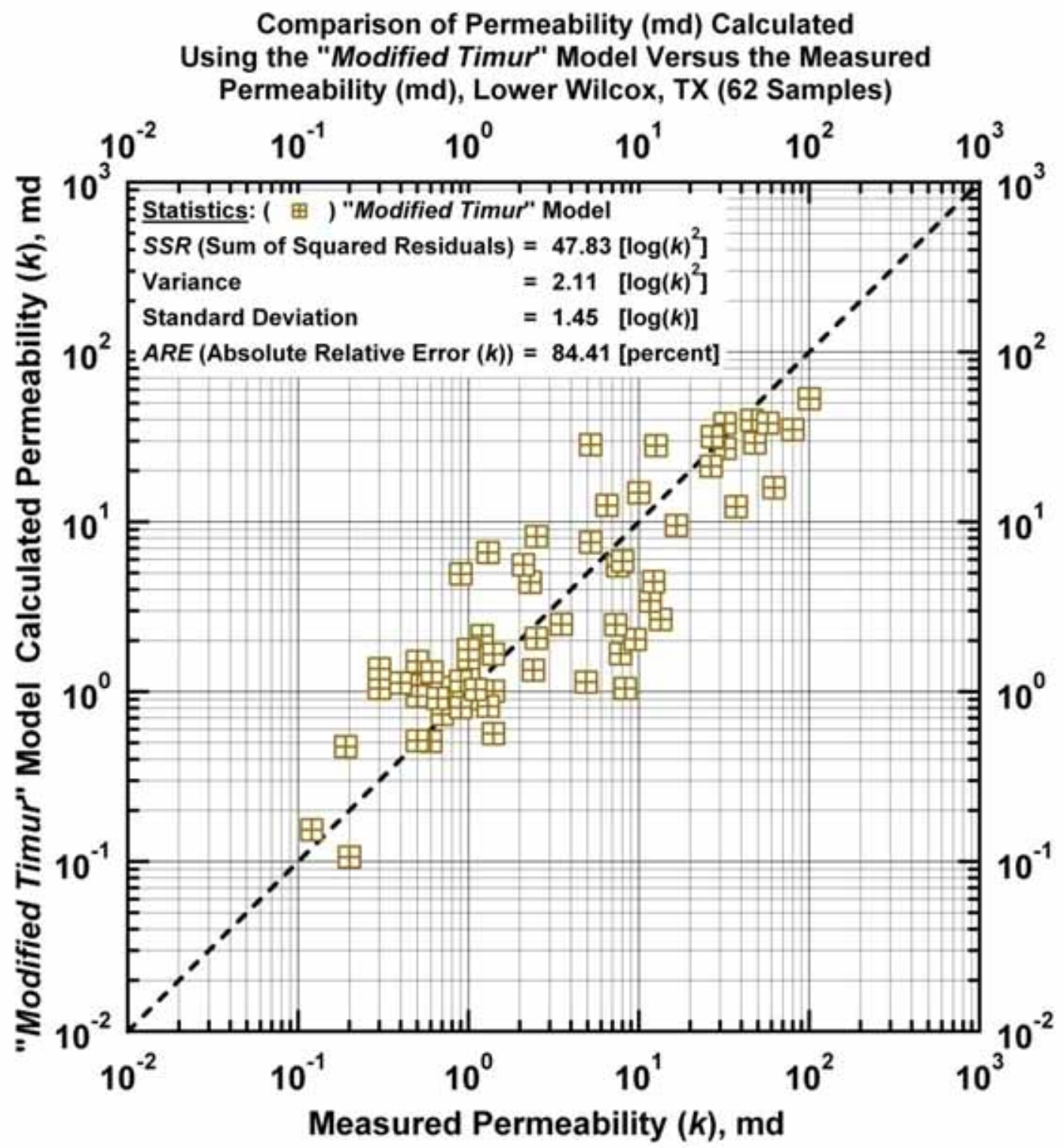

Figure I.10a - "Modified Timur Model:" Calculated versus measured permeability. [Case: Lower Wilcox S. TX (USA)] 


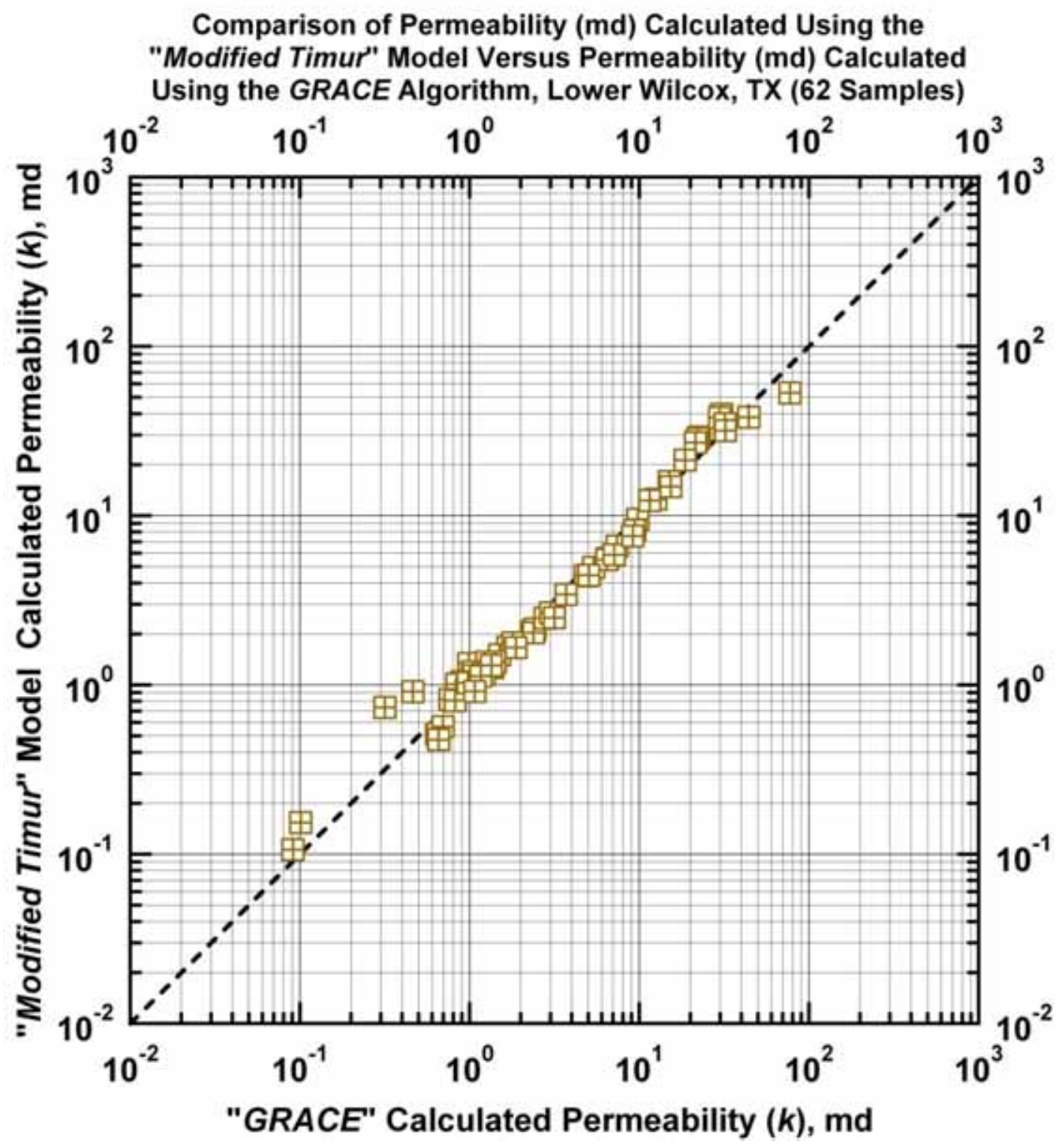

Figure I.10b - "Modified Timur Model:" Permeability calculated using the "Modified Timur Model" versus permeability calculated using the GRACE Algorithm. [Case: Lower Wilcox S. TX (USA)] 


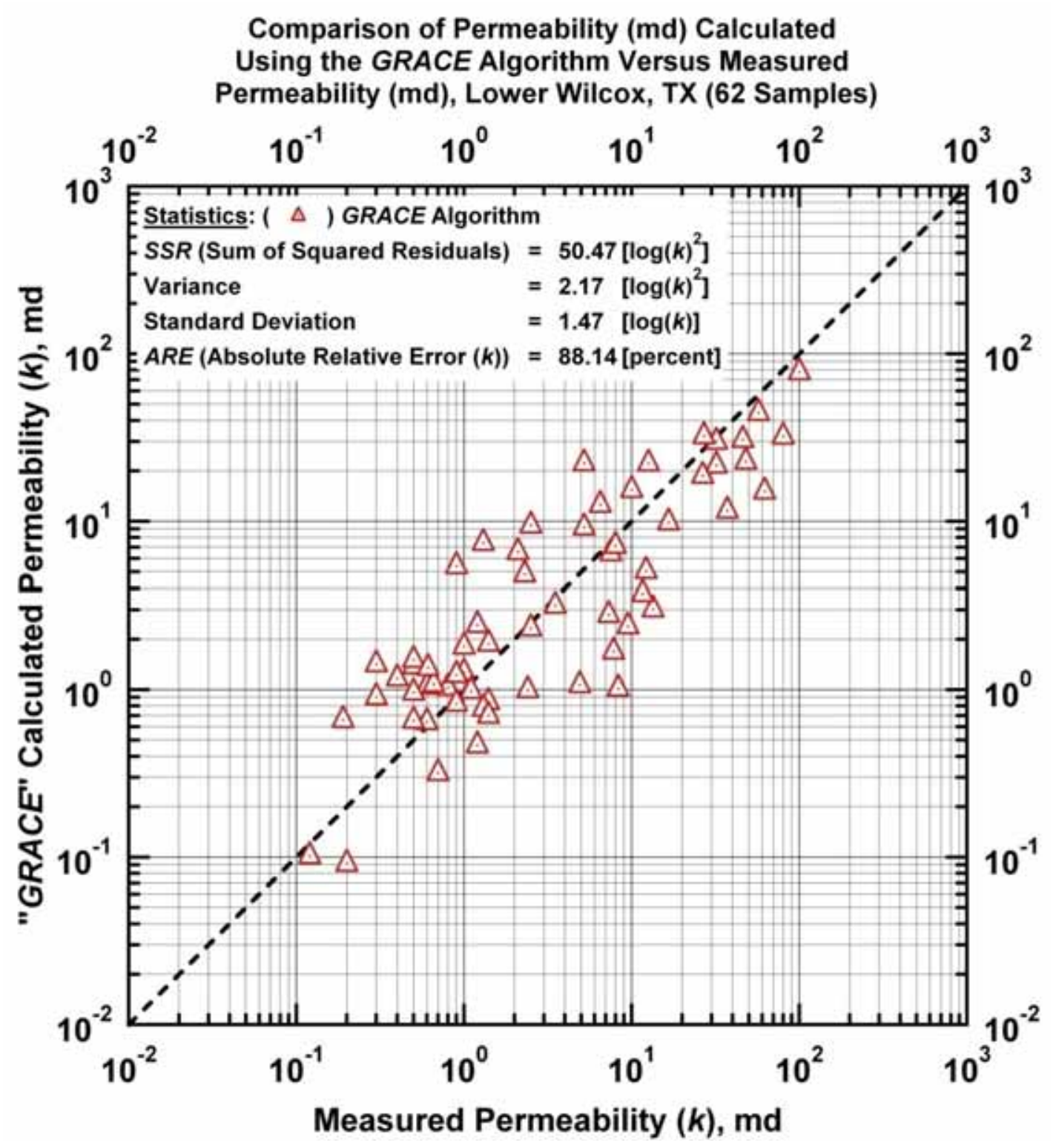

Figure I.11 - GRACE Model: Calculated versus measured permeability. [Case: Lower Wilcox S. TX (USA)] 


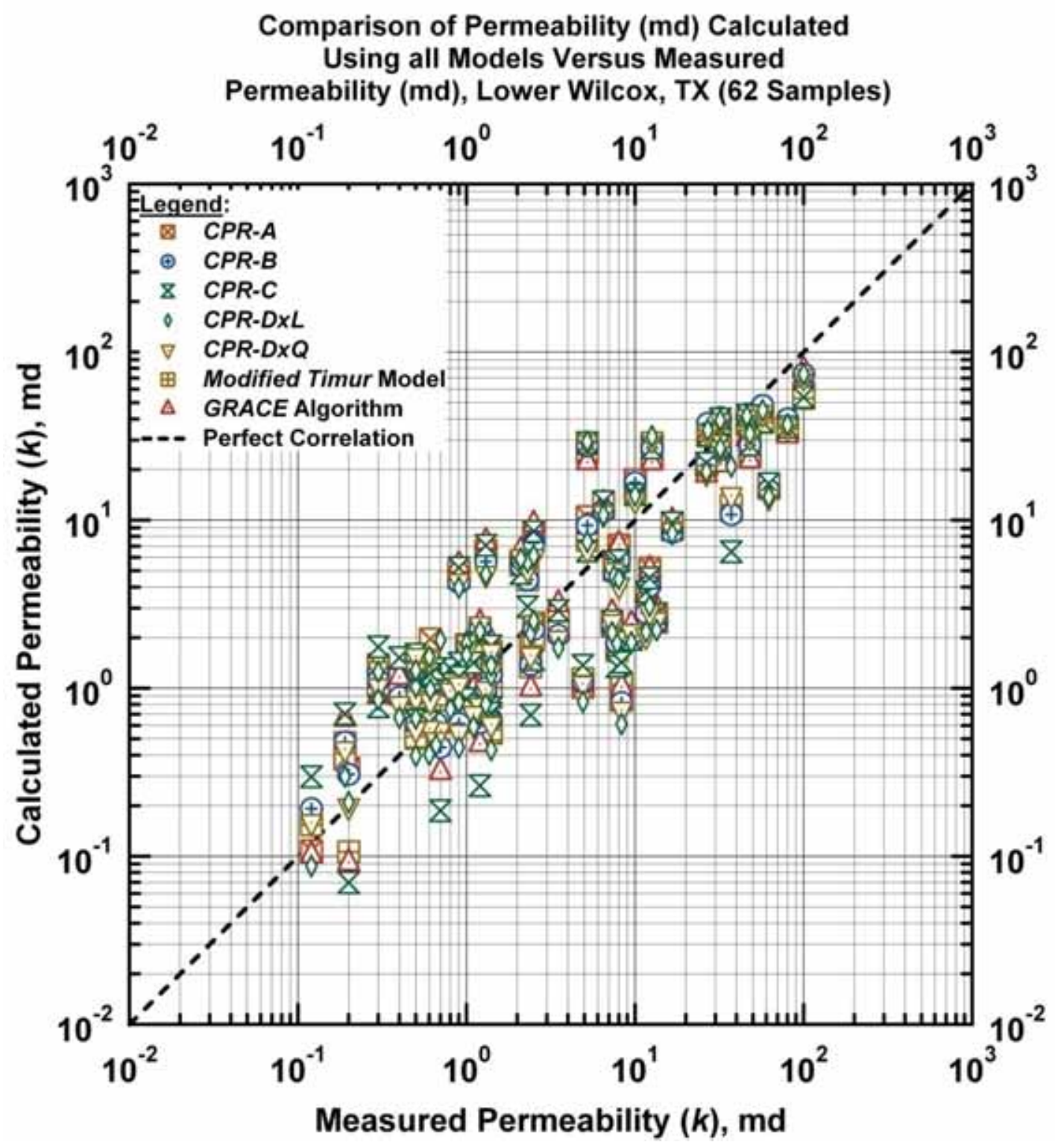

Figure I.12 - All Models: Calculated versus measured permeability. [Case: Lower Wilcox S. TX (USA)] 


\section{APPENDIX $\mathbf{J}$ \\ RESULTS FOR LOWER PALUXY CLEAN SANDSTONE (TEXAS - USA)}

In this Appendix we present the "Paluxy" Well, located in Texas (USA) — this is a clean sandstone reservoir (Ellis, 1987).

\section{Model CPJ-A: (Appendix J)}

The "characteristic permeability relation" (or CPR) Model CPJ-A (i.e., the modified Archie "Clean Sand" Model) is given as:

$$
k=a(\phi-c)^{b} \quad c=c_{\max } \exp \left[-c_{1} \phi^{c 2} F^{c_{3}}\right]
$$

Where a plot of $\log (k)$ versus $\log (\phi)$ is used to graphically calibrate Eq. J-1 in terms of the coefficients $a, b$, and $c_{\max }$. Fig. J.1 shows that the CPJ-A model performs quite well for this case. Figs. J.1a and $\mathbf{b}$ show the log-log and the semilog format of permeability versus porosity, respectively. In Fig. J.2 we provide the "error" plots for this case, where the measured and computed data are compared systematically. In Fig. J.2a we note a good correlation of the results obtained using Model CPJ-A versus the measured permeability data - there is good agreement with the perfect correlation trend (i.e., the 45degree line). In Fig. J.2b we provide a comparison with the GRACE algorithm correlation (Xue et al. 1997)), and we note that the CPJ-A model results correlate very well with the GRACE algorithm results for this case. Lastly, in Fig. J.2c we present the calculated and measured $c$-function - and again we note a reasonable correlation of the data about the perfect correlation trend.

\section{Model CPJ-B: (Appendix J)}

The "characteristic permeability relation" (or CPR) Model CPJ-B (i.e., the modified Archie "Dirty Sand" Model (power-law basis)) is given as:

$$
k=a(\phi+c)^{b} \quad c=c_{\max } \exp \left[-c_{1} \phi^{c_{2}} F^{c_{3}}\right]
$$

Where a plot of $\log (k)$ versus $\log (\phi)$ is used to graphically calibrate Eq. J-2 in terms of the coefficients $a, b$, and $c_{\max }$. The results for this case are presented in Figs. J.3 and 4. In Fig. J.3a (log-log format) we note that a very distinct "envelope" is formed by the CPJ-B model, essentially all of the data on the right and left flanks are well-matched (i.e., the "dirty sand" power law relation (applied on the right portion of the data) and its correction function (applied on the left portion of the data). Similarly, in Fig. J.3b (semilog format), we observe an excellent distribution of the power law model across the body of the data. The error analyses for this case are shown in Fig. J.4. In Fig. J.4a we present the results obtained using Model $C P J-B$ versus the measured permeability data. In this case we observe good agreement with the 
perfect correlation trend (i.e., the 45-degree line). We provide a comparison with the GRACE algorithm correlation in Fig. J.4b - the results using Model CPJ-B and the GRACE algorithm compare very well for this case. We compare the computed and data-derived values of the $c$-function for this case in Fig. J.4c and we note a reasonably "tight" correlation, except for the very lowest values of the $c$-function. In summary, we believe that Model $C P J-B$ is a very effective correlation model for this particular data case.

\section{Model CPJ-C: (Appendix J)}

The "characteristic permeability relation" (or $C P R$ ) Model CPJ-C (i.e., the modified Archie "Dirty Sand" Model (exponential basis)) is given as:

$$
k=c \exp [\beta \phi] \quad c=c_{\max } \exp \left[-c_{1} \phi^{c_{2}} F^{c_{3}}\right]
$$

Where a plot of $\log (k)$ versus $\phi$ is used to graphically calibrate Eq. J-3 in terms of the coefficients $-\beta$ and $C_{\max }$. Model $C P J-C$ is fundamentally different than Models $C P J-A$ and $C P J-B$, as the basis for Model $C P J-C$ is an exponential function, not a power law relation. In Figs. J.5 and 6 we present the results of applying Model $C P J-C$ for this case, and in general, we observe a very consistent performance of Model $C P J-C$. Fig. J.5b illustrates the excellent conformance of Model CPJ-C for this case - again recalling that this model has an exponential relationship with porosity as its basis.

In Figs. J.6a, J.6b, and J.6c we review the error analyses plots for this case, and we note good performance (visually) in terms of the correlation of the results using Model CPJ-C. The correlation of the $c$-function (see Fig. J.6c) appears to be consistent — but scatter and a slight off-center trend is evident. We believe that the $C P J-C$ model has performed well for this case, and the plots in Fig. J.6 confirm the value of this model as a "characteristic relation" for permeability.

\section{Model CPJ-D: (Appendix J)}

The "characteristic permeability relation" (or CPR) Model CPJ-D (i.e., the weighted power law-exponential model) is given in its fundamental form as:

$$
\left.k=x a \phi^{b}+(1-x) \alpha \exp [\beta \phi] \quad[0 \leq x \leq 1]\right]
$$

For Eq. J-4 there are two proposed models for the $x$-function (i.e., the weighting function) - these are:

$$
\begin{aligned}
& x=\exp \left[-\ln \left(x_{0}\right)+x_{1} \ln (\phi)+x_{2} \ln (F)+x_{3} \ln (\phi) \ln (F)\right](\text { Model CPJ-DxL) } \\
& x=\exp \left[\begin{array}{l}
-\ln \left(x_{0}\right)+x_{1} \ln (\phi)+x_{2} \ln (F)+x_{3} \ln (\phi) \ln (F) \\
+x_{4} \ln (\phi)^{2}+x_{5} \ln (F)^{2}+x_{6} \ln (\phi)^{2} \ln (F) \\
+x_{7} \ln (\phi) \ln (F)^{2}+x_{8} \ln (\phi)^{2} \ln (F)^{2}
\end{array}\right](\text { Model CPJ-DxL)......... }
\end{aligned}
$$

As noted in Eq. J-4b, Model CPJ-DxL utilizes a logarithmic linear model for the weights ( $x$-values) and as seen in Eq. J-4c, Model CPJ-DxQ utilizes a logarithmic quadratic model for the weights ( $x$-values). While these models (Eqs. J-4b and J-4c) are empirical, we have found generally good application of these relations for all cases. In this case, the base results using Models $C P J-D x L$ and $C P J-D x Q$ are presented in 
Fig. J.7 - where the power law and exponential basis functions are fitted to the appropriate portion of the data. The power law equation represents the "Archie clean sand" trend and is fitted to the leftmost data as shown in Fig. J.7a and in contrast, the exponential equation is thought to represent the "Archie dirty sand" trend and the exponential is fitted to the far rightmost portion of the data (see Fig. J.7b).

In Figs. J.8 and 9 we present the error analyses for this case, where we have employed the weighting functions (Eqs. J-4b and J-4c) as appropriate. In Fig. J.8a we find a good correlation of permeability (with the out-lying points noted in the middle of the trend). The comparison of the Model CPJ-DxL results are compared to the results obtained from the GRACE algorithm in Fig. J.8b, we note that the very good results obtained using Model CPJ-DxL. The computed weight function ( $x$-values) shown for the $C P J-D x L$ model in Fig. J.8c does exhibit more scatter than expected, but the trend is (relatively) centered about the perfect correlation line.

The results obtained using Model $C P J-D x Q$ are presented in Fig. J.9, and we note substantially improved behavior over that of Model CPJ-DxL. In particular, the base correlation of computed and measured permeabilities (Fig. J.9a) for Model CPJ-DxQ does indicate a slightly better correlation than that of Model CPJ-DxL. Also, the comparison of Model CPJ-DxQ with the results from the GRACE algorithm (Fig. J.9b) exhibits better agreement than the results of Model CPJ-DxL. Finally, the correlation of the $x$ function (Fig. J.9c) for Model CPJ-DxQ is better than the correlation for Model CPJ-DxL. These comparisons suggest that Model CPJ-DxQ (i.e., the combination of Eqs. J-4a and J-4c) has provided a better correlation of data, for this case, than Model CPJ-DxL.

"Modified Timur Model": (Appendix J)

The "Modified Timur Model" is given as:

$$
k_{\text {Timur }}=a \phi^{b} F^{c}
$$

In Fig. J.10 we present the results of the "Modified Timur Model" (Eq. J-5) which is essentially just a generalized power-law function relation in terms of $k, \phi$, and $F$. Figs. J.10a and b, provide the "Modified Timur Model" results of the data for this case.

\section{GRACE Algorithm: (Appendix J)}

In Fig. J.11 we present the results predicted by the GRACE algorithm, which is a non-parametric regression approach (see Xue et al. (1997) for details). In theory, the GRACE algorithm should provide the most unbiased correlation of the data - i.e., the GRACE algorithm is designed not to "fit the errors" as other regression approaches may. It is our contention that the GRACE algorithm is the statistical standard - and any algorithm/approach/model which achieves better regression statistics than the GRACE algorithm is actually "fitting the errors" in the data. Surprisingly all the cases have significantly better regression statistics than the GRACE algorithm — hence, we must label these cases as "over-fitted" in a statistical sense. 


\section{Comparison of All Models: (Appendix J)}

Our final graphic, Fig. J.12, illustrates all of the models on a single plot of calculated versus measured permeability. We also present a table of all statistical results in Table J.1.

Table J.1 Statistical Results for all Models (Paluxy TX (USA), $n=43$ ).

\begin{tabular}{|c|c|c|c|c|}
\hline Model & $\begin{array}{c}\text { Sum of } \\
\text { Squared } \\
\text { Residuals } \\
{\left[\log (k)^{2}\right]}\end{array}$ & $\begin{array}{l}\text { Variance } \\
{\left[\log (k)^{2}\right]}\end{array}$ & $\begin{array}{c}\text { Standard } \\
\text { Deviation } \\
{[\log (k)]}\end{array}$ & $\begin{array}{c}\text { Absolute } \\
\text { Relative } \\
\text { Error } \\
\text { [percent] }\end{array}$ \\
\hline CPJ-A & 18.60 & 5.10 & 2.26 & 45.26 \\
\hline$C P J-B$ & 16.00 & 4.90 & 2.21 & 42.69 \\
\hline$C P J-C$ & 15.38 & 5.05 & 2.25 & 44.37 \\
\hline$C P J-D x L$ & 15.52 & 4.91 & 2.22 & 46.13 \\
\hline$C P J-D x Q$ & 14.43 & 4.94 & 2.22 & 44.86 \\
\hline Modified Timur & 15.28 & 4.98 & 2.23 & 39.75 \\
\hline GRACE Algorithm & 15.73 & 4.88 & 2.21 & 47.68 \\
\hline
\end{tabular}

The coefficients for the models used in this case are provided in Table J.2.

Table J.2a - Model Coefficients (Paluxy TX (USA), $n=43$ ).

\begin{tabular}{|c|c|c|c|c|c|c|}
\hline Model & $a$ or $\alpha$ & $b$ or $\beta$ & $c$ or $c_{\max }$ & $c_{1}$ & $C_{2}$ & $c_{3}$ \\
\hline$C P J-A$ & $1.50 \times 10^{9}$ & 8.00 & 0.090 & $1.16 \times 10^{-3}$ & -6.21 & -1.47 \\
\hline$C P J-B$ & $1.50 \times 10^{7}$ & 8.00 & 0.110 & 2.62 & 3.21 & 1.38 \\
\hline$C P J-C$ & $1.50 \times 10^{-3}$ & 50.00 & 0.05 & $1.69 \times 10^{2}$ & 4.91 & 1.12 \\
\hline Modified Timur & $5.76 \times 10^{5}$ & 0.94 & -2.194 & - & - & - \\
\hline
\end{tabular}

Table J.2b - Model Coefficients (Paluxy TX (USA), $n=43$ ).

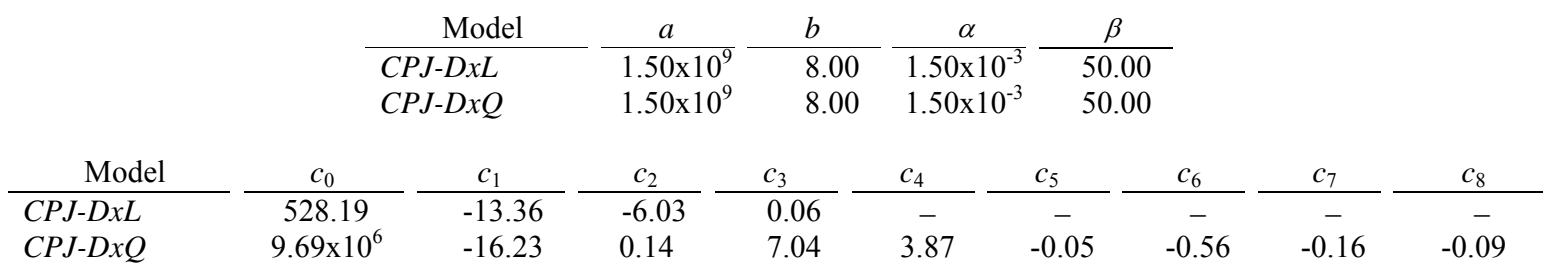

\section{Nomenclature: (Appendix J)}

$F=$ Archie Formation Factor (dimensionless)

$k=$ Formation Permeability, md (or any consistent units)

$\phi \quad=$ Porosity, fraction

\section{References: (Appendix J)}

Ellis, K.W.: "Extended Correlations of Porosity, Permeability, and Formation Resistivity Factor," MS thesis, Texas A\&M U., College Station, TX (1987).

Xue, G., Datta-Gupta, A., Valko, P., and Blasingame, T.A.: "Optimal Transformations for Multiple Regression: Application to Permeability Estimation from Well Logs," SPEFE (June 1997), 85-93. 


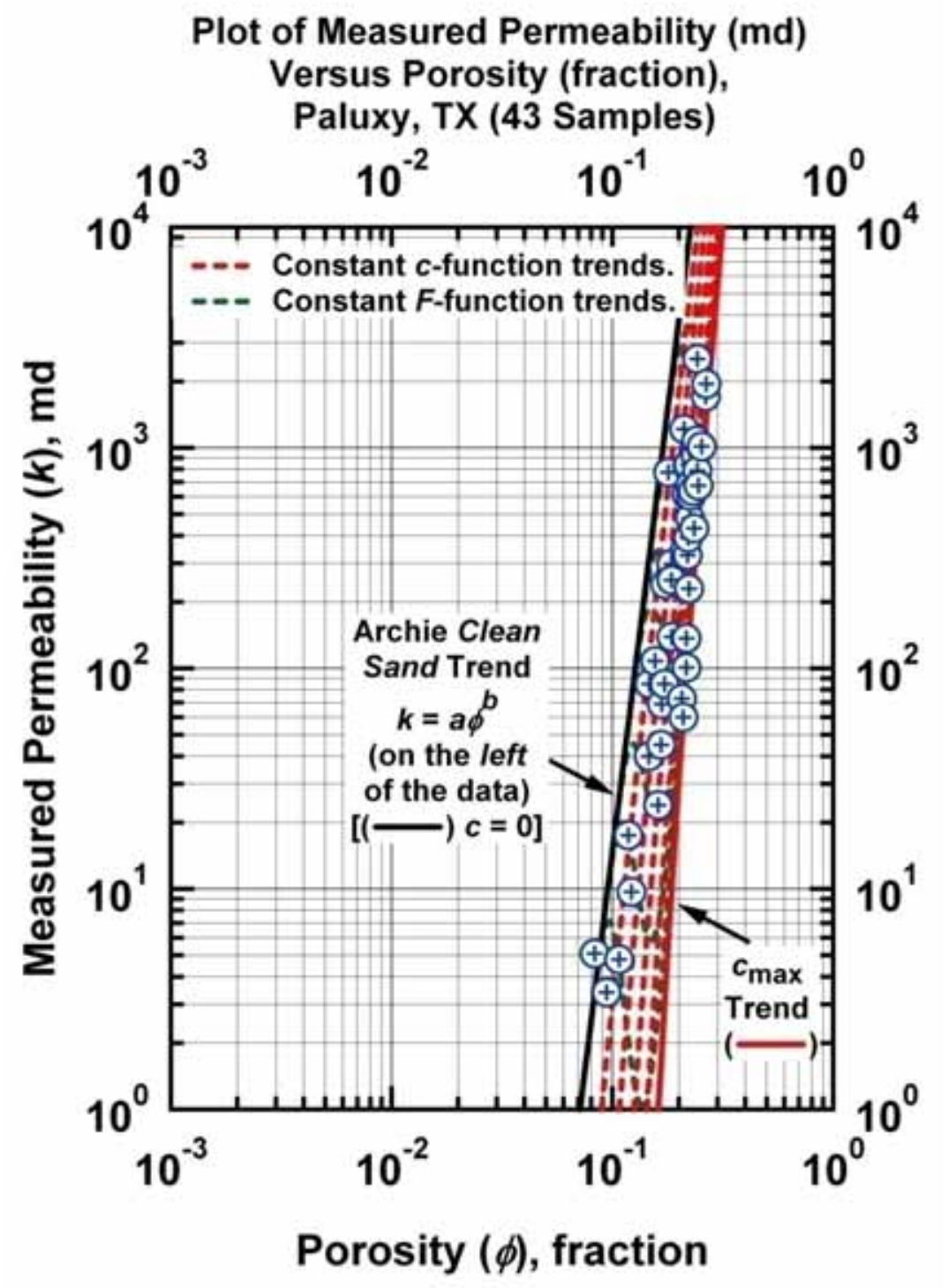

Figure J.1a - Model CPJ-A: $k=a(\phi-c)^{b}\left(c=c_{\max } \exp \left[-c_{1} \phi^{c 2} F^{c 3}\right]\right)$ - "Clean Sand" Plot (log-log format) - Archie "Clean Sand" trend is given by the straight-line trend at the far left of the data (power law model). [Case: Paluxy, TX (USA)] 


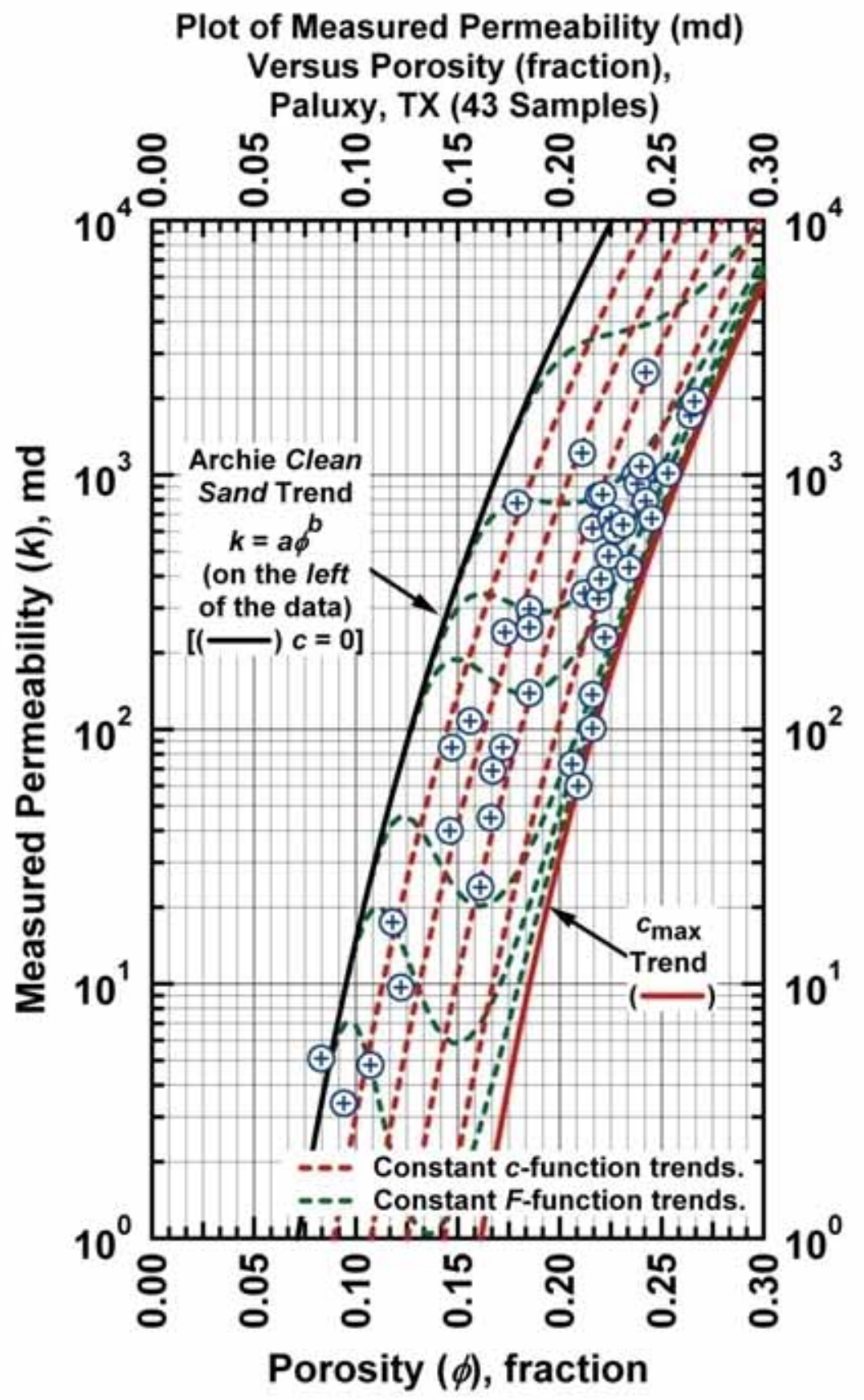

Figure J.1b - Model CPJ-A: $k=a(\phi-c)^{b}\left(c=c_{\max } \exp \left[-c_{1} \phi^{c 2} F^{c_{3}}\right]\right)$ - "Dirty Sand" Plot (semilog format). Archie "Clean Sand" trend is given by the curved trend at the far left of the data (power law model). [Case: Paluxy, TX (USA)] 


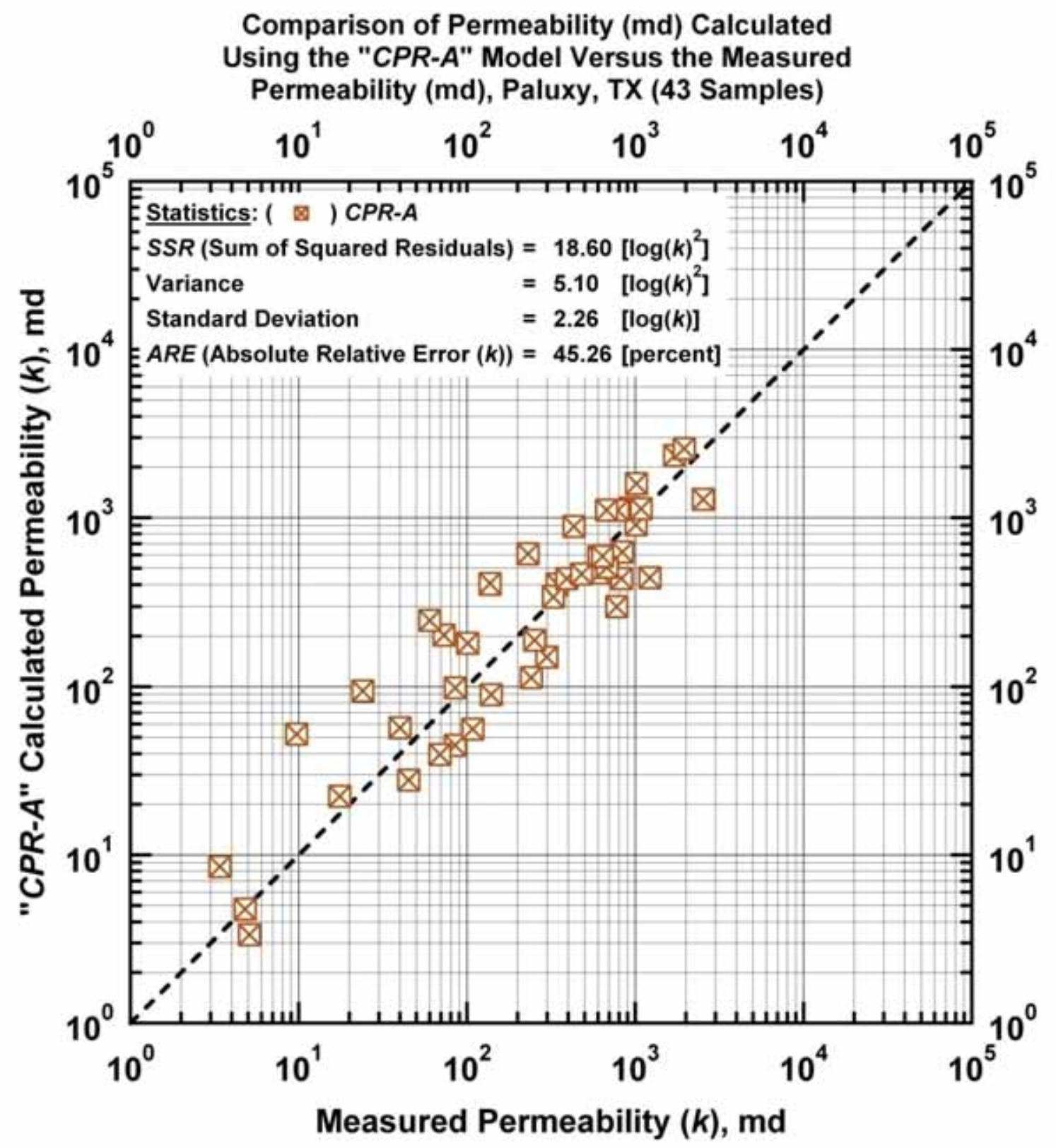

Figure J.2a - Model CPJ-A: calculated versus measured permeability. [Case: Paluxy, TX (USA)] 


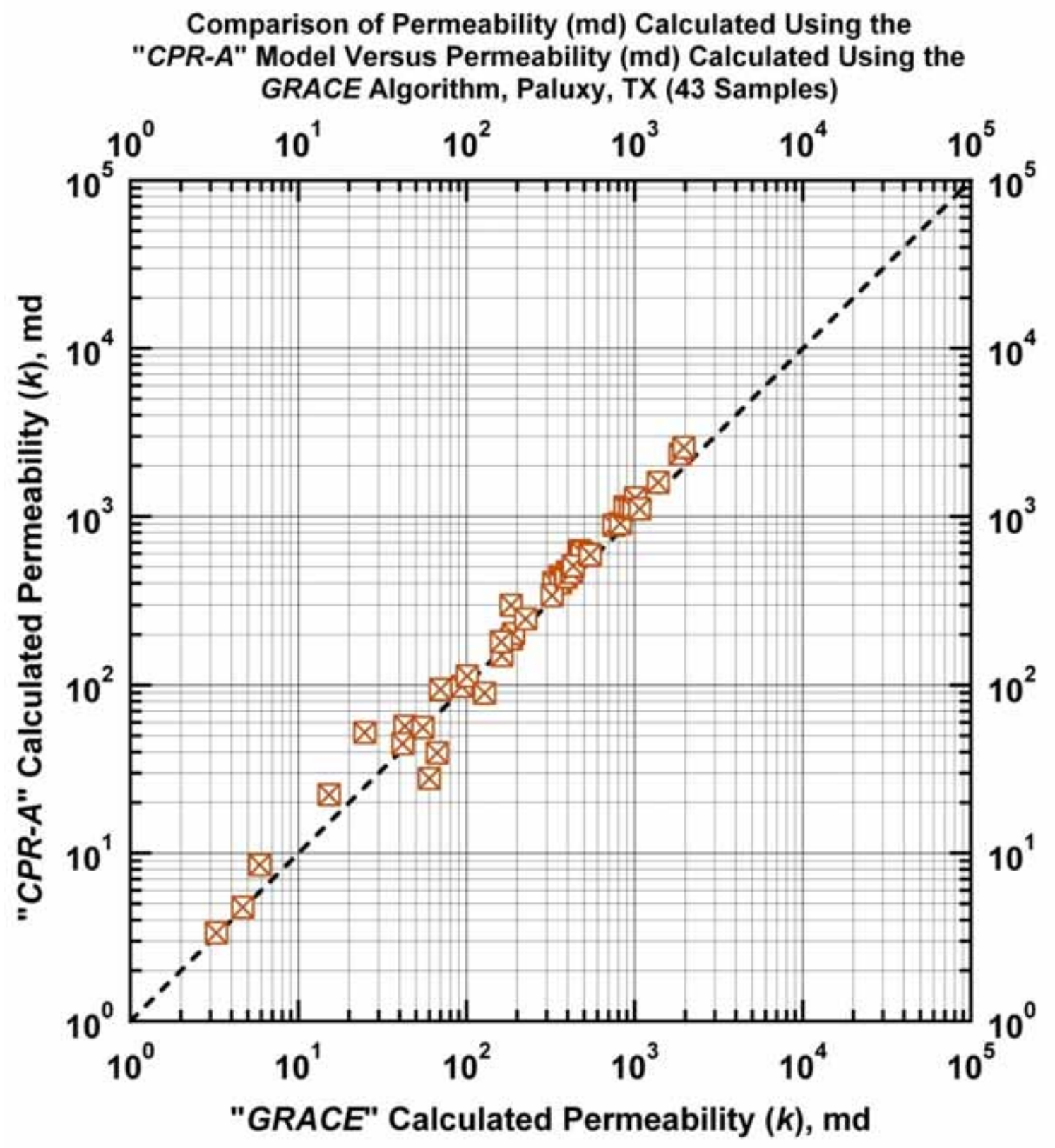

Figure J.2b - Model $C P J-A$ : Permeability calculated using Model $C P J-A$ versus permeability calculated using the GRACE Algorithm. [Case: Paluxy, TX (USA)] 


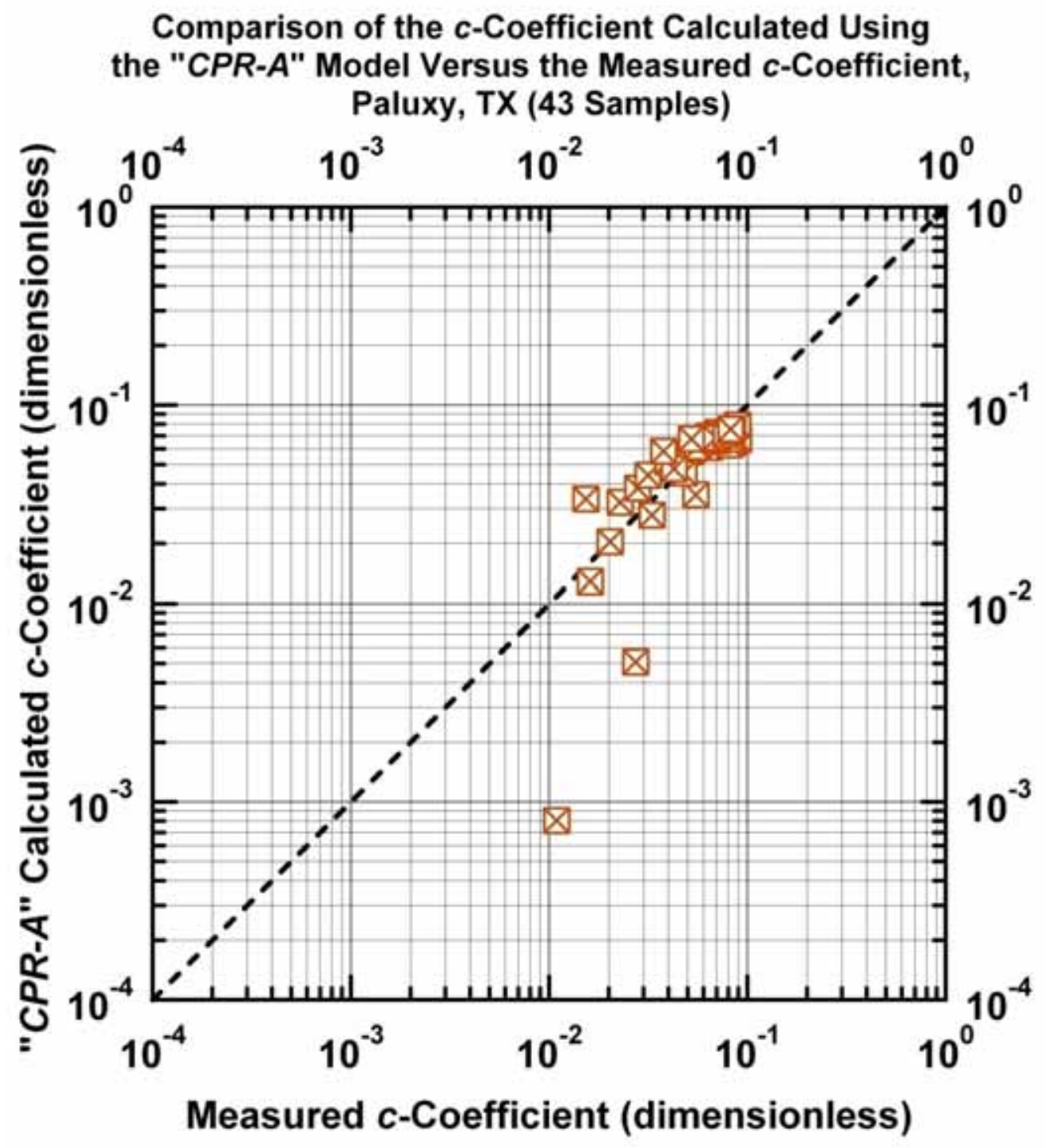

Figure J.2c - Model CPJ-A: Calculated $c$-function values versus measured $c$-function values. [Case: Paluxy, TX (USA)] 


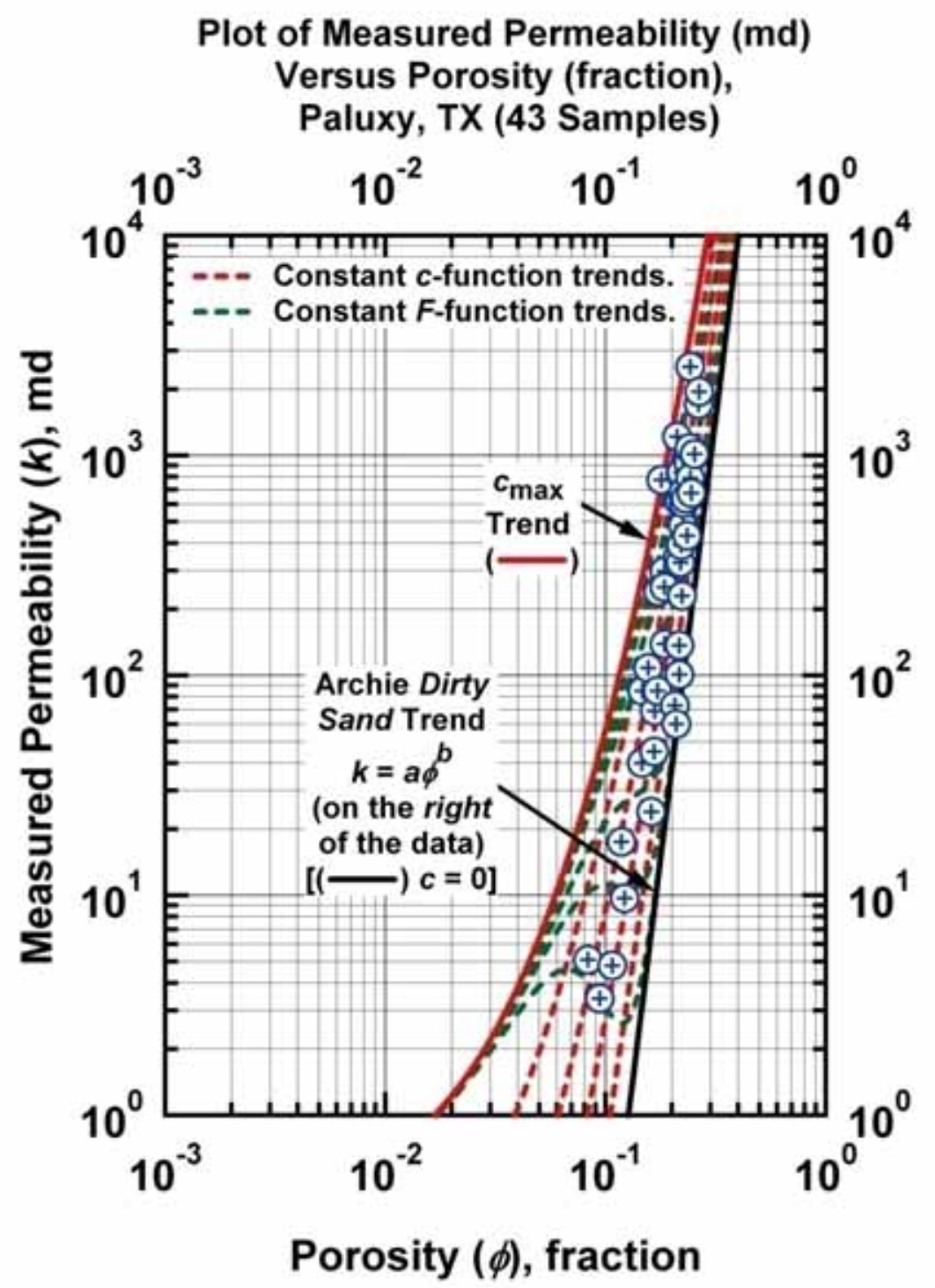

Figure J.3a - Model $C P J-B: \quad k=a(\phi+c)^{b} \quad c=c_{\max } \exp \left[-c_{1} \phi^{c_{2}} F^{c_{3}}\right]$ - "Clean Sand" Plot (log-log format) - Archie "Dirty Sand" trend is given by the straight-line trend at the far right of the data (power law model). [Case: Paluxy, TX (USA)] 


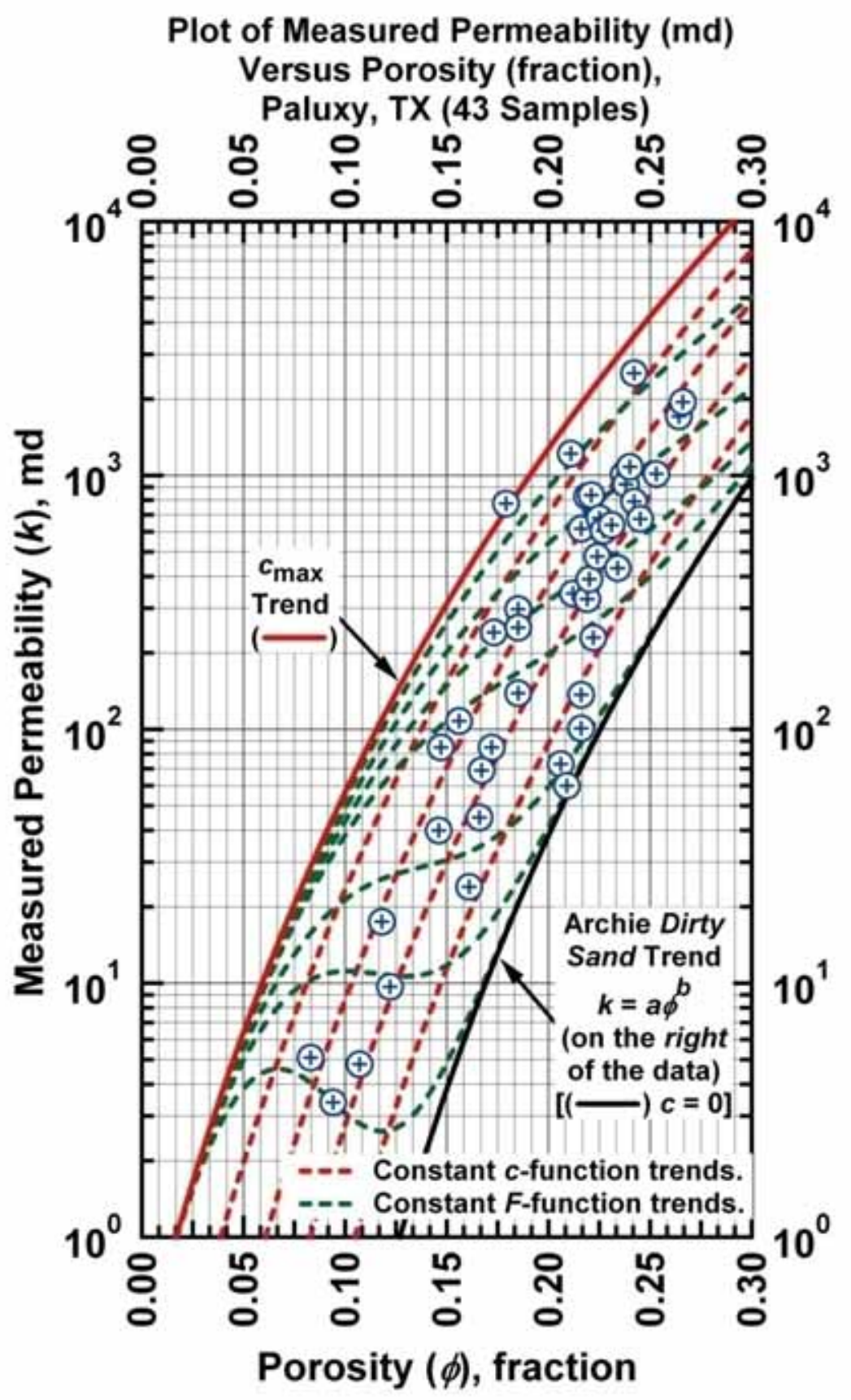

Figure J.3b - Model CPJ-B: $k=a(\phi+c)^{b} \quad c=c_{\max } \exp \left[-c_{1} \phi^{c_{2}} F^{c_{3}}\right]$ — "Dirty Sand" Plot (semilog format) - Archie "Dirty Sand" trend is given by the curved trend at the far right of the data (power law model). [Case: Paluxy, TX (USA)] 


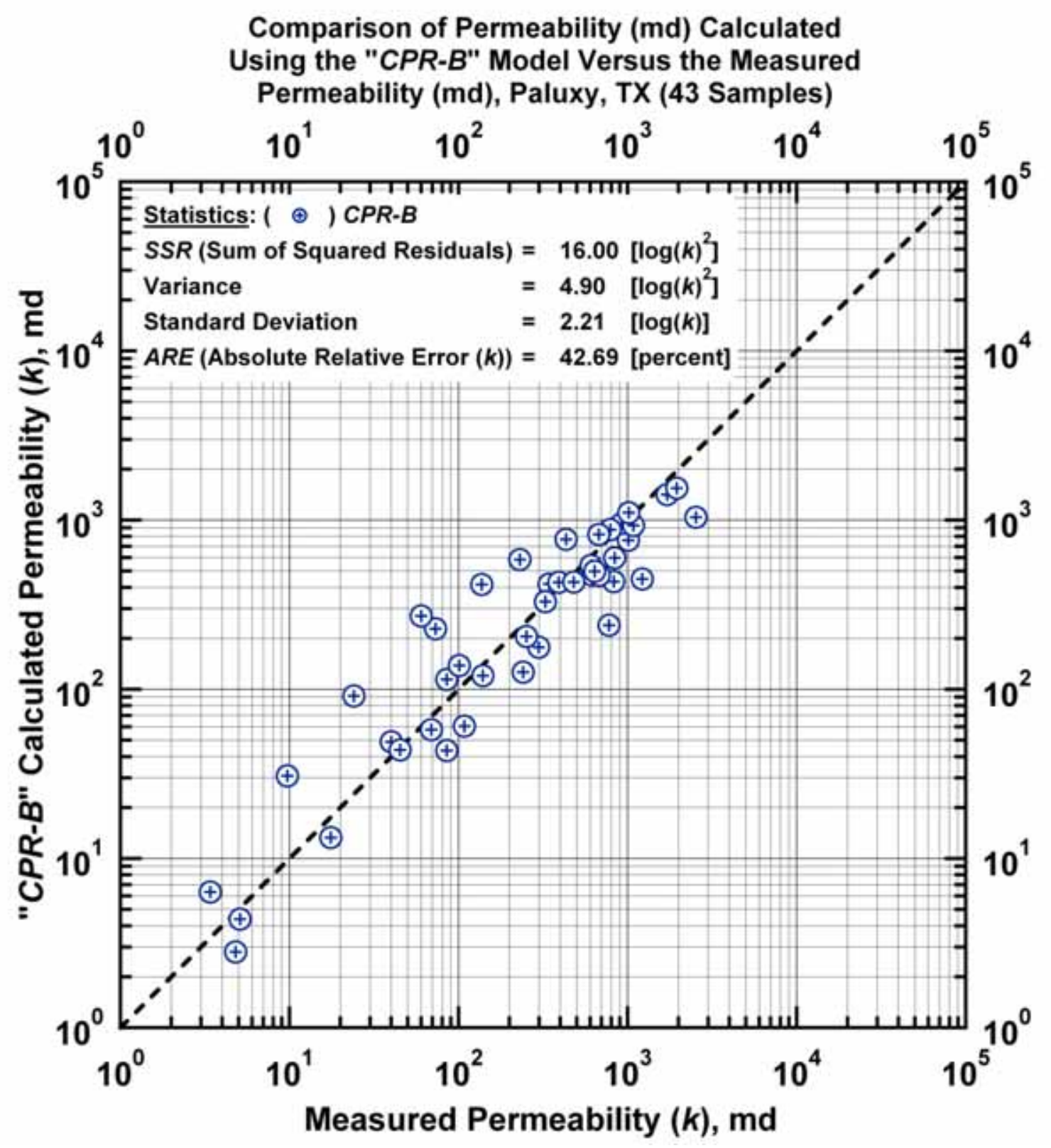

Figure J.4a - Model CPJ-B: calculated versus measured permeability. [Case: Paluxy, TX (USA)] 


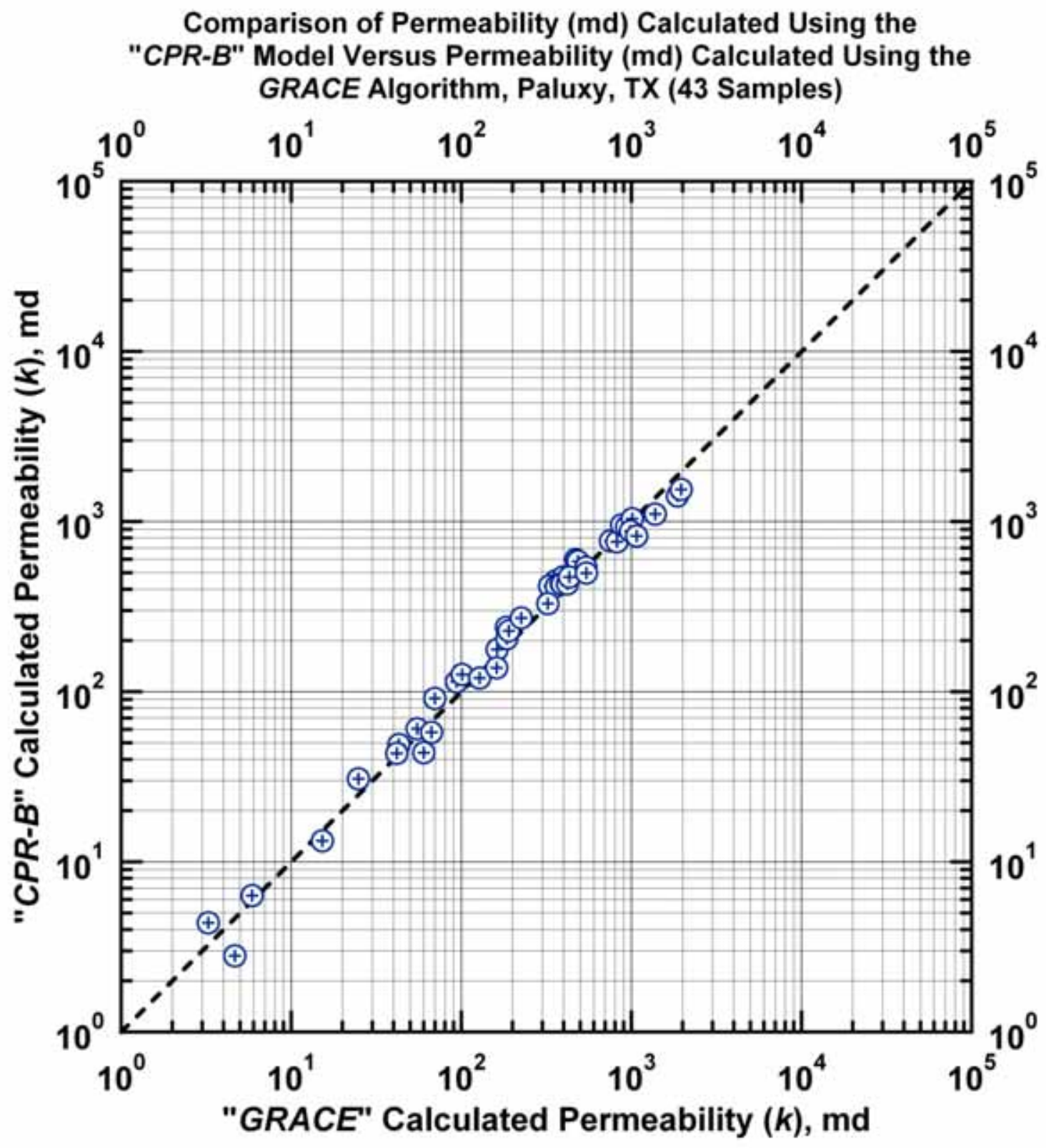

Figure J.4b - Model $C P J-B$ : Permeability calculated using Model $C P J-B$ versus permeability calculated using the GRACE Algorithm. [Case: Paluxy, TX (USA)] 


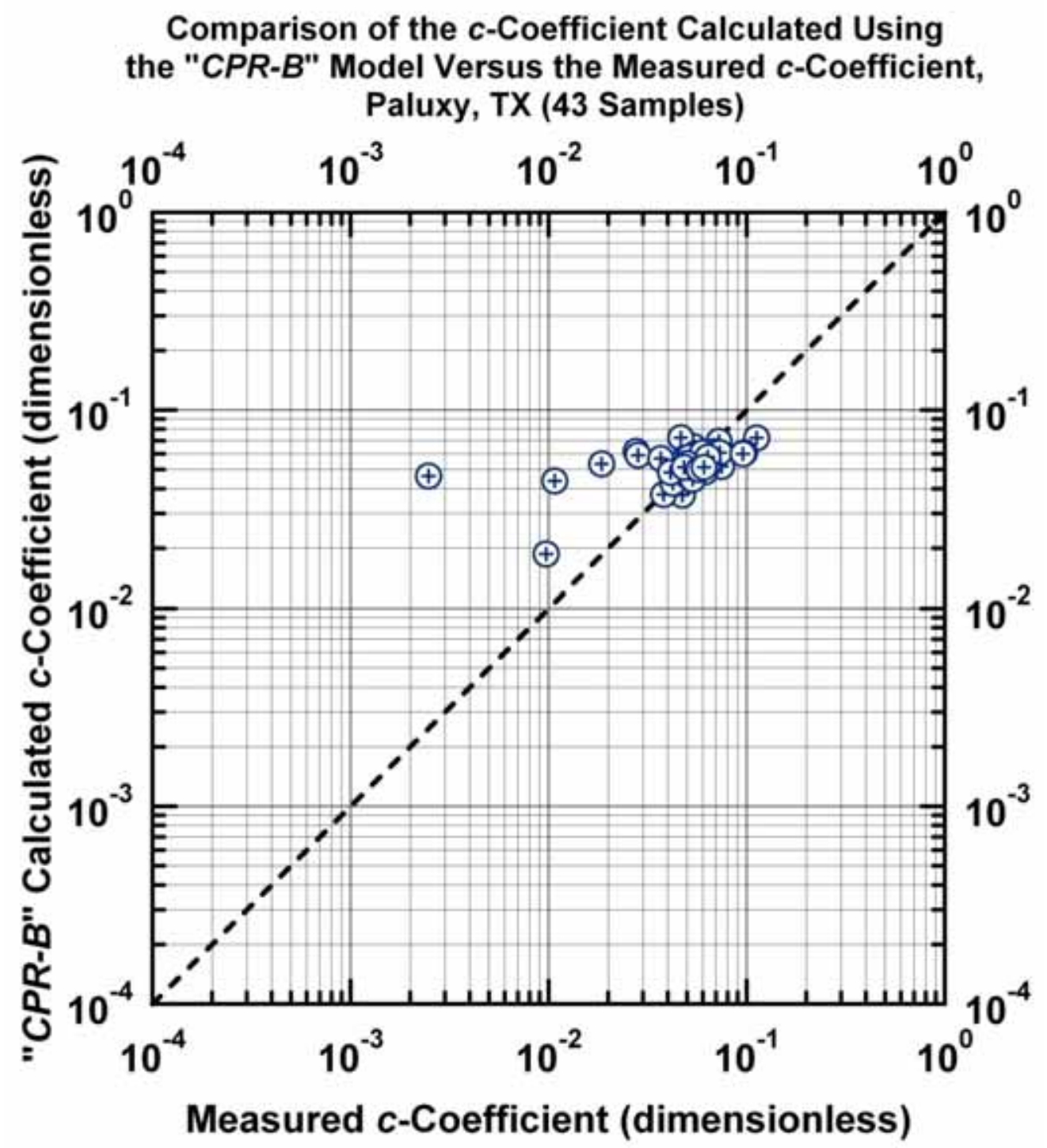

Figure J.4c - Model $C P J-B$ : Calculated $c$-function values versus measured $c$-function values. [Case: Paluxy, TX (USA)] 


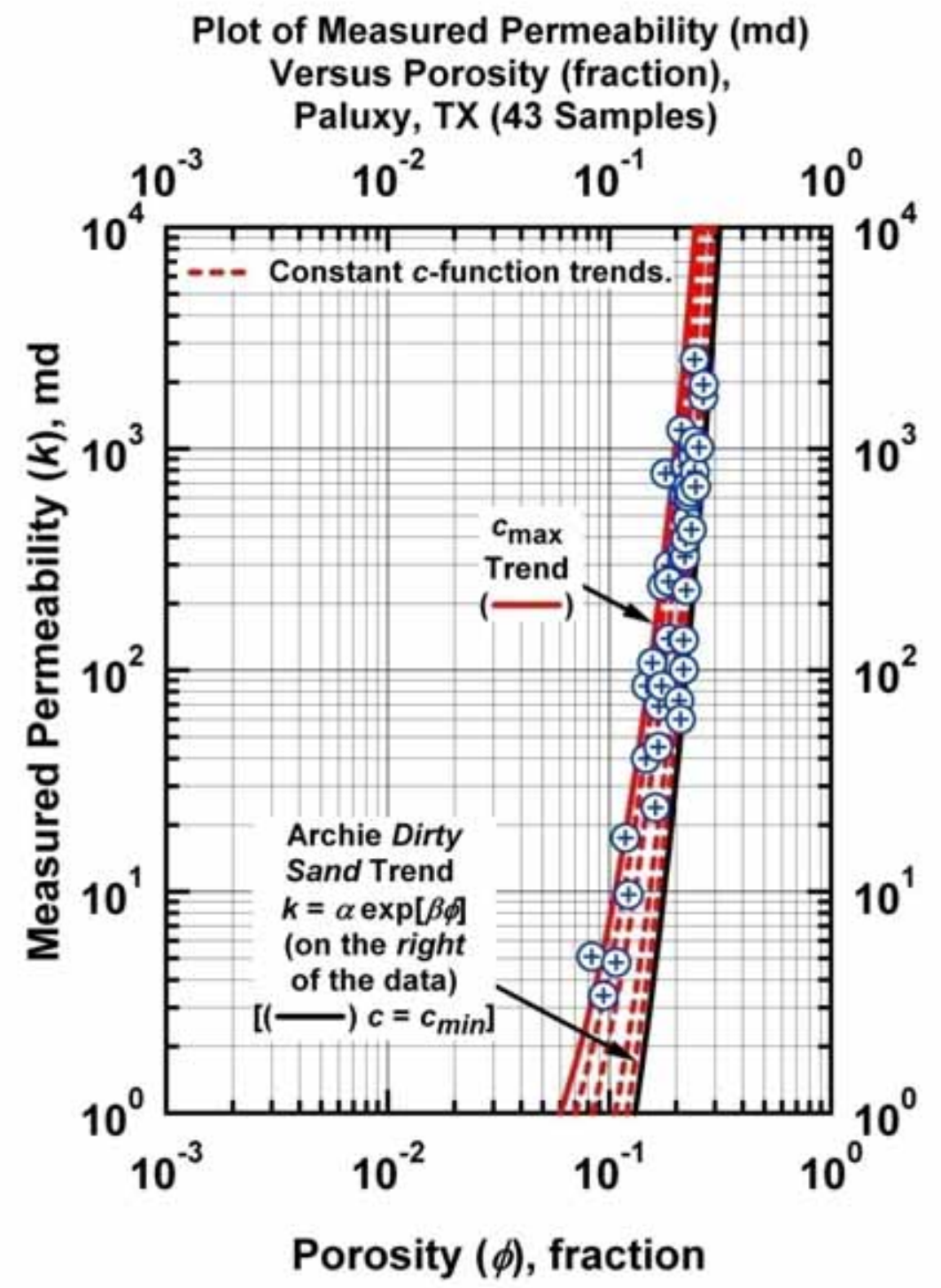

Figure J.5a - Model $C P J-C: \quad k=c \exp [\beta \phi] \quad c=c_{\max } \exp \left[-c_{1} \phi^{c_{2}} F^{c_{3}}\right]$ - "Clean Sand" Plot (log-log format) - Archie "Dirty Sand" trend is given by the curved trend at the far right of the data (exponential model). [Case: Paluxy, TX (USA)] 


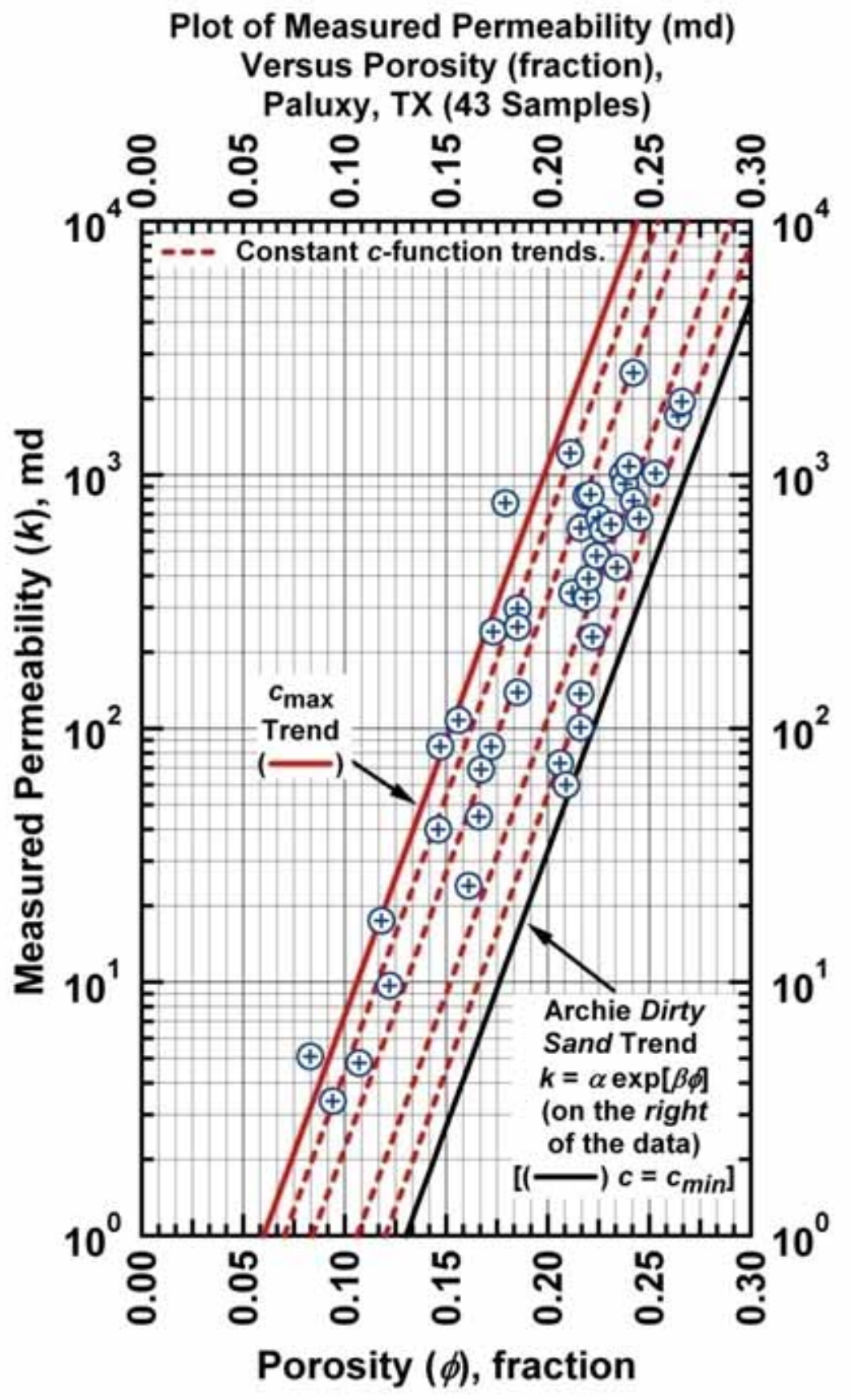

Figure J.5b - Model $C P J-C: \quad k=c \exp [\beta \phi] \quad c=c_{\max } \exp \left[-c_{1} \phi^{c 2} F^{c 3}\right]$ - "Dirty Sand" Plot (semilog format) - Archie "Dirty Sand" trend is given by the straight-line trend at the far right of the data (exponential model). [Case: Paluxy, TX (USA)] 


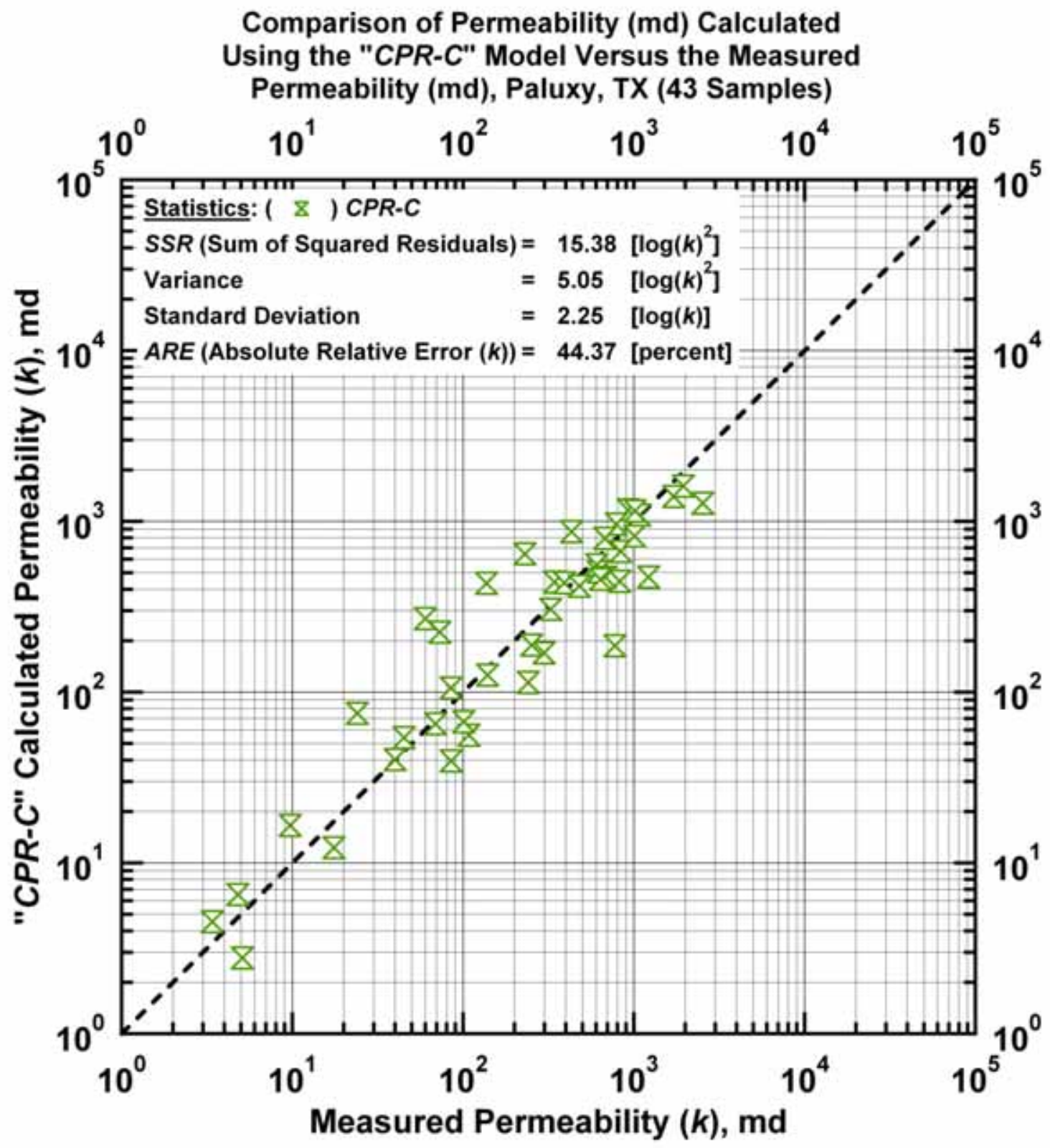

Figure J.6a - Model CPJ-C: calculated versus measured permeability. [Case: Paluxy, TX (USA)] 


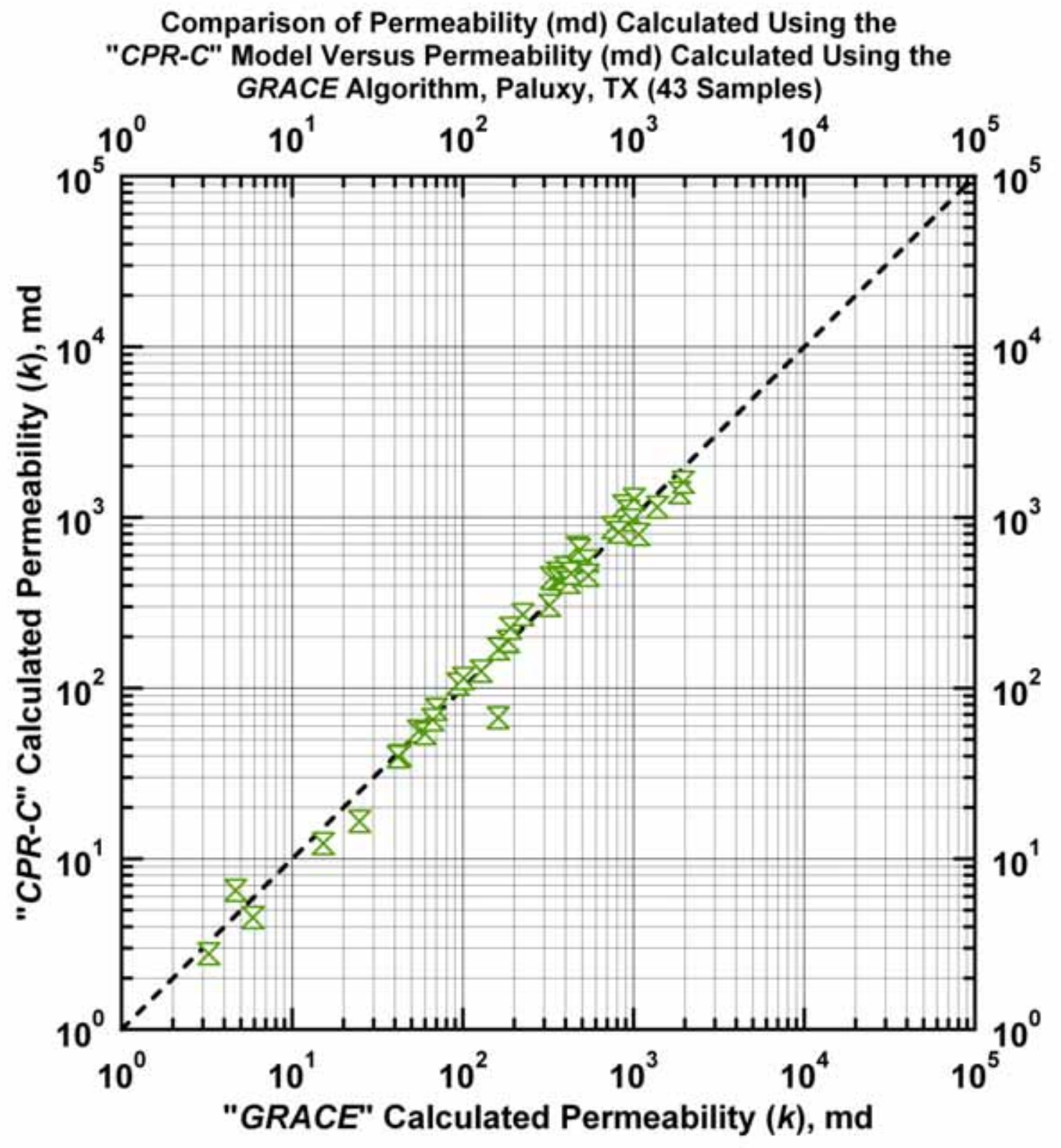

Figure J.6b - Model $C P J-C$ : Permeability calculated using Model $C P J-C$ versus permeability calculated using the GRACE Algorithm. [Case: Paluxy, TX (USA)] 
Comparison of the $c$-Coefficient Calculated Using the "CPR-C" Model Versus the Measured $c$-Coefficient, Paluxy, TX (43 Samples)

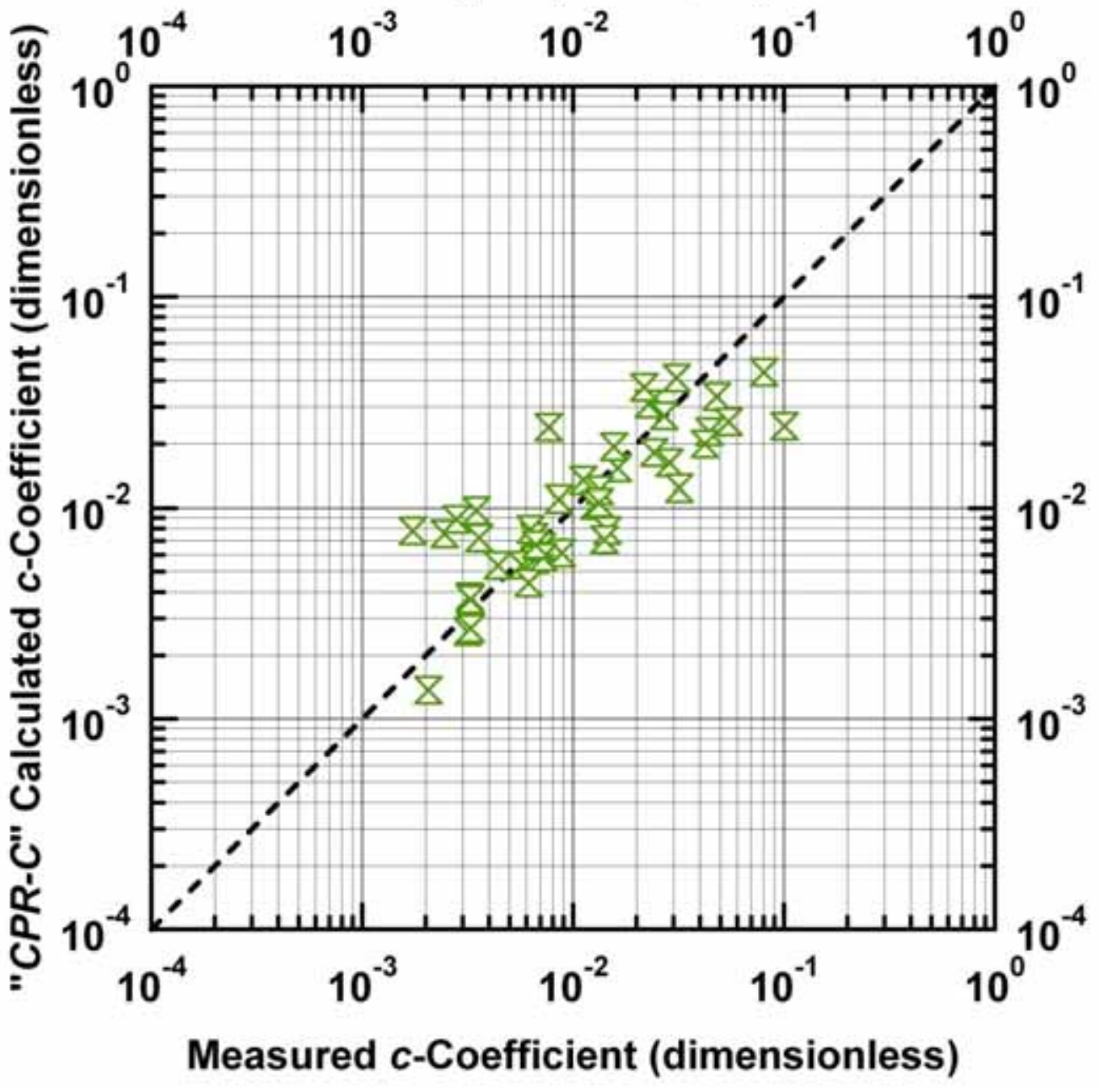

Figure J.6c - Model $C P J$-C: Calculated $c$-function values versus measured $c$-function values. [Case: Paluxy, TX (USA)] 


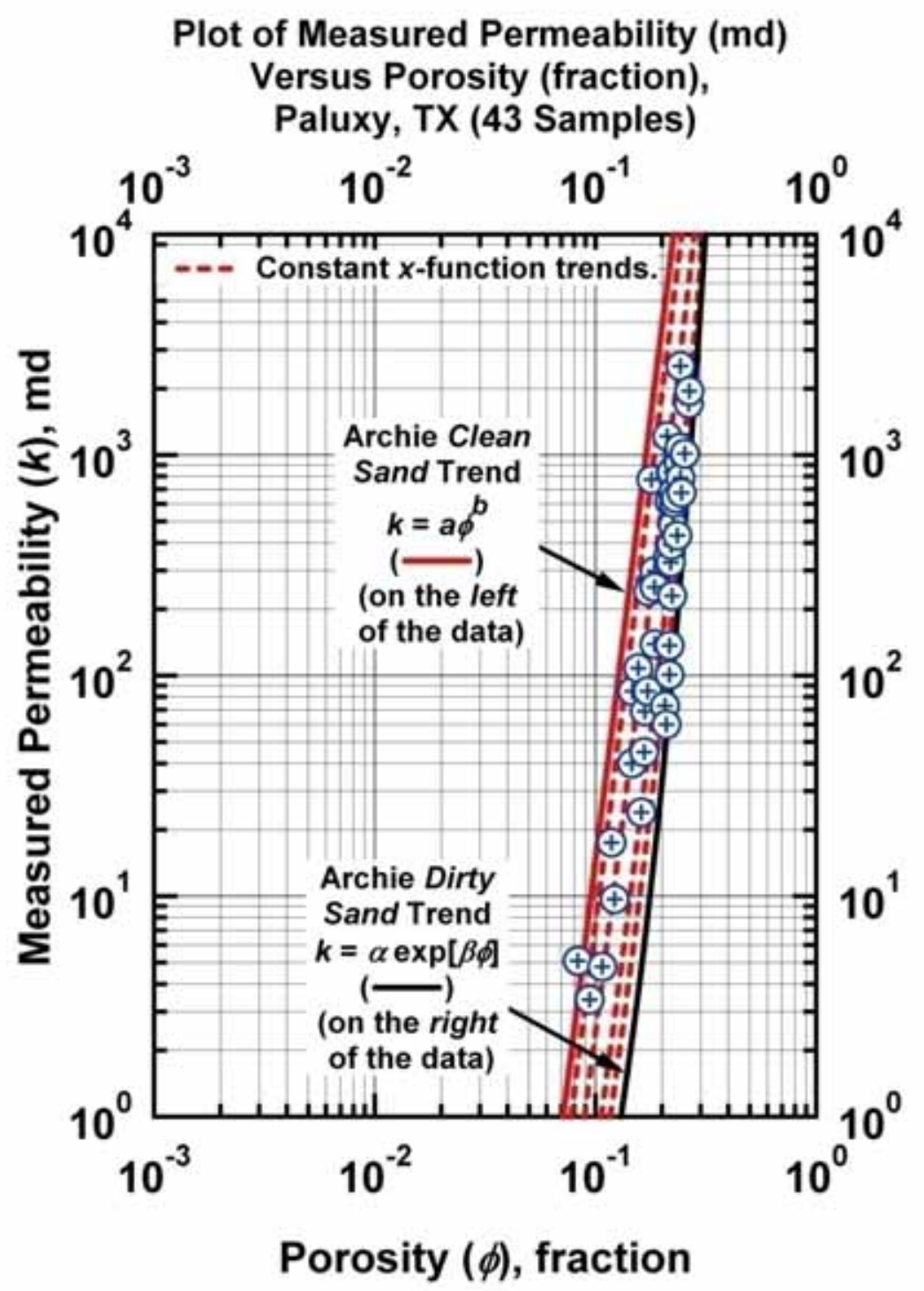

Figure J.7a - Model CPJ-D: $k=x a \phi^{b}+(1-x) \alpha \exp [\beta \phi] \quad[0 \leq x \leq 1]$ - Weighted Power Law-Exponential Model used to correlate permeability $(k)$ and porosity $(\phi)$. "Clean Sand" Plot - Archie "Clean Sand" trend is given by the straight-line trend at the far left of the data (power law model). [Case: Paluxy, TX (USA)] 


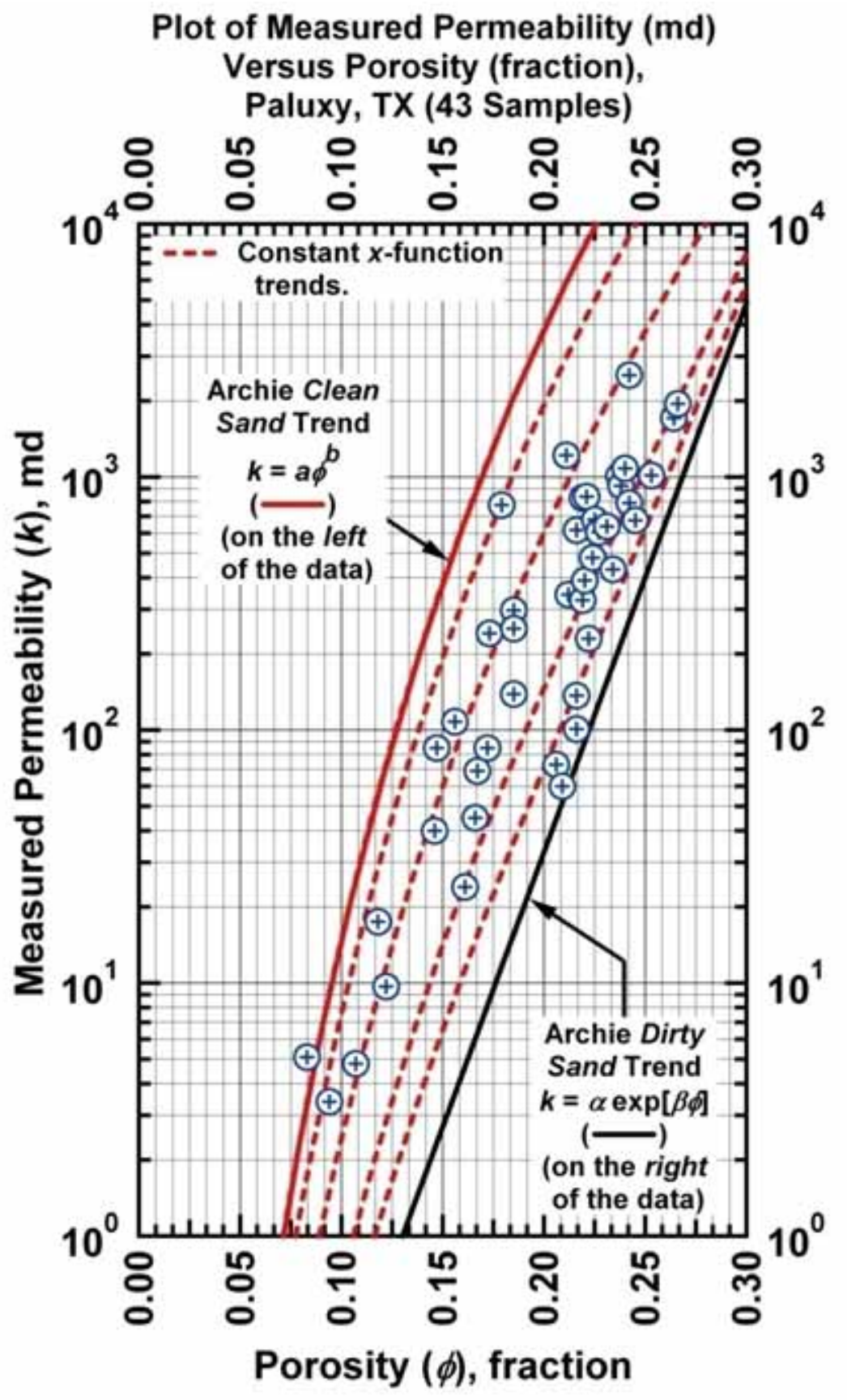

Figure J.7b - Model CPJ-D: $k=x a \phi^{b}+(1-x) \alpha \exp [\beta \phi] \quad[0 \leq x \leq 1]$ - Weighted Power Law-Exponential Model used to correlate permeability $(k)$ and porosity $(\phi)$. "Dirty Sand" Plot - Archie "Dirty Sand" trend is given by the straight-line trend at the far right of the data (exponential model). [Case: Paluxy, TX (USA)] 


\section{Comparison of Permeability (md) Calculated Using the "CPR-DxL" Model Versus the Measured Permeability (md), Paluxy, TX (43 Samples)}

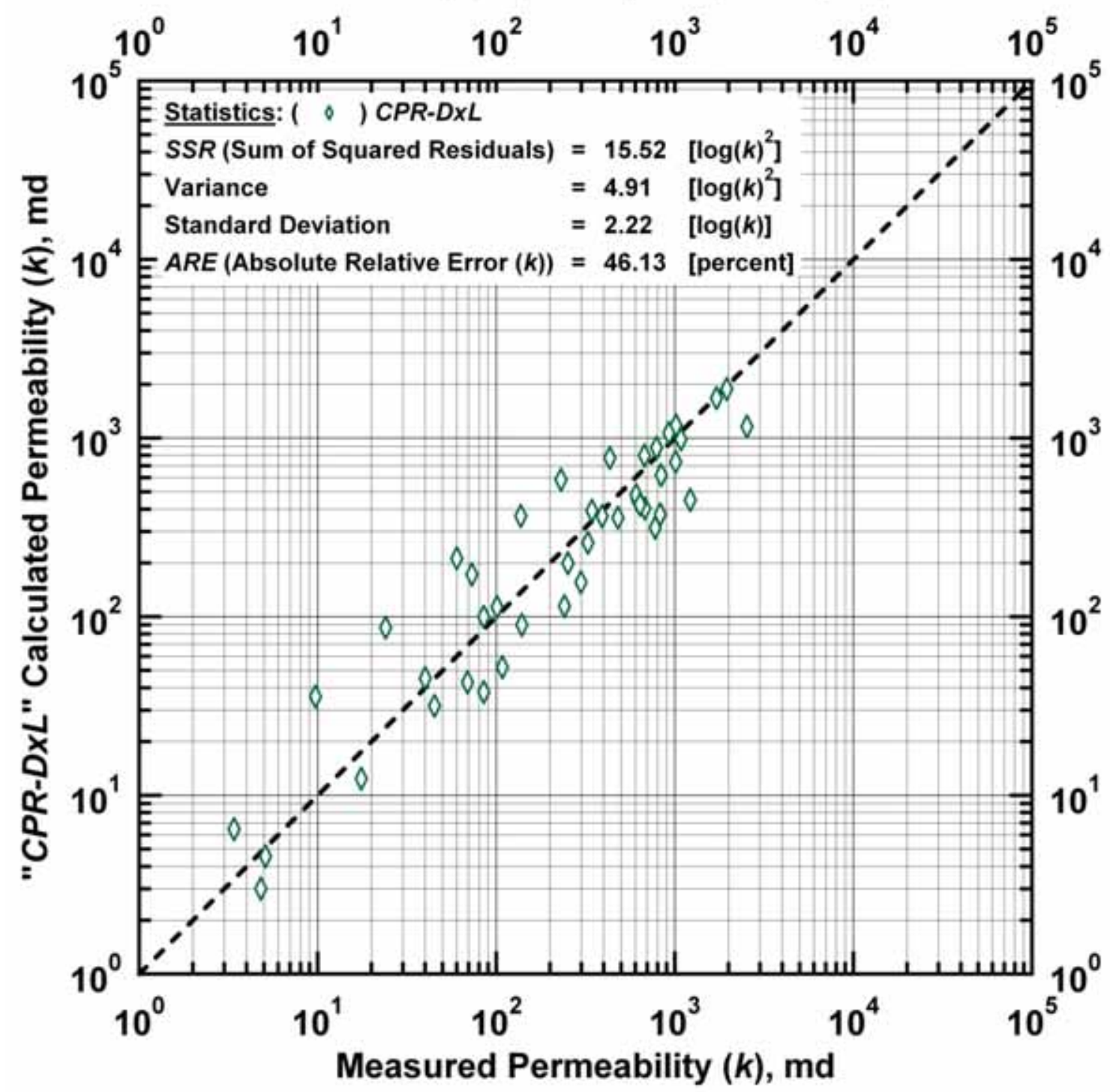

Figure J.8a - Model CPJ-DxL: Calculated versus measured permeability. [Case: Paluxy, TX (USA)] 


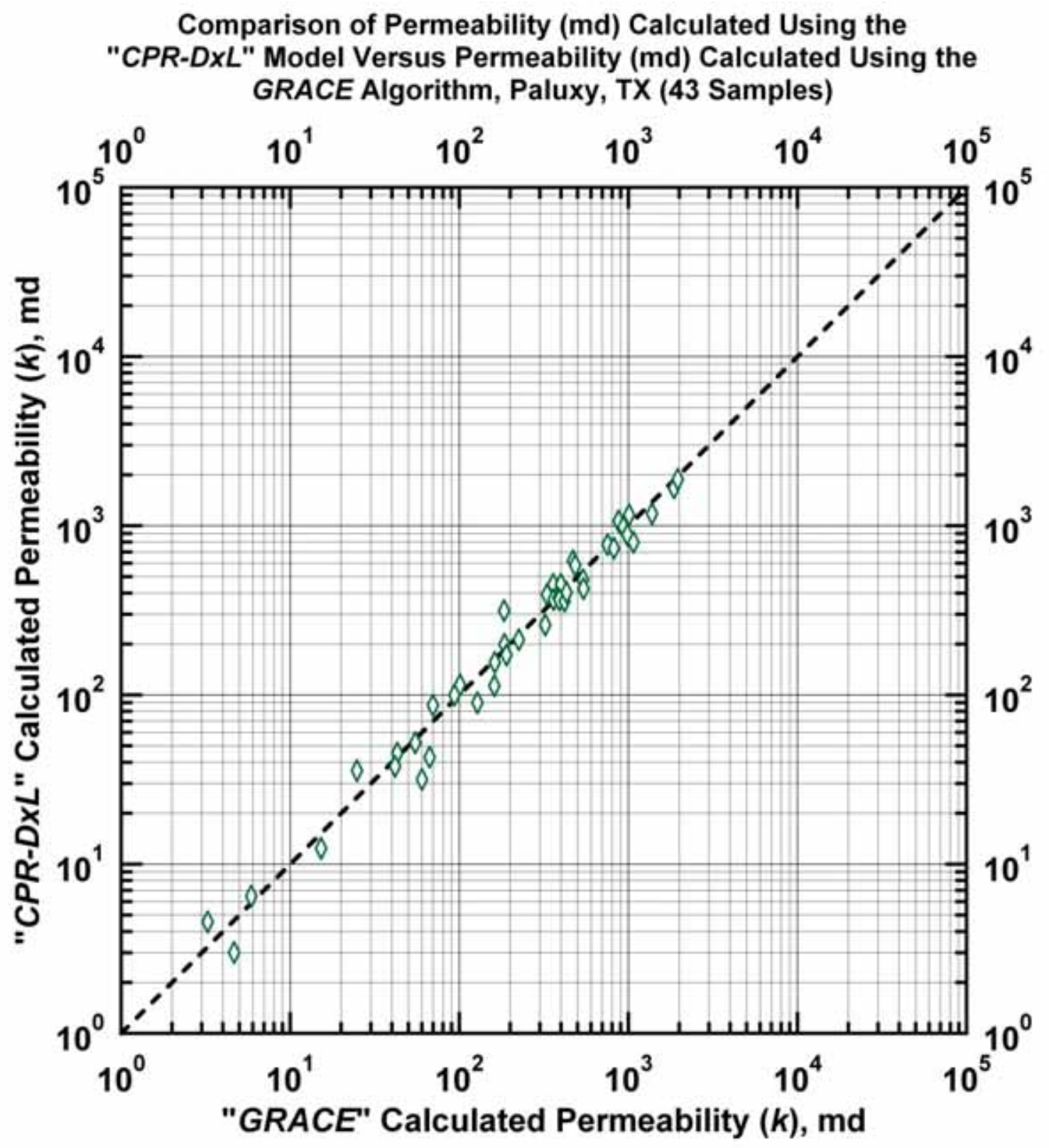

Figure J.8b - Model CPJ-DxL: Permeability calculated using Model $C P J-C$ versus permeability calculated using the GRACE Algorithm. [Case: Paluxy, TX (USA)] 


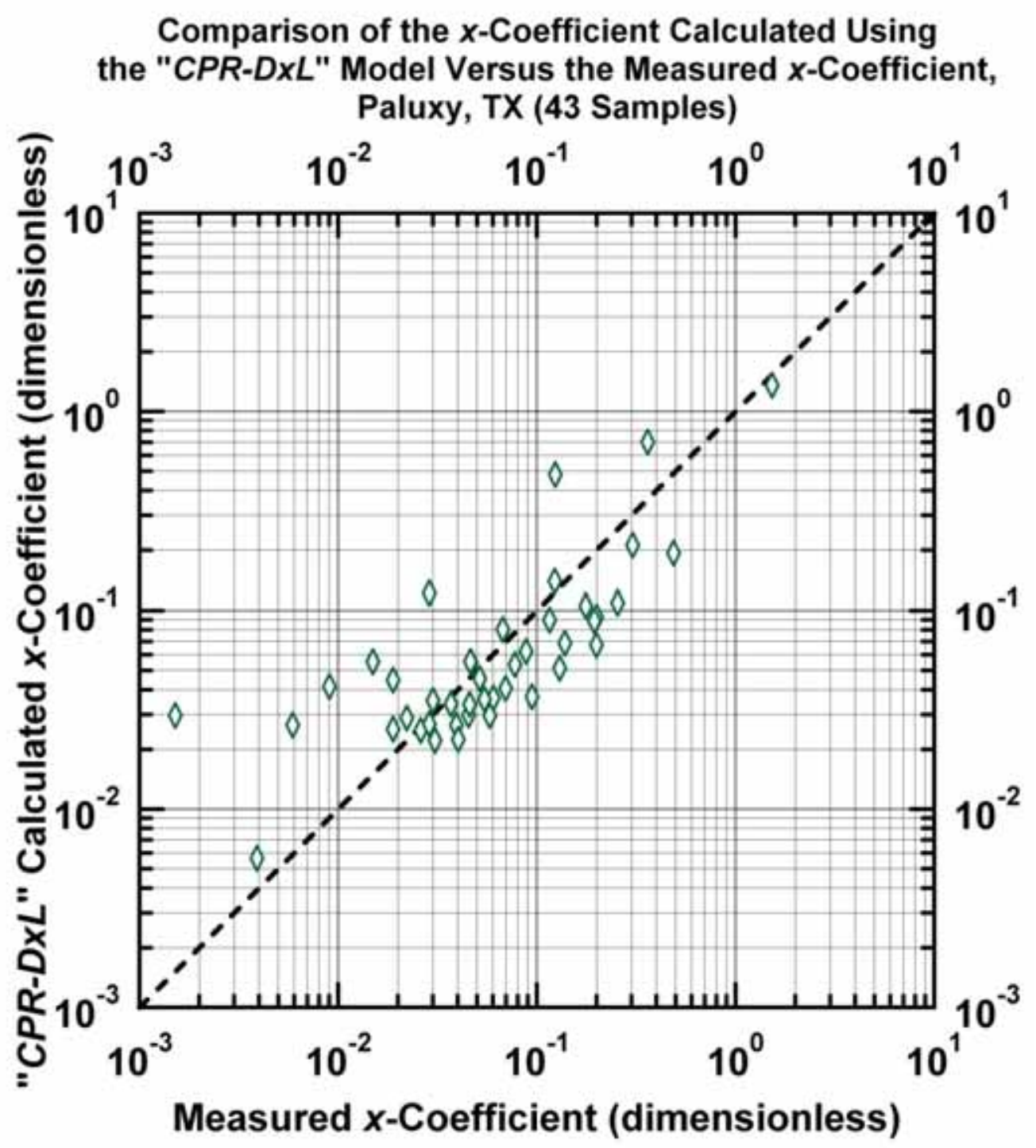

Figure J.8c - Model CPJ-DxL: Calculated $x$-function weight values versus measured $x$ function weight values. [Case: Paluxy, TX (USA)] 


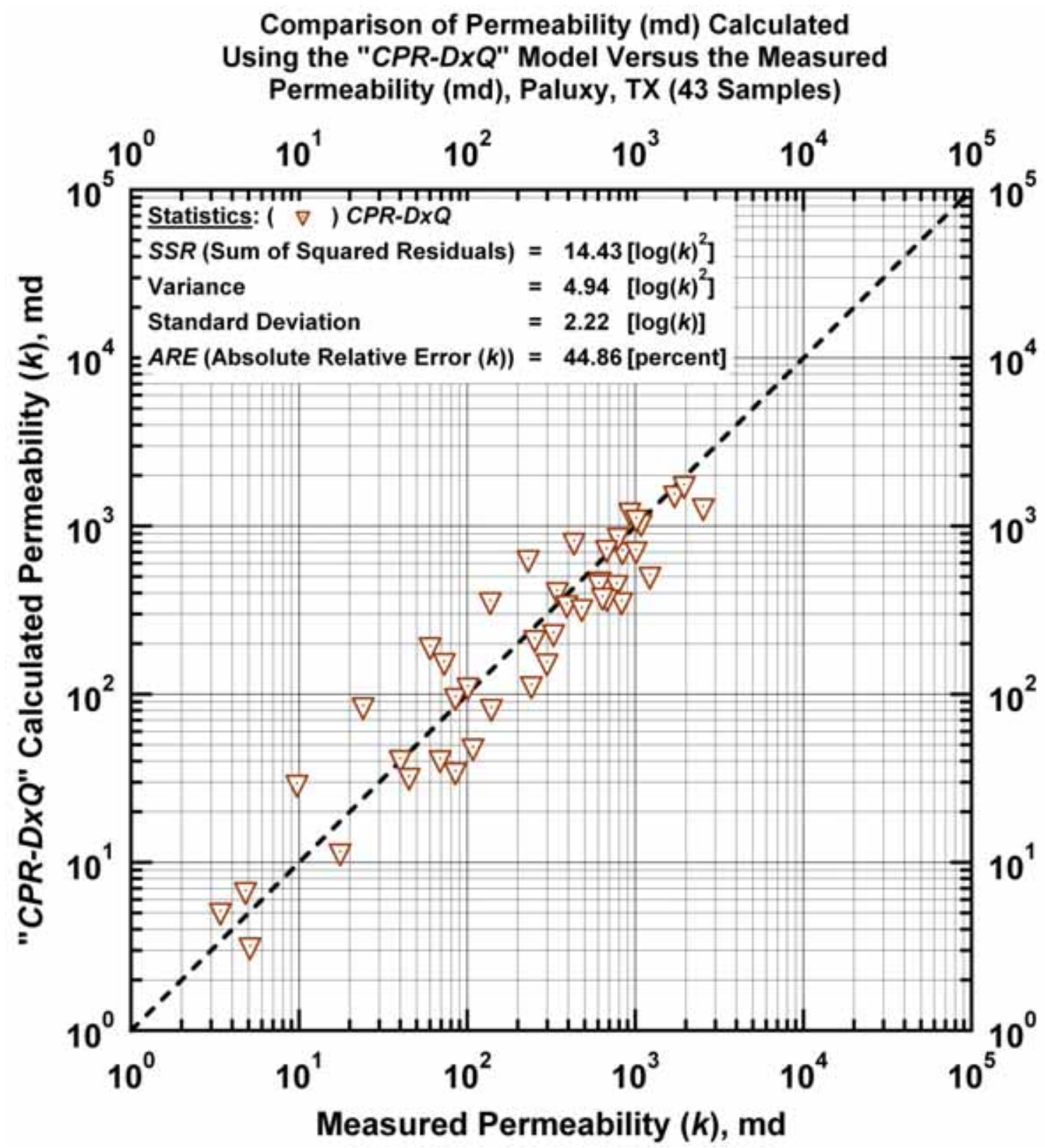

Figure J.9a - Model CPJ-DxQ: Calculated versus measured permeability. [Case: Paluxy, TX (USA)] 


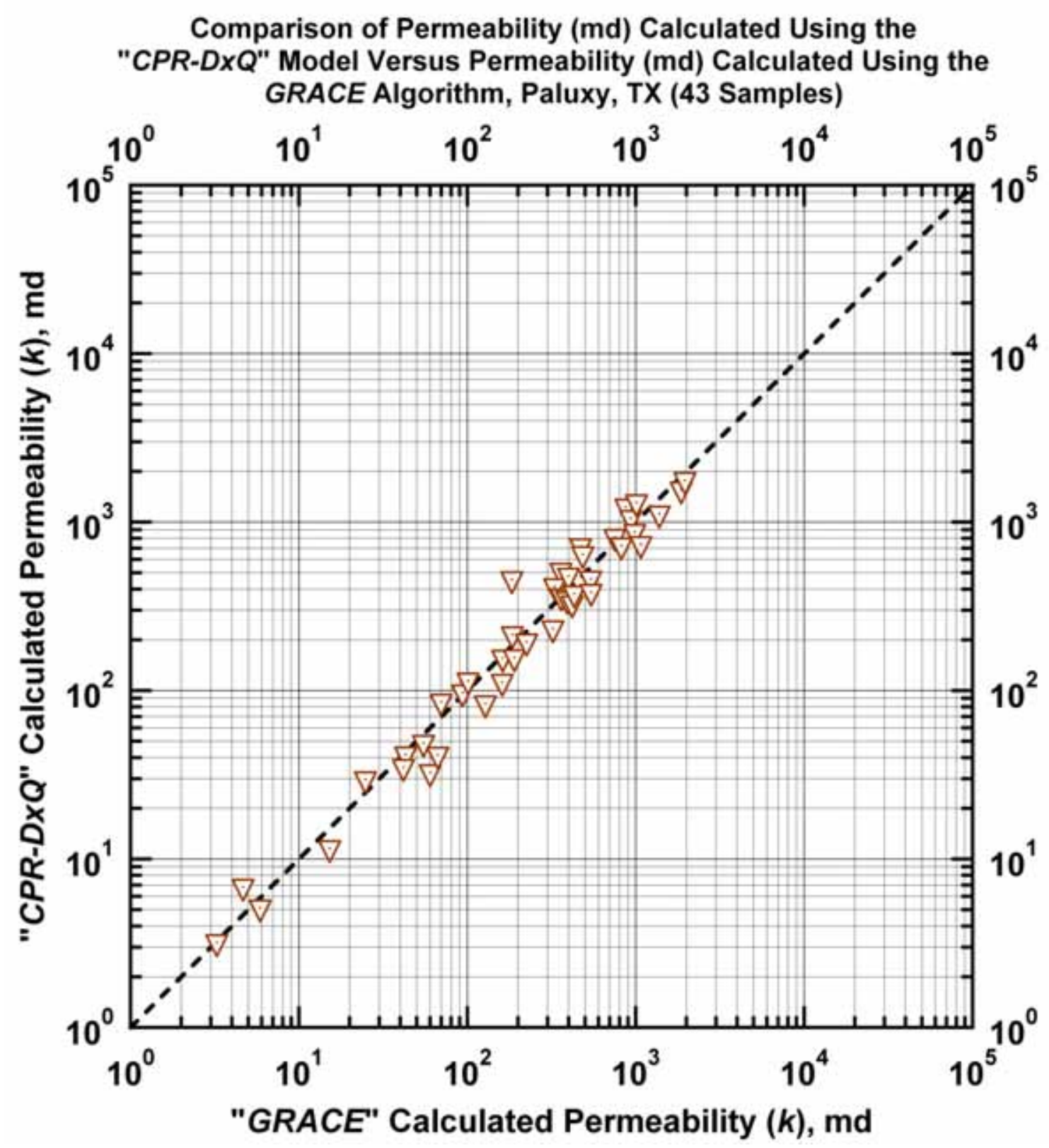

Figure J.9b - Model CPJ-DxQ: Permeability calculated using Model $C P J-C$ versus permeability calculated using the GRACE Algorithm. [Case: Paluxy, TX (USA)] 


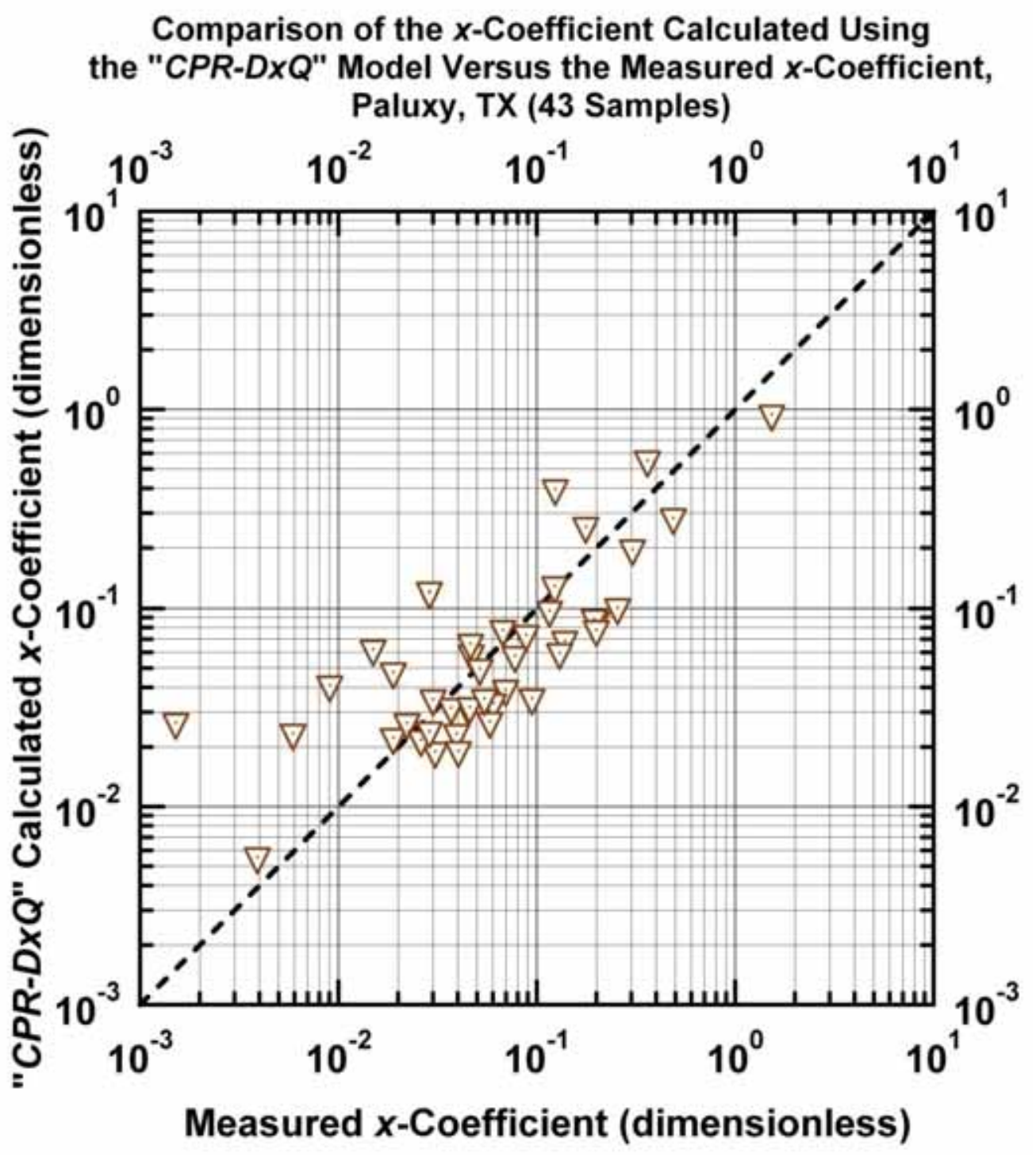

Figure J.9c - Model CPJ-DxQ: Calculated $x$-function weight values versus measured $x$ function weight values. [Case: Paluxy, TX (USA)] 
Comparison of Permeability (md) Calculated Using the "Modified Timur" Model Versus the Measured Permeability (md), Paluxy, TX (43 Samples)

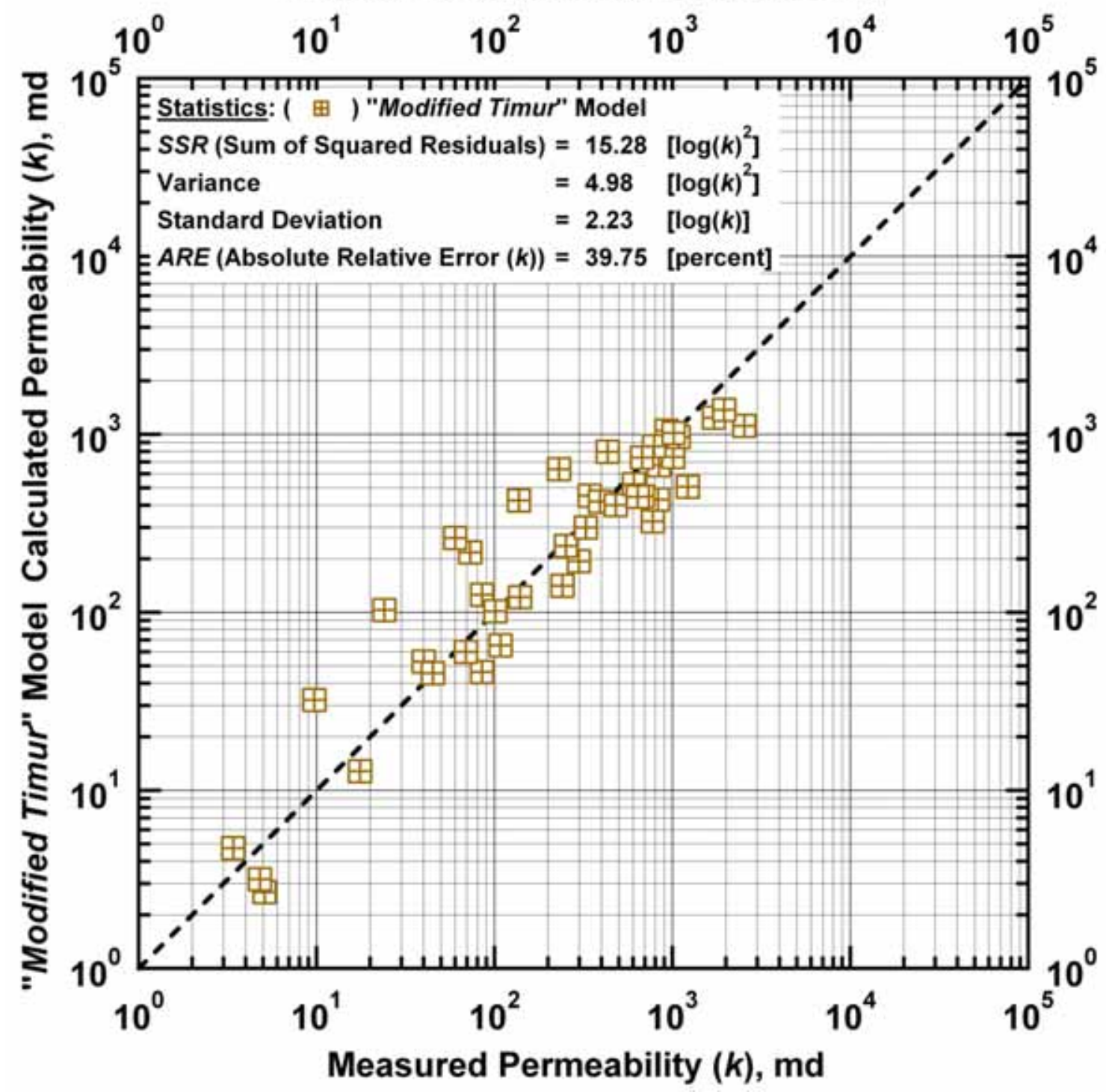

Figure J.10a — "Modified Timur Model:" Calculated versus measured permeability. [Case: Paluxy, TX (USA)] 


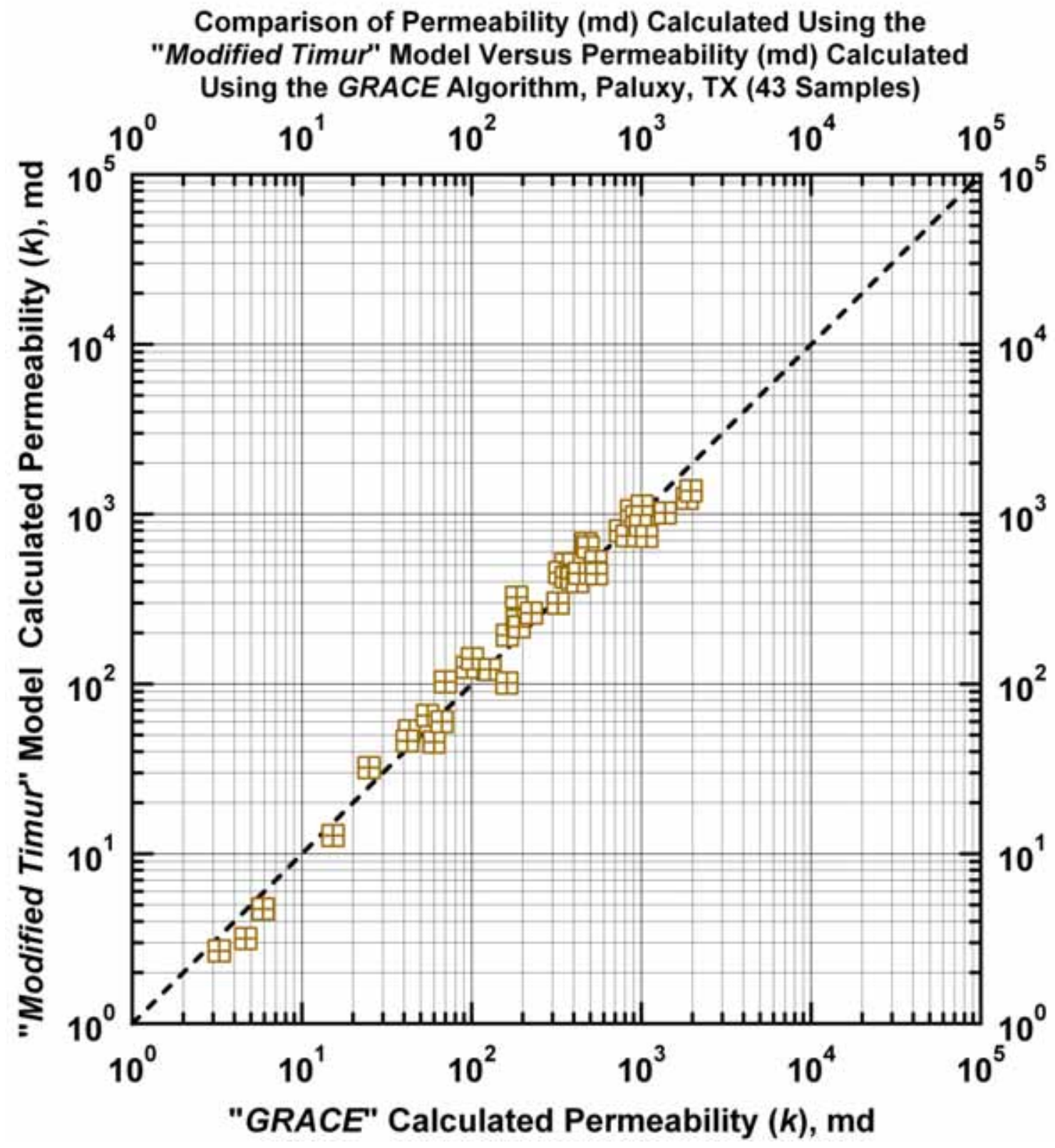

Figure J.10b — "Modified Timur Model:" Permeability calculated using the "Modified Timur Model" versus permeability calculated using the GRACE Algorithm. [Case: Paluxy, TX (USA)] 


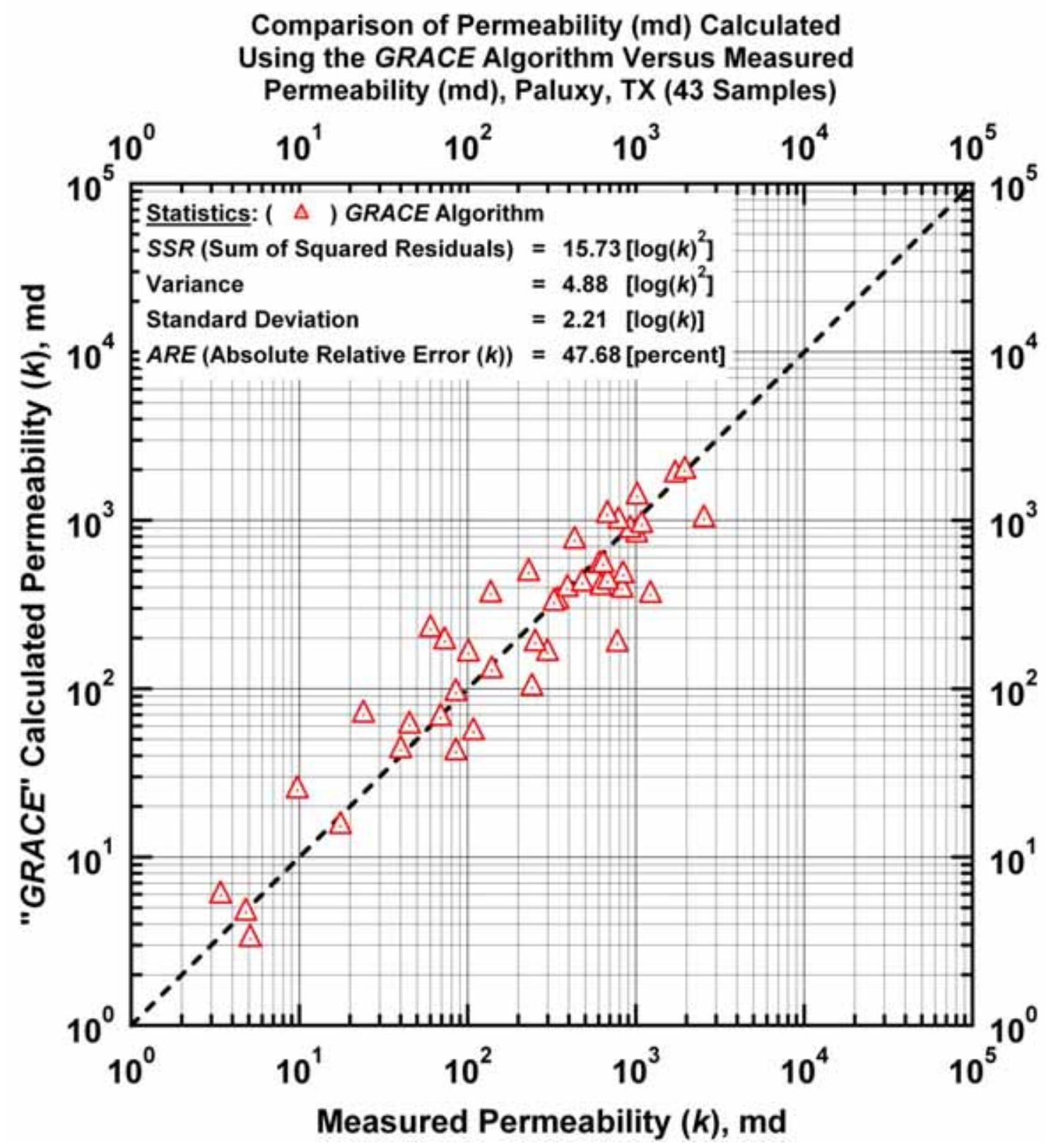

Figure J.11 - GRACE Model: Calculated versus measured permeability. [Case: Paluxy, TX (USA)] 


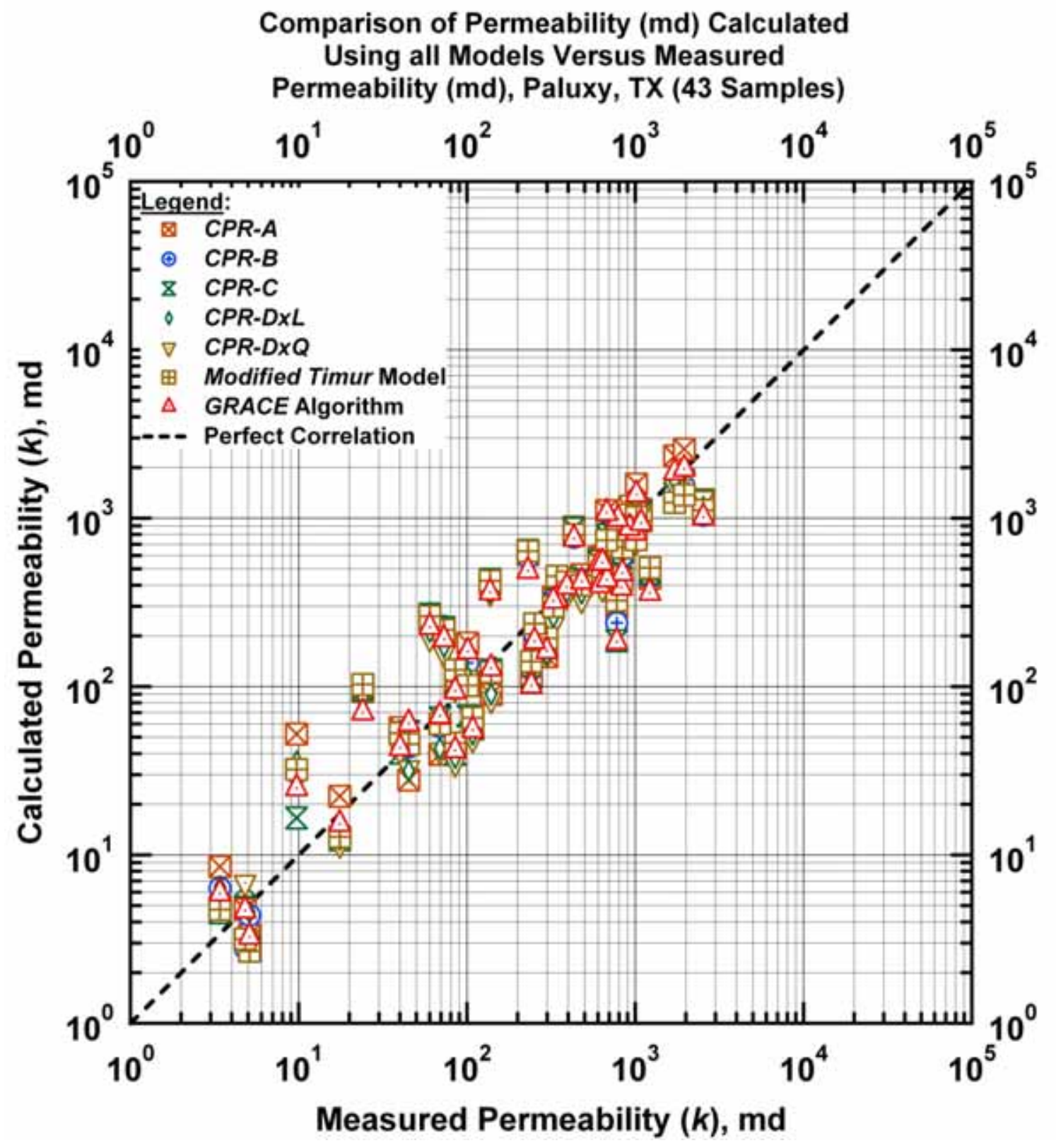

Figure J.12 - All Models: Calculated versus measured permeability. [Case: Paluxy, TX (USA)] 


\section{VITA}

Name:

Permanent Address:

E-mail Address:

Education:

Affiliations:
Adil Ahmed Siddiqui

A-301/C, Grey Garden,

Gulistan-e-Jauhar, Block-16

Karachi, 75290

PAKISTAN

sir_adilahmed@yahoo.com

Texas A\&M University, College Station, Texas, USA

Master of Science Degree in Petroleum Engineering

August 2008

IFP School, Rueil-Malmaison, France

Master of Science Degree in Reservoir Geoscience and Engineering

Expected September 2008

National University of Sciences \& Technology, Karachi, Pakistan

Bachelor of Engineering Degree in Mechanical Engineering

June 2005

Society of Petroleum Engineers 